\title{
REFINEMENT OF THE NEPHELINE DISCRIMINATOR: RESULTS OF A PHASE I STUDY
}

K. M. Fox

J. D. Newell

T.B. Edwards

D.R. Best

I.A. Reamer

R.J. Workman

November 2007

Process Science and Engineering

Savannah River National Laboratory

Aiken, SC 29808

Prepared for the U.S. Department of Energy Under

Contract Number DEAC09-96SR18500

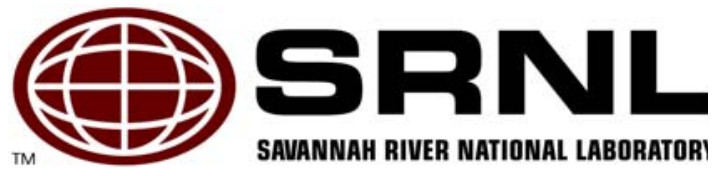


WSRC-STI-2007-00659

Revision 0

\section{DISCLAIMER}

This report was prepared by Washington Savannah River Company (WSRC) for the United States Department of Energy under Contract No. DE-AC09-96SR18500 and is an account of work performed under that contract. Neither the United States Department of Energy, nor WSRC, nor any of their employees makes any warranty, expressed or implied, or assumes any legal liability or responsibility for the accuracy, completeness, or usefulness, of any information, apparatus, or product or process disclosed herein or represents that its use will not infringe privately owned rights. Reference herein to any specific commercial product, process, or service by trademark, name, manufacturer or otherwise does not necessarily constitute or imply endorsement, recommendation, or favoring of same by WSRC or by the United States Government or any agency thereof. The views and opinions of the authors expressed herein do not necessarily state or reflect those of the United States Government or any agency thereof.

\section{Printed in the United States of America \\ Prepared For U.S. Department of Energy}

The Savannah River National Laboratory is operated for the U.S. Department of Energy by Washington Savannah River Company. 
WSRC-STI-2007-00659

Revision 0

Keywords: high level waste

glass, nepheline, durability

Retention: permanent

\section{REFINEMENT OF THE NEPHELINE DISCRIMINATOR: RESULTS OF A PHASE I STUDY}

K. M. Fox

J. D. Newell

T.B. Edwards

D.R. Best

I.A. Reamer

R.J. Workman

November 2007

Process Science and Engineering

Savannah River National Laboratory

Aiken, SC 29808

Prepared for the U.S. Department of Energy Under

Contract Number DEAC09-96SR18500

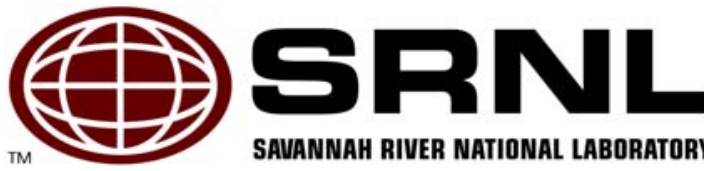


WSRC-STI-2007-00659

Revision 0

\section{REVIEWS AND APPROVALS}

\section{AUTHORS:}

K.M. Fox, Process Science and Engineering Section

Date

J.D. Newell, Process Science and Engineering Section

Date

T.B. Edwards, Statistical Consulting Section

Date

D.R. Best, Process Science and Engineering Section

Date

I.A. Reamer, Process Science and Engineering Section

Date

R.J. Workman, Process Science and Engineering Section

Date

\section{TECHNICAL REVIEWER:}

D.K. Peeler, Process Science and Engineering Section

Date

\section{APPROVERS:}

C.C. Herman, Manager, Process Engineering Technology Group

Date

R.E. Edwards, Manager, Process Science and Engineering Section

Date

J.E. Occhipinti, Manager, Waste Solidification Engineering

Date 
WSRC-STI-2007-00659

Revision 0

\section{EXECUTIVE SUMMARY}

The performance of a glass used for immobilization of high-level nuclear waste (HLW) is generally quantified by its resistance to chemical degradation, or durability. The durability of a HLW glass is dependent on its composition. If crystalline phases form within a glass during cooling, the composition of the residual glass network is altered, therefore affecting the durability of the glass. Crystallization of nepheline $\left(\mathrm{NaAlSiO}_{4}\right)$ has been shown to adversely impact the durability of HLW glasses since it removes glass forming species (in this case, $\mathrm{Al}$ and $\mathrm{Si}$ ) from the glass network. The propensity for nepheline crystallization in a HLW glass increases with increasing concentrations of $\mathrm{Al}_{2} \mathrm{O}_{3}$ and $\mathrm{Na}_{2} \mathrm{O}$ in the glass. Nepheline crystallization is therefore of concern for processing of HLW at the Defense Waste Processing Facility (DWPF) since the sludge waste streams at the Savannah River Site (SRS) can contain high concentrations of $\mathrm{Al}_{2} \mathrm{O}_{3}$ and $\mathrm{Na}_{2} \mathrm{O}$.

Currently, a 'nepheline discriminator' is included as a process control constraint at the DWPF. The nepheline discriminator relates the concentrations of $\mathrm{SiO}_{2}, \mathrm{Na}_{2} \mathrm{O}$ and $\mathrm{Al}_{2} \mathrm{O}_{3}$ (as weight percentages in glass) to a critical value of 0.62 . The discriminator defines a boundary line on the $\mathrm{SiO}_{2}-\mathrm{Na}_{2} \mathrm{O}-\mathrm{Al}_{2} \mathrm{O}_{3}$ ternary diagram above which (or toward the $\mathrm{SiO}_{2}$ corner of the ternary) nepheline is not predicted to crystallize in the glass upon quenching or slow cooling.

The current equation uses only the concentrations of the $\mathrm{SiO}_{2}, \mathrm{Na}_{2} \mathrm{O}$ and $\mathrm{Al}_{2} \mathrm{O}_{3}$ components in the glass in predicting whether or not nepheline is likely to crystallize. However, several other components have been shown to impact the propensity for nepheline crystallization, including $\mathrm{B}_{2} \mathrm{O}_{3}$ and $\mathrm{CaO}$ among others. Therefore, the potential exists to further refine the nepheline discriminator to include these components. In addition, recently studied HLW glasses with relatively high $\mathrm{Al}_{2} \mathrm{O}_{3}$ compositions of $25 \mathrm{wt} \%$ or greater and nepheline discriminator values well below 0.62 have been shown to be free of nepheline crystallization upon quenching and slow cooling. Thus, the current nepheline discriminator equation also appears to be conservative for some HLW glass compositions. Refining the nepheline discriminator to include other important components and to reduce conservatism may provide access to high $\mathrm{Al}_{2} \mathrm{O}_{3}$ concentration glass compositions for the DWPF, which could in turn allow access to higher waste loadings, decreased washing and improved waste throughput.

The objective of this study was to develop and characterize a series of HLW glass compositions based on a projected composition of Sludge Batch 5 (SB5), the next sludge batch to be processed in the DWPF. The selected glass compositions all had nepheline discriminator values below the current limit of 0.62 . They cover a range of locations on the $\mathrm{SiO}_{2}-\mathrm{Na}_{2} \mathrm{O}-\mathrm{Al}_{2} \mathrm{O}_{3}$ diagram. They also include varying amounts of $\mathrm{B}_{2} \mathrm{O}_{3}$ and $\mathrm{CaO}$ to support an evaluation of the impact of these components on the propensity for nepheline crystallization.

The results described in this report confirm that some conservatism exists in the current nepheline discriminator. Several glass compositions, particularly compositions that target higher $\mathrm{Al}_{2} \mathrm{O}_{3}$ concentrations, were shown to be very durable (i.e., PCT responses that were more than an order of magnitude better than that of the Environmental Assessment benchmark glass) while their nepheline discriminator values were well below the current nepheline discriminator limit of 0.62 . Increased concentrations of $\mathrm{B}_{2} \mathrm{O}_{3}$ and increased concentrations of $\mathrm{CaO}$ were shown to improve durability responses and suppress the formation of nepheline. This provides incentive to revise the nepheline discriminator to reduce some of this conservatism and incorporate the influence of $\mathrm{B}_{2} \mathrm{O}_{3}$. The revised nepheline discriminator could potentially change from a constant line on the $\mathrm{SiO}_{2}-\mathrm{Na}_{2} \mathrm{O}-\mathrm{Al}_{2} \mathrm{O}_{3}$ ternary diagram to a sloped line, where the slope varies with $\mathrm{B}_{2} \mathrm{O}_{3}$ 
WSRC-STI-2007-00659

Revision 0

concentration, thus allowing access to higher concentrations of $\mathrm{Al}_{2} \mathrm{O}_{3}$ in glass. Additional data will be required to support development and possible implementation of this revision. A Phase II study will be required to provide the necessary data, and recommendations for a Phase II study are included at the end of this report. 


\section{TABLE OF CONTENTS}

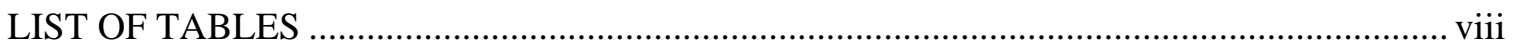

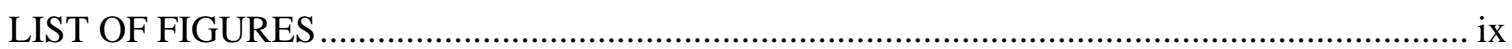

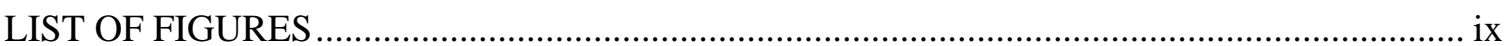

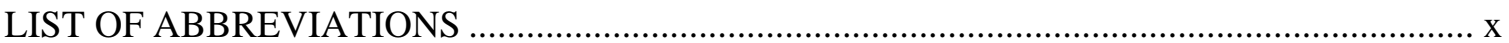

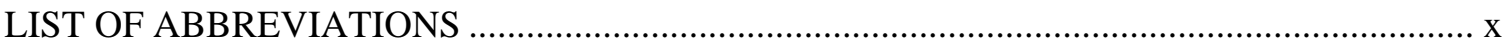

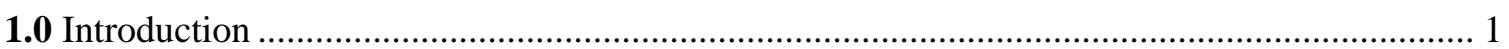

2.0 Experimental Procedure …............................................................................................. 3

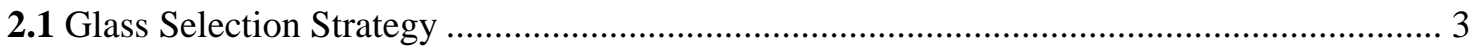

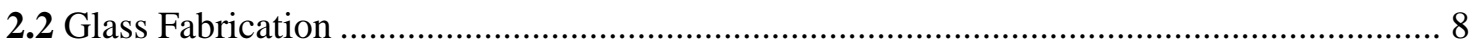

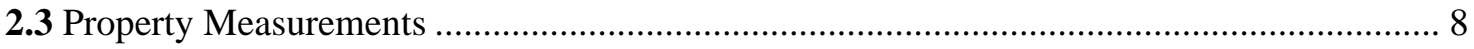

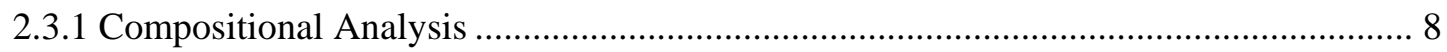

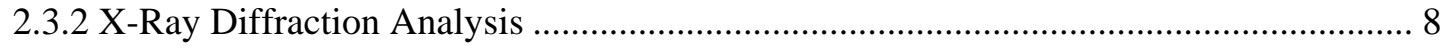

2.3.3 Product Consistency Test (PCT) ……........................................................................ 9

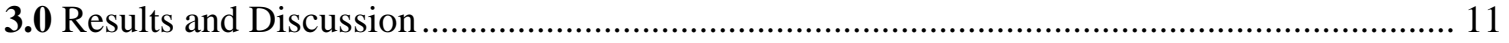

3.1 A Statistical Review of the Chemical Composition Measurements .................................... 11

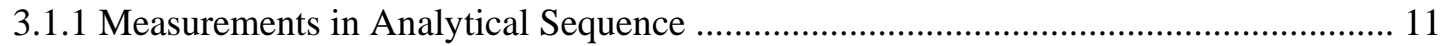

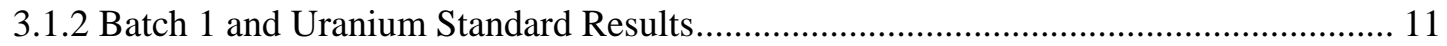

3.1.3 Composition Measurements by Glass Number .......................................................... 12

3.1.4 Measured versus Targeted Compositions ....................................................................... 13

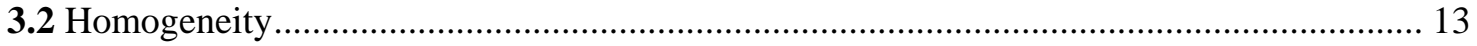

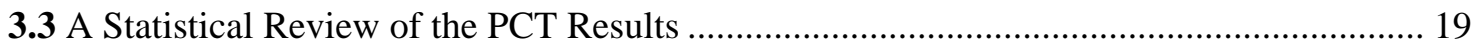

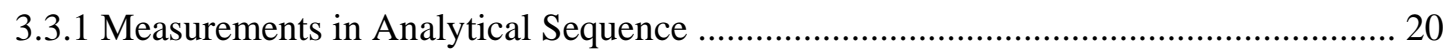

3.3.2 Results for the Samples of the Multi-Element Solution Standard................................. 20

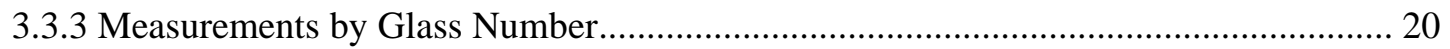

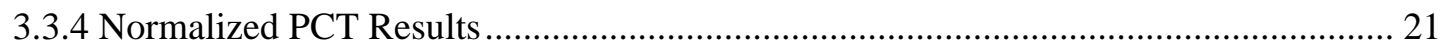

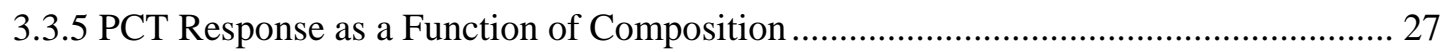

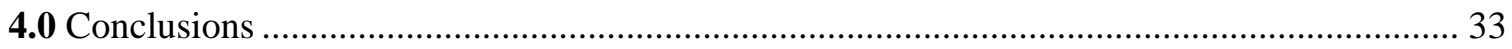

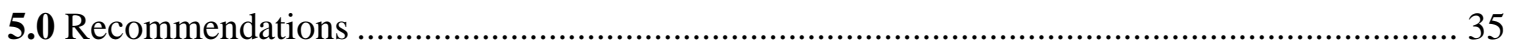

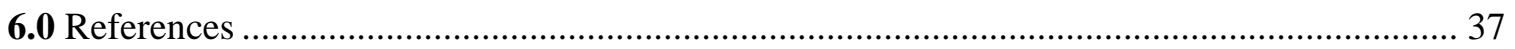

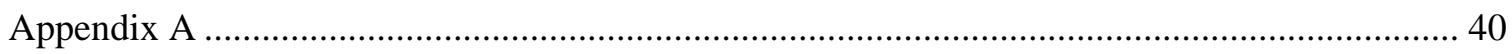

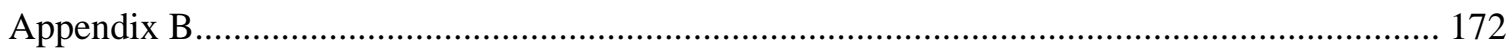




\section{LIST OF TABLES}

Table 2-1. Description of the ten composition points selected on the $\mathrm{SiO}_{2}-\mathrm{Na}_{2} \mathrm{O}-\mathrm{Al}_{2} \mathrm{O}_{3}$ ternary

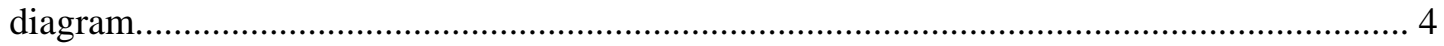

Table 2-2. Composition of the SB5 ‘Others' grouping. .............................................................. 5

Table 2-3. Glass compositions including the $\mathrm{B}_{2} \mathrm{O}_{3}$ and $\mathrm{CaO}$ components, and the Others

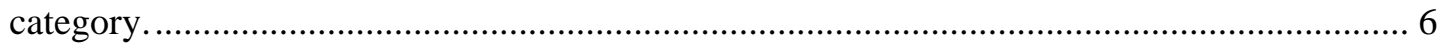

Table 2-4. Target compositions (wt \%) of the 40 nepheline study glasses. ................................... 7

Table 3-1. Summary of the visual observations and XRD results for the nepheline study glasses. 14

Table 3-2. Results from samples of the multi-element solution standard. .................................. 20

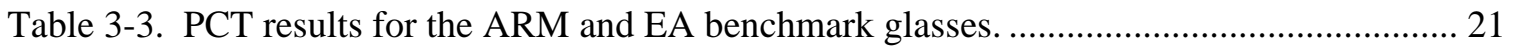

Table 3-4. Normalized PCT Results by Glass ID and Compositional View............................... 23 


\section{LIST OF FIGURES}

Figure 1-1. Ternary $\mathrm{SiO}_{2}-\mathrm{Na}_{2} \mathrm{O}-\mathrm{Al}_{2} \mathrm{O}_{3}$ diagram showing the location of the current nepheline discriminator. Glasses below the 0.62 line are considered prone to nepheline crystallization.

Figure 2-1. Location of the ten composition points selected on the $\mathrm{SiO}_{2}-\mathrm{Na}_{2} \mathrm{O}-\mathrm{Al}_{2} \mathrm{O}_{3}$ ternary diagram.

Figure 3-1. PCT responses (NL [B], in g/L) for the quenched versions of the study glasses as a function of location on the $\mathrm{SiO}_{2}-\mathrm{Na}_{2} \mathrm{O}-\mathrm{Al}_{2} \mathrm{O}_{3}$ ternary diagram and with varying concentrations of $\mathrm{B}_{2} \mathrm{O}_{3}$ and $\mathrm{CaO}$. Compositions where nepheline was detected by XRD are indicated in red.

Figure 3-2. PCT responses (NL [B], in g/L) for the CCC versions of the study glasses as a function of location on the $\mathrm{SiO}_{2}-\mathrm{Na}_{2} \mathrm{O}-\mathrm{Al}_{2} \mathrm{O}_{3}$ ternary diagram and with varying concentrations of $\mathrm{B}_{2} \mathrm{O}_{3}$ and $\mathrm{CaO}$. Compositions where nepheline was detected by XRD are indicated in red.

Figure 4-1. The current nepheline discriminator plotted on the $\mathrm{SiO}_{2}-\mathrm{Na}_{2} \mathrm{O}-\mathrm{Al}_{2} \mathrm{O}_{3}$ ternary diagram (a), and a potential example of the refined nepheline discriminator (b), where the slope of the line identifying the nepheline prone region increases with increasing $\mathrm{B}_{2} \mathrm{O}_{3}$ concentration... 33 


\section{LIST OF ABBREVIATIONS}

$\begin{array}{ll}\text { ANOVA } & \text { Analysis Of VAriance } \\ \text { ARM } & \text { Approved Reference Material } \\ \text { bc } & \text { bias-corrected } \\ \text { CCC } & \text { Canister Centerline Cooling } \\ \text { DWPF } & \text { Defense Waste Processing Facility } \\ \text { EA } & \text { Environmental Assessment } \\ \text { HLW } & \text { High Level Waste } \\ \text { ICP-AES } & \text { Inductively Coupled Plasma - Atomic Emission Spectroscopy } \\ \text { LM } & \text { Lithium Metaborate } \\ \text { LWO } & \text { Liquid Waste Organization } \\ \text { NL } & \text { Normalized Leachate } \\ \text { PCT } & \text { Product Consistency Test } \\ \text { PF } & \text { Peroxide Fusion } \\ \text { Ppm } & \text { parts per million } \\ \text { PSAL } & \text { Process Science Analytical Laboratory } \\ \text { SB5 } & \text { Sludge Batch 5 } \\ \text { SRS } & \text { Savannah River Site } \\ \text { XRD } & \text { X-ray Diffraction }\end{array}$


WSRC-STI-2007-00659

Revision 0

\subsection{Introduction}

The performance of a glass used for immobilization of high-level nuclear waste (HLW) is generally quantified by its resistance to chemical degradation, or durability. The durability of a HLW glass is dependent upon its composition. If crystalline phases form within a glass during cooling, the composition of the residual glass network is altered, therefore affecting the durability of the glass. Crystallization of nepheline $\left(\mathrm{NaAlSiO}_{4}\right)$ has been shown to adversely impact the durability of HLW glasses since it removes glass forming species (in this case, $\mathrm{Al}$ and $\mathrm{Si}$ ) from the glass network. ${ }^{1}$ The propensity for nepheline crystallization in a HLW glass increases with increasing concentrations of $\mathrm{Al}_{2} \mathrm{O}_{3}$ and $\mathrm{Na}_{2} \mathrm{O}$ in the glass. ${ }^{2}$ Nepheline crystallization is therefore of concern for processing of HLW at the Defense Waste Processing Facility (DWPF) since the sludge waste streams at the Savannah River Site (SRS) can contain high concentrations of $\mathrm{Al}_{2} \mathrm{O}_{3}$ and $\mathrm{Na}_{2} \mathrm{O}$.

Currently, a 'nepheline discriminator' is included as a process control constraint at the DWPF. ${ }^{3}$ This constraint uses the equation that was first proposed by $\mathrm{Li}^{2}$ and later verified as applicable to DWPF-type glasses. $^{4-8}$ The nepheline discriminator is given as Equation 1, and relates the concentrations of $\mathrm{SiO}_{2}, \mathrm{Na}_{2} \mathrm{O}$ and $\mathrm{Al}_{2} \mathrm{O}_{3}$ (as weight percentages in glass) to a critical value of 0.62 .

$$
\frac{\mathrm{SiO}_{2}}{\mathrm{SiO}_{2}+\mathrm{Na}_{2} \mathrm{O}+\mathrm{Al}_{2} \mathrm{O}_{3}}>0.62
$$

This equation defines a boundary line on the $\mathrm{SiO}_{2}-\mathrm{Na}_{2} \mathrm{O}-\mathrm{Al}_{2} \mathrm{O}_{3}$ ternary diagram above which (or toward the $\mathrm{SiO}_{2}$ corner of the ternary, see Figure 1-1) nepheline is not predicted to crystallize in the glass upon quenching or slow cooling.

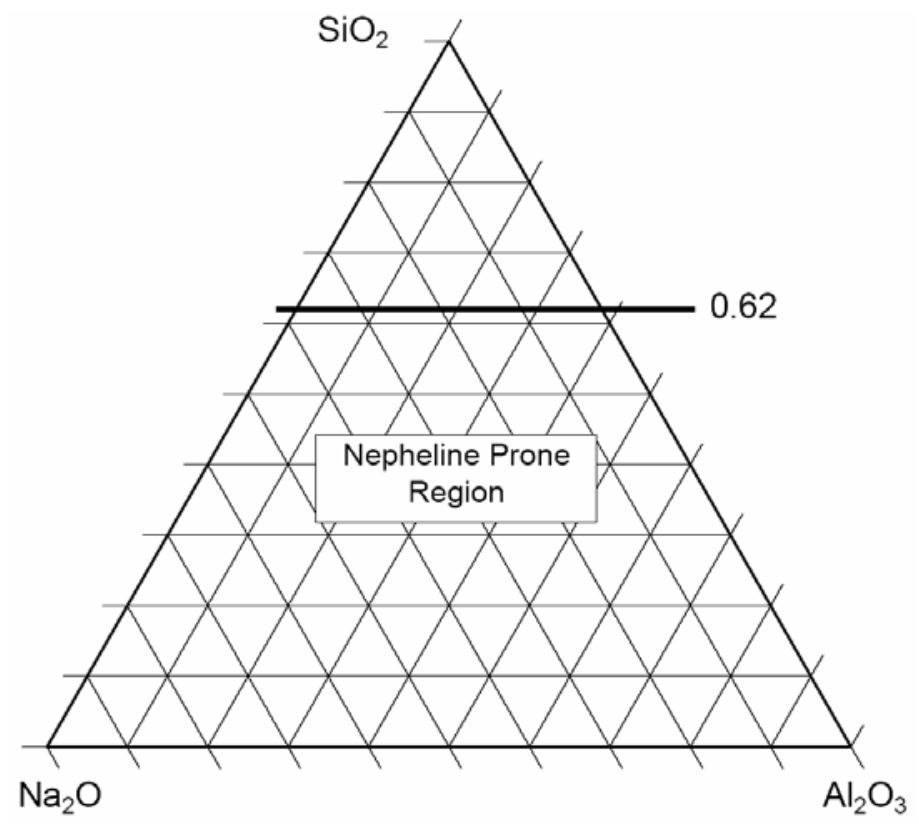

Figure 1-1. Ternary $\mathrm{SiO}_{2}-\mathrm{Na}_{2} \mathrm{O}-\mathrm{Al}_{2} \mathrm{O}_{3}$ diagram showing the location of the current nepheline discriminator. Glasses below the 0.62 line are considered prone to nepheline crystallization. 
The current equation uses only the concentrations of the $\mathrm{SiO}_{2}, \mathrm{Na}_{2} \mathrm{O}$ and $\mathrm{Al}_{2} \mathrm{O}_{3}$ components in the glass in predicting whether or not nepheline is likely to crystallize. However, several other components have been shown to impact the propensity for nepheline crystallization, including $\mathrm{B}_{2} \mathrm{O}_{3}$ and $\mathrm{CaO}$ (potential DWPF frit components), among others. ${ }^{1,2,5,9,10}$ Therefore, the potential exists to further refine the nepheline discriminator to include these components. In addition, recently studied HLW glasses with relatively high $\mathrm{Al}_{2} \mathrm{O}_{3}$ compositions of $25 \mathrm{wt} \%$ or greater and nepheline discriminator values well below 0.62 have been shown to be free of nepheline crystallization upon quenching and slow cooling. ${ }^{11}$ Thus, the current nepheline discriminator equation also appears to be conservative for some HLW glass compositions. Refining the nepheline discriminator to include other important components and to reduce conservatism may provide access to high $\mathrm{Al}_{2} \mathrm{O}_{3}$ concentration glass compositions for the DWPF, which could in turn allow access to higher waste loadings, decreased washing and improved waste throughput.

The objective of this study was to develop and characterize a series of HLW glass compositions based on a projected composition of Sludge Batch 5 (SB5), the next sludge batch to be processed in the DWPF. The selected glass compositions all had nepheline discriminator values below the current limit of 0.62. They cover a range of locations on the $\mathrm{SiO}_{2}-\mathrm{Na}_{2} \mathrm{O}-\mathrm{Al}_{2} \mathrm{O}_{3}$ diagram. They also include varying amounts of $\mathrm{B}_{2} \mathrm{O}_{3}$ and $\mathrm{CaO}$ to support an evaluation of the impact of these components on the propensity for nepheline crystallization. A more thorough discussion of the selection process and fabrication of these glasses will be given in Section 2.0. Section 3.0 describes the measured properties (chemical composition and crystalline phase content) and durability of each glass. The Product Consistency Test (PCT) was used to evaluate the durability of each glass, after both quenching and slow cooling. X-ray diffraction was used to identify any crystalline phases within the glasses. Section 0 provides an evaluation of these data, as well as a discussion of how the results may be used to guide refinement of the nepheline discriminator. Section 5.0 offers recommendations for the next phase of this study, which will provide the additional experimental data necessary to recommend implementation of a revised nepheline discriminator in the DWPF process control system.

This study is performed in response to Technical Task Request HLW-DWPF-TTR-2007-0007, ${ }^{12}$ under Task Technical and Quality Assurance Plan WSRC-STI-2006-00321. ${ }^{13}$ 
WSRC-STI-2007-00659

Revision 0

\subsection{Experimental Procedure}

\subsection{Glass Selection Strategy}

Glass compositions for this study were selected to have nepheline discriminator values that were intentionally below the current limit of 0.62. Ten points were selected on the $\mathrm{SiO}_{2}-\mathrm{Na}_{2} \mathrm{O}-\mathrm{Al}_{2} \mathrm{O}_{3}$ ternary diagram in order to cover a broad range of ratios of these components. The location of these ten points is shown graphically in Figure 2-1.

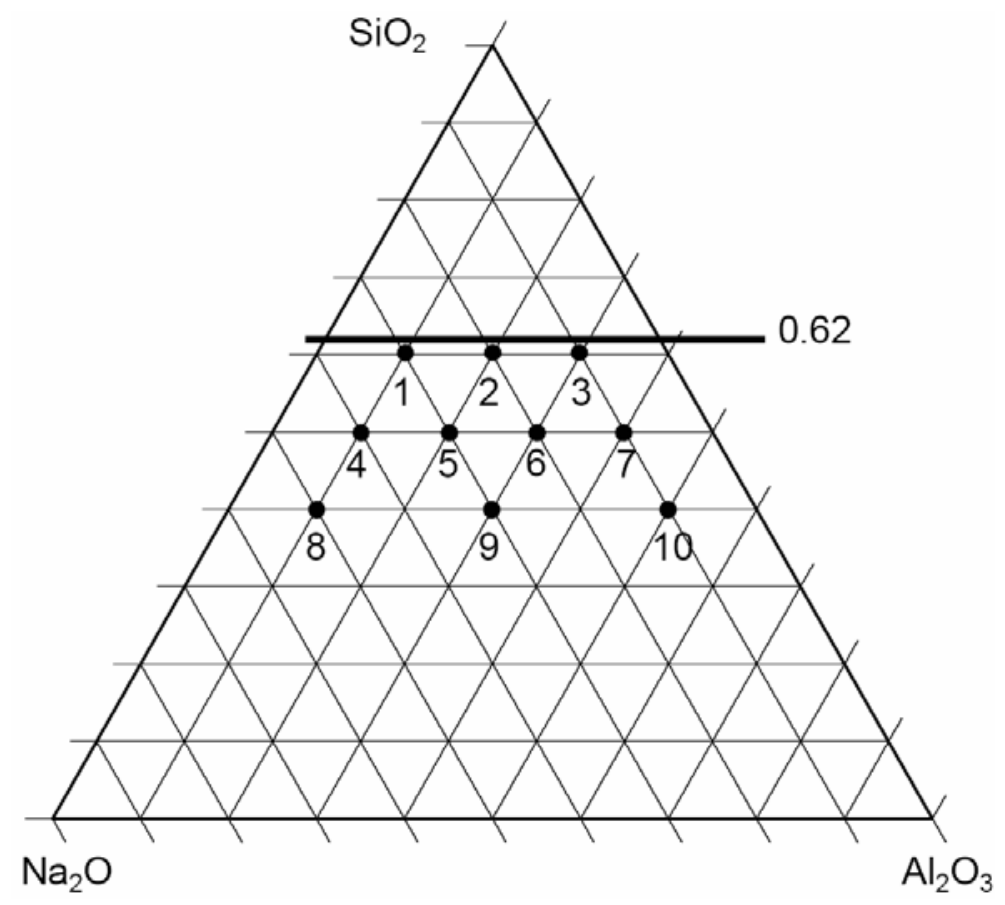

Figure 2-1. Location of the ten composition points selected on the $\mathrm{SiO}_{2}-\mathrm{Na}_{2} \mathrm{O}-\mathrm{Al}_{2} \mathrm{O}_{3}$ ternary diagram.

The nepheline discriminator values and the ratios of $\mathrm{SiO}_{2}$ to $\mathrm{Na}_{2} \mathrm{O}$ to $\mathrm{Al}_{2} \mathrm{O}_{3}$ for each of these points are given in Table 2-1. 
WSRC-STI-2007-00659

Revision 0

Table 2-1. Description of the ten composition points selected on the $\mathrm{SiO}_{2}-\mathrm{Na}_{2} \mathrm{O}-\mathrm{Al}_{2} \mathrm{O}_{3}$ ternary diagram.

\begin{tabular}{|c|c|c|c|c|}
\hline $\begin{array}{c}\text { Point on } \\
\text { Ternary }\end{array}$ & $\begin{array}{c}\text { Nepheline } \\
\text { Discriminator Value }\end{array}$ & $\begin{array}{c}\mathbf{N a}_{\mathbf{2}} \mathbf{O} \\
\text { (wt \%) }\end{array}$ & $\begin{array}{c}\mathbf{A l}_{\mathbf{2}} \mathbf{O}_{\mathbf{3}} \\
\mathbf{( w t} \text { \%) }\end{array}$ & $\begin{array}{c}\mathrm{SiO}_{\mathbf{2}} \\
\text { (wt \%) }\end{array}$ \\
\hline 1 & 0.60 & 30 & 10 & 60 \\
\hline 2 & 0.60 & 20 & 20 & 60 \\
\hline 3 & 0.60 & 10 & 30 & 60 \\
\hline 4 & 0.50 & 40 & 10 & 50 \\
\hline 5 & 0.50 & 30 & 20 & 50 \\
\hline 6 & 0.50 & 20 & 30 & 50 \\
\hline 7 & 0.50 & 10 & 40 & 50 \\
\hline 8 & 0.40 & 50 & 10 & 40 \\
\hline 9 & 0.40 & 10 & 50 & 40 \\
\hline 10 & 0.40 & &
\end{tabular}

The effects of $\mathrm{B}_{2} \mathrm{O}_{3}$ and $\mathrm{CaO}$ on the propensity for nepheline crystallization are of primary interest to this study. These components were combined with the $\mathrm{SiO}_{2}$ to $\mathrm{Na}_{2} \mathrm{O}$ to $\mathrm{Al}_{2} \mathrm{O}_{3}$ ratios described in Table 2-1 at concentrations of 4 and $18 \mathrm{wt} \%$ (for $\mathrm{B}_{2} \mathrm{O}_{3}$ ) and 2 and $12 \mathrm{wt} \%$ (for $\mathrm{CaO}$ ). It was also desirable that the glasses studied be similar in composition to SB5, the next HLW batch scheduled for processing in the DWPF. A projected SB5 composition provided by the Liquid Waste Organization (LWO) ${ }^{14}$ was used to develop a set of components, labeled 'Others,' to be included in the glasses. ${ }^{\text {a }}$ The projection was modified by first converting all the elemental concentrations to calcined oxides. Next the values for $\mathrm{ThO}_{2}$ and $\mathrm{U}_{3} \mathrm{O}_{8}$ were removed, since the study glasses will be non-radioactive simulants. The values for $\mathrm{Al}_{2} \mathrm{O}_{3}, \mathrm{CaO}, \mathrm{Na}_{2} \mathrm{O}$ and $\mathrm{SiO}_{2}$ were then removed, since these components are already included as part of the experimental design. The projected composition was then renormalized without these components to produce the 'Others' composition, which is given in Table 2-2.

\section{0}

${ }^{a}$ Note that this projection was issued in February 2007 and did not include the possible impacts of aluminum dissolution on SB5. 
WSRC-STI-2007-00659

Revision 0

Table 2-2. Composition of the SB5 'Others’ grouping.

\begin{tabular}{|c|c|c|}
\hline $\begin{array}{c}\text { Sludge } \\
\text { Component }\end{array}$ & $\begin{array}{c}\text { Projected SB5 Blend } \\
\text { as Calcined Oxides } \\
\text { (wt \%) }\end{array}$ & $\begin{array}{c}\text { 'Others' Composition } \\
\text { (SB5 Blend without } \mathrm{Al}_{2} \mathrm{O}_{3}, \mathrm{CaO} \text {, } \\
\mathrm{Na}_{2} \mathrm{O}, \mathrm{SiO}_{2}, \mathrm{ThO}_{2} \text { and } \mathrm{U}_{3} \mathrm{O}_{8} \text { ) } \\
\text { (wt \%) }\end{array}$ \\
\hline $\mathrm{Al}_{2} \mathrm{O}_{3}$ & 30.58 & - \\
\hline $\mathrm{BaO}$ & 0.11 & 0.306 \\
\hline $\mathrm{CaO}$ & 2.09 & - \\
\hline $\mathrm{Ce}_{2} \mathrm{O}_{3}$ & 0.23 & 0.642 \\
\hline $\mathrm{Cr}_{2} \mathrm{O}_{3}$ & 0.20 & 0.561 \\
\hline $\mathrm{CuO}$ & 0.07 & 0.206 \\
\hline $\mathrm{Fe}_{2} \mathrm{O}_{3}$ & 24.30 & 67.325 \\
\hline $\mathrm{K}_{2} \mathrm{O}$ & 0.16 & 0.429 \\
\hline $\mathrm{La}_{2} \mathrm{O}_{3}$ & 0.03 & 0.096 \\
\hline $\mathrm{MgO}$ & 1.41 & 3.911 \\
\hline $\mathrm{MnO}$ & 5.20 & 14.413 \\
\hline $\mathrm{Na}_{2} \mathrm{O}$ & 22.65 & - \\
\hline $\mathrm{NiO}$ & 2.31 & 6.387 \\
\hline $\mathrm{PbO}$ & 0.10 & 0.266 \\
\hline $\mathrm{SO}_{4}{ }^{2-}$ & 1.16 & 3.203 \\
\hline $\mathrm{SiO}_{2}$ & 1.82 & - \\
\hline $\mathrm{ThO}_{2}$ & 0.01 & - \\
\hline $\mathrm{TiO}_{2}$ & 0.51 & 1.418 \\
\hline $\mathrm{U}_{3} \mathrm{O}_{8}$ & 6.75 & - \\
\hline $\mathrm{ZnO}$ & 0.07 & 0.205 \\
\hline $\mathrm{ZrO}_{2}$ & 0.23 & 0.630 \\
\hline
\end{tabular}

The $\mathrm{SiO}_{2}$ to $\mathrm{Na}_{2} \mathrm{O}$ to $\mathrm{Al}_{2} \mathrm{O}_{3}$ ratios were combined with the $\mathrm{B}_{2} \mathrm{O}_{3}$ and $\mathrm{CaO}$ additions, plus the 'Others' components at a fixed concentration of $16 \mathrm{wt} \%$ to develop 40 glass compositions. These combinations are given in Table 2-3. The 'Others' category was then expanded to provide the complete glass compositions. The target compositions for the 40 study glasses are listed in Table 2-4. 
Table 2-3. Glass compositions including the $\mathrm{B}_{2} \mathrm{O}_{3}$ and $\mathrm{CaO}$ components, and the Others category.

\begin{tabular}{|c|c|c|c|c|c|c|c|}
\hline $\begin{array}{l}\text { Point on } \\
\text { Ternary }\end{array}$ & $\begin{array}{c}\mathrm{B}_{2} \mathrm{O}_{3} \\
\text { (wt \%) }\end{array}$ & $\begin{array}{c}\mathrm{CaO} \\
(\text { wt \%) }\end{array}$ & $\begin{array}{l}\mathrm{Na}_{2} \mathrm{O} \\
(w t \%)\end{array}$ & $\begin{array}{c}\mathrm{Al}_{2} \mathrm{O}_{3} \\
(\mathrm{wt} \%)\end{array}$ & $\begin{array}{c}\mathrm{SiO}_{2} \\
\text { (wt \%) }\end{array}$ & $\begin{array}{l}\text { Others } \\
\text { (wt \%) }\end{array}$ & $\begin{array}{c}\text { Sum } \\
\text { (wt \%) }\end{array}$ \\
\hline 1 & 4 & 2 & 23.4 & 7.8 & 46.8 & 16 & 100 \\
\hline 1 & 18 & 2 & 19.2 & 6.4 & 38.4 & 16 & 100 \\
\hline 1 & 4 & 12 & 20.4 & 6.8 & 40.8 & 16 & 100 \\
\hline 1 & 18 & 12 & 16.2 & 5.4 & 32.4 & 16 & 100 \\
\hline 2 & 4 & 2 & 15.6 & 15.6 & 46.8 & 16 & 100 \\
\hline 2 & 18 & 2 & 12.8 & 12.8 & 38.4 & 16 & 100 \\
\hline 2 & 4 & 12 & 13.6 & 13.6 & 40.8 & 16 & 100 \\
\hline 2 & 18 & 12 & 10.8 & 10.8 & 32.4 & 16 & 100 \\
\hline 3 & 4 & 2 & 7.8 & 23.4 & 46.8 & 16 & 100 \\
\hline 3 & 18 & 2 & 6.4 & 19.2 & 38.4 & 16 & 100 \\
\hline 3 & 4 & 12 & 6.8 & 20.4 & 40.8 & 16 & 100 \\
\hline 3 & 18 & 12 & 5.4 & 16.2 & 32.4 & 16 & 100 \\
\hline 4 & 4 & 2 & 31.2 & 7.8 & 39 & 16 & 100 \\
\hline 4 & 18 & 2 & 25.6 & 6.4 & 32 & 16 & 100 \\
\hline 4 & 4 & 12 & 27.2 & 6.8 & 34 & 16 & 100 \\
\hline 4 & 18 & 12 & 21.6 & 5.4 & 27 & 16 & 100 \\
\hline 5 & 4 & 2 & 23.4 & 15.6 & 39 & 16 & 100 \\
\hline 5 & 18 & 2 & 19.2 & 12.8 & 32 & 16 & 100 \\
\hline 5 & 4 & 12 & 20.4 & 13.6 & 34 & 16 & 100 \\
\hline 5 & 18 & 12 & 16.2 & 10.8 & 27 & 16 & 100 \\
\hline 6 & 4 & 2 & 15.6 & 23.4 & 39 & 16 & 100 \\
\hline 6 & 18 & 2 & 12.8 & 19.2 & 32 & 16 & 100 \\
\hline 6 & 4 & 12 & 13.6 & 20.4 & 34 & 16 & 100 \\
\hline 6 & 18 & 12 & 10.8 & 16.2 & 27 & 16 & 100 \\
\hline 7 & 4 & 2 & 7.8 & 31.2 & 39 & 16 & 100 \\
\hline 7 & 18 & 2 & 6.4 & 25.6 & 32 & 16 & 100 \\
\hline 7 & 4 & 12 & 6.8 & 27.2 & 34 & 16 & 100 \\
\hline 7 & 18 & 12 & 5.4 & 21.6 & 27 & 16 & 100 \\
\hline 8 & 4 & 2 & 39 & 7.8 & 31.2 & 16 & 100 \\
\hline 8 & 18 & 2 & 32 & 6.4 & 25.6 & 16 & 100 \\
\hline 8 & 4 & 12 & 34 & 6.8 & 27.2 & 16 & 100 \\
\hline 8 & 18 & 12 & 27 & 5.4 & 21.6 & 16 & 100 \\
\hline 9 & 4 & 2 & 23.4 & 23.4 & 31.2 & 16 & 100 \\
\hline 9 & 18 & 2 & 19.2 & 19.2 & 25.6 & 16 & 100 \\
\hline 9 & 4 & 12 & 20.4 & 20.4 & 27.2 & 16 & 100 \\
\hline 9 & 18 & 12 & 16.2 & 16.2 & 21.6 & 16 & 100 \\
\hline 10 & 4 & 2 & 7.8 & 39 & 31.2 & 16 & 100 \\
\hline 10 & 18 & 2 & 6.4 & 32 & 25.6 & 16 & 100 \\
\hline 10 & 4 & 12 & 6.8 & 34 & 27.2 & 16 & 100 \\
\hline 10 & 18 & 12 & 5.4 & 27 & 21.6 & 16 & 100 \\
\hline
\end{tabular}


Table 2-4. Target compositions (wt \%) of the 40 nepheline study glasses.

\begin{tabular}{|c|c|c|c|c|c|c|c|c|c|c|c|c|c|c|c|c|c|c|c|c|c|}
\hline Glass ID & Point & $\mathrm{Al}_{2} \mathbf{O}_{3}$ & $\mathbf{B}_{2} \mathbf{O}_{3}$ & $\mathbf{B a O}$ & $\mathrm{CaO}$ & $\mathrm{Ce}_{2} \mathrm{O}_{3}$ & $\mathrm{Cr}_{2} \mathrm{O}_{3}$ & $\mathrm{CuO}$ & $\mathrm{Fe}_{2} \mathrm{O}_{3}$ & $\mathrm{~K}_{2} \mathrm{O}$ & $\mathrm{La}_{2} \mathrm{O}_{3}$ & MgO & MnO & $\mathrm{Na}_{2} \mathrm{O}$ & $\mathrm{NiO}$ & $\mathbf{P b O}$ & $\mathrm{SO}_{4}{ }^{2-}$ & $\mathrm{SiO}_{2}$ & $\mathrm{TiO}_{2}$ & $\mathrm{ZnO}$ & $\mathrm{ZrO}_{2}$ \\
\hline SB5NEPH-01 & 1 & 7.80 & 4.00 & 0.05 & 2.00 & 0.10 & 0.09 & 0.03 & 10.77 & 0.07 & 0.02 & 0.63 & 2.31 & 23.40 & 1.02 & 0.04 & 0.51 & 46.80 & 0.23 & 0.03 & 0.10 \\
\hline SB5NEPH-02 & 1 & 6.40 & 18.00 & 0.05 & 2.00 & 0.10 & 0.09 & 0.03 & 10.77 & 0.07 & 0.02 & 0.63 & 2.31 & 19.20 & 1.02 & 0.04 & 0.51 & 38.40 & 0.23 & 0.03 & 0.10 \\
\hline SB5NEPH-03 & 1 & 6.80 & 4.00 & 0.05 & 12.00 & 0.10 & 0.09 & 0.03 & 10.77 & 0.07 & 0.02 & 0.63 & 2.31 & 20.40 & 1.02 & 0.04 & 0.51 & 40.80 & 0.23 & 0.03 & 0.10 \\
\hline SB5NEPH-04 & 1 & 5.40 & 18.00 & 0.05 & 12.00 & 0.10 & 0.09 & 0.03 & 10.77 & 0.07 & 0.02 & 0.63 & 2.31 & 16.20 & 1.02 & 0.04 & 0.51 & 32.40 & 0.23 & 0.03 & 0.10 \\
\hline SB5NEPH-05 & 2 & 15.60 & 4.00 & 0.05 & 2.00 & 0.10 & 0.09 & 0.03 & 10.77 & 0.07 & 0.02 & 0.63 & 2.31 & 15.60 & 1.02 & 0.04 & 0.51 & 46.80 & 0.23 & 0.03 & 0.10 \\
\hline SB5NEPH-06 & 2 & 12.80 & 18.00 & 0.05 & 2.00 & 0.10 & 0.09 & 0.03 & 10.77 & 0.07 & 0.02 & 0.63 & 2.31 & 12.80 & 1.02 & 0.04 & 0.51 & 38.40 & 0.23 & 0.03 & 0.10 \\
\hline SB5NEPH-07 & 2 & 13.60 & 4.00 & 0.05 & 12.00 & 0.10 & 0.09 & 0.03 & 10.77 & 0.07 & 0.02 & 0.63 & 2.31 & 13.60 & 1.02 & 0.04 & 0.51 & 40.80 & 0.23 & 0.03 & 0.10 \\
\hline SB5NEPH-08 & 2 & 10.80 & 18.00 & 0.05 & 12.00 & 0.10 & 0.09 & 0.03 & 10.77 & 0.07 & 0.02 & 0.63 & 2.31 & 10.80 & 1.02 & 0.04 & 0.51 & 32.40 & 0.23 & 0.03 & 0.10 \\
\hline SB5NEPH-09 & 3 & 23.40 & 4.00 & 0.05 & 2.00 & 0.10 & 0.09 & 0.03 & 10.77 & 0.07 & 0.02 & 0.63 & 2.31 & 7.80 & 1.02 & 0.04 & 0.51 & 46.80 & 0.23 & 0.03 & 0.10 \\
\hline SB5NEPH-10 & 3 & 19.20 & 18.00 & 0.05 & 2.00 & 0.10 & 0.09 & 0.03 & 10.77 & 0.07 & 0.02 & 0.63 & 2.31 & 6.40 & 1.02 & 0.04 & 0.51 & 38.40 & 0.23 & 0.03 & 0.10 \\
\hline SB5NEPH-11 & 3 & 20.40 & 4.00 & 0.05 & 12.00 & 0.10 & 0.09 & 0.03 & 10.77 & 0.07 & 0.02 & 0.63 & 2.31 & 6.80 & 1.02 & 0.04 & 0.51 & 40.80 & 0.23 & 0.03 & 0.10 \\
\hline SB5NEPH-12 & 3 & 16.20 & 18.00 & 0.05 & 12.00 & 0.10 & 0.09 & 0.03 & 10.77 & 0.07 & 0.02 & 0.63 & 2.31 & 5.40 & 1.02 & 0.04 & 0.51 & 32.40 & 0.23 & 0.03 & 0.10 \\
\hline SB5NEPH-13 & 4 & 7.80 & 4.00 & 0.05 & 2.00 & 0.10 & 0.09 & 0.03 & 10.77 & 0.07 & 0.02 & 0.63 & 2.31 & 31.20 & 1.02 & 0.04 & 0.51 & 39.00 & 0.23 & 0.03 & 0.10 \\
\hline SB5NEPH-14 & 4 & 6.40 & 18.00 & 0.05 & 2.00 & 0.10 & 0.09 & 0.03 & 10.77 & 0.07 & 0.02 & 0.63 & 2.31 & 25.60 & 1.02 & 0.04 & 0.51 & 32.00 & 0.23 & 0.03 & 0.10 \\
\hline SB5NEPH-15 & 4 & 6.80 & 4.00 & 0.05 & 12.00 & 0.10 & 0.09 & 0.03 & 10.77 & 0.07 & 0.02 & 0.63 & 2.31 & 27.20 & 1.02 & 0.04 & 0.51 & 34.00 & 0.23 & 0.03 & 0.10 \\
\hline SB5NEPH-16 & 4 & 5.40 & 18.00 & 0.05 & 12.00 & 0.10 & 0.09 & 0.03 & 10.77 & 0.07 & 0.02 & 0.63 & 2.31 & 21.60 & 1.02 & 0.04 & 0.51 & 27.00 & 0.23 & 0.03 & 0.10 \\
\hline SB5NEPH-17 & 5 & 15.60 & 4.00 & 0.05 & 2.00 & 0.10 & 0.09 & 0.03 & 10.77 & 0.07 & 0.02 & 0.63 & 2.31 & 23.40 & 1.02 & 0.04 & 0.51 & 39.00 & 0.23 & 0.03 & 0.10 \\
\hline SB5NEPH-18 & 5 & 12.80 & 18.00 & 0.05 & 2.00 & 0.10 & 0.09 & 0.03 & 10.77 & 0.07 & 0.02 & 0.63 & 2.31 & 19.20 & 1.02 & 0.04 & 0.51 & 32.00 & 0.23 & 0.03 & 0.10 \\
\hline SB5NEPH-19 & 5 & 13.60 & 4.00 & 0.05 & 12.00 & 0.10 & 0.09 & 0.03 & 10.77 & 0.07 & 0.02 & 0.63 & 2.31 & 20.40 & 1.02 & 0.04 & 0.51 & 34.00 & 0.23 & 0.03 & 0.10 \\
\hline SB5NEPH-20 & 5 & 10.80 & 18.00 & 0.05 & 12.00 & 0.10 & 0.09 & 0.03 & 10.77 & 0.07 & 0.02 & 0.63 & 2.31 & 16.20 & 1.02 & 0.04 & 0.51 & 27.00 & 0.23 & 0.03 & 0.10 \\
\hline SB5NEPH-21 & 6 & 23.40 & 4.00 & 0.05 & 2.00 & 0.10 & 0.09 & 0.03 & 10.77 & 0.07 & 0.02 & 0.63 & 2.31 & 15.60 & 1.02 & 0.04 & 0.51 & 39.00 & 0.23 & 0.03 & 0.10 \\
\hline SB5NEPH-22 & 6 & 19.20 & 18.00 & 0.05 & 2.00 & 0.10 & 0.09 & 0.03 & 10.77 & 0.07 & 0.02 & 0.63 & 2.31 & 12.80 & 1.02 & 0.04 & 0.51 & 32.00 & 0.23 & 0.03 & 0.10 \\
\hline SB5NEPH-23 & 6 & 20.40 & 4.00 & 0.05 & 12.00 & 0.10 & 0.09 & 0.03 & 10.77 & 0.07 & 0.02 & 0.63 & 2.31 & 13.60 & 1.02 & 0.04 & 0.51 & 34.00 & 0.23 & 0.03 & 0.10 \\
\hline SB5NEPH-24 & 6 & 16.20 & 18.00 & 0.05 & 12.00 & 0.10 & 0.09 & 0.03 & 10.77 & 0.07 & 0.02 & 0.63 & 2.31 & 10.80 & 1.02 & 0.04 & 0.51 & 27.00 & 0.23 & 0.03 & 0.10 \\
\hline SB5NEPH-25 & 7 & 31.20 & 4.00 & 0.05 & 2.00 & 0.10 & 0.09 & 0.03 & 10.77 & 0.07 & 0.02 & 0.63 & 2.31 & 7.80 & 1.02 & 0.04 & 0.51 & 39.00 & 0.23 & 0.03 & 0.10 \\
\hline SB5NEPH-26 & 7 & 25.60 & 18.00 & 0.05 & 2.00 & 0.10 & 0.09 & 0.03 & 10.77 & 0.07 & 0.02 & 0.63 & 2.31 & 6.40 & 1.02 & 0.04 & 0.51 & 32.00 & 0.23 & 0.03 & 0.10 \\
\hline SB5NEPH-27 & 7 & 27.20 & 4.00 & 0.05 & 12.00 & 0.10 & 0.09 & 0.03 & 10.77 & 0.07 & 0.02 & 0.63 & 2.31 & 6.80 & 1.02 & 0.04 & 0.51 & 34.00 & 0.23 & 0.03 & 0.10 \\
\hline SB5NEPH-28 & 7 & 21.60 & 18.00 & 0.05 & 12.00 & 0.10 & 0.09 & 0.03 & 10.77 & 0.07 & 0.02 & 0.63 & 2.31 & 5.40 & 1.02 & 0.04 & 0.51 & 27.00 & 0.23 & 0.03 & 0.10 \\
\hline SB5NEPH-29 & 8 & 7.80 & 4.00 & 0.05 & 2.00 & 0.10 & 0.09 & 0.03 & 10.77 & 0.07 & 0.02 & 0.63 & 2.31 & 39.00 & 1.02 & 0.04 & 0.51 & 31.20 & 0.23 & 0.03 & 0.10 \\
\hline SB5NEPH-30 & 8 & 6.40 & 18.00 & 0.05 & 2.00 & 0.10 & 0.09 & 0.03 & 10.77 & 0.07 & 0.02 & 0.63 & 2.31 & 32.00 & 1.02 & 0.04 & 0.51 & 25.60 & 0.23 & 0.03 & 0.10 \\
\hline SB5NEPH-31 & 8 & 6.80 & 4.00 & 0.05 & 12.00 & 0.10 & 0.09 & 0.03 & 10.77 & 0.07 & 0.02 & 0.63 & 2.31 & 34.00 & 1.02 & 0.04 & 0.51 & 27.20 & 0.23 & 0.03 & 0.10 \\
\hline SB5NEPH-32 & 8 & 5.40 & 18.00 & 0.05 & 12.00 & 0.10 & 0.09 & 0.03 & 10.77 & 0.07 & 0.02 & 0.63 & 2.31 & 27.00 & 1.02 & 0.04 & 0.51 & 21.60 & 0.23 & 0.03 & 0.10 \\
\hline SB5NEPH-33 & 9 & 23.40 & 4.00 & 0.05 & 2.00 & 0.10 & 0.09 & 0.03 & 10.77 & 0.07 & 0.02 & 0.63 & 2.31 & 23.40 & 1.02 & 0.04 & 0.51 & 31.20 & 0.23 & 0.03 & 0.10 \\
\hline SB5NEPH-34 & 9 & 19.20 & 18.00 & 0.05 & 2.00 & 0.10 & 0.09 & 0.03 & 10.77 & 0.07 & 0.02 & 0.63 & 2.31 & 19.20 & 1.02 & 0.04 & 0.51 & 25.60 & 0.23 & 0.03 & 0.10 \\
\hline SB5NEPH-35 & 9 & 20.40 & 4.00 & 0.05 & 12.00 & 0.10 & 0.09 & 0.03 & 10.77 & 0.07 & 0.02 & 0.63 & 2.31 & 20.40 & 1.02 & 0.04 & 0.51 & 27.20 & 0.23 & 0.03 & 0.10 \\
\hline SB5NEPH-36 & 9 & 16.20 & 18.00 & 0.05 & 12.00 & 0.10 & 0.09 & 0.03 & 10.77 & 0.07 & 0.02 & 0.63 & 2.31 & 16.20 & 1.02 & 0.04 & 0.51 & 21.60 & 0.23 & 0.03 & 0.10 \\
\hline SB5NEPH-37 & 10 & 39.00 & 4.00 & 0.05 & 2.00 & 0.10 & 0.09 & 0.03 & 10.77 & 0.07 & 0.02 & 0.63 & 2.31 & 7.80 & 1.02 & 0.04 & 0.51 & 31.20 & 0.23 & 0.03 & 0.10 \\
\hline SB5NEPH-38 & 10 & 32.00 & 18.00 & 0.05 & 2.00 & 0.10 & 0.09 & 0.03 & 10.77 & 0.07 & 0.02 & 0.63 & 2.31 & 6.40 & 1.02 & 0.04 & 0.51 & 25.60 & 0.23 & 0.03 & 0.10 \\
\hline SB5NEPH-39 & 10 & 34.00 & 4.00 & 0.05 & 12.00 & 0.10 & 0.09 & 0.03 & 10.77 & 0.07 & 0.02 & 0.63 & 2.31 & 6.80 & 1.02 & 0.04 & 0.51 & 27.20 & 0.23 & 0.03 & 0.10 \\
\hline SB5NEPH-40 & 10 & 27.00 & 18.00 & 0.05 & 12.00 & 0.10 & 0.09 & 0.03 & 10.77 & 0.07 & 0.02 & 0.63 & 2.31 & 5.40 & 1.02 & 0.04 & 0.51 & 21.60 & 0.23 & 0.03 & 0.10 \\
\hline
\end{tabular}


WSRC-STI-2007-00659

Revision 0

\subsection{Glass Fabrication}

Each of the study glasses was prepared from the proper proportions of reagent-grade metal oxides, carbonates, boric acid and salts in $150 \mathrm{~g}$ batches. ${ }^{15}$ The raw materials were thoroughly mixed and placed into platinum/rhodium, $250 \mathrm{ml}$ crucibles. The batch was placed into a high-temperature furnace at the target melt temperature of $1150^{\circ} \mathrm{C} .{ }^{16}$ The melt temperature had to be increased for some of the more refractory glass compositions, up to a maximum of $1450{ }^{\circ} \mathrm{C}$. Melt temperatures for each glass are included later in Table 3-1. The crucible was removed from the furnace after an isothermal hold at the melt temperature for 1 hour. The glass was poured onto a clean, stainless steel plate and allowed to air cool (quench). The glass pour patty was used as a sampling stock for the various property measurements, including chemical composition and durability testing.

Approximately $25 \mathrm{~g}$ of each glass was heat-treated to simulate cooling along the centerline of a DWPF-type canister ${ }^{17}$ to gauge the effects of thermal history on the product performance. This cooling schedule is referred to as the centerline canister cooling (CCC) curve. Visual observations on both quenched and CCC glasses were documented.

\subsection{Property Measurements}

This section provides a general discussion of the chemical composition analyses, the PCTs, and the XRD analyses of the study glasses.

\subsubsection{Compositional Analysis}

To confirm that the as-fabricated glasses met the target compositions, a representative sample from each quenched glass was submitted to the SRNL Process Science Analytical Laboratory (PSAL) for chemical analysis under the auspices of an analytical plan. ${ }^{18}$ The plan identified the cations to be analyzed and the two dissolution techniques, sodium peroxide fusion (PF) and lithium-metaborate (LM), to be used. The samples prepared by LM were used to measure aluminum ( $\mathrm{Al})$, barium $(\mathrm{Ba})$, calcium $(\mathrm{Ca})$, cerium $(\mathrm{Ce})$, chromium $(\mathrm{Cr})$, copper $(\mathrm{Cu})$, potassium $(\mathrm{K})$, lanthanum $(\mathrm{La})$, magnesium $(\mathrm{Mg})$, manganese $(\mathrm{Mn})$, sodium $(\mathrm{Na})$, nickel $(\mathrm{Ni})$, lead $(\mathrm{Pb})$, sulfur (S), titanium (Ti), zinc (Zn), and zirconium (Zr) concentrations. Samples prepared by PF were used to measure boron (B), iron (Fe), lithium ( $\mathrm{Li}$ ), and silicon ( $\mathrm{Si}$ ) concentrations. Each glass was prepared in duplicate for each of the two cation dissolution techniques. All of the prepared samples were analyzed twice for each element of interest by Inductively Coupled Plasma - Atomic Emission Spectroscopy (ICP-AES), with the instrumentation being re-calibrated between the duplicate analyses. The analytical plan was developed in such a way as to provide the opportunity to evaluate potential sources of bias and error. Glass standards were also intermittently measured to assess the performance of the ICP-AES instrument over the course of these analyses.

\subsubsection{X-Ray Diffraction Analysis}

Representative samples for all quenched and CCC glasses were submitted to SRNL Analytical Development for XRD analysis. Samples were run under conditions providing a detection limit of approximately $0.5 \mathrm{vol} \%$. That is, if crystals (or undissolved batch material) were present at $0.5 \mathrm{vol} \%$ or greater, the diffractometer would not only be capable of detecting the crystals but would also allow a qualitative determination of the type of crystal(s) present. Otherwise, a characteristically high background signal (amorphous hump) devoid of crystalline spectral peaks indicates that the glass product is free of crystallization, suggesting either a completely amorphous product or that the degree of crystallization is below the detection limit. 


\subsubsection{Product Consistency Test (PCT)}

The PCT Method- $\mathrm{A}^{19}$ was performed in triplicate on each quenched and CCC glass to assess chemical durability. Also included in the experimental test matrix was the Environmental Assessment (EA) benchmark glass, ${ }^{20}$ the Approved Reference Material (ARM) glass, and blanks from the sample cleaning batch. Samples were ground, washed, and prepared according to the standard procedure. ${ }^{19}$ Fifteen milliliters of Type-I ASTM water were added to $1.5 \mathrm{~g}$ of glass in stainless steel vessels. The vessels were closed, sealed, and placed in an oven at $90 \pm 2{ }^{\circ} \mathrm{C}$ where the samples were maintained at temperature for 7 days. Once cooled, the resulting solutions were sampled (filtered and acidified), then labeled and analyzed by PSAL under the auspices of an analytical plan. ${ }^{21}$ The aim of the plan was to provide an opportunity to assess the consistency (repeatability) of the PCT and analytical procedures in evaluating the chemical durability of the glasses. Normalized release rates were calculated based on target, measured, and bias-corrected (bc) compositions using the average of the common logarithms of the leachate concentrations. 
WSRC-STI-2007-00659

Revision 0

This page intentionally left blank. 
WSRC-STI-2007-00659

Revision 0

\subsection{Results and Discussion}

\subsection{A Statistical Review of the Chemical Composition Measurements}

In this section, the measured versus targeted compositions of the study glasses (Glass Identifiers SB5NEPH-01 through SB5NEPH-40) are presented and compared. The targeted compositions for these glasses are provided in Table 2-4, as well as Table A1 of Appendix A. Chemical composition measurements for these glasses were conducted by the PSAL under the auspices of analytical plans. ${ }^{18}$ For each study glass, measurements were obtained from samples prepared in duplicate by the LM and PF dissolution methods. All of the prepared samples were analyzed (twice for each element of interest) by ICP-AES, with the instrumentation being re-calibrated between the duplicate analyses.

Table A2 in Appendix A provides the elemental concentration measurements derived from the samples prepared using LM, and Table A3 in Appendix A provides the measurements derived from the samples prepared using PF. Measurements for the standard (Batch 1) samples that were included in the PSAL analytical plans are also provided in these two tables.

The elemental concentrations were converted to oxide concentrations by multiplying the values for each element by the gravimetric factor for the corresponding oxide. During this process, an elemental concentration that was determined to be below the detection limit of the analytical procedures used by the PSAL was reduced to half of that detection limit as the oxide concentration was determined.

In the sections that follow, the analytical sequences of the measurements are explored, the measurements of the standards are investigated and used for bias correction, the measurements for each glass are reviewed, the average chemical compositions (measured and bias-corrected) for each glass are determined, and comparisons are made between the measurements and the targeted compositions for the glasses.

\subsubsection{Measurements in Analytical Sequence}

Exhibit A1 in Appendix A provides plots of the measurements generated by the PSAL for samples prepared using the LM method. The plots are in analytical sequence with different symbols and colors being used to represent each of the study and standard glasses. Similar plots for the samples prepared using the PF method are provided in Exhibit A2 in Appendix A. These plots include all of the measurement data from Tables A2 and A3. While these plots provide only a limited opportunity for revealing patterns in these data, there is an indication of one glass, SB5NEPH-11, with $\mathrm{TiO}_{2}$ values near zero that stands out. In general, the review of these plots indicates no significant patterns or trends in the analytical process over the course of these measurements, and there appear to be no dramatic outliers in these chemical composition measurements.

\subsubsection{Batch 1 and Uranium Standard Results}

In this section, the PSAL measurements of the chemical compositions of the Batch 1 samples are reviewed. These measurements are investigated across the ICP-AES analytical sets and blocks, and the results are used to bias correct the measurements for the study glasses.

Exhibit A3 in Appendix A provides statistical analyses of the Batch 1 results generated by the LM prep method by set/block/sub-block for each oxide of interest. The results include analysis of variance (ANOVA) investigations looking for statistically significant differences between the 
means of these groups for each of the oxides for the standard. The reference value for the oxide concentration of the standard is given in the header for each set of measurements in the exhibit. The results from the statistical tests for the Batch 1 standard may be summarized as follows: $\mathrm{Al}_{2} \mathrm{O}_{3}, \mathrm{BaO}, \mathrm{CaO}, \mathrm{Cr}_{2} \mathrm{O}_{3}, \mathrm{CuO}, \mathrm{K}_{2} \mathrm{O}, \mathrm{MgO}, \mathrm{MnO}, \mathrm{Na}_{2} \mathrm{O}, \mathrm{NiO}, \mathrm{TiO}_{2}$, and $\mathrm{ZrO}_{2}$ have measurements that indicate a significant ICP-AES calibration effect on the block averages at the $5 \%$ significance level.

Exhibit A4 in Appendix A provides a similar set of analyses for the measurements derived from samples prepared via the PF method. The reference value for the oxide concentration of the standard is given in the header for each set of measurements in the exhibit. The results from the statistical tests for the Batch 1 standard may be summarized as follows: $\mathrm{B}_{2} \mathrm{O}_{3}, \mathrm{Fe}_{2} \mathrm{O}_{3}, \mathrm{Li}_{2} \mathrm{O}$ and $\mathrm{SiO}_{2}$ have measurements that indicate significant ICP-AES calibration effects on the block averages at the $5 \%$ significance level.

Thus, some of these results provide incentive for adjusting the measurements by the effects of the ICP-AES calibration. Therefore, the oxide measurements of the study glasses are to be bias corrected for the effect of the ICP-AES calibration on each of the analytical sets, blocks and subblocks. The basis for this bias correction is presented as part of Exhibits $\mathrm{A} 3$ and $\mathrm{A} 4$ - the average measurement for Batch 1 for each ICP-AES set/block/sub-block for $\mathrm{Al}_{2} \mathrm{O}_{3}, \mathrm{~B}_{2} \mathrm{O}_{3}, \mathrm{BaO}, \mathrm{CaO}$, $\mathrm{Cr}_{2} \mathrm{O}_{3}, \mathrm{CuO}, \mathrm{Fe}_{2} \mathrm{O}_{3}, \mathrm{Li}_{2} \mathrm{O}, \mathrm{MgO}, \mathrm{MnO}, \mathrm{Na}_{2} \mathrm{O}, \mathrm{NiO}, \mathrm{SiO}_{2}$, and $\mathrm{TiO}_{2}$. The Batch 1 results were used to conduct the bias correction as long as the reference value for the oxide concentration in the Batch 1 glass was greater than or equal to $0.1 \mathrm{wt} \%$. Thus, applying this approach and based upon the information in the exhibits, the Batch 1 results were used to bias correct the $\mathrm{Al}_{2} \mathrm{O}_{3}, \mathrm{~B}_{2} \mathrm{O}_{3}$, $\mathrm{BaO}, \mathrm{CaO}, \mathrm{Cr}_{2} \mathrm{O}_{3}, \mathrm{CuO}, \mathrm{Fe}_{2} \mathrm{O}_{3}, \mathrm{~K}_{2} \mathrm{O}, \mathrm{Li}_{2} \mathrm{O}, \mathrm{MgO}, \mathrm{MnO}, \mathrm{Na}_{2} \mathrm{O}, \mathrm{NiO}, \mathrm{SiO}_{2}$, and $\mathrm{TiO}_{2}$ measurements. No bias correction was conducted for $\mathrm{Ce}_{2} \mathrm{O}_{3}, \mathrm{La}_{2} \mathrm{O}_{3}, \mathrm{PbO}, \mathrm{SO}_{4}{ }^{2-}$, $\mathrm{ZnO}$, or $\mathrm{ZrO}_{2}$.

The bias correction was conducted as follows. For each oxide, let $\bar{a}_{i j}$ be the average measurement for the $i^{\text {th }}$ oxide at analytical block $j$ for Batch 1 , and let $t_{i}$ be the reference value for the $i^{\text {th }}$ oxide for Batch 1. (The averages and reference values are provided in Exhibits A3 and A4.) Let $\bar{C}_{i j k}$ be the average measurement for the $i^{\text {th }}$ oxide at analytical block $j$ for the $k^{\text {th }}$ glass. The bias adjustment was conducted following Equation 2:

$$
\bar{c}_{i j k} \bullet\left(1-\frac{\bar{a}_{i j}-t_{i}}{\bar{a}_{i j}}\right)=\bar{c}_{i j k} \bullet \frac{t_{i}}{\bar{a}_{i j}}
$$

Bias-corrected measurements are indicated by a "bc" suffix, and such adjustments were performed for all of the oxides of this study except for $\mathrm{Ce}_{2} \mathrm{O}_{3}, \mathrm{La}_{2} \mathrm{O}_{3}, \mathrm{PbO}, \mathrm{SO}_{4}{ }^{2-}, \mathrm{ZnO}$, and $\mathrm{ZrO}_{2}$. Both measured and measured "bc" values are included in the discussion that follows. In these discussions bias-corrected values for $\mathrm{Ce}_{2} \mathrm{O}_{3}, \mathrm{La}_{2} \mathrm{O}_{3}, \mathrm{PbO}, \mathrm{SO}_{4}{ }^{2-}, \mathrm{ZnO}$, and $\mathrm{ZrO}_{2}$ are included for completeness (e.g., to allow a sum of oxides to be computed for the bias-corrected results). These bias-corrected values are the same as the original $\mathrm{Ce}_{2} \mathrm{O}_{3}, \mathrm{La}_{2} \mathrm{O}_{3}, \mathrm{PbO}, \mathrm{SO}_{4}{ }^{2-}, \mathrm{ZnO}$, and $\mathrm{ZrO}_{2}$ values.

\subsubsection{Composition Measurements by Glass Number}

Exhibits A5 and A6 in Appendix A provide plots of the oxide concentration measurements by Lab ID within Glass ID (including Batch 1) grouped by target values for the measured and biascorrected values for the LM and PF preparation methods, respectively. Different symbols and colors are used to represent the different glasses. These plots show the individual measurements 
across the duplicates of each preparation method and the two ICP-AES calibrations. A review of the plots presented in these exhibits reveals the repeatability of the four individual, oxide values for each glass. A small amount of scatter is present in the $\mathrm{MnO}$ and $\mathrm{NiO}$ measurements. Scatter is present in the $\mathrm{Fe}_{2} \mathrm{O}_{3}$ measurements on the order of $0.5 \mathrm{wt} \%$. This is not expected to have a significant impact on the outcome of the study. For two glasses (SB5NEPH-9 and SB5NEPH-10) in Set 1, three glasses (SB5NEPH-25, SB5NEPH-26, and SB5NEPH-28) in Set 3, and four glasses (SB5NEPH-37 through SB5NEPH-40) in Set 4, the $\mathrm{SO}_{4}{ }^{2-}$ values reported were at the detection limit. This is likely due to the compositionally and thermally (melt temperature) dependent solubility of $\mathrm{SO}_{4}{ }^{2-}$ in these glasses, and will not have a significant influence on the outcome of this study. In Set 2, one of the glasses (SB5NEPH-11) had $\mathrm{TiO}_{2}$ measurements at the detection limit. It is possible that $\mathrm{TiO}_{2}$ was omitted from this glass during batching, which is not seen as having any significant impact on the outcome of this study.

\subsubsection{Measured versus Targeted Compositions}

The four measurements for each oxide for each glass (over both preparation methods) were averaged to determine a representative chemical composition for each glass. These determinations were conducted both for the measured and for the bias-corrected data. A sum of oxides was also computed for each glass based upon both the measured and bias-corrected values. Exhibit A7 in Appendix A provides plots showing results for each glass for each oxide to help highlight the comparisons among the measured, bias-corrected, and targeted values. Some scatter for the measured $\mathrm{Cr}_{2} \mathrm{O}_{3}, \mathrm{Fe}_{2} \mathrm{O}_{3}, \mathrm{MnO}, \mathrm{NiO}$ and $\mathrm{ZrO}_{2}$ concentrations is apparent in these plots, although it should not have a significant impact on the outcome of the study.

Table A4 in Appendix A provides a summary of the average compositions as well as the targeted compositions and some associated differences and relative differences. Notice that the targeted sums of oxides for the Batch 1 standard do not sum to $100 \%$ due to an incomplete coverage of the oxides in the Batch 1 (Glass \# 100) samples. All of the sums of oxides (both measured and biascorrected) for the study glasses fall within the interval of 95 to $105 \mathrm{wt} \%$ except for study glass SB5NEPH-31. Entries in Table A4 show the relative differences between the measured or biascorrected values and the targeted values. These differences are shaded when they are greater than or equal to 5\%. Overall, these comparisons between the measured and targeted compositions suggest only minor difficulties in hitting the targeted compositions for some of the oxides for some of the glasses, none of which should influence the conclusions of this report.

\subsection{Homogeneity}

Visual observations were recorded for each glass after melting and quenching, and also after completion of the CCC heat treatment. The surface of the glass pour patty and the glass remaining in the crucible were observed for the quenched glasses. Both the surface and a crosssectional fracture surface (described as 'bulk') were observed for the CCC versions of the glasses. In describing the appearance of the glasses, terms such as clean or shiny indicate that the glass was free of any visible crystallization. Terms such as metallic, silvery, dull or hazy are used to describe crystallization visible on the surface of the glasses. The visual observations for all of the glasses are summarized in Table 3-1. In addition, any crystalline phases identified by XRD are listed on the right side of the table. 
Table 3-1. Summary of the visual observations and XRD results for the nepheline study glasses.

\begin{tabular}{|c|c|c|c|c|}
\hline Glass ID & $\begin{array}{c}\text { Heat } \\
\text { Treatment }\end{array}$ & $\begin{array}{l}\mathbf{T}_{\text {Melt }} \\
\left({ }^{\circ} \mathbf{C}\right)\end{array}$ & Visual Observations & XRD Phase Identification \\
\hline SB5NEPH-01 & quenched & 1150 & $\begin{array}{l}\text { patty: black, shiny and homogeneous; crucible: } \\
\text { clean }\end{array}$ & amorphous \\
\hline SB5NEPH-02 & quenched & 1150 & $\begin{array}{l}\text { patty: black, shiny and homogeneous; crucible: } \\
\text { clean }\end{array}$ & amorphous \\
\hline SB5NEPH-03 & quenched & 1150 & $\begin{array}{l}\text { patty: black, shiny and homogeneous; crucible: } \\
\text { clean }\end{array}$ & amorphous \\
\hline SB5NEPH-04 & quenched & 1150 & $\begin{array}{l}\text { patty: black, shiny and homogeneous; crucible: } \\
\text { clean }\end{array}$ & amorphous \\
\hline SB5NEPH-05 & quenched & 1300 & $\begin{array}{l}\text { patty: black, shiny and homogeneous; crucible: } \\
\text { clean }\end{array}$ & amorphous \\
\hline SB5NEPH-06 & quenched & 1150 & $\begin{array}{l}\text { patty: clean; crucible: four spots of undissolved } \\
\text { material }\end{array}$ & Trevorite $\left[\mathrm{NiFe}_{2} \mathrm{O}_{4}\right]$ \\
\hline SB5NEPH-07 & quenched & 1300 & $\begin{array}{l}\text { patty: black, shiny and homogeneous; crucible: } \\
\text { clean }\end{array}$ & amorphous \\
\hline SB5NEPH-08 & quenched & 1150 & $\begin{array}{l}\text { patty: clean, black and shiny; crucible: four small } \\
\text { spots of undissolved material }\end{array}$ & amorphous \\
\hline SB5NEPH-09 & quenched & 1450 & $\begin{array}{l}\text { patty: chocolate brown color black and shiny bulk, } \\
\text { clean and shiny; crucible: line of foam at melt line }\end{array}$ & Trevorite $\left[\mathrm{NiFe}_{2} \mathrm{O}_{4}\right]$ \\
\hline SB5NEPH-10 & quenched & 1450 & $\begin{array}{l}\text { patty: chocolate brown surface, black and shiny } \\
\text { bulk; crucible: chocolate brown color on surface, no } \\
\text { undissolved material }\end{array}$ & Trevorite $\left[\mathrm{NiFe}_{2} \mathrm{O}_{4}\right]$ \\
\hline SB5NEPH-11 & quenched & 1300 & $\begin{array}{l}\text { patty: dark brown, shiny, bulk clean; crucible: one } \\
\text { spot of undissolved material }\end{array}$ & Trevorite $\left[\mathrm{NiFe}_{2} \mathrm{O}_{4}\right]$ \\
\hline SB5NEPH-12 & quenched & 1300 & patty: clean; crucible: clean & amorphous \\
\hline SB5NEPH-13 & quenched & 1150 & $\begin{array}{l}\text { patty: shiny/silver surface, bulk clean; crucible: } \\
\text { clean with bubbles }\end{array}$ & possible Gregoryite $\left[\mathrm{Na}_{2} \mathrm{CO}_{3}\right]$ \\
\hline SB5NEPH-14 & quenched & 1150 & patty: clean; crucible: clean & amorphous \\
\hline SB5NEPH-15 & quenched & 1150 & $\begin{array}{l}\text { patty: shiny, silvery surface, bulk clean; crucible: } \\
\text { silvery surface, slight yellow tint on walls }\end{array}$ & amorphous \\
\hline SB5NEPH-16 & quenched & 1150 & patty: dark brown, clean; crucible: clean & amorphous \\
\hline SB5NEPH-17 & quenched & 1300 & $\begin{array}{l}\text { patty: silvery/metallic surface, bulk clean; crucible: } \\
\text { clean with slight metallic haze on surface }\end{array}$ & amorphous \\
\hline
\end{tabular}


Table 3-1. Summary of the visual observations and XRD results for the nepheline study glasses. (continued)

\begin{tabular}{|c|c|c|c|c|}
\hline Glass ID & $\begin{array}{c}\text { Heat } \\
\text { Treatment }\end{array}$ & $\begin{array}{l}T_{\text {Melt }} \\
\left({ }^{\circ} \mathrm{C}\right)\end{array}$ & Visual Observations & XRD Phase Identification \\
\hline SB5NEPH-18 & quenched & 1300 & patty: dark brown, shiny and clean; crucible: clean & amorphous \\
\hline SB5NEPH-19 & quenched & 1300 & $\begin{array}{l}\text { patty: silvery/metallic haze on surface, bulk clean; } \\
\text { crucible: clean }\end{array}$ & amorphous \\
\hline SB5NEPH-20 & quenched & 1300 & patty: dark brown, shiny, clean; crucible: clean & amorphous \\
\hline SB5NEPH-21 & quenched & 1450 & $\begin{array}{l}\text { patty: dark brown, shiny surface, bulk crystallized; } \\
\text { crucible: chocolate brown }\end{array}$ & Magnetite $\left[\mathrm{Fe}^{2+} \mathrm{Fe}_{2}{ }^{3+} \mathrm{O}_{4}\right]$ \\
\hline SB5NEPH-22 & quenched & 1300 & $\begin{array}{l}\text { patty: chocolate brown, shiny, bulk chocolate } \\
\text { brown with crystals; crucible: chocolate brown }\end{array}$ & Magnetite $\left[\mathrm{Fe}^{2+} \mathrm{Fe}_{2}{ }^{3+} \mathrm{O}_{4}\right]$ \\
\hline SB5NEPH-23 & quenched & 1300 & $\begin{array}{l}\text { patty: silvery/metallic haze on surface, bulk clean; } \\
\text { crucible: metallic haze }\end{array}$ & Bunsenite [NiO] \\
\hline SB5NEPH-24 & quenched & 1150 & patty: black and shiny, bulk clean; crucible: clean & amorphous \\
\hline SB5NEPH-25 & quenched & 1450 & $\begin{array}{l}\text { patty: light haze, crystals throughout bulk; crucible: } \\
\text { haze but no undissolved material }\end{array}$ & Trevorite $\left[\mathrm{NiFe}_{2} \mathrm{O}_{4}\right]$, Corundum $\left[\mathrm{Al}_{2} \mathrm{O}_{3}\right]$ \\
\hline SB5NEPH-26 & quenched & 1300 & $\begin{array}{l}\text { patty: brownish haze, crystals in bulk; crucible: one } \\
\text { spot of undissolved material with haze on surface }\end{array}$ & Trevorite $\left[\mathrm{NiFe}_{2} \mathrm{O}_{4}\right]$, Hematite $\left[\mathrm{Fe}_{2} \mathrm{O}_{3}\right]$ \\
\hline SB5NEPH-27 & quenched & 1300 & $\begin{array}{l}\text { patty: black and shiny, crystals in bulk; crucible: } \\
\text { clean }\end{array}$ & Iron Aluminum Oxide $\left[\left(\mathrm{Fe}_{.78} \mathrm{Al}_{.22}\right)\left(\mathrm{Al}_{1.78} \mathrm{Fe}_{.22}\right) \mathrm{O}_{4}\right]$ \\
\hline SB5NEPH-28 & quenched & 1300 & patty: black and shiny, bulk clean; crucible: clean & amorphous \\
\hline SB5NEPH-29 & quenched & 1150 & $\begin{array}{l}\text { patty: golden/bronze haze with some green and } \\
\text { purple streaks on surface, bulk clean; crucible: haze } \\
\text { on surface, no undissolved material }\end{array}$ & Thermonatrite $\left[\mathrm{Na}_{2} \mathrm{CO}_{3} \cdot \mathrm{H}_{2} \mathrm{O}\right]$, Gregoryite $\left[\mathrm{Na}_{2} \mathrm{CO}_{3}\right]$ \\
\hline SB5NEPH-30 & quenched & 1150 & $\begin{array}{l}\text { patty: light, silvery haze on surface, bulk clean; } \\
\text { crucible: clean }\end{array}$ & amorphous \\
\hline SB5NEPH-31 & quenched & 1150 & $\begin{array}{l}\text { patty: bronze/golden haze with green and purple } \\
\text { swirls, bulk devitrified; crucible: bronze haze on } \\
\text { surface and devitrified }\end{array}$ & $\begin{array}{l}\text { Thermonatrite }\left[\mathrm{Na}_{2} \mathrm{CO}_{3} \cdot \mathrm{H}_{2} \mathrm{O}\right] \text {, Sodium aluminum silicate } \\
{\left[\left(\mathrm{Na}_{2} \mathrm{O}\right)_{.33} \mathrm{NaAlSiO}_{4}\right], \text { Calcium silicate }\left[\mathrm{Ca}_{2}\left(\mathrm{SiO}_{4}\right)\right]}\end{array}$ \\
\hline SB5NEPH-32 & quenched & 1150 & $\begin{array}{l}\text { patty: silvery haze on surface, bulk clean; crucible: } \\
\text { clean }\end{array}$ & amorphous \\
\hline SB5NEPH-33 & quenched & 1300 & $\begin{array}{l}\text { patty: chocolate brown, dull surface, bulk } \\
\text { devitrified; crucible: chocolate brown and } \\
\text { devitrified }\end{array}$ & $\begin{array}{l}\text { Sodium aluminum silicate }\left[\mathrm{Na}_{1.55} \mathrm{Al}_{1.55} \mathrm{Si}_{.45} \mathrm{O}_{4}\right] \text {, Magnetite } \\
{\left[\mathrm{Fe}^{2+} \mathrm{Fe}_{2}{ }^{3+} \mathrm{O}_{4}\right]}\end{array}$ \\
\hline
\end{tabular}


Table 3-1. Summary of the visual observations and XRD results for the nepheline study glasses. (continued)

\begin{tabular}{|c|c|c|c|c|}
\hline Glass ID & $\begin{array}{c}\text { Heat } \\
\text { Treatment }\end{array}$ & $\begin{array}{l}\mathbf{T}_{\text {Melt }} \\
\left({ }^{\circ} \mathrm{C}\right)\end{array}$ & Visual Observations & XRD Phase Identification \\
\hline SB5NEPH-34 & quenched & 1300 & $\begin{array}{l}\text { patty: dark brown, shiny surface, a few chocolate } \\
\text { brown swirls, bulk clean; crucible: clean }\end{array}$ & Bunsenite [NiO] \\
\hline SB5NEPH-35 & quenched & 1300 & $\begin{array}{l}\text { patty: shiny surface with hazy swirls, bulk } \\
\text { chocolate brown and crystallized; crucible: clean }\end{array}$ & $\begin{array}{l}\text { Sodium aluminum silicate }\left[\mathrm{Na}_{1.55} \mathrm{Al}_{1.55} \mathrm{Si}_{.45} \mathrm{O}_{4}\right] \text {, Calcium iron } \\
\text { oxide }\left[\mathrm{Ca}_{2} \mathrm{Fe}_{2} \mathrm{O}_{5}\right]\end{array}$ \\
\hline SB5NEPH-36 & quenched & 1300 & $\begin{array}{l}\text { patty: black, shiny and clean, bulk clean; crucible: } \\
\text { clean }\end{array}$ & $\begin{array}{l}\text { Nepheline }\left[\mathrm{NaAlSiO}_{4}\right] \text {, Jacobsite }\left[\mathrm{MnFe}_{2} \mathrm{O}_{4}\right] \text {, Calcium Borate } \\
{\left[\mathrm{Ca}_{2} \mathrm{~B}_{2} \mathrm{O}_{5}\right]}\end{array}$ \\
\hline SB5NEPH-37 & quenched & 1450 & $\begin{array}{l}\text { patty: completely crystallized surface and bulk; } \\
\text { crucible: crystallized }\end{array}$ & Trevorite $\left[\mathrm{NiFe}_{2} \mathrm{O}_{4}\right]$, Corundum $\left[\mathrm{Al}_{2} \mathrm{O}_{3}\right]$ \\
\hline SB5NEPH-38 & quenched & 1300 & $\begin{array}{l}\text { patty: dark, chocolate brown and completely } \\
\text { crystallized; crucible: crystallized }\end{array}$ & $\begin{array}{l}\text { Trevorite }\left[\mathrm{NiFe}_{2} \mathrm{O}_{4}\right] \text {, Corundum }\left[\mathrm{Al}_{2} \mathrm{O}_{3}\right] \text {, Hematite }\left[\mathrm{Fe}_{2} \mathrm{O}_{3}\right] \text {, } \\
\text { Mullite }\left[\mathrm{Al}_{2.4} \mathrm{Si}_{0.6} \mathrm{O}_{4.8}\right]\end{array}$ \\
\hline SB5NEPH-39 & quenched & 1450 & $\begin{array}{l}\text { patty: chocolate brown surface, bulk crystallized; } \\
\text { crucible: clean }\end{array}$ & Bunsenite [NiO], Magnetite $\left[\mathrm{Fe}^{2+} \mathrm{Fe}_{2}{ }^{3+} \mathrm{O}_{4}\right]$ \\
\hline SB5NEPH-40 & quenched & 1300 & patty: black, shiny and clean; crucible: clean & amorphous \\
\hline SB5NEPH-01 & CCC & 1150 & surface: golden/bronze, shiny; bulk: clean & amorphous \\
\hline SB5NEPH-02 & CCC & 1150 & surface: black, shiny and clean; bulk: clean & amorphous \\
\hline SB5NEPH-03 & CCC & 1150 & $\begin{array}{l}\text { silvery/hazy surface with lots of crystals; bulk: } \\
\text { clean }\end{array}$ & Combeite $\left[\mathrm{Na}_{4} \mathrm{Ca}_{4}\left(\mathrm{Si}_{6} \mathrm{O}_{18}\right)\right]$, Nepheline $\left[\mathrm{Na}_{6} \mathrm{~K}_{1.2} \mathrm{Al}_{7.2} \mathrm{Si}_{8.8} \mathrm{O}_{32}\right]$ \\
\hline SB5NEPH-04 & CCC & 1150 & surface: black, shiny and clean; bulk: clean & amorphous \\
\hline SB5NEPH-05 & CCC & 1300 & $\begin{array}{l}\text { surface: shiny film with crystals; bulk: chocolate } \\
\text { brown, full of crystals }\end{array}$ & Magnetite $\left[\mathrm{Fe}^{2+} \mathrm{Fe}_{2}{ }^{3+} \mathrm{O}_{4}\right]$ \\
\hline SB5NEPH-06 & CCC & 1150 & surface: black, shiny and clean; bulk: crystals & Magnetite $\left[\mathrm{Fe}^{2+} \mathrm{Fe}_{2}^{3+} \mathrm{O}_{4}\right]$ \\
\hline SB5NEPH-07 & CCC & 1300 & surface: spotty crystals; bulk: crystals & Magnetite $\left[\mathrm{Fe}^{2+} \mathrm{Fe}_{2}{ }^{3+} \mathrm{O}_{4}\right]$, Aegirine $\left[\mathrm{NaFe}^{3+}\left(\mathrm{SiO}_{3}\right)_{2}\right]$ \\
\hline SB5NEPH-08 & CCC & 1150 & $\begin{array}{l}\text { surface: black and shiny with splotches of crystals; } \\
\text { bulk: clean }\end{array}$ & amorphous \\
\hline SB5NEPH-09 & CCC & 1450 & $\begin{array}{l}\text { surface: dull silver matte finish; bulk: devitrified, } \\
\text { purple/brown color }\end{array}$ & Magnetite $\left[\mathrm{Fe}^{2+} \mathrm{Fe}_{2}{ }^{3+} \mathrm{O}_{4}\right]$, Hercynite $\left[\mathrm{Fe}^{2+} \mathrm{Al}_{2} \mathrm{O}_{4}\right]$ \\
\hline SB5NEPH-10 & CCC & 1450 & surface: swirls of hazy crystals; bulk: crystallized & Trevorite $\left[\mathrm{NiFe}_{2} \mathrm{O}_{4}\right]$ \\
\hline SB5NEPH-11 & CCC & 1300 & surface: dull, matte; bulk: devitrified & Magnetite $\left[\mathrm{Fe}^{2+} \mathrm{Fe}_{2}{ }^{3+} \mathrm{O}_{4}\right]$, Anorthite [Ca ${ }_{66} \mathrm{Na}_{34} \mathrm{Al}_{1.66} \mathrm{Si}_{2.34} \mathrm{O}_{8}$ ] \\
\hline SB5NEPH-12 & CCC & 1300 & $\begin{array}{l}\text { surface: black, shiny, small crystals; bulk: } \\
\text { crystallized }\end{array}$ & Magnetite $\left[\mathrm{Fe}^{2+} \mathrm{Fe}_{2}^{3+} \mathrm{O}_{4}\right]$ \\
\hline SB5NEPH-13 & CCC & 1150 & surface: dull/silvery, crystallized; bulk: clean & Sodium Magnesium Silicon Oxide $\left[\mathrm{Na}_{1.74}\left(\mathrm{Mg}_{0.865} \mathrm{Si}_{1.135} \mathrm{O}_{4}\right)\right]$ \\
\hline
\end{tabular}


Table 3-1. Summary of the visual observations and XRD results for the nepheline study glasses. (continued)

\begin{tabular}{|c|c|c|c|c|}
\hline Glass ID & $\begin{array}{c}\text { Heat } \\
\text { Treatment }\end{array}$ & $\begin{array}{l}\mathbf{T}_{\text {Melt }} \\
\left({ }^{\circ} \mathbf{C}\right)\end{array}$ & Visual Observations & XRD Phase Identification \\
\hline SB5NEPH-14 & $\mathrm{CCC}$ & 1150 & surface: golden/purple, shiny surface; bulk: clean & amorphous \\
\hline SB5NEPH-15 & CCC & 1150 & surface: dull, crystallized; bulk: devitrified & $\begin{array}{l}\text { Magnetite }\left[\mathrm{Fe}^{2+} \mathrm{Fe}_{2}{ }^{3+} \mathrm{O}_{4}\right] \text {, Sodium magnesium silicon oxide } \\
{\left[\mathrm{Na}_{1.74}\left(\mathrm{Mg}_{0.865} \mathrm{Si}_{1.135} \mathrm{O}_{4}\right)\right] \text {, Combeite }\left[\mathrm{Na}_{4} \mathrm{Ca}_{4}\left(\mathrm{Si}_{6} \mathrm{O}_{18}\right)\right]}\end{array}$ \\
\hline SB5NEPH-16 & CCC & 1150 & $\begin{array}{l}\text { surface: crystallized, shiny with gold flecks; bulk: } \\
\text { clean }\end{array}$ & amorphous \\
\hline SB5NEPH-17 & CCC & 1300 & $\begin{array}{l}\text { surface: dull, completely crystallized; bulk: } \\
\text { devitrified }\end{array}$ & Nepheline $\left[\mathrm{Na}_{6} \mathrm{~K}_{1.2} \mathrm{Al}_{7.2} \mathrm{Si}_{8.8} \mathrm{O}_{32}\right]$ \\
\hline SB5NEPH-18 & CCC & 1300 & $\begin{array}{l}\text { surface: shiny with a few splotches of crystals; } \\
\text { bulk: chocolate brown, crystallized }\end{array}$ & Bunsenite $[\mathrm{NiO}]$ \\
\hline SB5NEPH-19 & CCC & 1300 & surface: dull, matte, devitrified; bulk: devitrified & $\begin{array}{l}\text { Nepheline }\left[\mathrm{Na}\left(\mathrm{AlSiO}_{4}\right)\right] \text {, Combeite }\left[\mathrm{Na}_{4.24} \mathrm{Ca}_{3.8}\left(\mathrm{Si}_{6} \mathrm{O}_{18}\right)\right] \text {, } \\
\text { Magnetite }\left[\mathrm{Fe}^{2+} \mathrm{Fe}_{2}{ }^{3+} \mathrm{O}_{4}\right] \text {, Jacobsite }\left[\mathrm{MnFe}_{2} \mathrm{O}_{4}\right]\end{array}$ \\
\hline SB5NEPH-20 & CCC & 1300 & $\begin{array}{l}\text { surface: black, shiny with splotches of crystals; } \\
\text { bulk: clean }\end{array}$ & amorphous \\
\hline SB5NEPH-21 & CCC & 1450 & surface: dull, matte; bulk: devitrified & Nepheline $\left[\mathrm{NaAlSiO}_{4}\right]$, Magnetite $\left[\mathrm{Fe}^{2+} \mathrm{Fe}_{2}{ }^{3+} \mathrm{O}_{4}\right]$ \\
\hline SB5NEPH-22 & CCC & 1300 & $\begin{array}{l}\text { surface: black, shiny, very light swirls; bulk: } \\
\text { crystallized }\end{array}$ & Magnetite $\left[\mathrm{Fe}^{2+} \mathrm{Fe}_{2}{ }^{3+} \mathrm{O}_{4}\right]$ \\
\hline SB5NEPH-23 & CCC & 1300 & surface: devitrified; bulk: devitrified & $\begin{array}{l}\text { Magnetite }\left[\mathrm{Fe}^{2+} \mathrm{Fe}_{2}{ }^{3+} \mathrm{O}_{4}\right] \text {, Nepheline }\left[\mathrm{NaAlSiO}_{4}\right] \text {, Aegirine } \\
\mathrm{NaFe}^{3+}\left(\mathrm{SiO}_{3}\right)_{2}\end{array}$ \\
\hline SB5NEPH-24 & CCC & 1150 & $\begin{array}{l}\text { surface: black and shiny with a few swirls of } \\
\text { crystals; bulk: crystallized }\end{array}$ & Magnetite $\left[\mathrm{Fe}^{2+} \mathrm{Fe}_{2}{ }^{3+} \mathrm{O}_{4}\right]$ \\
\hline SB5NEPH-25 & CCC & 1450 & surface: dull silver and devitrified; bulk: devitrified & $\begin{array}{l}\text { Magnetite }\left[\mathrm{Fe}^{2+} \mathrm{Fe}_{2}{ }^{3+} \mathrm{O}_{4}\right] \text {, Nickel aluminum oxide }\left[\mathrm{NiAl}_{2} \mathrm{O}_{4}\right] \text {, } \\
\text { Corundum }\left[\mathrm{Al}_{2} \mathrm{O}_{3}\right]\end{array}$ \\
\hline SB5NEPH-26 & CCC & 1300 & surface: dull, matte; bulk: devitrified & Trevorite $\left[\mathrm{NiFe}_{2} \mathrm{O}_{4}\right]$, Aluminum borate $\left[\mathrm{Al}_{4} \mathrm{~B}_{2} \mathrm{O}_{9}\right]$ \\
\hline SB5NEPH-27 & CCC & 1300 & surface: dulle, matte; bulk: devitrified & $\begin{array}{l}\text { Nepheline }\left[\mathrm{NaAlSiO}_{4}\right] \text {, Anorthite }\left[\mathrm{Ca}\left(\mathrm{Al}_{2} \mathrm{Si}_{2} \mathrm{O}_{8}\right)\right] \text {, Magnetite } \\
{\left[\mathrm{Fe}^{2+} \mathrm{Fe}_{2}{ }^{3+} \mathrm{O}_{4}\right]}\end{array}$ \\
\hline SB5NEPH-28 & CCC & 1300 & $\begin{array}{l}\text { surface: black and shiny, a few crystals; bulk: } \\
\text { crystallized }\end{array}$ & Magnetite $\left[\mathrm{Fe}^{2+} \mathrm{Fe}_{2}{ }^{3+} \mathrm{O}_{4}\right]$ \\
\hline SB5NEPH-29 & CCC & 1150 & surface: dimpled and crystallized; bulk: devitrified & $\begin{array}{l}\text { Sodium silicate }\left[\mathrm{Na}_{2} \mathrm{SiO}_{3}\right] \text {, Sodium iron oxide }\left[\mathrm{NaFeO}_{2}\right] \text {, } \\
\text { Sodium magnesium aluminum silicate }\left[\mathrm{Na}_{3} \mathrm{MgAlSi}{ }_{2} \mathrm{O}_{8}\right] \text {, Silicon } \\
\text { oxide }\left[\mathrm{SiO}_{2}\right]\end{array}$ \\
\hline
\end{tabular}


Table 3-1. Summary of the visual observations and XRD results for the nepheline study glasses. (continued)

\begin{tabular}{|c|c|c|c|c|}
\hline Glass ID & $\begin{array}{c}\text { Heat } \\
\text { Treatment }\end{array}$ & $\begin{array}{l}\mathbf{T}_{\text {Melt }} \\
\left({ }^{\circ} \mathrm{C}\right)\end{array}$ & Visual Observations & XRD Phase Identification \\
\hline SB5NEPH-30 & CCC & 1150 & surface: dull, matte, crystallized; bulk: devitrified & $\begin{array}{l}\text { Sodium aluminum silicate }\left[\mathrm{Na}_{1.75} \mathrm{Al}_{1.75} \mathrm{Si}_{0.25} \mathrm{O}_{4}\right] \text {, Tincalconite } \\
{\left[\mathrm{Na}_{2} \mathrm{~B}_{4} \mathrm{O}_{7} \cdot 5 \mathrm{H}_{2} \mathrm{O}\right] \text {, Thermonatrite }\left[\mathrm{Na}_{2} \mathrm{CO}_{3} \cdot \mathrm{H}_{2} \mathrm{O}\right] \text {, Borax }} \\
{\left[\mathrm{Na}_{2} \mathrm{~B}_{4} \mathrm{O}_{5}(\mathrm{OH})_{4}\left(\mathrm{H}_{2} \mathrm{O}\right)_{8}\right] \text {, Sodium borate }\left[\mathrm{Na}_{4} \mathrm{~B}_{10} \mathrm{O}_{17}\right]}\end{array}$ \\
\hline SB5NEPH-31 & CCC & 1150 & surface: shiny but crystallized; bulk: devitrified & $\begin{array}{l}\text { Sodium aluminum silicate }\left[\left(\mathrm{Na}_{2} \mathrm{O}\right)_{0.33} \mathrm{NaAlSiO}_{4}\right] \text {, Thermonatrite } \\
{\left[\mathrm{Na}_{2} \mathrm{CO}_{3} \cdot \mathrm{H}_{2} \mathrm{O}\right] \text {, Rankinite }\left[\mathrm{Ca}_{3} \mathrm{Si}_{2} \mathrm{O}_{7}\right] \text {, Calcium silicate }} \\
{\left[\mathrm{Ca}_{2}\left(\mathrm{SiO}_{4}\right)\right]}\end{array}$ \\
\hline SB5NEPH-32 & CCC & 1150 & $\begin{array}{l}\text { surface: devitrified layer with golden crystals; bulk: } \\
\text { black and shiny with crystals }\end{array}$ & $\begin{array}{l}\text { Sodium aluminum silicate oxide }\left[\mathrm{Na}_{1.67} \mathrm{Al}\left(\mathrm{SiO}_{4}\right) \mathrm{O}_{0.33}\right] \text {, Jacobsite } \\
{\left[\mathrm{MnFe}_{2} \mathrm{O}_{4}\right] \text {, Calcium borate }\left[\mathrm{Ca}_{2} \mathrm{~B}_{2} \mathrm{O}_{5}\right] \text {, Calcium borate }} \\
{\left[\mathrm{Ca}\left(\mathrm{B}_{4} \mathrm{O}_{7}\right)\right] \text {, plus unidentifiable }}\end{array}$ \\
\hline SB5NEPH-33 & CCC & 1300 & surface: dull, matte brown; bulk: devitrified & $\begin{array}{l}\text { Nepheline }\left[\mathrm{NaAlSiO}_{4}\right], \text { Magnetite }\left[\mathrm{Fe}^{2+} \mathrm{Fe}_{2}{ }^{3+} \mathrm{O}_{4}\right] \text {, Aegirine } \\
{\left[\mathrm{NaFe}\left(\mathrm{Si}_{2} \mathrm{O}_{6}\right)\right]}\end{array}$ \\
\hline SB5NEPH-34 & CCC & 1300 & surface: crystallized; bulk: devitrified & $\begin{array}{l}\text { Nepheline }\left[\mathrm{NaAlSiO}_{4}\right] \text {, Magnetite }\left[\mathrm{Fe}^{2+} \mathrm{Fe}_{2}{ }^{3+} \mathrm{O}_{4}\right] \text {, Jacobsite } \\
{\left[\mathrm{MnFe}_{2} \mathrm{O}_{4}\right]}\end{array}$ \\
\hline SB5NEPH-35 & CCC & 1300 & surface: black, matte; bulk: devitrified & $\begin{array}{l}\text { Nepheline }\left[\mathrm{NaAlSiO}_{4}\right] \text {, Sodium aluminum silicate } \\
\left.\left[\mathrm{Na}_{1.55} \mathrm{Al}_{1.55} \mathrm{Si}_{.45} \mathrm{O}_{4}\right] \text {, Sodium aluminum silicate }\left[\mathrm{Na}_{6}(\mathrm{AlSiO})_{4}\right)_{6}\right] \\
\text { Nekoite }\left[\mathrm{Ca}_{3} \mathrm{Si}_{6} \mathrm{O}_{12}(\mathrm{OH})_{6} \cdot 5 \mathrm{H}_{2} \mathrm{O}\right] \text {, Manganese iron oxide } \\
{\left[\mathrm{Mn}_{.43} \mathrm{Fe}_{2.57} \mathrm{Fe}_{2.57} \mathrm{O}_{4}\right] \text {, Calcium silicate }\left[\mathrm{Ca}_{2} \mathrm{SiO}_{4}\right]}\end{array}$ \\
\hline SB5NEPH-36 & CCC & 1300 & surface: dull, matte, crystallized; bulk: devitrified & amorphous \\
\hline SB5NEPH-37 & CCC & 1450 & $\begin{array}{l}\text { did not melt completely, dull matte surface, bulk } \\
\text { devitrified }\end{array}$ & $\begin{array}{l}\text { Trevorite }\left[\mathrm{NiFe}_{2} \mathrm{O}_{4}\right] \text {, Aluminum oxide }\left[\mathrm{Al}_{2} \mathrm{O}_{3}\right] \text {, Hematite } \\
{\left[\mathrm{Fe}_{2} \mathrm{O}_{3}\right]}\end{array}$ \\
\hline SB5NEPH-38 & CCC & 1300 & did not melt completely, devitrified & $\begin{array}{l}\text { Trevorite }\left[\mathrm{NiFe}_{2} \mathrm{O}_{4}\right] \text {, Aluminum oxide }\left[\mathrm{Al}_{2} \mathrm{O}_{3}\right] \text {, Hematite } \\
{\left[\mathrm{Fe}_{2} \mathrm{O}_{3}\right] \text {, Aluminum borate }\left[\mathrm{Al}_{4} \mathrm{~B}_{2} \mathrm{O}_{9}\right]}\end{array}$ \\
\hline SB5NEPH-39 & CCC & 1450 & did not melt completely, devitrified & $\begin{array}{l}\left.\text { Nepheline [NaAlSiO } \mathrm{N}_{4}\right] \text {, Calcium aluminum iron oxide carbonate } \\
\text { hydroxide hydrate }\left[\mathrm{Ca}_{8} \mathrm{Al}_{2} \mathrm{Fe}_{2} \mathrm{O}_{12} \mathrm{CO}_{3}(\mathrm{OH})_{2} \cdot 22 \mathrm{H}_{2} \mathrm{O}\right] \text {, Calcium } \\
\text { aluminum oxide hydrate }\left[\mathrm{Ca}_{2} \mathrm{Al}_{2} \mathrm{O}_{5} \cdot 6 \mathrm{H}_{2} \mathrm{O}\right]\end{array}$ \\
\hline SB5NEPH-40 & CCC & 1300 & surface: black and shiny; bulk: crystallized & Trevorite $\left[\mathrm{NiFe}_{2} \mathrm{O}_{4}\right]$ \\
\hline
\end{tabular}


In general, the visual observations indicate that the amount of crystallization increased as the nepheline discriminator value of the glasses decreased. In some cases (e.g., the quenched versions of SB5NEPH-19, SB5NEPH-30, etc.), crystallization was noted during visual observation of the glasses but not identified by XRD. This is likely due to the volume fraction of crystallization being lower than what was detectible via XRD.

Nepheline or other crystalline phases that are expected to have the same impact on durability as nepheline (e.g., Anorthite [Ca $\left.{ }_{66} \mathrm{Na}_{34} \mathrm{Al}_{1.66} \mathrm{Si}_{2.34} \mathrm{O}_{8}\right]$ ), were detected in several of the glasses and are highlighted in Table 3-1. Several of the other crystalline phases detected, such as trevorite and magnetite, have been shown to have little impact on the durability of DWPF-type glasses ${ }^{22}$ and are not of concern to this study. Further discussion on the glasses that contained nepheline will be provided below in combination with the PCT results.

\subsection{A Statistical Review of the PCT Results}

The study glasses, after being batched and fabricated, were subjected to the 7-day PCT Method-A. ${ }^{19}$ Both heat treatments (quenched and CCC) for each study glass were subjected to the PCT in triplicate. PCTs were also conducted in triplicate for samples of the EA glass and for samples of the ARM glass. Blanks (samples consisting only of ASTM Type-I water) were also submitted for the PCT.

Four analytical plans were provided to the PSAL to support the measurement of the compositions of the solutions resulting from the PCTs. ${ }^{21}$ Samples of a multi-element, standard solution were included in the analytical plans (as a check on the accuracy of the ICP-AES used for these measurements). In this and the following sections, the measurements generated by the PSAL for these PCTs are presented and reviewed.

Table B1 in Appendix B provides the elemental leachate concentration measurements determined by the PSAL for the solution samples generated by the PCTs by analytical set. One of the quality control checkpoints for the PCT procedure is solution-weight loss over the course of the 7-day test. There were some water loss problems for the third set of PCTs. Sets 1, 2, and 4 had no problems. The following PCTs had water loss problems: all three solutions of the quenched version of SB5NEPH-26 (Lab IDs J32, J20, and J05), SB5NEPH-26CCC-2 and -3 (Lab IDs J23 and J02), and the first solution of the quenched version of SB5NEPH-27 (Lab ID J11). In addition, the PCTs for the CCC version of SB5-NEPH-29 (Lab IDs J58, J33, and J22) all formed gel, indicating significant leaching but not allowing for an appropriate measurement for the PCT. Any measurement in Table B1 below the detection limit of the analytical procedure (indicated by a " $<$ ”) was replaced by one half of the detection limit in subsequent analyses. In addition to adjustments for detection limits, the values were adjusted for the dilution factors: the factor for the study glasses, the blanks, and the ARM glass in Table B1 was 1.6667 and the factor for EA was 16.6667. Table B2 in Appendix B provides the resulting measurements.

In the sections that follow, the analytical sequence of the measurements over the four sets of PCTs is explored, the measurements of the standards are investigated and used to assess the overall accuracy of the ICP-AES measurement process, the measurements for each glass are reviewed, plots are provided that explore the effects of heat treatment on the PCTs for these glasses, and the PCTs are normalized using the compositions (targeted, measured, and biascorrected) presented in Table A4 in Appendix A. 


\subsubsection{Measurements in Analytical Sequence}

Exhibits B1 and B2 in Appendix B provide plots of the leachate concentrations (ppm) in analytical sequence as generated by the PSAL for all of the data and for the data from only the study glasses, respectively. A different color and symbol are used for each study glass or standard. No issues with the leachate measurements are seen in these plots.

\subsubsection{Results for the Samples of the Multi-Element Solution Standard}

Exhibit B3 in Appendix B provides analyses of the PSAL measurements of the samples of the multi-element solution standard by analytical set by ICP-AES block. An ANOVA investigating for statistically significant differences among the block averages for these samples for each element of interest is included in these exhibits. These results indicate a statistically significant (at approximately a 5\% level) difference among the Li average measurements, among the $\mathrm{Na}$ average measurements, and among the $\mathrm{Si}$ average measurements over these sets and blocks. However, no bias correction of the PCT results for the study glasses was conducted. This approach was taken since the triplicate PCTs for a single study glass were placed in different ICPAES blocks. Averaging the concentration measurements for each set of triplicates helps to minimize the impact of the ICP-AES effects.

Table 3-2 summarizes the average measurements and the reference values for the four primary elements of interest. The results indicate consistent and accurate measurements from the PSAL processes used to conduct these analyses.

Table 3-2. Results from samples of the multi-element solution standard.

\begin{tabular}{||c|c|c|c|c|c||}
\hline $\begin{array}{c}\text { Analytical } \\
\text { Set }\end{array}$ & $\begin{array}{c}\text { Analytical } \\
\text { Block }\end{array}$ & $\begin{array}{c}\text { Avg B } \\
(\mathbf{p p m})\end{array}$ & $\begin{array}{c}\text { Avg Li } \\
(\mathbf{p p m})\end{array}$ & $\begin{array}{c}\text { Avg Na } \\
(\mathbf{p p m})\end{array}$ & $\begin{array}{c}\text { Avg Si } \\
(\mathbf{p p m})\end{array}$ \\
\hline 1 & 1 & 20.8 & 9.7 & 81.9 & 49.8 \\
\hline & 2 & 21.0 & 10.3 & 83.8 & 51.9 \\
\hline & 3 & 20.3 & 9.7 & 85.0 & 48.0 \\
\hline 2 & 1 & 21.7 & 10.0 & 85.5 & 49.7 \\
\hline & 2 & 20.8 & 9.6 & 85.5 & 48.8 \\
\hline & 3 & 19.7 & 9.7 & 85.4 & 48.9 \\
\hline 3 & 1 & 22.0 & 9.8 & 82.8 & 48.9 \\
\hline & 2 & 20.1 & 9.7 & 80.2 & 48.7 \\
\hline & 3 & 20.7 & 9.7 & 81.8 & 48.1 \\
\hline 4 & 1 & 21.8 & 9.9 & 82.6 & 51.5 \\
\hline & 2 & 21.2 & 9.6 & 82.9 & 47.4 \\
\hline & 3 & 20.1 & 9.8 & 80.9 & 48.9 \\
\hline & $\begin{array}{c}\text { Grand } \\
\text { Average }\end{array}$ & $\mathbf{2 0 . 9}$ & $\mathbf{9 . 8}$ & $\mathbf{8 3 . 2}$ & $\mathbf{4 9 . 2}$ \\
\hline & $\begin{array}{c}\text { Reference } \\
\text { Value }\end{array}$ & $\mathbf{2 0}$ & $\mathbf{1 0}$ & $\mathbf{8 1}$ & $\mathbf{5 0}$ \\
\hline & \% Difference & $\mathbf{4 . 3 3 \%}$ & $\mathbf{- 2 . 0 5 \%}$ & $\mathbf{2 . 7 1 \%}$ & $\mathbf{- 1 . 5 7 \%}$ \\
\hline
\end{tabular}

\subsubsection{Measurements by Glass Number}

Exhibit B4 in Appendix B provides plots of the leachate concentrations for each type of submitted sample by analytical set: the study glasses by heat treatment and the benchmark glasses (EA (101), ARM (102), the multi-element solution standard (100), and blanks (103)). These plots allow for the assessment of the repeatability of the measurements, which suggests some scatter in 
the triplicate values for some analytes for some of the glasses. Also, note that some differences between the values for the two heat treatments for some glasses are evident.

PCT results for the ARM and EA benchmark glasses are provided in Table 3-3. All but two of the measured values for the ARM benchmark glass fell within the defined control limits. ${ }^{23}$ One of the ARM triplicate samples in each of the third and fourth PCT sets had a measured B concentration that was greater than the control limit. According to the ASTM standard, ${ }^{19}$ the PCT values reported in this study should be bias corrected using the measured values for the ARM glass before comparisons are made with other reports. This bias correction is expected to have no significant impact on the results presented here and was therefore not performed at this time. The average NL [B] for the EA glass measured in this study was $18.94 \mathrm{~g} / \mathrm{L}$, which is higher than the reference value of $16.695 \mathrm{~g} / \mathrm{L}{ }^{20}$ Some variation in the measured leach rate of the EA glass is apparent in other recent investigations, ${ }^{24-29}$ and should not impact the conclusions of this study.

Table 3-3. PCT results for the ARM and EA benchmark glasses.

\begin{tabular}{|c|c|c|c|c|c|c|c|c|c|}
\hline Set & $\begin{array}{c}\text { Glass } \\
\text { ID }\end{array}$ & $\begin{array}{c}\log N \mathrm{~L} \\
{[\mathrm{~B}(\mathrm{~g} / \mathrm{L})]}\end{array}$ & $\begin{array}{c}\log N L \\
{[\mathrm{Li}(\mathrm{g} / \mathrm{L})]}\end{array}$ & $\begin{array}{c}\log \mathrm{NL} \\
{[\mathrm{Na}(\mathrm{g} / \mathrm{L})]}\end{array}$ & $\begin{array}{c}\log N L \\
{[\mathrm{Si}(\mathrm{g} / \mathrm{L})]}\end{array}$ & $\begin{array}{c}\text { NL } \\
{[B(g / L)]}\end{array}$ & $\begin{array}{c}\mathrm{NL} \\
{[\mathrm{Li}(\mathrm{g} / \mathrm{L})]}\end{array}$ & $\begin{array}{c}\mathrm{NL} \\
{[\mathrm{Na}(\mathrm{g} / \mathrm{L})]}\end{array}$ & $\begin{array}{c}\text { NL } \\
{[\mathrm{Si}(\mathrm{g} / \mathrm{L})]}\end{array}$ \\
\hline 1 & ARM & -0.229 & -0.377 & 0.066 & -0.639 & 0.59 & 0.42 & 1.16 & 0.23 \\
\hline 2 & ARM & -0.229 & -0.383 & 0.076 & -0.646 & 0.59 & 0.41 & 1.19 & 0.23 \\
\hline 3 & ARM & -0.225 & -0.384 & 0.056 & -0.651 & 0.60 & 0.41 & 1.14 & 0.22 \\
\hline 4 & ARM & -0.223 & -0.384 & 0.059 & -0.645 & 0.60 & 0.41 & 1.15 & 0.23 \\
\hline 1 & EA & 1.250 & 0.973 & 1.132 & 0.593 & 17.77 & 9.40 & 13.56 & 3.92 \\
\hline 2 & EA & 1.291 & 0.984 & 1.249 & 0.598 & 19.54 & 9.63 & 17.75 & 3.96 \\
\hline 3 & EA & 1.271 & 0.982 & 1.169 & 0.588 & 18.68 & 9.60 & 14.77 & 3.88 \\
\hline 4 & EA & 1.296 & 0.979 & 1.139 & 0.596 & 19.77 & 9.52 & 13.77 & 3.95 \\
\hline
\end{tabular}

\subsubsection{Normalized PCT Results}

PCT leachate concentrations are typically normalized using the cation composition (expressed as a weight percent) in the glass to obtain a grams-per-liter ( $\mathrm{g} / \mathrm{L}$ ) leachate concentration. Two of the three replicate PCTs for a glass (whether the quenched or the CCC version) must be available for this computation to be completed. Otherwise, no PCT can be reported for the glass. As mentioned earlier, water loss and gelling issues prevent the reporting of normalized release rates for glasses SB5NEPH-26 quenched, SB5NEPH-26 CCC and SB5NEPH-29 CCC. The normalization of the PCTs is usually conducted using the measured compositions of the glasses. This is the preferred normalization process for the PCTs. For completeness, the targeted cation and the bias-corrected cation compositions were also used to conduct this normalization.

As is the usual convention, the common logarithm of the normalized PCT (normalized leachate, $\mathrm{NL}$ ) for each element of interest was determined and used for comparison. To accomplish this computation, one must:

1.Determine the common logarithm of the elemental leachate concentration for each of the triplicates and each of the elements of interest (these values are provided in Table B2 of Appendix B),

2. Average the common logarithms over the triplicates for each element of interest, and then

Normalizing Using Measured Composition (preferred method) 
3. Subtract a quantity equal to 1 plus the common logarithm of the average cation measured concentration (expressed as a weight percent of the glass) from the average computed in step 2.

Or Normalizing Using Target Composition

3. Subtract a quantity equal to 1 plus the common logarithm of the target cation concentration (expressed as a weight percent of the glass) from the average computed in step 2 .

Or Normalizing Using Measured Bias-Corrected Composition

3. Subtract a quantity equal to 1 plus the common logarithm of the measured bias-corrected cation concentration (expressed as a weight percent of the glass) from the average computed in step 2.

Exhibit B5 in Appendix B provides scatter plots for these results and offers an opportunity to investigate the consistency in the leaching across the elements for the glasses of this study. All combinations of the normalizations of the PCTs (i.e., those generated using the targeted, measured, and bias-corrected compositional views) and both heat treatments are represented in the series of scatter plots. Consistency in the leaching across the elements is typically demonstrated by a high degree of linear correlation among the values for pairs of these elements. The smallest correlation in this plot is between $\mathrm{B}$ and $\mathrm{Si}$, with a value of $\sim 58 \%$, which is a lower correlation than typically seen for a set of PCTs. This indicates some inconsistency in the degree of leaching that occurred for $\mathrm{B}$ relative to $\mathrm{Si}$, and is likely due to the large variation in the glass compositions studied. This inconsistency will not impact the conclusions of this study.

Table 3-4 summarizes the normalized PCTs for the glasses of this study. The glasses are listed by analytical set, glass identifier, heat treatment and compositional view. Normalized release data for $\mathrm{Li}$ are not shown since the study glasses did not contain $\mathrm{Li}$. 
Table 3-4. Normalized PCT Results by Glass ID and Compositional View.

\begin{tabular}{|c|c|c|c|c|c|c|c|c|c|}
\hline Set & Glass ID & $\begin{array}{c}\text { Heat } \\
\text { Treatment }\end{array}$ & $\begin{array}{c}\text { Compositional } \\
\text { View }\end{array}$ & $\begin{array}{c}\log N \mathrm{~L} \\
{[\mathrm{~B}(\mathrm{~g} / \mathrm{L})]}\end{array}$ & $\begin{array}{c}\log \mathbf{N L} \\
{[\mathrm{Na}(\mathrm{g} / \mathrm{L})]}\end{array}$ & $\begin{array}{c}\log \mathrm{NL} \\
{[\mathrm{Si}(\mathrm{g} / \mathrm{L})]}\end{array}$ & $\begin{array}{c}\text { NL } \\
{[B(g / L)]}\end{array}$ & $\begin{array}{c}\text { NL } \\
{[\mathrm{Na}(\mathrm{g} / \mathrm{L})]}\end{array}$ & $\begin{array}{c}\text { NL } \\
{[\mathrm{Si}(\mathrm{g} / \mathrm{L})]}\end{array}$ \\
\hline 1 & SB5NEPH-01 & $\mathrm{CCC}$ & targeted & 0.068 & 0.255 & -0.174 & 1.17 & 1.80 & 0.67 \\
\hline 1 & SB5NEPH-01 & CCC & measured & 0.067 & 0.255 & -0.173 & 1.17 & 1.80 & 0.67 \\
\hline 1 & SB5NEPH-01 & CCC & measured bc & 0.054 & 0.264 & -0.180 & 1.13 & 1.84 & 0.66 \\
\hline 1 & SB5NEPH-01 & quenched & targeted & -0.003 & 0.276 & -0.168 & 0.99 & 1.89 & 0.68 \\
\hline 1 & SB5NEPH-01 & quenched & measured & -0.004 & 0.276 & -0.166 & 0.99 & 1.89 & 0.68 \\
\hline 1 & SB5NEPH-01 & quenched & measured bc & -0.017 & 0.284 & -0.174 & 0.96 & 1.92 & 0.67 \\
\hline 1 & SB5NEPH-02 & $\mathrm{CCC}$ & targeted & 0.833 & 0.730 & -0.451 & 6.81 & 5.38 & 0.35 \\
\hline 1 & SB5NEPH-02 & CCC & measured & 0.822 & 0.734 & -0.454 & 6.64 & 5.42 & 0.35 \\
\hline 1 & SB5NEPH-02 & CCC & measured bc & 0.815 & 0.743 & -0.467 & 6.53 & 5.53 & 0.34 \\
\hline 1 & SB5NEPH-02 & quenched & targeted & 0.888 & 0.790 & -0.469 & 7.74 & 6.17 & 0.34 \\
\hline 1 & SB5NEPH-02 & quenched & measured & 0.878 & 0.794 & -0.472 & 7.55 & 6.22 & 0.34 \\
\hline 1 & SB5NEPH-02 & quenched & measured bc & 0.870 & 0.802 & -0.485 & 7.41 & 6.34 & 0.33 \\
\hline 1 & SB5NEPH-03 & CCC & targeted & 0.219 & 0.340 & -0.402 & 1.66 & 2.19 & 0.40 \\
\hline 1 & SB5NEPH-03 & $\mathrm{CCC}$ & measured & 0.220 & 0.339 & -0.406 & 1.66 & 2.18 & 0.39 \\
\hline 1 & SB5NEPH-03 & CCC & measured bc & 0.207 & 0.347 & -0.413 & 1.61 & 2.23 & 0.39 \\
\hline 1 & SB5NEPH-03 & quenched & targeted & -0.155 & 0.200 & -0.575 & 0.70 & 1.58 & 0.27 \\
\hline 1 & SB5NEPH-03 & quenched & measured & -0.154 & 0.199 & -0.579 & 0.70 & 1.58 & 0.26 \\
\hline 1 & SB5NEPH-03 & quenched & measured bc & -0.167 & 0.208 & -0.586 & 0.68 & 1.61 & 0.26 \\
\hline 1 & SB5NEPH-04 & CCC & targeted & 0.485 & 0.573 & -0.398 & 3.06 & 3.74 & 0.40 \\
\hline 1 & SB5NEPH-04 & CCC & measured & 0.478 & 0.570 & -0.407 & 3.01 & 3.72 & 0.39 \\
\hline 1 & SB5NEPH-04 & $\mathrm{CCC}$ & measured bc & 0.465 & 0.579 & -0.415 & 2.92 & 3.79 & 0.38 \\
\hline 1 & SB5NEPH-04 & quenched & targeted & 0.480 & 0.556 & -0.431 & 3.02 & 3.60 & 0.37 \\
\hline 1 & SB5NEPH-04 & quenched & measured & 0.473 & 0.553 & -0.440 & 2.97 & 3.57 & 0.36 \\
\hline 1 & SB5NEPH-04 & quenched & measured bc & 0.460 & 0.562 & -0.447 & 2.88 & 3.65 & 0.36 \\
\hline 1 & SB5NEPH-05 & CCC & targeted & -0.559 & -0.445 & -0.700 & 0.28 & 0.36 & 0.20 \\
\hline 1 & SB5NEPH-05 & CCC & measured & -0.547 & -0.450 & -0.695 & 0.28 & 0.35 & 0.20 \\
\hline 1 & SB5NEPH-05 & CCC & measured bc & -0.560 & -0.439 & -0.703 & 0.28 & 0.36 & 0.20 \\
\hline 1 & SB5NEPH-05 & quenched & targeted & -0.390 & -0.279 & -0.605 & 0.41 & 0.53 & 0.25 \\
\hline 1 & SB5NEPH-05 & quenched & measured & -0.378 & -0.285 & -0.600 & 0.42 & 0.52 & 0.25 \\
\hline 1 & SB5NEPH-05 & quenched & measured bc & -0.391 & -0.273 & -0.607 & 0.41 & 0.53 & 0.25 \\
\hline 1 & SB5NEPH-06 & CCC & targeted & 0.067 & -0.051 & -0.629 & 1.17 & 0.89 & 0.23 \\
\hline 1 & SB5NEPH-06 & CCC & measured & 0.056 & -0.063 & -0.637 & 1.14 & 0.87 & 0.23 \\
\hline 1 & SB5NEPH-06 & CCC & measured bc & 0.048 & -0.054 & -0.650 & 1.12 & 0.88 & 0.22 \\
\hline 1 & SB5NEPH-06 & quenched & targeted & -0.589 & -0.411 & -0.786 & 0.26 & 0.39 & 0.16 \\
\hline 1 & SB5NEPH-06 & quenched & measured & -0.599 & -0.423 & -0.794 & 0.25 & 0.38 & 0.16 \\
\hline 1 & SB5NEPH-06 & quenched & measured bc & -0.607 & -0.414 & -0.807 & 0.25 & 0.39 & 0.16 \\
\hline 1 & SB5NEPH-07 & CCC & targeted & -0.566 & -0.454 & -0.972 & 0.27 & 0.35 & 0.11 \\
\hline 1 & SB5NEPH-07 & $\mathrm{CCC}$ & measured & -0.575 & -0.462 & -0.972 & 0.27 & 0.34 & 0.11 \\
\hline 1 & SB5NEPH-07 & CCC & measured bc & -0.583 & -0.451 & -0.985 & 0.26 & 0.35 & 0.10 \\
\hline 1 & SB5NEPH-07 & quenched & targeted & -0.519 & -0.415 & -0.981 & 0.30 & 0.38 & 0.10 \\
\hline 1 & SB5NEPH-07 & quenched & measured & -0.529 & -0.423 & -0.981 & 0.30 & 0.38 & 0.10 \\
\hline 1 & SB5NEPH-07 & quenched & measured bc & -0.536 & -0.412 & -0.994 & 0.29 & 0.39 & 0.10 \\
\hline 1 & SB5NEPH-08 & CCC & targeted & -0.288 & -0.232 & -0.993 & 0.52 & 0.59 & 0.10 \\
\hline 1 & SB5NEPH-08 & $\mathrm{CCC}$ & measured & -0.293 & -0.243 & -1.004 & 0.51 & 0.57 & 0.10 \\
\hline 1 & SB5NEPH-08 & $\mathrm{CCC}$ & measured bc & -0.306 & -0.231 & -1.012 & 0.49 & 0.59 & 0.10 \\
\hline 1 & SB5NEPH-08 & quenched & targeted & -0.207 & -0.124 & -0.983 & 0.62 & 0.75 & 0.10 \\
\hline 1 & SB5NEPH-08 & quenched & measured & -0.212 & -0.135 & -0.994 & 0.61 & 0.73 & 0.10 \\
\hline 1 & SB5NEPH-08 & quenched & measured bc & -0.225 & -0.123 & -1.001 & 0.60 & 0.75 & 0.10 \\
\hline 1 & SB5NEPH-09 & CCC & targeted & -0.714 & -0.547 & -1.027 & 0.19 & 0.28 & 0.09 \\
\hline 1 & SB5NEPH-09 & $\mathrm{CCC}$ & measured & -0.735 & -0.568 & -1.014 & 0.18 & 0.27 & 0.10 \\
\hline
\end{tabular}


Table 3-4. Normalized PCT Results by Glass ID and Compositional View. (continued)

\begin{tabular}{|c|c|c|c|c|c|c|c|c|c|}
\hline Set & Glass ID & $\begin{array}{c}\text { Heat } \\
\text { Treatment }\end{array}$ & $\begin{array}{c}\text { Compositional } \\
\text { View }\end{array}$ & $\begin{array}{c}\log N L \\
{[B(g / L)]}\end{array}$ & $\begin{array}{c}\log \mathbf{N L} \\
{[\mathrm{Na}(\mathrm{g} / \mathrm{L})]}\end{array}$ & $\begin{array}{c}\log \mathrm{NL} \\
{[\mathrm{Si}(\mathrm{g} / \mathrm{L})]}\end{array}$ & $\begin{array}{c}\text { NL } \\
{[B(g / L)]}\end{array}$ & $\begin{array}{c}\text { NL } \\
{[\mathrm{Na}(\mathrm{g} / \mathrm{L})]}\end{array}$ & $\begin{array}{c}\text { NL } \\
{[\mathrm{Si}(\mathrm{g} / \mathrm{L})]}\end{array}$ \\
\hline 1 & SB5NEPH-09 & $\mathrm{CCC}$ & measured bc & -0.742 & -0.556 & -1.027 & 0.18 & 0.28 & 0.09 \\
\hline 1 & SB5NEPH-09 & quenched & targeted & -0.749 & -0.896 & -1.145 & 0.18 & 0.13 & 0.07 \\
\hline 1 & SB5NEPH-09 & quenched & measured & -0.770 & -0.917 & -1.131 & 0.17 & 0.12 & 0.07 \\
\hline 1 & SB5NEPH-09 & quenched & measured bc & -0.777 & -0.906 & -1.144 & 0.17 & 0.12 & 0.07 \\
\hline 1 & SB5NEPH-10 & CCC & targeted & -0.604 & -0.615 & -0.909 & 0.25 & 0.24 & 0.12 \\
\hline 1 & SB5NEPH-10 & $\mathrm{CCC}$ & measured & -0.618 & -0.641 & -0.908 & 0.24 & 0.23 & 0.12 \\
\hline 1 & SB5NEPH-10 & $\mathrm{CCC}$ & measured bc & -0.626 & -0.629 & -0.921 & 0.24 & 0.23 & 0.12 \\
\hline 1 & SB5NEPH-10 & quenched & targeted & -0.760 & -0.845 & -1.036 & 0.17 & 0.14 & 0.09 \\
\hline 1 & SB5NEPH-10 & quenched & measured & -0.775 & -0.870 & -1.034 & 0.17 & 0.13 & 0.09 \\
\hline 1 & SB5NEPH-10 & quenched & measured bc & -0.782 & -0.859 & -1.047 & 0.17 & 0.14 & 0.09 \\
\hline 2 & SB5NEPH-11 & $\mathrm{CCC}$ & targeted & -0.080 & -0.476 & -1.219 & 0.83 & 0.33 & 0.06 \\
\hline 2 & SB5NEPH-11 & $\mathrm{CCC}$ & measured & -0.089 & -0.496 & -1.232 & 0.82 & 0.32 & 0.06 \\
\hline 2 & SB5NEPH-11 & $\mathrm{CCC}$ & measured bc & -0.098 & -0.490 & -1.240 & 0.80 & 0.32 & 0.06 \\
\hline 2 & SB5NEPH-11 & quenched & targeted & -0.378 & -0.822 & -1.255 & 0.42 & 0.15 & 0.06 \\
\hline 2 & SB5NEPH-11 & quenched & measured & -0.387 & -0.842 & -1.268 & 0.41 & 0.14 & 0.05 \\
\hline 2 & SB5NEPH-11 & quenched & measured bc & -0.397 & -0.836 & -1.276 & 0.40 & 0.15 & 0.05 \\
\hline 2 & SB5NEPH-12 & CCC & targeted & -0.656 & -0.580 & -1.207 & 0.22 & 0.26 & 0.06 \\
\hline 2 & SB5NEPH-12 & CCC & measured & -0.665 & -0.593 & -1.215 & 0.22 & 0.26 & 0.06 \\
\hline 2 & SB5NEPH-12 & $\mathrm{CCC}$ & measured bc & -0.675 & -0.587 & -1.223 & 0.21 & 0.26 & 0.06 \\
\hline 2 & SB5NEPH-12 & quenched & targeted & -0.529 & -0.487 & -1.297 & 0.30 & 0.33 & 0.05 \\
\hline 2 & SB5NEPH-12 & quenched & measured & -0.539 & -0.500 & -1.305 & 0.29 & 0.32 & 0.05 \\
\hline 2 & SB5NEPH-12 & quenched & measured bc & -0.548 & -0.495 & -1.313 & 0.28 & 0.32 & 0.05 \\
\hline 2 & SB5NEPH-13 & CCC & targeted & 0.751 & 0.989 & 0.314 & 5.63 & 9.74 & 2.06 \\
\hline 2 & SB5NEPH-13 & $\mathrm{CCC}$ & measured & 0.747 & 1.001 & 0.313 & 5.59 & 10.03 & 2.05 \\
\hline 2 & SB5NEPH-13 & CCC & measured bc & 0.737 & 1.007 & 0.305 & 5.46 & 10.17 & 2.02 \\
\hline 2 & SB5NEPH-13 & quenched & targeted & 0.571 & 0.948 & 0.289 & 3.72 & 8.88 & 1.95 \\
\hline 2 & SB5NEPH-13 & quenched & measured & 0.567 & 0.961 & 0.288 & 3.69 & 9.14 & 1.94 \\
\hline 2 & SB5NEPH-13 & quenched & measured bc & 0.557 & 0.967 & 0.280 & 3.61 & 9.27 & 1.90 \\
\hline 2 & SB5NEPH-14 & CCC & targeted & 1.205 & 1.106 & 0.140 & 16.03 & 12.75 & 1.38 \\
\hline 2 & SB5NEPH-14 & $\mathrm{CCC}$ & measured & 1.204 & 1.118 & 0.134 & 16.01 & 13.11 & 1.36 \\
\hline 2 & SB5NEPH-14 & CCC & measured bc & 1.202 & 1.119 & 0.127 & 15.92 & 13.14 & 1.34 \\
\hline 2 & SB5NEPH-14 & quenched & targeted & 1.325 & 1.203 & 0.225 & 21.12 & 15.97 & 1.68 \\
\hline 2 & SB5NEPH-14 & quenched & measured & 1.324 & 1.215 & 0.219 & 21.09 & 16.41 & 1.65 \\
\hline 2 & SB5NEPH-14 & quenched & measured bc & 1.322 & 1.216 & 0.212 & 20.97 & 16.45 & 1.63 \\
\hline 2 & SB5NEPH-15 & CCC & targeted & 1.054 & 1.515 & 0.439 & 11.32 & 32.75 & 2.75 \\
\hline 2 & SB5NEPH-15 & CCC & measured & 1.048 & 1.537 & 0.445 & 11.16 & 34.46 & 2.79 \\
\hline 2 & SB5NEPH-15 & CCC & measured bc & 1.045 & 1.538 & 0.438 & 11.10 & 34.54 & 2.74 \\
\hline 2 & SB5NEPH-15 & quenched & targeted & 0.452 & 0.938 & 0.044 & 2.83 & 8.67 & 1.11 \\
\hline 2 & SB5NEPH-15 & quenched & measured & 0.446 & 0.960 & 0.050 & 2.79 & 9.12 & 1.12 \\
\hline 2 & SB5NEPH-15 & quenched & measured bc & 0.443 & 0.961 & 0.043 & 2.77 & 9.14 & 1.11 \\
\hline 2 & SB5NEPH-16 & CCC & targeted & 0.761 & 0.799 & -0.223 & 5.77 & 6.30 & 0.60 \\
\hline 2 & SB5NEPH-16 & $\mathrm{CCC}$ & measured & 0.765 & 0.838 & -0.223 & 5.82 & 6.89 & 0.60 \\
\hline 2 & SB5NEPH-16 & CCC & measured bc & 0.762 & 0.839 & -0.230 & 5.79 & 6.90 & 0.59 \\
\hline 2 & SB5NEPH-16 & quenched & targeted & 0.730 & 0.786 & -0.246 & 5.37 & 6.11 & 0.57 \\
\hline 2 & SB5NEPH-16 & quenched & measured & 0.733 & 0.825 & -0.247 & 5.41 & 6.69 & 0.57 \\
\hline 2 & SB5NEPH-16 & quenched & measured bc & 0.731 & 0.826 & -0.254 & 5.38 & 6.70 & 0.56 \\
\hline 2 & SB5NEPH-17 & CCC & targeted & 1.101 & 0.888 & 0.104 & 12.62 & 7.73 & 1.27 \\
\hline 2 & SB5NEPH-17 & CCC & measured & 1.096 & 0.893 & 0.094 & 12.47 & 7.81 & 1.24 \\
\hline 2 & SB5NEPH-17 & $\mathrm{CCC}$ & measured bc & 1.086 & 0.899 & 0.086 & 12.19 & 7.92 & 1.22 \\
\hline 2 & SB5NEPH-17 & quenched & targeted & -0.027 & 0.136 & -0.281 & 0.94 & 1.37 & 0.52 \\
\hline 2 & SB5NEPH-17 & quenched & measured & -0.033 & 0.141 & -0.291 & 0.93 & 1.38 & 0.51 \\
\hline 2 & SB5NEPH-17 & quenched & measured bc & -0.042 & 0.147 & -0.299 & 0.91 & 1.40 & 0.50 \\
\hline 2 & SB5NEPH-18 & CCC & targeted & 0.600 & 0.562 & -0.641 & 3.99 & 3.65 & 0.23 \\
\hline 2 & SB5NEPH-18 & CCC & measured & 0.592 & 0.569 & -0.650 & 3.91 & 3.71 & 0.22 \\
\hline 2 & SB5NEPH-18 & CCC & measured bc & 0.590 & 0.570 & -0.657 & 3.89 & 3.72 & 0.22 \\
\hline 2 & SB5NEPH-18 & quenched & targeted & 0.632 & 0.519 & -0.647 & 4.28 & 3.31 & 0.23 \\
\hline 2 & SB5NEPH-18 & quenched & measured & 0.623 & 0.526 & -0.656 & 4.20 & 3.36 & 0.22 \\
\hline 2 & SB5NEPH-18 & quenched & measured bc & 0.621 & 0.527 & -0.663 & 4.18 & 3.36 & 0.22 \\
\hline
\end{tabular}


Table 3-4. Normalized PCT Results by Glass ID and Compositional View. (continued)

\begin{tabular}{|c|c|c|c|c|c|c|c|c|c|}
\hline Set & Glass ID & $\begin{array}{c}\text { Heat } \\
\text { Treatment }\end{array}$ & $\begin{array}{c}\text { Compositional } \\
\text { View }\end{array}$ & $\begin{array}{c}\log \mathrm{NL} \\
{[\mathrm{B}(\mathrm{g} / \mathrm{L})]}\end{array}$ & $\begin{array}{c}\log \mathbf{N L} \\
{[\mathrm{Na}(\mathrm{g} / \mathrm{L})]}\end{array}$ & $\begin{array}{c}\log \mathrm{NL} \\
{[\mathrm{Si}(\mathrm{g} / \mathrm{L})]}\end{array}$ & $\begin{array}{c}\text { NL } \\
{[B(g / L)]}\end{array}$ & $\begin{array}{c}\text { NL } \\
{[\mathrm{Na}(\mathrm{g} / \mathrm{L})]}\end{array}$ & $\begin{array}{c}\mathrm{NL} \\
{[\mathrm{Si}(\mathrm{g} / \mathrm{L})]}\end{array}$ \\
\hline 2 & SB5NEPH-19 & $\mathrm{CCC}$ & targeted & 0.701 & 0.978 & -0.283 & 5.02 & 9.51 & 0.52 \\
\hline 2 & SB5NEPH-19 & $\mathrm{CCC}$ & measured & 0.686 & 0.991 & -0.292 & 4.85 & 9.79 & 0.51 \\
\hline 2 & SB5NEPH-19 & CCC & measured bc & 0.684 & 0.992 & -0.299 & 4.83 & 9.81 & 0.50 \\
\hline 2 & SB5NEPH-19 & quenched & targeted & -0.074 & 0.336 & -0.567 & 0.84 & 2.17 & 0.27 \\
\hline 2 & SB5NEPH-19 & quenched & measured & -0.089 & 0.348 & -0.576 & 0.81 & 2.23 & 0.27 \\
\hline 2 & SB5NEPH-19 & quenched & measured bc & -0.092 & 0.349 & -0.583 & 0.81 & 2.23 & 0.26 \\
\hline 2 & SB5NEPH-20 & CCC & targeted & 0.056 & 0.204 & -0.821 & 1.14 & 1.60 & 0.15 \\
\hline 2 & SB5NEPH-20 & $\mathrm{CCC}$ & measured & 0.053 & 0.201 & -0.829 & 1.13 & 1.59 & 0.15 \\
\hline 2 & SB5NEPH-20 & $\mathrm{CCC}$ & measured bc & 0.043 & 0.207 & -0.837 & 1.10 & 1.61 & 0.15 \\
\hline 2 & SB5NEPH-20 & quenched & targeted & 0.458 & 0.558 & -0.753 & 2.87 & 3.61 & 0.18 \\
\hline 2 & SB5NEPH-20 & quenched & measured & 0.454 & 0.555 & -0.760 & 2.85 & 3.59 & 0.17 \\
\hline 2 & SB5NEPH-20 & quenched & measured bc & 0.445 & 0.561 & -0.768 & 2.78 & 3.64 & 0.17 \\
\hline 3 & SB5NEPH-21 & CCC & targeted & 1.172 & 0.339 & -0.869 & 14.85 & 2.18 & 0.14 \\
\hline 3 & SB5NEPH-21 & CCC & measured & 1.187 & 0.337 & -0.873 & 15.37 & 2.17 & 0.13 \\
\hline 3 & SB5NEPH-21 & CCC & measured bc & 1.166 & 0.344 & -0.885 & 14.67 & 2.21 & 0.13 \\
\hline 3 & SB5NEPH-21 & quenched & targeted & -0.517 & -0.436 & -0.716 & 0.30 & 0.37 & 0.19 \\
\hline 3 & SB5NEPH-21 & quenched & measured & -0.502 & -0.438 & -0.720 & 0.31 & 0.36 & 0.19 \\
\hline 3 & SB5NEPH-21 & quenched & measured bc & -0.522 & -0.431 & -0.732 & 0.30 & 0.37 & 0.19 \\
\hline 3 & SB5NEPH-22 & CCC & targeted & 0.021 & -0.069 & -0.544 & 1.05 & 0.85 & 0.29 \\
\hline 3 & SB5NEPH-22 & $\mathrm{CCC}$ & measured & 0.011 & -0.078 & -0.560 & 1.03 & 0.84 & 0.28 \\
\hline 3 & SB5NEPH-22 & CCC & measured bc & -0.009 & -0.071 & -0.571 & 0.98 & 0.85 & 0.27 \\
\hline 3 & SB5NEPH-22 & quenched & targeted & -0.303 & -0.302 & -0.616 & 0.50 & 0.50 & 0.24 \\
\hline 3 & SB5NEPH-22 & quenched & measured & -0.312 & -0.311 & -0.632 & 0.49 & 0.49 & 0.23 \\
\hline 3 & SB5NEPH-22 & quenched & measured bc & -0.333 & -0.304 & -0.643 & 0.46 & 0.50 & 0.23 \\
\hline 3 & SB5NEPH-23 & CCC & targeted & -0.120 & -0.734 & -1.174 & 0.76 & 0.18 & 0.07 \\
\hline 3 & SB5NEPH-23 & $\mathrm{CCC}$ & measured & -0.109 & -0.738 & -1.178 & 0.78 & 0.18 & 0.07 \\
\hline 3 & SB5NEPH-23 & CCC & measured bc & -0.122 & -0.730 & -1.184 & 0.75 & 0.19 & 0.07 \\
\hline 3 & SB5NEPH-23 & quenched & targeted & -0.564 & -0.407 & -1.067 & 0.27 & 0.39 & 0.09 \\
\hline 3 & SB5NEPH-23 & quenched & measured & -0.553 & -0.411 & -1.071 & 0.28 & 0.39 & 0.08 \\
\hline 3 & SB5NEPH-23 & quenched & measured bc & -0.566 & -0.403 & -1.077 & 0.27 & 0.40 & 0.08 \\
\hline 3 & SB5NEPH-24 & CCC & targeted & -0.463 & -0.425 & -1.182 & 0.34 & 0.38 & 0.07 \\
\hline 3 & SB5NEPH-24 & CCC & measured & -0.467 & -0.429 & -1.185 & 0.34 & 0.37 & 0.07 \\
\hline 3 & SB5NEPH-24 & $\mathrm{CCC}$ & measured bc & -0.480 & -0.421 & -1.191 & 0.33 & 0.38 & 0.06 \\
\hline 3 & SB5NEPH-24 & quenched & targeted & -0.046 & 0.061 & -1.266 & 0.90 & 1.15 & 0.05 \\
\hline 3 & SB5NEPH-24 & quenched & measured & -0.051 & 0.057 & -1.270 & 0.89 & 1.14 & 0.05 \\
\hline 3 & SB5NEPH-24 & quenched & measured bc & -0.064 & 0.065 & -1.276 & 0.86 & 1.16 & 0.05 \\
\hline 3 & SB5NEPH-25 & CCC & targeted & -0.864 & -0.783 & -1.021 & 0.14 & 0.16 & 0.10 \\
\hline 3 & SB5NEPH-25 & CCC & measured & -0.875 & -0.800 & -1.037 & 0.13 & 0.16 & 0.09 \\
\hline 3 & SB5NEPH-25 & CCC & measured bc & -0.888 & -0.794 & -1.043 & 0.13 & 0.16 & 0.09 \\
\hline 3 & SB5NEPH-25 & quenched & targeted & -0.232 & -0.836 & -1.133 & 0.59 & 0.15 & 0.07 \\
\hline 3 & SB5NEPH-25 & quenched & measured & -0.242 & -0.854 & -1.149 & 0.57 & 0.14 & 0.07 \\
\hline 3 & SB5NEPH-25 & quenched & measured bc & -0.256 & -0.847 & -1.155 & 0.56 & 0.14 & 0.07 \\
\hline 3 & SB5NEPH-26 & CCC & targeted & . & . & . & & & \\
\hline 3 & SB5NEPH-26 & $\mathrm{CCC}$ & measured & . & . & . & & & \\
\hline 3 & SB5NEPH-26 & CCC & measured bc & . &. & . & & & \\
\hline 3 & SB5NEPH-26 & quenched & targeted & . & . & . & & & \\
\hline 3 & SB5NEPH-26 & quenched & measured & . &. & . & & & \\
\hline 3 & SB5NEPH-26 & quenched & measured bc & . &. &. & & & \\
\hline 3 & SB5NEPH-27 & CCC & targeted & 1.303 & 0.286 & -2.472 & 20.07 & 1.93 & 0.00 \\
\hline 3 & SB5NEPH-27 & $\mathrm{CCC}$ & measured & 1.310 & 0.282 & -2.477 & 20.39 & 1.91 & 0.00 \\
\hline 3 & SB5NEPH-27 & CCC & measured bc & 1.289 & 0.290 & -2.489 & 19.46 & 1.95 & 0.00 \\
\hline 3 & SB5NEPH-27 & quenched & targeted & -0.446 & -0.892 & -1.291 & 0.36 & 0.13 & 0.05 \\
\hline 3 & SB5NEPH-27 & quenched & measured & -0.439 & -0.897 & -1.296 & 0.36 & 0.13 & 0.05 \\
\hline 3 & SB5NEPH-27 & quenched & measured bc & -0.459 & -0.889 & -1.308 & 0.35 & 0.13 & 0.05 \\
\hline 3 & SB5NEPH-28 & CCC & targeted & -0.459 & -0.812 & -1.258 & 0.35 & 0.15 & 0.06 \\
\hline 3 & SB5NEPH-28 & $\mathrm{CCC}$ & measured & -0.467 & -0.823 & -1.259 & 0.34 & 0.15 & 0.06 \\
\hline 3 & SB5NEPH-28 & $\mathrm{CCC}$ & measured bc & -0.480 & -0.815 & -1.265 & 0.33 & 0.15 & 0.05 \\
\hline 3 & SB5NEPH-28 & quenched & targeted & -0.512 & -0.577 & -1.412 & 0.31 & 0.27 & 0.04 \\
\hline
\end{tabular}


Table 3-4. Normalized PCT Results by Glass ID and Compositional View. (continued)

\begin{tabular}{|c|c|c|c|c|c|c|c|c|c|}
\hline Set & Glass ID & $\begin{array}{c}\text { Heat } \\
\text { Treatment }\end{array}$ & $\begin{array}{c}\text { Compositional } \\
\text { View }\end{array}$ & $\begin{array}{c}\log N L \\
{[B(g / L)]}\end{array}$ & $\begin{array}{c}\log \mathbf{N L} \\
{[\mathrm{Na}(\mathrm{g} / \mathrm{L})]}\end{array}$ & $\begin{array}{c}\log \mathrm{NL} \\
{[\mathrm{Si}(\mathrm{g} / \mathrm{L})]}\end{array}$ & $\begin{array}{c}\text { NL } \\
{[B(g / L)]}\end{array}$ & $\begin{array}{c}\text { NL } \\
{[\mathrm{Na}(\mathrm{g} / \mathrm{L})]}\end{array}$ & $\begin{array}{c}\mathrm{NL} \\
{[\mathrm{Si}(\mathrm{g} / \mathrm{L})]}\end{array}$ \\
\hline 3 & SB5NEPH-28 & quenched & measured & -0.520 & -0.588 & -1.413 & 0.30 & 0.26 & 0.04 \\
\hline 3 & SB5NEPH-28 & quenched & measured bc & -0.533 & -0.580 & -1.419 & 0.29 & 0.26 & 0.04 \\
\hline 3 & SB5NEPH-29 & CCC & targeted &. &. &. & & & \\
\hline 3 & SB5NEPH-29 & CCC & measured & . & . & . & & & \\
\hline 3 & SB5NEPH-29 & $\mathrm{CCC}$ & measured bc & . & . & . & & & \\
\hline 3 & SB5NEPH-29 & quenched & targeted & 2.009 & 1.855 & 1.205 & 102.07 & 71.62 & 16.04 \\
\hline 3 & SB5NEPH-29 & quenched & measured & 2.029 & 1.887 & 1.218 & 107.01 & 77.07 & 16.53 \\
\hline 3 & SB5NEPH-29 & quenched & measured bc & 2.009 & 1.894 & 1.207 & 102.12 & 78.27 & 16.09 \\
\hline 3 & SB5NEPH-30 & $\mathrm{CCC}$ & targeted & 0.600 & 1.158 & 0.484 & 3.99 & 14.38 & 3.05 \\
\hline 3 & SB5NEPH-30 & CCC & measured & 0.605 & 1.180 & 0.484 & 4.03 & 15.13 & 3.05 \\
\hline 3 & SB5NEPH-30 & CCC & measured bc & 0.585 & 1.187 & 0.473 & 3.84 & 15.37 & 2.97 \\
\hline 3 & SB5NEPH-30 & quenched & targeted & 1.995 & 1.859 & 0.930 & 98.78 & 72.31 & 8.50 \\
\hline 3 & SB5NEPH-30 & quenched & measured & 1.999 & 1.881 & 0.930 & 99.77 & 76.07 & 8.52 \\
\hline 3 & SB5NEPH-30 & quenched & measured bc & 1.979 & 1.888 & 0.919 & 95.21 & 77.26 & 8.29 \\
\hline 4 & SB5NEPH-31 & CCC & targeted & 1.659 & 1.736 & 0.793 & 45.56 & 54.45 & 6.21 \\
\hline 4 & SB5NEPH-31 & $\mathrm{CCC}$ & measured & 1.685 & 1.776 & 0.815 & 48.37 & 59.71 & 6.52 \\
\hline 4 & SB5NEPH-31 & CCC & measured bc & 1.674 & 1.781 & 0.814 & 47.16 & 60.33 & 6.52 \\
\hline 4 & SB5NEPH-31 & quenched & targeted & 1.854 & 1.865 & 0.752 & 71.50 & 73.32 & 5.65 \\
\hline 4 & SB5NEPH-31 & quenched & measured & 1.880 & 1.905 & 0.773 & 75.92 & 80.41 & 5.93 \\
\hline 4 & SB5NEPH-31 & quenched & measured bc & 1.869 & 1.910 & 0.773 & 74.02 & 81.25 & 5.93 \\
\hline 4 & SB5NEPH-32 & CCC & targeted & 0.459 & 0.765 & 0.004 & 2.88 & 5.82 & 1.01 \\
\hline 4 & SB5NEPH-32 & $\mathrm{CCC}$ & measured & 0.462 & 0.769 & 0.003 & 2.90 & 5.88 & 1.01 \\
\hline 4 & SB5NEPH-32 & $\mathrm{CCC}$ & measured bc & 0.455 & 0.783 & 0.003 & 2.85 & 6.06 & 1.01 \\
\hline 4 & SB5NEPH-32 & quenched & targeted & 1.263 & 1.286 & -0.005 & 18.34 & 19.33 & 0.99 \\
\hline 4 & SB5NEPH-32 & quenched & measured & 1.266 & 1.291 & -0.007 & 18.46 & 19.53 & 0.98 \\
\hline 4 & SB5NEPH-32 & quenched & measured bc & 1.260 & 1.304 & -0.007 & 18.19 & 20.14 & 0.99 \\
\hline 4 & SB5NEPH-33 & CCC & targeted & 1.486 & 1.275 & 0.087 & 30.58 & 18.85 & 1.22 \\
\hline 4 & SB5NEPH-33 & CCC & measured & 1.491 & 1.275 & 0.085 & 30.95 & 18.86 & 1.21 \\
\hline 4 & SB5NEPH-33 & CCC & measured bc & 1.484 & 1.289 & 0.085 & 30.50 & 19.45 & 1.22 \\
\hline 4 & SB5NEPH-33 & quenched & targeted & 1.921 & 1.335 & -0.365 & 83.35 & 21.63 & 0.43 \\
\hline 4 & SB5NEPH-33 & quenched & measured & 1.926 & 1.335 & -0.368 & 84.35 & 21.64 & 0.43 \\
\hline 4 & SB5NEPH-33 & quenched & measured bc & 1.920 & 1.349 & -0.368 & 83.12 & 22.32 & 0.43 \\
\hline 4 & SB5NEPH-34 & CCC & targeted & 0.821 & 0.574 & -0.960 & 6.62 & 3.75 & 0.11 \\
\hline 4 & SB5NEPH-34 & CCC & measured & 0.815 & 0.582 & -0.972 & 6.53 & 3.82 & 0.11 \\
\hline 4 & SB5NEPH-34 & CCC & measured bc & 0.804 & 0.586 & -0.973 & 6.37 & 3.86 & 0.11 \\
\hline 4 & SB5NEPH-34 & quenched & targeted & 0.469 & 0.376 & -0.623 & 2.94 & 2.38 & 0.24 \\
\hline 4 & SB5NEPH-34 & quenched & measured & 0.463 & 0.384 & -0.635 & 2.91 & 2.42 & 0.23 \\
\hline 4 & SB5NEPH-34 & quenched & measured bc & 0.452 & 0.388 & -0.636 & 2.83 & 2.44 & 0.23 \\
\hline 4 & SB5NEPH-35 & CCC & targeted & 0.819 & 1.012 & -0.022 & 6.59 & 10.28 & 0.95 \\
\hline 4 & SB5NEPH-35 & $\mathrm{CCC}$ & measured & 0.824 & 1.008 & -0.020 & 6.67 & 10.19 & 0.95 \\
\hline 4 & SB5NEPH-35 & CCC & measured bc & 0.818 & 1.021 & -0.020 & 6.57 & 10.50 & 0.95 \\
\hline 4 & SB5NEPH-35 & quenched & targeted & 0.147 & 0.106 & -0.875 & 1.40 & 1.28 & 0.13 \\
\hline 4 & SB5NEPH-35 & quenched & measured & 0.152 & 0.102 & -0.872 & 1.42 & 1.27 & 0.13 \\
\hline 4 & SB5NEPH-35 & quenched & measured bc & 0.145 & 0.116 & -0.872 & 1.40 & 1.31 & 0.13 \\
\hline 4 & SB5NEPH-36 & CCC & targeted & 1.526 & 1.451 & -1.390 & 33.59 & 28.27 & 0.04 \\
\hline 4 & SB5NEPH-36 & CCC & measured & 1.531 & 1.444 & -1.393 & 34.00 & 27.79 & 0.04 \\
\hline 4 & SB5NEPH-36 & CCC & measured bc & 1.525 & 1.457 & -1.393 & 33.50 & 28.66 & 0.04 \\
\hline 4 & SB5NEPH-36 & quenched & targeted & 0.432 & 0.516 & -1.054 & 2.70 & 3.28 & 0.09 \\
\hline 4 & SB5NEPH-36 & quenched & measured & 0.437 & 0.508 & -1.056 & 2.74 & 3.22 & 0.09 \\
\hline 4 & SB5NEPH-36 & quenched & measured bc & 0.431 & 0.522 & -1.056 & 2.70 & 3.33 & 0.09 \\
\hline 4 & SB5NEPH-37 & CCC & targeted & 0.011 & -0.648 & -0.912 & 1.03 & 0.23 & 0.12 \\
\hline 4 & SB5NEPH-37 & CCC & measured & 0.008 & -0.667 & -0.929 & 1.02 & 0.22 & 0.12 \\
\hline 4 & SB5NEPH-37 & $\mathrm{CCC}$ & measured bc & -0.003 & -0.663 & -0.930 & 0.99 & 0.22 & 0.12 \\
\hline 4 & SB5NEPH-37 & quenched & targeted & -0.358 & -0.597 & -0.947 & 0.44 & 0.25 & 0.11 \\
\hline 4 & SB5NEPH-37 & quenched & measured & -0.361 & -0.617 & -0.965 & 0.44 & 0.24 & 0.11 \\
\hline 4 & SB5NEPH-37 & quenched & measured bc & -0.372 & -0.612 & -0.965 & 0.42 & 0.24 & 0.11 \\
\hline 4 & SB5NEPH-38 & CCC & targeted & 0.196 & 0.106 & -0.529 & 1.57 & 1.28 & 0.30 \\
\hline 4 & SB5NEPH-38 & CCC & measured & 0.179 & 0.084 & -0.545 & 1.51 & 1.21 & 0.29 \\
\hline
\end{tabular}


Table 3-4. Normalized PCT Results by Glass ID and Compositional View. (continued)

\begin{tabular}{|c|c|c|c|c|c|c|c|c|c|}
\hline Set & Glass ID & $\begin{array}{c}\text { Heat } \\
\text { Treatment }\end{array}$ & $\begin{array}{c}\text { Compositional } \\
\text { View }\end{array}$ & $\begin{array}{c}\log N L \\
{[B(g / L)]}\end{array}$ & $\begin{array}{c}\log \mathrm{NL} \\
{[\mathrm{Na}(\mathrm{g} / \mathrm{L})]}\end{array}$ & $\begin{array}{c}\log N L \\
{[\mathrm{Si}(\mathrm{g} / \mathrm{L})]}\end{array}$ & $\begin{array}{c}\text { NL } \\
{[B(g / L)]}\end{array}$ & $\begin{array}{c}\text { NL } \\
{[\mathrm{Na}(\mathrm{g} / \mathrm{L})]}\end{array}$ & $\begin{array}{c}\text { NL } \\
{[S i(g / L)]}\end{array}$ \\
\hline 4 & SB5NEPH-38 & CCC & measured bc & 0.168 & 0.088 & -0.545 & 1.47 & 1.23 & 0.28 \\
\hline 4 & SB5NEPH-38 & quenched & targeted & -0.479 & -0.530 & -0.963 & 0.33 & 0.29 & 0.11 \\
\hline 4 & SB5NEPH-38 & quenched & measured & -0.495 & -0.553 & -0.979 & 0.32 & 0.28 & 0.10 \\
\hline 4 & SB5NEPH-38 & quenched & measured bc & -0.506 & -0.548 & -0.980 & 0.31 & 0.28 & 0.10 \\
\hline 4 & SB5NEPH-39 & CCC & targeted & 1.394 & 0.296 & -2.183 & 24.76 & 1.98 & 0.01 \\
\hline 4 & SB5NEPH-39 & CCC & measured & 1.397 & 0.279 & -2.192 & 24.95 & 1.90 & 0.01 \\
\hline 4 & SB5NEPH-39 & CCC & measured bc & 1.386 & 0.284 & -2.193 & 24.33 & 1.92 & 0.01 \\
\hline 4 & SB5NEPH-39 & quenched & targeted & -0.399 & -0.891 & -1.293 & 0.40 & 0.13 & 0.05 \\
\hline 4 & SB5NEPH-39 & quenched & measured & -0.396 & -0.907 & -1.302 & 0.40 & 0.12 & 0.05 \\
\hline 4 & SB5NEPH-39 & quenched & measured bc & -0.407 & -0.903 & -1.302 & 0.39 & 0.13 & 0.05 \\
\hline 4 & SB5NEPH-40 & CCC & targeted & -0.501 & -0.748 & -1.261 & 0.32 & 0.18 & 0.05 \\
\hline 4 & SB5NEPH-40 & CCC & measured & -0.503 & -0.773 & -1.267 & 0.31 & 0.17 & 0.05 \\
\hline 4 & SB5NEPH-40 & $\mathrm{CCC}$ & measured bc & -0.509 & -0.760 & -1.266 & 0.31 & 0.17 & 0.05 \\
\hline 4 & SB5NEPH-40 & quenched & targeted & -0.386 & -0.552 & -1.508 & 0.41 & 0.28 & 0.03 \\
\hline 4 & SB5NEPH-40 & quenched & measured & -0.388 & -0.577 & -1.514 & 0.41 & 0.27 & 0.03 \\
\hline 4 & SB5NEPH-40 & quenched & measured bc & -0.395 & -0.563 & -1.514 & 0.40 & 0.27 & 0.03 \\
\hline
\end{tabular}

\subsubsection{PCT Response as a Function of Composition}

The PCT results may be easier to interpret when represented graphically. Figure 3-1 presents the measured normalized releases for boron as a function of each quenched glass composition's location on the $\mathrm{SiO}_{2}-\mathrm{Na}_{2} \mathrm{O}-\mathrm{Al}_{2} \mathrm{O}_{3}$ ternary diagram and the concentrations of $\mathrm{B}_{2} \mathrm{O}_{3}$ and $\mathrm{CaO}$. 


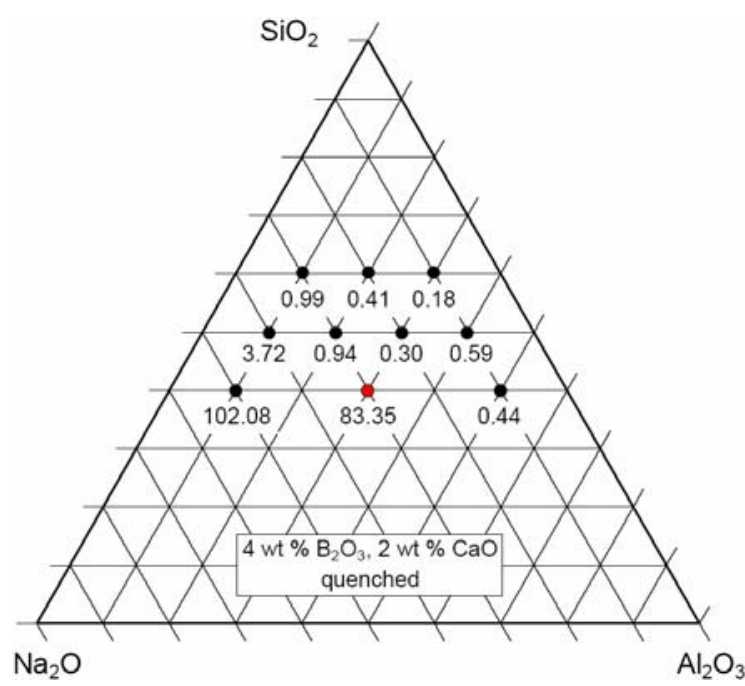

(a)

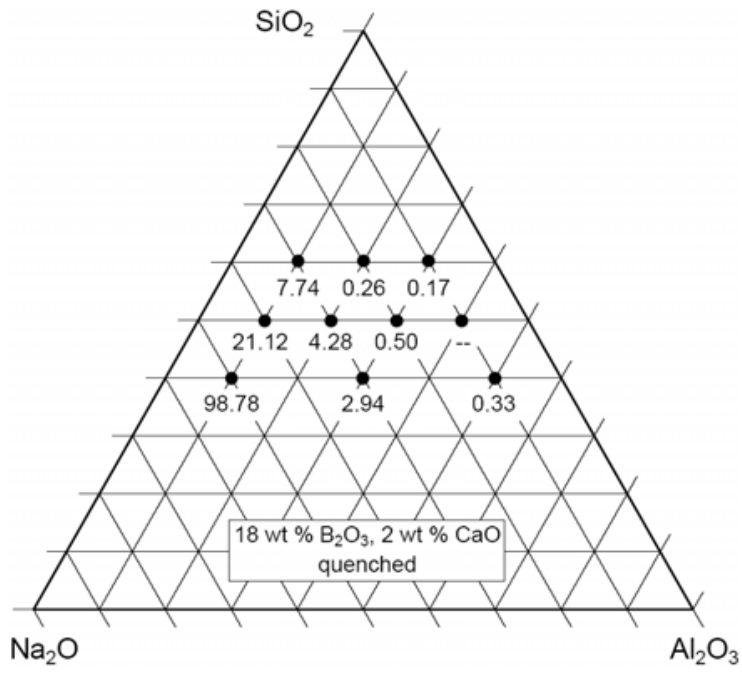

(c)

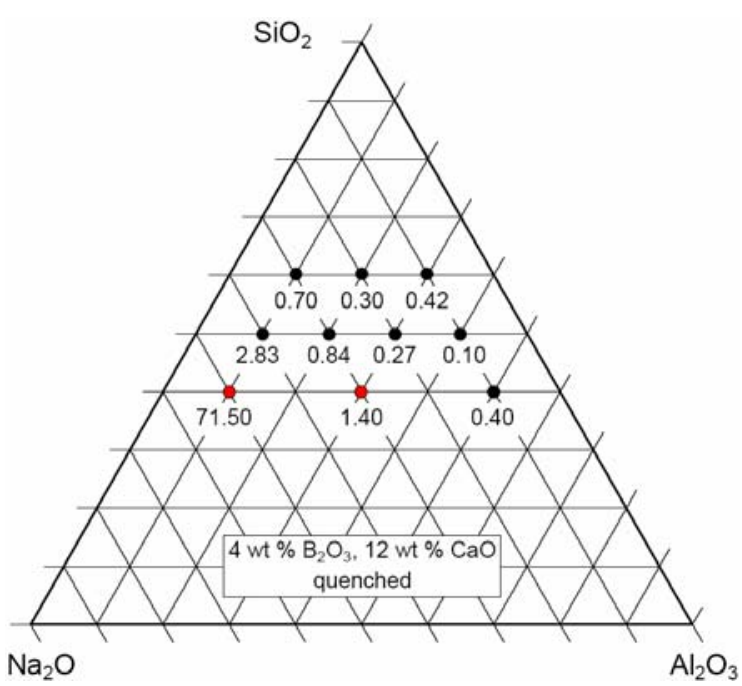

(b)

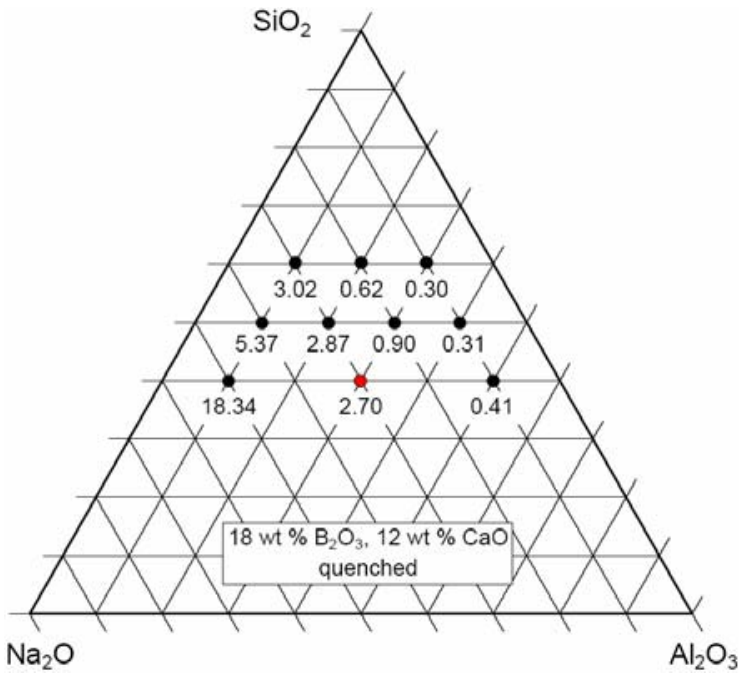

(d)

Figure 3-1. PCT responses (NL [B], in $\mathrm{g} / \mathrm{L}$ ) for the quenched versions of the study glasses as a function of location on the $\mathrm{SiO}_{2}-\mathrm{Na}_{2} \mathrm{O}-\mathrm{Al}_{2} \mathrm{O}_{3}$ ternary diagram and with varying concentrations of $\mathrm{B}_{2} \mathrm{O}_{3}$ and $\mathrm{CaO}$. Compositions where nepheline was detected by XRD are indicated in red.

A review of these figures shows that several of the quenched glass compositions with nepheline discriminator values below the current limit of 0.62 have NL [B] values that are well below that of the benchmark EA glass $(16.695 \mathrm{~g} / \mathrm{L}){ }^{20}$ This indicates the potential to target glass compositions with higher concentrations of $\mathrm{Al}_{2} \mathrm{O}_{3}$ while continuing to produce durable glasses. In general, the durability of all the quenched glasses decreases as the composition moves toward the $\mathrm{Na}_{2} \mathrm{O}$ corner of the ternary. All of the quenched glasses at Point 8 (refer to Figure 2-1), the closest to the $\mathrm{Na}_{2} \mathrm{O}$ corner of the ternary, have NL [B] values that are greater than that of the EA benchmark glass.

Increasing the $\mathrm{CaO}$ concentration improved the durability of most of the quenched glasses, particularly those with low $\mathrm{Al}_{2} \mathrm{O}_{3}$ concentrations (the left side of the ternary) and those with the 
highest $\mathrm{Na}_{2} \mathrm{O}$ concentrations. The most significant difference was seen at Point 9 on the ternary diagram, where the NL [B] was reduced from $83.35 \mathrm{~g} / \mathrm{L}(2 \mathrm{wt} \% \mathrm{CaO})$ to $1.40 \mathrm{~g} / \mathrm{L}(12 \mathrm{wt} \% \mathrm{CaO})$.

Increasing the $\mathrm{B}_{2} \mathrm{O}_{3}$ concentration from $4 \mathrm{wt} \%$ to $18 \mathrm{wt} \%$ appears to have had a mixed effect on the NL [B] values for the quenched glasses (compare Figure 3-1 (a) and (b) to (c) and (d)). When the $\mathrm{B}_{2} \mathrm{O}_{3}$ concentration is increased but the $\mathrm{CaO}$ concentration is kept constant, the number of compositions where nepheline was detected by XRD was reduced. However, several of the compositions had higher NL [B] values when the $\mathrm{B}_{2} \mathrm{O}_{3}$ concentration was increased, although the higher values remained below that of the EA benchmark glass (with one exception). A few compositions, particularly the composition closest to the $\mathrm{Na}_{2} \mathrm{O}$ corner of the ternary diagram, had reduced $\mathrm{NL}$ [B] values when the $\mathrm{B}_{2} \mathrm{O}_{3}$ concentration was increased. The $\mathrm{NL}$ [B] for the quenched glass at Point 8 with $18 \mathrm{wt} \% \mathrm{~B}_{2} \mathrm{O}_{3}$ and $12 \mathrm{wt} \% \mathrm{CaO}$ was reduced to $18.34 \mathrm{~g} / \mathrm{L}$. While still greater than EA, this is considerably better than the NL [B] of $71.50 \mathrm{~g} / \mathrm{L}$ at Point 8 for the $4 \mathrm{wt} \%$ $\mathrm{B}_{2} \mathrm{O}_{3}, 12 \mathrm{wt} \% \mathrm{CaO}$ case.

Compositions where nepheline (or crystalline phases that are expected to have a similar effect as nepheline, refer to Table 3-1) was detected by XRD are indicated in red in Figure 3-1. The detection of nepheline does not appear to correlate directly to the NL [B] response for these glasses. This may indicate that other compositional effects beyond crystallization - particularly very high alkali concentrations - influenced the durability of some of the study glasses.

Similar diagrams for the CCC versions of the glasses are shown in Figure 3-2. 


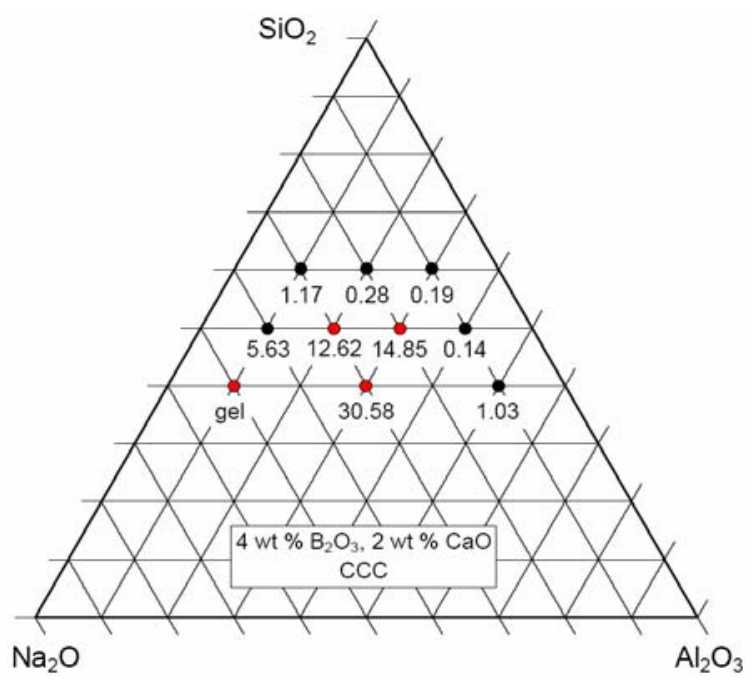

(a)

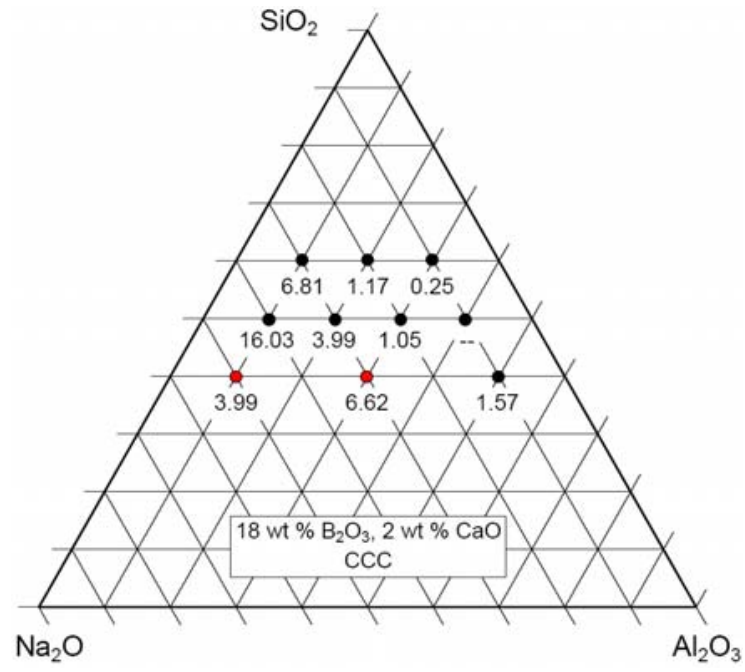

(c)

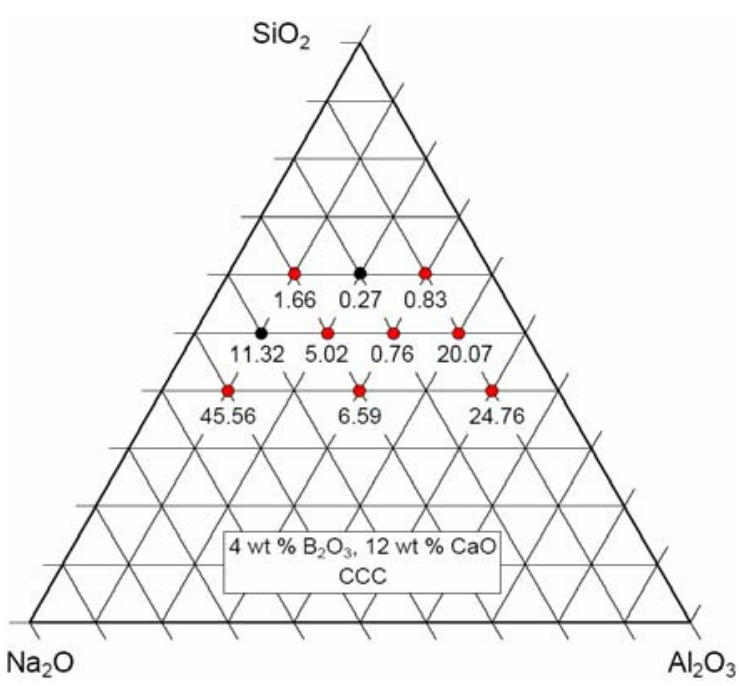

(b)

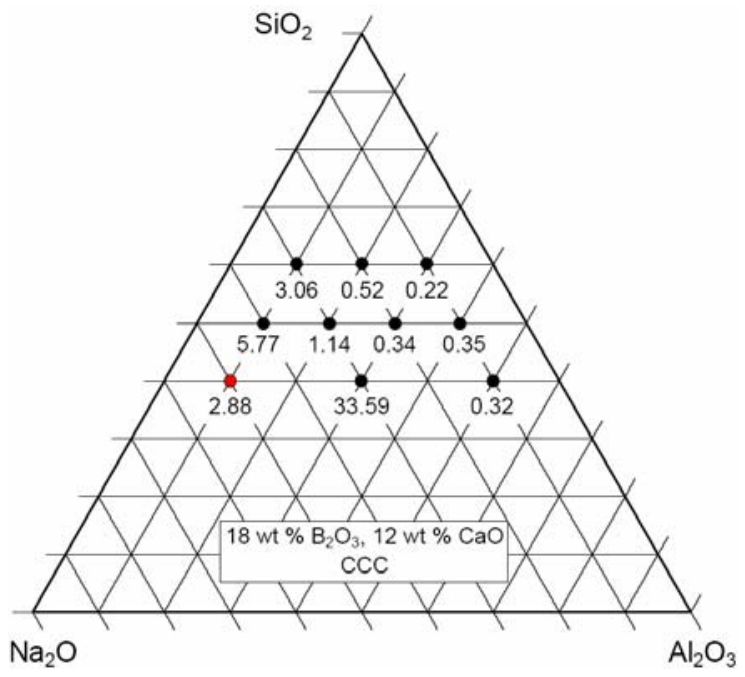

(d)

Figure 3-2. PCT responses (NL [B], in $\mathrm{g} / \mathrm{L}$ ) for the CCC versions of the study glasses as a function of location on the $\mathrm{SiO}_{2}-\mathrm{Na}_{2} \mathrm{O}-\mathrm{Al}_{2} \mathrm{O}_{3}$ ternary diagram and with varying concentrations of $\mathrm{B}_{2} \mathrm{O}_{3}$ and $\mathrm{CaO}$. Compositions where nepheline was detected by XRD are indicated in red.

Again, many of the study glasses with nepheline discriminator values below the current limit of 0.62 have NL [B] values that are well below that of the EA benchmark glass (16.695 g/L). The trend of increasing NL [B] as the glass composition moves toward the $\mathrm{Na}_{2} \mathrm{O}$ corner of the ternary diagram continues for the CCC versions of the study glasses. The durability of the glass at Point 8 for the 4 wt \% $\mathrm{B}_{2} \mathrm{O}_{3}$, 2 wt \% CaO case (Figure 3-2 (a)) was so poor that the glass gelled during preparation for the PCT. When the $\mathrm{B}_{2} \mathrm{O}_{3}$ concentration was increased to $18 \mathrm{wt} \%$, the glass compositions at this point had NL [B] values that were acceptable (Figure 3-2 (c) and (d)).

The effects of increasing $\mathrm{CaO}$ concentration from 2 to $12 \mathrm{wt} \%$ were mixed for the CCC glasses. The number of compositions where nepheline formed (as indicated by the red points in Figure 3-2) increased when the $\mathrm{CaO}$ concentration was increased and the $\mathrm{B}_{2} \mathrm{O}_{3}$ concentration was 
held at 4 wt \%. The changes in NL [B] response were mixed, depending on the location of the glass composition on the ternary diagram. The effects of increasing $\mathrm{B}_{2} \mathrm{O}_{3}$ concentration from 4 to 18 wt \% were clearer. The increased $\mathrm{B}_{2} \mathrm{O}_{3}$ concentration significantly reduced the number of glass compositions where nepheline crystallized. The NL [B] responses for most of the glasses were reduced to values below that of the EA benchmark glass, although there were a few exceptions.

There is improved correlation between the formation of nepheline and an increase in NL [B] values for the CCC versions of the study glasses, although some compositions continue to show elevated NL [B] values where no nepheline was detected. This is again likely to effects of the extremes in composition, particularly high $\mathrm{Na}_{2} \mathrm{O}$ concentrations, which are represented by some of the study glasses. Note that several of the CCC versions of the study glasses have NL [B] values that are lower than their quenched counterparts. This may be due to the type of crystalline phases that formed within the glasses. If crystalline phases that remove the alkali from the glass without removing the glass forming components form during the CCC thermal treatment, PCT response may improve. Quantitative XRD studies would aid in understanding this behavior. 
WSRC-STI-2007-00659

Revision 0

This page intentionally left blank. 
WSRC-STI-2007-00659

Revision 0

\subsection{Conclusions}

The results of this study confirm that some conservatism exists in the current nepheline discriminator. Several glass compositions, particularly compositions that target higher $\mathrm{Al}_{2} \mathrm{O}_{3}$ concentrations, were shown to be very durable (i.e., PCT responses that were more than an order of magnitude better than that of the EA benchmark glass) while their nepheline discriminator values were well below the current nepheline discriminator limit of 0.62. Increased concentrations of $\mathrm{B}_{2} \mathrm{O}_{3}$, and, to a degree, increased concentrations of $\mathrm{CaO}$ were shown to improve durability responses and suppress the formation of nepheline. Recently a smaller, yet similar study provided supporting results. ${ }^{11}$ These provide incentive to continue the investigation into the possibility of a revision to the nepheline discriminator to reduce some of this conservatism and incorporate the influence of $\mathrm{B}_{2} \mathrm{O}_{3}$ at a minimum.

Figure 4-1 shows an example of the potential change to the nepheline discriminator. The revised nepheline discriminator could potentially change from a constant line on the $\mathrm{SiO}_{2}-\mathrm{Na}_{2} \mathrm{O}-\mathrm{Al}_{2} \mathrm{O}_{3}$ ternary diagram (Figure 4-1 (a)) to a sloped line, where the slope varies with $\mathrm{B}_{2} \mathrm{O}_{3}$ concentration, thus allowing access to higher concentrations of $\mathrm{Al}_{2} \mathrm{O}_{3}$ in glass (Figure 4-1 (b)). Additional data will be required to support development and possible implementation of this revision. A Phase II study will be required to provide the necessary data, and recommendations for a Phase II study are included in the following section.

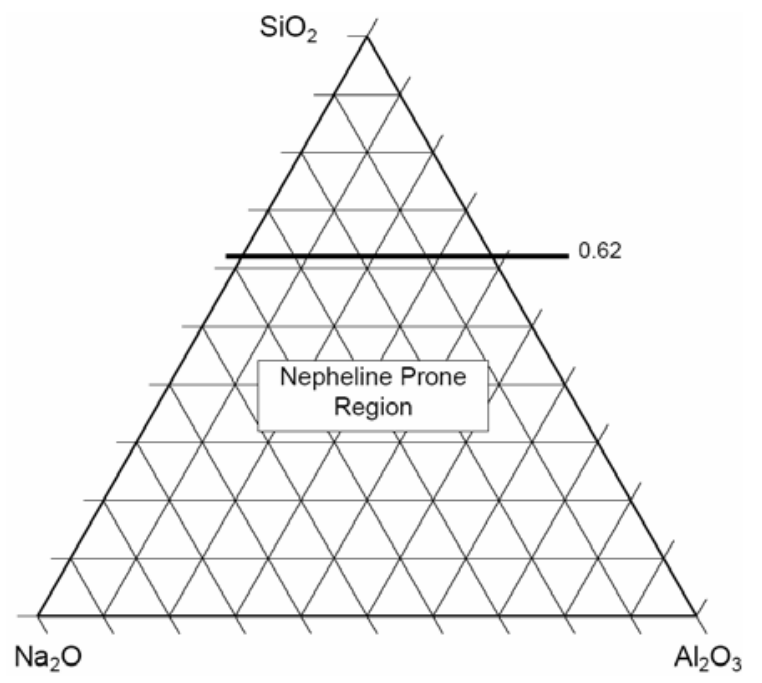

(a)

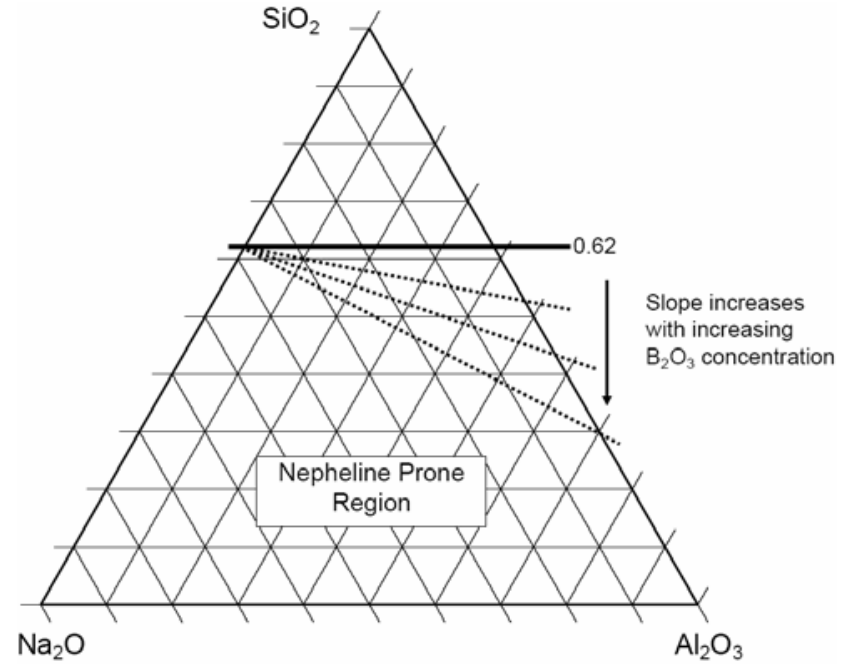

(b)

Figure 4-1. The current nepheline discriminator plotted on the $\mathrm{SiO}_{2}-\mathrm{Na}_{2} \mathrm{O}-\mathrm{Al}_{2} \mathrm{O}_{3}$ ternary diagram (a), and a potential example of the refined nepheline discriminator (b), where the slope of the line identifying the nepheline prone region increases with increasing $\mathrm{B}_{2} \mathrm{O}_{3}$ concentration. 
WSRC-STI-2007-00659

Revision 0

This page intentionally left blank. 
WSRC-STI-2007-00659

Revision 0

\subsection{Recommendations}

A second phase of this study should be undertaken to provide the data necessary to support the possible revision to the nepheline discriminator as currently implemented in the DWPF process control system. The study described here (Phase I) evaluated a relatively wide range of glass compositions in order to provide the data necessary to identify conservatism in, and compositional impacts on, the current nepheline discriminator. These glass compositions were intentionally chosen to challenge the nepheline discriminator and therefore represent compositional regions that would be restricted from processing at the DWPF by the current control models. The second phase should focus on a smaller range of glass compositions that more closely resemble those likely to be processed in completing SRS waste disposition missions.

The impact of $\mathrm{B}_{2} \mathrm{O}_{3}$ and $\mathrm{CaO}$ concentrations should continue to be an important focus of the study. Consideration should be given to the practicality of adding a relatively large amount of $\mathrm{B}_{2} \mathrm{O}_{3}$ to a frit for DWPF processing. The glass compositions did not contain $\mathrm{Li}_{2} \mathrm{O}$ during this phase in order to focus only on the effects of $\mathrm{Na}_{2} \mathrm{O}$. The second phase should include $\mathrm{Li}_{2} \mathrm{O}$ as a variable since the frits utilized at DWPF typically contain this component. Finally, the glasses in this study were melted over a range of temperatures, some of which were well above the operating temperature of the DWPF melter. This decision was made again to allow access to a wide range of glass compositions. The second phase study should more closely consider the effects of both melting temperature and the CCC thermal treatment on nepheline crystallization. 
WSRC-STI-2007-00659

Revision 0

This page intentionally left blank. 
WSRC-STI-2007-00659

Revision 0

\subsection{References}

1. Li, H., B. Jones, P. Hrma and J. D. Vienna, "Compositional Effects on Liquidus Temperature of Hanford Simulated High-Level Waste Glasses Precipitating Nepheline $\left(\mathrm{NaAlSiO}_{4}\right)$," Ceramic Transactions Vol. 87, edited by D. K. Peeler and J. C. Marra. American Ceramic Society, Westerville, OH, pp. 279-288 (1998).

2. Li, H., P. Hrma, J. D. Vienna, M. Qian, Y. Su and D. E. Smith, "Effects of $\mathrm{Al}_{2} \mathrm{O}_{3}, \mathrm{~B}_{2} \mathrm{O}_{3}, \mathrm{Na}_{2} \mathrm{O}$, and $\mathrm{SiO}_{2}$ on Nepheline Formation in Borosilicate Glasses: Chemical and Physical Correlations," J. Non-Crystalline Solids, 331 202-216 (2003).

3. Edwards, T. B., D. K. Peeler and K. M. Fox, "The Nepheline Discriminator: Justification and DWPF PCCS Implementation Details,” U.S. Department of Energy Report WSRC-STI-200600014, Revision 0, Washington Savannah River Company, Aiken, SC (2006).

4. Fox, K. M., T. B. Edwards and D. K. Peeler, "Nepheline Formation Potential in Sludge Batch 4 (SB4) and Its Impact on Durability: Selecting Glasses for a Phase 3 Study," U.S. Department of Energy Report WSRC-TR-2006-00053, Revision 0, Washington Savannah River Company, Aiken, SC (2006).

5. Fox, K. M., T. B. Edwards, D. K. Peeler, D. R. Best, I. A. Reamer and R. J. Workman, "Durability and Nepheline Crystallization Study for High Level Waste (HLW) Sludge Batch 4 (SB4) Glasses Formulated with Frit 503," U.S. Department of Energy Report WSRC-STI-200600009, Revision 0, Washington Savannah River Company, Aiken, SC (2006).

6. Fox, K. M., D. K. Peeler, T. B. Edwards, D. R. Best, I. A. Reamer and R. J. Workman, "Nepheline Formation Study for Sludge Batch 4 (SB4): Phase 3 Experimental Results," U.S. Department of Energy Report WSRC-TR-2006-00093, Revision 0, Washington Savannah River Company, Aiken, SC (2006).

7. Peeler, D. K., T. B. Edwards, D. R. Best, I. A. Reamer and R. J. Workman, “Nepheline Formation Study for Sludge Batch 4 (SB4): Phase 2 Experimental Results," U.S. Department of Energy Report WSRC-TR-2006-00006, Revision 0, Washington Savannah River Company, Aiken, SC (2006).

8. Peeler, D. K., T. B. Edwards and T. H. Lorier, "Nepheline Formation Potential in Sludge Batch (SB4) Glasses,” U.S. Department of Energy Report WSRC-TR-2005-00153, Revision 0, Westinghouse Savannah River Company, Aiken, SC (2005).

9. Besmann, T. M., K. E. Spear and E. C. Beahm, “Assessment of Nepheline Precipitation in Nuclear Waste Glass via Thermochemical Modeling,” Mater. Res. Soc. Symp. Proc., 608 715-720 (2000).

10. Li, H., J. D. Vienna, P. Hrma, D. E. Smith and M. J. Schweiger, "Nepheline Precipitation in High-Level Waste Glasses: Compositional Effects and Impact on the Waste Form Acceptability," Mat. Res. Soc. Proc., Vol. 465, pp. 261-268 (1997).

11. Fox, K. M. and D. K. Peeler, "Demonstration of Very High Aluminum Retention in Simulated HLW Glass,” U.S. Department of Energy Report SRNL-PSE-2007-00231, Washington Savannah River Company, Aiken, SC (2007). 
WSRC-STI-2007-00659

Revision 0

12. Culbertson, B. H., “Sludge Batch 5 Frit Optimization,” U.S. Department of Energy Report HLW-DWPF-TTR-2007-0007, Revision 0, Washington Savannah River Company, Aiken, SC (2006).

13. Peeler, D. K., "Sludge Batch 5 Frit Optimization,” U.S. Department of Energy Report WSRCSTI-2006-00321, Washington Savannah River Company, Aiken, SC (2007).

14. Shah, H. B., "Estimate of Sludge Batch 4 and 5 Sludge Calcine Compositions for SRNL," U.S. Department of Energy Report LWO-PIT-2007-00017, Revision 0, Washington Savannah River Company, Aiken, SC (2007).

15. SRNL, "Glass Batching," U.S. Department of Energy Report SRTC Procedure Manual, L29, ITS-0001, Westinghouse Savannah River Company, Aiken, SC (2002).

16. SRNL, “Glass Melting,” U.S. Department of Energy Report SRTC Procedure Manual, L29, ITS-0003, Westinghouse Savannah River Company, Aiken, SC (2002).

17. Marra, S. L. and C. M. Jantzen, "Characterization of Projected DWPF Glass Heat Treated to Simulate Canister Centerline Cooling," U.S. Department of Energy Report WSRC-TR-92-142, Revision 1, Westinghouse Savannah River Company, Aiken, SC (1993).

18. Edwards, T. B., "Analytical Plans for Measuring the Chemical Compositions of Glasses from the SB5 Nepheline Study,” U.S. Department of Energy Report SRNL-SCS-2007-00031, Washington Savannah River Company, Aiken, SC (2007).

19. ASTM, "Standard Test Methods for Determining Chemical Durability of Nuclear Waste Glasses: The Product Consistency Test (PCT),” ASTM C-1285, (2002).

20. Jantzen, C. M., N. E. Bibler, D. C. Beam, C. L. Crawford and M. A. Pickett, "Characterization of the Defense Waste Processing Facility (DWPF) Environmental Assessment (EA) Glass Standard Reference Material,” U.S. Department of Energy Report WSRC-TR-92-346, Revision 1, Westinghouse Savannah River Company, Aiken, SC (1993).

21. Edwards, T. B., "Analytical Plans for Measuring the PCT Solutions for the SB5 Nepheline Study Glasses,” U.S. Department of Energy Report SRNL-SCS-2007-00023, Washington Savannah River Company, Aiken, SC (2007).

22. Bickford, D. F. and C. M. Jantzen, "Devitrification of SRL Defense Waste Glass,” Sci. Basis for Nuclear Waste Management VII, edited by G. L. McVay. Elsevier, New York, pp. 557-565 (1984).

23. Jantzen, C. M., J. B. Picket, K. G. Brown, T. B. Edwards and D. C. Beam, "Process/Product Models for the Defense Waste Processing Facility (DWPF): Part I. Predicting Glass Durability from Composition Using a Thermodynamic Hydration Energy Reaction Model (THERMO)," U.S. Department of Energy Report WSRC-TR-93-672, Revision 1, Westinghouse Savannah River Company, Aiken, SC (1995).

24. Cozzi, A. D., N. E. Bibler and C. J. Bannochie, "Analytical Results of DWPF Glass Sample Taken During Filling of Canister S01913,” U.S. Department of Energy Report WSRC-TR-200400316, Revision 2, Washington Savannah River Company, Aiken, SC (2004). 
25. Pareizs, J. M., C. J. Bannochie, M. J. Barnes, N. E. Bibler, D. R. Click, E. K. Hansen, D. P. Lambert and M. E. Stone, "Demonstration of the DWPF Flowsheet in the SRNL Shielded Cells in Support of Sludge Batch 4 Qualification,” U.S. Department of Energy Report WSRC-STI-200700053, Revision 0, Washington Savannah River Company, Aiken, SC (2007).

26. Fox, K. M., E. N. Hoffman, C. L. Crawford, T. B. Edwards, D. R. Best and J. C. Marra, "Variability Study to Determine the Solubility of Impurities in Plutonium-Bearing, Lanthanide Borosilicate Glass,” U.S. Department of Energy Report WSRC-STI-2007-00477, Revision 0, Washington Savannah River Company, Aiken, SC (2007).

27. Fox, K. M., T. B. Edwards, D. R. Best, I. A. Reamer and R. J. Workman, "Sludge Batch 5 (SB5): Selection of Candidate Frits and Characterization of Preliminary Glass Systems," U.S. Department of Energy Report WSRC-STI-2007-00418, Rev. 0, Washington Savannah River Company, Aiken, SC (2007).

28. Gillam Jr., J. H., K. M. Fox, T. B. Edwards and D. K. Peeler, "Frit Selection to Support Steklo Metallicheskie Konstruktsii (SMK) Melter Testing with SRNL Feeds," U.S. Department of Energy Report WSRC-STI-2007-00363, Revision 0, Washington Savannah River Company, Aiken, SC (2007).

29. Fox, K. M., T. B. Edwards, D. K. Peeler, D. R. Best, I. A. Reamer and R. J. Workman, "High Level Waste (HLW) Sludge Batch 4 (SB4) Variability Study,” U.S. Department of Energy Report WSRC-STI-2006-00204, Revision 0, Washington Savannah River Company, Aiken, SC (2006). 
WSRC-STI-2007-00659

Revision 0

Appendix A

Tables and Exhibits Supporting the Analysis of the Chemical Composition Measurements of the SB5-Nepheline Study Glasses 
Table A1. Targeted Oxide Concentrations (wt \%) for the Nepheline Study Glasses.

\begin{tabular}{|c|c|c|c|c|c|c|c|c|c|c|c|c|c|c|c|c|c|c|c|c|c|}
\hline Glass ID & $\mathrm{Al}_{2} \mathrm{O}_{3}$ & $\mathrm{~B}_{2} \mathrm{O}_{3}$ & $\mathrm{BaO}$ & $\mathrm{CaO}$ & $\mathrm{Ce}_{2} \mathrm{O}_{3}$ & $\mathrm{Cr}_{2} \mathrm{O}_{3}$ & $\begin{array}{l}\mathrm{CuO} \\
\end{array}$ & $\mathrm{Fe}_{2} \mathrm{O}_{3}$ & $\mathrm{~K}_{2} \mathrm{O}$ & $\mathrm{La}_{2} \mathrm{O}_{3}$ & $\mathrm{MgO}$ & $\mathrm{MnO}$ & $\mathrm{Na}_{2} \mathrm{O}$ & $\mathrm{NiO}$ & $\begin{array}{l}\mathrm{PbO} \\
\end{array}$ & $\mathrm{SO}_{4}$ & $\mathrm{SiO}_{2}$ & $\mathrm{TiO}_{2}$ & $\mathrm{ZnO}$ & $\mathrm{ZrO}_{2}$ & Sum \\
\hline SB5NEPH-01 & 7.800 & 4.000 & 0.049 & 2.000 & 0.103 & 0.090 & 0.033 & 10.772 & 0.069 & 0.015 & 0.626 & 2.306 & 23.400 & 1.022 & 0.043 & 0.513 & 46.800 & 0.227 & 0.033 & 0.101 & 100.000 \\
\hline SB5NEPH-02 & 6.400 & 18.000 & 0.049 & 2.000 & 0.103 & 0.090 & 0.033 & 10.772 & 0.069 & 0.015 & 0.626 & 2.306 & 19.200 & 1.022 & 0.043 & 0.513 & 38.400 & 0.227 & 0.033 & 0.101 & 100.000 \\
\hline SB5NEPH-03 & 6.800 & 4.000 & 0.049 & 12.000 & 0.103 & 0.090 & 0.033 & 10.772 & 0.069 & 0.015 & 0.626 & 2.306 & 20.400 & 1.022 & 0.043 & 0.513 & 40.800 & 0.227 & 0.033 & 0.101 & 100.000 \\
\hline SB5NEPH-04 & 5.400 & 18.000 & 0.049 & 12.000 & 0.103 & 0.090 & 0.033 & 10.772 & 0.069 & 0.015 & 0.626 & 2.306 & 16.200 & 1.022 & 0.043 & 0.513 & 32.400 & 0.227 & 0.033 & 0.101 & 100.000 \\
\hline SB5NEPH-05 & 15.600 & 4.000 & 0.049 & 2.000 & 0.103 & 0.090 & 0.033 & 10.772 & 0.069 & 0.015 & 0.626 & 2.306 & 15.600 & 1.022 & 0.043 & 0.513 & 46.800 & 0.227 & 0.033 & 0.101 & 100.000 \\
\hline SB5NEPH-06 & 12.800 & 18.000 & 0.049 & 2.000 & 0.103 & 0.090 & 0.033 & 10.772 & 0.069 & 0.015 & 0.626 & 2.306 & 12.800 & 1.022 & 0.043 & 0.513 & 38.400 & 0.227 & 0.033 & 0.101 & 100.000 \\
\hline SB5NEPH-07 & 13.600 & 4.000 & 0.049 & 12.000 & 0.103 & 0.090 & 0.033 & 10.772 & 0.069 & 0.015 & 0.626 & 2.306 & 13.600 & 1.022 & 0.043 & 0.513 & 40.800 & 0.227 & 0.033 & 0.101 & 100.000 \\
\hline SB5NEPH-08 & 10.800 & 18.000 & 0.049 & 12.000 & 0.103 & 0.090 & 0.033 & 10.772 & 0.069 & 0.015 & 0.626 & 2.306 & 10.800 & 1.022 & 0.043 & 0.513 & 32.400 & 0.227 & 0.033 & 0.101 & 100.000 \\
\hline SB5NEPH-09 & 23.400 & 4.000 & 0.049 & 2.000 & 0.103 & 0.090 & 0.033 & 10.772 & 0.069 & 0.015 & 0.626 & 2.306 & 7.800 & 1.022 & 0.043 & 0.513 & 46.800 & 0.227 & 0.033 & 0.101 & 100.000 \\
\hline SB5NEPH-10 & 19.200 & 18.000 & 0.049 & 2.000 & 0.103 & 0.090 & 0.033 & 10.772 & 0.069 & 0.015 & 0.626 & 2.306 & 6.400 & 1.022 & 0.043 & 0.513 & 38.400 & 0.227 & 0.033 & 0.101 & 100.000 \\
\hline SB5NEPH-11 & 20.400 & 4.000 & 0.049 & 12.000 & 0.103 & 0.090 & 0.033 & 10.772 & 0.069 & 0.015 & 0.626 & 2.306 & 6.800 & 1.022 & 0.043 & 0.513 & 40.800 & 0.227 & 0.033 & 0.101 & 100.000 \\
\hline SB5NEPH-12 & 16.200 & 18.000 & 0.049 & 12.000 & 0.103 & 0.090 & 0.033 & 10.772 & 0.069 & 0.015 & 0.626 & 2.306 & 5.400 & 1.022 & 0.043 & 0.513 & 32.400 & 0.227 & 0.033 & 0.101 & 100.000 \\
\hline SB5NEPH-13 & 7.800 & 4.000 & 0.049 & 2.000 & 0.103 & 0.090 & 0.033 & 10.772 & 0.069 & 0.015 & 0.626 & 2.306 & 31.200 & 1.022 & 0.043 & 0.513 & 39.000 & 0.227 & 0.033 & 0.101 & 100.000 \\
\hline SB5NEPH-14 & 6.400 & 18.000 & 0.049 & 2.000 & 0.103 & 0.090 & 0.033 & 10.772 & 0.069 & 0.015 & 0.626 & 2.306 & 25.600 & 1.022 & 0.043 & 0.513 & 32.000 & 0.227 & 0.033 & 0.101 & 100.000 \\
\hline SB5NEPH-15 & 6.800 & 4.000 & 0.049 & 12.000 & 0.103 & 0.090 & 0.033 & 10.772 & 0.069 & 0.015 & 0.626 & 2.306 & 27.200 & 1.022 & 0.043 & 0.513 & 34.000 & 0.227 & 0.033 & 0.101 & 100.000 \\
\hline SB5NEPH-16 & 5.400 & 18.000 & 0.049 & 12.000 & 0.103 & 0.090 & 0.033 & 10.772 & 0.069 & 0.015 & 0.626 & 2.306 & 21.600 & 1.022 & 0.043 & 0.513 & 27.000 & 0.227 & 0.033 & 0.101 & 100.000 \\
\hline SB5NEPH-17 & 15.600 & 4.000 & 0.049 & 2.000 & 0.103 & 0.090 & 0.033 & 10.772 & 0.069 & 0.015 & 0.626 & 2.306 & 23.400 & 1.022 & 0.043 & 0.513 & 39.000 & 0.227 & 0.033 & 0.101 & 100.000 \\
\hline SB5NEPH-18 & 12.800 & 18.000 & 0.049 & 2.000 & 0.103 & 0.090 & 0.033 & 10.772 & 0.069 & 0.015 & 0.626 & 2.306 & 19.200 & 1.022 & 0.043 & 0.513 & 32.000 & 0.227 & 0.033 & 0.101 & 100.000 \\
\hline SB5NEPH-19 & 13.600 & 4.000 & 0.049 & 12.000 & 0.103 & 0.090 & 0.033 & 10.772 & 0.069 & 0.015 & 0.626 & 2.306 & 20.400 & 1.022 & 0.043 & 0.513 & 34.000 & 0.227 & 0.033 & 0.101 & 100.000 \\
\hline SB5NEPH-20 & 10.800 & 18.000 & 0.049 & 12.000 & 0.103 & 0.090 & 0.033 & 10.772 & 0.069 & 0.015 & 0.626 & 2.306 & 16.200 & 1.022 & 0.043 & 0.513 & 27.000 & 0.227 & 0.033 & 0.101 & 100.000 \\
\hline SB5NEPH-21 & 23.400 & 4.000 & 0.049 & 2.000 & 0.103 & 0.090 & 0.033 & 10.772 & 0.069 & 0.015 & 0.626 & 2.306 & 15.600 & 1.022 & 0.043 & 0.513 & 39.000 & 0.227 & 0.033 & 0.101 & 100.000 \\
\hline SB5NEPH-22 & 19.200 & 18.000 & 0.049 & 2.000 & 0.103 & 0.090 & 0.033 & 10.772 & 0.069 & 0.015 & 0.626 & 2.306 & 12.800 & 1.022 & 0.043 & 0.513 & 32.000 & 0.227 & 0.033 & 0.101 & 100.000 \\
\hline SB5NEPH-23 & 20.400 & 4.000 & 0.049 & 12.000 & 0.103 & 0.090 & 0.033 & 10.772 & 0.069 & 0.015 & 0.626 & 2.306 & 13.600 & 1.022 & 0.043 & 0.513 & 34.000 & 0.227 & 0.033 & 0.101 & 100.000 \\
\hline SB5NEPH-24 & 16.200 & 18.000 & 0.049 & 12.000 & 0.103 & 0.090 & 0.033 & 10.772 & 0.069 & 0.015 & 0.626 & 2.306 & 10.800 & 1.022 & 0.043 & 0.513 & 27.000 & 0.227 & 0.033 & 0.101 & 100.000 \\
\hline SB5NEPH-25 & 31.200 & 4.000 & 0.049 & 2.000 & 0.103 & 0.090 & 0.033 & 10.772 & 0.069 & 0.015 & 0.626 & 2.306 & 7.800 & 1.022 & 0.043 & 0.513 & 39.000 & 0.227 & 0.033 & 0.101 & 100.000 \\
\hline SB5NEPH-26 & 25.600 & 18.000 & 0.049 & 2.000 & 0.103 & 0.090 & 0.033 & 10.772 & 0.069 & 0.015 & 0.626 & 2.306 & 6.400 & 1.022 & 0.043 & 0.513 & 32.000 & 0.227 & 0.033 & 0.101 & 100.000 \\
\hline SB5NEPH-27 & 27.200 & 4.000 & 0.049 & 12.000 & 103 & 0.090 & 0.033 & 10.772 & 0.069 & 0.015 & 0.626 & 2.306 & 6.800 & 1.022 & 0.043 & 0.513 & 34.000 & 0.227 & 0.033 & 0.101 & 100.000 \\
\hline SB5NEPH-28 & 21.600 & 18.000 & 0.049 & 12.000 & 0.103 & 0.090 & 0.033 & 10.772 & 0.069 & 0.015 & 0.626 & 2.306 & 5.400 & 1.022 & 0.043 & 0.513 & 27.000 & 0.227 & 0.033 & 0.101 & 100.000 \\
\hline SB5NEPH-29 & 7.800 & 4.000 & 0.049 & 2.000 & 0.103 & 0.090 & 0.033 & 10.772 & 0.069 & 0.015 & 0.626 & 2.306 & 39.000 & 1.022 & 0.043 & 0.513 & 31.200 & 0.227 & 0.033 & 0.101 & 100.000 \\
\hline SB5NEPH-30 & 6.400 & 18.000 & 0.049 & 2.000 & 103 & 0.090 & 0.033 & 10.772 & 0.069 & 0.015 & 0.626 & 2.306 & 32.000 & 1.022 & 0.043 & 0.513 & 25.600 & 0.227 & 0.033 & 0.101 & 100.000 \\
\hline SB5NEPH-31 & 6.800 & 4.000 & 0.049 & 12.000 & 0.103 & 0.090 & 0.033 & 10.772 & 0.069 & 0.015 & 0.626 & 2.306 & 34.000 & 1.022 & 0.043 & 0.513 & 27.200 & 0.227 & 0.033 & 0.101 & 100.000 \\
\hline SB5NEPH-32 & 5.400 & 18.000 & 0.049 & 12.000 & & 0.090 & 0.033 & 10.772 & 0.069 & 0.015 & 0.626 & 2.306 & 27.000 & 1.022 & 0.043 & 0.513 & 21.600 & 0.227 & 0.033 & 0.101 & 100.000 \\
\hline SB5NEPH-33 & 23.400 & 4.000 & 0.049 & 2.000 & 0.103 & 0.090 & 0.033 & 10.772 & 0.069 & 0.015 & 0.626 & 2.306 & 23.400 & 1.022 & 0.043 & 0.513 & 31.200 & 0.227 & 0.033 & 0.101 & 100.000 \\
\hline SB5NEPH-34 & 19.200 & 18.000 & 0.049 & 2.000 & 0.103 & 0.090 & 0.033 & 10.772 & 0.069 & 0.015 & 0.626 & 2.306 & 19.200 & 1.022 & 0.043 & 0.513 & 25.600 & 0.227 & 0.033 & 0.101 & 100.000 \\
\hline SB5NEPH-35 & 20.400 & 4.000 & 0.049 & 12.000 & 0.103 & 0.090 & 0.033 & 10.772 & 0.069 & 0.015 & 0.626 & 2.306 & 20.400 & 1.022 & 0.043 & 0.513 & 27.200 & 0.227 & 0.033 & 0.101 & 100.000 \\
\hline SB5NEPH-36 & 16.200 & 18.000 & 0.049 & 12.000 & 0.103 & 0.090 & 0.033 & 10.772 & 0.069 & 0.015 & 0.626 & 2.306 & 16.200 & 1.022 & 0.043 & 0.513 & 21.600 & 0.227 & 0.033 & 0.101 & 100.000 \\
\hline SB5NEPH-37 & 39.000 & 4.000 & 0.049 & 2.000 & 0.103 & 0.090 & 0.033 & 10.772 & 0.069 & 0.015 & 0.626 & 2.306 & 7.800 & 1.022 & 0.043 & 0.513 & 31.200 & 0.227 & 0.033 & 0.101 & 100.000 \\
\hline SB5NEPH-38 & 32.000 & 18.000 & 0.049 & 2.000 & 0.103 & 0.090 & 0.033 & 10.772 & 0.069 & 0.015 & 0.626 & 2.306 & 6.400 & 1.022 & 0.043 & 0.513 & 25.600 & 0.227 & 0.033 & 0.101 & 100.000 \\
\hline SB5NEPH-39 & 34.000 & 4.000 & 0.049 & 12.000 & 0.103 & 0.090 & 0.033 & 10.772 & 0.069 & 0.015 & 0.626 & 2.306 & 6.800 & 1.022 & 0.043 & 0.513 & 27.200 & 0.227 & 0.033 & 0.101 & 100.000 \\
\hline SB5NEPH-40 & 27.000 & 18.000 & 0.049 & 12.000 & 0.103 & 0.090 & 0.033 & 10.772 & 0.069 & 0.015 & 0.626 & 2.306 & 5.400 & 1.022 & 0.043 & 0.513 & 21.600 & 0.227 & 0.033 & 0.101 & 100.000 \\
\hline
\end{tabular}


Table A2. Measured Elemental Concentrations (wt \%) for Samples Prepared Using Lithium Metaborate. (continued)

\begin{tabular}{|c|c|c|c|c|c|c|c|c|c|c|c|c|c|c|c|c|c|c|c|c|c|c|}
\hline Set & Glass ID & Block & $\begin{array}{c}\text { Sub } \\
\text { Block }\end{array}$ & Seq. & ID Check & $\begin{array}{c}\mathrm{Al} \\
\text { (wt\%) }\end{array}$ & $\begin{array}{c}\mathrm{Ba} \\
\text { (wt\%) }\end{array}$ & $\begin{array}{c}\mathrm{Ca} \\
\text { (wt\%) }\end{array}$ & $\begin{array}{c}\mathrm{Ce} \\
\text { (wt\%) }\end{array}$ & $\begin{array}{c}\mathrm{Cr} \\
(\mathrm{wt} \%)\end{array}$ & $\begin{array}{c}\mathrm{Cu} \\
(\mathrm{wt} \%)\end{array}$ & $\begin{array}{c}\mathrm{K} \\
(\mathrm{wt} \%)\end{array}$ & $\begin{array}{c}\mathrm{La} \\
\text { (wt\%) }\end{array}$ & $\begin{array}{c}\mathrm{Mg} \\
\text { (wt\%) }\end{array}$ & $\begin{array}{c}\begin{array}{c}\mathrm{Mn} \\
\text { (wt\%) }\end{array} \\
\end{array}$ & $\begin{array}{c}\mathrm{Na} \\
\text { (wt\%) }\end{array}$ & $\begin{array}{c}\mathrm{Ni} \\
\text { (wt\%) }\end{array}$ & $\begin{array}{c}\begin{array}{c}\mathrm{Pb} \\
(\mathrm{wt} \%)\end{array} \\
\end{array}$ & $\begin{array}{c}\mathrm{S} \\
\text { (wt\%) }\end{array}$ & $\begin{array}{c}\mathrm{Ti} \\
\text { (wt\%) }\end{array}$ & $\begin{array}{c}\mathrm{Zn} \\
(\mathrm{wt} \%)\end{array}$ & $\begin{array}{c}\mathrm{Zr} \\
\text { (wt\%) }\end{array}$ \\
\hline 1 & Batch 1 & 1 & 1 & 1 & BCHLM1111 & 2.49 & 0.126 & 0.818 & $<0.010$ & 0.074 & 0.312 & 2.57 & $<0.100$ & 0.836 & 1.32 & 6.61 & 0.558 & $<0.010$ & $<0.100$ & 0.388 & $<0.010$ & 0.063 \\
\hline 1 & SB5NEPH-02 & 1 & 1 & 2 & R06LM11 & 3.38 & 0.04 & 1.42 & 0.08 & 0.062 & 0.026 & 0.051 & $<0.100$ & 0.381 & 1.81 & 13.9 & 0.759 & 0.035 & 0.166 & 0.134 & 0.023 & 0.064 \\
\hline 1 & SB5NEPH-01 & 1 & 1 & 3 & R01LM21 & 4.17 & 0.039 & 1.42 & 0.078 & 0.062 & 0.032 & 0.049 & $<0.100$ & 0.384 & 1.82 & 17.1 & 0.757 & 0.035 & 0.169 & 0.138 & 0.022 & 0.068 \\
\hline 1 & SB5NEPH-06 & 1 & 1 & 4 & R03LM11 & 6.82 & 0.039 & 1.42 & 0.081 & 0.058 & 0.027 & 0.06 & $<0.100$ & 0.381 & 1.81 & 9.55 & 0.731 & 0.033 & 0.121 & 0.135 & 0.024 & 0.063 \\
\hline 1 & SB5NEPH-02 & 1 & 1 & 5 & R06LM21 & 3.41 & 0.039 & 1.44 & 0.08 & 0.063 & 0.028 & 0.049 & $<0.100$ & 0.38 & 1.81 & 14.2 & 0.753 & 0.032 & 0.163 & 0.133 & 0.023 & 0.067 \\
\hline 1 & SB5NEPH-04 & 1 & 1 & 6 & R07LM11 & 2.88 & 0.037 & 8.67 & 0.078 & 0.061 & 0.028 & 0.052 & $<0.100$ & 0.378 & 1.79 & 12 & 0.768 & 0.033 & 0.147 & 0.135 & 0.022 & 0.053 \\
\hline 1 & Batch 1 & 1 & 1 & 7 & BCHLM1112 & 2.49 & 0.124 & 0.823 & $<0.010$ & 0.074 & 0.313 & 2.59 & $<0.100$ & 0.82 & 1.33 & 6.68 & 0.551 & $<0.010$ & $<0.100$ & 0.389 & $<0.010$ & 0.062 \\
\hline 1 & SB5NEPH-01 & 1 & 1 & 8 & R01LM11 & 4.19 & 0.039 & 1.44 & 0.077 & 0.075 & 0.027 & 0.057 & $<0.100$ & 0.378 & 1.8 & 17.2 & 0.743 & 0.037 & 0.172 & 0.137 & 0.024 & 0.064 \\
\hline 1 & SB5NEPH-03 & 1 & 1 & 9 & R10LM11 & 3.67 & 0.038 & 8.7 & 0.078 & 0.061 & 0.024 & 0.05 & $<0.100$ & 0.376 & 1.8 & 15.3 & 0.742 & 0.029 & 0.174 & 0.134 & 0.023 & 0.068 \\
\hline 1 & SB5NEPH-06 & 1 & 1 & 10 & R03LM21 & 6.88 & 0.039 & 1.42 & 0.081 & 0.06 & 0.026 & 0.05 & $<0.100$ & 0.378 & 1.81 & 9.77 & 0.729 & 0.034 & 0.109 & 0.135 & 0.023 & 0.067 \\
\hline 1 & SB5NEPH-03 & 1 & 1 & 11 & R10LM21 & 3.61 & 0.038 & 8.58 & 0.078 & 0.061 & 0.029 & 0.05 & $<0.100$ & 0.373 & 1.77 & 15 & 0.744 & 0.029 & 0.172 & 0.133 & 0.023 & 0.068 \\
\hline 1 & SB5NEPH-04 & 1 & 1 & 12 & $\begin{array}{l}\text { R07LM21 } \\
\end{array}$ & 2.9 & 0.036 & 8.72 & 0.076 & 0.059 & 0.029 & 0.05 & $<0.100$ & 0.365 & 1.8 & 12 & 0.737 & 0.031 & 0.145 & 0.131 & 0.022 & 0.065 \\
\hline 1 & Batch 1 & 1 & 1 & 13 & BCHLM1113 & 2.55 & 0.125 & 0.842 & $<0.010$ & 0.074 & 0.321 & 2.67 & $<0.100$ & 0.829 & 1.32 & 6.82 & 0.555 & $<0.010$ & $<0.100$ & 0.391 & $<0.010$ & 0.062 \\
\hline 1 & $\begin{array}{l}\text { Batch } 1 \\
\end{array}$ & 2 & 1 & 1 & BCHLM1211 & 2.48 & 0.125 & 0.811 & $<0.010$ & 0.075 & 0.312 & 2.49 & $<0.100$ & 0.824 & 1.33 & 6.76 & 0.553 & $<0.010$ & $<0.100$ & 0.391 & $<0.010$ & 0.064 \\
\hline 1 & SB5NEPH-09 & 2 & 1 & 2 & R04LM11 & 12.8 & 0.042 & 1.44 & 0.084 & 0.061 & 0.026 & 0.054 & $<0.100$ & 0.395 & 1.87 & 6.06 & 0.749 & 0.032 & $<0.100$ & 0.137 & 0.025 & 0.071 \\
\hline 1 & SB5NEPH-08 & 2 & 1 & 3 & R08LM21 & 5.65 & 0.04 & 8.52 & 0.083 & 0.059 & 0.031 & 0.054 & $<0.100$ & 0.381 & 1.81 & 8.09 & 0.735 & 0.03 & 0.16 & 0.135 & 0.024 & 0.069 \\
\hline 1 & SB5NEPH-05 & 2 & 1 & 4 & R02LM21 & 8.59 & 0.041 & 1.43 & 0.084 & 0.055 & 0.027 & 0.06 & $<0.100$ & 0.393 & 1.82 & 11.8 & 0.747 & 0.035 & 0.129 & 0.143 & 0.026 & 0.069 \\
\hline 1 & SB5NEPH-08 & 2 & 1 & 5 & R08LM11 & 5.77 & 0.042 & 8.7 & 0.088 & 0.062 & 0.031 & 0.061 & $<0.100$ & 0.4 & 1.83 & 8.29 & 0.773 & 0.034 & 0.17 & 0.142 & 0.027 & 0.073 \\
\hline 1 & SB5NEPH-09 & 2 & 1 & 6 & R04LM21 & 12.9 & 0.042 & 1.44 & 0.085 & 0.062 & 0.026 & 0.056 & $<0.100$ & 0.397 & 1.86 & 6.12 & 0.742 & 0.032 & $<0.100$ & 0.138 & 0.025 & 0.071 \\
\hline 1 & Batch 1 & 2 & 1 & 7 & BCHLM1211 & 2.52 & 0.126 & 0.815 & $<0.010$ & 0.075 & 0.313 & 2.52 & $<0.100$ & 0.821 & 1.33 & 6.9 & 0.552 & $<0.010$ & $<0.100$ & 0.389 & $<0.010$ & 0.064 \\
\hline 1 & SB5NEPH-07 & 2 & 1 & 8 & R09LM11 & 7.18 & 0.042 & 8.48 & 0.085 & 0.067 & 0.027 & 0.056 & $<0.100$ & 0.389 & 1.79 & 10.2 & 0.755 & 0.036 & 0.132 & 0.138 & 0.022 & 0.074 \\
\hline 1 & SB5NEPH-10 & 2 & 1 & 9 & R05LM21 & 10.5 & 0.041 & 1.45 & 0.084 & 0.042 & 0.027 & 0.054 & $<0.100$ & 0.398 & 1.86 & 5.08 & 0.755 & 0.033 & $<0.100$ & 0.143 & 0.024 & 0.073 \\
\hline 1 & SB5NEPH-10 & 2 & 1 & 10 & R05LM11 & 10.4 & 0.04 & 1.43 & 0.082 & 0.042 & 0.024 & 0.053 & $<0.100$ & 0.391 & 1.86 & 5.06 & 0.756 & 0.032 & $<0.100$ & 0.14 & 0.024 & 0.071 \\
\hline 1 & SB5NEPH-05 & 2 & 1 & 11 & R02LM11 & 8.37 & 0.04 & 1.42 & 0.082 & 0.055 & 0.027 & 0.055 & $<0.100$ & 0.383 & 1.81 & 11.8 & 0.74 & 0.033 & 0.12 & 0.139 & 0.026 & 0.069 \\
\hline 1 & SB5NEPH-07 & 2 & 1 & 12 & R09LM21 & 7.28 & 0.04 & 8.62 & 0.081 & 0.063 & 0.027 & 0.058 & $<0.100$ & 0.368 & 1.79 & 10.3 & 0.71 & 0.034 & 0.135 & 0.132 & 0.024 & 0.07 \\
\hline 1 & Batch 1 & 2 & 1 & 13 & BCHLM1213 & 2.52 & 0.125 & 0.811 & $<0.010$ & 0.074 & 0.313 & 2.52 & $<0.100$ & 0.818 & 1.33 & 6.89 & 0.547 & $<0.010$ & $<0.100$ & 0.389 & $<0.010$ & 0.064 \\
\hline 1 & Batch 1 & 1 & 2 & 1 & BCHLM1121 & 2.5 & 0.126 & 0.815 & $<0.010$ & 0.075 & 0.312 & 2.55 & $<0.100$ & 0.822 & 1.33 & 6.88 & 0.555 & $<0.010$ & $<0.100$ & 0.388 & $<0.010$ & 0.063 \\
\hline 1 & SB5NEPH-02 & 1 & 2 & 2 & R06LM12 & 3.38 & 0.041 & 1.41 & 0.082 & 0.063 & 0.026 & 0.048 & $<0.100$ & 0.381 & 1.79 & 14 & 0.754 & 0.035 & 0.161 & 0.134 & 0.022 & 0.066 \\
\hline 1 & SB5NEPH-03 & 1 & 2 & 3 & R10LM12 & 3.65 & 0.04 & 8.72 & 0.08 & 0.062 & 0.025 & 0.047 & $<0.100$ & 0.377 & 1.8 & 15.1 & 0.743 & 0.03 & 0.17 & 0.135 & 0.022 & 0.07 \\
\hline 1 & SB5NEPH-01 & 1 & 2 & 4 & R01LM12 & 4.43 & 0.04 & 1.51 & 0.078 & 0.075 & 0.028 & 0.053 & $<0.100$ & 0.378 & 1.9 & 18.3 & 0.74 & 0.036 & 0.168 & 0.136 & 0.023 & 0.065 \\
\hline 1 & SB5NEPH-04 & 1 & 2 & 5 & R07LM22 & 2.91 & 0.038 & 8.73 & 0.078 & 0.059 & 0.03 & 0.048 & $<0.100$ & 0.367 & 1.81 & 12.1 & 0.74 & 0.032 & 0.143 & 0.133 & 0.022 & 0.066 \\
\hline 1 & SB5NEPH-06 & 1 & 2 & 6 & R03LM22 & 7.01 & 0.041 & 1.43 & 0.083 & 0.061 & 0.027 & 0.048 & $<0.100$ & 0.38 & 1.83 & 9.92 & 0.731 & 0.035 & 0.113 & 0.136 & 0.023 & 0.069 \\
\hline 1 & Batch 1 & 1 & 2 & 7 & BCHLM1122 & 2.54 & 0.126 & 0.826 & $<0.010$ & 0.074 & 0.314 & 2.57 & $<0.100$ & 0.822 & 1.33 & 6.95 & 0.553 & $<0.010$ & $<0.100$ & 0.389 & $<0.010$ & 0.064 \\
\hline 1 & SB5NEPH-01 & 1 & 2 & 8 & R01LM22 & 4.08 & 0.039 & 1.39 & 0.077 & 0.061 & 0.031 & 0.044 & $<0.100$ & 0.367 & 1.75 & 16.9 & 0.723 & 0.034 & 0.158 & 0.134 & 0.021 & 0.067 \\
\hline 1 & SB5NEPH-06 & 1 & 2 & 9 & R03LM12 & 6.95 & 0.04 & 1.44 & 0.082 & 0.059 & 0.028 & 0.056 & $<0.100$ & 0.378 & 1.81 & 9.78 & 0.725 & 0.034 & 0.117 & 0.135 & 0.023 & 0.064 \\
\hline 1 & SB5NEPH-04 & 1 & 2 & 10 & R07LM12 & 2.95 & 0.039 & 8.95 & 0.08 & 0.062 & 0.029 & 0.05 & $<0.100$ & 0.378 & 1.81 & 12.3 & 0.763 & 0.033 & 0.142 & 0.136 & 0.021 & 0.054 \\
\hline 1 & SB5NEPH-02 & 1 & 2 & 11 & R06LM22 & 3.44 & 0.041 & 1.44 & 0.083 & 0.064 & 0.029 & 0.048 & $<0.100$ & 0.38 & 1.81 & 14.4 & 0.753 & 0.032 & 0.162 & 0.134 & 0.022 & 0.069 \\
\hline 1 & SB5NEPH-03 & 1 & 2 & 12 & $\begin{array}{l}\text { R10LM22 } \\
\end{array}$ & 3.67 & 0.04 & 8.75 & 0.08 & 0.062 & 0.03 & 0.049 & $<0.100$ & 0.374 & 1.8 & 15.3 & 0.743 & 0.029 & 0.167 & 0.134 & 0.022 & 0.07 \\
\hline 1 & Batch 1 & 1 & 2 & 13 & BCHLM1123 & 2.54 & 0.125 & 0.816 & $<0.010$ & 0.074 & 0.312 & 2.58 & $<0.100$ & 0.819 & 1.33 & 6.95 & 0.549 & $<0.010$ & $<0.100$ & 0.391 & $<0.010$ & 0.063 \\
\hline 1 & Batch 1 & 2 & 2 & 1 & BCHLM1221 & 2.51 & 0.126 & 0.81 & $<0.010$ & 0.075 & 0.313 & 2.55 & $<0.100$ & 0.828 & 1.34 & 6.78 & 0.555 & $<0.010$ & $<0.100$ & 0.387 & $<0.010$ & 0.064 \\
\hline 1 & SB5NEPH-05 & 2 & 2 & 2 & $\begin{array}{l}\text { R02LM22 } \\
\end{array}$ & 8.41 & 0.039 & 1.41 & 0.081 & 0.053 & 0.026 & 0.056 & $<0.100$ & 0.383 & 1.81 & 11.6 & 0.726 & 0.034 & 0.126 & 0.137 & 0.025 & 0.067 \\
\hline 1 & SB5NEPH-08 & 2 & 2 & 3 & R08LM22 & 5.69 & 0.039 & 8.53 & 0.082 & 0.058 & 0.031 & 0.051 & $<0.100$ & 0.378 & 1.82 & 8.06 & 0.729 & 0.031 & 0.16 & 0.133 & 0.023 & 0.069 \\
\hline 1 & SB5NEPH-09 & 2 & 2 & 4 & R04LM12 & 12.8 & 0.041 & 1.45 & 0.085 & 0.061 & 0.026 & 0.053 & $<0.100$ & 0.399 & 1.87 & 6.06 & 0.753 & 0.032 & $<0.100$ & 0.139 & 0.025 & 0.073 \\
\hline 1 & SB5NEPH-07 & 2 & 2 & 5 & R09LM22 & 7.27 & 0.04 & 8.6 & 0.081 & 0.064 & 0.027 & 0.056 & $<0.100$ & 0.372 & 1.8 & 10.3 & 0.716 & 0.036 & 0.129 & 0.131 & 0.024 & 0.071 \\
\hline 1 & SB5NEPH-10 & 2 & 2 & 6 & $\begin{array}{l}\text { R05LM22 } \\
\end{array}$ & 10.4 & 0.04 & 1.43 & 0.083 & 0.041 & 0.027 & 0.051 & $<0.100$ & 0.394 & 1.86 & 5 & 0.747 & 0.033 & $<0.100$ & 0.141 & 0.024 & 0.073 \\
\hline 1 & Batch 1 & 2 & 2 & 7 & BCHLM1222 & 2.52 & 0.126 & 0.814 & $<0.010$ & 0.075 & 0.314 & 2.55 & $<0.100$ & 0.832 & 1.33 & 6.84 & 0.555 & $<0.010$ & $<0.100$ & 0.391 & $<0.010$ & 0.065 \\
\hline
\end{tabular}


Table A2. Measured Elemental Concentrations (wt \%) for Samples Prepared Using Lithium Metaborate. (continued)

\begin{tabular}{|c|c|c|c|c|c|c|c|c|c|c|c|c|c|c|c|c|c|c|c|c|c|c|}
\hline Set & Glass ID & Block & $\begin{array}{c}\text { Sub } \\
\text { Block }\end{array}$ & Seq. & ID Check & $\begin{array}{c}\mathrm{Al} \\
\text { (wt\%) }\end{array}$ & $\begin{array}{c}\mathrm{Ba} \\
\text { (wt\%) }\end{array}$ & $\begin{array}{c}\mathrm{Ca} \\
\text { (wt\%) }\end{array}$ & $\begin{array}{c}\begin{array}{c}\mathrm{Ce} \\
\text { (wt\%) }\end{array} \\
\text { (a) }\end{array}$ & $\begin{array}{c}\mathrm{Cr} \\
\text { (wt\%) }\end{array}$ & $\begin{array}{c}\mathrm{Cu} \\
(\mathrm{wt} \%)\end{array}$ & $\begin{array}{c}\mathrm{K} \\
(\mathrm{wt} \%)\end{array}$ & $\begin{array}{c}\mathrm{La} \\
\text { (wt\%) }\end{array}$ & $\begin{array}{c}\mathrm{Mg} \\
\text { (wt\%) }\end{array}$ & $\begin{array}{c}\mathrm{Mn} \\
\text { (wt\%) }\end{array}$ & $\begin{array}{c}\mathrm{Na} \\
\text { (wt\%) }\end{array}$ & $\begin{array}{c}\mathrm{Ni} \\
\text { (wt\%) }\end{array}$ & $\begin{array}{c}\mathrm{Pb} \\
\text { (wt\%) }\end{array}$ & $\begin{array}{c}\mathrm{S} \\
\text { (wt\%) }\end{array}$ & $\begin{array}{c}\mathrm{Ti} \\
(\mathrm{wt} \%)\end{array}$ & $\begin{array}{c}\mathrm{Zn} \\
\text { (wt\%) }\end{array}$ & $\begin{array}{c}\mathrm{Zr} \\
\text { (wt\%) }\end{array}$ \\
\hline 1 & SB5NEPH-05 & 2 & 2 & 8 & R02LM12 & 8.26 & 0.04 & 1.4 & 0.083 & 0.055 & 0.028 & 0.054 & $<0.100$ & 0.39 & 1.81 & 11.7 & 0.75 & 0.034 & 0.123 & 0.141 & 0.026 & 0.07 \\
\hline 1 & SB5NEPH-09 & 2 & 2 & 9 & R04LM22 & 12.7 & 0.04 & 1.43 & 0.084 & 0.06 & 0.026 & 0.055 & $<0.100$ & 0.392 & 1.86 & 6.05 & 0.732 & 0.031 & $<0.100$ & 0.136 & 0.025 & 0.071 \\
\hline 1 & SB5NEPH-07 & 2 & 2 & 10 & R09LM12 & 7.27 & 0.04 & 8.6 & 0.082 & 0.065 & 0.026 & 0.052 & $<0.100$ & 0.379 & 1.8 & 10.3 & 0.735 & 0.035 & 0.134 & 0.134 & 0.021 & 0.073 \\
\hline 1 & SB5NEPH-10 & 2 & 2 & 11 & R05LM12 & 10.3 & 0.04 & 1.43 & 0.083 & 0.042 & 0.024 & 0.052 & $<0.100$ & 0.397 & 1.86 & 5.01 & 0.765 & 0.032 & $<0.100$ & 0.144 & 0.024 & 0.072 \\
\hline 1 & SB5NEPH-08 & 2 & 2 & 12 & R08LM12 & 5.84 & 0.039 & 8.81 & 0.083 & 0.059 & 0.03 & 0.056 & $<0.100$ & 0.382 & 1.84 & 8.38 & 0.737 & 0.034 & 0.16 & 0.134 & 0.025 & 0.07 \\
\hline 1 & Batch 1 & 2 & 2 & 13 & BCHLM1223 & 2.56 & 0.126 & 0.819 & $<0.010$ & 0.075 & 0.316 & 2.56 & $<0.100$ & 0.824 & 1.34 & 6.98 & 0.553 & $<0.010$ & $<0.100$ & 0.389 & $<0.010$ & 0.065 \\
\hline 2 & Batch 1 & 1 & 1 & 1 & BCHLM2111 & 2.48 & 0.124 & 0.821 & $<0.010$ & 0.074 & 0.309 & 2.52 & $<0.100$ & 0.82 & 1.33 & 6.64 & 0.55 & $<0.010$ & $<0.100$ & 0.387 & $<0.010$ & 0.064 \\
\hline 2 & SB5NEPH-17 & 1 & 1 & 2 & $\begin{array}{l}\text { S05lLM21 } \\
\end{array}$ & 8.24 & 0.041 & 1.41 & 0.081 & 0.065 & 0.031 & 0.058 & $<0.100$ & 0.383 & 1.83 & 17 & 0.744 & 0.034 & 0.159 & 0.134 & 0.028 & 0.07 \\
\hline 2 & SB5NEPH-20 & 1 & 1 & 3 & S06LM11 & 5.74 & 0.04 & 8.59 & 0.08 & 0.062 & 0.031 & 0.05 & $<0.100$ & 0.38 & 1.82 & 12.1 & 0.75 & 0.033 & 0.101 & 0.133 & 0.024 & 0.067 \\
\hline 2 & SB5NEPH-12 & 1 & 1 & 4 & S04LM11 & 8.58 & 0.039 & 8.59 & 0.082 & 0.052 & 0.037 & 0.053 & $<0.100$ & 0.378 & 1.81 & 4.08 & 0.741 & 0.033 & 0.095 & 0.139 & 0.027 & 0.072 \\
\hline 2 & SB5NEPH-13 & 1 & 1 & 5 & S07LM11 & 4.04 & 0.039 & 1.43 & 0.08 & 0.062 & 0.039 & 0.053 & $<0.100$ & 0.384 & 1.81 & 22.3 & 0.751 & 0.032 & 0.168 & 0.135 & 0.022 & 0.072 \\
\hline 2 & SB5NEPH-13 & 1 & 1 & 6 & S07LM21 & 4.06 & 0.04 & 1.44 & 0.081 & 0.062 & 0.039 & 0.055 & $<0.100$ & 0.385 & 1.81 & 22.3 & 0.755 & 0.031 & 0.168 & 0.136 & 0.022 & 0.07 \\
\hline 2 & Batch 1 & 1 & 1 & 7 & BCHLM2112 & 2.52 & 0.126 & 0.837 & $<0.010$ & 0.075 & 0.313 & 2.51 & $<0.100$ & 0.825 & 1.34 & 6.8 & 0.553 & $<0.010$ & $<0.100$ & 0.391 & $<0.010$ & 0.066 \\
\hline 2 & SB5NEPH-12 & 1 & 1 & 8 & $\begin{array}{l}\text { S04LM21 } \\
\end{array}$ & 8.59 & 0.039 & 8.56 & 0.082 & 0.051 & 0.036 & 0.052 & $<0.100$ & 0.375 & 1.81 & 4.14 & 0.733 & 0.032 & 0.095 & 0.138 & 0.028 & 0.07 \\
\hline 2 & SB5NEPH-11 & 1 & 1 & 9 & S02LM11 & 11.1 & 0.04 & 8.67 & 0.081 & 0.049 & 0.028 & 0.056 & $<0.100$ & 0.374 & 1.83 & 5.37 & 0.707 & 0.033 & 0.132 & 0.003 & 0.023 & 0.085 \\
\hline 2 & SB5NEPH-17 & 1 & 1 & 10 & S05LM11 & 8.27 & 0.041 & 1.41 & 0.081 & 0.065 & 0.032 & 0.055 & $<0.100$ & 0.385 & 1.83 & 17.1 & 0.75 & 0.033 & 0.16 & 0.133 & 0.027 & 0.072 \\
\hline 2 & SB5NEPH-20 & 1 & 1 & 11 & S06LM21 & 5.79 & 0.039 & 8.69 & 0.08 & 0.061 & 0.034 & 0.051 & $<0.100$ & 0.378 & 1.84 & 12.1 & 0.745 & 0.031 & 0.099 & 0.133 & 0.024 & 0.067 \\
\hline 2 & SB5NEPH-11 & 1 & 1 & 12 & S02LM21 & 10.8 & 0.04 & 8.57 & 0.08 & 0.047 & 0.028 & 0.056 & $<0.100$ & 0.368 & 1.79 & 5.22 & 0.704 & 0.032 & 0.13 & 0.002 & 0.022 & 0.069 \\
\hline 2 & Batch 1 & 1 & 1 & 13 & BCHLM2113 & 2.52 & 0.124 & 0.843 & $<0.010$ & 0.074 & 0.308 & 2.5 & $<0.100$ & 0.819 & 1.34 & 6.79 & 0.55 & $<0.010$ & $<0.100$ & 0.389 & $<0.010$ & 0.065 \\
\hline 2 & Batch 1 & 2 & 1 & 1 & BCHLM2211 & 2.49 & 0.127 & 0.839 & $<0.010$ & 0.076 & 0.312 & 2.53 & $<0.100$ & 0.823 & 1.33 & 6.56 & 0.554 & $<0.010$ & $<0.100$ & 0.392 & $<0.010$ & 0.065 \\
\hline 2 & SB5NEPH-19 & 2 & 1 & 2 & S03LM11 & 7.12 & 0.041 & 8.44 & 0.082 & 0.063 & 0.037 & 0.058 & $<0.100$ & 0.39 & 1.8 & 14.6 & 0.754 & 0.034 & 0.172 & 0.14 & 0.026 & 0.072 \\
\hline 2 & SB5NEPH-19 & 2 & 1 & 3 & S03LM21 & 7.1 & 0.041 & 8.43 & 0.081 & 0.063 & 0.038 & 0.056 & $<0.100$ & 0.387 & 1.79 & 14.6 & 0.759 & 0.033 & 0.174 & 0.138 & 0.025 & 0.072 \\
\hline 2 & SB5NEPH-14 & 2 & 1 & 4 & S09LM21 & 3.32 & 0.037 & 1.44 & 0.08 & 0.065 & 0.03 & 0.056 & $<0.100$ & 0.392 & 1.81 & 18.2 & 0.768 & 0.037 & 0.176 & 0.137 & 0.025 & 0.071 \\
\hline 2 & SB5NEPH-15 & 2 & 1 & 5 & S08LM11 & 3.47 & 0.04 & 8.27 & 0.08 & 0.06 & 0.031 & 0.054 & $<0.100$ & 0.38 & 1.76 & 19 & 0.752 & 0.032 & 0.18 & 0.135 & 0.026 & 0.071 \\
\hline 2 & SB5NEPH-18 & 2 & 1 & 6 & S01LM11 & 6.69 & 0.044 & 1.42 & 0.082 & 0.065 & 0.029 & 0.054 & $<0.100$ & 0.395 & 1.8 & 13.9 & 0.784 & 0.035 & 0.129 & 0.141 & 0.026 & 0.076 \\
\hline 2 & Batch 1 & 2 & 1 & 7 & BCHLM2212 & 2.49 & 0.13 & 0.838 & $<0.010$ & 0.078 & 0.315 & 2.54 & $<0.100$ & 0.843 & 1.33 & 6.72 & 0.573 & $<0.010$ & $<0.100$ & 0.395 & $<0.010$ & 0.066 \\
\hline 2 & SB5NEPH-16 & 2 & 1 & 8 & S10LM21 & 2.71 & 0.041 & 8.04 & 0.08 & 0.062 & 0.041 & 0.055 & $<0.100$ & 0.387 & 1.7 & 14.8 & 0.774 & 0.035 & 0.183 & 0.136 & 0.031 & 0.07 \\
\hline 2 & SB5NEPH-14 & 2 & 1 & 9 & S09LM11 & 3.39 & 0.037 & 1.48 & 0.081 & 0.066 & 0.032 & 0.055 & $<0.100$ & 0.396 & 1.82 & 18.5 & 0.78 & 0.038 & 0.181 & 0.138 & 0.025 & 0.073 \\
\hline 2 & SB5NEPH-15 & 2 & 1 & 10 & S08LM21 & 3.52 & 0.04 & 8.39 & 0.08 & 0.061 & 0.034 & 0.056 & $<0.100$ & 0.383 & 1.78 & 19.2 & 0.762 & 0.033 & 0.177 & 0.137 & 0.027 & 0.072 \\
\hline 2 & SB5NEPH-16 & 2 & 1 & 11 & S10LM11 & 2.62 & 0.041 & 7.78 & 0.079 & 0.062 & 0.039 & 0.054 & $<0.100$ & 0.387 & 1.66 & 14.3 & 0.77 & 0.034 & 0.181 & 0.135 & 0.031 & 0.07 \\
\hline 2 & SB5NEPH-18 & 2 & 1 & 12 & S01LM21 & 6.75 & 0.044 & 1.44 & 0.082 & 0.066 & 0.028 & 0.053 & $<0.100$ & 0.394 & 1.8 & 14.1 & 0.785 & 0.035 & 0.126 & 0.139 & 0.026 & 0.073 \\
\hline 2 & Batch 1 & 2 & 1 & 13 & BCHLM2213 & 2.51 & 0.13 & 0.852 & $<0.010$ & 0.077 & 0.315 & 2.55 & $<0.100$ & 0.843 & 1.33 & 6.77 & 0.572 & $<0.010$ & $<0.100$ & 0.396 & $<0.010$ & 0.067 \\
\hline 2 & Batch 1 & 1 & 2 & 1 & BCHLM2121 & 2.51 & 0.126 & 0.834 & $<0.010$ & 0.075 & 0.311 & 2.53 & $<0.100$ & 0.825 & 1.33 & 6.69 & 0.555 & $<0.010$ & $<0.100$ & 0.392 & $<0.010$ & 0.064 \\
\hline 2 & SB5NEPH-13 & 1 & 2 & 2 & S07LM12 & 4.07 & 0.04 & 1.45 & 0.081 & 0.063 & 0.039 & 0.053 & $<0.100$ & 0.383 & 1.81 & 22.4 & 0.753 & 0.032 & 0.172 & 0.134 & 0.022 & 0.07 \\
\hline 2 & SB5NEPH-11 & 1 & 2 & 3 & S02LM22 & 10.8 & 0.04 & 8.48 & 0.08 & 0.048 & 0.028 & 0.054 & $<0.100$ & 0.368 & 1.79 & 5.15 & 0.705 & 0.033 & 0.128 & 0.002 & 0.023 & 0.067 \\
\hline 2 & SB5NEPH-13 & 1 & 2 & 4 & S07LM22 & 4.18 & 0.041 & 1.48 & 0.082 & 0.064 & 0.04 & 0.054 & $<0.100$ & 0.393 & 1.86 & 22.9 & 0.771 & 0.034 & 0.17 & 0.137 & 0.023 & 0.071 \\
\hline 2 & SB5NEPH-20 & 1 & 2 & 5 & S06LM12 & 5.79 & 0.04 & 8.66 & 0.08 & 0.062 & 0.031 & 0.05 & $<0.100$ & 0.379 & 1.84 & 12.1 & 0.748 & 0.033 & 0.097 & 0.133 & 0.025 & 0.067 \\
\hline 2 & SB5NEPH-12 & 1 & 2 & 6 & $\begin{array}{l}\text { S04LM22 } \\
\end{array}$ & 8.62 & 0.039 & 8.64 & 0.082 & 0.051 & 0.036 & 0.051 & $<0.100$ & 0.376 & 1.82 & 4.17 & 0.734 & 0.032 & 0.095 & 0.138 & 0.029 & 0.071 \\
\hline 2 & Batch 1 & 1 & 2 & 7 & BCHLM2122 & 2.54 & 0.127 & 0.848 & $<0.010$ & 0.076 & 0.312 & 2.52 & $<0.100$ & 0.82 & 1.35 & 6.85 & 0.554 & $<0.010$ & $<0.100$ & 0.391 & $<0.010$ & 0.065 \\
\hline 2 & SB5NEPH-17 & 1 & 2 & 8 & $\begin{array}{l}\text { S05LM22 } \\
\end{array}$ & 8.39 & 0.041 & 1.44 & 0.081 & 0.065 & 0.031 & 0.056 & $<0.100$ & 0.381 & 1.83 & 17.3 & 0.74 & 0.033 & 0.156 & 0.134 & 0.028 & 0.069 \\
\hline 2 & SB5NEPH-11 & 1 & 2 & 9 & S02LM12 & 11 & 0.041 & 8.69 & 0.081 & 0.05 & 0.028 & 0.055 & $<0.100$ & 0.374 & 1.82 & 5.38 & 0.709 & 0.034 & 0.136 & 0.002 & 0.023 & 0.084 \\
\hline 2 & SB5NEPH-17 & 1 & 2 & 10 & S05LM12 & 8.35 & 0.041 & 1.43 & 0.082 & 0.066 & 0.032 & 0.054 & $<0.100$ & 0.387 & 1.83 & 17.3 & 0.756 & 0.034 & 0.161 & 0.136 & 0.028 & 0.072 \\
\hline 2 & SB5NEPH-12 & 1 & 2 & 11 & S04LM12 & 8.63 & 0.04 & 8.68 & 0.083 & 0.052 & 0.037 & 0.052 & $<0.100$ & 0.38 & 1.82 & 4.12 & 0.745 & 0.033 & 0.101 & 0.139 & 0.028 & 0.07 \\
\hline 2 & SB5NEPH-20 & 1 & 2 & 12 & $\begin{array}{l}\text { S06LM22 } \\
\end{array}$ & 5.73 & 0.04 & 8.6 & 0.08 & 0.062 & 0.034 & 0.05 & $<0.100$ & 0.379 & 1.82 & 12.1 & 0.746 & 0.032 & 0.099 & 0.132 & 0.025 & 0.067 \\
\hline 2 & Batch 1 & 1 & 2 & 13 & BCHLM2123 & 2.54 & 0.126 & 0.851 & $<0.010$ & 0.075 & 0.312 & 2.54 & $<0.100$ & 0.825 & 1.34 & 6.84 & 0.554 & $<0.010$ & $<0.100$ & 0.391 & $<0.010$ & 0.066 \\
\hline 2 & Batch 1 & 2 & 2 & 1 & BCHLM2221 & 2.51 & 0.127 & 0.84 & $<0.010$ & 0.076 & 0.313 & 2.54 & $<0.100$ & 0.825 & 1.34 & 6.65 & 0.553 & $<0.010$ & $<0.100$ & 0.39 & $<0.010$ & 0.064 \\
\hline
\end{tabular}


Table A2. Measured Elemental Concentrations (wt \%) for Samples Prepared Using Lithium Metaborate. (continued)

\begin{tabular}{|c|c|c|c|c|c|c|c|c|c|c|c|c|c|c|c|c|c|c|c|c|c|c|}
\hline Set & Glass ID & Block & $\begin{array}{c}\text { Sub } \\
\text { Block }\end{array}$ & Seq. & ID Check & $\begin{array}{c}\mathrm{Al} \\
\text { (wt\%) }\end{array}$ & $\begin{array}{c}\mathrm{Ba} \\
\text { (wt\%) }\end{array}$ & $\begin{array}{c}\begin{array}{c}\mathrm{Ca} \\
\text { (wt\%) }\end{array} \\
\end{array}$ & $\begin{array}{c}\begin{array}{c}\mathrm{Ce} \\
(\mathrm{wt} \%)\end{array} \\
\end{array}$ & $\begin{array}{c}\mathrm{Cr} \\
(\mathrm{wt} \%)\end{array}$ & $\begin{array}{c}\mathrm{Cu} \\
(\mathrm{wt} \%)\end{array}$ & $\begin{array}{c}\begin{array}{c}\mathrm{K} \\
(\mathrm{wt} \%)\end{array} \\
\end{array}$ & $\begin{array}{c}\mathrm{La} \\
\text { (wt\%) }\end{array}$ & $\begin{array}{c}\mathrm{Mg} \\
\text { (wt\%) }\end{array}$ & $\begin{array}{c}\begin{array}{c}\mathrm{Mn} \\
\text { (wt\%) }\end{array} \\
\end{array}$ & $\begin{array}{c}\mathrm{Na} \\
\text { (wt\%) }\end{array}$ & $\begin{array}{c}\mathrm{Ni} \\
\text { (wt\%) }\end{array}$ & $\begin{array}{c}\mathrm{Pb} \\
\text { (wt\%) }\end{array}$ & $\begin{array}{c}\mathrm{S} \\
\text { (wt\%) }\end{array}$ & $\begin{array}{c}\mathrm{Ti} \\
\text { (wt\%) }\end{array}$ & $\begin{array}{c}\mathrm{Zn} \\
\text { (wt\%) }\end{array}$ & $\begin{array}{c}\mathrm{Zr} \\
\text { (wt\%) }\end{array}$ \\
\hline 2 & SB5NEPH-15 & 2 & 2 & 2 & S08LM12 & 3.53 & 0.039 & 8.48 & 0.08 & 0.058 & 0.032 & 0.054 & $<0.100$ & 0.371 & 1.77 & 19.2 & 0.729 & 0.03 & 0.166 & 0.133 & 0.026 & 0.069 \\
\hline 2 & SB5NEPH-19 & 2 & 2 & 3 & S03LM12 & 7.15 & 0.04 & 8.54 & 0.082 & 0.061 & 0.037 & 0.056 & $<0.100$ & 0.379 & 1.79 & 14.8 & 0.73 & 0.031 & 0.159 & 0.136 & 0.025 & 0.071 \\
\hline 2 & SB5NEPH-16 & 2 & 2 & 4 & S10LM22 & 2.73 & 0.04 & 8.12 & 0.079 & 0.06 & 0.041 & 0.054 & $<0.100$ & 0.378 & 1.71 & 14.8 & 0.747 & 0.033 & 0.174 & 0.135 & 0.03 & 0.069 \\
\hline 2 & SB5NEPH-15 & 2 & 2 & 5 & $\begin{array}{l}\text { S08LM22 } \\
\end{array}$ & 3.55 & 0.039 & 8.49 & 0.081 & 0.059 & 0.034 & 0.054 & $<0.100$ & 0.373 & 1.78 & 19.3 & 0.736 & 0.032 & 0.169 & 0.135 & 0.026 & 0.071 \\
\hline 2 & SB5NEPH-18 & 2 & 2 & 6 & $\begin{array}{l}\text { S01LM22 } \\
\end{array}$ & 6.77 & 0.042 & 1.44 & 0.082 & 0.064 & 0.028 & 0.051 & $<0.100$ & 0.384 & 1.81 & 14 & 0.759 & 0.032 & 0.119 & 0.136 & 0.025 & 0.072 \\
\hline 2 & Batch 1 & 2 & 2 & 7 & BCHLM2222 & 2.51 & 0.127 & 0.845 & $<0.010$ & 0.076 & 0.31 & 2.5 & $<0.100$ & 0.825 & 1.34 & 6.68 & 0.553 & $<0.010$ & $<0.100$ & 0.39 & $<0.010$ & 0.065 \\
\hline 2 & SB5NEPH-19 & 2 & 2 & 8 & S03LM22 & 7.21 & 0.04 & 8.56 & 0.081 & 0.061 & 0.038 & 0.055 & $<0.100$ & 0.377 & 1.8 & 14.8 & 0.737 & 0.031 & 0.164 & 0.135 & 0.025 & 0.07 \\
\hline 2 & SB5NEPH-14 & 2 & 2 & 9 & S09LM12 & 3.43 & 0.036 & 1.49 & 0.081 & 0.063 & 0.032 & 0.053 & $<0.100$ & 0.385 & 1.83 & 18.7 & 0.752 & 0.036 & 0.166 & 0.136 & 0.024 & 0.071 \\
\hline 2 & SB5NEPH-16 & 2 & 2 & 10 & S10LM12 & 2.7 & 0.04 & 8.04 & 0.08 & 0.06 & 0.04 & 0.052 & $<0.100$ & 0.375 & 1.69 & 14.7 & 0.742 & 0.033 & 0.168 & 0.133 & 0.03 & 0.069 \\
\hline 2 & SB5NEPH-14 & 2 & 2 & 11 & S09LM22 & 3.38 & 0.036 & 1.47 & 0.08 & 0.063 & 0.03 & 0.055 & $<0.100$ & 0.381 & 1.82 & 18.5 & 0.74 & 0.036 & 0.169 & 0.134 & 0.025 & 0.07 \\
\hline 2 & SB5NEPH-18 & 2 & 2 & 12 & S01LM12 & 6.79 & 0.042 & 1.43 & 0.082 & 0.063 & 0.029 & 0.052 & $<0.100$ & 0.382 & 1.82 & 14.1 & 0.749 & 0.033 & 0.118 & 0.135 & 0.025 & 0.075 \\
\hline 2 & Batch 1 & 2 & 2 & 13 & BCHLM2223 & 2.54 & 0.126 & 0.854 & $<0.010$ & 0.075 & 0.312 & 2.54 & $<0.100$ & 0.825 & 1.34 & 6.78 & 0.55 & $<0.010$ & $<0.100$ & 0.39 & $<0.010$ & 0.064 \\
\hline 3 & Batch 1 & 1 & 1 & 1 & BCHLM3111 & 2.52 & 0.127 & 0.838 & $<0.010$ & 0.075 & 0.313 & 2.53 & $<0.100$ & 0.823 & 1.34 & 6.74 & 0.553 & $<0.010$ & $<0.100$ & 0.39 & $<0.010$ & 0.065 \\
\hline 3 & SB5NEPH-28 & 1 & 1 & 2 & T07LM11 & 11.5 & 0.039 & 8.6 & 0.082 & 0.046 & 0.031 & 0.051 & $<0.100$ & 0.38 & 1.81 & 4.12 & 0.702 & 0.036 & $<0.100$ & 0.134 & 0.025 & 0.068 \\
\hline 3 & SB5NEPH-24 & 1 & 1 & 3 & T10LM11 & 8.51 & 0.041 & 8.45 & 0.08 & 0.063 & 0.025 & 0.053 & $<0.100$ & 0.373 & 1.81 & 8.07 & 0.732 & 0.029 & 0.106 & 0.133 & 0.024 & 0.071 \\
\hline 3 & SB5NEPH-27 & 1 & 1 & 4 & T09LM21 & 13.9 & 0.036 & 8.38 & 0.082 & 0.056 & 0.027 & 0.053 & $<0.100$ & 0.369 & 1.77 & 5 & 0.668 & 0.03 & 0.136 & 0.133 & 0.026 & 0.072 \\
\hline 3 & SB5NEPH-23 & 1 & 1 & 5 & T05LM11 & 10.7 & 0.038 & 8.41 & 0.08 & 0.05 & 0.026 & 0.053 & $<0.100$ & 0.364 & 1.77 & 10.19 & 0.713 & 0.037 & 0.121 & 0.128 & 0.023 & 0.069 \\
\hline 3 & SB5NEPH-23 & 1 & 1 & 6 & $\begin{array}{l}\text { T05LM21 } \\
\end{array}$ & 10.9 & 0.038 & 8.53 & 0.08 & 0.049 & 0.027 & 0.054 & $<0.100$ & 0.364 & 1.81 & 10.18 & 0.714 & 0.037 & 0.122 & 0.13 & 0.023 & 0.069 \\
\hline 3 & Batch 1 & 1 & 1 & 7 & BCHLM3112 & 2.51 & 0.126 & 0.842 & $<0.010$ & 0.075 & 0.309 & 2.5 & $<0.100$ & 0.819 & 1.33 & 6.81 & 0.552 & $<0.010$ & $<0.100$ & 0.387 & $<0.010$ & 0.064 \\
\hline 3 & SB5NEPH-28 & 1 & 1 & 8 & $\begin{array}{l}\text { T07LM21 } \\
\end{array}$ & 11.4 & 0.039 & 8.58 & 0.081 & 0.043 & 0.031 & 0.05 & $<0.100$ & 0.375 & 1.71 & 4.1 & 0.64 & 0.036 & $<0.100$ & 0.126 & 0.024 & 0.067 \\
\hline 3 & SB5NEPH-26 & 1 & 1 & 9 & T01LM11 & 13.6 & 0.042 & 1.43 & 0.082 & 0.047 & 0.027 & 0.054 & $<0.100$ & 0.383 & 1.82 & 4.85 & 0.677 & 0.03 & $<0.100$ & 0.126 & 0.023 & 0.068 \\
\hline 3 & SB5NEPH-27 & 1 & 1 & 10 & T09LM11 & 14.1 & 0.035 & 8.5 & 0.081 & 0.058 & 0.029 & 0.054 & $<0.100$ & 0.366 & 1.76 & 5.11 & 0.641 & 0.03 & 0.131 & 0.131 & 0.025 & 0.07 \\
\hline 3 & SB5NEPH-24 & 1 & 1 & 11 & T10LM21 & 8.42 & 0.041 & 8.41 & 0.08 & 0.061 & 0.025 & 0.051 & $<0.100$ & 0.369 & 1.72 & 7.92 & 0.695 & 0.029 & 0.11 & 0.129 & 0.023 & 0.069 \\
\hline 3 & SB5NEPH-26 & 1 & 1 & 12 & $\begin{array}{l}\text { T01LM21 } \\
\end{array}$ & 13.6 & 0.042 & 2.04 & 0.082 & 0.047 & 0.03 & 0.054 & $<0.100$ & 0.387 & 1.83 & 4.91 & 0.682 & 0.029 & $<0.100$ & 0.127 & 0.023 & 0.071 \\
\hline 3 & Batch 1 & 1 & 1 & 13 & BCHLM3113 & 2.52 & 0.127 & 0.855 & $<0.010$ & 0.076 & 0.311 & 2.52 & $<0.100$ & 0.815 & 1.33 & 6.88 & 0.554 & $<0.010$ & $<0.100$ & 0.385 & $<0.010$ & 0.065 \\
\hline 3 & Batch 1 & 2 & 1 & 1 & BCHLM3211 & 2.47 & 0.126 & 0.823 & $<0.010$ & 0.075 & 0.31 & 2.5 & $<0.100$ & 0.822 & 1.32 & 6.67 & 0.551 & $<0.010$ & $<0.100$ & 0.386 & $<0.010$ & 0.063 \\
\hline 3 & SB5NEPH-29 & 2 & 1 & 2 & $\begin{array}{l}\text { T02LM11 } \\
\end{array}$ & 4 & 0.04 & 1.41 & 0.078 & 0.06 & 0.027 & 0.051 & $<0.100$ & 0.362 & 1.73 & 27.02 & 0.718 & 0.031 & 0.167 & 0.126 & 0.023 & 0.067 \\
\hline 3 & SB5NEPH-30 & 2 & 1 & 3 & T08LM21 & 3.37 & 0.039 & 1.45 & 0.079 & 0.06 & 0.034 & 0.051 & $<0.100$ & 0.374 & 1.77 & 22.6 & 0.729 & 0.036 & 0.168 & 0.13 & 0.029 & 0.067 \\
\hline 3 & SB5NEPH-22 & 2 & 1 & 4 & T04LM11 & 10.2 & 0.045 & 1.42 & 0.079 & 0.046 & 0.027 & 0.05 & $<0.100$ & 0.374 & 1.76 & 9.7 & 0.621 & 0.032 & $<0.100$ & 0.138 & 0.022 & 0.071 \\
\hline 3 & SB5NEPH-25 & 2 & 1 & 5 & T03LM11 & 16 & 0.04 & 1.41 & 0.083 & 0.054 & 0.028 & 0.052 & $<0.100$ & 0.373 & 1.79 & 6.07 & 0.686 & 0.031 & $<0.100$ & 0.136 & 0.024 & 0.069 \\
\hline 3 & SB5NEPH-22 & 2 & 1 & 6 & T04LM21 & 10.1 & 0.045 & 1.41 & 0.079 & 0.046 & 0.028 & 0.05 & $<0.100$ & 0.372 & 1.75 & 9.56 & 0.625 & 0.031 & $<0.100$ & 0.136 & 0.022 & 0.071 \\
\hline 3 & Batch 1 & 2 & 1 & 7 & BCHLM3212 & 2.49 & 0.124 & 0.822 & $<0.010$ & 0.074 & 0.304 & 2.62 & $<0.100$ & 0.811 & 1.33 & 6.68 & 0.541 & $<0.010$ & $<0.100$ & 0.381 & $<0.010$ & 0.063 \\
\hline 3 & SB5NEPH-25 & 2 & 1 & 8 & $\begin{array}{l}\text { T03LM21 } \\
\end{array}$ & 15.7 & 0.04 & 1.37 & 0.082 & 0.051 & 0.027 & 0.051 & $<0.100$ & 0.372 & 1.71 & 5.85 & 0.629 & 0.032 & $<0.100$ & 0.126 & 0.023 & 0.066 \\
\hline 3 & SB5NEPH-21 & 2 & 1 & 9 & T06LM21 & 12.5 & 0.043 & 1.43 & 0.081 & 0.048 & 0.025 & 0.055 & $<0.100$ & 0.372 & 1.76 & 11.63 & 0.679 & 0.03 & $<0.100$ & 0.13 & 0.022 & 0.071 \\
\hline 3 & SB5NEPH-29 & 2 & 1 & 10 & T02LM21 & 4.02 & 0.039 & 1.38 & 0.074 & 0.054 & 0.027 & 0.049 & $<0.100$ & 0.36 & 1.55 & 26.72 & 0.63 & 0.032 & 0.16 & 0.11 & 0.022 & 0.064 \\
\hline 3 & SB5NEPH-30 & 2 & 1 & 11 & T08LM11 & 3.33 & 0.039 & 1.43 & 0.078 & 0.06 & 0.031 & 0.047 & $<0.100$ & 0.372 & 1.74 & 22.24 & 0.729 & 0.035 & 0.164 & 0.13 & 0.028 & 0.066 \\
\hline 3 & SB5NEPH-21 & 2 & 1 & 12 & T06LM11 & 12.3 & 0.043 & 1.4 & 0.081 & 0.049 & 0.028 & 0.052 & $<0.100$ & 0.372 & 1.74 & 11.48 & 0.679 & 0.031 & $<0.100$ & 0.131 & 0.022 & 0.075 \\
\hline 3 & Batch 1 & 2 & 1 & 13 & BCHLM3213 & 2.52 & 0.126 & 0.843 & $<0.010$ & 0.075 & 0.309 & 2.5 & $<0.100$ & 0.817 & 1.33 & 6.81 & 0.551 & $<0.010$ & $<0.100$ & 0.388 & $<0.010$ & 0.064 \\
\hline 3 & Batch 1 & 1 & 2 & 1 & BCHLM3121 & 2.49 & 0.128 & 0.846 & $<0.010$ & 0.076 & 0.31 & 2.51 & $<0.100$ & 0.827 & 1.32 & 6.83 & 0.553 & $<0.010$ & $<0.100$ & 0.392 & $<0.010$ & 0.065 \\
\hline 3 & SB5NEPH-27 & 1 & 2 & 2 & T09LM22 & 14.1 & 0.038 & 8.41 & 0.082 & 0.056 & 0.027 & 0.053 & $<0.100$ & 0.372 & 1.77 & 5.1 & 0.666 & 0.031 & 0.132 & 0.131 & 0.027 & 0.072 \\
\hline 3 & SB5NEPH-26 & 1 & 2 & 3 & T01LM12 & 13.7 & 0.044 & 1.47 & 0.084 & 0.048 & 0.028 & 0.055 & $<0.100$ & 0.393 & 1.84 & 5.03 & 0.684 & 0.031 & $<0.100$ & 0.128 & 0.024 & 0.069 \\
\hline 3 & SB5NEPH-23 & 1 & 2 & 4 & T05LM22 & 11 & 0.04 & 8.6 & 0.08 & 0.05 & 0.028 & 0.054 & $<0.100$ & 0.368 & 1.82 & 10.26 & 0.711 & 0.038 & 0.12 & 0.13 & 0.023 & 0.07 \\
\hline 3 & SB5NEPH-26 & 1 & 2 & 5 & T01LM22 & 13.7 & 0.044 & 2.07 & 0.083 & 0.047 & 0.031 & 0.054 & $<0.100$ & 0.389 & 1.84 & 4.96 & 0.678 & 0.03 & $<0.100$ & 0.127 & 0.024 & 0.072 \\
\hline 3 & SB5NEPH-28 & 1 & 2 & 6 & $\begin{array}{l}\text { T07LM22 } \\
\end{array}$ & 11.5 & 0.041 & 8.59 & 0.082 & 0.043 & 0.032 & 0.05 & $<0.100$ & 0.381 & 1.73 & 4.09 & 0.641 & 0.038 & $<0.100$ & 0.129 & 0.025 & 0.068 \\
\hline 3 & Batch 1 & 1 & 2 & 7 & BCHLM3122 & 2.52 & 0.127 & 0.853 & $<0.010$ & 0.076 & 0.309 & 2.48 & $<0.100$ & 0.822 & 1.33 & 6.71 & 0.55 & $<0.010$ & $<0.100$ & 0.385 & $<0.010$ & 0.066 \\
\hline 3 & SB5NEPH-24 & 1 & 2 & 8 & $\begin{array}{l}\text { T10LM12 } \\
\end{array}$ & 8.88 & 0.043 & 8.73 & 0.081 & 0.063 & 0.026 & 0.052 & $<0.100$ & 0.376 & 1.83 & 8.37 & 0.729 & $\begin{array}{ll}0.03 \\
\end{array}$ & 0.109 & 0.132 & 0.024 & 0.071 \\
\hline
\end{tabular}


Table A2. Measured Elemental Concentrations (wt \%) for Samples Prepared Using Lithium Metaborate. (continued)

\begin{tabular}{|c|c|c|c|c|c|c|c|c|c|c|c|c|c|c|c|c|c|c|c|c|c|c|}
\hline Set & Glass ID & Block & $\begin{array}{c}\text { Sub } \\
\text { Block }\end{array}$ & Seq. & ID Check & $\begin{array}{c}\mathrm{Al} \\
\text { (wt\%) }\end{array}$ & $\begin{array}{c}\mathrm{Ba} \\
\text { (wt\%) }\end{array}$ & $\begin{array}{c}\begin{array}{c}\mathrm{Ca} \\
\text { (wt\%) }\end{array} \\
\end{array}$ & $\begin{array}{c}\begin{array}{c}\mathrm{Ce} \\
(\mathrm{wt} \%)\end{array} \\
\end{array}$ & $\begin{array}{c}\mathrm{Cr} \\
(\mathrm{wt} \%)\end{array}$ & $\begin{array}{c}\mathrm{Cu} \\
(\mathrm{wt} \%)\end{array}$ & $\begin{array}{c}\begin{array}{c}\mathrm{K} \\
(\mathrm{wt} \%)\end{array} \\
\end{array}$ & $\begin{array}{c}\mathrm{La} \\
\text { (wt\%) }\end{array}$ & $\begin{array}{c}\mathrm{Mg} \\
\text { (wt\%) }\end{array}$ & $\begin{array}{c}\begin{array}{c}\mathrm{Mn} \\
\text { (wt\%) }\end{array} \\
\end{array}$ & $\begin{array}{c}\begin{array}{c}\mathrm{Na} \\
\text { (wt\%) }\end{array} \\
\end{array}$ & $\begin{array}{c}\mathrm{Ni} \\
\text { (wt\%) }\end{array}$ & $\begin{array}{c}\mathrm{Pb} \\
\text { (wt\%) }\end{array}$ & $\begin{array}{c}\mathrm{S} \\
\text { (wt\%) }\end{array}$ & $\begin{array}{c}\mathrm{Ti} \\
\text { (wt\%) }\end{array}$ & $\begin{array}{c}\mathrm{Zn} \\
\text { (wt\%) }\end{array}$ & $\begin{array}{c}\mathrm{Zr} \\
\text { (wt\%) }\end{array}$ \\
\hline 3 & SB5NEPH-23 & 1 & 2 & 9 & T05LM12 & 10.7 & 0.039 & 8.38 & 0.08 & 0.05 & 0.027 & 0.053 & $<0.100$ & 0.365 & 1.77 & 10.09 & 0.704 & 0.037 & 0.117 & 0.129 & 0.023 & 0.07 \\
\hline 3 & SB5NEPH-27 & 1 & 2 & 10 & T09LM12 & 14.3 & 0.038 & 8.58 & 0.083 & 0.058 & 0.03 & 0.054 & $<0.100$ & 0.372 & 1.77 & 5.17 & 0.645 & 0.031 & 0.13 & 0.132 & 0.026 & 0.072 \\
\hline 3 & SB5NEPH-28 & 1 & 2 & 11 & T07LM12 & 11.6 & 0.041 & 8.6 & 0.082 & 0.046 & 0.031 & 0.05 & $<0.100$ & 0.383 & 1.81 & 4.15 & 0.702 & 0.036 & $<0.100$ & 0.134 & 0.026 & 0.069 \\
\hline 3 & SB5NEPH-24 & 1 & 2 & 12 & T10LM22 & 8.52 & 0.043 & 8.44 & 0.08 & 0.062 & 0.026 & 0.052 & $<0.100$ & 0.373 & 1.73 & 7.97 & 0.694 & 0.03 & 0.105 & 0.13 & 0.023 & 0.07 \\
\hline 3 & Batch 1 & 1 & 2 & 13 & BCHLM3123 & 2.53 & 0.128 & 0.862 & $<0.010$ & 0.077 & 0.313 & 2.53 & $<0.100$ & 0.824 & 1.32 & 6.85 & 0.555 & $<0.010$ & $<0.100$ & 0.392 & $<0.010$ & 0.067 \\
\hline 3 & Batch 1 & 2 & 2 & 1 & BCHLM3221 & 2.53 & 0.125 & 0.861 & $<0.010$ & 0.075 & 0.309 & 2.54 & $<0.100$ & 0.819 & 1.35 & 6.88 & 0.551 & $<0.010$ & $<0.100$ & 0.386 & $<0.010$ & 0.064 \\
\hline 3 & SB5NEPH-30 & 2 & 2 & 2 & T08LM12 & 3.39 & 0.039 & 1.46 & 0.078 & 0.059 & 0.03 & 0.047 & $<0.100$ & 0.371 & 1.78 & 22.5 & 0.728 & 0.034 & 0.166 & 0.13 & 0.028 & 0.066 \\
\hline 3 & SB5NEPH-22 & 2 & 2 & 3 & T04LM22 & 10.3 & 0.046 & 1.44 & 0.079 & 0.046 & 0.027 & 0.05 & $<0.100$ & 0.373 & 1.78 & 9.65 & 0.624 & 0.032 & $<0.100$ & 0.136 & 0.022 & 0.072 \\
\hline 3 & SB5NEPH-30 & 2 & 2 & 4 & T08LM22 & 3.42 & 0.039 & 1.48 & 0.079 & 0.059 & 0.033 & 0.051 & $<0.100$ & 0.373 & 1.8 & 22.93 & 0.728 & 0.036 & 0.167 & 0.129 & 0.028 & 0.068 \\
\hline 3 & SB5NEPH-29 & 2 & 2 & 5 & T02LM22 & 4.05 & 0.039 & 1.4 & 0.073 & 0.054 & 0.025 & 0.048 & $<0.100$ & 0.357 & 1.57 & 26.62 & 0.628 & 0.032 & 0.163 & 0.109 & 0.022 & 0.066 \\
\hline 3 & SB5NEPH-21 & 2 & 2 & 6 & $\begin{array}{l}\text { T06LM22 } \\
\end{array}$ & 12.5 & 0.043 & 1.46 & 0.08 & 0.048 & 0.024 & 0.053 & $<0.100$ & 0.369 & 1.79 & 11.59 & 0.674 & 0.031 & $<0.100$ & 0.128 & 0.022 & 0.071 \\
\hline 3 & Batch 1 & 2 & 2 & 7 & BCHLM3222 & 2.58 & 0.125 & 0.89 & $<0.010$ & 0.075 & 0.311 & 2.55 & $<0.100$ & 0.818 & 1.36 & 6.86 & 0.55 & $<0.010$ & $<0.100$ & 0.387 & $<0.010$ & 0.065 \\
\hline 3 & SB5NEPH-21 & 2 & 2 & 8 & T06LM12 & 12.7 & 0.043 & 1.47 & 0.081 & 0.049 & 0.027 & 0.052 & $<0.100$ & 0.372 & 1.78 & 11.77 & 0.679 & 0.031 & $<0.100$ & 0.13 & 0.022 & 0.075 \\
\hline 3 & SB5NEPH-22 & 2 & 2 & 9 & T04LM12 & 10.4 & 0.045 & 1.48 & 0.079 & 0.046 & 0.026 & 0.049 & $<0.100$ & 0.372 & 1.79 & 9.81 & 0.618 & 0.032 & $<0.100$ & 0.137 & 0.021 & 0.07 \\
\hline 3 & SB5NEPH-29 & 2 & 2 & 10 & T02LM12 & 4.07 & 0.04 & 1.46 & 0.078 & 0.06 & 0.026 & 0.05 & $<0.100$ & 0.36 & 1.76 & 27.19 & 0.714 & 0.033 & 0.165 & 0.126 & 0.022 & 0.067 \\
\hline 3 & SB5NEPH-25 & 2 & 2 & 11 & T03LM12 & 16.5 & 0.04 & 1.46 & 0.082 & 0.054 & 0.027 & 0.052 & $<0.100$ & 0.372 & 1.83 & 6.17 & 0.684 & 0.031 & $<0.100$ & 0.134 & 0.024 & 0.07 \\
\hline 3 & SB5NEPH-25 & 2 & 2 & 12 & $\begin{array}{r}\text { T03LM22 } \\
\end{array}$ & 16.1 & 0.04 & 1.43 & 0.082 & 0.051 & 0.026 & 0.051 & $<0.100$ & 0.371 & 1.74 & 6.02 & 0.63 & 0.031 & $<0.100$ & 0.126 & 0.023 & 0.067 \\
\hline 3 & Batch 1 & 2 & 2 & 13 & BCHLM3223 & 2.57 & 0.124 & 0.903 & $<0.010$ & 0.074 & 0.306 & 2.66 & $<0.100$ & 0.805 & 1.36 & 6.8 & 0.537 & $<0.010$ & $<0.100$ & 0.382 & $<0.010$ & 0.063 \\
\hline 4 & Batch 1 & 1 & 1 & 1 & BCHLM4111 & 2.52 & 0.124 & 0.846 & $<0.010$ & 0.075 & 0.306 & 2.6 & $<0.100$ & 0.814 & 1.33 & 6.7 & 0.542 & $<0.010$ & $<0.100$ & 0.385 & $<0.010$ & 0.066 \\
\hline 4 & SB5NEPH-33 & 1 & 1 & 2 & U10LM21 & 12.3 & 0.039 & 1.4 & 0.081 & 0.06 & 0.027 & 0.053 & $<0.100$ & 0.381 & 1.81 & 17 & 0.738 & 0.033 & 0.151 & 0.137 & 0.025 & 0.07 \\
\hline 4 & SB5NEPH-35 & 1 & 1 & 3 & U04LM21 & 10.7 & 0.038 & 8.59 & 0.08 & 0.061 & 0.031 & 0.053 & $<0.100$ & 0.374 & 1.78 & 15.3 & 0.722 & 0.031 & 0.162 & 0.133 & 0.027 & 0.069 \\
\hline 4 & SB5NEPH-36 & 1 & 1 & 4 & U01LM11 & 8.49 & 0.039 & 8.52 & 0.082 & 0.059 & 0.038 & 0.051 & $<0.100$ & 0.378 & 1.79 & 12.2 & 0.718 & 0.033 & 0.116 & 0.133 & 0.029 & 0.071 \\
\hline 4 & SB5NEPH-33 & 1 & 1 & 5 & U10LM11 & 12.5 & 0.039 & 1.43 & 0.081 & 0.061 & 0.027 & 0.054 & $<0.100$ & 0.385 & 1.81 & 17.5 & 0.737 & 0.033 & 0.151 & 0.136 & 0.025 & 0.074 \\
\hline 4 & SB5NEPH-40 & 1 & 1 & 6 & $\begin{array}{l}\text { U06LM11 } \\
\end{array}$ & 14.5 & 0.04 & 8.69 & 0.081 & 0.044 & 0.029 & 0.055 & $<0.100$ & 0.381 & 1.81 & 4.24 & 0.661 & 0.033 & $<0.100$ & 0.134 & 0.025 & 0.072 \\
\hline 4 & Batch 1 & 1 & 1 & 7 & BCHLM4112 & 2.54 & 0.125 & 0.841 & $<0.010$ & 0.075 & 0.31 & 2.62 & $<0.100$ & 0.821 & 1.34 & 6.82 & 0.545 & $<0.010$ & $<0.100$ & 0.384 & $<0.010$ & 0.07 \\
\hline 4 & SB5NEPH-32 & 1 & 1 & 8 & U05LM21 & 2.93 & 0.037 & 8.55 & 0.08 & 0.062 & 0.028 & 0.052 & $<0.100$ & 0.377 & 1.78 & 19.7 & 0.734 & 0.033 & 0.176 & 0.133 & 0.022 & 0.067 \\
\hline 4 & SB5NEPH-40 & 1 & 1 & 9 & U06LM21 & 14.3 & 0.039 & 8.56 & 0.08 & 0.042 & 0.027 & 0.052 & $<0.100$ & 0.376 & 1.78 & 4.14 & 0.632 & 0.032 & $<0.100$ & 0.132 & 0.024 & 0.071 \\
\hline 4 & SB5NEPH-32 & 1 & 1 & 10 & U05LM11 & 2.93 & 0.037 & 8.61 & 0.08 & 0.062 & 0.029 & 0.052 & $<0.100$ & 0.378 & 1.79 & 19.9 & 0.735 & 0.034 & 0.171 & 0.133 & 0.022 & 0.069 \\
\hline 4 & SB5NEPH-35 & 1 & 1 & 11 & U04LM11 & 10.6 & 0.038 & 8.55 & 0.08 & 0.061 & 0.029 & 0.068 & $<0.100$ & 0.372 & 1.76 & 15.1 & 0.727 & 0.029 & 0.161 & 0.133 & 0.026 & 0.072 \\
\hline 4 & SB5NEPH-36 & 1 & 1 & 12 & U01LM21 & 8.49 & 0.039 & 8.49 & 0.082 & 0.06 & 0.035 & 0.051 & $<0.100$ & 0.375 & 1.79 & 12.1 & 0.719 & 0.035 & 0.116 & 0.133 & 0.028 & 0.076 \\
\hline 4 & Batch 1 & 1 & 1 & 13 & BCHLM4113 & 2.54 & 0.124 & 0.849 & $<0.010$ & 0.074 & 0.31 & 2.64 & $<0.100$ & 0.815 & 1.34 & 6.81 & 0.543 & $<0.010$ & $<0.100$ & 0.384 & $<0.010$ & 0.07 \\
\hline 4 & Batch 1 & 2 & 1 & 1 & BCHLM4211 & 2.46 & 0.124 & 0.777 & $<0.010$ & 0.075 & 0.306 & 2.6 & $<0.100$ & 0.817 & 1.32 & 6.67 & 0.541 & $<0.010$ & $<0.100$ & 0.385 & $<0.010$ & 0.065 \\
\hline 4 & SB5NEPH-31 & 2 & 1 & 2 & U08LM21 & 3.48 & 0.037 & 8.14 & 0.077 & 0.059 & 0.032 & 0.05 & $<0.100$ & 0.345 & 1.67 & 23 & 0.64 & 0.033 & 0.162 & 0.127 & 0.024 & 0.064 \\
\hline 4 & SB5NEPH-37 & 2 & 1 & 3 & U09LM11 & 20.2 & 0.04 & 1.36 & 0.083 & 0.051 & 0.026 & 0.053 & $<0.100$ & 0.357 & 1.81 & 6.07 & 0.741 & 0.032 & $<0.100$ & 0.134 & 0.024 & 0.06 \\
\hline 4 & SB5NEPH-34 & 2 & 1 & 4 & U02LM11 & 10.1 & 0.039 & 1.37 & 0.084 & 0.058 & 0.028 & 0.05 & $<0.100$ & 0.378 & 1.79 & 14 & 0.725 & 0.033 & 0.122 & 0.135 & 0.024 & 0.069 \\
\hline 4 & SB5NEPH-31 & 2 & 1 & 5 & U08LM11 & 3.47 & 0.037 & 8.19 & 0.077 & 0.058 & 0.031 & 0.05 & $<0.100$ & 0.344 & 1.65 & 23.3 & 0.635 & 0.032 & 0.159 & 0.128 & 0.024 & 0.067 \\
\hline 4 & SB5NEPH-34 & 2 & 1 & 6 & U02LM21 & 10.26 & 0.039 & 1.39 & 0.085 & 0.058 & 0.029 & 0.051 & $<0.100$ & 0.379 & 1.82 & 14.1 & 0.729 & 0.033 & 0.118 & 0.136 & 0.025 & 0.072 \\
\hline 4 & Batch 1 & 2 & 1 & 7 & BCHLM4212 & 2.43 & 0.124 & 0.817 & $<0.010$ & 0.075 & 0.309 & 2.61 & $<0.100$ & 0.822 & 1.32 & 6.78 & 0.541 & $<0.010$ & $<0.100$ & 0.388 & $<0.010$ & 0.068 \\
\hline 4 & SB5NEPH-39 & 2 & 1 & 8 & U03LM21 & 17.9 & 0.038 & 8.48 & 0.083 & 0.043 & 0.027 & 0.052 & $<0.100$ & 0.371 & 1.79 & 5.31 & 0.693 & 0.033 & $<0.100$ & 0.132 & 0.024 & 0.07 \\
\hline 4 & SB5NEPH-38 & 2 & 1 & 9 & U07LM11 & 16.8 & 0.043 & 1.39 & 0.085 & 0.059 & 0.028 & 0.052 & $<0.100$ & 0.39 & 1.81 & 5.01 & 0.763 & 0.033 & $<0.100$ & 0.137 & 0.025 & 0.07 \\
\hline 4 & SB5NEPH-37 & 2 & 1 & 10 & $\begin{array}{l}\text { U09LM21 } \\
\end{array}$ & 20.3 & 0.04 & 1.37 & 0.083 & 0.051 & 0.026 & 0.053 & $<0.100$ & 0.36 & 1.81 & 6.15 & 0.757 & 0.032 & $<0.100$ & 0.135 & 0.024 & 0.063 \\
\hline 4 & SB5NEPH-39 & 2 & 1 & 11 & U03LM11 & 17.6 & 0.037 & 8.46 & 0.083 & 0.044 & 0.027 & 0.051 & $<0.100$ & 0.372 & 1.78 & 5.2 & 0.692 & 0.033 & $<0.100$ & 0.131 & 0.024 & 0.069 \\
\hline 4 & SB5NEPH-38 & 2 & 1 & 12 & U07LM21 & 16.5 & 0.042 & 1.37 & 0.084 & 0.057 & 0.028 & 0.052 & $<0.100$ & 0.386 & 1.78 & 4.91 & 0.745 & 0.032 & $<0.100$ & 0.136 & 0.024 & 0.071 \\
\hline 4 & Batch 1 & 2 & 1 & 13 & BCHLM4213 & 2.49 & 0.125 & 0.783 & $<0.010$ & 0.075 & 0.311 & 2.61 & $<0.100$ & 0.828 & 1.31 & 6.78 & 0.542 & $<0.010$ & $<0.100$ & 0.391 & $<0.010$ & 0.069 \\
\hline 4 & Batch 1 & 1 & 2 & 1 & BCHLM4121 & 2.58 & 0.125 & 0.869 & $<0.010$ & 0.074 & 0.305 & 2.6 & $<0.100$ & 0.81 & 1.35 & 6.91 & 0.544 & $<0.010$ & $<0.100$ & 0.383 & $<0.010$ & 0.066 \\
\hline 4 & SB5NEPH-36 & 1 & 2 & 2 & $\begin{array}{l}\text { U01LM12 } \\
\end{array}$ & 8.74 & 0.04 & $\begin{array}{lll}8.7 \\
\end{array}$ & 0.081 & 0.058 & 0.038 & 0.051 & $<0.100$ & 0.373 & 1.83 & 12.3 & 0.714 & 0.032 & 0.117 & 0.132 & 0.028 & 0.067 \\
\hline
\end{tabular}


Table A2. Measured Elemental Concentrations (wt \%) for Samples Prepared Using Lithium Metaborate. (continued)

\begin{tabular}{|c|c|c|c|c|c|c|c|c|c|c|c|c|c|c|c|c|c|c|c|c|c|c|}
\hline Set & Glass ID & Block & $\begin{array}{c}\text { Sub } \\
\text { Block }\end{array}$ & Seq. & ID Check & $\begin{array}{c}\mathrm{Al} \\
\text { (wt\%) }\end{array}$ & $\begin{array}{c}\mathrm{Ba} \\
\text { (wt\%) }\end{array}$ & $\begin{array}{c}\mathrm{Ca} \\
(\mathrm{wt} \%)\end{array}$ & $\begin{array}{c}\mathrm{Ce} \\
\text { (wt\%) }\end{array}$ & $\begin{array}{c}\mathrm{Cr} \\
(\mathrm{wt} \%)\end{array}$ & $\begin{array}{c}\begin{array}{c}\mathrm{Cu} \\
\text { (wt\%) }\end{array} \\
\end{array}$ & $\begin{array}{c}\begin{array}{c}\mathrm{K} \\
(\mathrm{wt} \%)\end{array} \\
\end{array}$ & 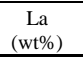 & $\begin{array}{c}\mathrm{Mg} \\
\text { (wt\%) }\end{array}$ & $\begin{array}{c}\mathrm{Mn} \\
\text { (wt\%) }\end{array}$ & $\begin{array}{c}\mathrm{Na} \\
\text { (wt\%) }\end{array}$ & $\begin{array}{c}\mathrm{Ni} \\
(\mathrm{wt} \%)\end{array}$ & 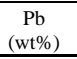 & $\begin{array}{c}\mathrm{S} \\
\text { (wt\%) }\end{array}$ & 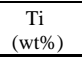 & $\begin{array}{c}\mathrm{Zn} \\
\text { (wt\%) }\end{array}$ & $\begin{array}{c}\begin{array}{c}\mathrm{Zr} \\
(\mathrm{wt} \%)\end{array} \\
\end{array}$ \\
\hline 4 & SB5NEPH-35 & 1 & 2 & 3 & U04LM22 & 11 & 0.039 & 8.84 & 0.08 & 0.06 & 0.032 & 0.054 & $<0.100$ & 0.371 & 1.81 & 15.4 & 0.718 & 0.029 & 0.166 & 0.133 & 0.026 & 0.07 \\
\hline 4 & SB5NEPH-33 & 1 & 2 & 4 & U10LM12 & 12.6 & 0.04 & 1.45 & 0.081 & 0.059 & 0.028 & 0.054 & $<0.100$ & 0.379 & 1.81 & 17.5 & 0.731 & 0.031 & 0.152 & 0.136 & 0.025 & 0.073 \\
\hline 4 & SB5NEPH-40 & 1 & 2 & 5 & U06LM12 & 14.9 & 0.04 & 8.88 & 0.08 & 0.043 & 0.029 & 0.055 & $<0.100$ & 0.379 & 1.83 & 4.37 & 0.658 & 0.031 & $<0.100$ & 0.134 & 0.025 & 0.071 \\
\hline 4 & SB5NEPH-32 & 1 & 2 & 6 & U05LM22 & 2.99 & 0.038 & 8.64 & 0.08 & 0.06 & 0.029 & 0.053 & $<0.100$ & 0.374 & 1.77 & 19.6 & 0.732 & 0.032 & 0.174 & 0.133 & 0.022 & 0.071 \\
\hline 4 & Batch 1 & 1 & 2 & 7 & BCHLM4122 & 2.73 & 0.124 & 0.934 & $<0.010$ & 0.074 & 0.306 & 2.62 & $<0.100$ & 0.819 & 1.37 & 7.29 & 0.545 & $<0.010$ & $<0.100$ & 0.386 & $<0.010$ & 0.068 \\
\hline 4 & SB5NEPH-36 & 1 & 2 & 8 & U01LM22 & 8.76 & 0.039 & 8.79 & 0.081 & 0.058 & 0.036 & 0.052 & $<0.100$ & 0.373 & 1.79 & 12.3 & 0.717 & 0.034 & 0.122 & 0.132 & 0.028 & 0.073 \\
\hline 4 & SB5NEPH-40 & 1 & 2 & 9 & U06LM22 & 14.7 & 0.04 & 8.81 & 0.08 & 0.041 & 0.028 & 0.054 & $<0.100$ & 0.374 & 1.78 & 4.23 & 0.63 & 0.031 & $<0.100$ & 0.132 & 0.024 & 0.071 \\
\hline 4 & SB5NEPH-35 & 1 & 2 & 10 & U04LM12 & 10.9 & 0.039 & 8.8 & 0.079 & 0.059 & 0.03 & 0.07 & $<0.100$ & 0.368 & 1.76 & 15.3 & 0.718 & 0.028 & 0.161 & 0.133 & 0.025 & 0.071 \\
\hline 4 & SB5NEPH-32 & 1 & 2 & 11 & U05LM12 & 3.03 & 0.038 & 8.88 & 0.08 & 0.06 & 0.03 & 0.052 & $<0.100$ & 0.374 & 1.78 & 20.1 & 0.731 & 0.032 & 0.175 & 0.132 & 0.021 & 0.068 \\
\hline 4 & SB5NEPH-33 & 1 & 2 & 12 & U10LM22 & 12.7 & 0.04 & 1.46 & 0.082 & 0.058 & 0.028 & 0.056 & $<0.100$ & 0.378 & 1.79 & 17.4 & 0.731 & 0.032 & 0.152 & 0.137 & 0.024 & 0.073 \\
\hline 4 & Batch 1 & 1 & 2 & 13 & BCHLM4123 & 2.61 & 0.124 & 0.893 & $<0.010$ & 0.074 & 0.305 & 2.62 & $<0.100$ & 0.813 & 1.33 & 6.8 & 0.542 & $<0.010$ & $<0.100$ & 0.381 & $<0.010$ & 0.067 \\
\hline 4 & Batch 1 & 2 & 2 & 1 & BCHLM4221 & 2.5 & 0.123 & 0.844 & $<0.010$ & 0.073 & 0.306 & 2.64 & $<0.100$ & 0.814 & 1.3 & 6.69 & 0.545 & $<0.010$ & $<0.100$ & 0.379 & $<0.010$ & 0.064 \\
\hline 4 & SB5NEPH-39 & 2 & 2 & 2 & U03LM12 & 17.8 & 0.036 & 8.57 & 0.079 & 0.042 & 0.024 & 0.048 & $<0.100$ & 0.366 & 1.77 & 5.27 & 0.695 & 0.031 & $<0.100$ & 0.129 & 0.023 & 0.065 \\
\hline 4 & SB5NEPH-34 & 2 & 2 & 3 & U02LM12 & 10.1 & 0.038 & 1.44 & 0.081 & 0.056 & 0.026 & 0.046 & $<0.100$ & 0.373 & 1.78 & 14 & 0.724 & 0.032 & $\begin{array}{ll}0.11 \\
\end{array}$ & 0.132 & 0.023 & 0.068 \\
\hline 4 & SB5NEPH-39 & 2 & 2 & 4 & U03LM22 & 17.7 & 0.036 & 8.52 & 0.079 & 0.042 & 0.025 & 0.049 & $<0.100$ & 0.367 & 1.78 & 5.18 & 0.698 & 0.032 & $<0.100$ & 0.129 & 0.023 & 0.071 \\
\hline 4 & SB5NEPH-37 & 2 & 2 & 5 & U09LM12 & 19.8 & 0.039 & 1.41 & 0.079 & 0.049 & 0.023 & 0.051 & $<0.100$ & 0.355 & 1.76 & 5.97 & 0.744 & 0.032 & $<0.100$ & 0.132 & 0.023 & 0.059 \\
\hline 4 & SB5NEPH-38 & 2 & 2 & 6 & $\begin{array}{l}\text { U07LM22 } \\
\end{array}$ & 16.3 & 0.041 & 1.42 & 0.079 & 0.055 & 0.025 & 0.05 & $<0.100$ & 0.379 & 1.75 & 5 & 0.749 & 0.03 & $<0.100$ & 0.132 & 0.023 & 0.067 \\
\hline 4 & Batch 1 & 2 & 2 & 7 & BCHLM4222 & 2.53 & 0.122 & 0.856 & $<0.010$ & 0.073 & 0.3 & 2.64 & $<0.100$ & 0.809 & 1.3 & 6.76 & 0.543 & $<0.010$ & $<0.100$ & 0.381 & $<0.010$ & 0.066 \\
\hline 4 & SB5NEPH-38 & 2 & 2 & 8 & U07LM12 & 16.4 & 0.041 & 1.44 & 0.08 & 0.057 & 0.025 & 0.05 & $<0.100$ & 0.383 & 1.75 & 5.06 & 0.771 & 0.031 & $<0.100$ & 0.134 & 0.024 & 0.065 \\
\hline 4 & SB5NEPH-34 & 2 & 2 & 9 & U02LM22 & 10.1 & 0.038 & 1.44 & 0.08 & 0.056 & 0.026 & 0.049 & $<0.100$ & 0.371 & 1.75 & 13.9 & 0.729 & 0.031 & 0.111 & 0.132 & 0.023 & 0.069 \\
\hline 4 & SB5NEPH-31 & 2 & 2 & 10 & U08LM22 & 3.49 & 0.036 & 8.2 & 0.073 & 0.057 & 0.03 & 0.048 & $<0.100$ & 0.34 & 1.61 & 22.7 & 0.643 & 0.031 & 0.162 & 0.125 & 0.023 & 0.065 \\
\hline 4 & SB5NEPH-31 & 2 & 2 & 11 & U08LM12 & 3.49 & 0.035 & 8.19 & 0.072 & 0.056 & 0.027 & 0.048 & $<0.100$ & 0.336 & 1.6 & 23 & 0.637 & 0.03 & 0.157 & 0.124 & 0.023 & 0.064 \\
\hline 4 & $\begin{array}{l}\text { SB5NEPH-37 } \\
\end{array}$ & 2 & 2 & 12 & U09LM22 & 20.1 & 0.038 & 1.43 & 0.078 & 0.049 & 0.023 & 0.052 & $<0.100$ & 0.35 & 1.75 & 6.02 & 0.755 & 0.031 & $<0.100$ & 0.131 & 0.023 & 0.061 \\
\hline 4 & Batch 1 & 2 & 2 & 13 & BCHLM 4223 & 2.55 & 0.121 & 0.868 & $<0.010$ & 0.073 & 0.299 & 2.66 & $<0.100$ & 0.803 & 1.3 & 6.81 & 0.541 & $<0.010$ & $<0.100$ & 0.377 & $<0.010$ & 0.064 \\
\hline
\end{tabular}


WSRC-STI-2007-00659

Revision 0

Appendix A

Table A3. Measured Elemental Concentrations (wt \%) for Samples

Prepared Using Peroxide Fusion. (continued)

\begin{tabular}{|c|c|c|c|c|c|c|c|c|c|}
\hline Set & Glass ID & Block & Sub-Block & Sequence & Lab ID & B (wt\%) & Fe (wt\%) & Li (wt\%) & Si (wt\%) \\
\hline 1 & Batch 1 & 1 & 1 & 1 & BCHPF1111 & 2.39 & 8.7 & 2 & 22.7 \\
\hline 1 & SB5NEPH-09 & 1 & 1 & 2 & R04PF21 & 1.3 & 8.11 & $<0.100$ & 21.6 \\
\hline 1 & SB5NEPH-06 & 1 & 1 & 3 & R03PF11 & 5.86 & 7.36 & $<0.100$ & 18.8 \\
\hline 1 & SB5NEPH-10 & 1 & 1 & 4 & R05PF11 & 5.84 & 8 & $<0.100$ & 18.2 \\
\hline 1 & SB5NEPH-02 & 1 & 1 & 5 & R06PF21 & 5.73 & 7.72 & $<0.100$ & 18.1 \\
\hline 1 & SB5NEPH-06 & 1 & 1 & 6 & R03PF21 & 5.73 & 7 & $<0.100$ & 18.2 \\
\hline 1 & Batch 1 & 1 & 1 & 7 & BCHPF1112 & 2.37 & 8.82 & 2.01 & 22.9 \\
\hline 1 & SB5NEPH-02 & 1 & 1 & 8 & R06PF11 & 5.77 & 7.62 & $<0.100$ & 18.2 \\
\hline 1 & SB5NEPH-10 & 1 & 1 & 9 & R05PF21 & 5.79 & 7.87 & $<0.100$ & 17.9 \\
\hline 1 & SB5NEPH-07 & 1 & 1 & 10 & R09PF11 & 1.3 & 7.69 & $<0.100$ & 19.5 \\
\hline 1 & SB5NEPH-09 & 1 & 1 & 11 & R04PF11 & 1.28 & 7.87 & $<0.100$ & 21.3 \\
\hline 1 & SB5NEPH-07 & 1 & 1 & 12 & R09PF21 & 1.21 & 7.42 & $<0.100$ & 19 \\
\hline 1 & Batch 1 & 1 & 1 & 13 & BCHPF1113 & 2.31 & 8.82 & 2.02 & 23 \\
\hline 1 & Batch 1 & 1 & 2 & 1 & BCHPF1121 & 2.41 & 8.54 & 2.02 & 22.6 \\
\hline 1 & SB5NEPH-09 & 1 & 2 & 2 & R04PF22 & 1.33 & 7.85 & $<0.100$ & 21.2 \\
\hline 1 & SB5NEPH-07 & 1 & 2 & 3 & R09PF22 & 1.27 & 7.19 & $<0.100$ & 18.9 \\
\hline 1 & SB5NEPH-10 & 1 & 2 & 4 & R05PF12 & 5.73 & 7.65 & $<0.100$ & 17.8 \\
\hline 1 & SB5NEPH-02 & 1 & 2 & 5 & R06PF12 & 5.71 & 7.46 & $<0.100$ & 18.2 \\
\hline 1 & SB5NEPH-10 & 1 & 2 & 6 & R05PF22 & 5.75 & 7.67 & $<0.100$ & 17.7 \\
\hline 1 & Batch 1 & 1 & 2 & 7 & BCHPF1122 & 2.39 & 8.53 & 2.03 & 22.7 \\
\hline 1 & SB5NEPH-02 & 1 & 2 & 8 & R06PF22 & 5.71 & 7.58 & $<0.100$ & 17.9 \\
\hline 1 & SB5NEPH-06 & 1 & 2 & 9 & R03PF12 & 5.71 & 6.84 & $<0.100$ & 18.2 \\
\hline 1 & SB5NEPH-07 & 1 & 2 & 10 & R09PF12 & 1.3 & 7.22 & $<0.100$ & 18.9 \\
\hline 1 & SB5NEPH-09 & 1 & 2 & 11 & R04PF12 & 1.3 & 7.64 & $<0.100$ & 20.8 \\
\hline 1 & SB5NEPH-06 & 1 & 2 & 12 & R03PF22 & 5.63 & 6.86 & $<0.100$ & 17.9 \\
\hline 1 & Batch 1 & 1 & 2 & 13 & BCHPF1123 & 2.37 & 8.62 & 2.02 & 22.8 \\
\hline 1 & Batch 1 & 2 & 1 & 1 & BCHPF1211 & 2.39 & 8.95 & 2.03 & 23.1 \\
\hline 1 & SB5NEPH-04 & 2 & 1 & 2 & R07PF11 & 5.77 & 7.58 & $<0.100$ & 15.6 \\
\hline 1 & SB5NEPH-03 & 2 & 1 & 3 & R10PF11 & 1.26 & 7.31 & $<0.100$ & 19.3 \\
\hline 1 & SB5NEPH-04 & 2 & 1 & 4 & R07PF21 & 5.76 & 7.64 & $<0.100$ & 15.7 \\
\hline 1 & SB5NEPH-01 & 2 & 1 & 5 & R01PF11 & 1.26 & 7.52 & $<0.100$ & 21.5 \\
\hline 1 & SB5NEPH-08 & 2 & 1 & 6 & R08PF11 & 5.65 & 7.34 & $<0.100$ & 15.6 \\
\hline 1 & Batch 1 & 2 & 1 & 7 & BCHPF1212 & 2.33 & 8.89 & 2.01 & 23.1 \\
\hline 1 & SB5NEPH-01 & 2 & 1 & 8 & R01PF21 & 1.28 & 7.63 & $<0.100$ & 22.3 \\
\hline 1 & SB5NEPH-05 & 2 & 1 & 9 & R02PF21 & 1.22 & 7.58 & $<0.100$ & 22.1 \\
\hline 1 & SB5NEPH-05 & 2 & 1 & 10 & R02PF11 & 1.21 & 7.56 & $<0.100$ & 21.4 \\
\hline 1 & SB5NEPH-08 & 2 & 1 & 11 & R08PF21 & 5.72 & 7.5 & $<0.100$ & 15.7 \\
\hline 1 & SB5NEPH-03 & 2 & 1 & 12 & R10PF21 & 1.21 & 7.25 & $<0.100$ & 19.1 \\
\hline 1 & Batch 1 & 2 & 1 & 13 & BCHPF1213 & 2.4 & 8.72 & 2.05 & 23.3 \\
\hline 1 & Batch 1 & 2 & 2 & 1 & BCHPF1221 & 2.36 & 8.88 & 2.03 & 23 \\
\hline 1 & SB5NEPH-04 & 2 & 2 & 2 & R07PF22 & 5.65 & 7.42 & $<0.100$ & 15.4 \\
\hline 1 & SB5NEPH-03 & 2 & 2 & 3 & R10PF12 & 1.25 & 7.52 & $<0.100$ & 19.5 \\
\hline 1 & SB5NEPH-05 & 2 & 2 & 4 & R02PF12 & 1.21 & 7.53 & $<0.100$ & 21.2 \\
\hline 1 & SB5NEPH-08 & 2 & 2 & 5 & R08PF22 & 5.63 & 7.46 & $<0.100$ & 15.4 \\
\hline 1 & SB5NEPH-08 & 2 & 2 & 6 & R08PF12 & 5.63 & 7.43 & $<0.100$ & 15.5 \\
\hline 1 & Batch 1 & 2 & 2 & 7 & BCHPF1222 & 2.3 & 8.85 & 2.02 & 23 \\
\hline 1 & SB5NEPH-03 & 2 & 2 & 8 & R10PF22 & 1.24 & 7.44 & $<0.100$ & 19.1 \\
\hline 1 & SB5NEPH-01 & 2 & 2 & 9 & R01PF22 & 1.23 & 7.55 & $<0.100$ & 21.9 \\
\hline 1 & SB5NEPH-01 & 2 & 2 & 10 & R01PF12 & 1.21 & 7.54 & $<0.100$ & 21.5 \\
\hline 1 & SB5NEPH-04 & 2 & 2 & 11 & R07PF12 & 5.56 & 7.34 & $<0.100$ & 15.2 \\
\hline 1 & SB5NEPH-05 & 2 & 2 & 12 & R02PF22 & 1.2 & 7.54 & $<0.100$ & 21.8 \\
\hline 1 & Batch 1 & 2 & 2 & 13 & BCHPF1223 & 2.29 & 8.82 & 2.02 & 23 \\
\hline 2 & Batch 1 & 1 & 1 & 1 & BCHPF2111 & 2.36 & 8.83 & 2.02 & 23.2 \\
\hline 2 & SB5NEPH-17 & 1 & 1 & 2 & S05PF21 & 1.22 & 7.43 & $<0.100$ & 18.6 \\
\hline 2 & SB5NEPH-20 & 1 & 1 & 3 & S06PF11 & 5.66 & 7.66 & $<0.100$ & 12.9 \\
\hline 2 & SB5NEPH-12 & 1 & 1 & 4 & S04PF11 & 5.67 & 7.68 & $<0.100$ & 15.4 \\
\hline 2 & SB5NEPH-13 & 1 & 1 & 5 & S07PF11 & 1.24 & 7.81 & $<0.100$ & 18.4 \\
\hline 2 & SB5NEPH-13 & 1 & 1 & 6 & S07PF21 & 1.21 & 7.6 & $<0.100$ & 18.4 \\
\hline 2 & Batch 1 & 1 & 1 & 7 & BCHPF2112 & 2.3 & 8.8 & 2.02 & 23.2 \\
\hline 2 & SB5NEPH-12 & 1 & 1 & 8 & S04PF21 & 5.6 & 7.49 & $<0.100$ & 15.2 \\
\hline 2 & SB5NEPH-11 & 1 & 1 & 9 & S02PF11 & 1.25 & 7.18 & $<0.100$ & 19.8 \\
\hline 2 & SB5NEPH-17 & 1 & 1 & 10 & S05PF11 & 1.22 & 7.55 & $<0.100$ & 18.7 \\
\hline 2 & SB5NEPH-20 & 1 & 1 & 11 & S06PF21 & 5.7 & 7.83 & $<0.100$ & 13.1 \\
\hline 2 & SB5NEPH-11 & 1 & 1 & 12 & S02PF21 & 1.25 & 7.24 & $<0.100$ & 20 \\
\hline 2 & Batch 1 & 1 & 1 & 13 & BCHPF2113 & 2.35 & 8.78 & 2.02 & 23.2 \\
\hline 2 & Batch 1 & 1 & 2 & 1 & BCHPF2121 & 2.43 & 8.79 & 2.04 & 23 \\
\hline 2 & SB5NEPH-13 & 1 & 2 & 2 & S07PF12 & 1.29 & 7.39 & $<0.100$ & 18.1 \\
\hline 2 & SB5NEPH-11 & 1 & 2 & 3 & S02PF22 & 1.29 & 6.85 & $<0.100$ & 19.4 \\
\hline
\end{tabular}


WSRC-STI-2007-00659

Revision 0

Appendix A

Table A3. Measured Elemental Concentrations (wt \%) for Samples

Prepared Using Peroxide Fusion. (continued)

\begin{tabular}{|c|c|c|c|c|c|c|c|c|c|}
\hline Set & "Glass ID & Block & Sub-Block & Sequence & Lab ID & $\mathrm{B}(\mathrm{wt} \%)$ & Fe (wt\%) & Li (wt\%) & Si (wt\%) \\
\hline 2 & SB5NEPH-13 & 1 & 2 & 4 & S07PF22 & 1.27 & 7.37 & $<0.100$ & 18.3 \\
\hline 2 & SB5NEPH-20 & 1 & 2 & 5 & S06PF12 & 5.59 & 7.18 & $<0.100$ & 12.6 \\
\hline 2 & SB5NEPH-12 & 1 & 2 & 6 & S04PF22 & 5.94 & 7.65 & $<0.100$ & 15.8 \\
\hline 2 & Batch 1 & 1 & 2 & 7 & BCHPF2122 & 2.37 & 8.55 & 2.04 & 22.8 \\
\hline 2 & SB5NEPH-17 & 1 & 2 & 8 & S05PF22 & 1.32 & 7.44 & $<0.100$ & 18.6 \\
\hline 2 & SB5NEPH-11 & 1 & 2 & 9 & S02PF12 & 1.28 & 6.9 & $<0.100$ & 19.4 \\
\hline 2 & SB5NEPH-17 & 1 & 2 & 10 & S05PF12 & 1.27 & 7.33 & $<0.100$ & 18.7 \\
\hline 2 & SB5NEPH-12 & 1 & 2 & 11 & S04PF12 & 5.65 & 7.33 & $<0.100$ & 15.3 \\
\hline 2 & SB5NEPH-20 & 1 & 2 & 12 & S06PF22 & 5.61 & 7.36 & $<0.100$ & 12.8 \\
\hline 2 & Batch 1 & 1 & 2 & 13 & BCHPF2123 & 2.36 & 8.66 & 2.05 & 22.9 \\
\hline 2 & Batch 1 & 2 & 1 & 1 & BCHPF2211 & 2.4 & 8.24 & 1.99 & 22.1 \\
\hline 2 & SB5NEPH-19 & 2 & 1 & 2 & S03PF11 & 1.3 & 6.76 & $<0.100$ & 15.7 \\
\hline 2 & SB5NEPH-19 & 2 & 1 & 3 & S03PF21 & 1.26 & 6.99 & $<0.100$ & 16 \\
\hline 2 & SB5NEPH-14 & 2 & 1 & 4 & S09PF21 & 5.35 & 6.99 & $<0.100$ & 14.4 \\
\hline 2 & SB5NEPH-15 & 2 & 1 & 5 & S08PF11 & 1.23 & 6.95 & $<0.100$ & 15.2 \\
\hline 2 & SB5NEPH-18 & 2 & 1 & 6 & S01PF11 & 5.58 & 7.34 & $<0.100$ & 14.8 \\
\hline 2 & Batch 1 & 2 & 1 & 7 & BCHPF2212 & 2.33 & 8.39 & 1.99 & 22.2 \\
\hline 2 & SB5NEPH-16 & 2 & 1 & 8 & S10PF21 & 5.49 & 7.43 & $<0.100$ & 12.4 \\
\hline 2 & SB5NEPH-14 & 2 & 1 & 9 & S09PF11 & 5.49 & 7.21 & $<0.100$ & 14.8 \\
\hline 2 & SB5NEPH-15 & 2 & 1 & 10 & S08PF21 & 1.27 & 7.27 & $<0.100$ & 15.3 \\
\hline 2 & SB5NEPH-16 & 2 & 1 & 11 & S10PF11 & 5.37 & 7.31 & $<0.100$ & 12.2 \\
\hline 2 & SB5NEPH-18 & 2 & 1 & 12 & S01PF21 & 5.65 & 7.67 & $<0.100$ & 15.1 \\
\hline 2 & Batch 1 & 2 & 1 & 13 & BCHPF2213 & 2.4 & 9.05 & 2.03 & 23.2 \\
\hline 2 & Batch 1 & 2 & 2 & 1 & BCHPF2221 & 2.43 & 9.11 & 2.06 & 23.5 \\
\hline 2 & SB5NEPH-15 & 2 & 2 & 2 & S08PF12 & 1.28 & 7.73 & $<0.100$ & 16.5 \\
\hline 2 & SB5NEPH-19 & 2 & 2 & 3 & S03PF12 & 1.29 & 7.42 & $<0.100$ & 16.6 \\
\hline 2 & SB5NEPH-16 & 2 & 2 & 4 & S10PF22 & 5.67 & 7.78 & $<0.100$ & 13 \\
\hline 2 & SB5NEPH-15 & 2 & 2 & 5 & S08PF22 & 1.26 & 7.42 & $<0.100$ & 15.7 \\
\hline 2 & SB5NEPH-18 & 2 & 2 & 6 & S01PF22 & 5.69 & 7.69 & $<0.100$ & 15.4 \\
\hline 2 & Batch 1 & 2 & 2 & 7 & BCHPF2222 & 2.39 & 8.99 & 2.05 & 23.6 \\
\hline 2 & SB5NEPH-19 & 2 & 2 & 8 & S03PF22 & 1.29 & 7.6 & $<0.100$ & 16.7 \\
\hline 2 & SB5NEPH-14 & 2 & 2 & 9 & S09PF12 & 5.73 & 7.89 & $<0.100$ & 15.7 \\
\hline 2 & SB5NEPH-16 & 2 & 2 & 10 & S10PF12 & 5.66 & 7.92 & $<0.100$ & 12.9 \\
\hline 2 & SB5NEPH-14 & 2 & 2 & 11 & S09PF22 & 5.82 & 8.04 & $<0.100$ & 15.8 \\
\hline 2 & SB5NEPH-18 & 2 & 2 & 12 & S01PF12 & 5.88 & 8.17 & $<0.100$ & 15.8 \\
\hline 2 & Batch 1 & 2 & 2 & 13 & BCHPF2223 & 2.46 & 9.45 & 2.06 & 24 \\
\hline 3 & Batch 1 & 1 & 1 & 1 & BCHPF3111 & 2.36 & 8.81 & 2.02 & 23.2 \\
\hline 3 & SB5NEPH-27 & 1 & 1 & 2 & T09PF21 & 1.24 & 7.11 & $<0.100$ & 16.4 \\
\hline 3 & SB5NEPH-21 & 1 & 1 & 3 & T06PF11 & 1.2 & 7.19 & $<0.100$ & 18.7 \\
\hline 3 & SB5NEPH-22 & 1 & 1 & 4 & T04PF11 & 5.69 & 6.81 & $<0.100$ & 15.5 \\
\hline 3 & SB5NEPH-29 & 1 & 1 & 5 & T02PF11 & 1.19 & 7.31 & $<0.100$ & 14.3 \\
\hline 3 & SB5NEPH-21 & 1 & 1 & 6 & T06PF21 & 1.19 & 7.18 & $<0.100$ & 18.6 \\
\hline 3 & Batch 1 & 1 & 1 & 7 & BCHPF3112 & 2.28 & 8.64 & 2 & 23 \\
\hline 3 & SB5NEPH-22 & 1 & 1 & 8 & T04PF21 & 5.75 & 6.92 & $<0.100$ & 15.6 \\
\hline 3 & SB5NEPH-27 & 1 & 1 & 9 & T09PF11 & 1.22 & 7.01 & $<0.100$ & 16.3 \\
\hline 3 & SB5NEPH-29 & 1 & 1 & 10 & T02PF21 & 1.15 & 7.28 & $<0.100$ & 14.2 \\
\hline 3 & SB5NEPH-30 & 1 & 1 & 11 & T08PF11 & 5.57 & 7.44 & $<0.100$ & 12.1 \\
\hline 3 & SB5NEPH-30 & 1 & 1 & 12 & T08PF21 & 5.62 & 7.39 & $<0.100$ & 12.1 \\
\hline 3 & Batch 1 & 1 & 1 & 13 & BCHPF3113 & 2.28 & 8.58 & 2.02 & 22.9 \\
\hline 3 & Batch 1 & 1 & 2 & 1 & BCHPF3121 & 2.36 & 8.65 & 2.01 & 22.9 \\
\hline 3 & SB5NEPH-29 & 1 & 2 & 2 & T02PF12 & 1.21 & 7.35 & $<0.100$ & 14.1 \\
\hline 3 & SB5NEPH-22 & 1 & 2 & 3 & T04PF22 & 5.71 & 6.92 & $<0.100$ & 15.5 \\
\hline 3 & SB5NEPH-30 & 1 & 2 & 4 & T08PF22 & 5.5 & 7.16 & $<0.100$ & 11.8 \\
\hline 3 & SB5NEPH-22 & 1 & 2 & 5 & T04PF12 & 5.7 & 6.88 & $<0.100$ & 15.4 \\
\hline 3 & SB5NEPH-29 & 1 & 2 & 6 & T02PF22 & 1.19 & 7.22 & $<0.100$ & 14 \\
\hline 3 & Batch 1 & 1 & 2 & 7 & BCHPF3122 & 2.31 & 8.73 & 2 & 22.8 \\
\hline 3 & SB5NEPH-21 & 1 & 2 & 8 & T06PF12 & 1.22 & 7 & $<0.100$ & 18.2 \\
\hline 3 & SB5NEPH-27 & 1 & 2 & 9 & T09PF22 & 1.23 & 6.86 & $<0.100$ & 15.9 \\
\hline 3 & SB5NEPH-27 & 1 & 2 & 10 & T09PF12 & 1.2 & 6.64 & $<0.100$ & 15.7 \\
\hline 3 & SB5NEPH-30 & 1 & 2 & 11 & T08PF12 & 5.45 & 7.16 & $<0.100$ & 11.8 \\
\hline 3 & SB5NEPH-21 & 1 & 2 & 12 & T06PF22 & 1.19 & 6.95 & $<0.100$ & 18.1 \\
\hline 3 & Batch 1 & 1 & 2 & 13 & BCHPF3123 & 2.24 & 8.38 & 1.99 & 22.3 \\
\hline 3 & Batch 1 & 2 & 1 & 1 & BCHPF3211 & 2.34 & 8.67 & 2.01 & 22.9 \\
\hline 3 & SB5NEPH-28 & 2 & 1 & 2 & T07PF11 & 5.69 & 7.35 & $<0.100$ & 12.7 \\
\hline 3 & SB5NEPH-25 & 2 & 1 & 3 & T03PF21 & 1.29 & 7.48 & $<0.100$ & 19.1 \\
\hline 3 & SB5NEPH-28 & 2 & 1 & 4 & T07PF21 & 5.71 & 7.37 & $<0.100$ & 12.7 \\
\hline 3 & SB5NEPH-26 & 2 & 1 & 5 & T01PF21 & 5.82 & 6.85 & $<0.100$ & 15.7 \\
\hline 3 & SB5NEPH-24 & 2 & 1 & 6 & T10PF21 & 5.66 & 7.51 & $<0.100$ & 12.9 \\
\hline
\end{tabular}


WSRC-STI-2007-00659

Revision 0

Appendix A

Table A3. Measured Elemental Concentrations (wt \%) for Samples

Prepared Using Peroxide Fusion. (continued)

\begin{tabular}{|c|c|c|c|c|c|c|c|c|c|}
\hline Set & Glass ID & Block & Sub-Block & Sequence & Lab ID & $\mathrm{B}(\mathrm{wt} \%)$ & Fe (wt\%) & Li (wt\%) & Si (wt\%) \\
\hline 3 & Batch 1 & 2 & 1 & 7 & BCHPF3212 & 2.35 & 8.9 & 2.04 & 23.3 \\
\hline 3 & SB5NEPH-24 & 2 & 1 & 8 & T10PF11 & 5.67 & 7.41 & $<0.100$ & 12.8 \\
\hline 3 & SB5NEPH-25 & 2 & 1 & 9 & T03PF11 & 1.29 & 7.47 & $<0.100$ & 18.9 \\
\hline 3 & SB5NEPH-23 & 2 & 1 & 10 & T05PF11 & 1.22 & 7.24 & $<0.100$ & 16.1 \\
\hline 3 & SB5NEPH-26 & 2 & 1 & 11 & T01PF11 & 5.75 & 6.82 & $<0.100$ & 15.5 \\
\hline 3 & SB5NEPH-23 & 2 & 1 & 12 & T05PF21 & 1.22 & 7.32 & $<0.100$ & 16.2 \\
\hline 3 & Batch 1 & 2 & 1 & 13 & BCHPF3213 & 2.28 & 8.7 & 2.03 & 23 \\
\hline 3 & Batch 1 & 2 & 2 & 1 & BCHPF3221 & 2.4 & 8.84 & 2.07 & 23.6 \\
\hline 3 & SB5NEPH-25 & 2 & 2 & 2 & T03PF22 & 1.27 & 7.42 & $<0.100$ & 18.9 \\
\hline 3 & SB5NEPH-25 & 2 & 2 & 3 & T03PF12 & 1.24 & 7.39 & $<0.100$ & 18.8 \\
\hline 3 & SB5NEPH-26 & 2 & 2 & 4 & T01PF22 & 5.78 & 6.8 & $<0.100$ & 15.5 \\
\hline 3 & SB5NEPH-28 & 2 & 2 & 5 & T07PF22 & 5.69 & 7.26 & $<0.100$ & 12.6 \\
\hline 3 & SB5NEPH-24 & 2 & 2 & 6 & T10PF12 & 5.63 & 7.22 & $<0.100$ & 12.6 \\
\hline 3 & Batch 1 & 2 & 2 & 7 & BCHPF3222 & 2.35 & 8.62 & 2.06 & 23.1 \\
\hline 3 & SB5NEPH-28 & 2 & 2 & 8 & T07PF12 & 5.68 & 7.14 & $<0.100$ & 12.6 \\
\hline 3 & SB5NEPH-23 & 2 & 2 & 9 & T05PF12 & 1.22 & 7.14 & $<0.100$ & 16.1 \\
\hline 3 & SB5NEPH-23 & 2 & 2 & 10 & T05PF22 & 1.19 & 7.08 & $<0.100$ & 15.8 \\
\hline 3 & SB5NEPH-26 & 2 & 2 & 11 & T01PF12 & 5.71 & 6.51 & $<0.100$ & 15.2 \\
\hline 3 & SB5NEPH-24 & 2 & 2 & 12 & T10PF22 & 5.62 & 7.17 & $<0.100$ & 12.6 \\
\hline 3 & Batch 1 & 2 & 2 & 13 & BCHPF3223 & 2.34 & 8.47 & 2.05 & 23.1 \\
\hline 4 & Batch 1 & 1 & 1 & 1 & BCHPF4111 & 2.42 & 9 & 2.07 & 23.6 \\
\hline 4 & SB5NEPH-33 & 1 & 1 & 2 & U10PF21 & 1.23 & 7.5 & $<0.100$ & 14.8 \\
\hline 4 & SB5NEPH-40 & 1 & 1 & 3 & U06PF21 & 5.66 & 7.36 & $<0.100$ & 10.4 \\
\hline 4 & SB5NEPH-32 & 1 & 1 & 4 & U05PF11 & 5.6 & 7.99 & $<0.100$ & 10.3 \\
\hline 4 & SB5NEPH-40 & 1 & 1 & 5 & U06PF11 & 5.72 & 7.38 & $<0.100$ & 10.4 \\
\hline 4 & SB5NEPH-33 & 1 & 1 & 6 & U10PF11 & 1.22 & 7.56 & $<0.100$ & 14.8 \\
\hline 4 & Batch 1 & 1 & 1 & 7 & BCHPF4112 & 2.36 & 9.17 & 2.07 & 23.7 \\
\hline 4 & SB5NEPH-35 & 1 & 1 & 8 & U04PF21 & 1.23 & 7.86 & $<0.100$ & 12.7 \\
\hline 4 & SB5NEPH-36 & 1 & 1 & 9 & U01PF11 & 5.51 & 7.76 & $<0.100$ & 10.2 \\
\hline 4 & SB5NEPH-32 & 1 & 1 & 10 & U05PF21 & 5.58 & 7.72 & $<0.100$ & 10.2 \\
\hline 4 & SB5NEPH-36 & 1 & 1 & 11 & U01PF21 & 5.61 & 7.72 & $<0.100$ & 10.3 \\
\hline 4 & SB5NEPH-35 & 1 & 1 & 12 & U04PF11 & 1.21 & 7.63 & $<0.100$ & 12.6 \\
\hline 4 & Batch 1 & 1 & 1 & 13 & BCHPF4113 & 2.35 & 8.75 & 2.08 & 23.4 \\
\hline 4 & Batch 1 & 1 & 2 & 1 & BCHPF4121 & 2.43 & 8.82 & 2.06 & 23.5 \\
\hline 4 & SB5NEPH-36 & 1 & 2 & 2 & U01PF22 & 5.58 & 7.61 & $<0.100$ & 10.2 \\
\hline 4 & SB5NEPH-33 & 1 & 2 & 3 & U10PF12 & 1.27 & 7.32 & $<0.100$ & 14.7 \\
\hline 4 & SB5NEPH-35 & 1 & 2 & 4 & U04PF12 & 1.24 & 7.57 & $<0.100$ & 12.6 \\
\hline 4 & SB5NEPH-32 & 1 & 2 & 5 & U05PF12 & 5.6 & 7.48 & $<0.100$ & 10.1 \\
\hline 4 & SB5NEPH-35 & 1 & 2 & 6 & U04PF22 & 1.23 & 7.59 & $<0.100$ & 12.7 \\
\hline 4 & Batch 1 & 1 & 2 & 7 & BCHPF4122 & 2.36 & 8.91 & 2.09 & 23.5 \\
\hline 4 & SB5NEPH-33 & 1 & 2 & 8 & U10PF22 & 1.19 & 7.14 & $<0.100$ & 14.4 \\
\hline 4 & SB5NEPH-40 & 1 & 2 & 9 & U06PF22 & 5.55 & 6.79 & $<0.100$ & 10.1 \\
\hline 4 & SB5NEPH-40 & 1 & 2 & 10 & U06PF12 & 5.54 & 6.75 & $<0.100$ & 10 \\
\hline 4 & SB5NEPH-36 & 1 & 2 & 11 & U01PF12 & 5.39 & 7.16 & $<0.100$ & 9.9 \\
\hline 4 & SB5NEPH-32 & 1 & 2 & 12 & U05PF22 & 5.44 & 7.1 & $<0.100$ & 9.9 \\
\hline 4 & Batch 1 & 1 & 2 & 13 & BCHPF4123 & 2.36 & 8.7 & 2.08 & 23.2 \\
\hline 4 & Batch 1 & 2 & 1 & 1 & BCHPF4211 & 2.42 & 9.16 & 2.06 & 23.8 \\
\hline 4 & SB5NEPH-38 & 2 & 1 & 2 & U07PF11 & 5.94 & 8.07 & $<0.100$ & 12.6 \\
\hline 4 & SB5NEPH-31 & 2 & 1 & 3 & U08PF11 & 1.18 & 7.32 & $<0.100$ & 12.2 \\
\hline 4 & SB5NEPH-39 & 2 & 1 & 4 & U03PF11 & 1.25 & 7.68 & $<0.100$ & 13.1 \\
\hline 4 & SB5NEPH-31 & 2 & 1 & 5 & U08PF21 & 1.16 & 7.36 & $<0.100$ & 12.2 \\
\hline 4 & SB5NEPH-39 & 2 & 1 & 6 & U03PF21 & 1.24 & 7.6 & $<0.100$ & 13.1 \\
\hline 4 & Batch 1 & 2 & 1 & 7 & BCHPF4212 & 2.36 & 9.18 & 2.07 & 23.6 \\
\hline 4 & SB5NEPH-38 & 2 & 1 & 8 & U07PF21 & 5.85 & 7.89 & $<0.100$ & 12.5 \\
\hline 4 & SB5NEPH-37 & 2 & 1 & 9 & U09PF21 & 1.28 & 7.52 & $<0.100$ & 15.5 \\
\hline 4 & SB5NEPH-34 & 2 & 1 & 10 & U02PF21 & 5.72 & 7.69 & $<0.100$ & 12.5 \\
\hline 4 & SB5NEPH-34 & 2 & 1 & 11 & U02PF11 & 5.72 & 7.52 & $<0.100$ & 12.3 \\
\hline 4 & SB5NEPH-37 & 2 & 1 & 12 & U09PF11 & 1.23 & 7.28 & $<0.100$ & 15.1 \\
\hline 4 & Batch 1 & 2 & 1 & 13 & BCHPF4213 & 2.3 & 8.86 & 2.06 & 23.4 \\
\hline 4 & Batch 1 & 2 & 2 & 1 & BCHPF4221 & 2.38 & 8.7 & 2.03 & 23 \\
\hline 4 & SB5NEPH-31 & 2 & 2 & 2 & U08PF22 & 1.18 & 6.89 & $<0.100$ & 12 \\
\hline 4 & SB5NEPH-31 & 2 & 2 & 3 & U08PF12 & 1.16 & 6.95 & $<0.100$ & 12 \\
\hline 4 & SB5NEPH-34 & 2 & 2 & 4 & U02PF12 & 5.64 & 7.39 & $<0.100$ & 12.3 \\
\hline 4 & SB5NEPH-38 & 2 & 2 & 5 & U07PF22 & 5.77 & 7.58 & $<0.100$ & 12.4 \\
\hline 4 & SB5NEPH-37 & 2 & 2 & 6 & U09PF22 & 1.25 & 7.28 & $<0.100$ & 15.2 \\
\hline 4 & Batch 1 & 2 & 2 & 7 & BCHPF4222 & 2.36 & 8.87 & 2.05 & 23.5 \\
\hline 4 & SB5NEPH-38 & 2 & 2 & 8 & U07PF12 & 5.68 & 7.27 & $<0.100$ & 12.2 \\
\hline 4 & SB5NEPH-34 & 2 & 2 & 9 & U02PF22 & 5.58 & 7.18 & $<0.100$ & 12.1 \\
\hline
\end{tabular}


WSRC-STI-2007-00659

Revision 0

Appendix A

Table A3. Measured Elemental Concentrations (wt \%) for Samples

Prepared Using Peroxide Fusion. (continued)

\begin{tabular}{|c|c|c|c|c|c|c|c|c|c|}
\hline Set & Glass ID & Block & Sub-Block & Sequence & Lab ID & B (wt\%) & Fe (wt\%) & Li (wt\%) & Si (wt\%) \\
\hline 4 & SB5NEPH-39 & 2 & 2 & 10 & U03PF12 & 1.23 & 7.14 & $<0.100$ & 12.7 \\
\hline 4 & SB5NEPH-37 & 2 & 2 & 11 & U09PF12 & 1.24 & 7.07 & $<0.100$ & 15 \\
\hline 4 & SB5NEPH-39 & 2 & 2 & 12 & U03PF22 & 1.21 & 7.36 & $<0.100$ & 13 \\
\hline 4 & Batch 1 & 2 & 2 & 13 & BCHPF4223 & 2.31 & 8.76 & 2.05 & 23.4 \\
\hline
\end{tabular}


WSRC-STI-2007-00659

Revision 0

Appendix A

Table A4. Average Measured and Bias-Corrected Chemical Compositions Versus Targeted Compositions by Oxide by SB4 Variability Study Glass. (continued)

(100 -Batch 1)

\begin{tabular}{|c|c|c|c|c|c|c|c|c|c|}
\hline Set & Glass \# & Oxide & $\begin{array}{l}\text { Measured } \\
\text { (wt \%) }\end{array}$ & $\begin{array}{c}\text { Measured Bias- } \\
\text { Corrected (wt \%) }\end{array}$ & $\begin{array}{c}\text { Targeted } \\
\text { (wt \%) }\end{array}$ & $\begin{array}{c}\text { Diff of } \\
\text { Measured }\end{array}$ & $\begin{array}{c}\text { Diff of } \\
\text { Meas BC }\end{array}$ & $\begin{array}{l}\% \text { Diff of } \\
\text { Measured }\end{array}$ & $\begin{array}{l}\text { \% Diff of } \\
\text { Meas BC }\end{array}$ \\
\hline 1 & 1 & $\mathrm{Al}_{2} \mathrm{O}_{3}$ & 7.9690 & 8.1675 & 7.8000 & 0.1690 & 0.3675 & $2.2 \%$ & $4.7 \%$ \\
\hline 1 & 1 & $\mathrm{~B}_{2} \mathrm{O}_{3}$ & 4.0088 & 4.1285 & 4.0000 & 0.0088 & 0.1285 & $0.2 \%$ & $3.2 \%$ \\
\hline 1 & 1 & $\mathrm{BaO}$ & 0.0438 & 0.0473 & 0.0490 & -0.0052 & -0.0017 & $-10.6 \%$ & $-3.5 \%$ \\
\hline 1 & 1 & $\mathrm{CaO}$ & 2.0148 & 2.1339 & 2.0000 & 0.0148 & 0.1339 & $0.7 \%$ & $6.7 \%$ \\
\hline 1 & 1 & $\mathrm{Ce}_{2} \mathrm{O}_{3}$ & 0.0908 & 0.0908 & 0.1030 & -0.0122 & -0.0122 & $-11.9 \%$ & $-11.9 \%$ \\
\hline 1 & 1 & $\mathrm{Cr}_{2} \mathrm{O}_{3}$ & 0.0998 & 0.0985 & 0.0900 & 0.0098 & 0.0085 & $10.8 \%$ & $9.4 \%$ \\
\hline 1 & 1 & $\mathrm{CuO}$ & 0.0369 & 0.0375 & 0.0330 & 0.0039 & 0.0045 & $11.9 \%$ & $13.6 \%$ \\
\hline 1 & 1 & $\mathrm{Fe}_{2} \mathrm{O}_{3}$ & 10.8085 & 10.9655 & 10.7720 & 0.0365 & 0.1935 & $0.3 \%$ & $1.8 \%$ \\
\hline 1 & 1 & $\mathrm{~K}_{2} \mathrm{O}$ & 0.0611 & 0.0652 & 0.0690 & -0.0079 & -0.0038 & $-11.4 \%$ & $-5.5 \%$ \\
\hline 1 & 1 & $\mathrm{La}_{2} \mathrm{O}_{3}$ & 0.0586 & 0.0586 & 0.0150 & 0.0436 & 0.0436 & $290.9 \%$ & $290.9 \%$ \\
\hline 1 & 1 & $\mathrm{Li}_{2} \mathrm{O}$ & 0.1076 & 0.1093 & 0.0000 & 0.1076 & 0.1093 & & \\
\hline 1 & 1 & $\mathrm{MgO}$ & 0.6248 & 0.6483 & 0.6260 & -0.0012 & 0.0223 & $-0.2 \%$ & $3.6 \%$ \\
\hline 1 & 1 & $\mathrm{MnO}$ & 2.3468 & 2.3646 & 2.3060 & 0.0408 & 0.0586 & $1.8 \%$ & $2.5 \%$ \\
\hline 1 & 1 & $\mathrm{Na}_{2} \mathrm{O}$ & 23.4215 & 22.9546 & 23.4000 & 0.0215 & -0.4454 & $0.1 \%$ & $-1.9 \%$ \\
\hline 1 & 1 & $\mathrm{NiO}$ & 0.9426 & 1.0050 & 1.0220 & -0.0794 & -0.0170 & $-7.8 \%$ & $-1.7 \%$ \\
\hline 1 & 1 & $\mathrm{PbO}$ & 0.0382 & 0.0382 & 0.0430 & -0.0048 & -0.0048 & $-11.1 \%$ & $-11.1 \%$ \\
\hline 1 & 1 & $\mathrm{SiO}_{2}$ & 46.6367 & 47.4278 & 46.8000 & -0.1633 & 0.6278 & $-0.3 \%$ & $1.3 \%$ \\
\hline 1 & 1 & $\mathrm{SO}_{4}$ & 0.4996 & 0.4996 & 0.5130 & -0.0134 & -0.0134 & $-2.6 \%$ & $-2.6 \%$ \\
\hline 1 & 1 & $\mathrm{TiO}_{2}$ & 0.2273 & 0.2369 & 0.2270 & 0.0003 & 0.0099 & $0.1 \%$ & $4.4 \%$ \\
\hline 1 & 1 & $\mathrm{ZnO}$ & 0.0280 & 0.0280 & 0.0330 & -0.0050 & -0.0050 & $-15.1 \%$ & $-15.1 \%$ \\
\hline 1 & 1 & $\mathrm{ZrO}_{2}$ & 0.0892 & 0.0892 & 0.1010 & -0.0118 & -0.0118 & $-11.7 \%$ & $-11.7 \%$ \\
\hline 1 & 1 & Sum & 100.1544 & 101.1947 & 100.0020 & 0.1524 & 1.1927 & $0.2 \%$ & $1.2 \%$ \\
\hline 1 & 2 & $\mathrm{Al}_{2} \mathrm{O}_{3}$ & 6.4290 & 6.5893 & 6.4000 & 0.0290 & 0.1893 & $0.5 \%$ & $3.0 \%$ \\
\hline 1 & 2 & $\mathrm{~B}_{2} \mathrm{O}_{3}$ & 18.4500 & 18.7776 & 18.0000 & 0.4500 & 0.7776 & $2.5 \%$ & $4.3 \%$ \\
\hline 1 & 2 & $\mathrm{BaO}$ & 0.0449 & 0.0485 & 0.0490 & -0.0041 & -0.0005 & $-8.3 \%$ & $-1.0 \%$ \\
\hline 1 & 2 & $\mathrm{CaO}$ & 1.9974 & 2.1153 & 2.0000 & -0.0026 & 0.1153 & $-0.1 \%$ & $5.8 \%$ \\
\hline 1 & 2 & $\mathrm{Ce}_{2} \mathrm{O}_{3}$ & 0.0952 & 0.0952 & 0.1030 & -0.0078 & -0.0078 & $-7.6 \%$ & $-7.6 \%$ \\
\hline 1 & 2 & $\mathrm{Cr}_{2} \mathrm{O}_{3}$ & 0.0921 & 0.0909 & 0.0900 & 0.0021 & 0.0009 & $2.3 \%$ & $1.0 \%$ \\
\hline 1 & 2 & $\mathrm{CuO}$ & 0.0341 & 0.0346 & 0.0330 & 0.0011 & 0.0016 & $3.4 \%$ & $4.9 \%$ \\
\hline 1 & 2 & $\mathrm{Fe}_{2} \mathrm{O}_{3}$ & 10.8586 & 11.2453 & 10.7720 & 0.0866 & 0.4733 & $0.8 \%$ & $4.4 \%$ \\
\hline 1 & 2 & $\mathrm{~K}_{2} \mathrm{O}$ & 0.0590 & 0.0630 & 0.0690 & -0.0100 & -0.0060 & $-14.5 \%$ & $-8.7 \%$ \\
\hline 1 & 2 & $\mathrm{La}_{2} \mathrm{O}_{3}$ & 0.0586 & 0.0586 & 0.0150 & 0.0436 & 0.0436 & $290.9 \%$ & $290.9 \%$ \\
\hline 1 & 2 & $\mathrm{Li}_{2} \mathrm{O}$ & 0.1076 & 0.1098 & 0.0000 & 0.1076 & 0.1098 & & \\
\hline 1 & 2 & $\mathrm{MgO}$ & 0.6310 & 0.6547 & 0.6260 & 0.0050 & 0.0287 & $0.8 \%$ & $4.6 \%$ \\
\hline 1 & 2 & $\mathrm{MnO}$ & 2.3306 & 2.3483 & 2.3060 & 0.0246 & 0.0423 & $1.1 \%$ & $1.8 \%$ \\
\hline 1 & 2 & $\mathrm{Na}_{2} \mathrm{O}$ & 19.0405 & 18.6633 & 19.2000 & -0.1595 & -0.5367 & $-0.8 \%$ & $-2.8 \%$ \\
\hline 1 & 2 & $\mathrm{NiO}$ & 0.9604 & 1.0241 & 1.0220 & -0.0616 & 0.0021 & $-6.0 \%$ & $0.2 \%$ \\
\hline 1 & 2 & $\mathrm{PbO}$ & 0.0361 & 0.0361 & 0.0430 & -0.0069 & -0.0069 & $-16.1 \%$ & $-16.1 \%$ \\
\hline 1 & 2 & $\mathrm{SiO}_{2}$ & 38.7213 & 39.8969 & 38.4000 & 0.3213 & 1.4969 & $0.8 \%$ & $3.9 \%$ \\
\hline 1 & 2 & $\mathrm{SO}_{4}$ & 0.4883 & 0.4883 & 0.5130 & -0.0247 & -0.0247 & $-4.8 \%$ & $-4.8 \%$ \\
\hline 1 & 2 & $\mathrm{TiO}_{2}$ & 0.2231 & 0.2326 & 0.2270 & -0.0039 & 0.0056 & $-1.7 \%$ & $2.5 \%$ \\
\hline 1 & 2 & $\mathrm{ZnO}$ & 0.0280 & 0.0280 & 0.0330 & -0.0050 & -0.0050 & $-15.1 \%$ & $-15.1 \%$ \\
\hline 1 & 2 & $\mathrm{ZrO}_{2}$ & 0.0898 & 0.0898 & 0.1010 & -0.0112 & -0.0112 & $-11.1 \%$ & $-11.1 \%$ \\
\hline 1 & 2 & Sum & 100.7758 & 102.6903 & 100.0020 & 0.7738 & 2.6883 & $0.8 \%$ & $2.7 \%$ \\
\hline 1 & 3 & $\mathrm{Al}_{2} \mathrm{O}_{3}$ & 6.8967 & 7.0686 & 6.8000 & 0.0967 & 0.2686 & $1.4 \%$ & $3.9 \%$ \\
\hline 1 & 3 & $\mathrm{~B}_{2} \mathrm{O}_{3}$ & 3.9927 & 4.1132 & 4.0000 & -0.0073 & 0.1132 & $-0.2 \%$ & $2.8 \%$ \\
\hline 1 & 3 & $\mathrm{BaO}$ & 0.0435 & 0.0470 & 0.0490 & -0.0055 & -0.0020 & $-11.1 \%$ & $-4.1 \%$ \\
\hline 1 & 3 & $\mathrm{CaO}$ & 12.1556 & 12.8737 & 12.0000 & 0.1556 & 0.8737 & $1.3 \%$ & $7.3 \%$ \\
\hline 1 & 3 & $\mathrm{Ce}_{2} \mathrm{O}_{3}$ & 0.0925 & 0.0925 & 0.1030 & -0.0105 & -0.0105 & $-10.2 \%$ & $-10.2 \%$ \\
\hline 1 & 3 & $\mathrm{Cr}_{2} \mathrm{O}_{3}$ & 0.0899 & 0.0887 & 0.0900 & -0.0001 & -0.0013 & $-0.1 \%$ & $-1.4 \%$ \\
\hline 1 & 3 & $\mathrm{CuO}$ & 0.0338 & 0.0343 & 0.0330 & 0.0008 & 0.0013 & $2.4 \%$ & $4.0 \%$ \\
\hline 1 & 3 & $\mathrm{Fe}_{2} \mathrm{O}_{3}$ & 10.5512 & 10.7044 & 10.7720 & -0.2208 & -0.0676 & $-2.0 \%$ & $-0.6 \%$ \\
\hline 1 & 3 & $\mathrm{~K}_{2} \mathrm{O}$ & 0.0590 & 0.0630 & 0.0690 & -0.0100 & -0.0060 & $-14.5 \%$ & $-8.7 \%$ \\
\hline 1 & 3 & $\mathrm{La}_{2} \mathrm{O}_{3}$ & 0.0586 & 0.0586 & 0.0150 & 0.0436 & 0.0436 & $290.9 \%$ & $290.9 \%$ \\
\hline 1 & 3 & $\mathrm{Li}_{2} \mathrm{O}$ & 0.1076 & 0.1093 & 0.0000 & 0.1076 & 0.1093 & & \\
\hline 1 & 3 & $\mathrm{MgO}$ & 0.6219 & 0.6453 & 0.6260 & -0.0041 & 0.0193 & $-0.7 \%$ & $3.1 \%$ \\
\hline 1 & 3 & $\mathrm{MnO}$ & 2.3145 & 2.3320 & 2.3060 & 0.0085 & 0.0260 & $0.4 \%$ & $1.1 \%$ \\
\hline 1 & 3 & $\mathrm{Na}_{2} \mathrm{O}$ & 20.4559 & 20.0519 & 20.4000 & 0.0559 & -0.3481 & $0.3 \%$ & $-1.7 \%$ \\
\hline 1 & 3 & $\mathrm{NiO}$ & 0.9455 & 1.0081 & 1.0220 & -0.0765 & -0.0139 & $-7.5 \%$ & $-1.4 \%$ \\
\hline 1 & 3 & $\mathrm{PbO}$ & 0.0315 & 0.0315 & 0.0430 & -0.0115 & -0.0115 & $-26.7 \%$ & $-26.7 \%$ \\
\hline 1 & 3 & $\mathrm{SiO}_{2}$ & 41.1815 & 41.8812 & 40.8000 & 0.3815 & 1.0812 & $0.9 \%$ & $2.6 \%$ \\
\hline 1 & 3 & $\mathrm{SO}_{4}$ & 0.5115 & 0.5115 & 0.5130 & -0.0015 & -0.0015 & $-0.3 \%$ & $-0.3 \%$ \\
\hline 1 & 3 & $\mathrm{TiO}_{2}$ & 0.2235 & 0.2330 & 0.2270 & -0.0035 & 0.0060 & $-1.5 \%$ & $2.6 \%$ \\
\hline 1 & 3 & $\mathrm{ZnO}$ & 0.0280 & 0.0280 & 0.0330 & -0.0050 & -0.0050 & $-15.1 \%$ & $-15.1 \%$ \\
\hline 1 & 3 & $\mathrm{ZrO}_{2}$ & 0.0932 & 0.0932 & 0.1010 & -0.0078 & -0.0078 & $-7.7 \%$ & $-7.7 \%$ \\
\hline
\end{tabular}


WSRC-STI-2007-00659

Revision 0

Appendix A

Table A4. Average Measured and Bias-Corrected Chemical Compositions Versus Targeted Compositions by Oxide by SB4 Variability Study Glass. (continued)

(100 -Batch 1)

\begin{tabular}{|c|c|c|c|c|c|c|c|c|c|}
\hline Set & Glass \# & Oxide & $\begin{array}{l}\text { Measured } \\
\text { (wt \%) }\end{array}$ & $\begin{array}{c}\text { Measured Bias- } \\
\text { Corrected (wt \%) }\end{array}$ & $\begin{array}{c}\text { Targeted } \\
\text { (wt \%) }\end{array}$ & $\begin{array}{c}\text { Diff of } \\
\text { Measured }\end{array}$ & $\begin{array}{c}\text { Diff of } \\
\text { Meas BC }\end{array}$ & $\begin{array}{l}\text { \% Diff of } \\
\text { Measured }\end{array}$ & $\begin{array}{l}\% \text { Diff of } \\
\text { Meas BC }\end{array}$ \\
\hline 1 & 3 & Sum & 100.4882 & 102.0691 & 100.0020 & 0.4862 & 2.0671 & $0.5 \%$ & $2.1 \%$ \\
\hline 1 & 4 & $\mathrm{Al}_{2} \mathrm{O}_{3}$ & 5.4984 & 5.6354 & 5.4000 & 0.0984 & 0.2354 & $1.8 \%$ & $4.4 \%$ \\
\hline 1 & 4 & $\mathrm{~B}_{2} \mathrm{O}_{3}$ & 18.3051 & 18.8534 & 18.0000 & 0.3051 & 0.8534 & $1.7 \%$ & $4.7 \%$ \\
\hline 1 & 4 & $\mathrm{BaO}$ & 0.0419 & 0.0452 & 0.0490 & -0.0071 & -0.0038 & $-14.6 \%$ & $-7.8 \%$ \\
\hline 1 & 4 & $\mathrm{CaO}$ & 12.2675 & 12.9924 & 12.0000 & 0.2675 & 0.9924 & $2.2 \%$ & $8.3 \%$ \\
\hline 1 & 4 & $\mathrm{Ce}_{2} \mathrm{O}_{3}$ & 0.0914 & 0.0914 & 0.1030 & -0.0116 & -0.0116 & $-11.3 \%$ & $-11.3 \%$ \\
\hline 1 & 4 & $\mathrm{Cr}_{2} \mathrm{O}_{3}$ & 0.0881 & 0.0869 & 0.0900 & -0.0019 & -0.0031 & $-2.2 \%$ & $-3.4 \%$ \\
\hline 1 & 4 & $\mathrm{CuO}$ & 0.0363 & 0.0369 & 0.0330 & 0.0033 & 0.0039 & $10.0 \%$ & $11.7 \%$ \\
\hline 1 & 4 & $\mathrm{Fe}_{2} \mathrm{O}_{3}$ & 10.7156 & 10.8712 & 10.7720 & -0.0564 & 0.0992 & $-0.5 \%$ & $0.9 \%$ \\
\hline 1 & 4 & $\mathrm{~K}_{2} \mathrm{O}$ & 0.0602 & 0.0643 & 0.0690 & -0.0088 & -0.0047 & $-12.7 \%$ & $-6.9 \%$ \\
\hline 1 & 4 & $\mathrm{La}_{2} \mathrm{O}_{3}$ & 0.0586 & 0.0586 & 0.0150 & 0.0436 & 0.0436 & $290.9 \%$ & $290.9 \%$ \\
\hline 1 & 4 & $\mathrm{Li}_{2} \mathrm{O}$ & 0.1076 & 0.1093 & 0.0000 & 0.1076 & 0.1093 & & \\
\hline 1 & 4 & $\mathrm{MgO}$ & 0.6169 & 0.6401 & 0.6260 & -0.0091 & 0.0141 & $-1.5 \%$ & $2.3 \%$ \\
\hline 1 & 4 & $\mathrm{MnO}$ & 2.3274 & 2.3451 & 2.3060 & 0.0214 & 0.0391 & $0.9 \%$ & $1.7 \%$ \\
\hline 1 & 4 & $\mathrm{Na}_{2} \mathrm{O}$ & 16.3108 & 15.9869 & 16.2000 & 0.1108 & -0.2131 & $0.7 \%$ & $-1.3 \%$ \\
\hline 1 & 4 & $\mathrm{NiO}$ & 0.9569 & 1.0203 & 1.0220 & -0.0651 & -0.0017 & $-6.4 \%$ & $-0.2 \%$ \\
\hline 1 & 4 & $\mathrm{PbO}$ & 0.0347 & 0.0347 & 0.0430 & -0.0083 & -0.0083 & $-19.2 \%$ & $-19.2 \%$ \\
\hline 1 & 4 & $\mathrm{SiO}_{2}$ & 33.1057 & 33.6664 & 32.4000 & 0.7057 & 1.2664 & $2.2 \%$ & $3.9 \%$ \\
\hline 1 & 4 & $\mathrm{SO}_{4}$ & 0.4322 & 0.4322 & 0.5130 & -0.0808 & -0.0808 & $-15.8 \%$ & $-15.8 \%$ \\
\hline 1 & 4 & $\mathrm{TiO}_{2}$ & 0.2231 & 0.2326 & 0.2270 & -0.0039 & 0.0056 & $-1.7 \%$ & $2.5 \%$ \\
\hline 1 & 4 & $\mathrm{ZnO}$ & 0.0271 & 0.0271 & 0.0330 & -0.0059 & -0.0059 & $-18.0 \%$ & $-18.0 \%$ \\
\hline 1 & 4 & $\mathrm{ZrO}_{2}$ & 0.0804 & 0.0804 & 0.1010 & -0.0206 & -0.0206 & $-20.4 \%$ & $-20.4 \%$ \\
\hline 1 & 4 & Sum & 101.3859 & 103.3107 & 100.0020 & 1.3839 & 3.3087 & $1.4 \%$ & $3.3 \%$ \\
\hline 1 & 5 & $\mathrm{Al}_{2} \mathrm{O}_{3}$ & 15.8860 & 16.2829 & 15.6000 & 0.2860 & 0.6829 & $1.8 \%$ & $4.4 \%$ \\
\hline 1 & 5 & $\mathrm{~B}_{2} \mathrm{O}_{3}$ & 3.8961 & 4.0133 & 4.0000 & -0.1039 & 0.0133 & $-2.6 \%$ & $0.3 \%$ \\
\hline 1 & 5 & $\mathrm{BaO}$ & 0.0447 & 0.0481 & 0.0490 & -0.0043 & -0.0009 & $-8.9 \%$ & $-1.9 \%$ \\
\hline 1 & 5 & $\mathrm{CaO}$ & 1.9799 & 2.1225 & 2.0000 & -0.0201 & 0.1225 & $-1.0 \%$ & $6.1 \%$ \\
\hline 1 & 5 & $\mathrm{Ce}_{2} \mathrm{O}_{3}$ & 0.0966 & 0.0966 & 0.1030 & -0.0064 & -0.0064 & $-6.2 \%$ & $-6.2 \%$ \\
\hline 1 & 5 & $\mathrm{Cr}_{2} \mathrm{O}_{3}$ & 0.0797 & 0.0779 & 0.0900 & -0.0103 & -0.0121 & $-11.5 \%$ & $-13.4 \%$ \\
\hline 1 & 5 & $\mathrm{CuO}$ & 0.0338 & 0.0344 & 0.0330 & 0.0008 & 0.0014 & $2.4 \%$ & $4.1 \%$ \\
\hline 1 & 5 & $\mathrm{Fe}_{2} \mathrm{O}_{3}$ & 10.7978 & 10.9546 & 10.7720 & 0.0258 & 0.1826 & $0.2 \%$ & $1.7 \%$ \\
\hline 1 & 5 & $\mathrm{~K}_{2} \mathrm{O}$ & 0.0678 & 0.0739 & 0.0690 & -0.0012 & 0.0049 & $-1.8 \%$ & $7.2 \%$ \\
\hline 1 & 5 & $\mathrm{La}_{2} \mathrm{O}_{3}$ & 0.0586 & 0.0586 & 0.0150 & 0.0436 & 0.0436 & $290.9 \%$ & $290.9 \%$ \\
\hline 1 & 5 & $\mathrm{Li}_{2} \mathrm{O}$ & 0.1076 & 0.1093 & 0.0000 & 0.1076 & 0.1093 & & \\
\hline 1 & 5 & $\mathrm{MgO}$ & 0.6422 & 0.6665 & 0.6260 & 0.0162 & 0.0405 & $2.6 \%$ & $6.5 \%$ \\
\hline 1 & 5 & $\mathrm{MnO}$ & 2.3403 & 2.3463 & 2.3060 & 0.0343 & 0.0403 & $1.5 \%$ & $1.7 \%$ \\
\hline 1 & 5 & $\mathrm{Na}_{2} \mathrm{O}$ & 15.8053 & 15.3917 & 15.6000 & 0.2053 & -0.2083 & $1.3 \%$ & $-1.3 \%$ \\
\hline 1 & 5 & $\mathrm{NiO}$ & 0.9426 & 1.0069 & 1.0220 & -0.0794 & -0.0151 & $-7.8 \%$ & $-1.5 \%$ \\
\hline 1 & 5 & $\mathrm{PbO}$ & 0.0366 & 0.0366 & 0.0430 & -0.0064 & -0.0064 & $-14.8 \%$ & $-14.8 \%$ \\
\hline 1 & 5 & $\mathrm{SiO}_{2}$ & 46.2624 & 47.0469 & 46.8000 & -0.5376 & 0.2469 & $-1.1 \%$ & $0.5 \%$ \\
\hline 1 & 5 & $\mathrm{SO}_{4}$ & 0.3730 & 0.3730 & 0.5130 & -0.1400 & -0.1400 & $-27.3 \%$ & $-27.3 \%$ \\
\hline 1 & 5 & $\mathrm{TiO}_{2}$ & 0.2335 & 0.2434 & 0.2270 & 0.0065 & 0.0164 & $2.9 \%$ & $7.2 \%$ \\
\hline 1 & 5 & $\mathrm{ZnO}$ & 0.0321 & 0.0321 & 0.0330 & -0.0009 & -0.0009 & $-2.9 \%$ & $-2.9 \%$ \\
\hline 1 & 5 & $\mathrm{ZrO}_{2}$ & 0.0929 & 0.0929 & 0.1010 & -0.0081 & -0.0081 & $-8.1 \%$ & $-8.1 \%$ \\
\hline 1 & 5 & Sum & 99.8093 & 101.1084 & 100.0020 & -0.1927 & 1.1064 & $-0.2 \%$ & $1.1 \%$ \\
\hline 1 & 6 & $\mathrm{Al}_{2} \mathrm{O}_{3}$ & 13.0659 & 13.3913 & 12.8000 & 0.2659 & 0.5913 & $2.1 \%$ & $4.6 \%$ \\
\hline 1 & 6 & $\mathrm{~B}_{2} \mathrm{O}_{3}$ & 18.4581 & 18.7868 & 18.0000 & 0.4581 & 0.7868 & $2.5 \%$ & $4.4 \%$ \\
\hline 1 & 6 & $\mathrm{BaO}$ & 0.0444 & 0.0479 & 0.0490 & -0.0046 & -0.0011 & $-9.4 \%$ & $-2.3 \%$ \\
\hline 1 & 6 & $\mathrm{CaO}$ & 1.9974 & 2.1154 & 2.0000 & -0.0026 & 0.1154 & $-0.1 \%$ & $5.8 \%$ \\
\hline 1 & 6 & $\mathrm{Ce}_{2} \mathrm{O}_{3}$ & 0.0958 & 0.0958 & 0.1030 & -0.0072 & -0.0072 & $-7.0 \%$ & $-7.0 \%$ \\
\hline 1 & 6 & $\mathrm{Cr}_{2} \mathrm{O}_{3}$ & 0.0870 & 0.0858 & 0.0900 & -0.0030 & -0.0042 & $-3.4 \%$ & $-4.6 \%$ \\
\hline 1 & 6 & $\mathrm{CuO}$ & 0.0338 & 0.0343 & 0.0330 & 0.0008 & 0.0013 & $2.4 \%$ & $4.0 \%$ \\
\hline 1 & 6 & $\mathrm{Fe}_{2} \mathrm{O}_{3}$ & 10.0293 & 10.3848 & 10.7720 & -0.7427 & -0.3872 & $-6.9 \%$ & $-3.6 \%$ \\
\hline 1 & 6 & $\mathrm{~K}_{2} \mathrm{O}$ & 0.0644 & 0.0688 & 0.0690 & -0.0046 & -0.0002 & $-6.6 \%$ & $-0.4 \%$ \\
\hline 1 & 6 & $\mathrm{La}_{2} \mathrm{O}_{3}$ & 0.0586 & 0.0586 & 0.0150 & 0.0436 & 0.0436 & $290.9 \%$ & $290.9 \%$ \\
\hline 1 & 6 & $\mathrm{Li}_{2} \mathrm{O}$ & 0.1076 & 0.1098 & 0.0000 & 0.1076 & 0.1098 & & \\
\hline 1 & 6 & $\mathrm{MgO}$ & 0.6289 & 0.6526 & 0.6260 & 0.0029 & 0.0266 & $0.5 \%$ & $4.2 \%$ \\
\hline 1 & 6 & $\mathrm{MnO}$ & 2.3435 & 2.3613 & 2.3060 & 0.0375 & 0.0553 & $1.6 \%$ & $2.4 \%$ \\
\hline 1 & 6 & $\mathrm{Na}_{2} \mathrm{O}$ & 13.1497 & 12.8883 & 12.8000 & 0.3497 & 0.0883 & $2.7 \%$ & $0.7 \%$ \\
\hline 1 & 6 & $\mathrm{NiO}$ & 0.9277 & 0.9891 & 1.0220 & -0.0943 & -0.0329 & $-9.2 \%$ & $-3.2 \%$ \\
\hline 1 & 6 & $\mathrm{PbO}$ & 0.0366 & 0.0366 & 0.0430 & -0.0064 & -0.0064 & $-14.8 \%$ & $-14.8 \%$ \\
\hline 1 & 6 & $\mathrm{SiO}_{2}$ & 39.0957 & 40.2813 & 38.4000 & 0.6957 & 1.8813 & $1.8 \%$ & $4.9 \%$ \\
\hline 1 & 6 & $\mathrm{SO}_{4}$ & 0.3445 & 0.3445 & 0.5130 & -0.1685 & -0.1685 & $-32.8 \%$ & $-32.8 \%$ \\
\hline 1 & 6 & $\mathrm{TiO}_{2}$ & 0.2256 & 0.2352 & 0.2270 & -0.0014 & 0.0082 & $-0.6 \%$ & $3.6 \%$ \\
\hline 1 & 6 & $\mathrm{ZnO}$ & 0.0289 & 0.0289 & 0.0330 & -0.0041 & -0.0041 & $-12.3 \%$ & $-12.3 \%$ \\
\hline
\end{tabular}


WSRC-STI-2007-00659

Revision 0

Appendix A

Table A4. Average Measured and Bias-Corrected Chemical Compositions Versus Targeted Compositions by Oxide by SB4 Variability Study Glass. (continued)

(100 -Batch 1)

\begin{tabular}{|c|c|c|c|c|c|c|c|c|c|}
\hline Set & Glass \# & Oxide & $\begin{array}{l}\text { Measured } \\
\text { (wt \%) }\end{array}$ & $\begin{array}{c}\text { Measured Bias- } \\
\text { Corrected (wt \%) }\end{array}$ & $\begin{array}{c}\text { Targeted } \\
\text { (wt \%) }\end{array}$ & $\begin{array}{c}\text { Diff of } \\
\text { Measured }\end{array}$ & $\begin{array}{c}\text { Diff of } \\
\text { Meas BC }\end{array}$ & $\begin{array}{l}\text { \% Diff of } \\
\text { Measured }\end{array}$ & $\begin{array}{l}\% \text { Diff of } \\
\text { Meas BC }\end{array}$ \\
\hline 1 & 6 & $\mathrm{ZrO}_{2}$ & 0.0888 & 0.0888 & 0.1010 & -0.0122 & -0.0122 & $-12.1 \%$ & $-12.1 \%$ \\
\hline 1 & 6 & Sum & 100.9123 & 103.0859 & 100.0020 & 0.9103 & 3.0839 & $0.9 \%$ & $3.1 \%$ \\
\hline 1 & 7 & $\mathrm{Al}_{2} \mathrm{O}_{3}$ & 13.6989 & 14.0405 & 13.6000 & 0.0989 & 0.4405 & $0.7 \%$ & $3.2 \%$ \\
\hline 1 & 7 & $\mathrm{~B}_{2} \mathrm{O}_{3}$ & 4.0893 & 4.1614 & 4.0000 & 0.0893 & 0.1614 & $2.2 \%$ & $4.0 \%$ \\
\hline 1 & 7 & $\mathrm{BaO}$ & 0.0452 & 0.0487 & 0.0490 & -0.0038 & -0.0003 & $-7.7 \%$ & $-0.7 \%$ \\
\hline 1 & 7 & $\mathrm{CaO}$ & 11.9981 & 12.8625 & 12.0000 & -0.0019 & 0.8625 & $0.0 \%$ & $7.2 \%$ \\
\hline 1 & 7 & $\mathrm{Ce}_{2} \mathrm{O}_{3}$ & 0.0963 & 0.0963 & 0.1030 & -0.0067 & -0.0067 & $-6.5 \%$ & $-6.5 \%$ \\
\hline 1 & 7 & $\mathrm{Cr}_{2} \mathrm{O}_{3}$ & 0.0946 & 0.0926 & 0.0900 & 0.0046 & 0.0026 & $5.2 \%$ & $2.9 \%$ \\
\hline 1 & 7 & $\mathrm{CuO}$ & 0.0335 & 0.0340 & 0.0330 & 0.0005 & 0.0010 & $1.5 \%$ & $3.2 \%$ \\
\hline 1 & 7 & $\mathrm{Fe}_{2} \mathrm{O}_{3}$ & 10.5512 & 10.9251 & 10.7720 & -0.2208 & 0.1531 & $-2.0 \%$ & $1.4 \%$ \\
\hline 1 & 7 & $\mathrm{~K}_{2} \mathrm{O}$ & 0.0669 & 0.0730 & 0.0690 & -0.0021 & 0.0040 & $-3.1 \%$ & $5.7 \%$ \\
\hline 1 & 7 & $\mathrm{La}_{2} \mathrm{O}_{3}$ & 0.0586 & 0.0586 & 0.0150 & 0.0436 & 0.0436 & $290.9 \%$ & $290.9 \%$ \\
\hline 1 & 7 & $\mathrm{Li}_{2} \mathrm{O}$ & 0.1076 & 0.1098 & 0.0000 & 0.1076 & 0.1098 & & \\
\hline 1 & 7 & $\mathrm{MgO}$ & 0.6252 & 0.6489 & 0.6260 & -0.0008 & 0.0229 & $-0.1 \%$ & $3.7 \%$ \\
\hline 1 & 7 & $\mathrm{MnO}$ & 2.3177 & 2.3236 & 2.3060 & 0.0117 & 0.0176 & $0.5 \%$ & $0.8 \%$ \\
\hline 1 & 7 & $\mathrm{Na}_{2} \mathrm{O}$ & 13.8507 & 13.4881 & 13.6000 & 0.2507 & -0.1119 & $1.8 \%$ & $-0.8 \%$ \\
\hline 1 & 7 & $\mathrm{NiO}$ & 0.9277 & 0.9909 & 1.0220 & -0.0943 & -0.0311 & $-9.2 \%$ & $-3.0 \%$ \\
\hline 1 & 7 & $\mathrm{PbO}$ & 0.0380 & 0.0380 & 0.0430 & -0.0050 & -0.0050 & $-11.7 \%$ & $-11.7 \%$ \\
\hline 1 & 7 & $\mathrm{SiO}_{2}$ & 40.8071 & 42.0451 & 40.8000 & 0.0071 & 1.2451 & $0.0 \%$ & $3.1 \%$ \\
\hline 1 & 7 & $\mathrm{SO}_{4}$ & 0.3970 & 0.3970 & 0.5130 & -0.1160 & -0.1160 & $-22.6 \%$ & $-22.6 \%$ \\
\hline 1 & 7 & $\mathrm{TiO}_{2}$ & 0.2231 & 0.2326 & 0.2270 & -0.0039 & 0.0056 & $-1.7 \%$ & $2.5 \%$ \\
\hline 1 & 7 & $\mathrm{ZnO}$ & 0.0283 & 0.0283 & 0.0330 & -0.0047 & -0.0047 & $-14.2 \%$ & $-14.2 \%$ \\
\hline 1 & 7 & $\mathrm{ZrO}_{2}$ & 0.0973 & 0.0973 & 0.1010 & -0.0037 & -0.0037 & $-3.7 \%$ & $-3.7 \%$ \\
\hline 1 & 7 & Sum & 100.1523 & 102.7921 & 100.0020 & 0.1503 & 2.7901 & $0.2 \%$ & $2.8 \%$ \\
\hline 1 & 8 & $\mathrm{Al}_{2} \mathrm{O}_{3}$ & 10.8410 & 11.1112 & 10.8000 & 0.0410 & 0.3112 & $0.4 \%$ & $2.9 \%$ \\
\hline 1 & 8 & $\mathrm{~B}_{2} \mathrm{O}_{3}$ & 18.2166 & 18.7643 & 18.0000 & 0.2166 & 0.7643 & $1.2 \%$ & $4.2 \%$ \\
\hline 1 & 8 & $\mathrm{BaO}$ & 0.0447 & 0.0481 & 0.0490 & -0.0043 & -0.0009 & $-8.9 \%$ & $-1.9 \%$ \\
\hline 1 & 8 & $\mathrm{CaO}$ & 12.0891 & 12.9600 & 12.0000 & 0.0891 & 0.9600 & $0.7 \%$ & $8.0 \%$ \\
\hline 1 & 8 & $\mathrm{Ce}_{2} \mathrm{O}_{3}$ & 0.0984 & 0.0984 & 0.1030 & -0.0046 & -0.0046 & $-4.5 \%$ & $-4.5 \%$ \\
\hline 1 & 8 & $\mathrm{Cr}_{2} \mathrm{O}_{3}$ & 0.0870 & 0.0851 & 0.0900 & -0.0030 & -0.0049 & $-3.4 \%$ & $-5.5 \%$ \\
\hline 1 & 8 & $\mathrm{CuO}$ & 0.0385 & 0.0391 & 0.0330 & 0.0055 & 0.0061 & $16.6 \%$ & $18.6 \%$ \\
\hline 1 & 8 & $\mathrm{Fe}_{2} \mathrm{O}_{3}$ & 10.6262 & 10.7806 & 10.7720 & -0.1458 & 0.0086 & $-1.4 \%$ & $0.1 \%$ \\
\hline 1 & 8 & $\mathrm{~K}_{2} \mathrm{O}$ & 0.0669 & 0.0730 & 0.0690 & -0.0021 & 0.0040 & $-3.1 \%$ & $5.7 \%$ \\
\hline 1 & 8 & $\mathrm{La}_{2} \mathrm{O}_{3}$ & 0.0586 & 0.0586 & 0.0150 & 0.0436 & 0.0436 & $290.9 \%$ & $290.9 \%$ \\
\hline 1 & 8 & $\mathrm{Li}_{2} \mathrm{O}$ & 0.1076 & 0.1093 & 0.0000 & 0.1076 & 0.1093 & & \\
\hline 1 & 8 & $\mathrm{MgO}$ & 0.6389 & 0.6631 & 0.6260 & 0.0129 & 0.0371 & $2.1 \%$ & $5.9 \%$ \\
\hline 1 & 8 & $\mathrm{MnO}$ & 2.3564 & 2.3625 & 2.3060 & 0.0504 & 0.0565 & $2.2 \%$ & $2.4 \%$ \\
\hline 1 & 8 & $\mathrm{Na}_{2} \mathrm{O}$ & 11.0603 & 10.7708 & 10.8000 & 0.2603 & -0.0292 & $2.4 \%$ & $-0.3 \%$ \\
\hline 1 & 8 & $\mathrm{NiO}$ & 0.9461 & 1.0107 & 1.0220 & -0.0759 & -0.0113 & $-7.4 \%$ & $-1.1 \%$ \\
\hline 1 & 8 & $\mathrm{PbO}$ & 0.0347 & 0.0347 & 0.0430 & -0.0083 & -0.0083 & $-19.2 \%$ & $-19.2 \%$ \\
\hline 1 & 8 & $\mathrm{SiO}_{2}$ & 33.2661 & 33.8302 & 32.4000 & 0.8661 & 1.4302 & $2.7 \%$ & $4.4 \%$ \\
\hline 1 & 8 & $\mathrm{SO}_{4}$ & 0.4868 & 0.4868 & 0.5130 & -0.0262 & -0.0262 & $-5.1 \%$ & $-5.1 \%$ \\
\hline 1 & 8 & $\mathrm{TiO}_{2}$ & 0.2268 & 0.2365 & 0.2270 & -0.0002 & 0.0095 & $-0.1 \%$ & $4.2 \%$ \\
\hline 1 & 8 & $\mathrm{ZnO}$ & 0.0308 & 0.0308 & 0.0330 & -0.0022 & -0.0022 & $-6.6 \%$ & $-6.6 \%$ \\
\hline 1 & 8 & $\mathrm{ZrO}_{2}$ & 0.0949 & 0.0949 & 0.1010 & -0.0061 & -0.0061 & $-6.0 \%$ & $-6.0 \%$ \\
\hline 1 & 8 & Sum & 101.4166 & 103.6485 & 100.0020 & 1.4146 & 3.6465 & $1.4 \%$ & $3.6 \%$ \\
\hline 1 & 9 & $\mathrm{Al}_{2} \mathrm{O}_{3}$ & 24.1856 & 24.7894 & 23.4000 & 0.7856 & 1.3894 & $3.4 \%$ & $5.9 \%$ \\
\hline 1 & 9 & $\mathrm{~B}_{2} \mathrm{O}_{3}$ & 4.1939 & 4.2680 & 4.0000 & 0.1939 & 0.2680 & $4.8 \%$ & $6.7 \%$ \\
\hline 1 & 9 & $\mathrm{BaO}$ & 0.0461 & 0.0496 & 0.0490 & -0.0029 & 0.0006 & $-6.0 \%$ & $1.2 \%$ \\
\hline 1 & 9 & $\mathrm{CaO}$ & 2.0148 & 2.1600 & 2.0000 & 0.0148 & 0.1600 & $0.7 \%$ & $8.0 \%$ \\
\hline 1 & 9 & $\mathrm{Ce}_{2} \mathrm{O}_{3}$ & 0.0990 & 0.0990 & 0.1030 & -0.0040 & -0.0040 & $-3.9 \%$ & $-3.9 \%$ \\
\hline 1 & 9 & $\mathrm{Cr}_{2} \mathrm{O}_{3}$ & 0.0892 & 0.0872 & 0.0900 & -0.0008 & -0.0028 & $-0.9 \%$ & $-3.1 \%$ \\
\hline 1 & 9 & $\mathrm{CuO}$ & 0.0325 & 0.0331 & 0.0330 & -0.0005 & 0.0001 & $-1.4 \%$ & $0.3 \%$ \\
\hline 1 & 9 & $\mathrm{Fe}_{2} \mathrm{O}_{3}$ & 11.2482 & 11.6479 & 10.7720 & 0.4762 & 0.8759 & $4.4 \%$ & $8.1 \%$ \\
\hline 1 & 9 & $\mathrm{~K}_{2} \mathrm{O}$ & 0.0657 & 0.0716 & 0.0690 & -0.0033 & 0.0026 & $-4.9 \%$ & $3.8 \%$ \\
\hline 1 & 9 & $\mathrm{La}_{2} \mathrm{O}_{3}$ & 0.0586 & 0.0586 & 0.0150 & 0.0436 & 0.0436 & $290.9 \%$ & $290.9 \%$ \\
\hline 1 & 9 & $\mathrm{Li}_{2} \mathrm{O}$ & 0.1076 & 0.1098 & 0.0000 & 0.1076 & 0.1098 & & \\
\hline 1 & 9 & $\mathrm{MgO}$ & 0.6563 & 0.6811 & 0.6260 & 0.0303 & 0.0551 & $4.8 \%$ & $8.8 \%$ \\
\hline 1 & 9 & $\mathrm{MnO}$ & 2.4081 & 2.4143 & 2.3060 & 0.1021 & 0.1083 & $4.4 \%$ & $4.7 \%$ \\
\hline 1 & 9 & $\mathrm{Na}_{2} \mathrm{O}$ & 8.1857 & 7.9715 & 7.8000 & 0.3857 & 0.1715 & $4.9 \%$ & $2.2 \%$ \\
\hline 1 & 9 & $\mathrm{NiO}$ & 0.9467 & 1.0113 & 1.0220 & -0.0753 & -0.0107 & $-7.4 \%$ & $-1.0 \%$ \\
\hline 1 & 9 & $\mathrm{PbO}$ & 0.0342 & 0.0342 & 0.0430 & -0.0088 & -0.0088 & $-20.5 \%$ & $-20.5 \%$ \\
\hline 1 & 9 & $\mathrm{SiO}_{2}$ & 45.4066 & 46.7839 & 46.8000 & -1.3934 & -0.0161 & $-3.0 \%$ & $0.0 \%$ \\
\hline 1 & 9 & $\mathrm{SO}_{4}$ & 0.1498 & 0.1498 & 0.5130 & -0.3632 & -0.3632 & $-70.8 \%$ & $-70.8 \%$ \\
\hline 1 & 9 & $\mathrm{TiO}_{2}$ & 0.2294 & 0.2391 & 0.2270 & 0.0023 & 0.0121 & $1.0 \%$ & $5.3 \%$ \\
\hline
\end{tabular}


WSRC-STI-2007-00659

Revision 0

Appendix A

Table A4. Average Measured and Bias-Corrected Chemical Compositions Versus Targeted Compositions by Oxide by SB4 Variability Study Glass. (continued)

(100 -Batch 1)

\begin{tabular}{|c|c|c|c|c|c|c|c|c|c|}
\hline Set & Glass \# & Oxide & $\begin{array}{l}\text { Measured } \\
\text { (wt \%) }\end{array}$ & $\begin{array}{c}\text { Measured Bias- } \\
\text { Corrected (wt \%) }\end{array}$ & $\begin{array}{c}\text { Targeted } \\
\text { (wt \%) }\end{array}$ & $\begin{array}{c}\text { Diff of } \\
\text { Measured }\end{array}$ & $\begin{array}{c}\text { Diff of } \\
\text { Meas BC }\end{array}$ & $\begin{array}{l}\text { \% Diff of } \\
\text { Measured }\end{array}$ & $\begin{array}{l}\% \text { Diff of } \\
\text { Meas BC }\end{array}$ \\
\hline 1 & 9 & $\mathrm{ZnO}$ & 0.0311 & 0.0311 & 0.0330 & -0.0019 & -0.0019 & $-5.7 \%$ & $-5.7 \%$ \\
\hline 1 & 9 & $\mathrm{ZrO}_{2}$ & 0.0966 & 0.0966 & 0.1010 & -0.0044 & -0.0044 & $-4.4 \%$ & $-4.4 \%$ \\
\hline 1 & 9 & Sum & 100.2857 & 102.7871 & 100.0020 & 0.2837 & 2.7851 & $0.3 \%$ & $2.8 \%$ \\
\hline 1 & 10 & $\mathrm{Al}_{2} \mathrm{O}_{3}$ & 19.6508 & 20.1415 & 19.2000 & 0.4508 & 0.9415 & $2.3 \%$ & $4.9 \%$ \\
\hline 1 & 10 & $\mathrm{~B}_{2} \mathrm{O}_{3}$ & 18.6030 & 18.9337 & 18.0000 & 0.6030 & 0.9337 & $3.3 \%$ & $5.2 \%$ \\
\hline 1 & 10 & $\mathrm{BaO}$ & 0.0449 & 0.0484 & 0.0490 & -0.0041 & -0.0006 & $-8.3 \%$ & $-1.3 \%$ \\
\hline 1 & 10 & $\mathrm{CaO}$ & 2.0079 & 2.1525 & 2.0000 & 0.0079 & 0.1525 & $0.4 \%$ & $7.6 \%$ \\
\hline 1 & 10 & $\mathrm{Ce}_{2} \mathrm{O}_{3}$ & 0.0972 & 0.0972 & 0.1030 & -0.0058 & -0.0058 & $-5.6 \%$ & $-5.6 \%$ \\
\hline 1 & 10 & $\mathrm{Cr}_{2} \mathrm{O}_{3}$ & 0.0610 & 0.0597 & 0.0900 & -0.0290 & -0.0303 & $-32.2 \%$ & $-33.7 \%$ \\
\hline 1 & 10 & $\mathrm{CuO}$ & 0.0319 & 0.0325 & 0.0330 & -0.0011 & -0.0005 & $-3.3 \%$ & $-1.7 \%$ \\
\hline 1 & 10 & $\mathrm{Fe}_{2} \mathrm{O}_{3}$ & 11.1481 & 11.5440 & 10.7720 & 0.3761 & 0.7720 & $3.5 \%$ & $7.2 \%$ \\
\hline 1 & 10 & $\mathrm{~K}_{2} \mathrm{O}$ & 0.0632 & 0.0690 & 0.0690 & -0.0058 & 0.0000 & $-8.3 \%$ & $0.0 \%$ \\
\hline 1 & 10 & $\mathrm{La}_{2} \mathrm{O}_{3}$ & 0.0586 & 0.0586 & 0.0150 & 0.0436 & 0.0436 & $290.9 \%$ & $290.9 \%$ \\
\hline 1 & 10 & $\mathrm{Li}_{2} \mathrm{O}$ & 0.1076 & 0.1098 & 0.0000 & 0.1076 & 0.1098 & & \\
\hline 1 & 10 & $\mathrm{MgO}$ & 0.6550 & 0.6798 & 0.6260 & 0.0290 & 0.0538 & $4.6 \%$ & $8.6 \%$ \\
\hline 1 & 10 & $\mathrm{MnO}$ & 2.4016 & 2.4078 & 2.3060 & 0.0956 & 0.1018 & $4.1 \%$ & $4.4 \%$ \\
\hline 1 & 10 & $\mathrm{Na}_{2} \mathrm{O}$ & 6.7906 & 6.6128 & 6.4000 & 0.3905 & 0.2128 & $6.1 \%$ & $3.3 \%$ \\
\hline 1 & 10 & $\mathrm{NiO}$ & 0.9617 & 1.0273 & 1.0220 & -0.0603 & 0.0053 & $-5.9 \%$ & $0.5 \%$ \\
\hline 1 & 10 & $\mathrm{PbO}$ & 0.0350 & 0.0350 & 0.0430 & -0.0080 & -0.0080 & $-18.6 \%$ & $-18.6 \%$ \\
\hline 1 & 10 & $\mathrm{SiO}_{2}$ & 38.2935 & 39.4553 & 38.4000 & -0.1065 & 1.0553 & $-0.3 \%$ & $2.7 \%$ \\
\hline 1 & 10 & $\mathrm{SO}_{4}$ & 0.1498 & 0.1498 & 0.5130 & -0.3632 & -0.3632 & $-70.8 \%$ & $-70.8 \%$ \\
\hline 1 & 10 & $\mathrm{TiO}_{2}$ & 0.2369 & 0.2469 & 0.2270 & 0.0099 & 0.0199 & $4.3 \%$ & $8.8 \%$ \\
\hline 1 & 10 & $\mathrm{ZnO}$ & 0.0299 & 0.0299 & 0.0330 & -0.0031 & -0.0031 & $-9.5 \%$ & $-9.5 \%$ \\
\hline 1 & 10 & $\mathrm{ZrO}_{2}$ & 0.0976 & 0.0976 & 0.1010 & -0.0034 & -0.0034 & $-3.4 \%$ & $-3.4 \%$ \\
\hline 1 & 10 & Sum & 101.5258 & 103.9890 & 100.0020 & 1.5238 & 3.9870 & $1.5 \%$ & $4.0 \%$ \\
\hline 2 & 11 & $\mathrm{Al}_{2} \mathrm{O}_{3}$ & 20.6428 & 21.1580 & 20.4000 & 0.2428 & 0.7580 & $1.2 \%$ & $3.7 \%$ \\
\hline 2 & 11 & $\mathrm{~B}_{2} \mathrm{O}_{3}$ & 4.0812 & 4.1738 & 4.0000 & 0.0812 & 0.1738 & $2.0 \%$ & $4.3 \%$ \\
\hline 2 & 11 & $\mathrm{BaO}$ & 0.0449 & 0.0484 & 0.0490 & -0.0041 & -0.0006 & $-8.3 \%$ & $-1.2 \%$ \\
\hline 2 & 11 & $\mathrm{CaO}$ & 12.0366 & 12.5097 & 12.0000 & 0.0366 & 0.5097 & $0.3 \%$ & $4.2 \%$ \\
\hline 2 & 11 & $\mathrm{Ce}_{2} \mathrm{O}_{3}$ & 0.0943 & 0.0943 & 0.1030 & -0.0087 & -0.0087 & $-8.5 \%$ & $-8.5 \%$ \\
\hline 2 & 11 & $\mathrm{Cr}_{2} \mathrm{O}_{3}$ & 0.0709 & 0.0693 & 0.0900 & -0.0191 & -0.0207 & $-21.2 \%$ & $-22.9 \%$ \\
\hline 2 & 11 & $\mathrm{CuO}$ & 0.0351 & 0.0359 & 0.0330 & 0.0021 & 0.0029 & $6.2 \%$ & $8.9 \%$ \\
\hline 2 & 11 & $\mathrm{Fe}_{2} \mathrm{O}_{3}$ & 10.0687 & 10.3500 & 10.7720 & -0.7033 & -0.4220 & $-6.5 \%$ & $-3.9 \%$ \\
\hline 2 & 11 & $\mathrm{~K}_{2} \mathrm{O}$ & 0.0666 & 0.0729 & 0.0690 & -0.0024 & 0.0039 & $-3.5 \%$ & $5.7 \%$ \\
\hline 2 & 11 & $\mathrm{La}_{2} \mathrm{O}_{3}$ & 0.0586 & 0.0586 & 0.0150 & 0.0436 & 0.0436 & $290.9 \%$ & $290.9 \%$ \\
\hline 2 & 11 & $\mathrm{Li}_{2} \mathrm{O}$ & 0.1076 & 0.1090 & 0.0000 & 0.1076 & 0.1090 & & \\
\hline 2 & 11 & $\mathrm{MgO}$ & 0.6152 & 0.6402 & 0.6260 & -0.0108 & 0.0142 & $-1.7 \%$ & $2.3 \%$ \\
\hline 2 & 11 & $\mathrm{MnO}$ & 2.3338 & 2.3311 & 2.3060 & 0.0278 & 0.0251 & $1.2 \%$ & $1.1 \%$ \\
\hline 2 & 11 & $\mathrm{Na}_{2} \mathrm{O}$ & 7.1174 & 7.0234 & 6.8000 & 0.3174 & 0.2234 & $4.7 \%$ & $3.3 \%$ \\
\hline 2 & 11 & $\mathrm{NiO}$ & 0.8987 & 0.9597 & 1.0220 & -0.1233 & -0.0623 & $-12.1 \%$ & $-6.1 \%$ \\
\hline 2 & 11 & $\mathrm{PbO}$ & 0.0355 & 0.0355 & 0.0430 & -0.0075 & -0.0075 & $-17.3 \%$ & $-17.3 \%$ \\
\hline 2 & 11 & $\mathrm{SiO}_{2}$ & 42.0372 & 42.8105 & 40.8000 & 1.2372 & 2.0105 & $3.0 \%$ & $4.9 \%$ \\
\hline 2 & 11 & $\mathrm{SO}_{4}$ & 0.3940 & 0.3940 & 0.5130 & -0.1190 & -0.1190 & $-23.2 \%$ & $-23.2 \%$ \\
\hline 2 & 11 & $\mathrm{TiO}_{2}$ & 0.0038 & 0.0039 & 0.2270 & -0.2232 & -0.2231 & $-98.3 \%$ & $-98.3 \%$ \\
\hline 2 & 11 & $\mathrm{ZnO}$ & 0.0283 & 0.0283 & 0.0330 & -0.0047 & -0.0047 & $-14.2 \%$ & $-14.2 \%$ \\
\hline 2 & 11 & $\mathrm{ZrO}_{2}$ & 0.1030 & 0.1030 & 0.1010 & 0.0020 & 0.0020 & $2.0 \%$ & $2.0 \%$ \\
\hline 2 & 11 & Sum & 100.8743 & 103.0097 & 100.0020 & 0.8723 & 3.0077 & $0.9 \%$ & $3.0 \%$ \\
\hline 2 & 12 & $\mathrm{Al}_{2} \mathrm{O}_{3}$ & 16.2591 & 16.6646 & 16.2000 & 0.0591 & 0.4646 & $0.4 \%$ & $2.9 \%$ \\
\hline 2 & 12 & $\mathrm{~B}_{2} \mathrm{O}_{3}$ & 18.4017 & 18.8189 & 18.0000 & 0.4017 & 0.8189 & $2.2 \%$ & $4.5 \%$ \\
\hline 2 & 12 & $\mathrm{BaO}$ & 0.0438 & 0.0472 & 0.0490 & -0.0052 & -0.0018 & $-10.6 \%$ & $-3.6 \%$ \\
\hline 2 & 12 & $\mathrm{CaO}$ & 12.0576 & 12.5309 & 12.0000 & 0.0576 & 0.5309 & $0.5 \%$ & $4.4 \%$ \\
\hline 2 & 12 & $\mathrm{Ce}_{2} \mathrm{O}_{3}$ & 0.0963 & 0.0963 & 0.1030 & -0.0067 & -0.0067 & $-6.5 \%$ & $-6.5 \%$ \\
\hline 2 & 12 & $\mathrm{Cr}_{2} \mathrm{O}_{3}$ & 0.0753 & 0.0736 & 0.0900 & -0.0147 & -0.0164 & $-16.4 \%$ & $-18.2 \%$ \\
\hline 2 & 12 & $\mathrm{CuO}$ & 0.0457 & 0.0469 & 0.0330 & 0.0127 & 0.0139 & $38.5 \%$ & $42.0 \%$ \\
\hline 2 & 12 & $\mathrm{Fe}_{2} \mathrm{O}_{3}$ & 10.7764 & 11.0790 & 10.7720 & 0.0044 & 0.3070 & $0.0 \%$ & $2.9 \%$ \\
\hline 2 & 12 & $\mathrm{~K}_{2} \mathrm{O}$ & 0.0626 & 0.0687 & 0.0690 & -0.0064 & -0.0003 & $-9.2 \%$ & $-0.5 \%$ \\
\hline 2 & 12 & $\mathrm{La}_{2} \mathrm{O}_{3}$ & 0.0586 & 0.0586 & 0.0150 & 0.0436 & 0.0436 & $290.9 \%$ & $290.9 \%$ \\
\hline 2 & 12 & $\mathrm{Li}_{2} \mathrm{O}$ & 0.1076 & 0.1090 & 0.0000 & 0.1076 & 0.1090 & & \\
\hline 2 & 12 & $\mathrm{MgO}$ & 0.6256 & 0.6510 & 0.6260 & -0.0004 & 0.0250 & $-0.1 \%$ & $4.0 \%$ \\
\hline 2 & 12 & $\mathrm{MnO}$ & 2.3435 & 2.3407 & 2.3060 & 0.0375 & 0.0347 & $1.6 \%$ & $1.5 \%$ \\
\hline 2 & 12 & $\mathrm{Na}_{2} \mathrm{O}$ & 5.5639 & 5.4902 & 5.4000 & 0.1639 & 0.0902 & $3.0 \%$ & $1.7 \%$ \\
\hline 2 & 12 & $\mathrm{NiO}$ & 0.9394 & 1.0032 & 1.0220 & -0.0826 & -0.0188 & $-8.1 \%$ & $-1.8 \%$ \\
\hline 2 & 12 & $\mathrm{PbO}$ & 0.0350 & 0.0350 & 0.0430 & -0.0080 & -0.0080 & $-18.6 \%$ & $-18.6 \%$ \\
\hline 2 & 12 & $\mathrm{SiO}_{2}$ & 32.9987 & 33.6103 & 32.4000 & 0.5987 & 1.2103 & $1.8 \%$ & $3.7 \%$ \\
\hline 2 & 12 & $\mathrm{SO}_{4}$ & 0.2891 & 0.2891 & 0.5130 & -0.2239 & -0.2239 & $-43.6 \%$ & $-43.6 \%$ \\
\hline
\end{tabular}


WSRC-STI-2007-00659

Revision 0

Appendix A

Table A4. Average Measured and Bias-Corrected Chemical Compositions Versus Targeted Compositions by Oxide by SB4 Variability Study Glass. (continued)

(100 -Batch 1)

\begin{tabular}{|c|c|c|c|c|c|c|c|c|c|}
\hline Set & Glass \# & Oxide & $\begin{array}{l}\text { Measured } \\
\text { (wt \%) }\end{array}$ & $\begin{array}{c}\text { Measured Bias- } \\
\text { Corrected (wt \%) }\end{array}$ & $\begin{array}{c}\text { Targeted } \\
\text { (wt \%) }\end{array}$ & $\begin{array}{c}\text { Diff of } \\
\text { Measured }\end{array}$ & $\begin{array}{c}\text { Diff of } \\
\text { Meas BC }\end{array}$ & $\begin{array}{l}\text { \% Diff of } \\
\text { Measured }\end{array}$ & $\begin{array}{l}\% \text { Diff of } \\
\text { Meas BC }\end{array}$ \\
\hline 2 & 12 & $\mathrm{TiO}_{2}$ & 0.2310 & 0.2403 & 0.2270 & 0.0040 & 0.0133 & $1.8 \%$ & $5.9 \%$ \\
\hline 2 & 12 & $\mathrm{ZnO}$ & 0.0349 & 0.0349 & 0.0330 & 0.0019 & 0.0019 & $5.6 \%$ & $5.6 \%$ \\
\hline 2 & 12 & $\mathrm{ZrO}_{2}$ & 0.0956 & 0.0956 & 0.1010 & -0.0054 & -0.0054 & $-5.4 \%$ & $-5.4 \%$ \\
\hline 2 & 12 & Sum & 101.1416 & 103.3841 & 100.0020 & 1.1396 & 3.3821 & $1.1 \%$ & $3.4 \%$ \\
\hline 2 & 13 & $\mathrm{Al}_{2} \mathrm{O}_{3}$ & 7.7233 & 7.9157 & 7.8000 & -0.0767 & 0.1157 & $-1.0 \%$ & $1.5 \%$ \\
\hline 2 & 13 & $\mathrm{~B}_{2} \mathrm{O}_{3}$ & 4.0329 & 4.1240 & 4.0000 & 0.0329 & 0.1240 & $0.8 \%$ & $3.1 \%$ \\
\hline 2 & 13 & $\mathrm{BaO}$ & 0.0447 & 0.0481 & 0.0490 & -0.0043 & -0.0009 & $-8.9 \%$ & $-1.8 \%$ \\
\hline 2 & 13 & $\mathrm{CaO}$ & 2.0288 & 2.1084 & 2.0000 & 0.0288 & 0.1084 & $1.4 \%$ & $5.4 \%$ \\
\hline 2 & 13 & $\mathrm{Ce}_{2} \mathrm{O}_{3}$ & 0.0949 & 0.0949 & 0.1030 & -0.0081 & -0.0081 & $-7.9 \%$ & $-7.9 \%$ \\
\hline 2 & 13 & $\mathrm{Cr}_{2} \mathrm{O}_{3}$ & 0.0917 & 0.0897 & 0.0900 & 0.0017 & -0.0003 & $1.9 \%$ & $-0.3 \%$ \\
\hline 2 & 13 & $\mathrm{CuO}$ & 0.0491 & 0.0504 & 0.0330 & 0.0161 & 0.0174 & $48.9 \%$ & $52.7 \%$ \\
\hline 2 & 13 & $\mathrm{Fe}_{2} \mathrm{O}_{3}$ & 10.7835 & 11.0850 & 10.7720 & 0.0115 & 0.3130 & $0.1 \%$ & $2.9 \%$ \\
\hline 2 & 13 & $\mathrm{~K}_{2} \mathrm{O}$ & 0.0647 & 0.0710 & 0.0690 & -0.0043 & 0.0020 & $-6.2 \%$ & $2.8 \%$ \\
\hline 2 & 13 & $\mathrm{La}_{2} \mathrm{O}_{3}$ & 0.0586 & 0.0586 & 0.0150 & 0.0436 & 0.0436 & $290.9 \%$ & $290.9 \%$ \\
\hline 2 & 13 & $\mathrm{Li}_{2} \mathrm{O}$ & 0.1076 & 0.1090 & 0.0000 & 0.1076 & 0.1090 & & \\
\hline 2 & 13 & $\mathrm{MgO}$ & 0.6405 & 0.6665 & 0.6260 & 0.0145 & 0.0405 & $2.3 \%$ & $6.5 \%$ \\
\hline 2 & 13 & $\mathrm{MnO}$ & 2.3532 & 2.3504 & 2.3060 & 0.0472 & 0.0444 & $2.0 \%$ & $1.9 \%$ \\
\hline 2 & 13 & $\mathrm{Na}_{2} \mathrm{O}$ & 30.2963 & 29.8950 & 31.2000 & -0.9037 & -1.3050 & $-2.9 \%$ & $-4.2 \%$ \\
\hline 2 & 13 & $\mathrm{NiO}$ & 0.9639 & 1.0293 & 1.0220 & -0.0581 & 0.0073 & $-5.7 \%$ & $0.7 \%$ \\
\hline 2 & 13 & $\mathrm{PbO}$ & 0.0347 & 0.0347 & 0.0430 & -0.0083 & -0.0083 & $-19.2 \%$ & $-19.2 \%$ \\
\hline 2 & 13 & $\mathrm{SiO}_{2}$ & 39.1492 & 39.8712 & 39.0000 & 0.1492 & 0.8712 & $0.4 \%$ & $2.2 \%$ \\
\hline 2 & 13 & $\mathrm{SO}_{4}$ & 0.5078 & 0.5078 & 0.5130 & -0.0052 & -0.0052 & $-1.0 \%$ & $-1.0 \%$ \\
\hline 2 & 13 & $\mathrm{TiO}_{2}$ & 0.2260 & 0.2351 & 0.2270 & -0.0010 & 0.0081 & $-0.4 \%$ & $3.6 \%$ \\
\hline 2 & 13 & $\mathrm{ZnO}$ & 0.0277 & 0.0277 & 0.0330 & -0.0053 & -0.0053 & $-16.1 \%$ & $-16.1 \%$ \\
\hline 2 & 13 & $\mathrm{ZrO}_{2}$ & 0.0956 & 0.0956 & 0.1010 & -0.0054 & -0.0054 & $-5.4 \%$ & $-5.4 \%$ \\
\hline 2 & 13 & Sum & 99.3750 & 100.4682 & 100.0020 & -0.6270 & 0.4662 & $-0.6 \%$ & $0.5 \%$ \\
\hline 2 & 14 & $\mathrm{Al}_{2} \mathrm{O}_{3}$ & 6.3865 & 6.5717 & 6.4000 & -0.0135 & 0.1717 & $-0.2 \%$ & $2.7 \%$ \\
\hline 2 & 14 & $\mathrm{~B}_{2} \mathrm{O}_{3}$ & 18.0234 & 18.1216 & 18.0000 & 0.0234 & 0.1216 & $0.1 \%$ & $0.7 \%$ \\
\hline 2 & 14 & $\mathrm{BaO}$ & 0.0408 & 0.0431 & 0.0490 & -0.0082 & -0.0059 & $-16.8 \%$ & $-12.0 \%$ \\
\hline 2 & 14 & $\mathrm{CaO}$ & 2.0568 & 2.1232 & 2.0000 & 0.0568 & 0.1232 & $2.8 \%$ & $6.2 \%$ \\
\hline 2 & 14 & $\mathrm{Ce}_{2} \mathrm{O}_{3}$ & 0.0943 & 0.0943 & 0.1030 & -0.0087 & -0.0087 & $-8.5 \%$ & $-8.5 \%$ \\
\hline 2 & 14 & $\mathrm{Cr}_{2} \mathrm{O}_{3}$ & 0.0939 & 0.0901 & 0.0900 & 0.0039 & 0.0001 & $4.3 \%$ & $0.1 \%$ \\
\hline 2 & 14 & $\mathrm{CuO}$ & 0.0388 & 0.0395 & 0.0330 & 0.0058 & 0.0065 & $17.6 \%$ & $19.8 \%$ \\
\hline 2 & 14 & $\mathrm{Fe}_{2} \mathrm{O}_{3}$ & 10.7692 & 10.8924 & 10.7720 & -0.0028 & 0.1204 & $0.0 \%$ & $1.1 \%$ \\
\hline 2 & 14 & $\mathrm{~K}_{2} \mathrm{O}$ & 0.0660 & 0.0719 & 0.0690 & -0.0030 & 0.0029 & $-4.4 \%$ & $4.2 \%$ \\
\hline 2 & 14 & $\mathrm{La}_{2} \mathrm{O}_{3}$ & 0.0586 & 0.0586 & 0.0150 & 0.0436 & 0.0436 & $290.9 \%$ & $290.9 \%$ \\
\hline 2 & 14 & $\mathrm{Li}_{2} \mathrm{O}$ & 0.1076 & 0.1091 & 0.0000 & 0.1076 & 0.1091 & & \\
\hline 2 & 14 & $\mathrm{MgO}$ & 0.6442 & 0.6636 & 0.6260 & 0.0182 & 0.0376 & $2.9 \%$ & $6.0 \%$ \\
\hline 2 & 14 & $\mathrm{MnO}$ & 2.3500 & 2.3531 & 2.3060 & 0.0440 & 0.0471 & $1.9 \%$ & $2.0 \%$ \\
\hline 2 & 14 & $\mathrm{Na}_{2} \mathrm{O}$ & 24.9043 & 24.8500 & 25.6000 & -0.6957 & -0.7500 & $-2.7 \%$ & $-2.9 \%$ \\
\hline 2 & 14 & $\mathrm{NiO}$ & 0.9671 & 1.0207 & 1.0220 & -0.0549 & -0.0013 & $-5.4 \%$ & $-0.1 \%$ \\
\hline 2 & 14 & $\mathrm{PbO}$ & 0.0396 & 0.0396 & 0.0430 & -0.0034 & -0.0034 & $-7.9 \%$ & $-7.9 \%$ \\
\hline 2 & 14 & $\mathrm{SiO}_{2}$ & 32.4639 & 32.9806 & 32.0000 & 0.4639 & 0.9806 & $1.4 \%$ & $3.1 \%$ \\
\hline 2 & 14 & $\mathrm{SO}_{4}$ & 0.5183 & 0.5183 & 0.5130 & 0.0053 & 0.0053 & $1.0 \%$ & $1.0 \%$ \\
\hline 2 & 14 & $\mathrm{TiO}_{2}$ & 0.2273 & 0.2352 & 0.2270 & 0.0003 & 0.0082 & $0.1 \%$ & $3.6 \%$ \\
\hline 2 & 14 & $\mathrm{ZnO}$ & 0.0308 & 0.0308 & 0.0330 & -0.0022 & -0.0022 & $-6.6 \%$ & $-6.6 \%$ \\
\hline 2 & 14 & $\mathrm{ZrO}_{2}$ & 0.0962 & 0.0962 & 0.1010 & -0.0048 & -0.0048 & $-4.7 \%$ & $-4.7 \%$ \\
\hline 2 & 14 & Sum & 99.9776 & 101.0037 & 100.0020 & -0.0244 & 1.0017 & $0.0 \%$ & $1.0 \%$ \\
\hline 2 & 15 & $\mathrm{Al}_{2} \mathrm{O}_{3}$ & 6.6463 & 6.8391 & 6.8000 & -0.1537 & 0.0391 & $-2.3 \%$ & $0.6 \%$ \\
\hline 2 & 15 & $\mathrm{~B}_{2} \mathrm{O}_{3}$ & 4.0571 & 4.0802 & 4.0000 & 0.0571 & 0.0802 & $1.4 \%$ & $2.0 \%$ \\
\hline 2 & 15 & $\mathrm{BaO}$ & 0.0441 & 0.0467 & 0.0490 & -0.0049 & -0.0023 & $-10.0 \%$ & $-4.8 \%$ \\
\hline 2 & 15 & $\mathrm{CaO}$ & 11.7638 & 12.1433 & 12.0000 & -0.2362 & 0.1433 & $-2.0 \%$ & $1.2 \%$ \\
\hline 2 & 15 & $\mathrm{Ce}_{2} \mathrm{O}_{3}$ & 0.0940 & 0.0940 & 0.1030 & -0.0090 & -0.0090 & $-8.7 \%$ & $-8.7 \%$ \\
\hline 2 & 15 & $\mathrm{Cr}_{2} \mathrm{O}_{3}$ & 0.0870 & 0.0834 & 0.0900 & -0.0030 & -0.0066 & $-3.4 \%$ & $-7.3 \%$ \\
\hline 2 & 15 & $\mathrm{CuO}$ & 0.0410 & 0.0418 & 0.0330 & 0.0080 & 0.0088 & $24.2 \%$ & $26.6 \%$ \\
\hline 2 & 15 & $\mathrm{Fe}_{2} \mathrm{O}_{3}$ & 10.4976 & 10.6273 & 10.7720 & -0.2744 & -0.1447 & $-2.5 \%$ & $-1.3 \%$ \\
\hline 2 & 15 & $\mathrm{~K}_{2} \mathrm{O}$ & 0.0657 & 0.0716 & 0.0690 & -0.0033 & 0.0026 & $-4.9 \%$ & $3.7 \%$ \\
\hline 2 & 15 & $\mathrm{La}_{2} \mathrm{O}_{3}$ & 0.0586 & 0.0586 & 0.0150 & 0.0436 & 0.0436 & $290.9 \%$ & $290.9 \%$ \\
\hline 2 & 15 & $\mathrm{Li}_{2} \mathrm{O}$ & 0.1076 & 0.1091 & 0.0000 & 0.1076 & 0.1091 & & \\
\hline 2 & 15 & $\mathrm{MgO}$ & 0.6248 & 0.6436 & 0.6260 & -0.0012 & 0.0176 & $-0.2 \%$ & $2.8 \%$ \\
\hline 2 & 15 & $\mathrm{MnO}$ & 2.2887 & 2.2917 & 2.3060 & -0.0173 & -0.0143 & $-0.8 \%$ & $-0.6 \%$ \\
\hline 2 & 15 & $\mathrm{Na}_{2} \mathrm{O}$ & 25.8479 & 25.7916 & 27.2000 & -1.3521 & -1.4084 & $-5.0 \%$ & $-5.2 \%$ \\
\hline 2 & 15 & $\mathrm{NiO}$ & 0.9477 & 1.0002 & 1.0220 & -0.0743 & -0.0218 & $-7.3 \%$ & $-2.1 \%$ \\
\hline 2 & 15 & $\mathrm{PbO}$ & 0.0342 & 0.0342 & 0.0430 & -0.0088 & -0.0088 & $-20.5 \%$ & $-20.5 \%$ \\
\hline 2 & 15 & $\mathrm{SiO}_{2}$ & 33.5335 & 34.0768 & 34.0000 & -0.4665 & 0.0768 & $-1.4 \%$ & $0.2 \%$ \\
\hline
\end{tabular}


WSRC-STI-2007-00659

Revision 0

Appendix A

Table A4. Average Measured and Bias-Corrected Chemical Compositions Versus Targeted Compositions by Oxide by SB4 Variability Study Glass. (continued)

(100 -Batch 1)

\begin{tabular}{|c|c|c|c|c|c|c|c|c|c|}
\hline Set & Glass \# & Oxide & $\begin{array}{l}\text { Measured } \\
\text { (wt \%) }\end{array}$ & $\begin{array}{c}\text { Measured Bias- } \\
\text { Corrected (wt \%) }\end{array}$ & $\begin{array}{c}\text { Targeted } \\
\text { (wt \%) }\end{array}$ & $\begin{array}{c}\text { Diff of } \\
\text { Measured }\end{array}$ & $\begin{array}{c}\text { Diff of } \\
\text { Meas BC }\end{array}$ & $\begin{array}{l}\text { \% Diff of } \\
\text { Measured }\end{array}$ & $\begin{array}{l}\% \text { Diff of } \\
\text { Meas BC }\end{array}$ \\
\hline 2 & 15 & $\mathrm{SO}_{4}$ & 0.5183 & 0.5183 & 0.5130 & 0.0053 & 0.0053 & $1.0 \%$ & $1.0 \%$ \\
\hline 2 & 15 & $\mathrm{TiO}_{2}$ & 0.2252 & 0.2330 & 0.2270 & -0.0018 & 0.0060 & $-0.8 \%$ & $2.7 \%$ \\
\hline 2 & 15 & $\mathrm{ZnO}$ & 0.0327 & 0.0327 & 0.0330 & -0.0003 & -0.0003 & $-1.0 \%$ & $-1.0 \%$ \\
\hline 2 & 15 & $\mathrm{ZrO}_{2}$ & 0.0956 & 0.0956 & 0.1010 & -0.0054 & -0.0054 & $-5.4 \%$ & $-5.4 \%$ \\
\hline 2 & 15 & Sum & 97.6112 & 98.9127 & 100.0020 & -2.3908 & -1.0893 & $-2.4 \%$ & $-1.1 \%$ \\
\hline 2 & 16 & $\mathrm{Al}_{2} \mathrm{O}_{3}$ & 5.0828 & 5.2301 & 5.4000 & -0.3172 & -0.1699 & $-5.9 \%$ & $-3.1 \%$ \\
\hline 2 & 16 & $\mathrm{~B}_{2} \mathrm{O}_{3}$ & 17.8624 & 17.9617 & 18.0000 & -0.1376 & -0.0383 & $-0.8 \%$ & $-0.2 \%$ \\
\hline 2 & 16 & $\mathrm{BaO}$ & 0.0452 & 0.0478 & 0.0490 & -0.0038 & -0.0012 & $-7.7 \%$ & $-2.4 \%$ \\
\hline 2 & 16 & $\mathrm{CaO}$ & 11.1866 & 11.5474 & 12.0000 & -0.8134 & -0.4526 & $-6.8 \%$ & $-3.8 \%$ \\
\hline 2 & 16 & $\mathrm{Ce}_{2} \mathrm{O}_{3}$ & 0.0931 & 0.0931 & 0.1030 & -0.0099 & -0.0099 & $-9.6 \%$ & $-9.6 \%$ \\
\hline 2 & 16 & $\mathrm{Cr}_{2} \mathrm{O}_{3}$ & 0.0892 & 0.0855 & 0.0900 & -0.0008 & -0.0045 & $-0.9 \%$ & $-5.0 \%$ \\
\hline 2 & 16 & $\mathrm{CuO}$ & 0.0504 & 0.0513 & 0.0330 & 0.0174 & 0.0183 & $52.7 \%$ & $55.6 \%$ \\
\hline 2 & 16 & $\mathrm{Fe}_{2} \mathrm{O}_{3}$ & 10.8800 & 11.0145 & 10.7720 & 0.1080 & 0.2425 & $1.0 \%$ & $2.3 \%$ \\
\hline 2 & 16 & $\mathrm{~K}_{2} \mathrm{O}$ & 0.0647 & 0.0706 & 0.0690 & -0.0043 & 0.0016 & $-6.2 \%$ & $2.3 \%$ \\
\hline 2 & 16 & $\mathrm{La}_{2} \mathrm{O}_{3}$ & 0.0586 & 0.0586 & 0.0150 & 0.0436 & 0.0436 & $290.9 \%$ & $290.9 \%$ \\
\hline 2 & 16 & $\mathrm{Li}_{2} \mathrm{O}$ & 0.1076 & 0.1091 & 0.0000 & 0.1076 & 0.1091 & & \\
\hline 2 & 16 & $\mathrm{MgO}$ & 0.6331 & 0.6521 & 0.6260 & 0.0071 & 0.0261 & $1.1 \%$ & $4.2 \%$ \\
\hline 2 & 16 & $\mathrm{MnO}$ & 2.1821 & 2.1850 & 2.3060 & -0.1239 & -0.1210 & $-5.4 \%$ & $-5.2 \%$ \\
\hline 2 & 16 & $\mathrm{Na}_{2} \mathrm{O}$ & 19.7482 & 19.7051 & 21.6000 & -1.8518 & -1.8949 & $-8.6 \%$ & $-8.8 \%$ \\
\hline 2 & 16 & $\mathrm{NiO}$ & 0.9649 & 1.0183 & 1.0220 & -0.0571 & -0.0037 & $-5.6 \%$ & $-0.4 \%$ \\
\hline 2 & 16 & $\mathrm{PbO}$ & 0.0364 & 0.0364 & 0.0430 & -0.0066 & -0.0066 & $-15.5 \%$ & $-15.5 \%$ \\
\hline 2 & 16 & $\mathrm{SiO}_{2}$ & 27.0087 & 27.4472 & 27.0000 & 0.0087 & 0.4472 & $0.0 \%$ & $1.7 \%$ \\
\hline 2 & 16 & $\mathrm{SO}_{4}$ & 0.5288 & 0.5288 & 0.5130 & 0.0158 & 0.0158 & $3.1 \%$ & $3.1 \%$ \\
\hline 2 & 16 & $\mathrm{TiO}_{2}$ & 0.2248 & 0.2326 & 0.2270 & -0.0022 & 0.0056 & $-1.0 \%$ & $2.5 \%$ \\
\hline 2 & 16 & $\mathrm{ZnO}$ & 0.0380 & 0.0380 & 0.0330 & 0.0050 & 0.0050 & $15.0 \%$ & $15.0 \%$ \\
\hline 2 & 16 & $\mathrm{ZrO}_{2}$ & 0.0939 & 0.0939 & 0.1010 & -0.0071 & -0.0071 & $-7.0 \%$ & $-7.0 \%$ \\
\hline 2 & 16 & Sum & 96.9793 & 98.2072 & 100.0020 & -3.0227 & -1.7948 & $-3.0 \%$ & $-1.8 \%$ \\
\hline 2 & 17 & $\mathrm{Al}_{2} \mathrm{O}_{3}$ & 15.7065 & 16.0978 & 15.6000 & 0.1065 & 0.4978 & $0.7 \%$ & $3.2 \%$ \\
\hline 2 & 17 & $\mathrm{~B}_{2} \mathrm{O}_{3}$ & 4.0490 & 4.1401 & 4.0000 & 0.0490 & 0.1401 & $1.2 \%$ & $3.5 \%$ \\
\hline 2 & 17 & $\mathrm{BaO}$ & 0.0458 & 0.0493 & 0.0490 & -0.0032 & 0.0003 & $-6.6 \%$ & $0.7 \%$ \\
\hline 2 & 17 & $\mathrm{CaO}$ & 1.9904 & 2.0684 & 2.0000 & -0.0096 & 0.0684 & $-0.5 \%$ & $3.4 \%$ \\
\hline 2 & 17 & $\mathrm{Ce}_{2} \mathrm{O}_{3}$ & 0.0952 & 0.0952 & 0.1030 & -0.0078 & -0.0078 & $-7.6 \%$ & $-7.6 \%$ \\
\hline 2 & 17 & $\mathrm{Cr}_{2} \mathrm{O}_{3}$ & 0.0954 & 0.0933 & 0.0900 & 0.0054 & 0.0033 & $6.0 \%$ & $3.7 \%$ \\
\hline 2 & 17 & $\mathrm{CuO}$ & 0.0394 & 0.0404 & 0.0330 & 0.0064 & 0.0074 & $19.5 \%$ & $22.5 \%$ \\
\hline 2 & 17 & $\mathrm{Fe}_{2} \mathrm{O}_{3}$ & 10.6334 & 10.9320 & 10.7720 & -0.1386 & 0.1600 & $-1.3 \%$ & $1.5 \%$ \\
\hline 2 & 17 & $\mathrm{~K}_{2} \mathrm{O}$ & 0.0672 & 0.0736 & 0.0690 & -0.0018 & 0.0046 & $-2.7 \%$ & $6.7 \%$ \\
\hline 2 & 17 & $\mathrm{La}_{2} \mathrm{O}_{3}$ & 0.0586 & 0.0586 & 0.0150 & 0.0436 & 0.0436 & $290.9 \%$ & $290.9 \%$ \\
\hline 2 & 17 & $\mathrm{Li}_{2} \mathrm{O}$ & 0.1076 & 0.1090 & 0.0000 & 0.1076 & 0.1090 & & \\
\hline 2 & 17 & $\mathrm{MgO}$ & 0.6368 & 0.6626 & 0.6260 & 0.0108 & 0.0366 & $1.7 \%$ & $5.9 \%$ \\
\hline 2 & 17 & $\mathrm{MnO}$ & 2.3629 & 2.3601 & 2.3060 & 0.0569 & 0.0541 & $2.5 \%$ & $2.3 \%$ \\
\hline 2 & 17 & $\mathrm{Na}_{2} \mathrm{O}$ & 23.1519 & 22.8453 & 23.4000 & -0.2481 & -0.5547 & $-1.1 \%$ & $-2.4 \%$ \\
\hline 2 & 17 & $\mathrm{NiO}$ & 0.9512 & 1.0158 & 1.0220 & -0.0708 & -0.0062 & $-6.9 \%$ & $-0.6 \%$ \\
\hline 2 & 17 & $\mathrm{PbO}$ & 0.0361 & 0.0361 & 0.0430 & -0.0069 & -0.0069 & $-16.1 \%$ & $-16.1 \%$ \\
\hline 2 & 17 & $\mathrm{SiO}_{2}$ & 39.8979 & 40.6353 & 39.0000 & 0.8979 & 1.6353 & $2.3 \%$ & $4.2 \%$ \\
\hline 2 & 17 & $\mathrm{SO}_{4}$ & 0.4763 & 0.4763 & 0.5130 & -0.0367 & -0.0367 & $-7.1 \%$ & $-7.1 \%$ \\
\hline 2 & 17 & $\mathrm{TiO}_{2}$ & 0.2239 & 0.2329 & 0.2270 & -0.0031 & 0.0059 & $-1.4 \%$ & $2.6 \%$ \\
\hline 2 & 17 & $\mathrm{ZnO}$ & 0.0345 & 0.0345 & 0.0330 & 0.0015 & 0.0015 & $4.7 \%$ & $4.7 \%$ \\
\hline 2 & 17 & $\mathrm{ZrO}_{2}$ & 0.0956 & 0.0956 & 0.1010 & -0.0054 & -0.0054 & $-5.4 \%$ & $-5.4 \%$ \\
\hline 2 & 17 & Sum & 100.7556 & 102.1523 & 100.0020 & 0.7536 & 2.1503 & $0.8 \%$ & $2.2 \%$ \\
\hline 2 & 18 & $\mathrm{Al}_{2} \mathrm{O}_{3}$ & 12.7541 & 13.1242 & 12.8000 & -0.0459 & 0.3242 & $-0.4 \%$ & $2.5 \%$ \\
\hline 2 & 18 & $\mathrm{~B}_{2} \mathrm{O}_{3}$ & 18.3534 & 18.4567 & 18.0000 & 0.3534 & 0.4567 & $2.0 \%$ & $2.5 \%$ \\
\hline 2 & 18 & $\mathrm{BaO}$ & 0.0480 & 0.0508 & 0.0490 & -0.0010 & 0.0018 & $-2.0 \%$ & $3.6 \%$ \\
\hline 2 & 18 & $\mathrm{CaO}$ & 2.0044 & 2.0690 & 2.0000 & 0.0044 & 0.0690 & $0.2 \%$ & $3.5 \%$ \\
\hline 2 & 18 & $\mathrm{Ce}_{2} \mathrm{O}_{3}$ & 0.0960 & 0.0960 & 0.1030 & -0.0070 & -0.0070 & $-6.8 \%$ & $-6.8 \%$ \\
\hline 2 & 18 & $\mathrm{Cr}_{2} \mathrm{O}_{3}$ & 0.0943 & 0.0904 & 0.0900 & 0.0043 & 0.0004 & $4.7 \%$ & $0.5 \%$ \\
\hline 2 & 18 & $\mathrm{CuO}$ & 0.0357 & 0.0364 & 0.0330 & 0.0027 & 0.0034 & $8.1 \%$ & $10.2 \%$ \\
\hline 2 & 18 & $\mathrm{Fe}_{2} \mathrm{O}_{3}$ & 11.0337 & 11.1717 & 10.7720 & 0.2617 & 0.3997 & $2.4 \%$ & $3.7 \%$ \\
\hline 2 & 18 & $\mathrm{~K}_{2} \mathrm{O}$ & 0.0632 & 0.0689 & 0.0690 & -0.0058 & -0.0001 & $-8.3 \%$ & $-0.1 \%$ \\
\hline 2 & 18 & $\mathrm{La}_{2} \mathrm{O}_{3}$ & 0.0586 & 0.0586 & 0.0150 & 0.0436 & 0.0436 & $290.9 \%$ & $290.9 \%$ \\
\hline 2 & 18 & $\mathrm{Li}_{2} \mathrm{O}$ & 0.1076 & 0.1091 & 0.0000 & 0.1076 & 0.1091 & & \\
\hline 2 & 18 & $\mathrm{MgO}$ & 0.6447 & 0.6641 & 0.6260 & 0.0187 & 0.0381 & $3.0 \%$ & $6.1 \%$ \\
\hline 2 & 18 & $\mathrm{MnO}$ & 2.3338 & 2.3369 & 2.3060 & 0.0278 & 0.0309 & $1.2 \%$ & $1.3 \%$ \\
\hline 2 & 18 & $\mathrm{Na}_{2} \mathrm{O}$ & 18.9057 & 18.8646 & 19.2000 & -0.2943 & -0.3354 & $-1.5 \%$ & $-1.7 \%$ \\
\hline 2 & 18 & $\mathrm{NiO}$ & 0.9789 & 1.0331 & 1.0220 & -0.0431 & 0.0111 & $-4.2 \%$ & $1.1 \%$ \\
\hline 2 & 18 & $\mathrm{PbO}$ & 0.0364 & 0.0364 & 0.0430 & -0.0066 & -0.0066 & $-15.5 \%$ & $-15.5 \%$ \\
\hline
\end{tabular}


WSRC-STI-2007-00659

Revision 0

Appendix A

Table A4. Average Measured and Bias-Corrected Chemical Compositions Versus Targeted Compositions by Oxide by SB4 Variability Study Glass. (continued)

(100 -Batch 1)

\begin{tabular}{|c|c|c|c|c|c|c|c|c|c|}
\hline Set & Glass \# & Oxide & $\begin{array}{l}\text { Measured } \\
\text { (wt \%) }\end{array}$ & $\begin{array}{c}\text { Measured Bias- } \\
\text { Corrected (wt \%) }\end{array}$ & $\begin{array}{c}\text { Targeted } \\
\text { (wt \%) }\end{array}$ & $\begin{array}{c}\text { Diff of } \\
\text { Measured }\end{array}$ & $\begin{array}{c}\text { Diff of } \\
\text { Meas BC }\end{array}$ & $\begin{array}{l}\% \text { Diff of } \\
\text { Measured }\end{array}$ & $\begin{array}{l}\text { \% Diff of } \\
\text { Meas BC }\end{array}$ \\
\hline 2 & 18 & $\mathrm{SiO}_{2}$ & 32.6778 & 33.2123 & 32.0000 & 0.6778 & 1.2123 & $2.1 \%$ & $3.8 \%$ \\
\hline 2 & 18 & $\mathrm{SO}_{4}$ & 0.3685 & 0.3685 & 0.5130 & -0.1445 & -0.1445 & $-28.2 \%$ & $-28.2 \%$ \\
\hline 2 & 18 & $\mathrm{TiO}_{2}$ & 0.2298 & 0.2378 & 0.2270 & 0.0028 & 0.0108 & $1.2 \%$ & $4.8 \%$ \\
\hline 2 & 18 & $\mathrm{ZnO}$ & 0.0317 & 0.0317 & 0.0330 & -0.0013 & -0.0013 & $-3.8 \%$ & $-3.8 \%$ \\
\hline 2 & 18 & $\mathrm{ZrO}_{2}$ & 0.1000 & 0.1000 & 0.1010 & -0.0010 & -0.0010 & $-1.0 \%$ & $-1.0 \%$ \\
\hline 2 & 18 & Sum & 100.9564 & 102.2171 & 100.0020 & 0.9544 & 2.2151 & $1.0 \%$ & $2.2 \%$ \\
\hline 2 & 19 & $\mathrm{Al}_{2} \mathrm{O}_{3}$ & 13.5005 & 13.8921 & 13.6000 & -0.0995 & 0.2921 & $-0.7 \%$ & $2.1 \%$ \\
\hline 2 & 19 & $\mathrm{~B}_{2} \mathrm{O}_{3}$ & 4.1376 & 4.1613 & 4.0000 & 0.1376 & 0.1613 & $3.4 \%$ & $4.0 \%$ \\
\hline 2 & 19 & $\mathrm{BaO}$ & 0.0452 & 0.0478 & 0.0490 & -0.0038 & -0.0012 & $-7.7 \%$ & $-2.4 \%$ \\
\hline 2 & 19 & $\mathrm{CaO}$ & 11.8827 & 12.2661 & 12.0000 & -0.1173 & 0.2661 & $-1.0 \%$ & $2.2 \%$ \\
\hline 2 & 19 & $\mathrm{Ce}_{2} \mathrm{O}_{3}$ & 0.0955 & 0.0955 & 0.1030 & -0.0075 & -0.0075 & $-7.3 \%$ & $-7.3 \%$ \\
\hline 2 & 19 & $\mathrm{Cr}_{2} \mathrm{O}_{3}$ & 0.0906 & 0.0869 & 0.0900 & 0.0006 & -0.0031 & $0.7 \%$ & $-3.4 \%$ \\
\hline 2 & 19 & $\mathrm{CuO}$ & 0.0469 & 0.0478 & 0.0330 & 0.0139 & 0.0148 & $42.3 \%$ & $44.9 \%$ \\
\hline 2 & 19 & $\mathrm{Fe}_{2} \mathrm{O}_{3}$ & 10.2831 & 10.4056 & 10.7720 & -0.4889 & -0.3664 & $-4.5 \%$ & $-3.4 \%$ \\
\hline 2 & 19 & $\mathrm{~K}_{2} \mathrm{O}$ & 0.0678 & 0.0739 & 0.0690 & -0.0012 & 0.0049 & $-1.8 \%$ & $7.1 \%$ \\
\hline 2 & 19 & $\mathrm{La}_{2} \mathrm{O}_{3}$ & 0.0586 & 0.0586 & 0.0150 & 0.0436 & 0.0436 & $290.9 \%$ & $290.9 \%$ \\
\hline 2 & 19 & $\mathrm{Li}_{2} \mathrm{O}$ & 0.1076 & 0.1091 & 0.0000 & 0.1076 & 0.1091 & & \\
\hline 2 & 19 & $\mathrm{MgO}$ & 0.6355 & 0.6547 & 0.6260 & 0.0095 & 0.0287 & $1.5 \%$ & $4.6 \%$ \\
\hline 2 & 19 & $\mathrm{MnO}$ & 2.3177 & 2.3208 & 2.3060 & 0.0117 & 0.0148 & $0.5 \%$ & $0.6 \%$ \\
\hline 2 & 19 & $\mathrm{Na}_{2} \mathrm{O}$ & 19.8156 & 19.7724 & 20.4000 & -0.5844 & -0.6276 & $-2.9 \%$ & $-3.1 \%$ \\
\hline 2 & 19 & $\mathrm{NiO}$ & 0.9480 & 1.0006 & 1.0220 & -0.0740 & -0.0214 & $-7.2 \%$ & $-2.1 \%$ \\
\hline 2 & 19 & $\mathrm{PbO}$ & 0.0347 & 0.0347 & 0.0430 & -0.0083 & -0.0083 & $-19.2 \%$ & $-19.2 \%$ \\
\hline 2 & 19 & $\mathrm{SiO}_{2}$ & 34.7636 & 35.3292 & 34.0000 & 0.7636 & 1.3292 & $2.2 \%$ & $3.9 \%$ \\
\hline 2 & 19 & $\mathrm{SO}_{4}$ & 0.5011 & 0.5011 & 0.5130 & -0.0119 & -0.0119 & $-2.3 \%$ & $-2.3 \%$ \\
\hline 2 & 19 & $\mathrm{TiO}_{2}$ & 0.2289 & 0.2369 & 0.2270 & 0.0019 & 0.0099 & $0.9 \%$ & $4.4 \%$ \\
\hline 2 & 19 & $\mathrm{ZnO}$ & 0.0314 & 0.0314 & 0.0330 & -0.0016 & -0.0016 & $-4.8 \%$ & $-4.8 \%$ \\
\hline 2 & 19 & $\mathrm{ZrO}_{2}$ & 0.0962 & 0.0962 & 0.1010 & -0.0048 & -0.0048 & $-4.7 \%$ & $-4.7 \%$ \\
\hline 2 & 19 & Sum & 99.6891 & 101.2227 & 100.0020 & -0.3129 & 1.2207 & $-0.3 \%$ & $1.2 \%$ \\
\hline 2 & 20 & $\mathrm{Al}_{2} \mathrm{O}_{3}$ & 10.8882 & 11.1599 & 10.8000 & 0.0882 & 0.3599 & $0.8 \%$ & $3.3 \%$ \\
\hline 2 & 20 & $\mathrm{~B}_{2} \mathrm{O}_{3}$ & 18.1602 & 18.5761 & 18.0000 & 0.1602 & 0.5761 & $0.9 \%$ & $3.2 \%$ \\
\hline 2 & 20 & $\mathrm{BaO}$ & 0.0444 & 0.0478 & 0.0490 & -0.0046 & -0.0012 & $-9.4 \%$ & $-2.4 \%$ \\
\hline 2 & 20 & $\mathrm{CaO}$ & 12.0821 & 12.5568 & 12.0000 & 0.0821 & 0.5568 & $0.7 \%$ & $4.6 \%$ \\
\hline 2 & 20 & $\mathrm{Ce}_{2} \mathrm{O}_{3}$ & 0.0937 & 0.0937 & 0.1030 & -0.0093 & -0.0093 & $-9.0 \%$ & $-9.0 \%$ \\
\hline 2 & 20 & $\mathrm{Cr}_{2} \mathrm{O}_{3}$ & 0.0903 & 0.0883 & 0.0900 & 0.0003 & -0.0017 & $0.3 \%$ & $-1.9 \%$ \\
\hline 2 & 20 & $\mathrm{CuO}$ & 0.0407 & 0.0417 & 0.0330 & 0.0077 & 0.0087 & $23.3 \%$ & $26.4 \%$ \\
\hline 2 & 20 & $\mathrm{Fe}_{2} \mathrm{O}_{3}$ & 10.7335 & 11.0327 & 10.7720 & -0.0385 & 0.2607 & $-0.4 \%$ & $2.4 \%$ \\
\hline 2 & 20 & $\mathrm{~K}_{2} \mathrm{O}$ & 0.0605 & 0.0663 & 0.0690 & -0.0085 & -0.0027 & $-12.3 \%$ & $-3.8 \%$ \\
\hline 2 & 20 & $\mathrm{La}_{2} \mathrm{O}_{3}$ & 0.0586 & 0.0586 & 0.0150 & 0.0436 & 0.0436 & $290.9 \%$ & $290.9 \%$ \\
\hline 2 & 20 & $\mathrm{Li}_{2} \mathrm{O}$ & 0.1076 & 0.1090 & 0.0000 & 0.1076 & 0.1090 & & \\
\hline 2 & 20 & $\mathrm{MgO}$ & 0.6285 & 0.6540 & 0.6260 & 0.0025 & 0.0280 & $0.4 \%$ & $4.5 \%$ \\
\hline 2 & 20 & $\mathrm{MnO}$ & 2.3629 & 2.3601 & 2.3060 & 0.0569 & 0.0541 & $2.5 \%$ & $2.3 \%$ \\
\hline 2 & 20 & $\mathrm{Na}_{2} \mathrm{O}$ & 16.3108 & 16.0952 & 16.2000 & 0.1108 & -0.1048 & $0.7 \%$ & $-0.6 \%$ \\
\hline 2 & 20 & $\mathrm{NiO}$ & 0.9509 & 1.0154 & 1.0220 & -0.0711 & -0.0066 & $-7.0 \%$ & $-0.6 \%$ \\
\hline 2 & 20 & $\mathrm{PbO}$ & 0.0347 & 0.0347 & 0.0430 & -0.0083 & -0.0083 & $-19.2 \%$ & $-19.2 \%$ \\
\hline 2 & 20 & $\mathrm{SiO}_{2}$ & 27.4900 & 27.9959 & 27.0000 & 0.4900 & 0.9959 & $1.8 \%$ & $3.7 \%$ \\
\hline 2 & 20 & $\mathrm{SO}_{4}$ & 0.2966 & 0.2966 & 0.5130 & -0.2164 & -0.2164 & $-42.2 \%$ & $-42.2 \%$ \\
\hline 2 & 20 & $\mathrm{TiO}_{2}$ & 0.2214 & 0.2303 & 0.2270 & -0.0056 & 0.0033 & $-2.5 \%$ & $1.5 \%$ \\
\hline 2 & 20 & $\mathrm{ZnO}$ & 0.0305 & 0.0305 & 0.0330 & -0.0025 & -0.0025 & $-7.6 \%$ & $-7.6 \%$ \\
\hline 2 & 20 & $\mathrm{ZrO}_{2}$ & 0.0905 & 0.0905 & 0.1010 & -0.0105 & -0.0105 & $-10.4 \%$ & $-10.4 \%$ \\
\hline 2 & 20 & Sum & 100.7767 & 102.6343 & 100.0020 & 0.7747 & 2.6323 & $0.8 \%$ & $2.6 \%$ \\
\hline 3 & 21 & $\mathrm{Al}_{2} \mathrm{O}_{3}$ & 23.6188 & 24.1293 & 23.4000 & 0.2188 & 0.7293 & $0.9 \%$ & $3.1 \%$ \\
\hline 3 & 21 & $\mathrm{~B}_{2} \mathrm{O}_{3}$ & 3.8639 & 4.0488 & 4.0000 & -0.1361 & 0.0488 & $-3.4 \%$ & $1.2 \%$ \\
\hline 3 & 21 & $\mathrm{BaO}$ & 0.0480 & 0.0519 & 0.0490 & -0.0010 & 0.0029 & $-2.0 \%$ & $6.0 \%$ \\
\hline 3 & 21 & $\mathrm{CaO}$ & 2.0148 & 2.0509 & 2.0000 & 0.0148 & 0.0509 & $0.7 \%$ & $2.5 \%$ \\
\hline 3 & 21 & $\mathrm{Ce}_{2} \mathrm{O}_{3}$ & 0.0946 & 0.0946 & 0.1030 & -0.0084 & -0.0084 & $-8.2 \%$ & $-8.2 \%$ \\
\hline 3 & 21 & $\mathrm{Cr}_{2} \mathrm{O}_{3}$ & 0.0709 & 0.0695 & 0.0900 & -0.0191 & -0.0205 & $-21.2 \%$ & $-22.8 \%$ \\
\hline 3 & 21 & $\mathrm{CuO}$ & 0.0325 & 0.0337 & 0.0330 & -0.0005 & 0.0007 & $-1.4 \%$ & $2.0 \%$ \\
\hline 3 & 21 & $\mathrm{Fe}_{2} \mathrm{O}_{3}$ & 10.1223 & 10.5305 & 10.7720 & -0.6497 & -0.2415 & $-6.0 \%$ & $-2.2 \%$ \\
\hline 3 & 21 & $\mathrm{~K}_{2} \mathrm{O}$ & 0.0638 & 0.0688 & 0.0690 & -0.0052 & -0.0002 & $-7.5 \%$ & $-0.2 \%$ \\
\hline 3 & 21 & $\mathrm{La}_{2} \mathrm{O}_{3}$ & 0.0586 & 0.0586 & 0.0150 & 0.0436 & 0.0436 & $290.9 \%$ & $290.9 \%$ \\
\hline 3 & 21 & $\mathrm{Li}_{2} \mathrm{O}$ & 0.1076 & 0.1104 & 0.0000 & 0.1076 & 0.1104 & & \\
\hline 3 & 21 & $\mathrm{MgO}$ & 0.6156 & 0.6461 & 0.6260 & -0.0104 & 0.0201 & $-1.7 \%$ & $3.2 \%$ \\
\hline 3 & 21 & $\mathrm{MnO}$ & 2.2822 & 2.2738 & 2.3060 & -0.0238 & -0.0322 & $-1.0 \%$ & $-1.4 \%$ \\
\hline 3 & 21 & $\mathrm{Na}_{2} \mathrm{O}$ & 15.6604 & 15.4196 & 15.6000 & 0.0604 & -0.1804 & $0.4 \%$ & $-1.2 \%$ \\
\hline 3 & 21 & $\mathrm{NiO}$ & 0.8624 & 0.9308 & 1.0220 & -0.1596 & -0.0912 & $-15.6 \%$ & $-8.9 \%$ \\
\hline
\end{tabular}


WSRC-STI-2007-00659

Revision 0

Appendix A

Table A4. Average Measured and Bias-Corrected Chemical Compositions Versus Targeted Compositions by Oxide by SB4 Variability Study Glass. (continued)

(100 -Batch 1)

\begin{tabular}{|c|c|c|c|c|c|c|c|c|c|}
\hline Set & Glass \# & Oxide & $\begin{array}{l}\text { Measured } \\
\text { (wt \%) }\end{array}$ & $\begin{array}{c}\text { Measured Bias- } \\
\text { Corrected (wt \%) }\end{array}$ & $\begin{array}{c}\text { Targeted } \\
\text { (wt \%) }\end{array}$ & $\begin{array}{c}\text { Diff of } \\
\text { Measured }\end{array}$ & $\begin{array}{c}\text { Diff of } \\
\text { Meas BC }\end{array}$ & $\begin{array}{l}\text { \% Diff of } \\
\text { Measured }\end{array}$ & $\begin{array}{l}\% \text { Diff of } \\
\text { Meas BC }\end{array}$ \\
\hline 3 & 21 & $\mathrm{PbO}$ & 0.0331 & 0.0331 & 0.0430 & -0.0099 & -0.0099 & $-23.0 \%$ & $-23.0 \%$ \\
\hline 3 & 21 & $\mathrm{SiO}_{2}$ & 39.3631 & 40.4379 & 39.0000 & 0.3631 & 1.4379 & $0.9 \%$ & $3.7 \%$ \\
\hline 3 & 21 & $\mathrm{SO}_{4}$ & 0.1498 & 0.1498 & 0.5130 & -0.3632 & -0.3632 & $-70.8 \%$ & $-70.8 \%$ \\
\hline 3 & 21 & $\mathrm{TiO}_{2}$ & 0.2164 & 0.2282 & 0.2270 & -0.0106 & 0.0012 & $-4.7 \%$ & $0.5 \%$ \\
\hline 3 & 21 & $\mathrm{ZnO}$ & 0.0274 & 0.0274 & 0.0330 & -0.0056 & -0.0056 & $-17.0 \%$ & $-17.0 \%$ \\
\hline 3 & 21 & $\mathrm{ZrO}_{2}$ & 0.0986 & 0.0986 & 0.1010 & -0.0024 & -0.0024 & $-2.4 \%$ & $-2.4 \%$ \\
\hline 3 & 21 & Sum & 99.4050 & 101.4924 & 100.0020 & -0.5970 & 1.4904 & $-0.6 \%$ & $1.5 \%$ \\
\hline 3 & 22 & $\mathrm{Al}_{2} \mathrm{O}_{3}$ & 19.3674 & 19.7856 & 19.2000 & 0.1674 & 0.5856 & $0.9 \%$ & $3.0 \%$ \\
\hline 3 & 22 & $\mathrm{~B}_{2} \mathrm{O}_{3}$ & 18.3937 & 19.2738 & 18.0000 & 0.3937 & 1.2738 & $2.2 \%$ & $7.1 \%$ \\
\hline 3 & 22 & $\mathrm{BaO}$ & 0.0505 & 0.0547 & 0.0490 & 0.0015 & 0.0057 & $3.1 \%$ & $11.6 \%$ \\
\hline 3 & 22 & $\mathrm{CaO}$ & 2.0114 & 2.0475 & 2.0000 & 0.0114 & 0.0475 & $0.6 \%$ & $2.4 \%$ \\
\hline 3 & 22 & $\mathrm{Ce}_{2} \mathrm{O}_{3}$ & 0.0925 & 0.0925 & 0.1030 & -0.0105 & -0.0105 & $-10.2 \%$ & $-10.2 \%$ \\
\hline 3 & 22 & $\mathrm{Cr}_{2} \mathrm{O}_{3}$ & 0.0672 & 0.0659 & 0.0900 & -0.0228 & -0.0241 & $-25.3 \%$ & $-26.8 \%$ \\
\hline 3 & 22 & $\mathrm{CuO}$ & 0.0338 & 0.0350 & 0.0330 & 0.0008 & 0.0020 & $2.4 \%$ & $5.9 \%$ \\
\hline 3 & 22 & $\mathrm{Fe}_{2} \mathrm{O}_{3}$ & 9.8399 & 10.2377 & 10.7720 & -0.9321 & -0.5343 & $-8.7 \%$ & $-5.0 \%$ \\
\hline 3 & 22 & $\mathrm{~K}_{2} \mathrm{O}$ & 0.0599 & 0.0646 & 0.0690 & -0.0091 & -0.0044 & $-13.1 \%$ & $-6.3 \%$ \\
\hline 3 & 22 & $\mathrm{La}_{2} \mathrm{O}_{3}$ & 0.0586 & 0.0586 & 0.0150 & 0.0436 & 0.0436 & $290.9 \%$ & $290.9 \%$ \\
\hline 3 & 22 & $\mathrm{Li}_{2} \mathrm{O}$ & 0.1076 & 0.1104 & 0.0000 & 0.1076 & 0.1104 & & \\
\hline 3 & 22 & $\mathrm{MgO}$ & 0.6181 & 0.6487 & 0.6260 & -0.0079 & 0.0227 & $-1.3 \%$ & $3.6 \%$ \\
\hline 3 & 22 & $\mathrm{MnO}$ & 2.2854 & 2.2771 & 2.3060 & -0.0206 & -0.0289 & $-0.9 \%$ & $-1.3 \%$ \\
\hline 3 & 22 & $\mathrm{Na}_{2} \mathrm{O}$ & 13.0486 & 12.8480 & 12.8000 & 0.2486 & 0.0480 & $1.9 \%$ & $0.4 \%$ \\
\hline 3 & 22 & $\mathrm{NiO}$ & 0.7915 & 0.8542 & 1.0220 & -0.2305 & -0.1678 & $-22.6 \%$ & $-16.4 \%$ \\
\hline 3 & 22 & $\mathrm{PbO}$ & 0.0342 & 0.0342 & 0.0430 & -0.0088 & -0.0088 & $-20.5 \%$ & $-20.5 \%$ \\
\hline 3 & 22 & $\mathrm{SiO}_{2}$ & 33.1592 & 34.0674 & 32.0000 & 1.1592 & 2.0674 & $3.6 \%$ & $6.5 \%$ \\
\hline 3 & 22 & $\mathrm{SO}_{4}$ & 0.1498 & 0.1498 & 0.5130 & -0.3632 & -0.3632 & $-70.8 \%$ & $-70.8 \%$ \\
\hline 3 & 22 & $\mathrm{TiO}_{2}$ & 0.2281 & 0.2405 & 0.2270 & 0.0011 & 0.0135 & $0.5 \%$ & $5.9 \%$ \\
\hline 3 & 22 & $\mathrm{ZnO}$ & 0.0271 & 0.0271 & 0.0330 & -0.0059 & -0.0059 & $-18.0 \%$ & $-18.0 \%$ \\
\hline 3 & 22 & $\mathrm{ZrO}_{2}$ & 0.0959 & 0.0959 & 0.1010 & -0.0051 & -0.0051 & $-5.0 \%$ & $-5.0 \%$ \\
\hline 3 & 22 & Sum & 100.5205 & 103.0691 & 100.0020 & 0.5185 & 3.0671 & $0.5 \%$ & $3.1 \%$ \\
\hline 3 & 23 & $\mathrm{Al}_{2} \mathrm{O}_{3}$ & 20.4538 & 20.9915 & 20.4000 & 0.0538 & 0.5915 & $0.3 \%$ & $2.9 \%$ \\
\hline 3 & 23 & $\mathrm{~B}_{2} \mathrm{O}_{3}$ & 3.9041 & 4.0245 & 4.0000 & -0.0959 & 0.0245 & $-2.4 \%$ & $0.6 \%$ \\
\hline 3 & 23 & $\mathrm{BaO}$ & 0.0433 & 0.0460 & 0.0490 & -0.0057 & -0.0030 & $-11.7 \%$ & $-6.1 \%$ \\
\hline 3 & 23 & $\mathrm{CaO}$ & 11.8652 & 12.1811 & 12.0000 & -0.1348 & 0.1811 & $-1.1 \%$ & $1.5 \%$ \\
\hline 3 & 23 & $\mathrm{Ce}_{2} \mathrm{O}_{3}$ & 0.0937 & 0.0937 & 0.1030 & -0.0093 & -0.0093 & $-9.0 \%$ & $-9.0 \%$ \\
\hline 3 & 23 & $\mathrm{Cr}_{2} \mathrm{O}_{3}$ & 0.0727 & 0.0702 & 0.0900 & -0.0173 & -0.0198 & $-19.2 \%$ & $-22.0 \%$ \\
\hline 3 & 23 & $\mathrm{CuO}$ & 0.0338 & 0.0347 & 0.0330 & 0.0008 & 0.0017 & $2.4 \%$ & $5.0 \%$ \\
\hline 3 & 23 & $\mathrm{Fe}_{2} \mathrm{O}_{3}$ & 10.2867 & 10.6176 & 10.7720 & -0.4853 & -0.1544 & $-4.5 \%$ & $-1.4 \%$ \\
\hline 3 & 23 & $\mathrm{~K}_{2} \mathrm{O}$ & 0.0644 & 0.0709 & 0.0690 & -0.0046 & 0.0019 & $-6.6 \%$ & $2.7 \%$ \\
\hline 3 & 23 & $\mathrm{La}_{2} \mathrm{O}_{3}$ & 0.0586 & 0.0586 & 0.0150 & 0.0436 & 0.0436 & $290.9 \%$ & $290.9 \%$ \\
\hline 3 & 23 & $\mathrm{Li}_{2} \mathrm{O}$ & 0.1076 & 0.1084 & 0.0000 & 0.1076 & 0.1084 & & \\
\hline 3 & 23 & $\mathrm{MgO}$ & 0.6057 & 0.6308 & 0.6260 & -0.0203 & 0.0048 & $-3.2 \%$ & $0.8 \%$ \\
\hline 3 & 23 & $\mathrm{MnO}$ & 2.3145 & 2.3292 & 2.3060 & 0.0085 & 0.0232 & $0.4 \%$ & $1.0 \%$ \\
\hline 3 & 23 & $\mathrm{Na}_{2} \mathrm{O}$ & 13.7226 & 13.4714 & 13.6000 & 0.1226 & -0.1286 & $0.9 \%$ & $-0.9 \%$ \\
\hline 3 & 23 & $\mathrm{NiO}$ & 0.9041 & 0.9652 & 1.0220 & -0.1179 & -0.0568 & $-11.5 \%$ & $-5.6 \%$ \\
\hline 3 & 23 & $\mathrm{PbO}$ & 0.0401 & 0.0401 & 0.0430 & -0.0029 & -0.0029 & $-6.7 \%$ & $-6.7 \%$ \\
\hline 3 & 23 & $\mathrm{SiO}_{2}$ & 34.3358 & 34.7943 & 34.0000 & 0.3358 & 0.7943 & $1.0 \%$ & $2.3 \%$ \\
\hline 3 & 23 & $\mathrm{SO}_{4}$ & 0.3595 & 0.3595 & 0.5130 & -0.1535 & -0.1535 & $-29.9 \%$ & $-29.9 \%$ \\
\hline 3 & 23 & $\mathrm{TiO}_{2}$ & 0.2156 & 0.2252 & 0.2270 & -0.0114 & -0.0018 & $-5.0 \%$ & $-0.8 \%$ \\
\hline 3 & 23 & $\mathrm{ZnO}$ & 0.0286 & 0.0286 & 0.0330 & -0.0044 & -0.0044 & $-13.2 \%$ & $-13.2 \%$ \\
\hline 3 & 23 & $\mathrm{ZrO}_{2}$ & 0.0939 & 0.0939 & 0.1010 & -0.0071 & -0.0071 & $-7.0 \%$ & $-7.0 \%$ \\
\hline 3 & 23 & Sum & 99.6045 & 101.2354 & 100.0020 & -0.3975 & 1.2334 & $-0.4 \%$ & $1.2 \%$ \\
\hline 3 & 24 & $\mathrm{Al}_{2} \mathrm{O}_{3}$ & 16.2166 & 16.6430 & 16.2000 & 0.0166 & 0.4430 & $0.1 \%$ & $2.7 \%$ \\
\hline 3 & 24 & $\mathrm{~B}_{2} \mathrm{O}_{3}$ & 18.1763 & 18.7364 & 18.0000 & 0.1763 & 0.7364 & $1.0 \%$ & $4.1 \%$ \\
\hline 3 & 24 & $\mathrm{BaO}$ & 0.0469 & 0.0499 & 0.0490 & -0.0021 & 0.0009 & $-4.3 \%$ & $1.8 \%$ \\
\hline 3 & 24 & $\mathrm{CaO}$ & 11.9037 & 12.2201 & 12.0000 & -0.0963 & 0.2201 & $-0.8 \%$ & $1.8 \%$ \\
\hline 3 & 24 & $\mathrm{Ce}_{2} \mathrm{O}_{3}$ & 0.0940 & 0.0940 & 0.1030 & -0.0090 & -0.0090 & $-8.7 \%$ & $-8.7 \%$ \\
\hline 3 & 24 & $\mathrm{Cr}_{2} \mathrm{O}_{3}$ & 0.0910 & 0.0878 & 0.0900 & 0.0010 & -0.0022 & $1.1 \%$ & $-2.4 \%$ \\
\hline 3 & 24 & $\mathrm{CuO}$ & 0.0319 & 0.0327 & 0.0330 & -0.0011 & -0.0003 & $-3.3 \%$ & $-0.8 \%$ \\
\hline 3 & 24 & $\mathrm{Fe}_{2} \mathrm{O}_{3}$ & 10.4761 & 10.8127 & 10.7720 & -0.2959 & 0.0407 & $-2.7 \%$ & $0.4 \%$ \\
\hline 3 & 24 & $\mathrm{~K}_{2} \mathrm{O}$ & 0.0626 & 0.0689 & 0.0690 & -0.0064 & -0.0001 & $-9.2 \%$ & $-0.2 \%$ \\
\hline 3 & 24 & $\mathrm{La}_{2} \mathrm{O}_{3}$ & 0.0586 & 0.0586 & 0.0150 & 0.0436 & 0.0436 & $290.9 \%$ & $290.9 \%$ \\
\hline 3 & 24 & $\mathrm{Li}_{2} \mathrm{O}$ & 0.1076 & 0.1084 & 0.0000 & 0.1076 & 0.1084 & & \\
\hline 3 & 24 & $\mathrm{MgO}$ & 0.6181 & 0.6437 & 0.6260 & -0.0079 & 0.0177 & $-1.3 \%$ & $2.8 \%$ \\
\hline 3 & 24 & $\mathrm{MnO}$ & 2.2887 & 2.3032 & 2.3060 & -0.0173 & -0.0028 & $-0.8 \%$ & $-0.1 \%$ \\
\hline 3 & 24 & $\mathrm{Na}_{2} \mathrm{O}$ & 10.8952 & 10.6959 & 10.8000 & 0.0952 & -0.1041 & $0.9 \%$ & $-1.0 \%$ \\
\hline
\end{tabular}


WSRC-STI-2007-00659

Revision 0

Appendix A

Table A4. Average Measured and Bias-Corrected Chemical Compositions Versus Targeted Compositions by Oxide by SB4 Variability Study Glass. (continued)

(100 -Batch 1)

\begin{tabular}{|c|c|c|c|c|c|c|c|c|c|}
\hline Set & Glass \# & Oxide & $\begin{array}{l}\text { Measured } \\
\text { (wt \%) }\end{array}$ & $\begin{array}{c}\text { Measured Bias- } \\
\text { Corrected (wt \%) }\end{array}$ & $\begin{array}{c}\text { Targeted } \\
\text { (wt \%) }\end{array}$ & $\begin{array}{c}\text { Diff of } \\
\text { Measured }\end{array}$ & $\begin{array}{c}\text { Diff of } \\
\text { Meas BC }\end{array}$ & $\begin{array}{l}\text { \% Diff of } \\
\text { Measured }\end{array}$ & $\begin{array}{l}\text { \% Diff of } \\
\text { Meas BC }\end{array}$ \\
\hline 3 & 24 & $\mathrm{NiO}$ & 0.9067 & 0.9679 & 1.0220 & -0.1153 & -0.0541 & $-11.3 \%$ & $-5.3 \%$ \\
\hline 3 & 24 & $\mathrm{PbO}$ & 0.0318 & 0.0318 & 0.0430 & -0.0112 & -0.0112 & $-26.1 \%$ & $-26.1 \%$ \\
\hline 3 & 24 & $\mathrm{SiO}_{2}$ & 27.2226 & 27.5866 & 27.0000 & 0.2226 & 0.5866 & $0.8 \%$ & $2.2 \%$ \\
\hline 3 & 24 & $\mathrm{SO}_{4}$ & 0.3221 & 0.3221 & 0.5130 & -0.1909 & -0.1909 & $-37.2 \%$ & $-37.2 \%$ \\
\hline 3 & 24 & $\mathrm{TiO}_{2}$ & 0.2185 & 0.2283 & 0.2270 & -0.0085 & 0.0013 & $-3.7 \%$ & $0.6 \%$ \\
\hline 3 & 24 & $\mathrm{ZnO}$ & 0.0293 & 0.0293 & 0.0330 & -0.0037 & -0.0037 & $-11.4 \%$ & $-11.4 \%$ \\
\hline 3 & 24 & $\mathrm{ZrO}_{2}$ & 0.0949 & 0.0949 & 0.1010 & -0.0061 & -0.0061 & $-6.0 \%$ & $-6.0 \%$ \\
\hline 3 & 24 & Sum & 99.8932 & 101.8162 & 100.0020 & -0.1088 & 1.8142 & $-0.1 \%$ & $1.8 \%$ \\
\hline 3 & 25 & $\mathrm{Al}_{2} \mathrm{O}_{3}$ & 30.3737 & 31.0278 & 31.2000 & -0.8263 & -0.1722 & $-2.6 \%$ & $-0.6 \%$ \\
\hline 3 & 25 & $\mathrm{~B}_{2} \mathrm{O}_{3}$ & 4.0973 & 4.2239 & 4.0000 & 0.0973 & 0.2239 & $2.4 \%$ & $5.6 \%$ \\
\hline 3 & 25 & $\mathrm{BaO}$ & 0.0447 & 0.0483 & 0.0490 & -0.0043 & -0.0007 & $-8.9 \%$ & $-1.4 \%$ \\
\hline 3 & 25 & $\mathrm{CaO}$ & 1.9834 & 2.0188 & 2.0000 & -0.0166 & 0.0188 & $-0.8 \%$ & $0.9 \%$ \\
\hline 3 & 25 & $\mathrm{Ce}_{2} \mathrm{O}_{3}$ & 0.0963 & 0.0963 & 0.1030 & -0.0067 & -0.0067 & $-6.5 \%$ & $-6.5 \%$ \\
\hline 3 & 25 & $\mathrm{Cr}_{2} \mathrm{O}_{3}$ & 0.0767 & 0.0752 & 0.0900 & -0.0133 & -0.0148 & $-14.7 \%$ & $-16.4 \%$ \\
\hline 3 & 25 & $\mathrm{CuO}$ & 0.0338 & 0.0350 & 0.0330 & 0.0008 & 0.0020 & $2.4 \%$ & $5.9 \%$ \\
\hline 3 & 25 & $\mathrm{Fe}_{2} \mathrm{O}_{3}$ & 10.6370 & 10.9797 & 10.7720 & -0.1350 & 0.2077 & $-1.3 \%$ & $1.9 \%$ \\
\hline 3 & 25 & $\mathrm{~K}_{2} \mathrm{O}$ & 0.0620 & 0.0669 & 0.0690 & -0.0070 & -0.0021 & $-10.1 \%$ & $-3.1 \%$ \\
\hline 3 & 25 & $\mathrm{La}_{2} \mathrm{O}_{3}$ & 0.0586 & 0.0586 & 0.0150 & 0.0436 & 0.0436 & $290.9 \%$ & $290.9 \%$ \\
\hline 3 & 25 & $\mathrm{Li}_{2} \mathrm{O}$ & 0.1076 & 0.1084 & 0.0000 & 0.1076 & 0.1084 & & \\
\hline 3 & 25 & $\mathrm{MgO}$ & 0.6169 & 0.6474 & 0.6260 & -0.0091 & 0.0214 & $-1.5 \%$ & $3.4 \%$ \\
\hline 3 & 25 & $\mathrm{MnO}$ & 2.2822 & 2.2738 & 2.3060 & -0.0238 & -0.0322 & $-1.0 \%$ & $-1.4 \%$ \\
\hline 3 & 25 & $\mathrm{Na}_{2} \mathrm{O}$ & 8.1251 & 7.9997 & 7.8000 & 0.3251 & 0.1997 & $4.2 \%$ & $2.6 \%$ \\
\hline 3 & 25 & $\mathrm{NiO}$ & 0.8364 & 0.9026 & 1.0220 & -0.1856 & -0.1194 & $-18.2 \%$ & $-11.7 \%$ \\
\hline 3 & 25 & $\mathrm{PbO}$ & 0.0337 & 0.0337 & 0.0430 & -0.0093 & -0.0093 & $-21.7 \%$ & $-21.7 \%$ \\
\hline 3 & 25 & $\mathrm{SiO}_{2}$ & 40.4863 & 41.0265 & 39.0000 & 1.4863 & 2.0265 & $3.8 \%$ & $5.2 \%$ \\
\hline 3 & 25 & $\mathrm{SO}_{4}$ & 0.1498 & 0.1498 & 0.5130 & -0.3632 & -0.3632 & $-70.8 \%$ & $-70.8 \%$ \\
\hline 3 & 25 & $\mathrm{TiO}_{2}$ & 0.2177 & 0.2295 & 0.2270 & -0.0093 & 0.0025 & $-4.1 \%$ & $1.1 \%$ \\
\hline 3 & 25 & $\mathrm{ZnO}$ & 0.0293 & 0.0293 & 0.0330 & -0.0037 & -0.0037 & $-11.4 \%$ & $-11.4 \%$ \\
\hline 3 & 25 & $\mathrm{ZrO}_{2}$ & 0.0919 & 0.0919 & 0.1010 & -0.0091 & -0.0091 & $-9.1 \%$ & $-9.1 \%$ \\
\hline 3 & 25 & Sum & 100.4402 & 102.1231 & 100.0020 & 0.4382 & 2.1211 & $0.4 \%$ & $2.1 \%$ \\
\hline 3 & 26 & $\mathrm{Al}_{2} \mathrm{O}_{3}$ & 25.7917 & 26.4697 & 25.6000 & 0.1917 & 0.8697 & $0.7 \%$ & $3.4 \%$ \\
\hline 3 & 26 & $\mathrm{~B}_{2} \mathrm{O}_{3}$ & 18.5627 & 19.1347 & 18.0000 & 0.5627 & 1.1347 & $3.1 \%$ & $6.3 \%$ \\
\hline 3 & 26 & $\mathrm{BaO}$ & 0.0480 & 0.0511 & 0.0490 & -0.0010 & 0.0021 & $-2.0 \%$ & $4.2 \%$ \\
\hline 3 & 26 & $\mathrm{CaO}$ & 2.4521 & 2.5173 & 2.0000 & 0.4521 & 0.5173 & $22.6 \%$ & $25.9 \%$ \\
\hline 3 & 26 & $\mathrm{Ce}_{2} \mathrm{O}_{3}$ & 0.0969 & 0.0969 & 0.1030 & -0.0061 & -0.0061 & $-5.9 \%$ & $-5.9 \%$ \\
\hline 3 & 26 & $\mathrm{Cr}_{2} \mathrm{O}_{3}$ & 0.0691 & 0.0667 & 0.0900 & -0.0209 & -0.0233 & $-23.3 \%$ & $-25.9 \%$ \\
\hline 3 & 26 & $\mathrm{CuO}$ & 0.0363 & 0.0372 & 0.0330 & 0.0033 & 0.0042 & $10.0 \%$ & $12.8 \%$ \\
\hline 3 & 26 & $\mathrm{Fe}_{2} \mathrm{O}_{3}$ & 9.6433 & 9.9535 & 10.7720 & -1.1287 & -0.8185 & $-10.5 \%$ & $-7.6 \%$ \\
\hline 3 & 26 & $\mathrm{~K}_{2} \mathrm{O}$ & 0.0653 & 0.0719 & 0.0690 & -0.0037 & 0.0029 & $-5.3 \%$ & $4.1 \%$ \\
\hline 3 & 26 & $\mathrm{La}_{2} \mathrm{O}_{3}$ & 0.0586 & 0.0586 & 0.0150 & 0.0436 & 0.0436 & $290.9 \%$ & $290.9 \%$ \\
\hline 3 & 26 & $\mathrm{Li}_{2} \mathrm{O}$ & 0.1076 & 0.1084 & 0.0000 & 0.1076 & 0.1084 & & \\
\hline 3 & 26 & $\mathrm{MgO}$ & 0.6434 & 0.6701 & 0.6260 & 0.0174 & 0.0441 & $2.8 \%$ & $7.0 \%$ \\
\hline 3 & 26 & $\mathrm{MnO}$ & 2.3661 & 2.3812 & 2.3060 & 0.0601 & 0.0752 & $2.6 \%$ & $3.3 \%$ \\
\hline 3 & 26 & $\mathrm{Na}_{2} \mathrm{O}$ & 6.6558 & 6.5340 & 6.4000 & 0.2558 & 0.1340 & $4.0 \%$ & $2.1 \%$ \\
\hline 3 & 26 & $\mathrm{NiO}$ & 0.8656 & 0.9241 & 1.0220 & -0.1564 & -0.0979 & $-15.3 \%$ & $-9.6 \%$ \\
\hline 3 & 26 & $\mathrm{PbO}$ & 0.0323 & 0.0323 & 0.0430 & -0.0107 & -0.0107 & $-24.8 \%$ & $-24.8 \%$ \\
\hline 3 & 26 & $\mathrm{SiO}_{2}$ & 33.1057 & 33.5480 & 32.0000 & 1.1057 & 1.5480 & $3.5 \%$ & $4.8 \%$ \\
\hline 3 & 26 & $\mathrm{SO}_{4}$ & 0.1498 & 0.1498 & 0.5130 & -0.3632 & -0.3632 & $-70.8 \%$ & $-70.8 \%$ \\
\hline 3 & 26 & $\mathrm{TiO}_{2}$ & 0.2118 & 0.2213 & 0.2270 & -0.0152 & -0.0057 & $-6.7 \%$ & $-2.5 \%$ \\
\hline 3 & 26 & $\mathrm{ZnO}$ & 0.0293 & 0.0293 & 0.0330 & -0.0037 & -0.0037 & $-11.4 \%$ & $-11.4 \%$ \\
\hline 3 & 26 & $\mathrm{ZrO}_{2}$ & 0.0946 & 0.0946 & 0.1010 & -0.0064 & -0.0064 & $-6.4 \%$ & $-6.4 \%$ \\
\hline 3 & 26 & Sum & 101.0861 & 103.1504 & 100.0020 & 1.0841 & 3.1484 & $1.1 \%$ & $3.1 \%$ \\
\hline 3 & 27 & $\mathrm{Al}_{2} \mathrm{O}_{3}$ & 26.6420 & 27.3424 & 27.2000 & -0.5580 & 0.1424 & $-2.1 \%$ & $0.5 \%$ \\
\hline 3 & 27 & $\mathrm{~B}_{2} \mathrm{O}_{3}$ & 3.9363 & 4.1247 & 4.0000 & -0.0637 & 0.1247 & $-1.6 \%$ & $3.1 \%$ \\
\hline 3 & 27 & $\mathrm{BaO}$ & 0.0410 & 0.0436 & 0.0490 & -0.0080 & -0.0054 & $-16.3 \%$ & $-11.0 \%$ \\
\hline 3 & 27 & $\mathrm{CaO}$ & 11.8477 & 12.1630 & 12.0000 & -0.1523 & 0.1630 & $-1.3 \%$ & $1.4 \%$ \\
\hline 3 & 27 & $\mathrm{Ce}_{2} \mathrm{O}_{3}$ & 0.0960 & 0.0960 & 0.1030 & -0.0070 & -0.0070 & $-6.8 \%$ & $-6.8 \%$ \\
\hline 3 & 27 & $\mathrm{Cr}_{2} \mathrm{O}_{3}$ & 0.0833 & 0.0804 & 0.0900 & -0.0067 & -0.0096 & $-7.4 \%$ & $-10.6 \%$ \\
\hline 3 & 27 & $\mathrm{CuO}$ & 0.0354 & 0.0363 & 0.0330 & 0.0024 & 0.0033 & $7.2 \%$ & $9.9 \%$ \\
\hline 3 & 27 & $\mathrm{Fe}_{2} \mathrm{O}_{3}$ & 9.8721 & 10.2698 & 10.7720 & -0.8999 & -0.5022 & $-8.4 \%$ & $-4.7 \%$ \\
\hline 3 & 27 & $\mathrm{~K}_{2} \mathrm{O}$ & 0.0644 & 0.0709 & 0.0690 & -0.0046 & 0.0019 & $-6.6 \%$ & $2.7 \%$ \\
\hline 3 & 27 & $\mathrm{La}_{2} \mathrm{O}_{3}$ & 0.0586 & 0.0586 & 0.0150 & 0.0436 & 0.0436 & $290.9 \%$ & $290.9 \%$ \\
\hline 3 & 27 & $\mathrm{Li}_{2} \mathrm{O}$ & 0.1076 & 0.1104 & 0.0000 & 0.1076 & 0.1104 & & \\
\hline 3 & 27 & $\mathrm{MgO}$ & 0.6132 & 0.6385 & 0.6260 & -0.0128 & 0.0125 & $-2.1 \%$ & $2.0 \%$ \\
\hline 3 & 27 & $\mathrm{MnO}$ & 2.2822 & 2.2967 & 2.3060 & -0.0238 & -0.0093 & $-1.0 \%$ & $-0.4 \%$ \\
\hline
\end{tabular}


WSRC-STI-2007-00659

Revision 0

Appendix A

Table A4. Average Measured and Bias-Corrected Chemical Compositions Versus Targeted Compositions by Oxide by SB4 Variability Study Glass. (continued)

(100 -Batch 1)

\begin{tabular}{|c|c|c|c|c|c|c|c|c|c|}
\hline Set & Glass \# & Oxide & $\begin{array}{l}\text { Measured } \\
\text { (wt \%) }\end{array}$ & $\begin{array}{c}\text { Measured Bias- } \\
\text { Corrected (wt \%) }\end{array}$ & $\begin{array}{c}\text { Targeted } \\
\text { (wt \%) }\end{array}$ & $\begin{array}{c}\text { Diff of } \\
\text { Measured }\end{array}$ & $\begin{array}{c}\text { Diff of } \\
\text { Meas BC }\end{array}$ & $\begin{array}{l}\text { \% Diff of } \\
\text { Measured }\end{array}$ & $\begin{array}{l}\% \text { Diff of } \\
\text { Meas BC }\end{array}$ \\
\hline 3 & 27 & $\mathrm{Na}_{2} \mathrm{O}$ & 6.8681 & 6.7424 & 6.8000 & 0.0681 & -0.0576 & $1.0 \%$ & $-0.8 \%$ \\
\hline 3 & 27 & $\mathrm{NiO}$ & 0.8335 & 0.8898 & 1.0220 & -0.1885 & -0.1322 & $-18.4 \%$ & $-12.9 \%$ \\
\hline 3 & 27 & $\mathrm{PbO}$ & 0.0329 & 0.0329 & 0.0430 & -0.0101 & -0.0101 & $-23.6 \%$ & $-23.6 \%$ \\
\hline 3 & 27 & $\mathrm{SiO}_{2}$ & 34.3892 & 35.3272 & 34.0000 & 0.3892 & 1.3272 & $1.1 \%$ & $3.9 \%$ \\
\hline 3 & 27 & $\mathrm{SO}_{4}$ & 0.3962 & 0.3962 & 0.5130 & -0.1168 & -0.1168 & $-22.8 \%$ & $-22.8 \%$ \\
\hline 3 & 27 & $\mathrm{TiO}_{2}$ & 0.2198 & 0.2296 & 0.2270 & -0.0072 & 0.0026 & $-3.2 \%$ & $1.1 \%$ \\
\hline 3 & 27 & $\mathrm{ZnO}$ & 0.0324 & 0.0324 & 0.0330 & -0.0006 & -0.0006 & $-1.9 \%$ & $-1.9 \%$ \\
\hline 3 & 27 & $\mathrm{ZrO}_{2}$ & 0.0966 & 0.0966 & 0.1010 & -0.0044 & -0.0044 & $-4.4 \%$ & $-4.4 \%$ \\
\hline 3 & 27 & Sum & 98.5485 & 101.0783 & 100.0020 & -1.4535 & 1.0763 & $-1.5 \%$ & $1.1 \%$ \\
\hline 3 & 28 & $\mathrm{Al}_{2} \mathrm{O}_{3}$ & 21.7293 & 22.3005 & 21.6000 & 0.1292 & 0.7005 & $0.6 \%$ & $3.2 \%$ \\
\hline 3 & 28 & $\mathrm{~B}_{2} \mathrm{O}_{3}$ & 18.3293 & 18.8937 & 18.0000 & 0.3293 & 0.8937 & $1.8 \%$ & $5.0 \%$ \\
\hline 3 & 28 & $\mathrm{BaO}$ & 0.0447 & 0.0475 & 0.0490 & -0.0043 & -0.0015 & $-8.9 \%$ & $-3.1 \%$ \\
\hline 3 & 28 & $\mathrm{CaO}$ & 12.0226 & 12.3427 & 12.0000 & 0.0226 & 0.3427 & $0.2 \%$ & $2.9 \%$ \\
\hline 3 & 28 & $\mathrm{Ce}_{2} \mathrm{O}_{3}$ & 0.0958 & 0.0958 & 0.1030 & -0.0072 & -0.0072 & $-7.0 \%$ & $-7.0 \%$ \\
\hline 3 & 28 & $\mathrm{Cr}_{2} \mathrm{O}_{3}$ & 0.0650 & 0.0628 & 0.0900 & -0.0250 & -0.0272 & $-27.7 \%$ & $-30.2 \%$ \\
\hline 3 & 28 & $\mathrm{CuO}$ & 0.0391 & 0.0401 & 0.0330 & 0.0061 & 0.0071 & $18.5 \%$ & $21.6 \%$ \\
\hline 3 & 28 & $\mathrm{Fe}_{2} \mathrm{O}_{3}$ & 10.4082 & 10.7431 & 10.7720 & -0.3638 & -0.0289 & $-3.4 \%$ & $-0.3 \%$ \\
\hline 3 & 28 & $\mathrm{~K}_{2} \mathrm{O}$ & 0.0605 & 0.0666 & 0.0690 & -0.0085 & -0.0024 & $-12.3 \%$ & $-3.5 \%$ \\
\hline 3 & 28 & $\mathrm{La}_{2} \mathrm{O}_{3}$ & 0.0586 & 0.0586 & 0.0150 & 0.0436 & 0.0436 & $290.9 \%$ & $290.9 \%$ \\
\hline 3 & 28 & $\mathrm{Li}_{2} \mathrm{O}$ & 0.1076 & 0.1084 & 0.0000 & 0.1076 & 0.1084 & & \\
\hline 3 & 28 & $\mathrm{MgO}$ & 0.6297 & 0.6558 & 0.6260 & 0.0037 & 0.0298 & $0.6 \%$ & $4.8 \%$ \\
\hline 3 & 28 & $\mathrm{MnO}$ & 2.2790 & 2.2934 & 2.3060 & -0.0270 & -0.0126 & $-1.2 \%$ & $-0.5 \%$ \\
\hline 3 & 28 & $\mathrm{Na}_{2} \mathrm{O}$ & 5.5470 & 5.4455 & 5.4000 & 0.1470 & 0.0455 & $2.7 \%$ & $0.8 \%$ \\
\hline 3 & 28 & $\mathrm{NiO}$ & 0.8542 & 0.9119 & 1.0220 & -0.1678 & -0.1101 & $-16.4 \%$ & $-10.8 \%$ \\
\hline 3 & 28 & $\mathrm{PbO}$ & 0.0393 & 0.0393 & 0.0430 & -0.0037 & -0.0037 & $-8.6 \%$ & $-8.6 \%$ \\
\hline 3 & 28 & $\mathrm{SiO}_{2}$ & 27.0621 & 27.4233 & 27.0000 & 0.0621 & 0.4233 & $0.2 \%$ & $1.6 \%$ \\
\hline 3 & 28 & $\mathrm{SO}_{4}$ & 0.1498 & 0.1498 & 0.5130 & -0.3632 & -0.3632 & $-70.8 \%$ & $-70.8 \%$ \\
\hline 3 & 28 & $\mathrm{TiO}_{2}$ & 0.2181 & 0.2278 & 0.2270 & -0.0089 & 0.0008 & $-3.9 \%$ & $0.4 \%$ \\
\hline 3 & 28 & $\mathrm{ZnO}$ & 0.0311 & 0.0311 & 0.0330 & -0.0019 & -0.0019 & $-5.7 \%$ & $-5.7 \%$ \\
\hline 3 & 28 & $\mathrm{ZrO}_{2}$ & 0.0919 & 0.0919 & 0.1010 & -0.0091 & -0.0091 & $-9.1 \%$ & $-9.1 \%$ \\
\hline 3 & 28 & Sum & 99.8630 & 102.0296 & 100.0020 & -0.1390 & 2.0276 & $-0.1 \%$ & $2.0 \%$ \\
\hline 3 & 29 & $\mathrm{Al}_{2} \mathrm{O}_{3}$ & 7.6241 & 7.7891 & 7.8000 & -0.1759 & -0.0109 & $-2.3 \%$ & $-0.1 \%$ \\
\hline 3 & 29 & $\mathrm{~B}_{2} \mathrm{O}_{3}$ & 3.8156 & 3.9982 & 4.0000 & -0.1844 & -0.0018 & $-4.6 \%$ & $0.0 \%$ \\
\hline 3 & 29 & $\mathrm{BaO}$ & 0.0441 & 0.0477 & 0.0490 & -0.0049 & -0.0013 & $-10.0 \%$ & $-2.6 \%$ \\
\hline 3 & 29 & $\mathrm{CaO}$ & 1.9764 & 2.0121 & 2.0000 & -0.0236 & 0.0121 & $-1.2 \%$ & $0.6 \%$ \\
\hline 3 & 29 & $\mathrm{Ce}_{2} \mathrm{O}_{3}$ & 0.0887 & 0.0887 & 0.1030 & -0.0143 & -0.0143 & $-13.9 \%$ & $-13.9 \%$ \\
\hline 3 & 29 & $\mathrm{Cr}_{2} \mathrm{O}_{3}$ & 0.0833 & 0.0817 & 0.0900 & -0.0067 & -0.0083 & $-7.4 \%$ & $-9.2 \%$ \\
\hline 3 & 29 & $\mathrm{CuO}$ & 0.0329 & 0.0340 & 0.0330 & -0.0001 & 0.0010 & $-0.4 \%$ & $3.0 \%$ \\
\hline 3 & 29 & $\mathrm{Fe}_{2} \mathrm{O}_{3}$ & 10.4225 & 10.8436 & 10.7720 & -0.3495 & 0.0716 & $-3.2 \%$ & $0.7 \%$ \\
\hline 3 & 29 & $\mathrm{~K}_{2} \mathrm{O}$ & 0.0596 & 0.0643 & 0.0690 & -0.0094 & -0.0047 & $-13.6 \%$ & $-6.8 \%$ \\
\hline 3 & 29 & $\mathrm{La}_{2} \mathrm{O}_{3}$ & 0.0586 & 0.0586 & 0.0150 & 0.0436 & 0.0436 & $290.9 \%$ & $290.9 \%$ \\
\hline 3 & 29 & $\mathrm{Li}_{2} \mathrm{O}$ & 0.1076 & 0.1104 & 0.0000 & 0.1076 & 0.1104 & & \\
\hline 3 & 29 & $\mathrm{MgO}$ & 0.5966 & 0.6261 & 0.6260 & -0.0294 & 0.0001 & $-4.7 \%$ & $0.0 \%$ \\
\hline 3 & 29 & $\mathrm{MnO}$ & 2.1337 & 2.1260 & 2.3060 & -0.1723 & -0.1800 & $-7.5 \%$ & $-7.8 \%$ \\
\hline 3 & 29 & $\mathrm{Na}_{2} \mathrm{O}$ & 36.2444 & 35.6886 & 39.0000 & -2.7557 & -3.3114 & $-7.1 \%$ & $-8.5 \%$ \\
\hline 3 & 29 & $\mathrm{NiO}$ & 0.8558 & 0.9236 & 1.0220 & -0.1662 & -0.0984 & $-16.3 \%$ & $-9.6 \%$ \\
\hline 3 & 29 & $\mathrm{PbO}$ & 0.0345 & 0.0345 & 0.0430 & -0.0085 & -0.0085 & $-19.8 \%$ & $-19.8 \%$ \\
\hline 3 & 29 & $\mathrm{SiO}_{2}$ & 30.2711 & 31.0993 & 31.2000 & -0.9289 & -0.1007 & $-3.0 \%$ & $-0.3 \%$ \\
\hline 3 & 29 & $\mathrm{SO}_{4}$ & 0.4906 & 0.4906 & 0.5130 & -0.0224 & -0.0224 & $-4.4 \%$ & $-4.4 \%$ \\
\hline 3 & 29 & $\mathrm{TiO}_{2}$ & 0.1964 & 0.2071 & 0.2270 & -0.0306 & -0.0199 & $-13.5 \%$ & $-8.8 \%$ \\
\hline 3 & 29 & $\mathrm{ZnO}$ & 0.0277 & 0.0277 & 0.0330 & -0.0053 & -0.0053 & $-16.1 \%$ & $-16.1 \%$ \\
\hline 3 & 29 & $\mathrm{ZrO}_{2}$ & 0.0892 & 0.0892 & 0.1010 & -0.0118 & -0.0118 & $-11.7 \%$ & $-11.7 \%$ \\
\hline 3 & 29 & Sum & 95.2533 & 96.4409 & 100.0020 & -4.7487 & -3.5611 & $-4.7 \%$ & $-3.6 \%$ \\
\hline 3 & 30 & $\mathrm{Al}_{2} \mathrm{O}_{3}$ & 6.3818 & 6.5197 & 6.4000 & -0.0182 & 0.1197 & $-0.3 \%$ & $1.9 \%$ \\
\hline 3 & 30 & $\mathrm{~B}_{2} \mathrm{O}_{3}$ & 17.8221 & 18.6748 & 18.0000 & -0.1779 & 0.6748 & $-1.0 \%$ & $3.7 \%$ \\
\hline 3 & 30 & $\mathrm{BaO}$ & 0.0435 & 0.0471 & 0.0490 & -0.0055 & -0.0019 & $-11.1 \%$ & $-3.9 \%$ \\
\hline 3 & 30 & $\mathrm{CaO}$ & 2.0358 & 2.0728 & 2.0000 & 0.0358 & 0.0728 & $1.8 \%$ & $3.6 \%$ \\
\hline 3 & 30 & $\mathrm{Ce}_{2} \mathrm{O}_{3}$ & 0.0919 & 0.0919 & 0.1030 & -0.0111 & -0.0111 & $-10.7 \%$ & $-10.7 \%$ \\
\hline 3 & 30 & $\mathrm{Cr}_{2} \mathrm{O}_{3}$ & 0.0870 & 0.0853 & 0.0900 & -0.0030 & -0.0047 & $-3.4 \%$ & $-5.3 \%$ \\
\hline 3 & 30 & $\mathrm{CuO}$ & 0.0401 & 0.0414 & 0.0330 & 0.0071 & 0.0084 & $21.4 \%$ & $25.6 \%$ \\
\hline 3 & 30 & $\mathrm{Fe}_{2} \mathrm{O}_{3}$ & 10.4189 & 10.8390 & 10.7720 & -0.3531 & 0.0670 & $-3.3 \%$ & $0.6 \%$ \\
\hline 3 & 30 & $\mathrm{~K}_{2} \mathrm{O}$ & 0.0590 & 0.0636 & 0.0690 & -0.0100 & -0.0054 & $-14.5 \%$ & $-7.8 \%$ \\
\hline 3 & 30 & $\mathrm{La}_{2} \mathrm{O}_{3}$ & 0.0586 & 0.0586 & 0.0150 & 0.0436 & 0.0436 & $290.9 \%$ & $290.9 \%$ \\
\hline 3 & 30 & $\mathrm{Li}_{2} \mathrm{O}$ & 0.1076 & 0.1104 & 0.0000 & 0.1076 & 0.1104 & & \\
\hline 3 & 30 & $\mathrm{MgO}$ & 0.6177 & 0.6483 & 0.6260 & -0.0083 & 0.0223 & $-1.3 \%$ & $3.6 \%$ \\
\hline
\end{tabular}


WSRC-STI-2007-00659

Revision 0

Appendix A

Table A4. Average Measured and Bias-Corrected Chemical Compositions Versus Targeted Compositions by Oxide by SB4 Variability Study Glass. (continued)

(100 -Batch 1)

\begin{tabular}{|c|c|c|c|c|c|c|c|c|c|}
\hline Set & Glass \# & Oxide & $\begin{array}{l}\text { Measured } \\
\text { (wt \%) }\end{array}$ & $\begin{array}{c}\text { Measured Bias- } \\
\text { Corrected (wt \%) }\end{array}$ & $\begin{array}{c}\text { Targeted } \\
\text { (wt \%) }\end{array}$ & $\begin{array}{c}\text { Diff of } \\
\text { Measured }\end{array}$ & $\begin{array}{c}\text { Diff of } \\
\text { Meas BC }\end{array}$ & $\begin{array}{l}\text { \% Diff of } \\
\text { Measured }\end{array}$ & $\begin{array}{l}\% \text { Diff of } \\
\text { Meas BC }\end{array}$ \\
\hline 3 & 30 & $\mathrm{MnO}$ & 2.2887 & 2.2803 & 2.3060 & -0.0173 & -0.0257 & $-0.8 \%$ & $-1.1 \%$ \\
\hline 3 & 30 & $\mathrm{Na}_{2} \mathrm{O}$ & 30.4210 & 29.9529 & 32.0000 & -1.5790 & -2.0471 & $-4.9 \%$ & $-6.4 \%$ \\
\hline 3 & 30 & $\mathrm{NiO}$ & 0.9270 & 1.0005 & 1.0220 & -0.0950 & -0.0215 & $-9.3 \%$ & $-2.1 \%$ \\
\hline 3 & 30 & $\mathrm{PbO}$ & 0.0380 & 0.0380 & 0.0430 & -0.0050 & -0.0050 & $-11.7 \%$ & $-11.7 \%$ \\
\hline 3 & 30 & $\mathrm{SiO}_{2}$ & 25.5646 & 26.2629 & 25.6000 & -0.0354 & 0.6629 & $-0.1 \%$ & $2.6 \%$ \\
\hline 3 & 30 & $\mathrm{SO}_{4}$ & 0.4981 & 0.4981 & 0.5130 & -0.0149 & -0.0149 & $-2.9 \%$ & $-2.9 \%$ \\
\hline 3 & 30 & $\mathrm{TiO}_{2}$ & 0.2164 & 0.2282 & 0.2270 & -0.0106 & 0.0012 & $-4.7 \%$ & $0.5 \%$ \\
\hline 3 & 30 & $\mathrm{ZnO}$ & 0.0352 & 0.0352 & 0.0330 & 0.0022 & 0.0022 & $6.6 \%$ & $6.6 \%$ \\
\hline 3 & 30 & $\mathrm{ZrO}_{2}$ & 0.0902 & 0.0902 & 0.1010 & -0.0108 & -0.0108 & $-10.7 \%$ & $-10.7 \%$ \\
\hline 3 & 30 & Sum & 97.8433 & 99.6390 & 100.0020 & -2.1587 & -0.3630 & $-2.2 \%$ & $-0.4 \%$ \\
\hline 4 & 31 & $\mathrm{Al}_{2} \mathrm{O}_{3}$ & 6.5802 & 6.8128 & 6.8000 & -0.2198 & 0.0128 & $-3.2 \%$ & $0.2 \%$ \\
\hline 4 & 31 & $\mathrm{~B}_{2} \mathrm{O}_{3}$ & 3.7673 & 3.8637 & 4.0000 & -0.2327 & -0.1363 & $-5.8 \%$ & $-3.4 \%$ \\
\hline 4 & 31 & $\mathrm{BaO}$ & 0.0405 & 0.0444 & 0.0490 & -0.0085 & -0.0046 & $-17.4 \%$ & $-9.3 \%$ \\
\hline 4 & 31 & $\mathrm{CaO}$ & 11.4455 & 12.1259 & 12.0000 & -0.5545 & 0.1259 & $-4.6 \%$ & $1.0 \%$ \\
\hline 4 & 31 & $\mathrm{Ce}_{2} \mathrm{O}_{3}$ & 0.0876 & 0.0876 & 0.1030 & -0.0154 & -0.0154 & $-15.0 \%$ & $-15.0 \%$ \\
\hline 4 & 31 & $\mathrm{Cr}_{2} \mathrm{O}_{3}$ & 0.0840 & 0.0831 & 0.0900 & -0.0060 & -0.0069 & $-6.6 \%$ & $-7.6 \%$ \\
\hline 4 & 31 & $\mathrm{CuO}$ & 0.0376 & 0.0392 & 0.0330 & 0.0046 & 0.0062 & $13.8 \%$ & $18.8 \%$ \\
\hline 4 & 31 & $\mathrm{Fe}_{2} \mathrm{O}_{3}$ & 10.1938 & 10.2584 & 10.7720 & -0.5782 & -0.5136 & $-5.4 \%$ & $-4.8 \%$ \\
\hline 4 & 31 & $\mathrm{~K}_{2} \mathrm{O}$ & 0.0590 & 0.0621 & 0.0690 & -0.0100 & -0.0069 & $-14.5 \%$ & $-10.0 \%$ \\
\hline 4 & 31 & $\mathrm{La}_{2} \mathrm{O}_{3}$ & 0.0586 & 0.0586 & 0.0150 & 0.0436 & 0.0436 & $290.9 \%$ & $290.9 \%$ \\
\hline 4 & 31 & $\mathrm{Li}_{2} \mathrm{O}$ & 0.1076 & 0.1079 & 0.0000 & 0.1076 & 0.1079 & & \\
\hline 4 & 31 & $\mathrm{MgO}$ & 0.5659 & 0.5938 & 0.6260 & -0.0601 & -0.0322 & $-9.6 \%$ & $-5.1 \%$ \\
\hline 4 & 31 & $\mathrm{MnO}$ & 2.1079 & 2.1535 & 2.3060 & -0.1981 & -0.1525 & $-8.6 \%$ & $-6.6 \%$ \\
\hline 4 & 31 & $\mathrm{Na}_{2} \mathrm{O}$ & 31.0040 & 30.6846 & 34.0000 & -2.9960 & -3.3154 & $-8.8 \%$ & $-9.8 \%$ \\
\hline 4 & 31 & $\mathrm{NiO}$ & 0.8128 & 0.8848 & 1.0220 & -0.2092 & -0.1372 & $-20.5 \%$ & $-13.4 \%$ \\
\hline 4 & 31 & $\mathrm{PbO}$ & 0.0339 & 0.0339 & 0.0430 & -0.0091 & -0.0091 & $-21.1 \%$ & $-21.1 \%$ \\
\hline 4 & 31 & $\mathrm{SiO}_{2}$ & 25.8855 & 25.9128 & 27.2000 & -1.3145 & -1.2872 & $-4.8 \%$ & $-4.7 \%$ \\
\hline 4 & 31 & $\mathrm{SO}_{4}$ & 0.4793 & 0.4793 & 0.5130 & -0.0337 & -0.0337 & $-6.6 \%$ & $-6.6 \%$ \\
\hline 4 & 31 & $\mathrm{TiO}_{2}$ & 0.2102 & 0.2224 & 0.2270 & -0.0168 & -0.0046 & $-7.4 \%$ & $-2.0 \%$ \\
\hline 4 & 31 & $\mathrm{ZnO}$ & 0.0293 & 0.0293 & 0.0330 & -0.0037 & -0.0037 & $-11.4 \%$ & $-11.4 \%$ \\
\hline 4 & 31 & $\mathrm{ZrO}_{2}$ & 0.0878 & 0.0878 & 0.1010 & -0.0132 & -0.0132 & $-13.1 \%$ & $-13.1 \%$ \\
\hline 4 & 31 & Sum & 93.6782 & 94.6261 & 100.0020 & -6.3238 & -5.3759 & $-6.3 \%$ & $-5.4 \%$ \\
\hline 4 & 32 & $\mathrm{Al}_{2} \mathrm{O}_{3}$ & 5.6118 & 5.6006 & 5.4000 & 0.2118 & 0.2006 & $3.9 \%$ & $3.7 \%$ \\
\hline 4 & 32 & $\mathrm{~B}_{2} \mathrm{O}_{3}$ & 17.8865 & 18.1520 & 18.0000 & -0.1135 & 0.1520 & $-0.6 \%$ & $0.8 \%$ \\
\hline 4 & 32 & $\mathrm{BaO}$ & 0.0419 & 0.0455 & 0.0490 & -0.0071 & -0.0035 & $-14.6 \%$ & $-7.1 \%$ \\
\hline 4 & 32 & $\mathrm{CaO}$ & 12.1311 & 12.1375 & 12.0000 & 0.1311 & 0.1375 & $1.1 \%$ & $1.1 \%$ \\
\hline 4 & 32 & $\mathrm{Ce}_{2} \mathrm{O}_{3}$ & 0.0937 & 0.0937 & 0.1030 & -0.0093 & -0.0093 & $-9.0 \%$ & $-9.0 \%$ \\
\hline 4 & 32 & $\mathrm{Cr}_{2} \mathrm{O}_{3}$ & 0.0892 & 0.0878 & 0.0900 & -0.0008 & -0.0022 & $-0.9 \%$ & $-2.4 \%$ \\
\hline 4 & 32 & $\mathrm{CuO}$ & 0.0363 & 0.0377 & 0.0330 & 0.0033 & 0.0047 & $10.0 \%$ & $14.2 \%$ \\
\hline 4 & 32 & $\mathrm{Fe}_{2} \mathrm{O}_{3}$ & 10.8264 & 10.9314 & 10.7720 & 0.0544 & 0.1594 & $0.5 \%$ & $1.5 \%$ \\
\hline 4 & 32 & $\mathrm{~K}_{2} \mathrm{O}$ & 0.0629 & 0.0664 & 0.0690 & -0.0061 & -0.0026 & $-8.8 \%$ & $-3.7 \%$ \\
\hline 4 & 32 & $\mathrm{La}_{2} \mathrm{O}_{3}$ & 0.0586 & 0.0586 & 0.0150 & 0.0436 & 0.0436 & $290.9 \%$ & $290.9 \%$ \\
\hline 4 & 32 & $\mathrm{Li}_{2} \mathrm{O}$ & 0.1076 & 0.1067 & 0.0000 & 0.1076 & 0.1067 & & \\
\hline 4 & 32 & $\mathrm{MgO}$ & 0.6231 & 0.6539 & 0.6260 & -0.0029 & 0.0279 & $-0.5 \%$ & $4.5 \%$ \\
\hline 4 & 32 & $\mathrm{MnO}$ & 2.2983 & 2.2871 & 2.3060 & -0.0077 & -0.0189 & $-0.3 \%$ & $-0.8 \%$ \\
\hline 4 & 32 & $\mathrm{Na}_{2} \mathrm{O}$ & 26.7241 & 25.9174 & 27.0000 & -0.2759 & -1.0826 & $-1.0 \%$ & $-4.0 \%$ \\
\hline 4 & 32 & $\mathrm{NiO}$ & 0.9327 & 1.0128 & 1.0220 & -0.0893 & -0.0092 & $-8.7 \%$ & $-0.9 \%$ \\
\hline 4 & 32 & $\mathrm{PbO}$ & 0.0353 & 0.0353 & 0.0430 & -0.0077 & -0.0077 & $-18.0 \%$ & $-18.0 \%$ \\
\hline 4 & 32 & $\mathrm{SiO}_{2}$ & 21.6604 & 21.6520 & 21.6000 & 0.0604 & 0.0520 & $0.3 \%$ & $0.2 \%$ \\
\hline 4 & 32 & $\mathrm{SO}_{4}$ & 0.5213 & 0.5213 & 0.5130 & 0.0083 & 0.0083 & $1.6 \%$ & $1.6 \%$ \\
\hline 4 & 32 & $\mathrm{TiO}_{2}$ & 0.2214 & 0.2341 & 0.2270 & -0.0056 & 0.0071 & $-2.5 \%$ & $3.1 \%$ \\
\hline 4 & 32 & $\mathrm{ZnO}$ & 0.0271 & 0.0271 & 0.0330 & -0.0059 & -0.0059 & $-18.0 \%$ & $-18.0 \%$ \\
\hline 4 & 32 & $\mathrm{ZrO}_{2}$ & 0.0929 & 0.0929 & 0.1010 & -0.0081 & -0.0081 & $-8.1 \%$ & $-8.1 \%$ \\
\hline 4 & 32 & Sum & 100.0827 & 99.7520 & 100.0020 & 0.0807 & -0.2500 & $0.1 \%$ & $-0.2 \%$ \\
\hline 4 & 33 & $\mathrm{Al}_{2} \mathrm{O}_{3}$ & 23.6660 & 23.6203 & 23.4000 & 0.2660 & 0.2203 & $1.1 \%$ & $0.9 \%$ \\
\hline 4 & 33 & $\mathrm{~B}_{2} \mathrm{O}_{3}$ & 3.9524 & 4.0110 & 4.0000 & -0.0476 & 0.0110 & $-1.2 \%$ & $0.3 \%$ \\
\hline 4 & 33 & $\mathrm{BaO}$ & 0.0441 & 0.0480 & 0.0490 & -0.0049 & -0.0010 & $-10.0 \%$ & $-2.1 \%$ \\
\hline 4 & 33 & $\mathrm{CaO}$ & 2.0079 & 2.0087 & 2.0000 & 0.0079 & 0.0087 & $0.4 \%$ & $0.4 \%$ \\
\hline 4 & 33 & $\mathrm{Ce}_{2} \mathrm{O}_{3}$ & 0.0952 & 0.0952 & 0.1030 & -0.0078 & -0.0078 & $-7.6 \%$ & $-7.6 \%$ \\
\hline 4 & 33 & $\mathrm{Cr}_{2} \mathrm{O}_{3}$ & 0.0870 & 0.0856 & 0.0900 & -0.0030 & -0.0044 & $-3.4 \%$ & $-4.8 \%$ \\
\hline 4 & 33 & $\mathrm{CuO}$ & 0.0344 & 0.0357 & 0.0330 & 0.0014 & 0.0027 & $4.3 \%$ & $8.3 \%$ \\
\hline 4 & 33 & $\mathrm{Fe}_{2} \mathrm{O}_{3}$ & 10.5512 & 10.6552 & 10.7720 & -0.2208 & -0.1168 & $-2.0 \%$ & $-1.1 \%$ \\
\hline 4 & 33 & $\mathrm{~K}_{2} \mathrm{O}$ & 0.0653 & 0.0690 & 0.0690 & -0.0037 & 0.0000 & $-5.3 \%$ & $0.0 \%$ \\
\hline 4 & 33 & $\mathrm{La}_{2} \mathrm{O}_{3}$ & 0.0586 & 0.0586 & 0.0150 & 0.0436 & 0.0436 & $290.9 \%$ & $290.9 \%$ \\
\hline 4 & 33 & $\mathrm{Li}_{2} \mathrm{O}$ & 0.1076 & 0.1067 & 0.0000 & 0.1076 & 0.1067 & & \\
\hline
\end{tabular}


WSRC-STI-2007-00659

Revision 0

Appendix A

Table A4. Average Measured and Bias-Corrected Chemical Compositions Versus Targeted Compositions by Oxide by SB4 Variability Study Glass. (continued)

(100 -Batch 1)

\begin{tabular}{|c|c|c|c|c|c|c|c|c|c|}
\hline Set & Glass \# & Oxide & $\begin{array}{l}\text { Measured } \\
\text { (wt \%) }\end{array}$ & $\begin{array}{c}\text { Measured Bias- } \\
\text { Corrected (wt \%) }\end{array}$ & $\begin{array}{c}\text { Targeted } \\
\text { (wt \%) }\end{array}$ & $\begin{array}{c}\text { Diff of } \\
\text { Measured }\end{array}$ & $\begin{array}{c}\text { Diff of } \\
\text { Meas BC }\end{array}$ & $\begin{array}{l}\text { \% Diff of } \\
\text { Measured }\end{array}$ & $\begin{array}{l}\% \text { Diff of } \\
\text { Meas BC }\end{array}$ \\
\hline 4 & 33 & $\mathrm{MgO}$ & 0.6314 & 0.6626 & 0.6260 & 0.0054 & 0.0366 & $0.9 \%$ & $5.9 \%$ \\
\hline 4 & 33 & $\mathrm{MnO}$ & 2.3306 & 2.3193 & 2.3060 & 0.0246 & 0.0133 & $1.1 \%$ & $0.6 \%$ \\
\hline 4 & 33 & $\mathrm{Na}_{2} \mathrm{O}$ & 23.3878 & 22.6802 & 23.4000 & -0.0122 & -0.7198 & $-0.1 \%$ & $-3.1 \%$ \\
\hline 4 & 33 & $\mathrm{NiO}$ & 0.9343 & 1.0146 & 1.0220 & -0.0877 & -0.0074 & $-8.6 \%$ & $-0.7 \%$ \\
\hline 4 & 33 & $\mathrm{PbO}$ & 0.0347 & 0.0347 & 0.0430 & -0.0083 & -0.0083 & $-19.2 \%$ & $-19.2 \%$ \\
\hline 4 & 33 & $\mathrm{SiO}_{2}$ & 31.3942 & 31.3825 & 31.2000 & 0.1942 & 0.1825 & $0.6 \%$ & $0.6 \%$ \\
\hline 4 & 33 & $\mathrm{SO}_{4}$ & 0.4539 & 0.4539 & 0.5130 & -0.0591 & -0.0591 & $-11.5 \%$ & $-11.5 \%$ \\
\hline 4 & 33 & $\mathrm{TiO}_{2}$ & 0.2277 & 0.2408 & 0.2270 & 0.0007 & 0.0138 & $0.3 \%$ & $6.1 \%$ \\
\hline 4 & 33 & $\mathrm{ZnO}$ & 0.0308 & 0.0308 & 0.0330 & -0.0022 & -0.0022 & $-6.6 \%$ & $-6.6 \%$ \\
\hline 4 & 33 & $\mathrm{ZrO}_{2}$ & 0.0979 & 0.0979 & 0.1010 & -0.0031 & -0.0031 & $-3.0 \%$ & $-3.0 \%$ \\
\hline 4 & 33 & Sum & 100.1932 & 99.7113 & 100.0020 & 0.1912 & -0.2907 & $0.2 \%$ & $-0.3 \%$ \\
\hline 4 & 34 & $\mathrm{Al}_{2} \mathrm{O}_{3}$ & 19.1595 & 19.8386 & 19.2000 & -0.0405 & 0.6386 & $-0.2 \%$ & $3.3 \%$ \\
\hline 4 & 34 & $\mathrm{~B}_{2} \mathrm{O}_{3}$ & 18.2407 & 18.7074 & 18.0000 & 0.2407 & 0.7074 & $1.3 \%$ & $3.9 \%$ \\
\hline 4 & 34 & $\mathrm{BaO}$ & 0.0430 & 0.0472 & 0.0490 & -0.0060 & -0.0018 & $-12.3 \%$ & $-3.7 \%$ \\
\hline 4 & 34 & $\mathrm{CaO}$ & 1.9729 & 2.0886 & 2.0000 & -0.0271 & 0.0886 & $-1.4 \%$ & $4.4 \%$ \\
\hline 4 & 34 & $\mathrm{Ce}_{2} \mathrm{O}_{3}$ & 0.0966 & 0.0966 & 0.1030 & -0.0064 & -0.0064 & $-6.2 \%$ & $-6.2 \%$ \\
\hline 4 & 34 & $\mathrm{Cr}_{2} \mathrm{O}_{3}$ & 0.0833 & 0.0824 & 0.0900 & -0.0067 & -0.0076 & $-7.4 \%$ & $-8.4 \%$ \\
\hline 4 & 34 & $\mathrm{CuO}$ & 0.0341 & 0.0356 & 0.0330 & 0.0011 & 0.0026 & $3.4 \%$ & $7.9 \%$ \\
\hline 4 & 34 & $\mathrm{Fe}_{2} \mathrm{O}_{3}$ & 10.6441 & 10.7130 & 10.7720 & -0.1279 & -0.0590 & $-1.2 \%$ & $-0.5 \%$ \\
\hline 4 & 34 & $\mathrm{~K}_{2} \mathrm{O}$ & 0.0590 & 0.0621 & 0.0690 & -0.0100 & -0.0069 & $-14.5 \%$ & $-10.0 \%$ \\
\hline 4 & 34 & $\mathrm{La}_{2} \mathrm{O}_{3}$ & 0.0586 & 0.0586 & 0.0150 & 0.0436 & 0.0436 & $290.9 \%$ & $290.9 \%$ \\
\hline 4 & 34 & $\mathrm{Li}_{2} \mathrm{O}$ & 0.1076 & 0.1079 & 0.0000 & 0.1076 & 0.1079 & & \\
\hline 4 & 34 & $\mathrm{MgO}$ & 0.6223 & 0.6529 & 0.6260 & -0.0037 & 0.0269 & $-0.6 \%$ & $4.3 \%$ \\
\hline 4 & 34 & $\mathrm{MnO}$ & 2.3048 & 2.3548 & 2.3060 & -0.0012 & 0.0488 & $-0.1 \%$ & $2.1 \%$ \\
\hline 4 & 34 & $\mathrm{Na}_{2} \mathrm{O}$ & 18.8720 & 18.6776 & 19.2000 & -0.3280 & -0.5224 & $-1.7 \%$ & $-2.7 \%$ \\
\hline 4 & 34 & $\mathrm{NiO}$ & 0.9248 & 1.0067 & 1.0220 & -0.0972 & -0.0153 & $-9.5 \%$ & $-1.5 \%$ \\
\hline 4 & 34 & $\mathrm{PbO}$ & 0.0347 & 0.0347 & 0.0430 & -0.0083 & -0.0083 & $-19.2 \%$ & $-19.2 \%$ \\
\hline 4 & 34 & $\mathrm{SiO}_{2}$ & 26.3134 & 26.3411 & 25.6000 & 0.7134 & 0.7411 & $2.8 \%$ & $2.9 \%$ \\
\hline 4 & 34 & $\mathrm{SO}_{4}$ & 0.3453 & 0.3453 & 0.5130 & -0.1677 & -0.1677 & $-32.7 \%$ & $-32.7 \%$ \\
\hline 4 & 34 & $\mathrm{TiO}_{2}$ & 0.2231 & 0.2361 & 0.2270 & -0.0039 & 0.0091 & $-1.7 \%$ & $4.0 \%$ \\
\hline 4 & 34 & $\mathrm{ZnO}$ & 0.0296 & 0.0296 & 0.0330 & -0.0034 & -0.0034 & $-10.4 \%$ & $-10.4 \%$ \\
\hline 4 & 34 & $\mathrm{ZrO}_{2}$ & 0.0939 & 0.0939 & 0.1010 & -0.0071 & -0.0071 & $-7.0 \%$ & $-7.0 \%$ \\
\hline 4 & 34 & Sum & 100.2634 & 101.6107 & 100.0020 & 0.2614 & 1.6087 & $0.3 \%$ & $1.6 \%$ \\
\hline 4 & 35 & $\mathrm{Al}_{2} \mathrm{O}_{3}$ & 20.4066 & 20.3656 & 20.4000 & 0.0066 & -0.0344 & $0.0 \%$ & $-0.2 \%$ \\
\hline 4 & 35 & $\mathrm{~B}_{2} \mathrm{O}_{3}$ & 3.9524 & 4.0110 & 4.0000 & -0.0476 & 0.0110 & $-1.2 \%$ & $0.3 \%$ \\
\hline 4 & 35 & $\mathrm{BaO}$ & 0.0430 & 0.0468 & 0.0490 & -0.0060 & -0.0022 & $-12.3 \%$ & $-4.6 \%$ \\
\hline 4 & 35 & $\mathrm{CaO}$ & 12.1660 & 12.1711 & 12.0000 & 0.1660 & 0.1711 & $1.4 \%$ & $1.4 \%$ \\
\hline 4 & 35 & $\mathrm{Ce}_{2} \mathrm{O}_{3}$ & 0.0934 & 0.0934 & 0.1030 & -0.0096 & -0.0096 & $-9.3 \%$ & $-9.3 \%$ \\
\hline 4 & 35 & $\mathrm{Cr}_{2} \mathrm{O}_{3}$ & 0.0881 & 0.0867 & 0.0900 & -0.0019 & -0.0033 & $-2.2 \%$ & $-3.6 \%$ \\
\hline 4 & 35 & $\mathrm{CuO}$ & 0.0382 & 0.0396 & 0.0330 & 0.0052 & 0.0066 & $15.7 \%$ & $20.1 \%$ \\
\hline 4 & 35 & $\mathrm{Fe}_{2} \mathrm{O}_{3}$ & 10.9551 & 11.0640 & 10.7720 & 0.1831 & 0.2920 & $1.7 \%$ & $2.7 \%$ \\
\hline 4 & 35 & $\mathrm{~K}_{2} \mathrm{O}$ & 0.0738 & 0.0779 & 0.0690 & 0.0048 & 0.0089 & $6.9 \%$ & $12.9 \%$ \\
\hline 4 & 35 & $\mathrm{La}_{2} \mathrm{O}_{3}$ & 0.0586 & 0.0586 & 0.0150 & 0.0436 & 0.0436 & $290.9 \%$ & $290.9 \%$ \\
\hline 4 & 35 & $\mathrm{Li}_{2} \mathrm{O}$ & 0.1076 & 0.1067 & 0.0000 & 0.1076 & 0.1067 & & \\
\hline 4 & 35 & $\mathrm{MgO}$ & 0.6156 & 0.6461 & 0.6260 & -0.0104 & 0.0201 & $-1.7 \%$ & $3.2 \%$ \\
\hline 4 & 35 & $\mathrm{MnO}$ & 2.2951 & 2.2839 & 2.3060 & -0.0109 & -0.0221 & $-0.5 \%$ & $-1.0 \%$ \\
\hline 4 & 35 & $\mathrm{Na}_{2} \mathrm{O}$ & 20.5907 & 19.9680 & 20.4000 & 0.1907 & -0.4320 & $0.9 \%$ & $-2.1 \%$ \\
\hline 4 & 35 & $\mathrm{NiO}$ & 0.9178 & 0.9966 & 1.0220 & -0.1042 & -0.0254 & $-10.2 \%$ & $-2.5 \%$ \\
\hline 4 & 35 & $\mathrm{PbO}$ & 0.0315 & 0.0315 & 0.0430 & -0.0115 & -0.0115 & $-26.7 \%$ & $-26.7 \%$ \\
\hline 4 & 35 & $\mathrm{SiO}_{2}$ & 27.0621 & 27.0528 & 27.2000 & -0.1379 & -0.1472 & $-0.5 \%$ & $-0.5 \%$ \\
\hline 4 & 35 & $\mathrm{SO}_{4}$ & 0.4868 & 0.4868 & 0.5130 & -0.0262 & -0.0262 & $-5.1 \%$ & $-5.1 \%$ \\
\hline 4 & 35 & $\mathrm{TiO}_{2}$ & 0.2218 & 0.2346 & 0.2270 & -0.0052 & 0.0076 & $-2.3 \%$ & $3.3 \%$ \\
\hline 4 & 35 & $\mathrm{ZnO}$ & 0.0324 & 0.0324 & 0.0330 & -0.0006 & -0.0006 & $-1.9 \%$ & $-1.9 \%$ \\
\hline 4 & 35 & $\mathrm{ZrO}_{2}$ & 0.0952 & 0.0952 & 0.1010 & -0.0058 & -0.0058 & $-5.7 \%$ & $-5.7 \%$ \\
\hline 4 & 35 & Sum & 100.3320 & 99.9493 & 100.0020 & 0.3300 & -0.0527 & $0.3 \%$ & $-0.1 \%$ \\
\hline 4 & 36 & $\mathrm{Al}_{2} \mathrm{O}_{3}$ & 16.2875 & 16.2543 & 16.2000 & 0.0875 & 0.0543 & $0.5 \%$ & $0.3 \%$ \\
\hline 4 & 36 & $\mathrm{~B}_{2} \mathrm{O}_{3}$ & 17.7819 & 18.0458 & 18.0000 & -0.2181 & 0.0458 & $-1.2 \%$ & $0.3 \%$ \\
\hline 4 & 36 & $\mathrm{BaO}$ & 0.0438 & 0.0477 & 0.0490 & -0.0052 & -0.0013 & $-10.6 \%$ & $-2.7 \%$ \\
\hline 4 & 36 & $\mathrm{CaO}$ & 12.0681 & 12.0732 & 12.0000 & 0.0681 & 0.0732 & $0.6 \%$ & $0.6 \%$ \\
\hline 4 & 36 & $\mathrm{Ce}_{2} \mathrm{O}_{3}$ & 0.0955 & 0.0955 & 0.1030 & -0.0075 & -0.0075 & $-7.3 \%$ & $-7.3 \%$ \\
\hline 4 & 36 & $\mathrm{Cr}_{2} \mathrm{O}_{3}$ & 0.0859 & 0.0846 & 0.0900 & -0.0041 & -0.0054 & $-4.6 \%$ & $-6.0 \%$ \\
\hline 4 & 36 & $\mathrm{CuO}$ & 0.0460 & 0.0478 & 0.0330 & 0.0130 & 0.0148 & $39.4 \%$ & $44.7 \%$ \\
\hline 4 & 36 & $\mathrm{Fe}_{2} \mathrm{O}_{3}$ & 10.8121 & 10.9183 & 10.7720 & 0.0401 & 0.1463 & $0.4 \%$ & $1.4 \%$ \\
\hline 4 & 36 & $\mathrm{~K}_{2} \mathrm{O}$ & 0.0617 & 0.0652 & 0.0690 & -0.0073 & -0.0038 & $-10.5 \%$ & $-5.6 \%$ \\
\hline 4 & 36 & $\mathrm{La}_{2} \mathrm{O}_{3}$ & 0.0586 & 0.0586 & 0.0150 & 0.0436 & 0.0436 & $290.9 \%$ & $290.9 \%$ \\
\hline
\end{tabular}


WSRC-STI-2007-00659

Revision 0

Appendix A

Table A4. Average Measured and Bias-Corrected Chemical Compositions Versus Targeted Compositions by Oxide by SB4 Variability Study Glass. (continued)

(100 -Batch 1)

\begin{tabular}{|c|c|c|c|c|c|c|c|c|c|}
\hline Set & Glass \# & Oxide & $\begin{array}{l}\text { Measured } \\
\text { (wt \%) }\end{array}$ & $\begin{array}{c}\text { Measured Bias- } \\
\text { Corrected (wt \%) }\end{array}$ & $\begin{array}{c}\text { Targeted } \\
\text { (wt \%) }\end{array}$ & $\begin{array}{c}\text { Diff of } \\
\text { Measured }\end{array}$ & $\begin{array}{c}\text { Diff of } \\
\text { Meas BC }\end{array}$ & $\begin{array}{l}\text { \% Diff of } \\
\text { Measured }\end{array}$ & $\begin{array}{l}\% \text { Diff of } \\
\text { Meas BC }\end{array}$ \\
\hline 4 & 36 & $\mathrm{Li}_{2} \mathrm{O}$ & 0.1076 & 0.1067 & 0.0000 & 0.1076 & 0.1067 & & \\
\hline 4 & 36 & $\mathrm{MgO}$ & 0.6214 & 0.6522 & 0.6260 & -0.0046 & 0.0262 & $-0.7 \%$ & $4.2 \%$ \\
\hline 4 & 36 & $\mathrm{MnO}$ & 2.3242 & 2.3127 & 2.3060 & 0.0182 & 0.0067 & $0.8 \%$ & $0.3 \%$ \\
\hline 4 & 36 & $\mathrm{Na}_{2} \mathrm{O}$ & 16.4793 & 15.9806 & 16.2000 & 0.2793 & -0.2194 & $1.7 \%$ & $-1.4 \%$ \\
\hline 4 & 36 & $\mathrm{NiO}$ & 0.9124 & 0.9907 & 1.0220 & -0.1096 & -0.0313 & $-10.7 \%$ & $-3.1 \%$ \\
\hline 4 & 36 & $\mathrm{PbO}$ & 0.0361 & 0.0361 & 0.0430 & -0.0069 & -0.0069 & $-16.1 \%$ & $-16.1 \%$ \\
\hline 4 & 36 & $\mathrm{SiO}_{2}$ & 21.7139 & 21.7057 & 21.6000 & 0.1139 & 0.1057 & $0.5 \%$ & $0.5 \%$ \\
\hline 4 & 36 & $\mathrm{SO}_{4}$ & 0.3528 & 0.3528 & 0.5130 & -0.1602 & -0.1602 & $-31.2 \%$ & $-31.2 \%$ \\
\hline 4 & 36 & $\mathrm{TiO}_{2}$ & 0.2210 & 0.2337 & 0.2270 & -0.0060 & 0.0067 & $-2.6 \%$ & $3.0 \%$ \\
\hline 4 & 36 & $\mathrm{ZnO}$ & 0.0352 & 0.0352 & 0.0330 & 0.0022 & 0.0022 & $6.6 \%$ & $6.6 \%$ \\
\hline 4 & 36 & $\mathrm{ZrO}_{2}$ & 0.0969 & 0.0969 & 0.1010 & -0.0041 & -0.0041 & $-4.0 \%$ & $-4.0 \%$ \\
\hline 4 & 36 & Sum & 100.2419 & 100.1943 & 100.0020 & 0.2399 & 0.1923 & $0.2 \%$ & $0.2 \%$ \\
\hline 4 & 37 & $\mathrm{Al}_{2} \mathrm{O}_{3}$ & 37.9790 & 39.3269 & 39.0000 & -1.0211 & 0.3269 & $-2.6 \%$ & $0.8 \%$ \\
\hline 4 & 37 & $\mathrm{~B}_{2} \mathrm{O}_{3}$ & 4.0249 & 4.1279 & 4.0000 & 0.0249 & 0.1279 & $0.6 \%$ & $3.2 \%$ \\
\hline 4 & 37 & $\mathrm{BaO}$ & 0.0438 & 0.0481 & 0.0490 & -0.0052 & -0.0009 & $-10.6 \%$ & $-1.8 \%$ \\
\hline 4 & 37 & $\mathrm{CaO}$ & 1.9484 & 2.0628 & 2.0000 & -0.0516 & 0.0628 & $-2.6 \%$ & $3.1 \%$ \\
\hline 4 & 37 & $\mathrm{Ce}_{2} \mathrm{O}_{3}$ & 0.0946 & 0.0946 & 0.1030 & -0.0084 & -0.0084 & $-8.2 \%$ & $-8.2 \%$ \\
\hline 4 & 37 & $\mathrm{Cr}_{2} \mathrm{O}_{3}$ & 0.0731 & 0.0723 & 0.0900 & -0.0169 & -0.0177 & $-18.8 \%$ & $-19.7 \%$ \\
\hline 4 & 37 & $\mathrm{CuO}$ & 0.0307 & 0.0320 & 0.0330 & -0.0023 & -0.0010 & $-7.1 \%$ & $-3.0 \%$ \\
\hline 4 & 37 & $\mathrm{Fe}_{2} \mathrm{O}_{3}$ & 10.4189 & 10.4874 & 10.7720 & -0.3531 & -0.2846 & $-3.3 \%$ & $-2.6 \%$ \\
\hline 4 & 37 & $\mathrm{~K}_{2} \mathrm{O}$ & 0.0629 & 0.0662 & 0.0690 & -0.0061 & -0.0028 & $-8.8 \%$ & $-4.1 \%$ \\
\hline 4 & 37 & $\mathrm{La}_{2} \mathrm{O}_{3}$ & 0.0586 & 0.0586 & 0.0150 & 0.0436 & 0.0436 & $290.9 \%$ & $290.9 \%$ \\
\hline 4 & 37 & $\mathrm{Li}_{2} \mathrm{O}$ & 0.1076 & 0.1079 & 0.0000 & 0.1076 & 0.1079 & & \\
\hline 4 & 37 & $\mathrm{MgO}$ & 0.5895 & 0.6186 & 0.6260 & -0.0365 & -0.0074 & $-5.8 \%$ & $-1.2 \%$ \\
\hline 4 & 37 & $\mathrm{MnO}$ & 2.3016 & 2.3514 & 2.3060 & -0.0044 & 0.0454 & $-0.2 \%$ & $2.0 \%$ \\
\hline 4 & 37 & $\mathrm{Na}_{2} \mathrm{O}$ & 8.1588 & 8.0747 & 7.8000 & 0.3588 & 0.2747 & $4.6 \%$ & $3.5 \%$ \\
\hline 4 & 37 & $\mathrm{NiO}$ & 0.9534 & 1.0379 & 1.0220 & -0.0686 & 0.0159 & $-6.7 \%$ & $1.6 \%$ \\
\hline 4 & 37 & $\mathrm{PbO}$ & 0.0342 & 0.0342 & 0.0430 & -0.0088 & -0.0088 & $-20.5 \%$ & $-20.5 \%$ \\
\hline 4 & 37 & $\mathrm{SiO}_{2}$ & 32.5174 & 32.5519 & 31.2000 & 1.3174 & 1.3519 & $4.2 \%$ & $4.3 \%$ \\
\hline 4 & 37 & $\mathrm{SO}_{4}$ & 0.1498 & 0.1498 & 0.5130 & -0.3632 & -0.3632 & $-70.8 \%$ & $-70.8 \%$ \\
\hline 4 & 37 & $\mathrm{TiO}_{2}$ & 0.2218 & 0.2348 & 0.2270 & -0.0052 & 0.0078 & $-2.3 \%$ & $3.4 \%$ \\
\hline 4 & 37 & $\mathrm{ZnO}$ & 0.0293 & 0.0293 & 0.0330 & -0.0037 & -0.0037 & $-11.4 \%$ & $-11.4 \%$ \\
\hline 4 & 37 & $\mathrm{ZrO}_{2}$ & 0.0821 & 0.0821 & 0.1010 & -0.0189 & -0.0189 & $-18.8 \%$ & $-18.8 \%$ \\
\hline 4 & 37 & Sum & 99.8803 & 101.6493 & 100.0020 & -0.1217 & 1.6473 & $-0.1 \%$ & $1.6 \%$ \\
\hline 4 & 38 & $\mathrm{Al}_{2} \mathrm{O}_{3}$ & 31.1768 & 32.2840 & 32.0000 & -0.8233 & 0.2840 & $-2.6 \%$ & $0.9 \%$ \\
\hline 4 & 38 & $\mathrm{~B}_{2} \mathrm{O}_{3}$ & 18.7076 & 19.1861 & 18.0000 & 0.7076 & 1.1861 & $3.9 \%$ & $6.6 \%$ \\
\hline 4 & 38 & $\mathrm{BaO}$ & 0.0466 & 0.0512 & 0.0490 & -0.0024 & 0.0022 & $-4.9 \%$ & $4.5 \%$ \\
\hline 4 & 38 & $\mathrm{CaO}$ & 1.9659 & 2.0815 & 2.0000 & -0.0341 & 0.0815 & $-1.7 \%$ & $4.1 \%$ \\
\hline 4 & 38 & $\mathrm{Ce}_{2} \mathrm{O}_{3}$ & 0.0960 & 0.0960 & 0.1030 & -0.0070 & -0.0070 & $-6.8 \%$ & $-6.8 \%$ \\
\hline 4 & 38 & $\mathrm{Cr}_{2} \mathrm{O}_{3}$ & 0.0833 & 0.0824 & 0.0900 & -0.0067 & -0.0076 & $-7.4 \%$ & $-8.4 \%$ \\
\hline 4 & 38 & $\mathrm{CuO}$ & 0.0332 & 0.0346 & 0.0330 & 0.0002 & 0.0016 & $0.5 \%$ & $4.9 \%$ \\
\hline 4 & 38 & $\mathrm{Fe}_{2} \mathrm{O}_{3}$ & 11.0123 & 11.0810 & 10.7720 & 0.2403 & 0.3090 & $2.2 \%$ & $2.9 \%$ \\
\hline 4 & 38 & $\mathrm{~K}_{2} \mathrm{O}$ & 0.0614 & 0.0646 & 0.0690 & -0.0076 & -0.0044 & $-11.0 \%$ & $-6.4 \%$ \\
\hline 4 & 38 & $\mathrm{La}_{2} \mathrm{O}_{3}$ & 0.0586 & 0.0586 & 0.0150 & 0.0436 & 0.0436 & $290.9 \%$ & $290.9 \%$ \\
\hline 4 & 38 & $\mathrm{Li}_{2} \mathrm{O}$ & 0.1076 & 0.1079 & 0.0000 & 0.1076 & 0.1079 & & \\
\hline 4 & 38 & $\mathrm{MgO}$ & 0.6376 & 0.6690 & 0.6260 & 0.0116 & 0.0430 & $1.9 \%$ & $6.9 \%$ \\
\hline 4 & 38 & $\mathrm{MnO}$ & 2.2887 & 2.3383 & 2.3060 & -0.0173 & 0.0323 & $-0.8 \%$ & $1.4 \%$ \\
\hline 4 & 38 & $\mathrm{Na}_{2} \mathrm{O}$ & 6.7333 & 6.6638 & 6.4000 & 0.3333 & 0.2638 & $5.2 \%$ & $4.1 \%$ \\
\hline 4 & 38 & $\mathrm{NiO}$ & 0.9633 & 1.0486 & 1.0220 & -0.0587 & 0.0266 & $-5.7 \%$ & $2.6 \%$ \\
\hline 4 & 38 & $\mathrm{PbO}$ & 0.0339 & 0.0339 & 0.0430 & -0.0091 & -0.0091 & $-21.1 \%$ & $-21.1 \%$ \\
\hline 4 & 38 & $\mathrm{SiO}_{2}$ & 26.5808 & 26.6085 & 25.6000 & 0.9808 & 1.0085 & $3.8 \%$ & $3.9 \%$ \\
\hline 4 & 38 & $\mathrm{SO}_{4}$ & 0.1498 & 0.1498 & 0.5130 & -0.3632 & -0.3632 & $-70.8 \%$ & $-70.8 \%$ \\
\hline 4 & 38 & $\mathrm{TiO}_{2}$ & 0.2248 & 0.2379 & 0.2270 & -0.0022 & 0.0109 & $-1.0 \%$ & $4.8 \%$ \\
\hline 4 & 38 & $\mathrm{ZnO}$ & 0.0299 & 0.0299 & 0.0330 & -0.0031 & -0.0031 & $-9.5 \%$ & $-9.5 \%$ \\
\hline 4 & 38 & $\mathrm{ZrO}_{2}$ & 0.0922 & 0.0922 & 0.1010 & -0.0088 & -0.0088 & $-8.7 \%$ & $-8.7 \%$ \\
\hline 4 & 38 & Sum & 101.0835 & 102.9997 & 100.0020 & 1.0815 & 2.9977 & $1.1 \%$ & $3.0 \%$ \\
\hline 4 & 39 & $\mathrm{Al}_{2} \mathrm{O}_{3}$ & 33.5386 & 34.7255 & 34.0000 & -0.4614 & 0.7255 & $-1.4 \%$ & $2.1 \%$ \\
\hline 4 & 39 & $\mathrm{~B}_{2} \mathrm{O}_{3}$ & 3.9685 & 4.0701 & 4.0000 & -0.0315 & 0.0701 & $-0.8 \%$ & $1.8 \%$ \\
\hline 4 & 39 & $\mathrm{BaO}$ & 0.0410 & 0.0451 & 0.0490 & -0.0080 & -0.0039 & $-16.3 \%$ & $-8.1 \%$ \\
\hline 4 & 39 & $\mathrm{CaO}$ & 11.9037 & 12.6102 & 12.0000 & -0.0963 & 0.6102 & $-0.8 \%$ & $5.1 \%$ \\
\hline 4 & 39 & $\mathrm{Ce}_{2} \mathrm{O}_{3}$ & 0.0949 & 0.0949 & 0.1030 & -0.0081 & -0.0081 & $-7.9 \%$ & $-7.9 \%$ \\
\hline 4 & 39 & $\mathrm{Cr}_{2} \mathrm{O}_{3}$ & 0.0625 & 0.0618 & 0.0900 & -0.0275 & -0.0282 & $-30.6 \%$ & $-31.3 \%$ \\
\hline 4 & 39 & $\mathrm{CuO}$ & 0.0322 & 0.0337 & 0.0330 & -0.0008 & 0.0007 & $-2.3 \%$ & $2.0 \%$ \\
\hline 4 & 39 & $\mathrm{Fe}_{2} \mathrm{O}_{3}$ & 10.6441 & 10.7122 & 10.7720 & -0.1279 & -0.0598 & $-1.2 \%$ & $-0.6 \%$ \\
\hline 4 & 39 & $\mathrm{~K}_{2} \mathrm{O}$ & 0.0602 & 0.0633 & 0.0690 & -0.0088 & -0.0057 & $-12.7 \%$ & $-8.2 \%$ \\
\hline
\end{tabular}


WSRC-STI-2007-00659

Revision 0

Appendix A

Table A4. Average Measured and Bias-Corrected Chemical Compositions Versus Targeted Compositions by Oxide by SB4 Variability Study Glass. (continued)

(100 -Batch 1)

\begin{tabular}{|c|c|c|c|c|c|c|c|c|c|}
\hline Set & Glass \# & Oxide & $\begin{array}{l}\text { Measured } \\
\text { (wt \%) }\end{array}$ & $\begin{array}{c}\text { Measured Bias- } \\
\text { Corrected (wt \%) }\end{array}$ & $\begin{array}{c}\text { Targeted } \\
\text { (wt \%) }\end{array}$ & $\begin{array}{c}\text { Diff of } \\
\text { Measured }\end{array}$ & $\begin{array}{c}\text { Diff of } \\
\text { Meas BC }\end{array}$ & $\begin{array}{l}\% \text { Diff of } \\
\text { Measured }\end{array}$ & $\begin{array}{l}\text { \% Diff of } \\
\text { Meas BC }\end{array}$ \\
\hline 4 & 39 & $\mathrm{La}_{2} \mathrm{O}_{3}$ & 0.0586 & 0.0586 & 0.0150 & 0.0436 & 0.0436 & $290.9 \%$ & $290.9 \%$ \\
\hline 4 & 39 & $\mathrm{Li}_{2} \mathrm{O}$ & 0.1076 & 0.1079 & 0.0000 & 0.1076 & 0.1079 & & \\
\hline 4 & 39 & $\mathrm{MgO}$ & 0.6119 & 0.6421 & 0.6260 & -0.0141 & 0.0161 & $-2.3 \%$ & $2.6 \%$ \\
\hline 4 & 39 & $\mathrm{MnO}$ & 2.2983 & 2.3483 & 2.3060 & -0.0077 & 0.0423 & $-0.3 \%$ & $1.8 \%$ \\
\hline 4 & 39 & $\mathrm{Na}_{2} \mathrm{O}$ & 7.0635 & 6.9907 & 6.8000 & 0.2635 & 0.1907 & $3.9 \%$ & $2.8 \%$ \\
\hline 4 & 39 & $\mathrm{NiO}$ & 0.8838 & 0.9620 & 1.0220 & -0.1382 & -0.0600 & $-13.5 \%$ & $-5.9 \%$ \\
\hline 4 & 39 & $\mathrm{PbO}$ & 0.0347 & 0.0347 & 0.0430 & -0.0083 & -0.0083 & $-19.2 \%$ & $-19.2 \%$ \\
\hline 4 & 39 & $\mathrm{SiO}_{2}$ & 27.7574 & 27.7864 & 27.2000 & 0.5574 & 0.5864 & $2.0 \%$ & $2.2 \%$ \\
\hline 4 & 39 & $\mathrm{SO}_{4}$ & 0.1498 & 0.1498 & 0.5130 & -0.3632 & -0.3632 & $-70.8 \%$ & $-70.8 \%$ \\
\hline 4 & 39 & $\mathrm{TiO}_{2}$ & 0.2173 & 0.2299 & 0.2270 & -0.0097 & 0.0029 & $-4.3 \%$ & $1.3 \%$ \\
\hline 4 & 39 & $\mathrm{ZnO}$ & 0.0293 & 0.0293 & 0.0330 & -0.0037 & -0.0037 & $-11.4 \%$ & $-11.4 \%$ \\
\hline 4 & 39 & $\mathrm{ZrO}_{2}$ & 0.0929 & 0.0929 & 0.1010 & -0.0081 & -0.0081 & $-8.1 \%$ & $-8.1 \%$ \\
\hline 4 & 39 & Sum & 99.6510 & 101.8493 & 100.0020 & -0.3510 & 1.8473 & $-0.4 \%$ & $1.8 \%$ \\
\hline 4 & 40 & $\mathrm{Al}_{2} \mathrm{O}_{3}$ & 27.5867 & 27.5313 & 27.0000 & 0.5867 & 0.5313 & $2.2 \%$ & $2.0 \%$ \\
\hline 4 & 40 & $\mathrm{~B}_{2} \mathrm{O}_{3}$ & 18.0878 & 18.3564 & 18.0000 & 0.0878 & 0.3564 & $0.5 \%$ & $2.0 \%$ \\
\hline 4 & 40 & $\mathrm{BaO}$ & 0.0444 & 0.0483 & 0.0490 & -0.0046 & -0.0007 & $-9.4 \%$ & $-1.5 \%$ \\
\hline 4 & 40 & $\mathrm{CaO}$ & 12.2220 & 12.2277 & 12.0000 & 0.2220 & 0.2277 & $1.9 \%$ & $1.9 \%$ \\
\hline 4 & 40 & $\mathrm{Ce}_{2} \mathrm{O}_{3}$ & 0.0940 & 0.0940 & 0.1030 & -0.0090 & -0.0090 & $-8.7 \%$ & $-8.7 \%$ \\
\hline 4 & 40 & $\mathrm{Cr}_{2} \mathrm{O}_{3}$ & 0.0621 & 0.0612 & 0.0900 & -0.0279 & -0.0288 & $-31.0 \%$ & $-32.0 \%$ \\
\hline 4 & 40 & $\mathrm{CuO}$ & 0.0354 & 0.0367 & 0.0330 & 0.0024 & 0.0037 & $7.2 \%$ & $11.3 \%$ \\
\hline 4 & 40 & $\mathrm{Fe}_{2} \mathrm{O}_{3}$ & 10.1080 & 10.2055 & 10.7720 & -0.6640 & -0.5665 & $-6.2 \%$ & $-5.3 \%$ \\
\hline 4 & 40 & $\mathrm{~K}_{2} \mathrm{O}$ & 0.0650 & 0.0687 & 0.0690 & -0.0040 & -0.0003 & $-5.7 \%$ & $-0.5 \%$ \\
\hline 4 & 40 & $\mathrm{La}_{2} \mathrm{O}_{3}$ & 0.0586 & 0.0586 & 0.0150 & 0.0436 & 0.0436 & $290.9 \%$ & $290.9 \%$ \\
\hline 4 & 40 & $\mathrm{Li}_{2} \mathrm{O}$ & 0.1076 & 0.1067 & 0.0000 & 0.1076 & 0.1067 & & \\
\hline 4 & 40 & $\mathrm{MgO}$ & 0.6260 & 0.6570 & 0.6260 & 0.0000 & 0.0310 & $0.0 \%$ & $5.0 \%$ \\
\hline 4 & 40 & $\mathrm{MnO}$ & 2.3242 & 2.3128 & 2.3060 & 0.0182 & 0.0068 & $0.8 \%$ & $0.3 \%$ \\
\hline 4 & 40 & $\mathrm{Na}_{2} \mathrm{O}$ & 5.7223 & 5.5485 & 5.4000 & 0.3223 & 0.1485 & $6.0 \%$ & $2.7 \%$ \\
\hline 4 & 40 & $\mathrm{NiO}$ & 0.8211 & 0.8916 & 1.0220 & -0.2009 & -0.1304 & $-19.7 \%$ & $-12.8 \%$ \\
\hline 4 & 40 & $\mathrm{PbO}$ & 0.0342 & 0.0342 & 0.0430 & -0.0088 & -0.0088 & $-20.5 \%$ & $-20.5 \%$ \\
\hline 4 & 40 & $\mathrm{SiO}_{2}$ & 21.8743 & 21.8655 & 21.6000 & 0.2743 & 0.2655 & $1.3 \%$ & $1.2 \%$ \\
\hline 4 & 40 & $\mathrm{SO}_{4}$ & 0.1498 & 0.1498 & 0.5130 & -0.3632 & -0.3632 & $-70.8 \%$ & $-70.8 \%$ \\
\hline 4 & 40 & $\mathrm{TiO}_{2}$ & 0.2218 & 0.2346 & 0.2270 & -0.0052 & 0.0076 & $-2.3 \%$ & $3.3 \%$ \\
\hline 4 & 40 & $\mathrm{ZnO}$ & 0.0305 & 0.0305 & 0.0330 & -0.0025 & -0.0025 & $-7.6 \%$ & $-7.6 \%$ \\
\hline 4 & 40 & $\mathrm{ZrO}_{2}$ & 0.0962 & 0.0962 & 0.1010 & -0.0048 & -0.0048 & $-4.7 \%$ & $-4.7 \%$ \\
\hline 4 & 40 & Sum & 100.3721 & 100.6158 & 100.0020 & 0.3701 & 0.6138 & $0.4 \%$ & $0.6 \%$ \\
\hline 1 & 100 & $\mathrm{Al}_{2} \mathrm{O}_{3}$ & 4.7584 & 4.8770 & 4.8770 & -0.1186 & 0.0000 & $-2.4 \%$ & $0.0 \%$ \\
\hline 1 & 100 & $\mathrm{~B}_{2} \mathrm{O}_{3}$ & 7.5963 & 7.7770 & 7.7770 & -0.1807 & 0.0000 & $-2.3 \%$ & $0.0 \%$ \\
\hline 1 & 100 & $\mathrm{BaO}$ & 0.1401 & 0.1510 & 0.1510 & -0.0109 & 0.0000 & $-7.2 \%$ & $0.0 \%$ \\
\hline 1 & 100 & $\mathrm{CaO}$ & 1.1450 & 1.2200 & 1.2200 & -0.0750 & 0.0000 & $-6.1 \%$ & $0.0 \%$ \\
\hline 1 & 100 & $\mathrm{Ce}_{2} \mathrm{O}_{3}$ & 0.0059 & 0.0059 & 0.0000 & 0.0059 & 0.0059 & & \\
\hline 1 & 100 & $\mathrm{Cr}_{2} \mathrm{O}_{3}$ & 0.1089 & 0.1070 & 0.1070 & 0.0019 & 0.0000 & $1.8 \%$ & $0.0 \%$ \\
\hline 1 & 100 & $\mathrm{CuO}$ & 0.3928 & 0.3990 & 0.3990 & -0.0062 & 0.0000 & $-1.6 \%$ & $0.0 \%$ \\
\hline 1 & 100 & $\mathrm{Fe}_{2} \mathrm{O}_{3}$ & 12.5266 & 12.8390 & 12.8390 & -0.3124 & 0.0000 & $-2.4 \%$ & $0.0 \%$ \\
\hline 1 & 100 & $\mathrm{~K}_{2} \mathrm{O}$ & 3.0838 & 3.3270 & 3.3270 & -0.2432 & 0.0000 & $-7.3 \%$ & $0.0 \%$ \\
\hline 1 & 100 & $\mathrm{La}_{2} \mathrm{O}_{3}$ & 0.0586 & 0.0586 & 0.0000 & 0.0586 & 0.0586 & & \\
\hline 1 & 100 & $\mathrm{Li}_{2} \mathrm{O}$ & 4.3524 & 4.4290 & 4.4290 & -0.0766 & 0.0000 & $-1.7 \%$ & $0.0 \%$ \\
\hline 1 & 100 & $\mathrm{MgO}$ & 1.3674 & 1.4190 & 1.4190 & -0.0516 & 0.0000 & $-3.6 \%$ & $0.0 \%$ \\
\hline 1 & 100 & $\mathrm{MnO}$ & 1.7173 & 1.7260 & 1.7260 & -0.0087 & 0.0000 & $-0.5 \%$ & $0.0 \%$ \\
\hline 1 & 100 & $\mathrm{Na}_{2} \mathrm{O}$ & 9.2158 & 9.0030 & 9.0030 & 0.2128 & 0.0000 & $2.4 \%$ & $0.0 \%$ \\
\hline 1 & 100 & $\mathrm{NiO}$ & 0.7037 & 0.7510 & 0.7510 & -0.0473 & 0.0000 & $-6.3 \%$ & $0.0 \%$ \\
\hline 1 & 100 & $\mathrm{PbO}$ & 0.0054 & 0.0054 & 0.0000 & 0.0054 & 0.0054 & & \\
\hline 1 & 100 & $\mathrm{SiO}_{2}$ & 49.0613 & 50.2200 & 50.2200 & -1.1587 & 0.0000 & $-2.3 \%$ & $0.0 \%$ \\
\hline 1 & 100 & $\mathrm{SO}_{4}$ & 0.1498 & 0.1498 & 0.0000 & 0.1498 & 0.1498 & & \\
\hline 1 & 100 & $\mathrm{TiO}_{2}$ & 0.6494 & 0.6770 & 0.6770 & -0.0276 & 0.0000 & $-4.1 \%$ & $0.0 \%$ \\
\hline 1 & 100 & $\mathrm{ZnO}$ & 0.0062 & 0.0062 & 0.0000 & 0.0062 & 0.0062 & & \\
\hline 1 & 100 & $\mathrm{ZrO}_{2}$ & 0.0859 & 0.0859 & 0.0980 & -0.0121 & -0.0121 & $-12.4 \%$ & $-12.4 \%$ \\
\hline 1 & 100 & Sum & 97.1309 & 99.2338 & 99.0200 & -1.8891 & 0.2138 & $-1.9 \%$ & $0.2 \%$ \\
\hline 2 & 100 & $\mathrm{Al}_{2} \mathrm{O}_{3}$ & 4.7489 & 4.8770 & 4.8770 & -0.1281 & 0.0000 & $-2.6 \%$ & $0.0 \%$ \\
\hline 2 & 100 & $\mathrm{~B}_{2} \mathrm{O}_{3}$ & 7.6687 & 7.7770 & 7.7770 & -0.1083 & 0.0000 & $-1.4 \%$ & $0.0 \%$ \\
\hline 2 & 100 & $\mathrm{BaO}$ & 0.1414 & 0.1510 & 0.1510 & -0.0096 & 0.0000 & $-6.3 \%$ & $0.0 \%$ \\
\hline 2 & 100 & $\mathrm{CaO}$ & 1.1779 & 1.2200 & 1.2200 & -0.0421 & 0.0000 & $-3.5 \%$ & $0.0 \%$ \\
\hline 2 & 100 & $\mathrm{Ce}_{2} \mathrm{O}_{3}$ & 0.0059 & 0.0059 & 0.0000 & 0.0059 & 0.0059 & & \\
\hline 2 & 100 & $\mathrm{Cr}_{2} \mathrm{O}_{3}$ & 0.1105 & 0.1070 & 0.1070 & 0.0035 & 0.0000 & $3.2 \%$ & $0.0 \%$ \\
\hline 2 & 100 & $\mathrm{CuO}$ & 0.3904 & 0.3990 & 0.3990 & -0.0086 & 0.0000 & $-2.2 \%$ & $0.0 \%$ \\
\hline 2 & 100 & $\mathrm{Fe}_{2} \mathrm{O}_{3}$ & 12.5861 & 12.8390 & 12.8390 & -0.2529 & 0.0000 & $-2.0 \%$ & $0.0 \%$ \\
\hline
\end{tabular}


WSRC-STI-2007-00659

Revision 0

Appendix A

Table A4. Average Measured and Bias-Corrected Chemical Compositions Versus Targeted Compositions by Oxide by SB4 Variability Study Glass. (continued)

(100 -Batch 1)

\begin{tabular}{|c|c|c|c|c|c|c|c|c|c|}
\hline Set & Glass \# & Oxide & $\begin{array}{l}\text { Measured } \\
\text { (wt \%) }\end{array}$ & $\begin{array}{c}\text { Measured Bias- } \\
\text { Corrected (wt \%) }\end{array}$ & $\begin{array}{c}\text { Targeted } \\
\text { (wt \%) }\end{array}$ & $\begin{array}{c}\text { Diff of } \\
\text { Measured }\end{array}$ & $\begin{array}{c}\text { Diff of } \\
\text { Meas BC }\end{array}$ & $\begin{array}{l}\% \text { Diff of } \\
\text { Measured }\end{array}$ & $\begin{array}{l}\text { \% Diff of } \\
\text { Meas BC }\end{array}$ \\
\hline 2 & 100 & $\mathrm{~K}_{2} \mathrm{O}$ & 3.0436 & 3.3270 & 3.3270 & -0.2834 & 0.0000 & $-8.5 \%$ & $0.0 \%$ \\
\hline 2 & 100 & $\mathrm{La}_{2} \mathrm{O}_{3}$ & 0.0586 & 0.0586 & 0.0000 & 0.0586 & 0.0586 & & \\
\hline 2 & 100 & $\mathrm{Li}_{2} \mathrm{O}$ & 4.3722 & 4.4290 & 4.4290 & -0.0568 & 0.0000 & $-1.3 \%$ & $0.0 \%$ \\
\hline 2 & 100 & $\mathrm{MgO}$ & 1.3706 & 1.4190 & 1.4190 & -0.0484 & 0.0000 & $-3.4 \%$ & $0.0 \%$ \\
\hline 2 & 100 & $\mathrm{MnO}$ & 1.7259 & 1.7260 & 1.7260 & -0.0001 & 0.0000 & $0.0 \%$ & $0.0 \%$ \\
\hline 2 & 100 & $\mathrm{Na}_{2} \mathrm{O}$ & 9.0732 & 9.0030 & 9.0030 & 0.0702 & 0.0000 & $0.8 \%$ & $0.0 \%$ \\
\hline 2 & 100 & $\mathrm{NiO}$ & 0.7074 & 0.7510 & 0.7510 & -0.0436 & 0.0000 & $-5.8 \%$ & $0.0 \%$ \\
\hline 2 & 100 & $\mathrm{PbO}$ & 0.0054 & 0.0054 & 0.0000 & 0.0054 & 0.0054 & & \\
\hline 2 & 100 & $\mathrm{SiO}_{2}$ & 49.3643 & 50.2200 & 50.2200 & -0.8557 & 0.0000 & $-1.7 \%$ & $0.0 \%$ \\
\hline 2 & 100 & $\mathrm{SO}_{4}$ & 0.1498 & 0.1498 & 0.0000 & 0.1498 & 0.1498 & & \\
\hline 2 & 100 & $\mathrm{TiO}_{2}$ & 0.6525 & 0.6770 & 0.6770 & -0.0245 & 0.0000 & $-3.6 \%$ & $0.0 \%$ \\
\hline 2 & 100 & $\mathrm{ZnO}$ & 0.0062 & 0.0062 & 0.0000 & 0.0062 & 0.0062 & & \\
\hline 2 & 100 & $\mathrm{ZrO}_{2}$ & 0.0879 & 0.0879 & 0.0980 & -0.0101 & -0.0101 & $-10.3 \%$ & $-10.3 \%$ \\
\hline 2 & 100 & Sum & 97.4474 & 99.2358 & 99.0200 & -1.5726 & 0.2158 & $-1.6 \%$ & $0.2 \%$ \\
\hline 3 & 100 & $\mathrm{Al}_{2} \mathrm{O}_{3}$ & 4.7631 & 4.8770 & 4.8770 & -0.1139 & 0.0000 & $-2.3 \%$ & $0.0 \%$ \\
\hline 3 & 100 & $\mathrm{~B}_{2} \mathrm{O}_{3}$ & 7.4836 & 7.7770 & 7.7770 & -0.2934 & 0.0000 & $-3.8 \%$ & $0.0 \%$ \\
\hline 3 & 100 & $\mathrm{BaO}$ & 0.1408 & 0.1510 & 0.1510 & -0.0102 & 0.0000 & $-6.8 \%$ & $0.0 \%$ \\
\hline 3 & 100 & $\mathrm{CaO}$ & 1.1938 & 1.2200 & 1.2200 & -0.0262 & 0.0000 & $-2.2 \%$ & $0.0 \%$ \\
\hline 3 & 100 & $\mathrm{Ce}_{2} \mathrm{O}_{3}$ & 0.0059 & 0.0059 & 0.0000 & 0.0059 & 0.0059 & & \\
\hline 3 & 100 & $\mathrm{Cr}_{2} \mathrm{O}_{3}$ & 0.1100 & 0.1070 & 0.1070 & 0.0030 & 0.0000 & $2.8 \%$ & $0.0 \%$ \\
\hline 3 & 100 & $\mathrm{CuO}$ & 0.3874 & 0.3990 & 0.3990 & -0.0116 & 0.0000 & $-2.9 \%$ & $0.0 \%$ \\
\hline 3 & 100 & $\mathrm{Fe}_{2} \mathrm{O}_{3}$ & 12.3895 & 12.8390 & 12.8390 & -0.4495 & 0.0000 & $-3.5 \%$ & $0.0 \%$ \\
\hline 3 & 100 & $\mathrm{~K}_{2} \mathrm{O}$ & 3.0557 & 3.3270 & 3.3270 & -0.2713 & 0.0000 & $-8.2 \%$ & $0.0 \%$ \\
\hline 3 & 100 & $\mathrm{La}_{2} \mathrm{O}_{3}$ & 0.0586 & 0.0586 & 0.0000 & 0.0586 & 0.0586 & & \\
\hline 3 & 100 & $\mathrm{Li}_{2} \mathrm{O}$ & 4.3596 & 4.4290 & 4.4290 & -0.0694 & 0.0000 & $-1.6 \%$ & $0.0 \%$ \\
\hline 3 & 100 & $\mathrm{MgO}$ & 1.3573 & 1.4190 & 1.4190 & -0.0617 & 0.0000 & $-4.3 \%$ & $0.0 \%$ \\
\hline 3 & 100 & $\mathrm{MnO}$ & 1.7238 & 1.7260 & 1.7260 & -0.0022 & 0.0000 & $-0.1 \%$ & $0.0 \%$ \\
\hline 3 & 100 & $\mathrm{Na}_{2} \mathrm{O}$ & 9.1574 & 9.0030 & 9.0030 & 0.1544 & 0.0000 & $1.7 \%$ & $0.0 \%$ \\
\hline 3 & 100 & $\mathrm{NiO}$ & 0.6997 & 0.7510 & 0.7510 & -0.0513 & 0.0000 & $-6.8 \%$ & $0.0 \%$ \\
\hline 3 & 100 & $\mathrm{PbO}$ & 0.0054 & 0.0054 & 0.0000 & 0.0054 & 0.0054 & & \\
\hline 3 & 100 & $\mathrm{SiO}_{2}$ & 49.2217 & 50.2200 & 50.2200 & -0.9983 & 0.0000 & $-2.0 \%$ & $0.0 \%$ \\
\hline 3 & 100 & $\mathrm{SO}_{4}$ & 0.1498 & 0.1498 & 0.0000 & 0.1498 & 0.1498 & & \\
\hline 3 & 100 & $\mathrm{TiO}_{2}$ & 0.6451 & 0.6770 & 0.6770 & -0.0319 & 0.0000 & $-4.7 \%$ & $0.0 \%$ \\
\hline 3 & 100 & $\mathrm{ZnO}$ & 0.0062 & 0.0062 & 0.0000 & 0.0062 & 0.0062 & & \\
\hline 3 & 100 & $\mathrm{ZrO}_{2}$ & 0.0871 & 0.0871 & 0.0980 & -0.0109 & -0.0109 & $-11.1 \%$ & $-11.1 \%$ \\
\hline 3 & 100 & Sum & 97.0015 & 99.2350 & 99.0200 & -2.0185 & 0.2150 & $-2.0 \%$ & $0.2 \%$ \\
\hline 4 & 100 & $\mathrm{Al}_{2} \mathrm{O}_{3}$ & 4.7993 & 4.8770 & 4.8770 & -0.0777 & 0.0000 & $-1.6 \%$ & $0.0 \%$ \\
\hline 4 & 100 & $\mathrm{~B}_{2} \mathrm{O}_{3}$ & 7.6231 & 7.7770 & 7.7770 & -0.1539 & 0.0000 & $-2.0 \%$ & $0.0 \%$ \\
\hline 4 & 100 & $\mathrm{BaO}$ & 0.1382 & 0.1510 & 0.1510 & -0.0128 & 0.0000 & $-8.5 \%$ & $0.0 \%$ \\
\hline 4 & 100 & $\mathrm{CaO}$ & 1.1866 & 1.2200 & 1.2200 & -0.0334 & 0.0000 & $-2.7 \%$ & $0.0 \%$ \\
\hline 4 & 100 & $\mathrm{Ce}_{2} \mathrm{O}_{3}$ & 0.0059 & 0.0059 & 0.0000 & 0.0059 & 0.0059 & & \\
\hline 4 & 100 & $\mathrm{Cr}_{2} \mathrm{O}_{3}$ & 0.1084 & 0.1070 & 0.1070 & 0.0014 & 0.0000 & $1.3 \%$ & $0.0 \%$ \\
\hline 4 & 100 & $\mathrm{CuO}$ & 0.3832 & 0.3990 & 0.3990 & -0.0158 & 0.0000 & $-4.0 \%$ & $0.0 \%$ \\
\hline 4 & 100 & $\mathrm{Fe}_{2} \mathrm{O}_{3}$ & 12.7339 & 12.8390 & 12.8390 & -0.1051 & 0.0000 & $-0.8 \%$ & $0.0 \%$ \\
\hline 4 & 100 & $\mathrm{~K}_{2} \mathrm{O}$ & 3.1581 & 3.3270 & 3.3270 & -0.1689 & 0.0000 & $-5.1 \%$ & $0.0 \%$ \\
\hline 4 & 100 & $\mathrm{La}_{2} \mathrm{O}_{3}$ & 0.0586 & 0.0586 & 0.0000 & 0.0586 & 0.0586 & & \\
\hline 4 & 100 & $\mathrm{Li}_{2} \mathrm{O}$ & 4.4439 & 4.4290 & 4.4290 & 0.0149 & 0.0000 & $0.3 \%$ & $0.0 \%$ \\
\hline 4 & 100 & $\mathrm{MgO}$ & 1.3522 & 1.4190 & 1.4190 & -0.0668 & 0.0000 & $-4.7 \%$ & $0.0 \%$ \\
\hline 4 & 100 & $\mathrm{MnO}$ & 1.7119 & 1.7260 & 1.7260 & -0.0141 & 0.0000 & $-0.8 \%$ & $0.0 \%$ \\
\hline 4 & 100 & $\mathrm{Na}_{2} \mathrm{O}$ & 9.1911 & 9.0030 & 9.0030 & 0.1881 & 0.0000 & $2.1 \%$ & $0.0 \%$ \\
\hline 4 & 100 & $\mathrm{NiO}$ & 0.6908 & 0.7510 & 0.7510 & -0.0602 & 0.0000 & $-8.0 \%$ & $0.0 \%$ \\
\hline 4 & 100 & $\mathrm{PbO}$ & 0.0054 & 0.0054 & 0.0000 & 0.0054 & 0.0054 & & \\
\hline 4 & 100 & $\mathrm{SiO}_{2}$ & 50.2022 & 50.2200 & 50.2200 & -0.0178 & 0.0000 & $0.0 \%$ & $0.0 \%$ \\
\hline 4 & 100 & $\mathrm{SO}_{4}$ & 0.1498 & 0.1498 & 0.0000 & 0.1498 & 0.1498 & & \\
\hline 4 & 100 & $\mathrm{TiO}_{2}$ & 0.6400 & 0.6770 & 0.6770 & -0.0370 & 0.0000 & $-5.5 \%$ & $0.0 \%$ \\
\hline 4 & 100 & $\mathrm{ZnO}$ & 0.0062 & 0.0062 & 0.0000 & 0.0062 & 0.0062 & & \\
\hline 4 & 100 & $\mathrm{ZrO}_{2}$ & 0.0904 & 0.0904 & 0.0980 & -0.0076 & -0.0076 & $-7.8 \%$ & $-7.8 \%$ \\
\hline 4 & 100 & Sum & 98.6791 & 99.2383 & 99.0200 & -0.3409 & 0.2183 & $-0.3 \%$ & $0.2 \%$ \\
\hline
\end{tabular}




\section{Exhibit A1. Oxide Measurements in Analytical Sequence for Samples Prepared Using the LM Method. (continued)}
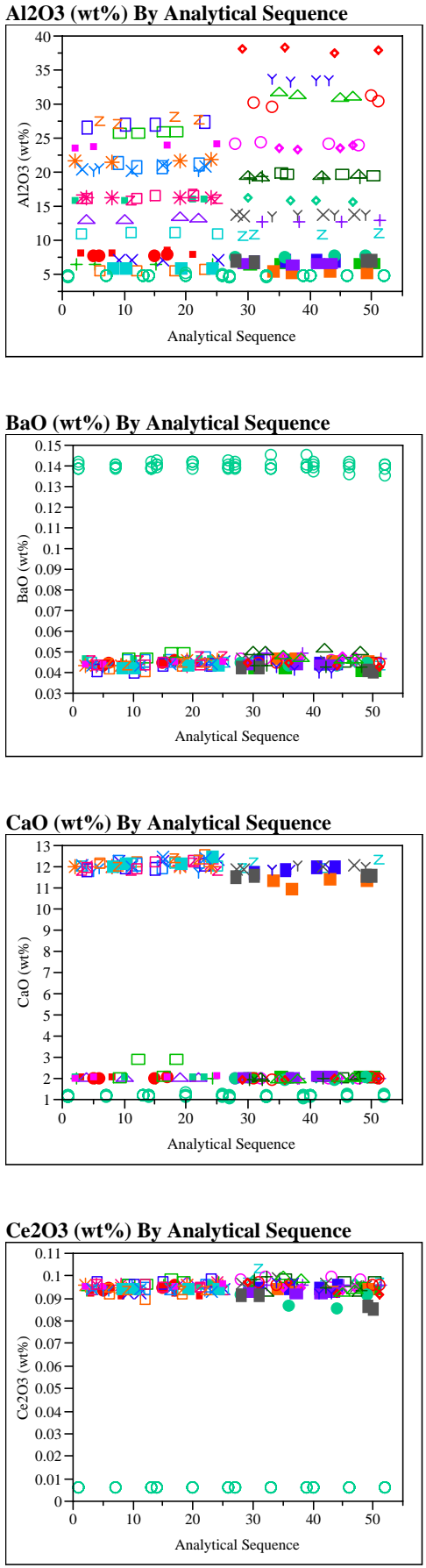
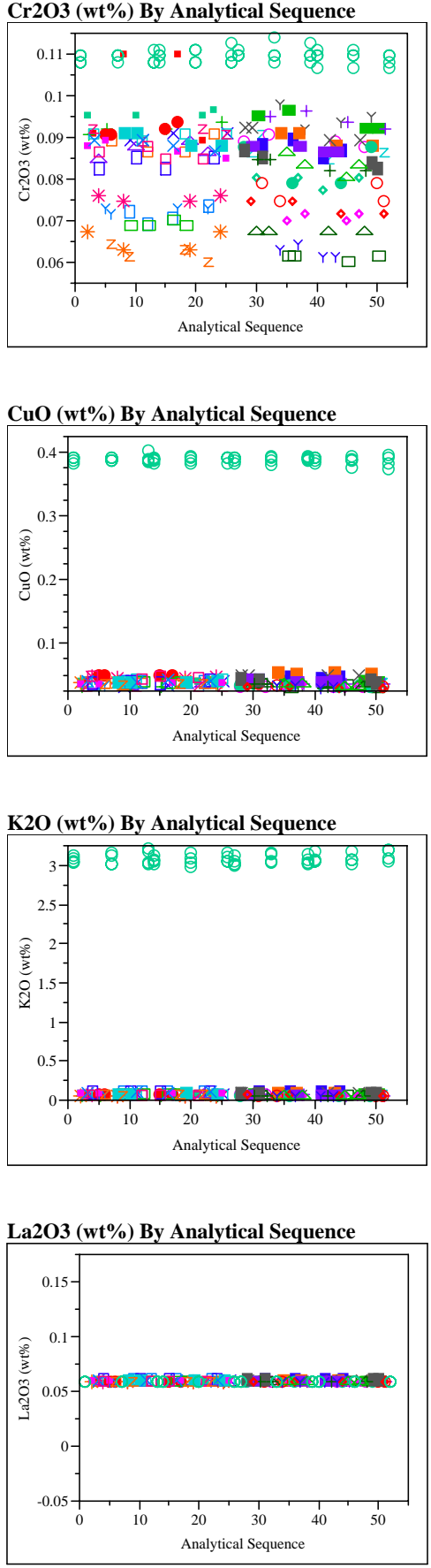
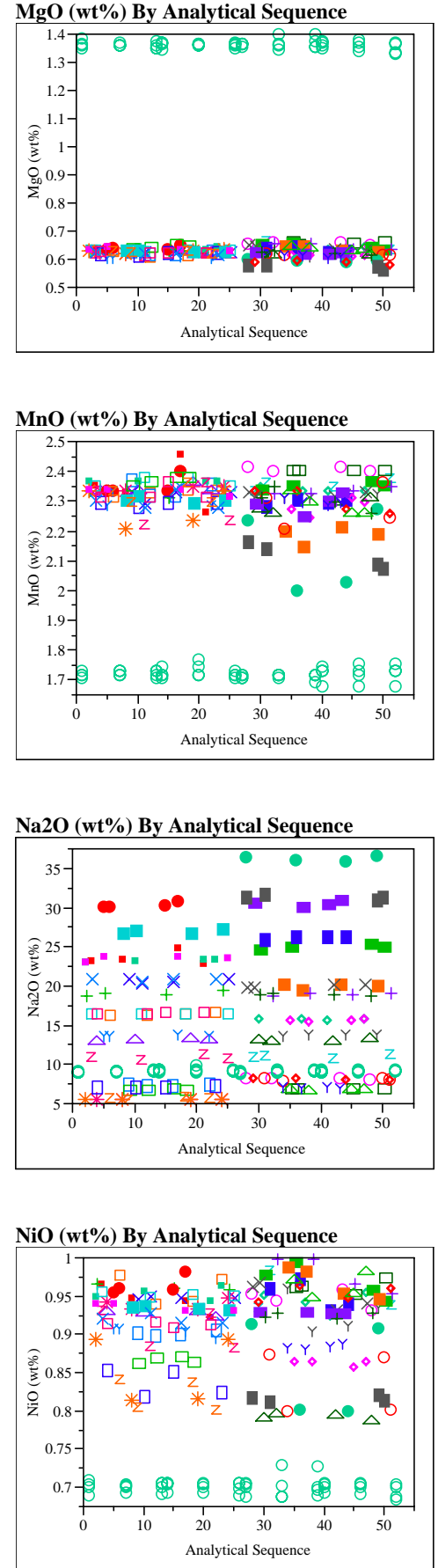


\section{Exhibit A1. Oxide Measurements in Analytical Sequence for Samples Prepared Using the LM Method. (continued)}
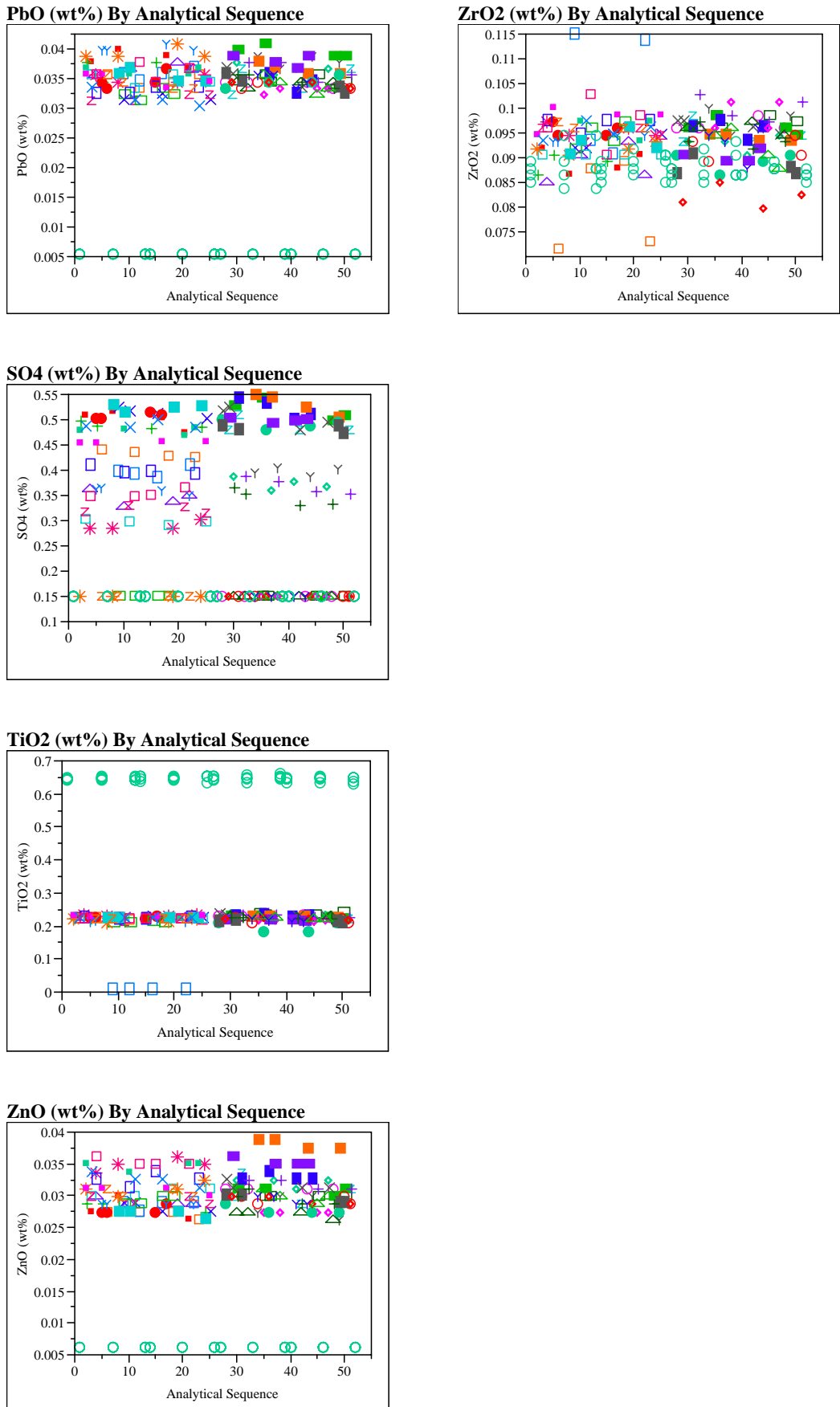
WSRC-STI-2007-00659

Revision 0

Appendix A

Exhibit A2. Oxide Measurements in Analytical Sequence for Samples Prepared Using the PF Method.

B2O3 (wt\%) By Analytical Sequence

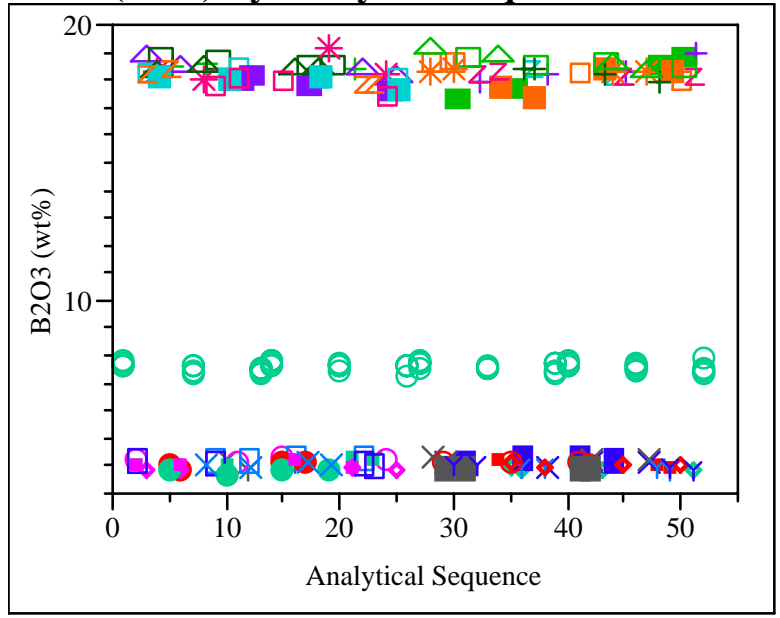

Fe2O3 (wt\%) By Analytical Sequence

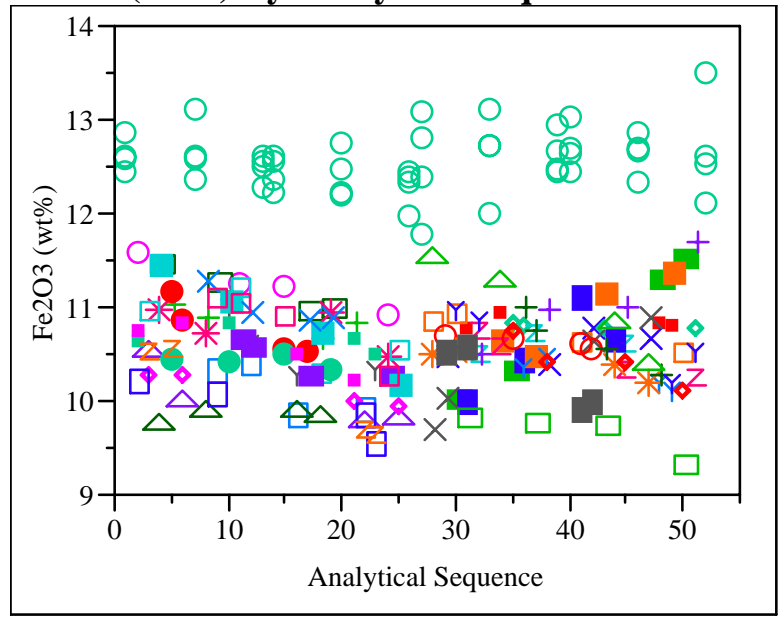

Li2O (wt\%) By Analytical Sequence

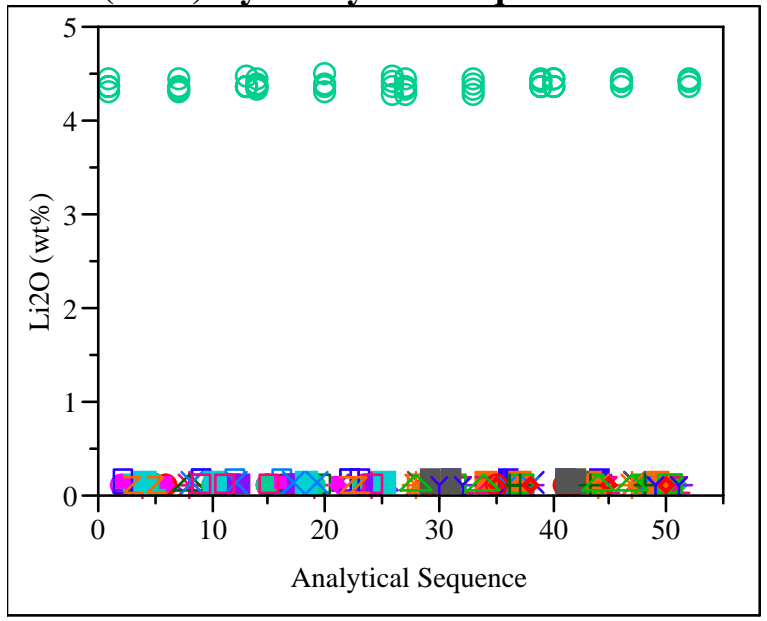

SiO2 (wt\%) By Analytical Sequence

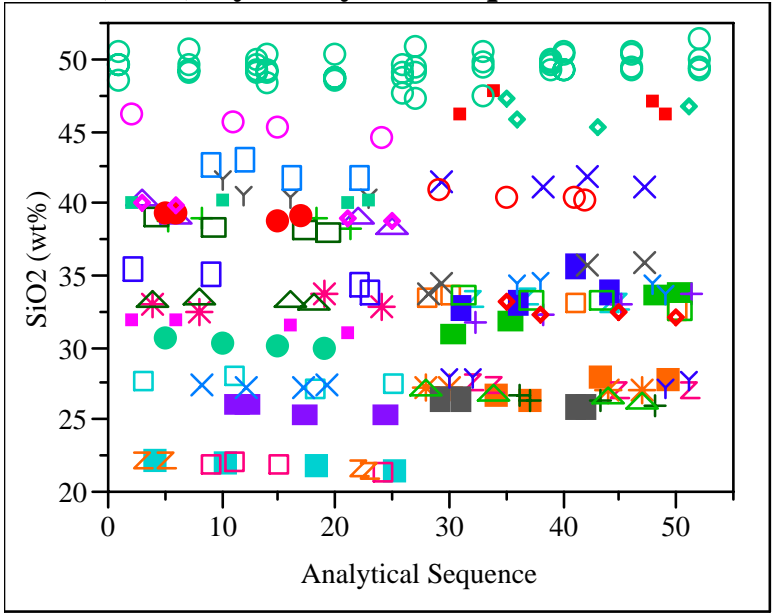




\section{Exhibit A3. PSAL Measurements by Analytical Block for Samples of the Batch 1 Standard Glass Prepared Using the LM Method. (continued)}

Oneway Analysis of Al2O3 (wt\%) By Block/Sub-Block

Reference Value $=\mathbf{4 . 8 7 7} \mathrm{wt} \%$

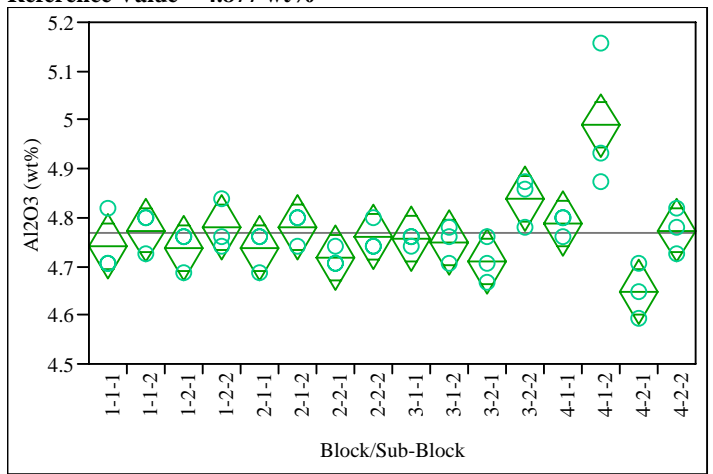

Oneway Anova

Summary of Fit

$\begin{array}{lr}\text { Rsquare } & 0.699026 \\ \text { Adj Rsquare } & 0.557944 \\ \text { Root Mean Square Error } & 0.055892 \\ \text { Mean of Response } & 4.767445 \\ \text { Observations (or Sum Wgts) } & 48\end{array}$

Analysis of Variance

Source DF Sum of Squares Mean Square F Ratio Prob $>$ F

$\begin{array}{llllll}\text { Block/Sub-Block } & 15 & 0.23217524 & 0.015478 & 4.9548 & <.0001\end{array}$

Error

$\begin{array}{lll}32 & 0.09996589 & 0.003124\end{array}$

Means for Oneway Anova

Level Number Mean Std Error Lower 95\% Upper 95\%

$\begin{array}{llllll}1-1-1 & 3 & 4.74265 & 0.03227 & 4.6769 & 4.8084\end{array}$

$\begin{array}{llllll}1-1-2 & 3 & 4.77414 & 0.03227 & 4.7084 & 4.8399\end{array}$

$\begin{array}{llllll}1-2-1 & 3 & 4.73635 & 0.03227 & 4.6706 & 4.8021\end{array}$

$\begin{array}{llllll}1-2-2 & 3 & 4.78044 & 0.03227 & 4.7147 & 4.8462\end{array}$

$\begin{array}{llllll}2-1-1 & 3 & 4.73635 & 0.03227 & 4.6706 & 4.8021\end{array}$

$\begin{array}{llllll}2-1-2 & 3 & 4.78044 & 0.03227 & 4.7147 & 4.8462\end{array}$

$\begin{array}{llllll}2-2-1 & 3 & 4.71745 & 0.03227 & 4.6517 & 4.7832\end{array}$

$\begin{array}{llllll}2-2-2 & 3 & 4.76154 & 0.03227 & 4.6958 & 4.8273\end{array}$

$\begin{array}{llllll}3-1-1 & 3 & 4.75524 & 0.03227 & 4.6895 & 4.8210\end{array}$

$\begin{array}{llllll}3-1-2 & 3 & 4.74894 & 0.03227 & 4.6832 & 4.8147\end{array}$

$\begin{array}{llllll}3-2-1 & 3 & 4.71115 & 0.03227 & 4.6454 & 4.7769\end{array}$

$\begin{array}{llllll}3-2-2 & 3 & 4.83712 & 0.03227 & 4.7714 & 4.9029\end{array}$

$\begin{array}{llllll}4-1-1 & 3 & 4.78673 & 0.03227 & 4.7210 & 4.8525\end{array}$

$\begin{array}{llllll}4-1-2 & 3 & 4.98828 & 0.03227 & 4.9225 & 5.0540\end{array}$

$\begin{array}{llllll}4-2-1 & 3 & 4.64817 & 0.03227 & 4.5824 & 4.7139\end{array}$

$\begin{array}{llllll}4-2-2 & 3 & 4.77414 & 0.03227 & 4.7084 & 4.8399\end{array}$

Std Error uses a pooled estimate of error variance
Oneway Analysis of BaO (wt\%) By Block/Sub-Block Reference Value $=0.151$ wt $\%$

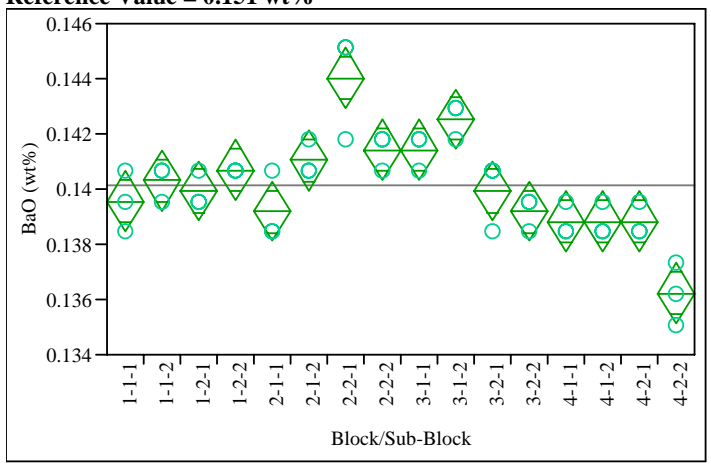

Oneway Anova

Summary of Fit

Rsquare

0.84058

Adj Rsquare

0.765851

Root Mean Square Error $\quad 0.000926$

Mean of Response $\quad 0.140121$

Observations (or Sum Wgts)

48

Analysis of Variance

$\begin{array}{lrrrrr}\text { Source } & \text { DF } & \text { Sum of Squares } & \text { Mean Square } & \text { F Ratio } & \text { Prob }>\text { F } \\ \text { Block/Sub-Block } & 15 & 0.00014460 & 9.6402 \mathrm{e}-6 & 11.2485 & <.0001 \\ \text { Error } & 32 & 0.00002742 & 8.5702 \mathrm{e}-7 & & \\ \text { C. Total } & 47 & 0.00017203 & & & \end{array}$

Means for Oneway Anova

Level Number Mean Std Error Lower 95\% Upper 95\%

$\begin{array}{llllll}1-1-1 & 3 & 0.139563 & 0.00053 & 0.13847 & 0.14065\end{array}$

$\begin{array}{llllll}1-1-2 & 3 & 0.140307 & 0.00053 & 0.13922 & 0.14140\end{array}$

$\begin{array}{llllll}1-2-1 & 3 & 0.139935 & 0.00053 & 0.13885 & 0.14102\end{array}$

$\begin{array}{llllll}1-2-2 & 3 & 0.140679 & 0.00053 & 0.13959 & 0.14177\end{array}$

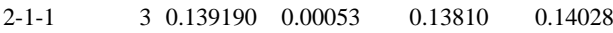

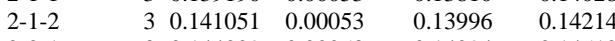

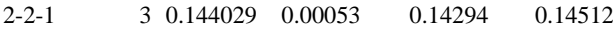

$\begin{array}{llllll}2-2-2 & 3 & 0.141423 & 0.00053 & 0.14033 & 0.14251\end{array}$

$\begin{array}{llllll}3-1-1 & 3 & 0.141423 & 0.00053 & 0.14033 & 0.14251\end{array}$

$\begin{array}{llllll}3-1-2 & 3 & 0.142540 & 0.00053 & 0.14145 & 0.14363\end{array}$

$\begin{array}{llllll}3-2-1 & 3 & 0.139935 & 0.00053 & 0.13885 & 0.14102\end{array}$

$\begin{array}{llllll}3-2-2 & 3 & 0.139190 & 0.00053 & 0.13810 & 0.14028\end{array}$

$\begin{array}{llllll}4-1-1 & 3 & 0.138818 & 0.00053 & 0.13773 & 0.13991\end{array}$

$\begin{array}{llllll}4-1-2 & 3 & 0.138818 & 0.00053 & 0.13773 & 0.13991\end{array}$

$\begin{array}{llllll}4-2-1 & 3 & 0.138818 & 0.00053 & 0.13773 & 0.13991\end{array}$

$\begin{array}{llllll}4-2-2 & 3 & 0.136213 & 0.00053 & 0.13512 & 0.13730\end{array}$

Std Error uses a pooled estimate of error variance 


\section{Exhibit A3. PSAL Measurements by Analytical Block for Samples of the Batch 1 Standard Glass Prepared Using the LM Method. (continued)}

Oneway Analysis of $\mathrm{CaO}$ (wt \%) By Block/Sub-Block

Reference Value $=1.220 \mathrm{wt} \%$

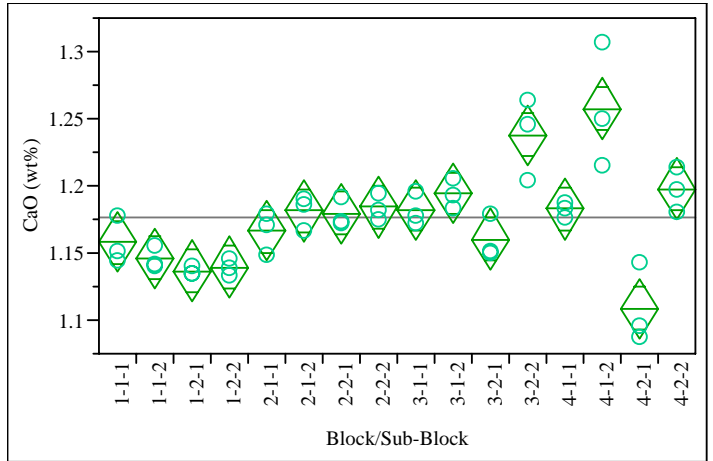

Oneway Anova

Summary of Fit

Rsquare $\quad 0.839348$

Adj Rsquare $\quad 0.764043$

Root Mean Square Error $\quad 0.019153$

Mean of Response 1.175824

Observations (or Sum Wgts) $\quad 48$

Analysis of Variance

Source DF Sum of Squares Mean Square F Ratio Prob > F

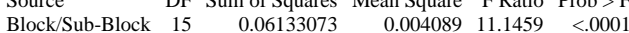

$\begin{array}{llll}\text { Block/Sub-Block } & 15 & 0.06133073 & 0.004089 \\ \text { Error } & 32 & 0.01173873 & 0.000367\end{array}$

C. Total

$32-0.01173873$

Means for Oneway Anova

Level Number Mean Std Error Lower 95\% Upper 95\%

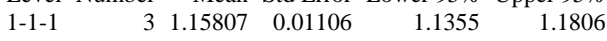

$\begin{array}{llllll}1-1-2 & 3 & 1.14594 & 0.01106 & 1.1234 & 1.1685\end{array}$

$\begin{array}{llllll}1-2-1 & 3 & 1.13662 & 0.01106 & 1.1141 & 1.1591\end{array}$

$\begin{array}{llllll}1-2-2 & 3 & 1.13942 & 0.01106 & 1.1169 & 1.1619\end{array}$

$\begin{array}{llllll}1-2-2 & 3 & 1.13942 & 0.01106 & 1.1169 & 1.1619 \\ 2-1-1 & 3 & 1.16647 & 0.01106 & 1.1439 & 1.1890\end{array}$

$\begin{array}{llllll}2-1-2 & 3 & 1.18139 & 0.01106 & 1.1589 & 1.2039\end{array}$

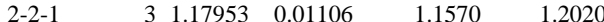

$\begin{array}{llllll}2-2-2 & 3 & 1.18419 & 0.01106 & 1.1617 & 1.2067\end{array}$

$\begin{array}{llllll}3-1-1 & 3 & 1.18232 & 0.01106 & 1.1598 & 1.2048\end{array}$

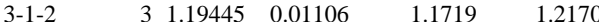

$\begin{array}{llllll}3-2-1 & 3 & 1.16040 & 0.01106 & 1.1379 & 1.1829\end{array}$

$\begin{array}{llllll}3-2-2 & 3 & 1.23783 & 0.01106 & 1.2153 & 1.2603\end{array}$

$\begin{array}{llllll}4-1-1 & 3 & 1.18279 & 0.01106 & 1.1603 & 1.2053\end{array}$

$\begin{array}{llllll}4-1-2 & 3 & 1.25741 & 0.01106 & 1.2349 & 1.2799\end{array}$

$\begin{array}{llllll}4-2-1 & 3 & 1.10863 & 0.01106 & 1.0861 & 1.1312\end{array}$

$\begin{array}{llllll}4-2-2 & 3 & 1.19772 & 0.01106 & 1.1752 & 1.2202\end{array}$

Std Error uses a pooled estimate of error variance
Oneway Analysis of $\mathrm{Ce} 2 \mathrm{O} 3$ (wt\%) By Block/Sub-Block

Reference Value $=0.0 \mathrm{wt} \%$

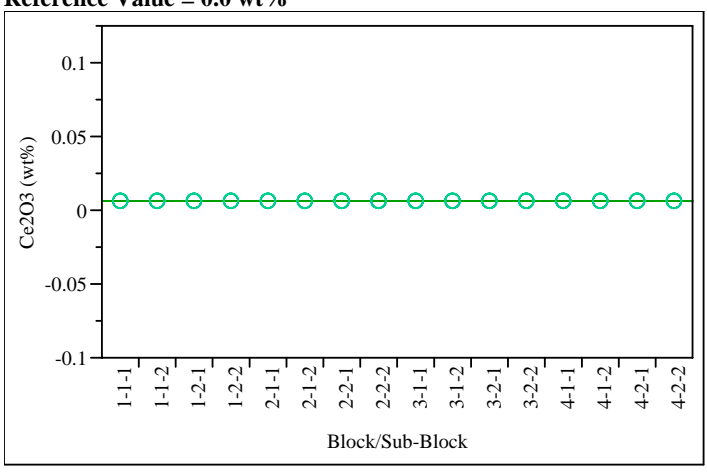

Oneway Anova

Summary of Fit

Rsquare

Adj Rsquare

Root Mean Square Error

Mean of Response

Analysis of Variance

$\begin{array}{lrrrrr}\text { Source } & \text { DF } & \text { Sum of Squares } & \text { Mean Square } & \text { F Ratio } & \text { Prob > F } \\ \text { Block/Sub-Block } & 15 & 3.25 \mathrm{e}-34 & 2.167 \mathrm{e}-35 & \text {. } & \text {. } \\ \text { Error } & 32 & 0 & 0 & & \\ \text { C. Total } & 47 & 3.25 \mathrm{e}-34 & & \end{array}$

$47 \quad 3.25 \mathrm{e}-34$

Means for Oneway Anova

Level Number Mean Std Error Lower 95\% Upper 95\%

$\begin{array}{llllll}1-1-1 & 3 & 0.005857 & 0 & 0.00586 & 0.00586\end{array}$

$\begin{array}{llllll}1-1-2 & 3 & 0.005857 & 0 & 0.00586 & 0.00586\end{array}$

$\begin{array}{llllll}1-2-1 & 3 & 0.005857 & 0 & 0.00586 & 0.00586\end{array}$

$\begin{array}{llllll}1-2-2 & 3 & 0.005857 & 0 & 0.00586 & 0.00586\end{array}$

$\begin{array}{llllll}2-1-1 & 3 & 0.005857 & 0 & 0.00586 & 0.00586\end{array}$

$\begin{array}{llllll}2-1-2 & 3 & 0.005857 & 0 & 0.00586 & 0.00586\end{array}$

$\begin{array}{llllll}2-2-1 & 3 & 0.005857 & 0 & 0.00586 & 0.00586\end{array}$

$\begin{array}{llllll}2-2-2 & 3 & 0.005857 & 0 & 0.00586 & 0.00586\end{array}$

$\begin{array}{llllll}3-1-1 & 3 & 0.005857 & 0 & 0.00586 & 0.00586\end{array}$

$\begin{array}{llllll}3-1-2 & 3 & 0.005857 & 0 & 0.00586 & 0.00586\end{array}$

$\begin{array}{llllll}3-2-1 & 3 & 0.005857 & 0 & 0.00586 & 0.00586\end{array}$

$\begin{array}{llllll}3-2-2 & 3 & 0.005857 & 0 & 0.00586 & 0.00586\end{array}$

$\begin{array}{llllll}4-1-1 & 3 & 0.005857 & 0 & 0.00586 & 0.00586\end{array}$

$\begin{array}{llllll}4-1-2 & 3 & 0.005857 & 0 & 0.00586 & 0.00586\end{array}$

$\begin{array}{llllll}4-2-1 & 3 & 0.005857 & 0 & 0.00586 & 0.00586\end{array}$

$\begin{array}{llllll}4-2-2 & 3 & 0.005857 & 0 & 0.00586 & 0.00586\end{array}$

Std Error uses a pooled estimate of error variance 


\section{Exhibit A3. PSAL Measurements by Analytical Block for Samples of the Batch 1 Standard Glass Prepared Using the LM Method. (continued)}

Oneway Analysis of Cr2O3 (wt \%) By Block/Sub-Block

Reference Value $=\mathbf{0 . 1 0 7} \mathrm{wt} \%$

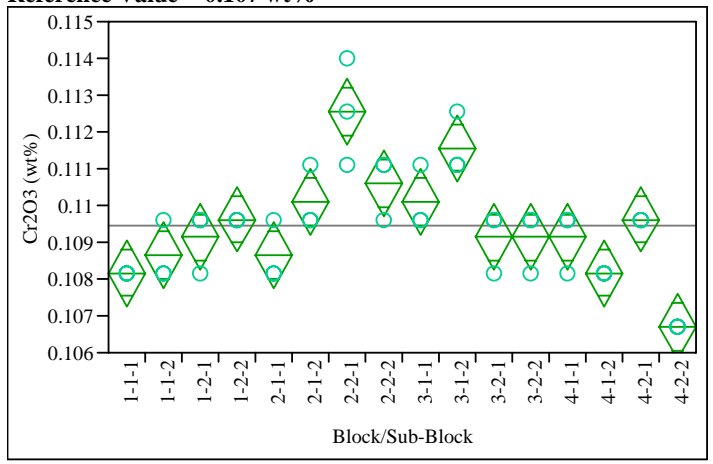

Oneway Anova

Summary of Fit

$\begin{array}{lr}\text { Rsquare } & 0.824027 \\ \text { Adj Rsquare } & 0.74154 \\ \text { Root Mean Square Error } & 0.000761 \\ \text { Mean of Response } & 0.109437 \\ \text { Observations (or Sum Wgts) } & 48\end{array}$

Analysis of Variance

Source DF Sum of Squares Mean Square F Ratio Prob > F

Block/Sub-Block $15 \quad 0.00008670 \quad 5.7798 \mathrm{e}-6 \quad 9.9897 \quad<.0001$

\begin{tabular}{lll} 
Error & 32 & 0.0000185 \\
\hline
\end{tabular}

Means for Oneway Anova

Level Number Mean Std Error Lower 95\% Upper 95\%

$\begin{array}{llllll}1-1-1 & 3 & 0.108158 & 0.00044 & 0.10726 & 0.10905\end{array}$

$\begin{array}{llllll}1-1-2 & 3 & 0.108646 & 0.00044 & 0.10775 & 0.10954\end{array}$

$\begin{array}{llllll}1-2-1 & 3 & 0.109133 & 0.00044 & 0.10824 & 0.11003\end{array}$

$\begin{array}{llllll}1-2-2 & 3 & 0.109620 & 0.00044 & 0.10873 & 0.11051\end{array}$

$\begin{array}{llllll}2-1-1 & 3 & 0.108646 & 0.00044 & 0.10775 & 0.10954\end{array}$

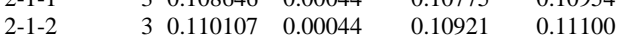

$\begin{array}{llllll}2-2-1 & 3 & 0.112543 & 0.00044 & 0.11165 & 0.11344\end{array}$

$\begin{array}{llllll}2-2-2 & 3 & 0.110594 & 0.00044 & 0.10970 & 0.11149\end{array}$

$\begin{array}{llllll}3-1-1 & 3 & 0.110107 & 0.00044 & 0.10921 & 0.11100\end{array}$

$\begin{array}{llllll}3-1-2 & 3 & 0.111569 & 0.00044 & 0.11067 & 0.11246\end{array}$

$\begin{array}{llllll}3-2-1 & 3 & 0.109133 & 0.00044 & 0.10824 & 0.11003\end{array}$

$\begin{array}{llllll}3-2-2 & 3 & 0.109133 & 0.00044 & 0.10824 & 0.11003\end{array}$

$\begin{array}{llllll}3-2-2 & 3 & 0.109133 & 0.00044 & 0.10824 & 0.11003 \\ 4-1-1 & 3 & 0.109133 & 0.00044 & 0.10824 & 0.11003\end{array}$

$\begin{array}{llllll}4-1-2 & 3 & 0.108158 & 0.00044 & 0.10726 & 0.10905\end{array}$

$\begin{array}{llllll}4-2-1 & 3 & 0.109620 & 0.00044 & 0.10873 & 0.11051\end{array}$

$\begin{array}{llllll}4-2-2 & 3 & 0.106697 & 0.00044 & 0.10580 & 0.10759\end{array}$

Std Error uses a pooled estimate of error variance
Oneway Analysis of CuO (wt\%) By Block/Sub-Block Reference Value $=0.399 \mathrm{wt} \%$

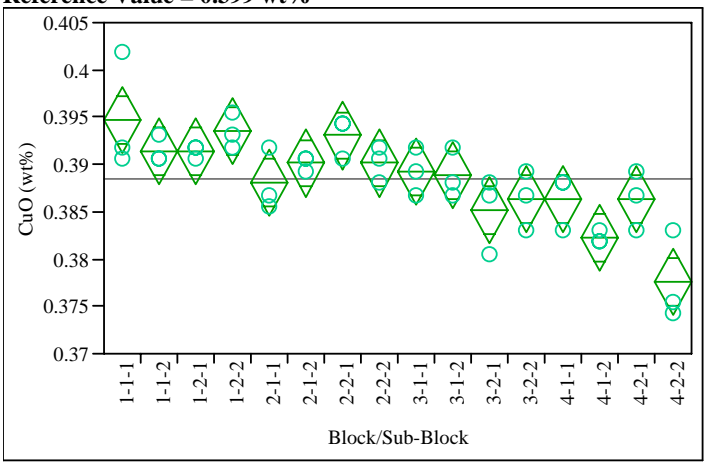

Oneway Anova

Summary of Fit

Rsquare

Adj Rsquare

0.633433

Root Mean Square Error $\quad 0.003007$

Mean of Response

0.388423

Observations (or Sum Wgts)

\section{Analysis of Variance}

$\begin{array}{lrrrr}\text { Source } & \text { DF Sum of Squares Mean Square F Ratio Prob }>\text { F }\end{array}$

$\begin{array}{llll}\text { Error } & 32 & 0.00028937 & 0.000058 \\ \text { C. } & 47 & 0.0015945 & \end{array}$

$\begin{array}{lll}\text { C. Total } & 47 & 0.00115945\end{array}$

Means for Oneway Anova

Level Number Mean Std Error Lower 95\% Upper 95\%

$\begin{array}{llllll}1-1-1 & 3 & 0.394734 & 0.00174 & 0.39120 & 0.39827\end{array}$

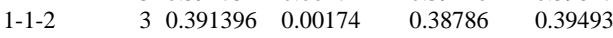

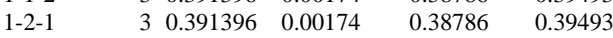

$\begin{array}{llllll}1-2-2 & 3 & 0.393482 & 0.00174 & 0.38995 & 0.39702\end{array}$

$\begin{array}{llllll}2-1-1 & 3 & 0.388058 & 0.00174 & 0.38452 & 0.39159\end{array}$

$\begin{array}{llllll}2-1-2 & 3 & 0.390144 & 0.00174 & 0.38661 & 0.39368\end{array}$

$\begin{array}{llllll}2-2-1 & 3 & 0.393065 & 0.00174 & 0.38953 & 0.39660\end{array}$

$\begin{array}{llllll}2-2-2 & 3 & 0.390144 & 0.00174 & 0.38661 & 0.39368\end{array}$

$\begin{array}{llllll}3-1-1 & 3 & 0.389310 & 0.00174 & 0.38577 & 0.39285\end{array}$

$\begin{array}{llllll}3-1-2 & 3 & 0.388893 & 0.00174 & 0.38536 & 0.39243\end{array}$

$\begin{array}{llllll}3-2-1 & 3 & 0.385137 & 0.00174 & 0.38160 & 0.38867\end{array}$

$\begin{array}{llllll}3-2-2 & 3 & 0.386389 & 0.00174 & 0.38285 & 0.38993\end{array}$

$\begin{array}{llllll}4-1-1 & 3 & 0.386389 & 0.00174 & 0.38285 & 0.38993\end{array}$

$\begin{array}{llllll}4-1-2 & 3 & 0.382216 & 0.00174 & 0.37868 & 0.38575\end{array}$

$\begin{array}{llllll}4-2-1 & 3 & 0.386389 & 0.00174 & 0.38285 & 0.38993\end{array}$

$\begin{array}{llllll}4-2-2 & 3 & 0.377626 & 0.00174 & 0.37409 & 0.38116\end{array}$

Std Error uses a pooled estimate of error variance 


\section{Exhibit A3. PSAL Measurements by Analytical Block for Samples of the Batch 1 Standard Glass Prepared Using the LM Method. (continued)}

Oneway Analysis of K2O (wt\%) By Block/Sub-Block

Reference Value $=3.327 \mathrm{wt} \%$

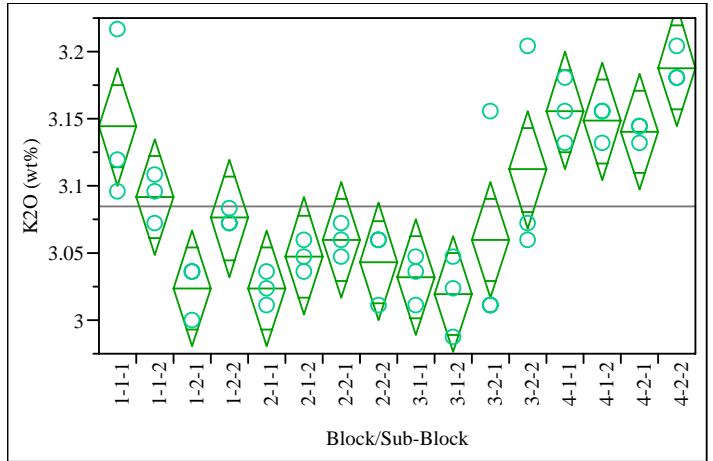

Oneway Anova

Summary of Fit

Rsquare $\quad 0.760622$

Adj Rsquare $\quad 0.648414$

Root Mean Square Error $\quad 0.036883$

Mean of Response 3.085282

Observations (or Sum Wgts) $\quad 48$

Analysis of Variance

Source $\quad$ DF Sum of Squares Mean Square F Ratio Prob $>$ F

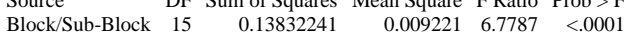

$\begin{array}{llll}\text { Block/Sub-Block } & 15 & 0.13832241 & 0.009221 \\ \text { Error } & 32 & 0.04353183 & 0.001360\end{array}$

C. Total

$\begin{array}{ll}32 & 0.04353183 \\ 47 & 0.18185424\end{array}$

Means for Oneway Anova

Level Number Mean Std Error Lower 95\% Upper 95\%

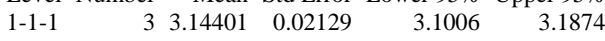

$\begin{array}{llllll}1-1-2 & 3 & 3.09181 & 0.02129 & 3.0484 & 3.1352\end{array}$

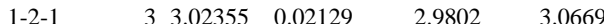

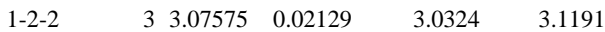

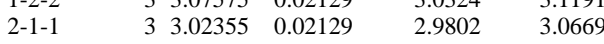

$\begin{array}{llllll}2-1-2 & 3 & 3.04764 & 0.02129 & 3.0043 & 3.0910\end{array}$

$\begin{array}{llllll}2-2-1 & 3 & 3.05968 & 0.02129 & 3.0163 & 3.1031\end{array}$

$\begin{array}{llllll}2-2-2 & 3 & 3.04362 & 0.02129 & 3.0002 & 3.0870\end{array}$

$\begin{array}{llllll}3-1-1 & 3 & 3.03158 & 0.02129 & 2.9882 & 3.0750\end{array}$

$\begin{array}{llllll}3-1-2 & 3 & 3.01953 & 0.02129 & 2.9762 & 3.0629\end{array}$

$\begin{array}{llllll}3-2-1 & 3 & 3.05968 & 0.02129 & 3.0163 & 3.1031\end{array}$

$\begin{array}{llllll}3-2-2 & 3 & 3.11188 & 0.02129 & 3.0685 & 3.1553\end{array}$

$\begin{array}{llllll}4-1-1 & 3 & 3.15605 & 0.02129 & 3.1127 & 3.1994\end{array}$

$\begin{array}{llllll}4-1-2 & 3 & 3.14802 & 0.02129 & 3.1046 & 3.1914\end{array}$

$\begin{array}{llllll}4-2-1 & 3 & 3.13999 & 0.02129 & 3.0966 & 3.1834\end{array}$

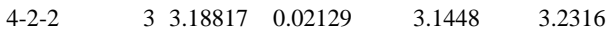

Std Error uses a pooled estimate of error variance
Oneway Analysis of La2O3 (wt\%) By Block/Sub-Block

Reference Value $=0.0 \mathrm{wt} \%$

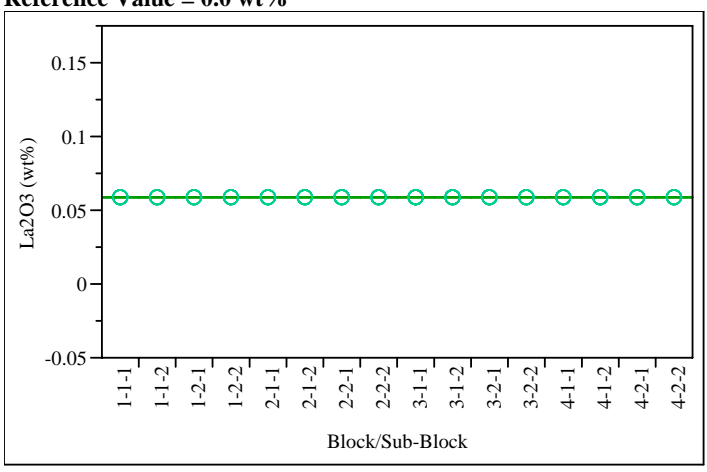

Oneway Anova

Summary of Fit

Rsquare

Adj Rsquare

Root Mean Square Error

Mean of Response

Analysis of Variance

Source DF Sum of Squares Mean Square F Ratio Prob $>$ F

Block/Sub-Block 15

$9.2445 \mathrm{e}-33 \quad 6.163 \mathrm{e}-34$

Error

32
47

Means for Oneway Anova

Level Number Mean Std Error Lower 95\% Upper 95\%

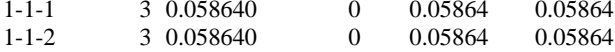

$\begin{array}{llllll}1-2-1 & 3 & 0.058640 & 0 & 0.05864 & 0.05864\end{array}$

$\begin{array}{llllll}1-2-2 & 3 & 0.058640 & 0 & 0.05864 & 0.05864\end{array}$

$\begin{array}{llllll}2-1-1 & 3 & 0.058640 & 0 & 0.05864 & 0.05864\end{array}$

$\begin{array}{llllll}2-1-2 & 3 & 0.058640 & 0 & 0.05864 & 0.05864\end{array}$

$\begin{array}{llllll}2-2-1 & 3 & 0.058640 & 0 & 0.05864 & 0.05864\end{array}$

$\begin{array}{llllll}2-2-2 & 3 & 0.058640 & 0 & 0.05864 & 0.05864\end{array}$

$\begin{array}{llllll}3-1-1 & 3 & 0.058640 & 0 & 0.05864 & 0.05864\end{array}$

$\begin{array}{llllll}3-1-2 & 3 & 0.058640 & 0 & 0.05864 & 0.05864\end{array}$

$\begin{array}{llllll}3-2-1 & 3 & 0.058640 & 0 & 0.05864 & 0.05864\end{array}$

$\begin{array}{llllll}3-2-2 & 3 & 0.058640 & 0 & 0.05864 & 0.05864\end{array}$

$\begin{array}{llllll}4-1-1 & 3 & 0.058640 & 0 & 0.05864 & 0.05864\end{array}$

$\begin{array}{llllll}4-1-2 & 3 & 0.058640 & 0 & 0.05864 & 0.05864\end{array}$

$\begin{array}{llllll}4-2-1 & 3 & 0.058640 & 0 & 0.05864 & 0.05864\end{array}$

$\begin{array}{llllll}4-2-2 & 3 & 0.058640 & 0 & 0.05864 & 0.05864\end{array}$

Std Error uses a pooled estimate of error variance 


\section{Exhibit A3. PSAL Measurements by Analytical Block for Samples of the Batch 1 Standard Glass Prepared Using the LM Method. (continued)}

Oneway Analysis of MgO (wt\%) By Block/Sub-Block Reference Value $=1.419 \mathrm{wt} \%$

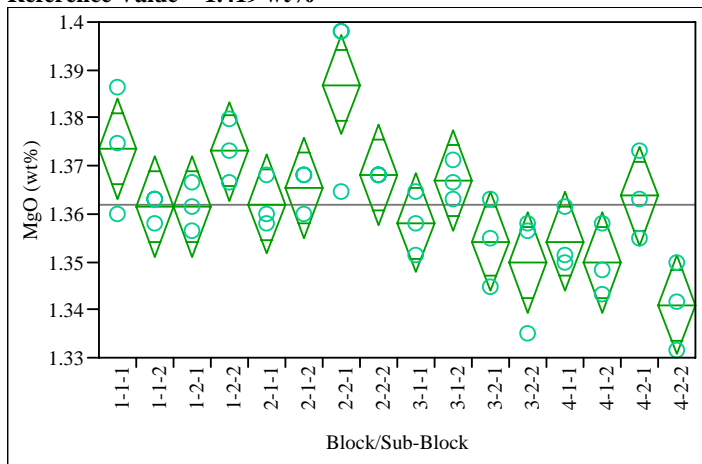

Oneway Anova

Summary of Fit

Rsquare $\quad 0.686572$

Adj Rsquare $\quad 0.539653$

Root Mean Square Error $\quad 0.008833$

Mean of Response 1.361879

Observations (or Sum Wgts) $\quad 48$

Analysis of Variance

Source DF Sum of Squares Mean Square F Ratio Prob $>$ F

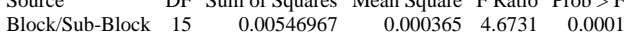

$\begin{array}{llll}\text { Block/Sub-Block } & 15 & 0.00546967 & 0.000365 \\ \text { Error } & 32 & 0.00249696 & 0.000078\end{array}$

C. Total

$32-0.00249696$

Means for Oneway Anova

Level Number Mean Std Error Lower 95\% Upper 95\%

$\begin{array}{lrrrrr}1-1-1 & 3 & 1.37363 & 0.00510 & 1.3632 & 1.3840\end{array}$

$\begin{array}{llllll}1-1-2 & 3 & 1.36146 & 0.00510 & 1.3511 & 1.3719\end{array}$

$\begin{array}{llllll}1-2-1 & 3 & 1.36146 & 0.00510 & 1.3511 & 1.3719\end{array}$

$\begin{array}{llllll}1-2-2 & 3 & 1.37307 & 0.00510 & 1.3627 & 1.3835\end{array}$

$\begin{array}{llllll}1-2-2 & 3 & 1.37307 & 0.00510 & 1.3627 & 1.3835 \\ 2-1-1 & 3 & 1.36202 & 0.00510 & 1.3516 & 1.3724\end{array}$

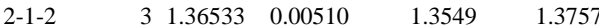

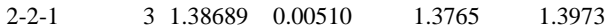

$\begin{array}{llllll}2-2-2 & 3 & 1.36810 & 0.00510 & 1.3577 & 1.3785\end{array}$

$\begin{array}{llllll}3-1-1 & 3 & 1.35815 & 0.00510 & 1.3478 & 1.3685\end{array}$

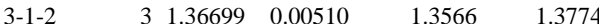

$\begin{array}{llllll}3-2-1 & 3 & 1.35428 & 0.00510 & 1.3439 & 1.3647\end{array}$

$\begin{array}{llllll}3-2-2 & 3 & 1.34986 & 0.00510 & 1.3395 & 1.3602\end{array}$

$\begin{array}{llllll}4-1-1 & 3 & 1.35428 & 0.00510 & 1.3439 & 1.3647\end{array}$

$\begin{array}{llllll}4-1-2 & 3 & 1.34986 & 0.00510 & 1.3395 & 1.3602\end{array}$

$\begin{array}{llllll}4-2-1 & 3 & 1.36368 & 0.00510 & 1.3533 & 1.3741\end{array}$

$\begin{array}{llllll}4-2-2 & 3 & 1.34101 & 0.00510 & 1.3306 & 1.3514\end{array}$

Std Error uses a pooled estimate of error variance
Oneway Analysis of $\mathrm{MnO}$ (wt\%) By Block/Sub-Block

Reference Value $=1.726 \mathrm{wt} \%$

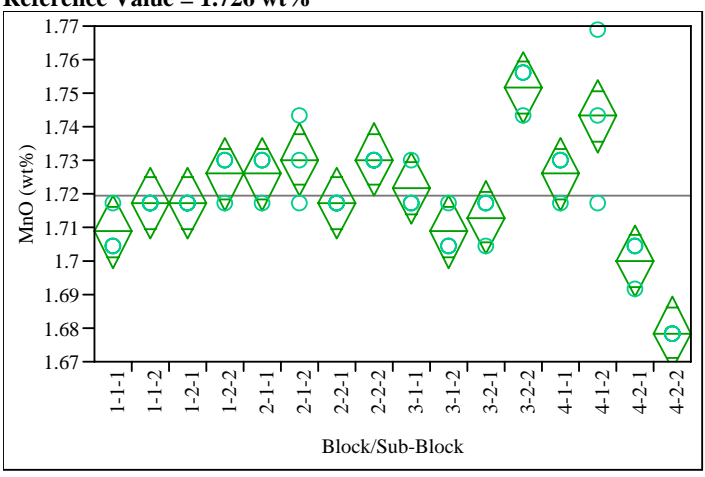

Oneway Anova

Summary of Fit

Rsquare

0.828533

Adj Rsquare

0.748158

Root Mean Square Error $\quad 0.00913$

Mean of Response

1.719717

Observations (or Sum Wgts)

48

Analysis of Variance

Source DF Sum of Squares Mean Square F Ratio Prob > F

$\begin{array}{lrrrrr}\text { Block/Sub-Block } & 15 & 0.01288952 & 0.000859 & 10.3083 & <.0001\end{array}$

$\begin{array}{lll}15 & 0.01288952 & 0.000859 \\ 32 & 0.00266752 & 0.000083\end{array}$

C. Total

0.01555704

0.000083

Means for Oneway Anova

Level Number Mean Std Error Lower 95\% Upper 95\%

$\begin{array}{llllll}1-1-1 & 3 & 1.70869 & 0.00527 & 1.6980 & 1.7194\end{array}$

$\begin{array}{llllll}1-1-2 & 3 & 1.71730 & 0.00527 & 1.7066 & 1.7280\end{array}$

$\begin{array}{llllll}1-2-1 & 3 & 1.71730 & 0.00527 & 1.7066 & 1.7280\end{array}$

$\begin{array}{llllll}1-2-2 & 3 & 1.72590 & 0.00527 & 1.7152 & 1.7366\end{array}$

$\begin{array}{llllll}2-1-1 & 3 & 1.72590 & 0.00527 & 1.7152 & 1.7366\end{array}$

$\begin{array}{llllll}2-1-2 & 3 & 1.73021 & 0.00527 & 1.7195 & 1.7409\end{array}$

$\begin{array}{llllll}2-2-1 & 3 & 1.71730 & 0.00527 & 1.7066 & 1.7280\end{array}$

$\begin{array}{llllll}2-2-2 & 3 & 1.73021 & 0.00527 & 1.7195 & 1.7409\end{array}$

$\begin{array}{llllll}3-1-1 & 3 & 1.72160 & 0.00527 & 1.7109 & 1.7323\end{array}$

$\begin{array}{llllll}3-1-2 & 3 & 1.70869 & 0.00527 & 1.6980 & 1.7194\end{array}$

$\begin{array}{llllll}3-2-1 & 3 & 1.71299 & 0.00527 & 1.7023 & 1.7237\end{array}$

$\begin{array}{llllll}3-2-2 & 3 & 1.75173 & 0.00527 & 1.7410 & 1.7625\end{array}$

$\begin{array}{llllll}4-1-1 & 3 & 1.72590 & 0.00527 & 1.7152 & 1.7366\end{array}$

$\begin{array}{llllll}4-1-2 & 3 & 1.74312 & 0.00527 & 1.7324 & 1.7539\end{array}$

$\begin{array}{llllll}4-2-1 & 3 & 1.70008 & 0.00527 & 1.6893 & 1.7108\end{array}$

$\begin{array}{llllll}4-2-2 & 3 & 1.67856 & 0.00527 & 1.6678 & 1.6893\end{array}$

Std Error uses a pooled estimate of error variance 


\section{Exhibit A3. PSAL Measurements by Analytical Block for Samples of the Batch 1 Standard Glass Prepared Using the LM Method. (continued)}

Oneway Analysis of Na2O (wt\%) By Block/Sub-Block Reference Value $=\mathbf{9 . 0 0 3} \mathrm{wt} \%$

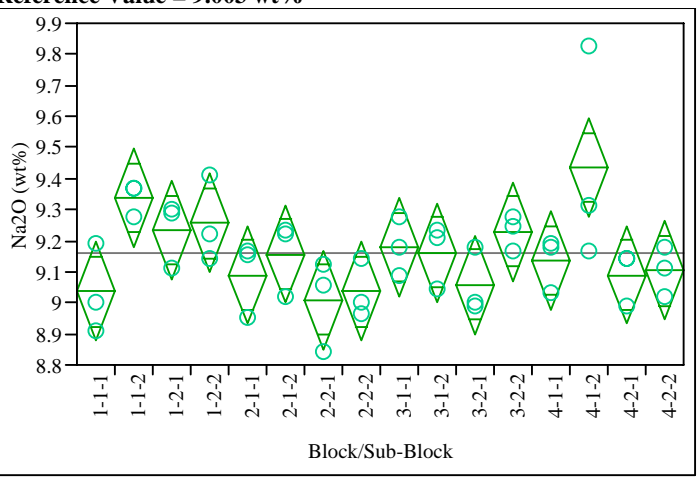

Oneway Anova

Summary of Fit

$\begin{array}{lr}\text { Rsquare } & 0.515233 \\ \text { Adj Rsquare } & 0.287999 \\ \text { Root Mean Square Error } & 0.13442 \\ \text { Mean of Response } & 9.159379 \\ \text { Observations (or Sum Wgts) } & 48\end{array}$

Analysis of Variance

Source DF Sum of Squares Mean Square F Ratio Prob $>$ F

$\begin{array}{llllll}\text { Block/Sub-Block } & 15 & 0.6145408 & 0.040969 & 2.2674 & 0.0255\end{array}$

Error

$\begin{array}{lll}32 & 0.5782025 & 0.018069\end{array}$

Means for Oneway Anova

Level Number Mean Std Error Lower 95\% Upper 95\%

$\begin{array}{llllll}1-1-1 & 3 & 9.03609 & 0.07761 & 8.8780 & 9.1942\end{array}$

$\begin{array}{llllll}1-1-2 & 3 & 9.33715 & 0.07761 & 9.1791 & 9.4952\end{array}$

$\begin{array}{llllll}1-2-1 & 3 & 9.23380 & 0.07761 & 9.0757 & 9.3919\end{array}$

$\begin{array}{llllll}1-2-2 & 3 & 9.25627 & 0.07761 & 9.0982 & 9.4143\end{array}$

$\begin{array}{llllll}2-1-1 & 3 & 9.09001 & 0.07761 & 8.9319 & 9.2481\end{array}$

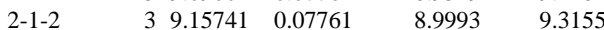

$\begin{array}{llllll}2-2-1 & 3 & 9.00913 & 0.07761 & 8.8511 & 9.1672\end{array}$

$\begin{array}{llllll}2-2-2 & 3 & 9.03609 & 0.07761 & 8.8780 & 9.1942\end{array}$

$\begin{array}{llllll}3-1-1 & 3 & 9.17988 & 0.07761 & 9.0218 & 9.3380\end{array}$

$\begin{array}{llllll}3-1-2 & 3 & 9.16191 & 0.07761 & 9.0038 & 9.3200\end{array}$

$\begin{array}{llllll}3-2-1 & 3 & 9.05856 & 0.07761 & 8.9005 & 9.2166\end{array}$

$\begin{array}{llllll}3-2-2 & 3 & 9.22931 & 0.07761 & 9.0712 & 9.3874\end{array}$

$\begin{array}{llllll}4-1-1 & 3 & 9.13495 & 0.07761 & 8.9769 & 9.2930\end{array}$

$\begin{array}{llllll}4-1-2 & 3 & 9.43600 & 0.07761 & 9.2779 & 9.5941\end{array}$

$\begin{array}{llllll}4-2-1 & 3 & 9.09001 & 0.07761 & 8.9319 & 9.2481\end{array}$

$\begin{array}{llllll}4-2-2 & 3 & 9.10349 & 0.07761 & 8.9454 & 9.2616\end{array}$

Std Error uses a pooled estimate of error variance
Oneway Analysis of $\mathrm{NiO}$ (wt\%) By Block/Sub-Block Reference Value $=\mathbf{0 . 7 5 1} \mathbf{w t} \%$

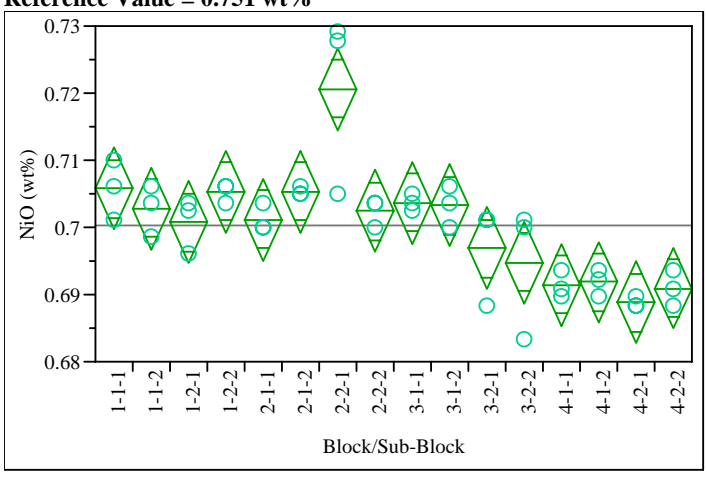

Oneway Anova

Summary of Fit

Rsquare

0.766965

Adj Rsquare

0.65773

Root Mean Square Error $\quad 0.005175$

Mean of Response

0.700379

Observations (or Sum Wgts)

Analysis of Variance

Source DF Sum of Squares Mean Square F Ratio Prob $>$ F

$\begin{array}{lrrrrr}\text { Block/Sub-Block } & 15 & 0.00282098 & 0.000188 & 7.0212 & <.0001\end{array}$

$\begin{array}{llll}\text { Error } & 32 & 0.00085713 & 0.000027\end{array}$

C. Total

Means for Oneway Anova

Level Number Mean Std Error Lower 95\% Upper 95\%

$\begin{array}{llllll}1-1-1 & 3 & 0.705813 & 0.00299 & 0.69973 & 0.71190\end{array}$

$\begin{array}{llllll}1-1-2 & 3 & 0.702844 & 0.00299 & 0.69676 & 0.70893\end{array}$

$\begin{array}{llllll}1-2-1 & 3 & 0.700723 & 0.00299 & 0.69464 & 0.70681\end{array}$

$\begin{array}{llllll}1-2-2 & 3 & 0.705389 & 0.00299 & 0.69930 & 0.71148\end{array}$

$\begin{array}{llllll}1-1-1 & 3 & 0.701148 & 0.00299 & 0.69506 & 0.70723\end{array}$

$\begin{array}{llllll}2-1-2 & 3 & 0.705389 & 0.00299 & 0.69930 & 0.71148\end{array}$

$\begin{array}{llllll}2-2-1 & 3 & 0.720659 & 0.00299 & 0.71457 & 0.72675\end{array}$

$\begin{array}{llllll}2-2-2 & 3 & 0.702420 & 0.00299 & 0.69633 & 0.70851\end{array}$

$\begin{array}{llllll}3-1-1 & 3 & 0.703693 & 0.00299 & 0.69761 & 0.70978\end{array}$

$\begin{array}{llllll}3-1-2 & 3 & 0.703268 & 0.00299 & 0.69718 & 0.70935\end{array}$

$\begin{array}{llllll}3-2-1 & 3 & 0.696906 & 0.00299 & 0.69082 & 0.70299\end{array}$

$\begin{array}{llllll}3-2-2 & 3 & 0.694785 & 0.00299 & 0.68870 & 0.70087\end{array}$

$\begin{array}{llllll}4-1-1 & 3 & 0.691392 & 0.00299 & 0.68531 & 0.69748\end{array}$

$\begin{array}{llllll}4-1-2 & 3 & 0.691816 & 0.00299 & 0.68573 & 0.69790\end{array}$

$\begin{array}{llllll}4-2-1 & 3 & 0.688847 & 0.00299 & 0.68276 & 0.69493\end{array}$

$\begin{array}{llllll}4-2-2 & 3 & 0.690968 & 0.00299 & 0.68488 & 0.69705\end{array}$

Std Error uses a pooled estimate of error variance 


\section{Exhibit A3. PSAL Measurements by Analytical Block for Samples of the Batch 1 Standard Glass Prepared Using the LM Method. (continued)}

Oneway Analysis of PbO (wt \%) By Block/Sub-Block Reference Value $=0.0 \mathrm{wt} \%$

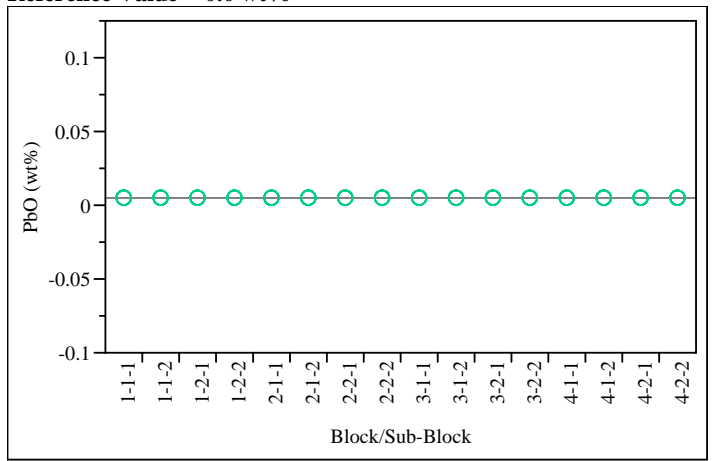

Oneway Anova

Summary of Fit

$\begin{array}{lr}\text { Rsquare } & 4 \\ \text { Adj Rsquare } & 5.40625 \\ \text { Root Mean Square Error } & . \\ \text { Mean of Response } & 0.005386 \\ \text { Observations (or Sum Wgts) } & 48\end{array}$

Analysis of Variance

Source DF Sum of Squares Mean Square F Ratio Prob $>$ F

Block/Sub-Block $15 \quad 1.4444 \mathrm{e}-34 \quad 9.63 \mathrm{e}-36 \quad-2.8444 \quad 0.0000$

Error $\quad 32 \quad-1.083 \mathrm{e}-34 \quad-3.39 \mathrm{e}-36$

$\begin{array}{lll}\text { C. Total } & 47 & 3.6111 \mathrm{e}-35\end{array}$

Means for Oneway Anova

Level Number Mean Std Error Lower 95\% Upper 95\%

1-1-1 30.005386

$1-1-2 \quad 3 \quad 0.005386$

$\begin{array}{lll}1-2-1 & 3 & 0.005386\end{array}$

$1-2-2 \quad 3 \quad 0.005386$

2-1-1 30.005386

$2-1-2 \quad 3 \quad 0.005386$

$2-2-1 \quad 3 \quad 0.005386$

$\begin{array}{lll}2-2-2 & 3 & 0.005386\end{array}$

$3-1-1 \quad 3 \quad 0.005386$

$3-1-2 \quad 3 \quad 0.005386$

$3-2-1 \quad 30.005386$

3-2-2 30.005386

$\begin{array}{lll}4-1-1 & 3 & 0.005386\end{array}$

$4-1-2 \quad 3 \quad 0.005386$

4-2-1 30.005386

4-2-2 $\quad 30.005386$

Std Error uses a pooled estimate of error variance
Oneway Analysis of SO4 (wt\%) By Block/Sub-Block

Reference Value $=0.0 \mathrm{wt} \%$

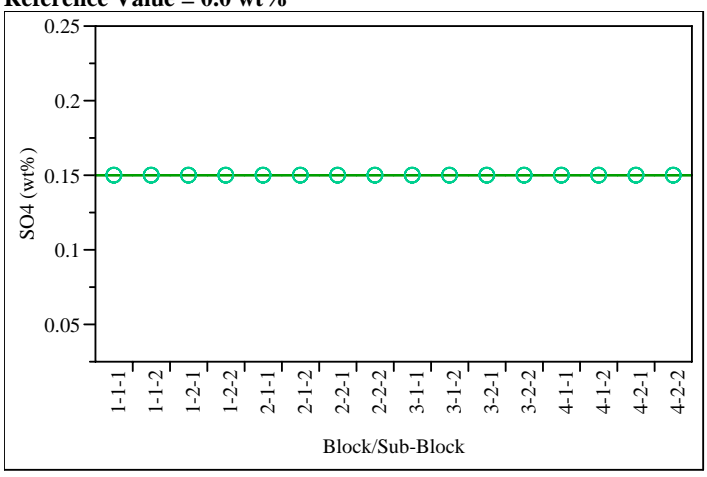

Oneway Anova

Summary of Fit

Rsquare

Adj Rsquare

Root Mean Square Error

Mean of Response

Observations (or Sum Wgts)

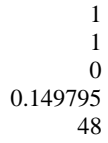

Analysis of Variance

$\begin{array}{lrrrrr}\text { Source } & \text { DF } & \text { Sum of Squares } & \text { Mean Square } & \text { F Ratio } & \text { Prob > F } \\ \text { Block/Sub-Block } & 15 & 3.328 \mathrm{e}-31 & 2.219 \mathrm{e}-32 & \text {. } & \text {. } \\ \text { Error } & 32 & 0 & 0 & & \\ \text { C. Total } & 47 & 3.328 \mathrm{e}-31 & & \end{array}$

Means for Oneway Anova

Level Number Mean Std Error Lower 95\% Upper 95\%

$\begin{array}{llllll}1-1-1 & 3 & 0.149795 & 0 & 0.14980 & 0.14980\end{array}$

$\begin{array}{llllll}1-1-2 & 3 & 0.149795 & 0 & 0.14980 & 0.14980\end{array}$

$\begin{array}{llllll}1-2-1 & 3 & 0.149795 & 0 & 0.14980 & 0.14980\end{array}$

$\begin{array}{llllll}1-2-2 & 3 & 0.149795 & 0 & 0.14980 & 0.14980\end{array}$

$\begin{array}{llllll}1-2-2 & 3 & 0.149795 & 0 & 0.14980 & 0.14980 \\ 2-1-1 & 3 & 0.149795 & 0 & 0.14980 & 0.14980\end{array}$

$\begin{array}{llllll}2-1-2 & 3 & 0.149795 & 0 & 0.14980 & 0.14980\end{array}$

$\begin{array}{llllll}2-2-1 & 3 & 0.149795 & 0 & 0.14980 & 0.14980\end{array}$

$\begin{array}{llllll}2-2-2 & 3 & 0.149795 & 0 & 0.14980 & 0.14980\end{array}$

$\begin{array}{llllll}3-1-1 & 3 & 0.149795 & 0 & 0.14980 & 0.14980\end{array}$

$\begin{array}{llllll}3-1-2 & 3 & 0.149795 & 0 & 0.14980 & 0.14980\end{array}$

$\begin{array}{llllll}3-2-1 & 3 & 0.149795 & 0 & 0.14980 & 0.14980\end{array}$

$\begin{array}{llllll}3-2-2 & 3 & 0.149795 & 0 & 0.14980 & 0.14980\end{array}$

$\begin{array}{llllll}4-1-1 & 3 & 0.149795 & 0 & 0.14980 & 0.14980\end{array}$

$\begin{array}{llllll}4-1-2 & 3 & 0.149795 & 0 & 0.14980 & 0.14980\end{array}$

$\begin{array}{llllll}4-2-1 & 3 & 0.149795 & 0 & 0.14980 & 0.14980\end{array}$

$\begin{array}{llllll}4-2-2 & 3 & 0.149795 & 0 & 0.14980 & 0.14980\end{array}$

Std Error uses a pooled estimate of error variance 


\section{Exhibit A3. PSAL Measurements by Analytical Block for Samples of the Batch 1 Standard Glass Prepared Using the LM Method. (continued)}

Oneway Analysis of TiO2 (wt\%) By Block/Sub-Block Reference Value $=0.677 \mathrm{wt} \%$

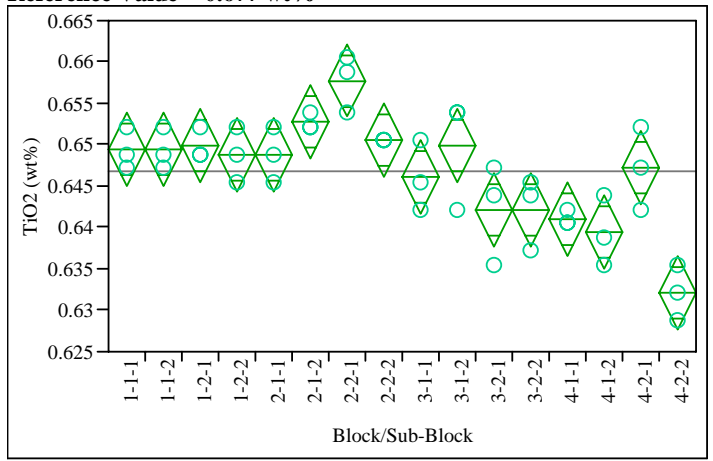

Oneway Anova

Summary of Fit

$\begin{array}{lr}\text { Rsquare } & 0.787256 \\ \text { Adj Rsquare } & 0.687532 \\ \text { Root Mean Square Error } & 0.003753 \\ \text { Mean of Response } & 0.646732 \\ \text { Observations (or Sum Wgts) } & 48\end{array}$

Analysis of Variance

Source DF Sum of Squares Mean Square F Ratio Prob $>$ F

Block/Sub-Block $15 \quad 0.00166789 \quad 0.000111 \quad 7.8944<.0001$

$\begin{array}{llll}\text { Error } & 32 & 0.00045072 & 0.000014\end{array}$

$\begin{array}{lll}\text { C. Total } & 47 & 0.00211861\end{array}$

Means for Oneway Anova

Level Number Mean Std Error Lower 95\% Upper 95\%

$\begin{array}{llllll}1-1-1 & 3 & 0.649408 & 0.00217 & 0.64499 & 0.65382\end{array}$

$\begin{array}{llllll}1-1-2 & 3 & 0.649408 & 0.00217 & 0.64499 & 0.65382\end{array}$

$\begin{array}{llllll}1-2-1 & 3 & 0.649964 & 0.00217 & 0.64555 & 0.65438\end{array}$

$\begin{array}{llllll}1-2-2 & 3 & 0.648852 & 0.00217 & 0.64444 & 0.65327\end{array}$

$\begin{array}{llllll}2-1-1 & 3 & 0.648852 & 0.00217 & 0.64444 & 0.65327\end{array}$

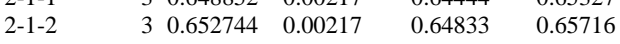

$\begin{array}{llllll}2-2-1 & 3 & 0.657748 & 0.00217 & 0.65333 & 0.66216\end{array}$

$\begin{array}{llllll}2-2-2 & 3 & 0.650520 & 0.00217 & 0.64611 & 0.65493\end{array}$

$\begin{array}{llllll}3-1-1 & 3 & 0.646072 & 0.00217 & 0.64166 & 0.65049\end{array}$

$\begin{array}{llllll}3-1-2 & 3 & 0.649964 & 0.00217 & 0.64555 & 0.65438\end{array}$

$\begin{array}{llllll}3-2-1 & 3 & 0.642180 & 0.00217 & 0.63777 & 0.64659\end{array}$

$\begin{array}{llllll}3-2-2 & 3 & 0.642180 & 0.00217 & 0.63777 & 0.64659\end{array}$

$\begin{array}{llllll}3-1-1 & 3 & 0.641068 & 0.00217 & 0.63665 & 0.64548\end{array}$

$\begin{array}{llllll}4-1-2 & 3 & 0.639400 & 0.00217 & 0.63499 & 0.64381\end{array}$

$\begin{array}{llllll}4-2-1 & 3 & 0.647184 & 0.00217 & 0.64277 & 0.65160\end{array}$

$\begin{array}{llllll}4-2-2 & 3 & 0.632172 & 0.00217 & 0.62776 & 0.63659\end{array}$

Std Error uses a pooled estimate of error variance
Oneway Analysis of ZnO (wt\%) By Block/Sub-Block

Reference Value $=0.0 \mathrm{wt} \%$

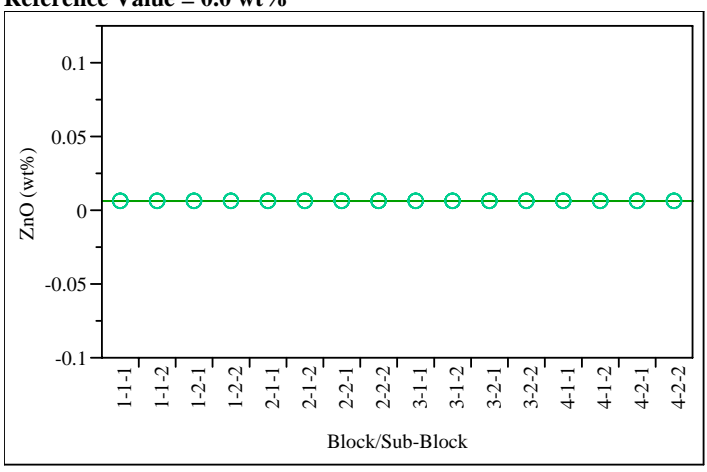

Oneway Anova

Summary of Fit

Rsquare

Adj Rsquare

Root Mean Square Error

Mean of Response

Analysis of Variance

$\begin{array}{lrrrrr}\text { Source } & \text { DF } & \begin{array}{r}\text { Sum of } \\ \text { Squares }\end{array} & \begin{array}{r}\text { Mean } \\ \text { Square }\end{array} & \begin{array}{r}\text { F } \\ \text { Ratio }\end{array} & \text { Prob > } \\ \text { Block/Sub- } & 15 & 3.6111 \mathrm{e}-35 & 2.407 \mathrm{e}-36 & . & \text {. } \\ \text { Block } & & & & & \\ \text { Error } & 32 & 0 & 0 & & \\ \text { C. Total } & 47 & 3.6111 \mathrm{e}-35 & & & \end{array}$

Means for Oneway Anova

Level Number Mean Std Error Lower 95\% Upper 95\%

$\begin{array}{llllrr}1-1-1 & 3 & 0.006224 & 0 & 0.00622 & 0.00622\end{array}$

$\begin{array}{llllll}1-1-2 & 3 & 0.006224 & 0 & 0.00622 & 0.00622\end{array}$

$\begin{array}{llllll}1-2-1 & 3 & 0.006224 & 0 & 0.00622 & 0.00622\end{array}$

$\begin{array}{llllll}1-2-2 & 3 & 0.006224 & 0 & 0.00622 & 0.00622\end{array}$

$\begin{array}{llllll}2-1-1 & 3 & 0.006224 & 0 & 0.00622 & 0.00622\end{array}$

$\begin{array}{llllll}2-1-2 & 3 & 0.006224 & 0 & 0.00622 & 0.00622\end{array}$

$\begin{array}{llllll}2-2-1 & 3 & 0.006224 & 0 & 0.00622 & 0.00622\end{array}$

$\begin{array}{llllll}2-2-2 & 3 & 0.006224 & 0 & 0.00622 & 0.00622\end{array}$

$\begin{array}{llllll}3-1-1 & 3 & 0.006224 & 0 & 0.00622 & 0.00622\end{array}$

$\begin{array}{llllll}3-1-2 & 3 & 0.006224 & 0 & 0.00622 & 0.00622\end{array}$

$\begin{array}{llllll}3-2-1 & 3 & 0.006224 & 0 & 0.00622 & 0.00622\end{array}$

$\begin{array}{llllll}3-2-2 & 3 & 0.006224 & 0 & 0.00622 & 0.00622\end{array}$

$\begin{array}{llllll}4-1-1 & 3 & 0.006224 & 0 & 0.00622 & 0.00622\end{array}$

$\begin{array}{llllll}4-1-2 & 3 & 0.006224 & 0 & 0.00622 & 0.00622\end{array}$

$\begin{array}{llllll}4-1-2 & 3 & 0.006224 & 0 & 0.00622 & 0.00622\end{array}$

$\begin{array}{llllll}4-2-2 & 3 & 0.006224 & 0 & 0.00622 & 0.00622\end{array}$

Std Error uses a pooled estimate of error variance 
WSRC-STI-2007-00659

Revision 0

Appendix A

Exhibit A3. PSAL Measurements by Analytical Block for Samples of the

Batch 1 Standard Glass Prepared Using the LM Method. (continued)

Oneway Analysis of ZrO2 (wt\%) By Block/Sub-Block

Reference Value $=\mathbf{0 . 0 9 8}$ wt $\%$

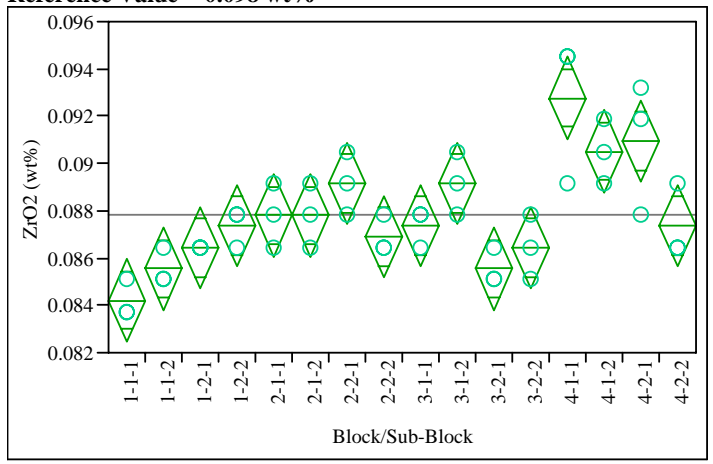

Oneway Anova

Summary of Fit

$\begin{array}{lr}\text { Rsquare } & 0.760975 \\ \text { Adj Rsquare } & 0.648932 \\ \text { Root Mean Square Error } & 0.001472 \\ \text { Mean of Response } & 0.08783 \\ \text { Observations (or Sum Wgts) } & 48\end{array}$

Analysis of Variance

$\begin{array}{lcrrrr}\text { Source } & \text { DF } & \begin{array}{r}\text { Sum of } \\ \text { Squares }\end{array} & \begin{array}{r}\text { Mean } \\ \text { Square }\end{array} & \begin{array}{r}\text { F } \\ \text { Ratio }\end{array} & \text { Prob }> \\ \text { Block/Sub- } & 15 & 0.00022075 & 0.000015 & 6.7918 & <.0001 \\ \text { Block } & & & & & \\ \text { Error } & 32 & 0.00006934 & 2.167 \mathrm{e}-6 & & \\ \text { C. Total } & 47 & 0.00029008 & & & \end{array}$

Means for Oneway Anova

Level Number Mean Std Error Lower 95\% Upper 95\%

$\begin{array}{llllll}1-1-1 & 3 & 0.084200 & 0.00085 & 0.08247 & 0.08593\end{array}$

$\begin{array}{llllll}1-1-2 & 3 & 0.085551 & 0.00085 & 0.08382 & 0.08728\end{array}$

$\begin{array}{llllll}1-2-1 & 3 & 0.086451 & 0.00085 & 0.08472 & 0.08818\end{array}$

$\begin{array}{llllll}1-2-2 & 3 & 0.087352 & 0.00085 & 0.08562 & 0.08908\end{array}$

$\begin{array}{llllll}1-1-1 & 3 & 0.087802 & 0.00085 & 0.08607 & 0.08953\end{array}$

$\begin{array}{llllll}2-1-2 & 3 & 0.087802 & 0.00085 & 0.08607 & 0.08953\end{array}$

$\begin{array}{llllll}2-2-1 & 3 & 0.089153 & 0.00085 & 0.08742 & 0.09088\end{array}$

$\begin{array}{llllll}2-2-2 & 3 & 0.086901 & 0.00085 & 0.08517 & 0.08863\end{array}$

$\begin{array}{llllll}3-1-1 & 3 & 0.087352 & 0.00085 & 0.08562 & 0.08908\end{array}$

$\begin{array}{llllll}3-1-2 & 3 & 0.089153 & 0.00085 & 0.08742 & 0.09088\end{array}$

$\begin{array}{llllll}3-2-1 & 3 & 0.085551 & 0.00085 & 0.08382 & 0.08728\end{array}$

$\begin{array}{llllll}3-2-1 & 3 & 0.086451 & 0.00085 & 0.08472 & 0.08818\end{array}$

$\begin{array}{llllll}4-1-1 & 3 & 0.092755 & 0.00085 & 0.09102 & 0.09449\end{array}$

$\begin{array}{llllll}4-1-2 & 3 & 0.090504 & 0.00085 & 0.08877 & 0.09223\end{array}$

$\begin{array}{llllll}4-2-1 & 3 & 0.090954 & 0.00085 & 0.08922 & 0.09268\end{array}$

$\begin{array}{llllll}4-2-2 & 3 & 0.087352 & 0.00085 & 0.08562 & 0.08908\end{array}$

Std Error uses a pooled estimate of error variance 


\section{Exhibit A4: PSAL Measurements by Analytical Block for Samples of the Batch 1 Standard Glass Prepared Using the PF Method. (continued)}

Oneway Analysis of B2O3 (wt\%) By Block/Sub-Block Reference Value $=7.777 \mathrm{wt} \%$

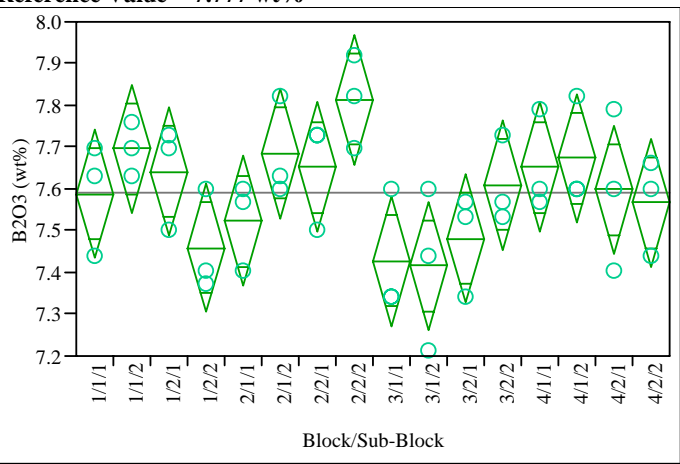

Oneway Anova

Summary of Fit

$\begin{array}{lr}\text { Rsquare } & 0.493306 \\ \text { Adj Rsquare } & 0.255794 \\ \text { Root Mean Square Error } & 0.131123 \\ \text { Mean of Response } & 7.592927 \\ \text { Observations (or Sum Wgts) } & 48\end{array}$

Analysis of Variance

Source DF Sum of Squares Mean Square F Ratio Prob $>$ F

$\begin{array}{llllll}\text { Block/Sub-Block } & 15 & 0.5356458 & 0.035710 & 2.0770 & 0.0406\end{array}$

Error

$\begin{array}{lll}15 & 0.5356458 & 0.035710 \\ 32 & 0.5501823 & 0.017193\end{array}$

$\begin{array}{lll}\text { C. Total } & 47 & 1.0858280\end{array}$

Means for Oneway Anova

Level Number Mean Std Error Lower 95\% Upper 95\%

$\begin{array}{llrrrr}1 / 1 / 1 & 3 & 7.58823 & 0.07570 & 7.4340 & 7.7424\end{array}$

$\begin{array}{llllll}1 / 1 / 2 & 3 & 7.69556 & 0.07570 & 7.5414 & 7.8498\end{array}$

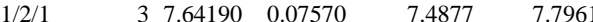

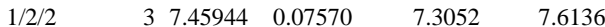

$\begin{array}{llllll}1 / 2 / 2 & 3 & 7.45944 & 0.07570 & 7.3052 & 7.6136 \\ 2 / 1 / 1 & 3 & 7.52383 & 0.07570 & 7.3696 & 7.6780\end{array}$

$\begin{array}{llllll}2 / 1 / 2 & 3 & 7.68483 & 0.07570 & 7.5306 & 7.8390\end{array}$

$\begin{array}{llllll}2 / 2 / 1 & 3 & 7.65263 & 0.07570 & 7.4984 & 7.8068\end{array}$

$\begin{array}{llllll}2 / 2 / 2 & 3 & 7.81362 & 0.07570 & 7.6594 & 7.9678\end{array}$

$\begin{array}{llllll}2 / 2 / 2 & 3 & 7.42724 & 0.07570 & 7.2730 & 7.5814\end{array}$

$\begin{array}{llllll}3 / 1 / 2 & 3 & 7.41650 & 0.07570 & 7.2623 & 7.5707\end{array}$

$\begin{array}{llllll}3 / 2 / 1 & 3 & 7.48090 & 0.07570 & 7.3267 & 7.6351\end{array}$

$\begin{array}{llllll}3 / 2 / 2 & 3 & 7.60970 & 0.07570 & 7.4555 & 7.7639\end{array}$

$\begin{array}{llllll}4 / 1 / 1 & 3 & 7.65263 & 0.07570 & 7.4984 & 7.8068\end{array}$

$\begin{array}{llllll}4 / 1 / 2 & 3 & 7.67410 & 0.07570 & 7.5199 & 7.8283\end{array}$

$\begin{array}{llllll}4 / 2 / 1 & 3 & 7.59896 & 0.07570 & 7.4448 & 7.7532\end{array}$

$\begin{array}{llllll}4 / 2 / 2 & 3 & 7.56677 & 0.07570 & 7.4126 & 7.7210\end{array}$

Std Error uses a pooled estimate of error variance
Oneway Analysis of Fe2O3 (wt\%) By Block/Sub-Block Reference Value $=12.839$ wt $\%$

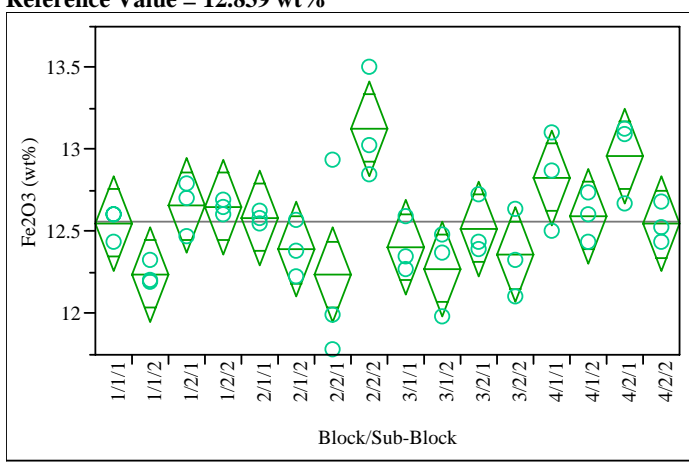

Oneway Anova

Summary of Fit

$\begin{array}{lr}\text { Rsquare } & 0.597771 \\ \text { Adj Rsquare } & 0.409226 \\ \text { Root Mean Square Error } & 0.245949 \\ \text { Mean of Response } & 12.55902 \\ \text { Observations (or Sum Wgts) } & 48\end{array}$

Antysis of Variance

$\begin{array}{lrrrrr}\text { Source } & \text { DF } & \text { Sum of Squares } & \text { Mean Square } & \text { F Ratio } & \text { Prob > F } \\ \text { Block/Sub-Block } & 15 & 2.8767465 & 0.191783 & 3.1704 & 0.0030 \\ \text { Error } & 32 & 1.9357079 & 0.060491 & & \\ \text { C. Total } & 47 & 4.8124544 & & & \end{array}$

Means for Oneway Anova

Level Number Mean Std Error Lower 95\% Upper 95\%

$\begin{array}{llllll}1 / 1 / 1 & 3 & 12.5528 & 0.14200 & 12.264 & 12.842\end{array}$

$\begin{array}{llllll}1 / 1 / 2 & 3 & 12.2430 & 0.14200 & 11.954 & 12.532\end{array}$

$\begin{array}{llllll}1 / 2 / 1 & 3 & 12.6576 & 0.14200 & 12.368 & 12.947\end{array}$

$\begin{array}{llllll}1 / 2 / 2 & 3 & 12.6528 & 0.14200 & 12.364 & 12.942\end{array}$

$\begin{array}{llllll}2 / 1 / 1 & 3 & 12.5861 & 0.14200 & 12.297 & 12.875\end{array}$

$\begin{array}{llllll}2 / 1 / 2 & 3 & 12.3907 & 0.14200 & 12.101 & 12.680\end{array}$

$\begin{array}{llllll}2 / 2 / 1 & 3 & 12.2382 & 0.14200 & 11.949 & 12.527\end{array}$

$\begin{array}{llllll}2 / 2 / 2 & 3 & 13.1294 & 0.14200 & 12.840 & 13.419\end{array}$

$\begin{array}{llllll}3 / 1 / 1 & 3 & 12.4050 & 0.14200 & 12.116 & 12.694\end{array}$

$\begin{array}{llllll}3 / 1 / 2 & 3 & 12.2764 & 0.14200 & 11.987 & 12.566\end{array}$

$\begin{array}{llllll}3 / 2 / 1 & 3 & 12.5194 & 0.14200 & 12.230 & 12.809\end{array}$

$\begin{array}{llllll}3 / 2 / 2 & 3 & 12.3574 & 0.14200 & 12.068 & 12.647\end{array}$

$\begin{array}{llllll}4 / 1 / 1 & 3 & 12.8292 & 0.14200 & 12.540 & 13.118\end{array}$

$\begin{array}{llllll}4 / 1 / 2 & 3 & 12.5957 & 0.14200 & 12.306 & 12.885\end{array}$

$\begin{array}{llllll}4 / 2 / 1 & 3 & 12.9626 & 0.14200 & 12.673 & 13.252\end{array}$

$\begin{array}{llllll}4 / 2 / 2 & 3 & 12.5480 & 0.14200 & 12.259 & 12.837\end{array}$

Std Error uses a pooled estimate of error variance 


\section{Exhibit A4: PSAL Measurements by Analytical Block for Samples of the Batch 1 Standard Glass Prepared Using the PF Method. (continued)}

Oneway Analysis of Li2O (wt\%) By Block/Sub-Block Reference Value $=4.429 \mathrm{wt} \%$

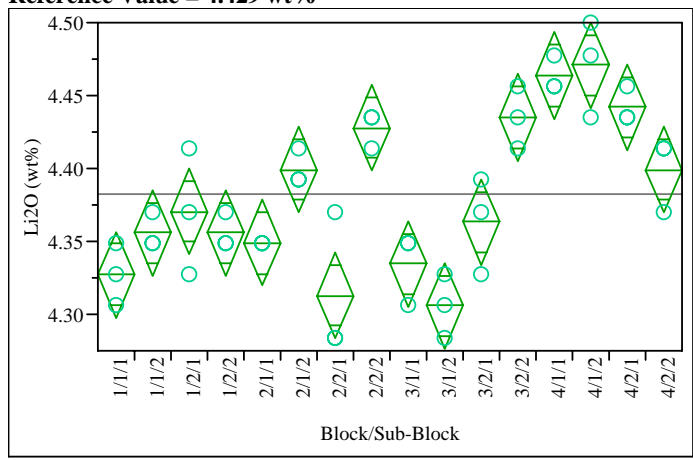

Oneway Anova

Summary of Fit

$\begin{array}{lr}\text { Rsquare } & 0.863696 \\ \text { Adj Rsquare } & 0.799803 \\ \text { Root Mean Square Error } & 0.025053 \\ \text { Mean of Response } & 4.382049 \\ \text { Observations (or Sum Wgts) } & 48\end{array}$

Analysis of Variance

Source DF Sum of Squares Mean Square F Ratio Prob > F

Block/Sub-Block $15 \quad 0.12726878 \quad 0.00848513 .5179<.0001$

C. Total

$\begin{array}{ll}32 & 0.02008491 \\ 47 & 0.14735369\end{array}$

Means for Oneway Anova

Level Number Mean Std Error Lower 95\% Upper 95\%

$\begin{array}{llllll}1 / 1 / 1 & 3 & 4.32733 & 0.01446 & 4.2979 & 4.3568\end{array}$

$\begin{array}{llllll}1 / 1 / 2 & 3 & 4.35603 & 0.01446 & 4.3266 & 4.3855\end{array}$

$\begin{array}{llllll}1 / 2 / 1 & 3 & 4.37039 & 0.01446 & 4.3409 & 4.3998\end{array}$

$\begin{array}{llllll}1 / 2 / 2 & 3 & 4.35603 & 0.01446 & 4.3266 & 4.3855\end{array}$

$\begin{array}{llllll}2 / 1 / 1 & 3 & 4.34886 & 0.01446 & 4.3194 & 4.3783\end{array}$

$\begin{array}{llllll}2 / 1 / 2 & 3 & 4.39909 & 0.01446 & 4.3696 & 4.4286\end{array}$

$\begin{array}{llllll}2 / 2 / 1 & 3 & 4.31298 & 0.01446 & 4.2835 & 4.3424\end{array}$

$\begin{array}{llllll}2 / 2 / 2 & 3 & 4.42780 & 0.01446 & 4.3983 & 4.4573\end{array}$

$\begin{array}{llllll}3 / 1 / 1 & 3 & 4.33451 & 0.01446 & 4.3050 & 4.3640\end{array}$

$\begin{array}{llllll}3 / 1 / 2 & 3 & 4.30580 & 0.01446 & 4.2763 & 4.3353\end{array}$

$\begin{array}{llllll}3 / 2 / 1 & 3 & 4.36321 & 0.01446 & 4.3337 & 4.3927\end{array}$

$\begin{array}{llllll}3 / 2 / 2 & 3 & 4.43497 & 0.01446 & 4.4055 & 4.4644\end{array}$

$\begin{array}{llllll}4 / 1 / 1 & 3 & 4.46368 & 0.01446 & 4.4342 & 4.4931\end{array}$

$\begin{array}{llllll}4 / 1 / 2 & 3 & 4.47086 & 0.01446 & 4.4414 & 4.5003\end{array}$

$4 / 2 / 1 \quad 34.44215 \quad 0.01446 \quad-4.4127-4.4716$

$\begin{array}{llllll}4 / 2 / 2 & 3 & 4.39909 & 0.01446 & 4.3696 & 4.4286\end{array}$

Std Error uses a pooled estimate of error variance
Oneway Analysis of SiO2 (wt\%) By Block/Sub-Block

Reference Value $=\mathbf{5 0 . 2 2} \mathbf{w t} \%$

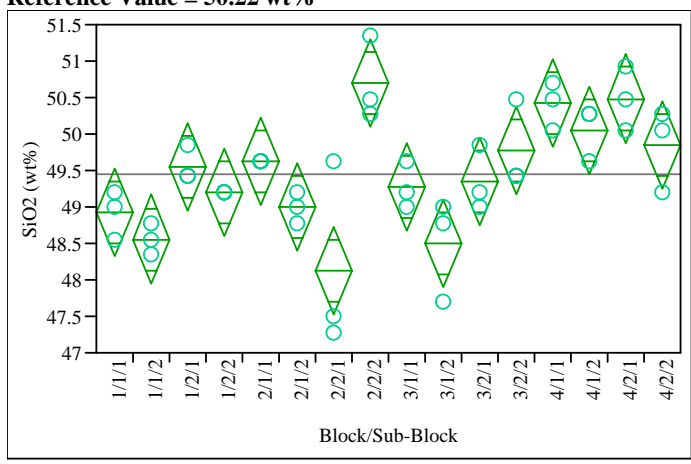

Oneway Anova

Summary of Fit

$\begin{array}{lr}\text { Rsquare } & 0.748364 \\ \text { Adj Rsquare } & 0.63041 \\ \text { Root Mean Square Error } & 0.511124 \\ \text { Mean of Response } & 49.4624 \\ \text { Observations (or Sum Wgts) } & 48\end{array}$

Analysis of Variance

$\begin{array}{lrrrrr}\text { Source } & \text { DF } & \text { Sum of Squares } & \text { Mean Square } & \text { F Ratio } & \text { Prob > F } \\ \text { Block/Sub-Block } & 15 & 24.862404 & 1.65749 & 6.3445 & <.0001 \\ \text { Error } & 32 & 8.359931 & 0.26125 & & \\ \text { C. Total } & 47 & 33.222335 & & & \end{array}$

Means for Oneway Anova

Level Number Mean Std Error Lower 95\% Upper 95\%

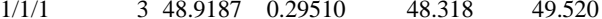

$\begin{array}{llllll}1 / 1 / 2 & 3 & 48.5621 & 0.29510 & 47.961 & 49.163\end{array}$

$\begin{array}{llllll}1 / 2 / 1 & 3 & 49.5605 & 0.29510 & 48.959 & 50.162\end{array}$

$\begin{array}{llllll}1 / 2 / 2 & 3 & 49.2039 & 0.29510 & 48.603 & 49.805\end{array}$

$\begin{array}{llllll}2 / 1 / 1 & 3 & 49.6318 & 0.29510 & 49.031 & 50.233\end{array}$

$\begin{array}{llllll}2 / 1 / 2 & 3 & 48.9900 & 0.29510 & 48.389 & 49.591\end{array}$

$\begin{array}{llllll}2 / 2 / 1 & 3 & 48.1343 & 0.29510 & 47.533 & 48.735\end{array}$

$\begin{array}{llllll}2 / 2 / 2 & 3 & 50.7014 & 0.29510 & 50.100 & 51.303\end{array}$

$\begin{array}{llllll}3 / 1 / 1 & 3 & 49.2752 & 0.29510 & 48.674 & 49.876\end{array}$

$\begin{array}{llllll}3 / 1 / 2 & 3 & 48.4908 & 0.29510 & 47.890 & 49.092\end{array}$

$\begin{array}{llllll}3 / 2 / 1 & 3 & 49.3465 & 0.29510 & 48.745 & 49.948\end{array}$

$\begin{array}{llllll}3 / 2 / 2 & 3 & 49.7744 & 0.29510 & 49.173 & 50.375\end{array}$

$\begin{array}{llllll}4 / 1 / 1 & 3 & 50.4162 & 0.29510 & 49.815 & 51.017\end{array}$

$\begin{array}{llllll}4 / 1 / 2 & 3 & 50.0596 & 0.29510 & 49.459 & 50.661\end{array}$

$\begin{array}{llll}4 / 2 / 1 & 3 & 50.4875 & 0.29510\end{array}$

$\begin{array}{llllll}4 / 2 / 2 & 3 & 49.8457 & 0.29510 & 49.245 & 50.447\end{array}$

Std Error uses a pooled estimate of error variance 


\section{Exhibit A5. Measured and Measured Bias-Corrected Oxide Weight Percents}

by Lab ID within Glass ID for the Glasses Prepared Using the LM Method. (continued)

Variability Gage Set $=1$, Oxide=Al2O3 (wt $\%$ )

Variability Chart for Measured

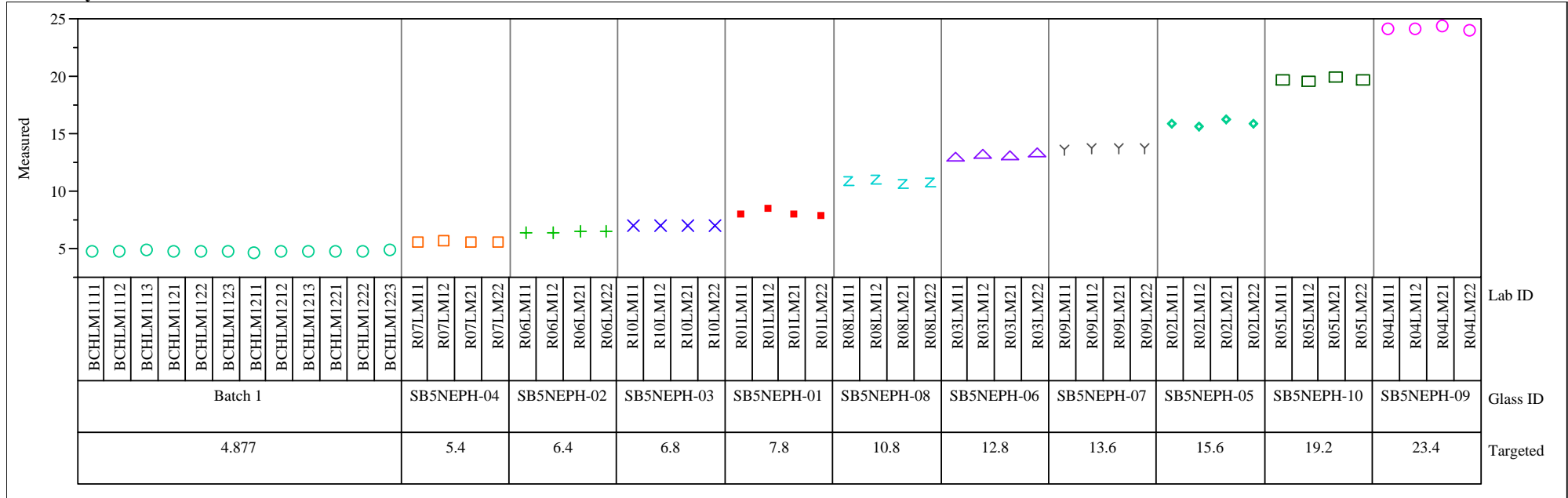

Variability Chart for Measured bc

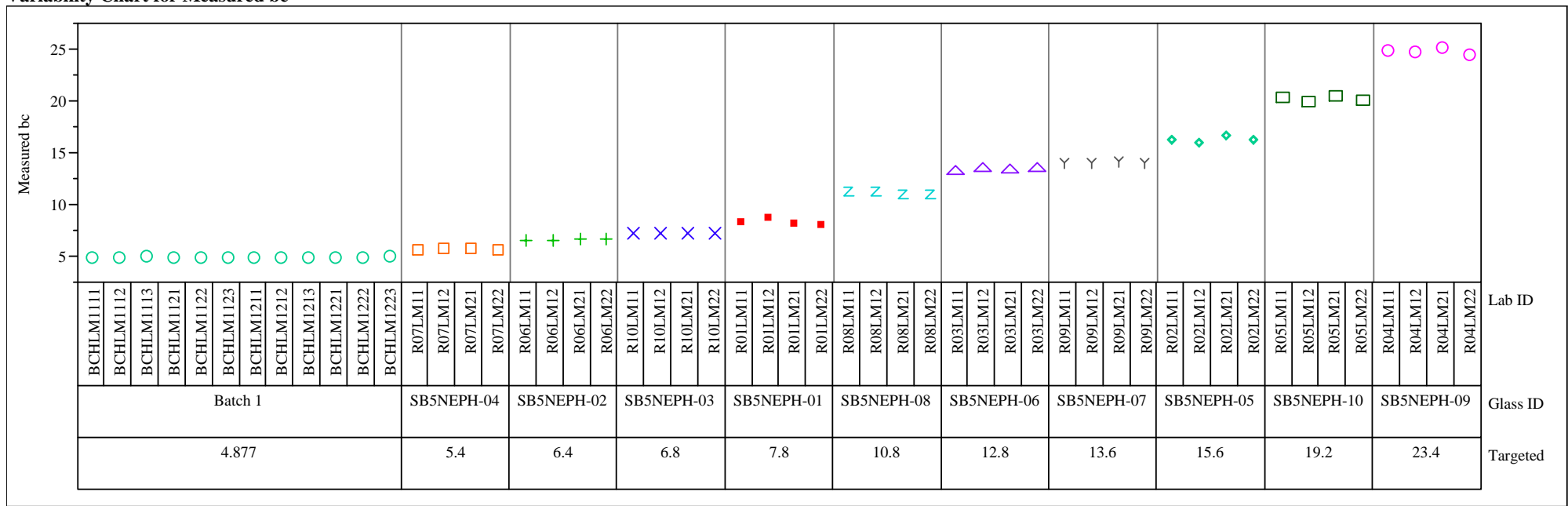


Exhibit A5. Measured and Measured Bias-Corrected Oxide Weight Percents

by Lab ID within Glass ID for the Glasses Prepared Using the LM Method. (continued)

Variability Gage Set $=1$, Oxide $=\mathrm{BaO}$ ( $\mathrm{wt} \%$ )

Variability Chart for Measured

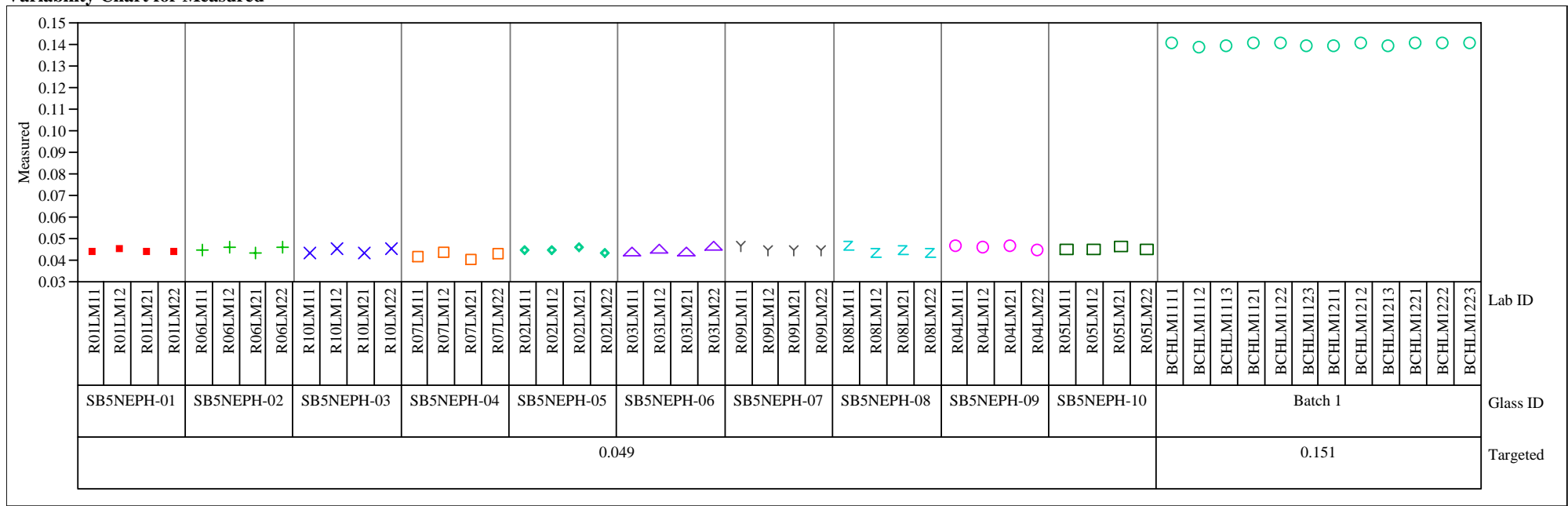

Variability Chart for Measured bc

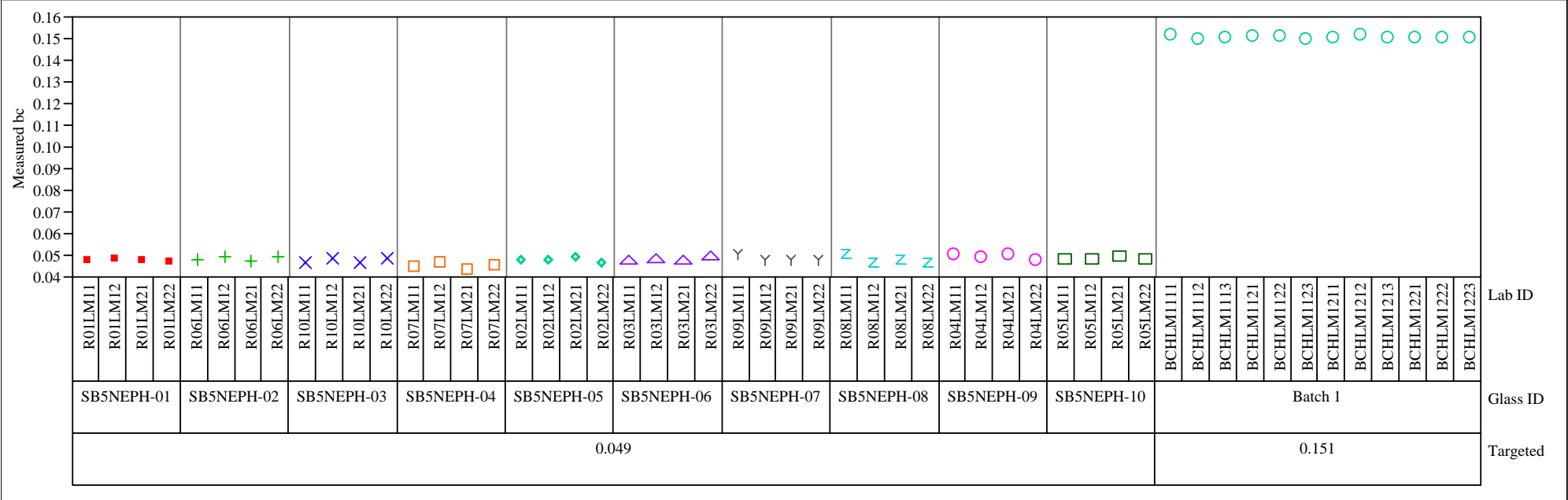


Exhibit A5. Measured and Measured Bias-Corrected Oxide Weight Percents

by Lab ID within Glass ID for the Glasses Prepared Using the LM Method. (continued)

Variability Gage Set $=1$, Oxide $=\mathrm{CaO}$ (wt $\%$ )

Variability Chart for Measured

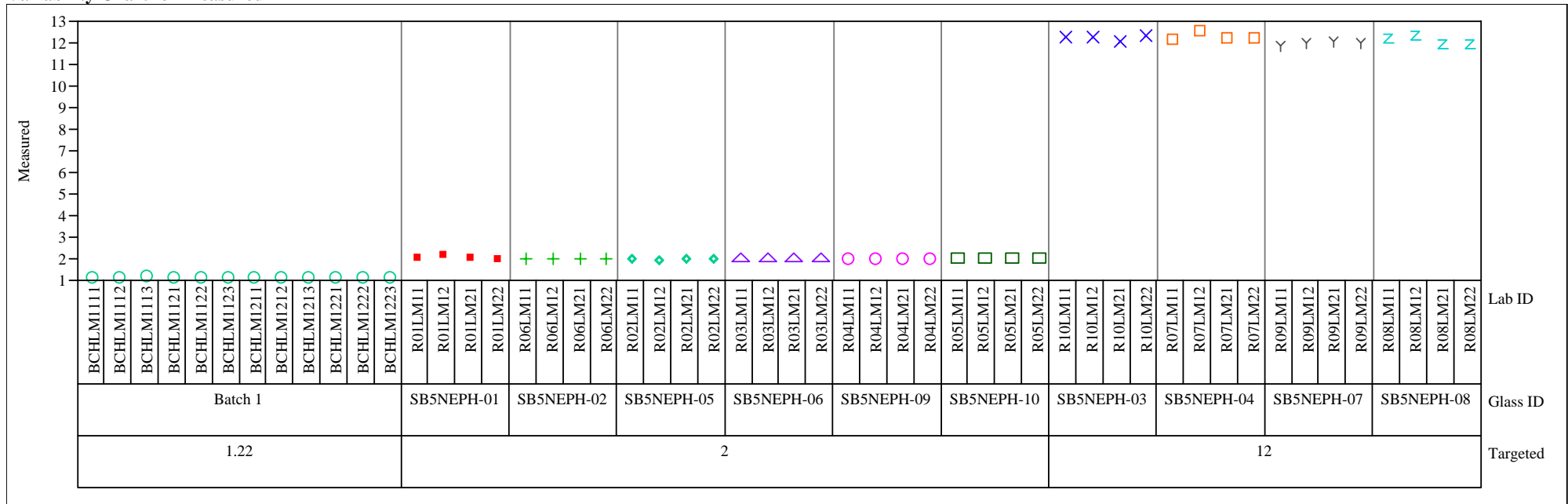

Variability Chart for Measured bc

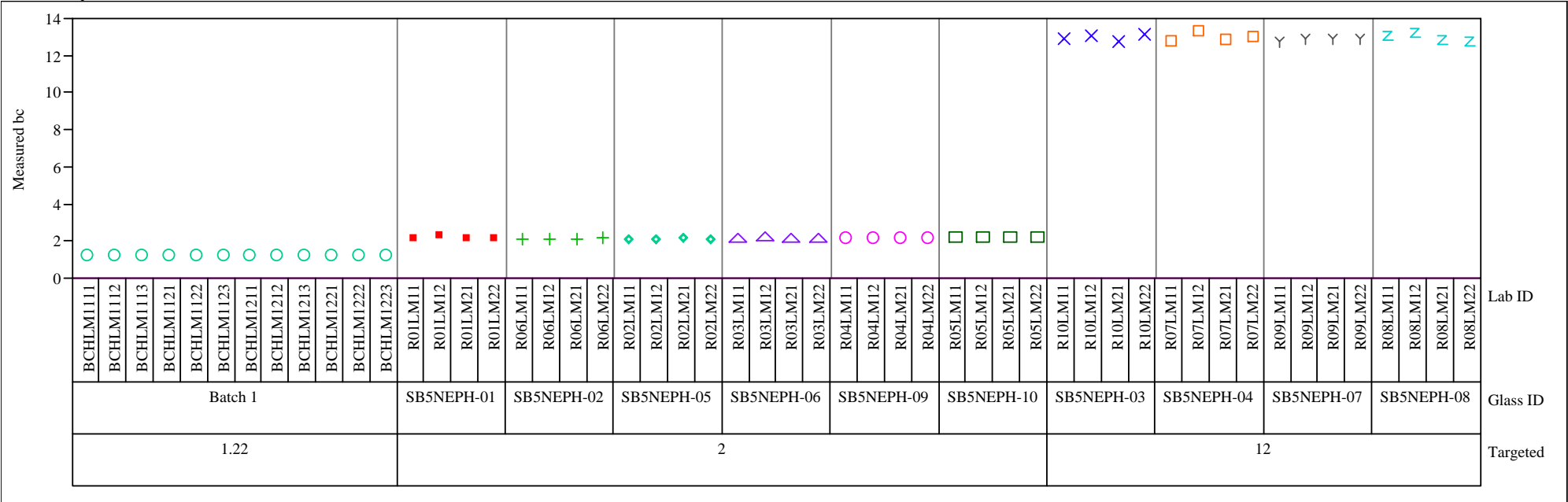


Exhibit A5. Measured and Measured Bias-Corrected Oxide Weight Percents

by Lab ID within Glass ID for the Glasses Prepared Using the LM Method. (continued)

Variability Gage Set $=1$, Oxide $=$ Ce2O3 $(w t \%)$

Variability Chart for Measured

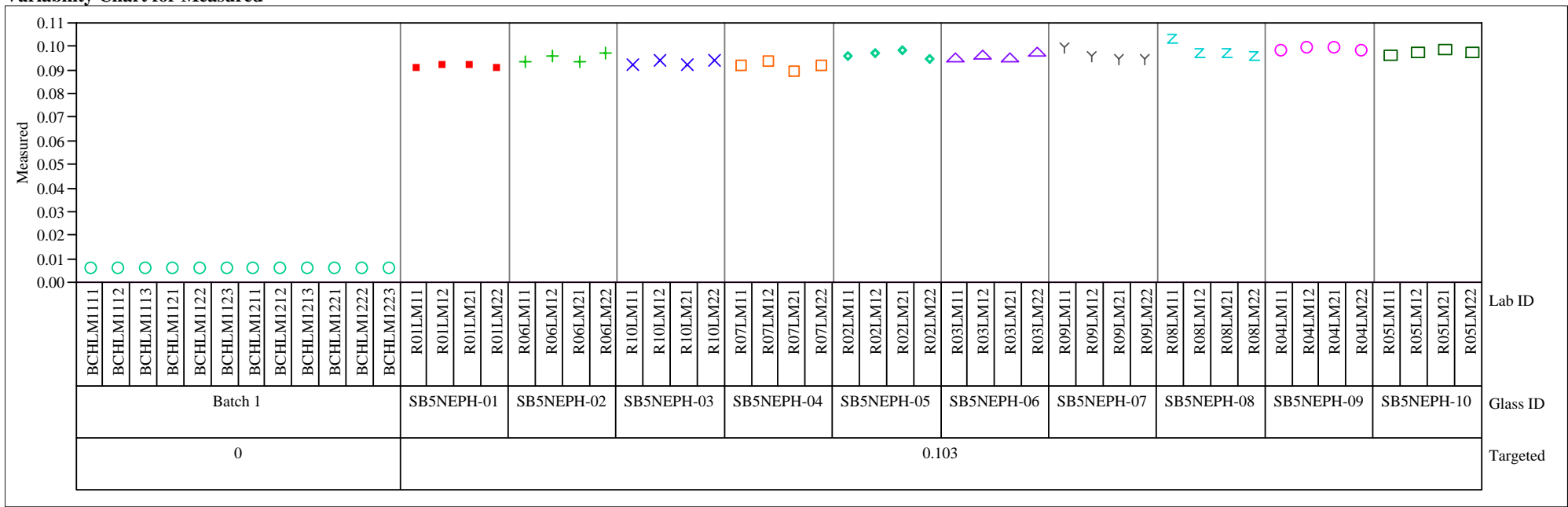

Variability Chart for Measured bc

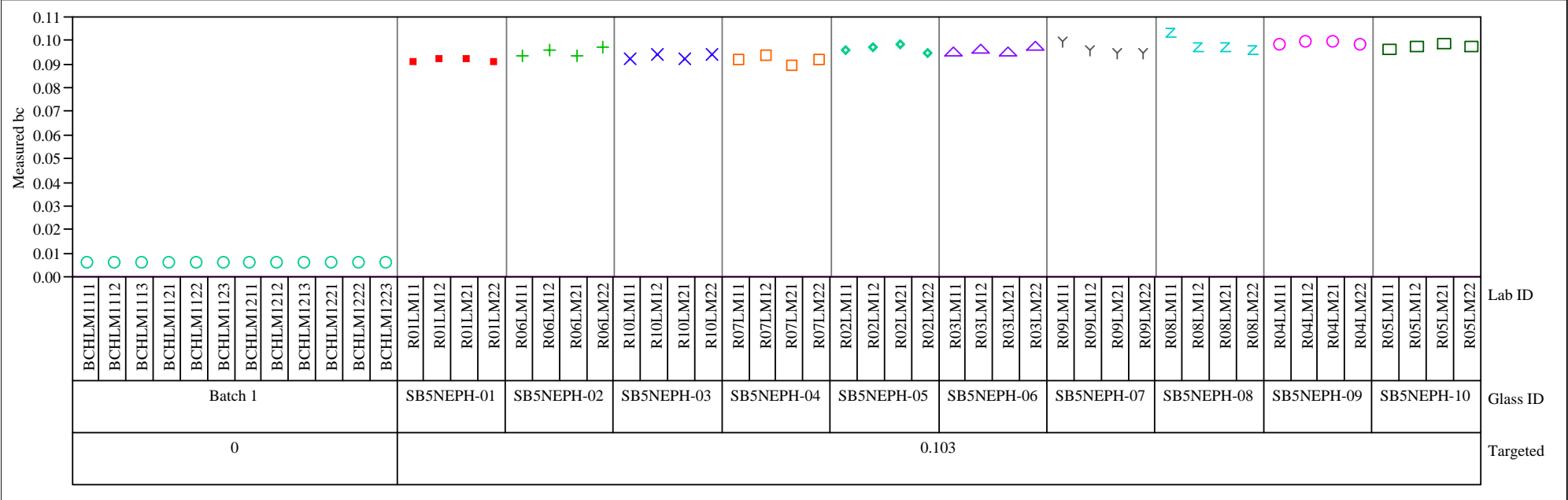


Exhibit A5. Measured and Measured Bias-Corrected Oxide Weight Percents

by Lab ID within Glass ID for the Glasses Prepared Using the LM Method. (continued)

Variability Gage Set $=1$, Oxide $=$ Cr2O3 (wt $\%)$

Variability Chart for Measured

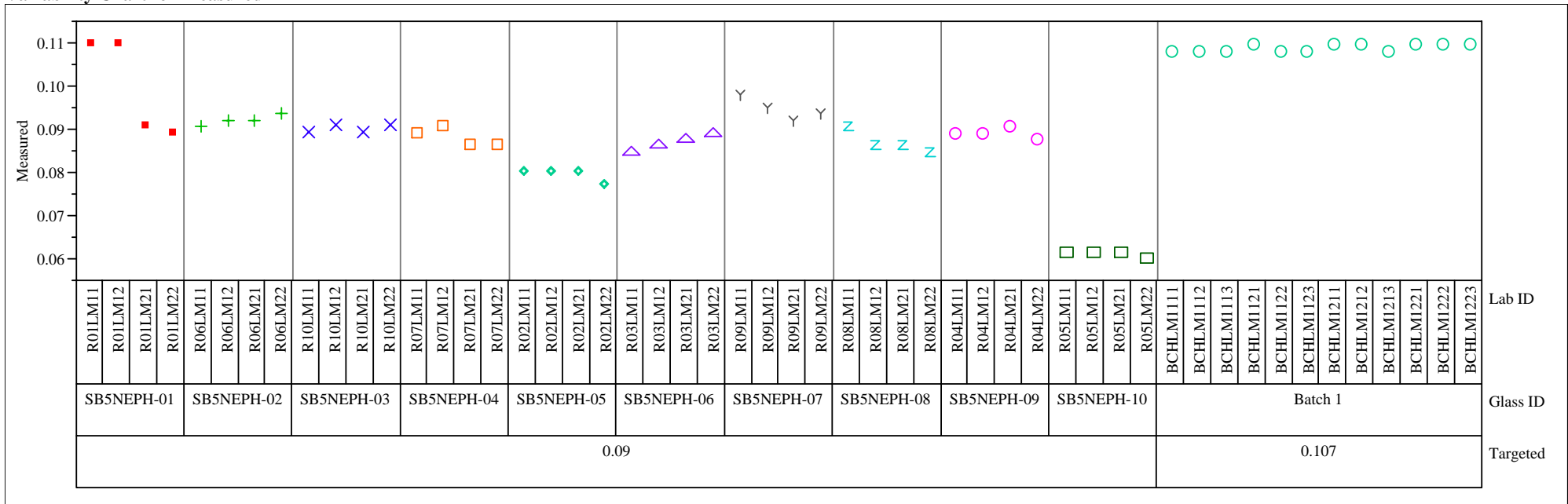

Variability Chart for Measured bc

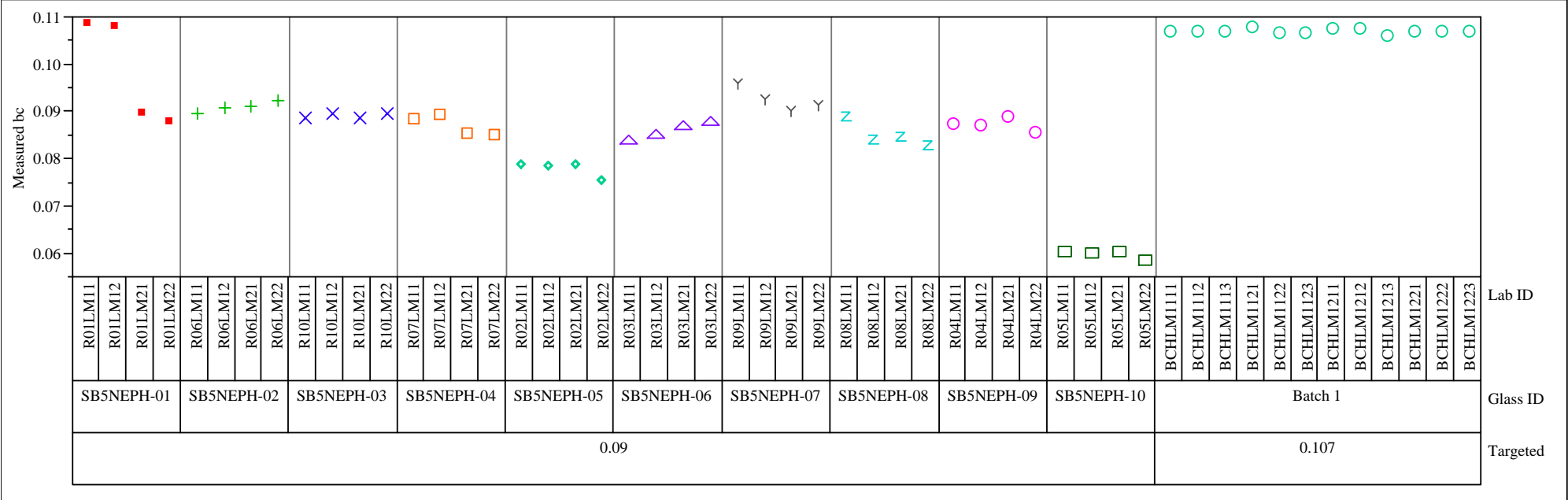


Exhibit A5. Measured and Measured Bias-Corrected Oxide Weight Percents

by Lab ID within Glass ID for the Glasses Prepared Using the LM Method. (continued)

Variability Gage Set $=1$, Oxide $=\mathrm{CuO}(\mathrm{wt} \%)$

Variability Chart for Measured

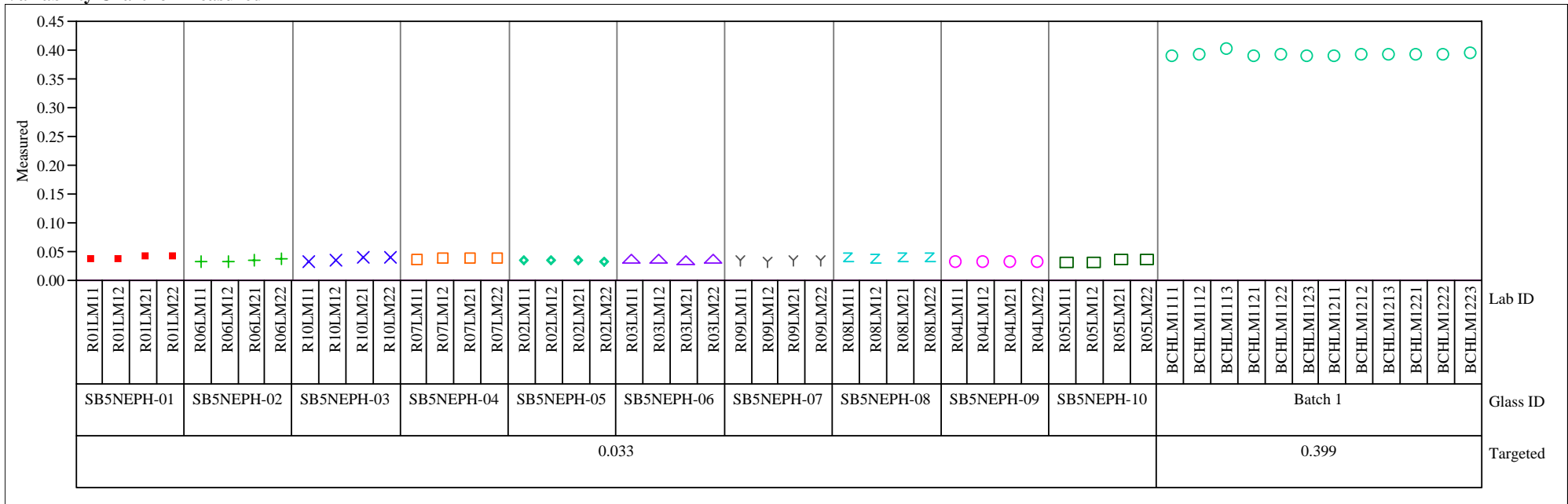

Variability Chart for Measured bc

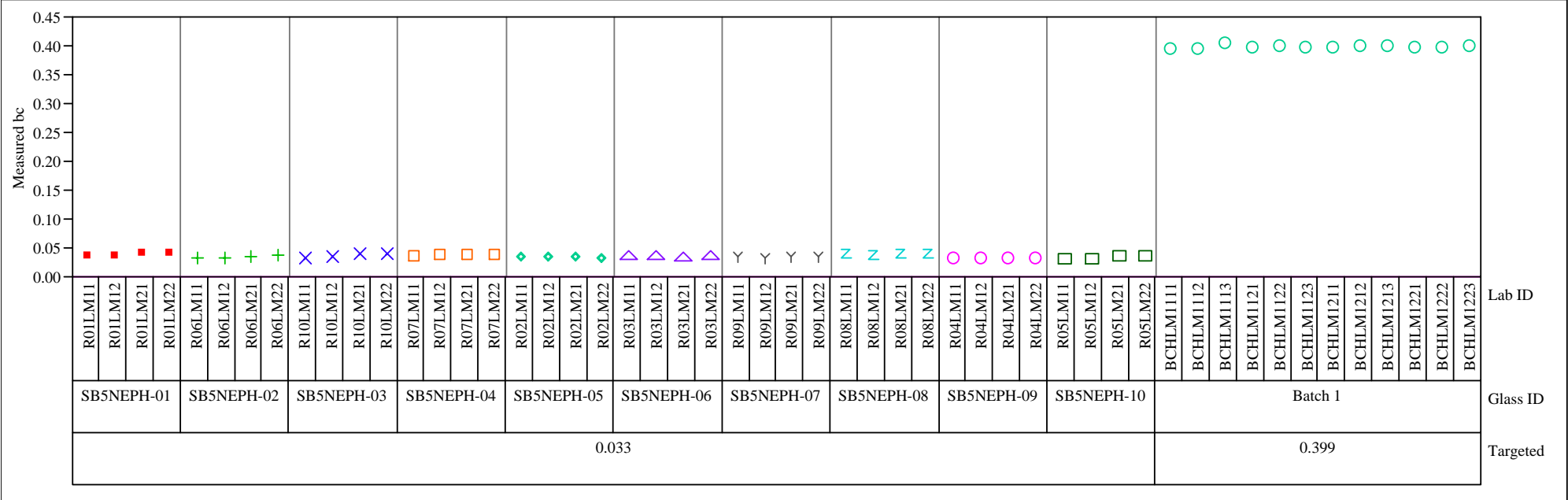


Exhibit A5. Measured and Measured Bias-Corrected Oxide Weight Percents

by Lab ID within Glass ID for the Glasses Prepared Using the LM Method. (continued)

Variability Gage Set $=1$, Oxide $=$ K2O (wt $\%$ )

Variability Chart for Measured

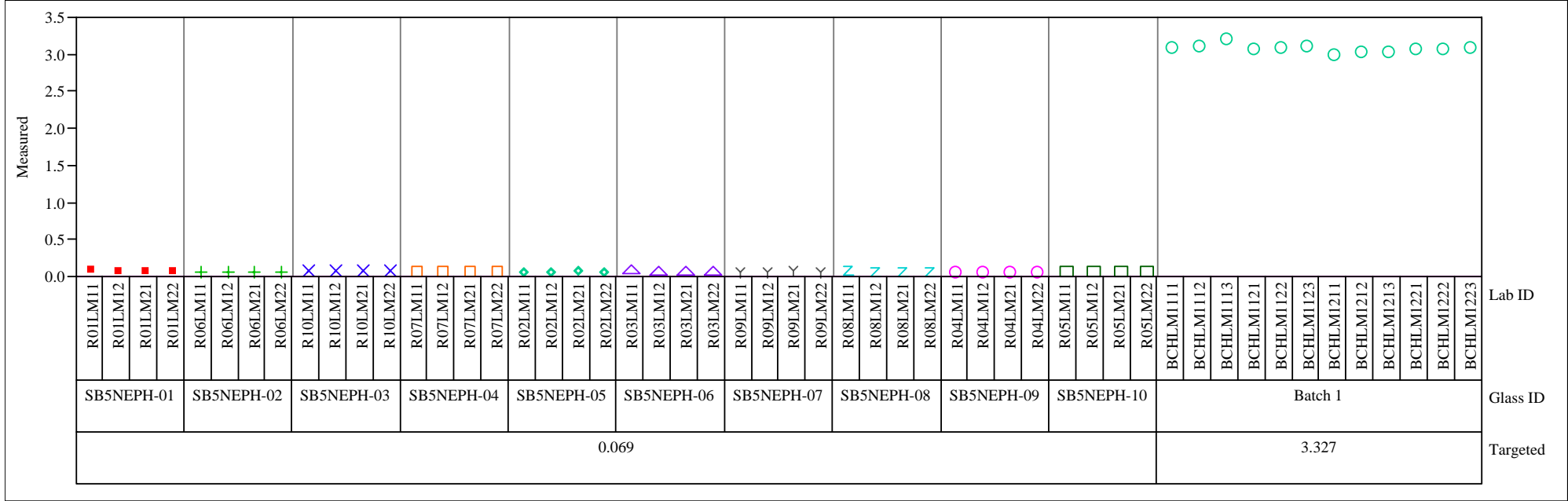

Variability Chart for Measured bc

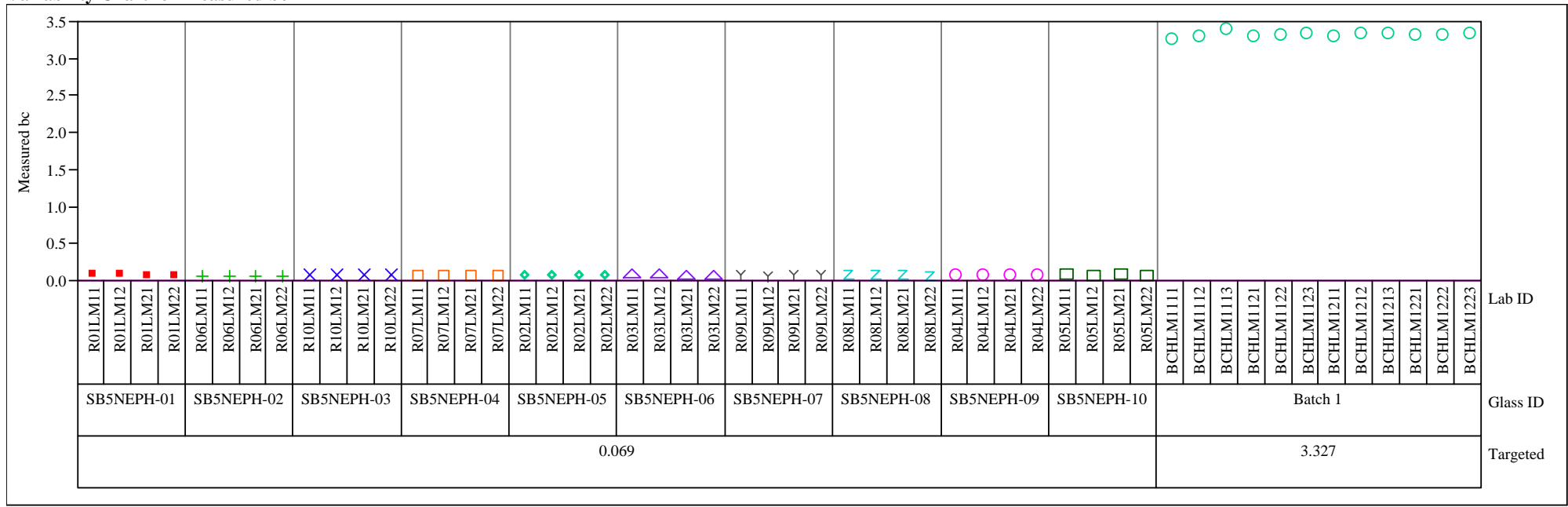


Exhibit A5. Measured and Measured Bias-Corrected Oxide Weight Percents

by Lab ID within Glass ID for the Glasses Prepared Using the LM Method. (continued)

Variability Gage Set $=1$, Oxide $=$ La2O3 (wt $\%$ )

Variability Chart for Measured

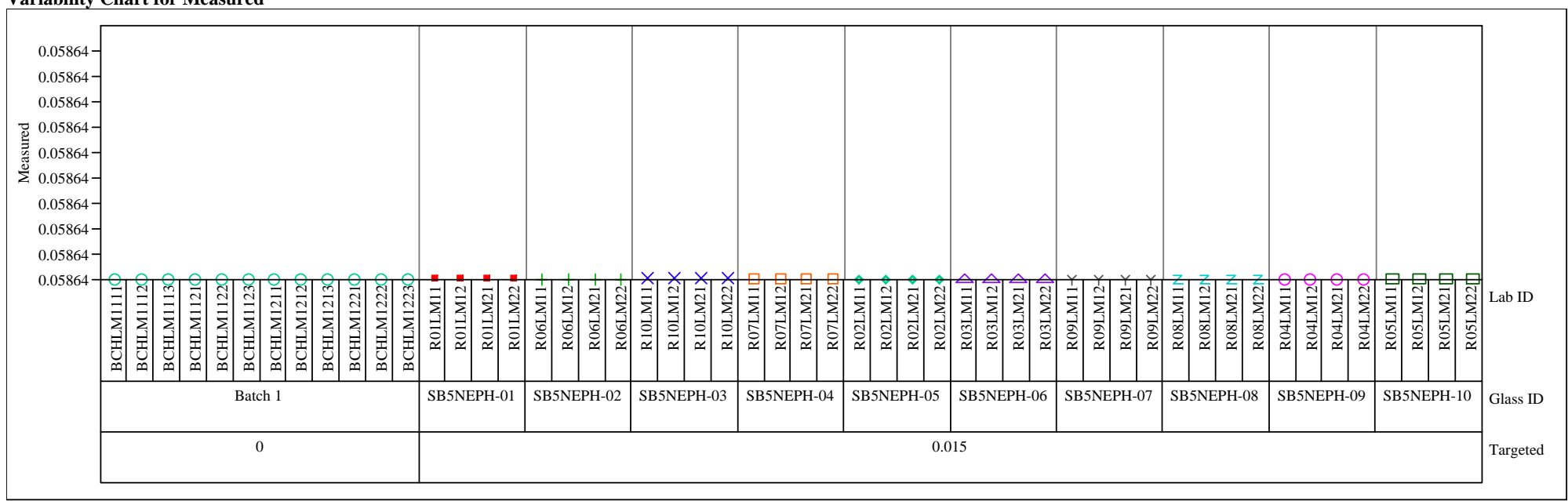

Variability Chart for Measured bc

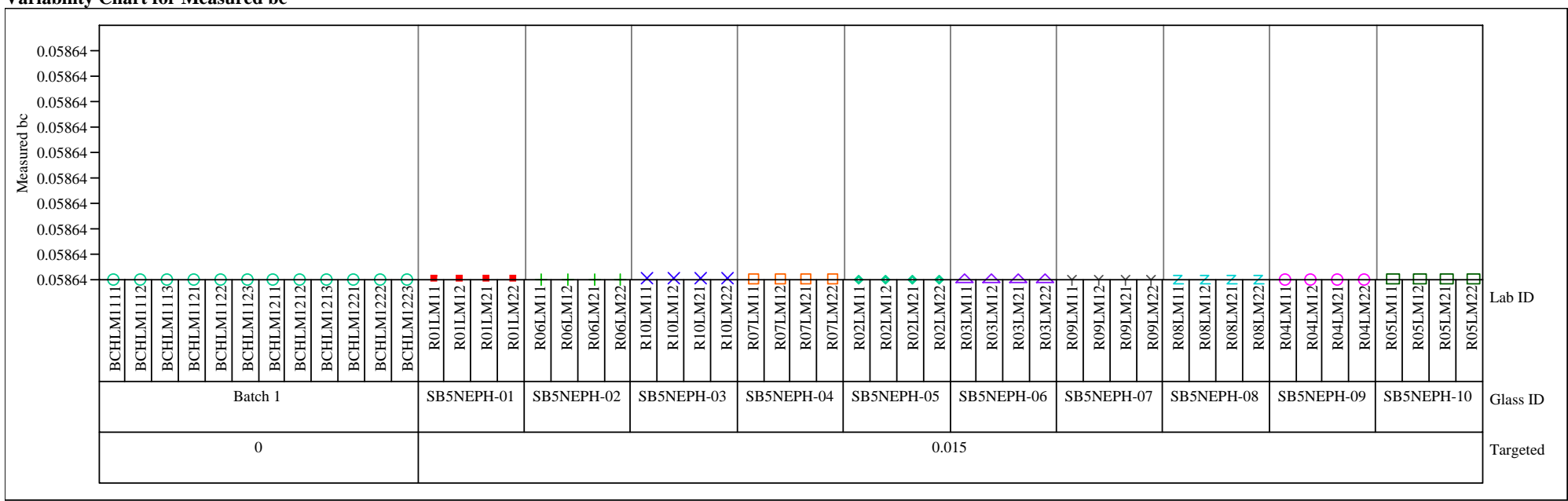


Exhibit A5. Measured and Measured Bias-Corrected Oxide Weight Percents

by Lab ID within Glass ID for the Glasses Prepared Using the LM Method. (continued)

Variability Gage Set $=1$, Oxide $=\mathrm{MgO}(\mathrm{wt} \%)$

Variability Chart for Measured

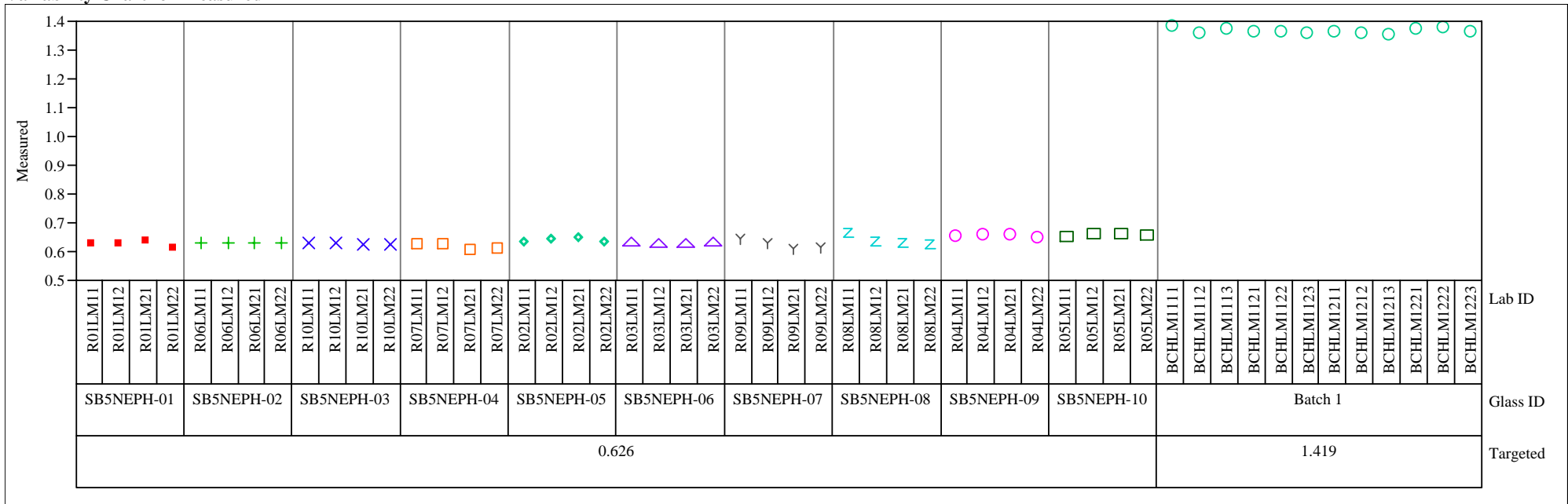

Variability Chart for Measured bc

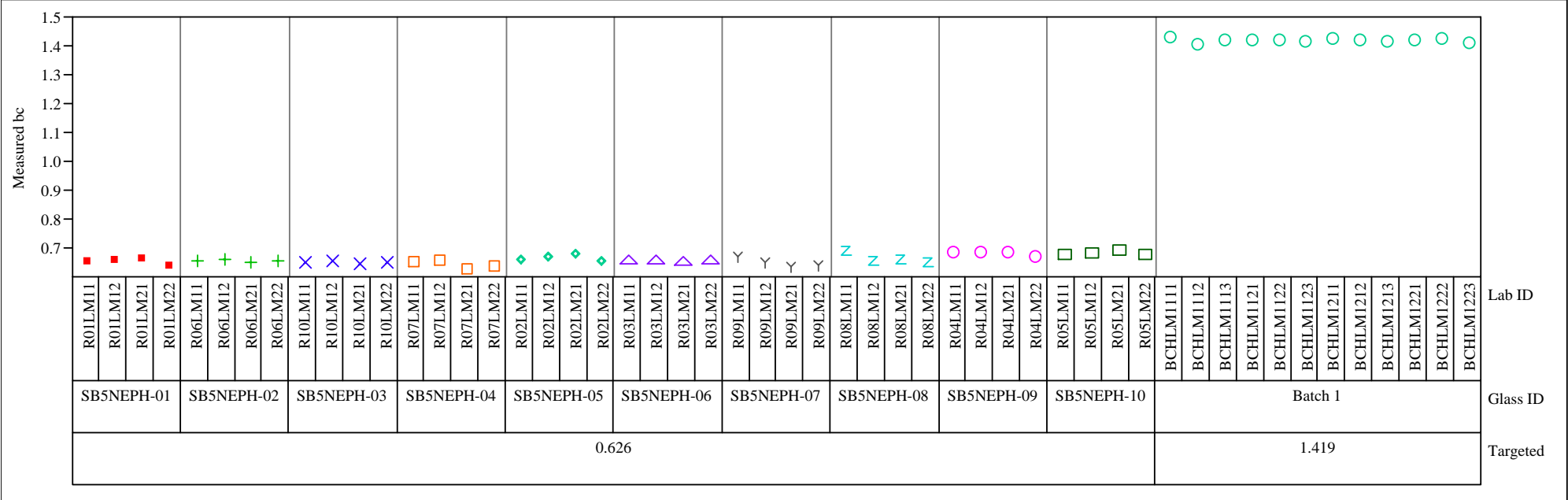


Exhibit A5. Measured and Measured Bias-Corrected Oxide Weight Percents

by Lab ID within Glass ID for the Glasses Prepared Using the LM Method. (continued)

Variability Gage Set=1, Oxide $=\mathrm{MnO}(\mathrm{wt} \%)$

Variability Chart for Measured

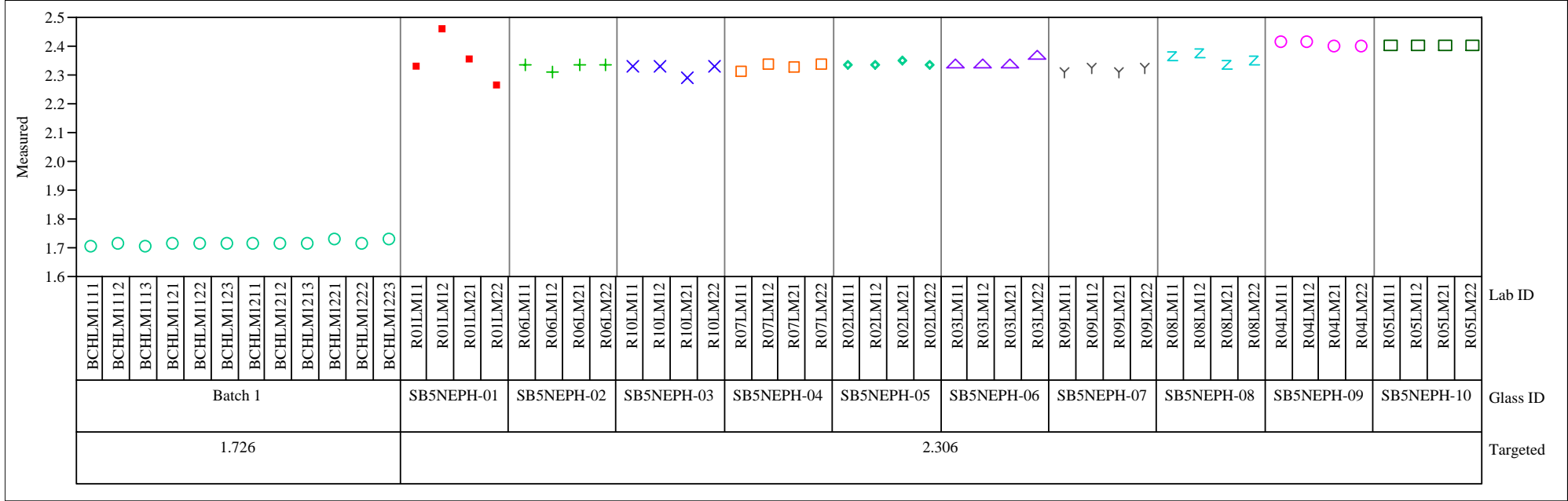

Variability Chart for Measured bc

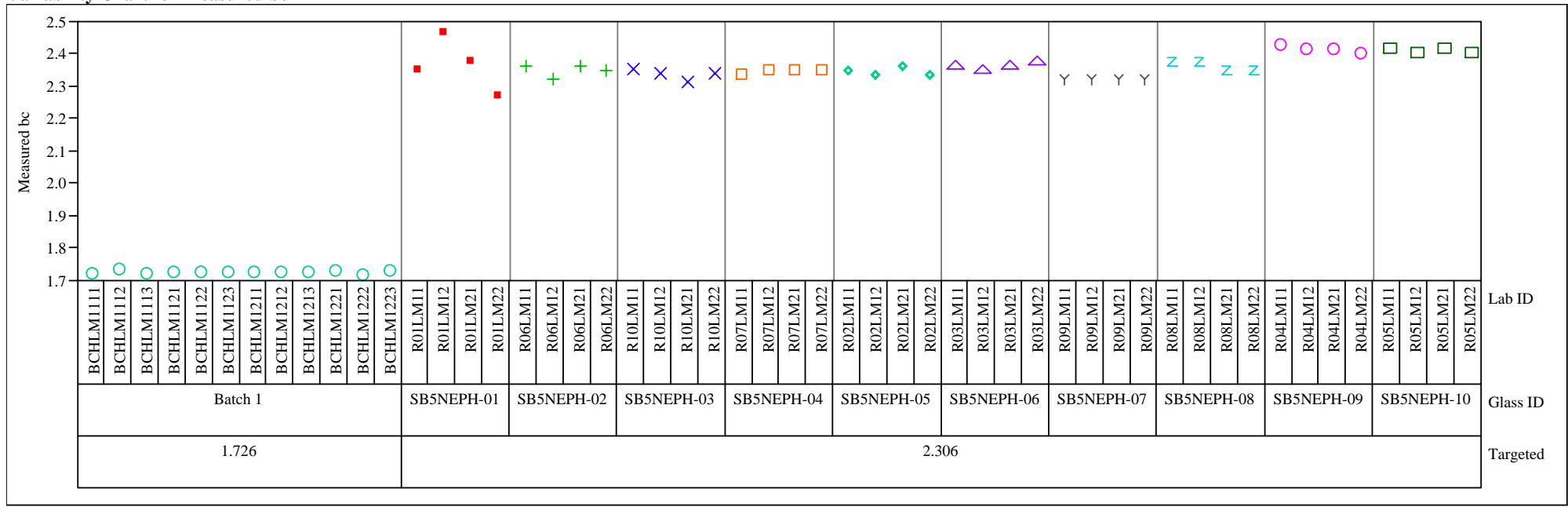


Exhibit A5. Measured and Measured Bias-Corrected Oxide Weight Percents

by Lab ID within Glass ID for the Glasses Prepared Using the LM Method. (continued)

Variability Gage Set $=1$, Oxide $=\mathrm{Na} 2 \mathrm{O}(\mathrm{wt} \%)$

Variability Chart for Measured

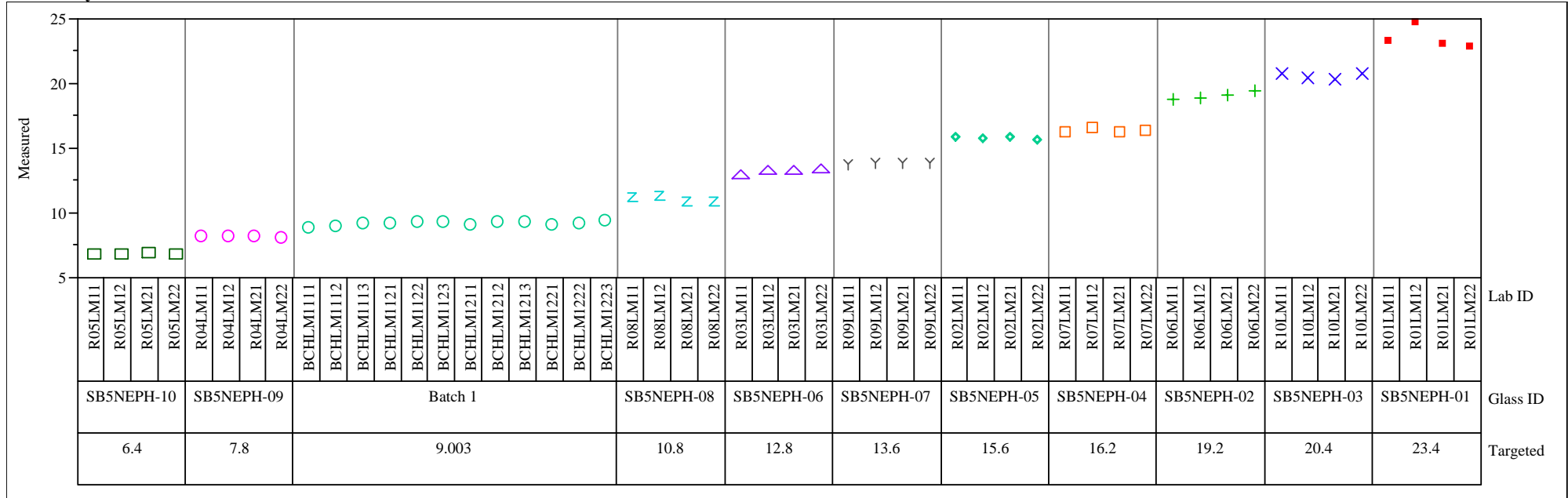

Variability Chart for Measured bc

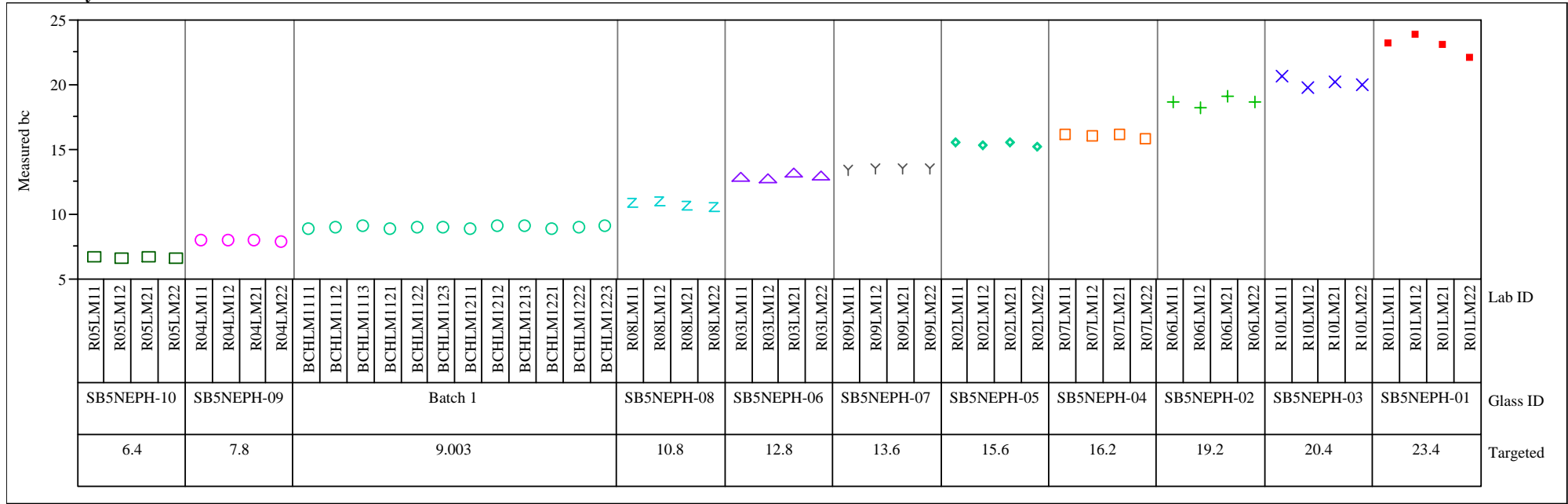


Exhibit A5. Measured and Measured Bias-Corrected Oxide Weight Percents

by Lab ID within Glass ID for the Glasses Prepared Using the LM Method. (continued)

Variability Gage Set $=1$, Oxide $=\mathrm{NiO}$ (wt $\%$ )

Variability Chart for Measured

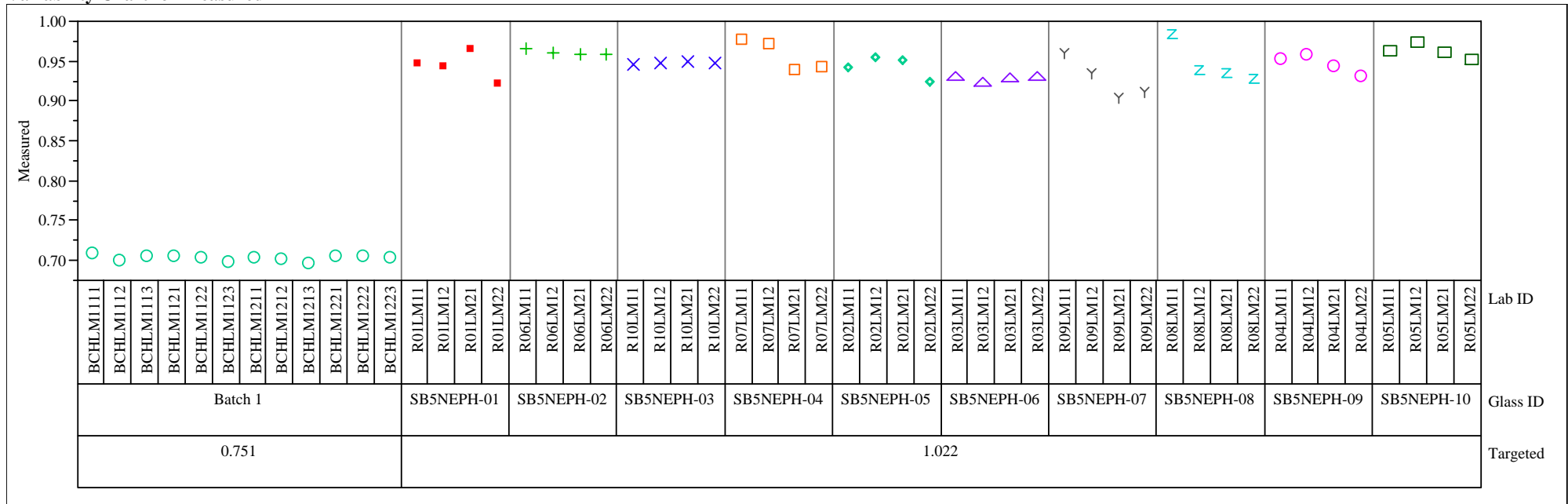

Variability Chart for Measured bc

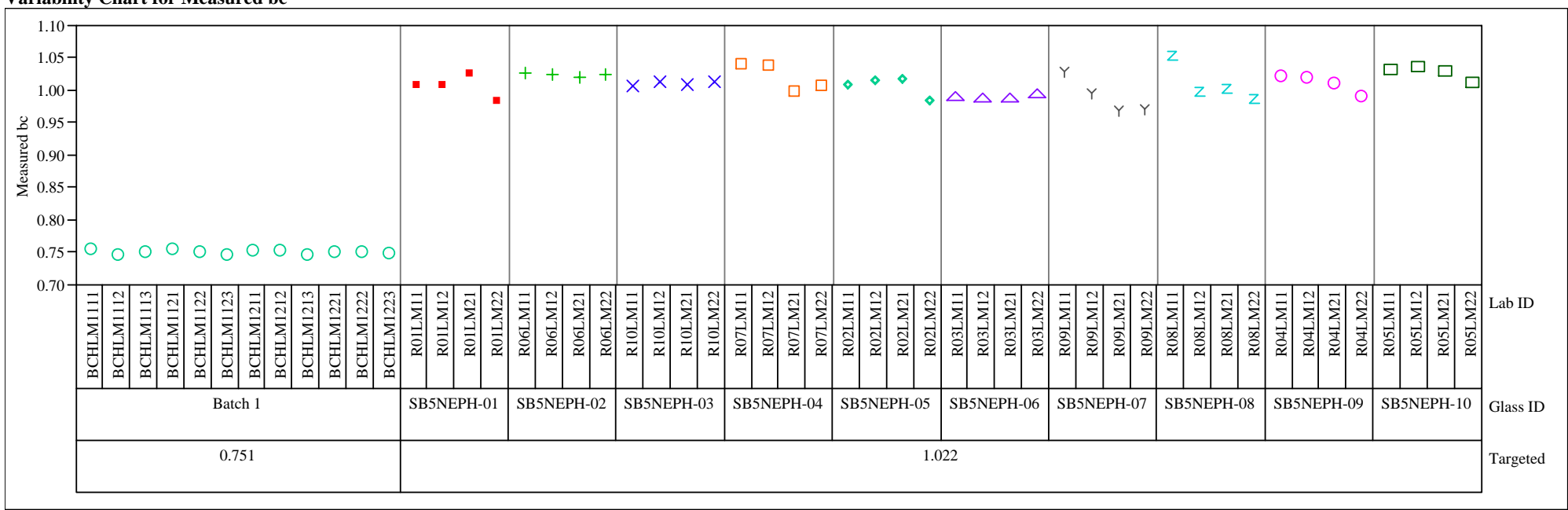


Exhibit A5. Measured and Measured Bias-Corrected Oxide Weight Percents

by Lab ID within Glass ID for the Glasses Prepared Using the LM Method. (continued)

Variability Gage Set $=1$, Oxide $=\mathbf{P b O}$ (wt $\%$ )

Variability Chart for Measured
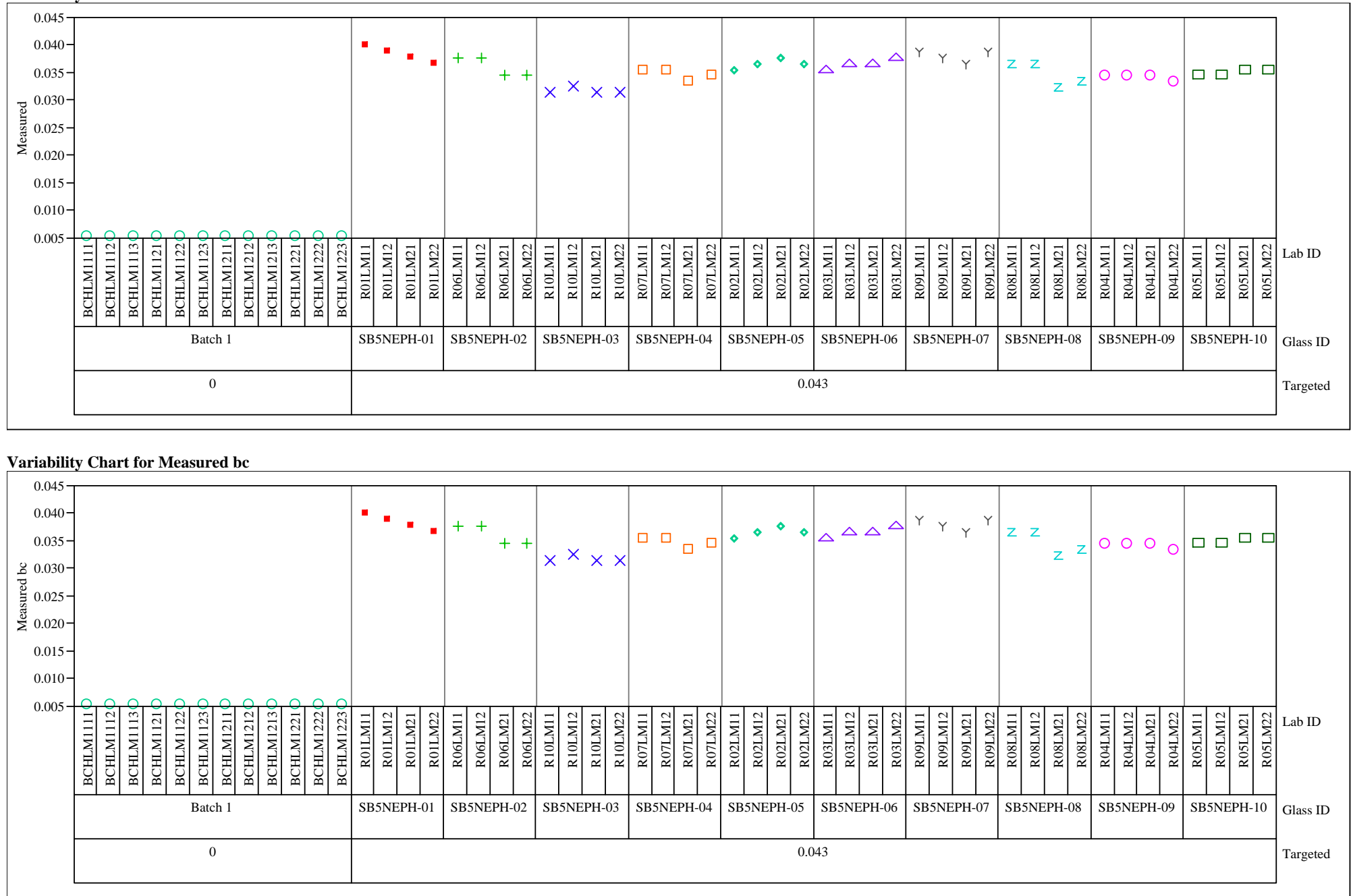
Exhibit A5. Measured and Measured Bias-Corrected Oxide Weight Percents

by Lab ID within Glass ID for the Glasses Prepared Using the LM Method. (continued)

Variability Gage Set=1, Oxide=SO4 (wt\%)

Variability Chart for Measured

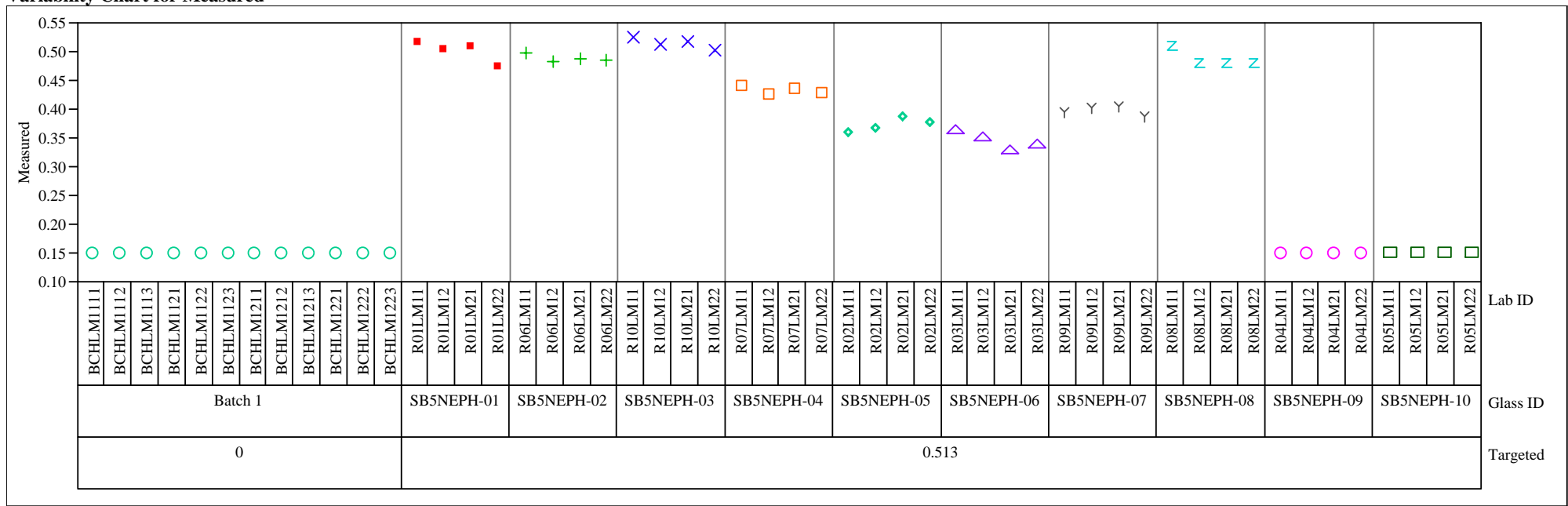

Variability Chart for Measured bc

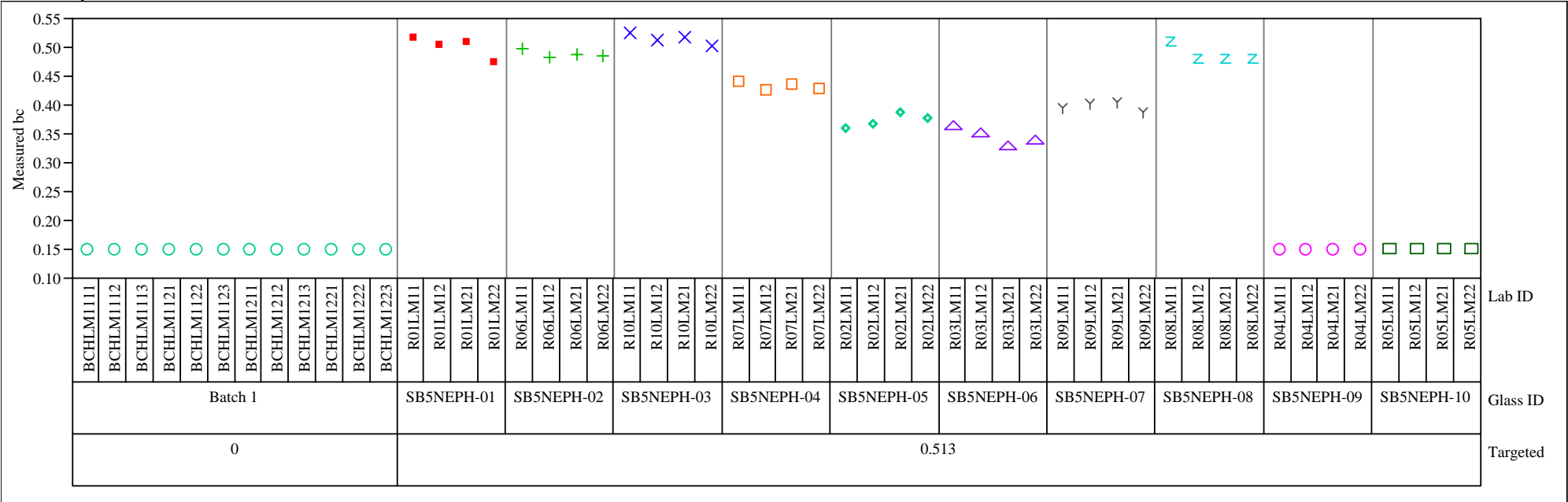


Exhibit A5. Measured and Measured Bias-Corrected Oxide Weight Percents

by Lab ID within Glass ID for the Glasses Prepared Using the LM Method. (continued)

Variability Gage Set $=1$, Oxide $=$ TiO2 $(w t \%)$

Variability Chart for Measured

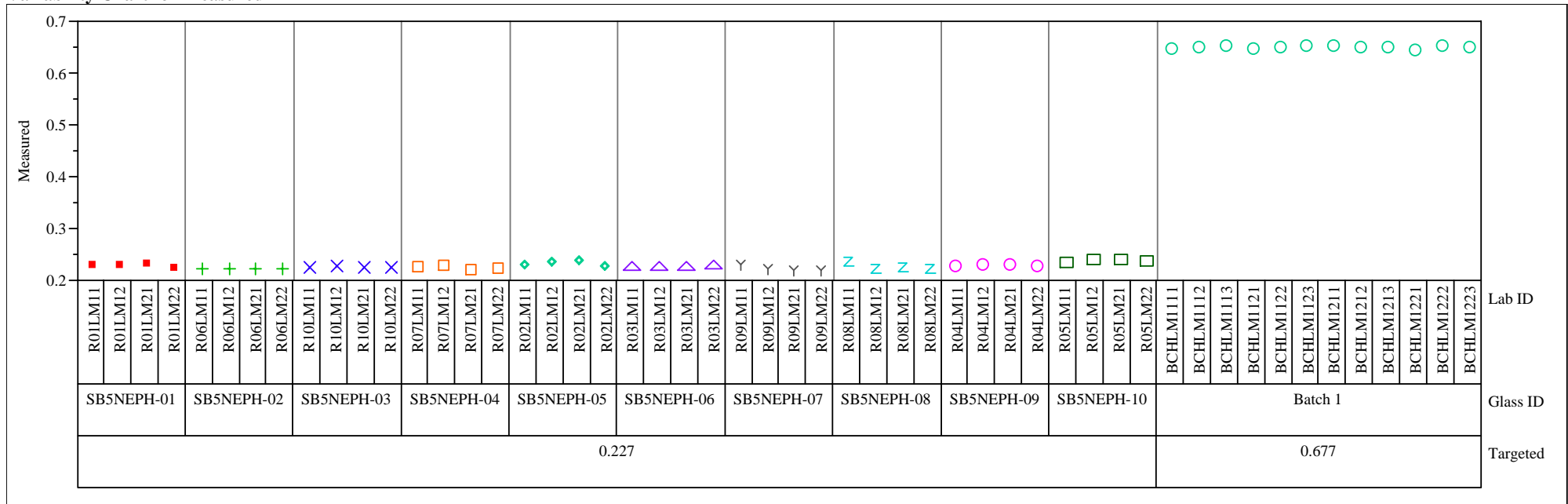

Variability Chart for Measured bc

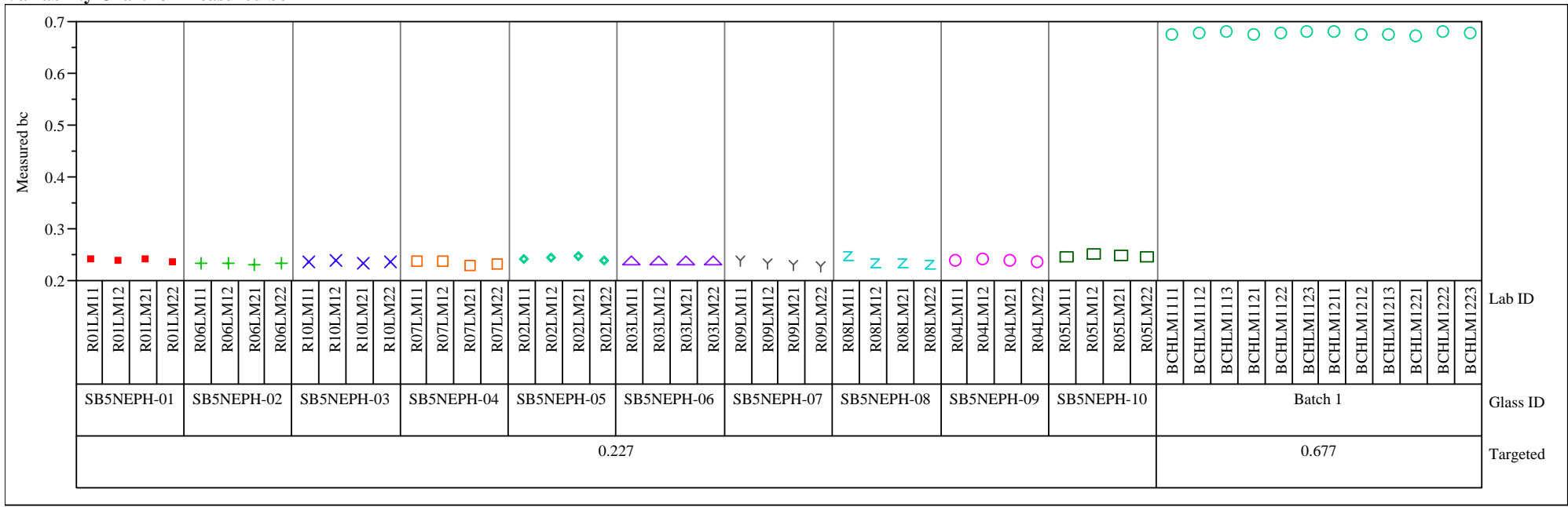


Exhibit A5. Measured and Measured Bias-Corrected Oxide Weight Percents

by Lab ID within Glass ID for the Glasses Prepared Using the LM Method. (continued)

Variability Gage Set $=1$, Oxide $=\mathrm{ZnO}(\mathrm{wt} \%)$

Variability Chart for Measured
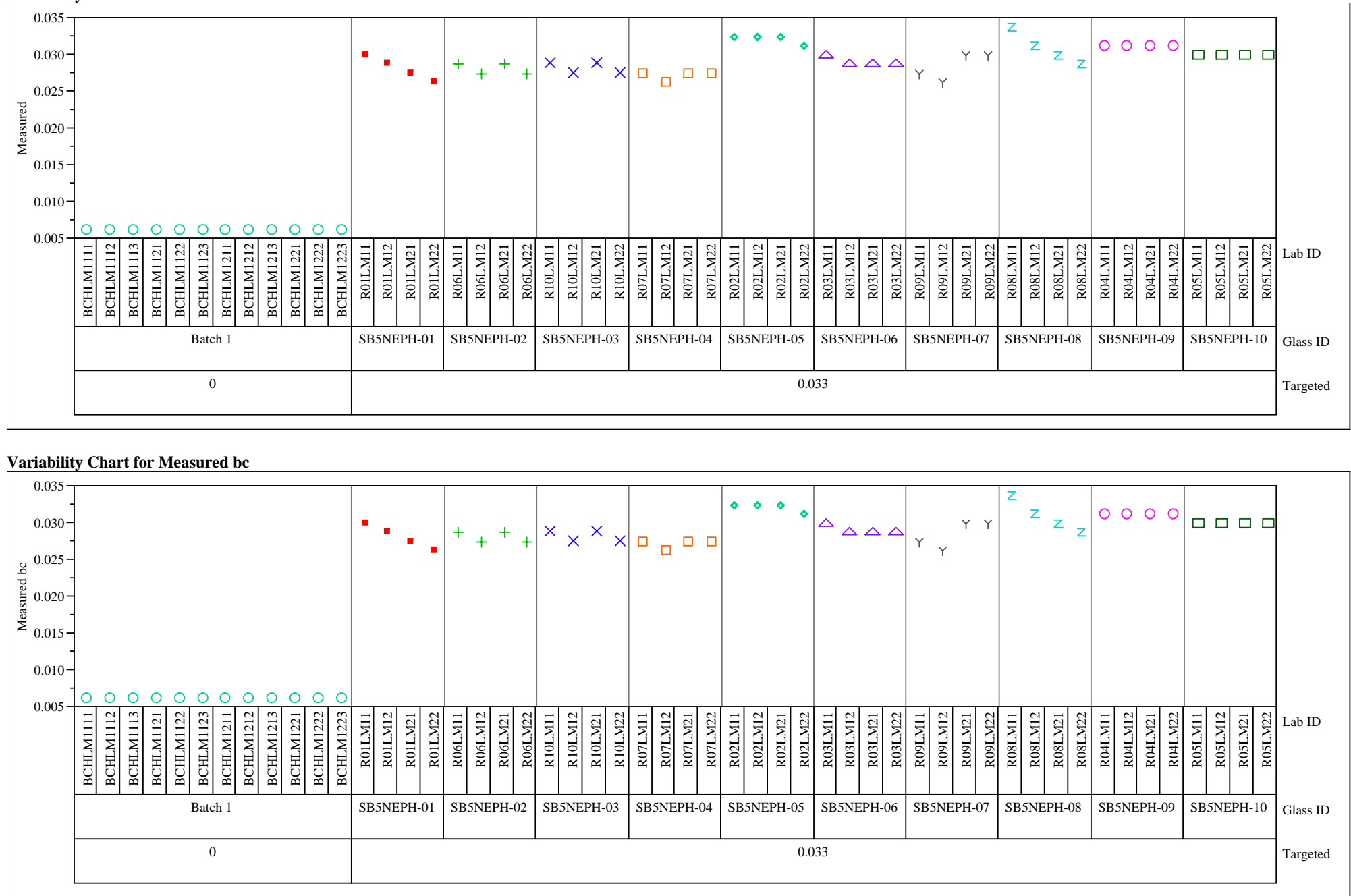
Exhibit A5. Measured and Measured Bias-Corrected Oxide Weight Percents

by Lab ID within Glass ID for the Glasses Prepared Using the LM Method. (continued)

Variability Gage Set $=1$, Oxide $=\mathrm{ZrO} 2(\mathrm{wt} \%)$

Variability Chart for Measured

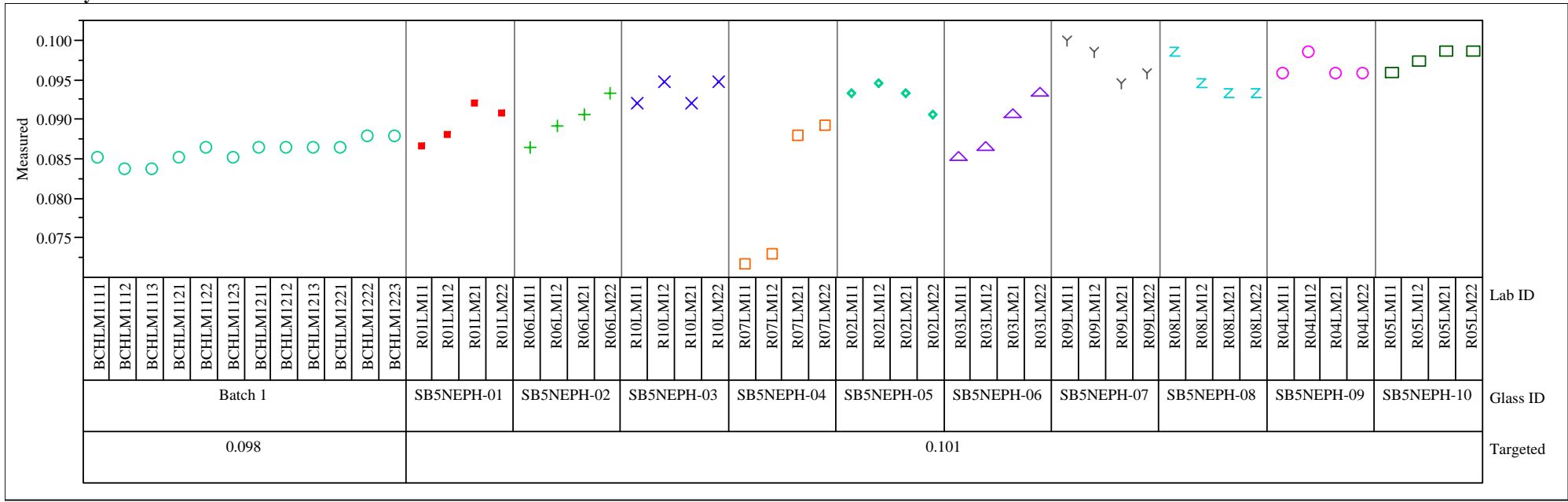

Variability Chart for Measured bc

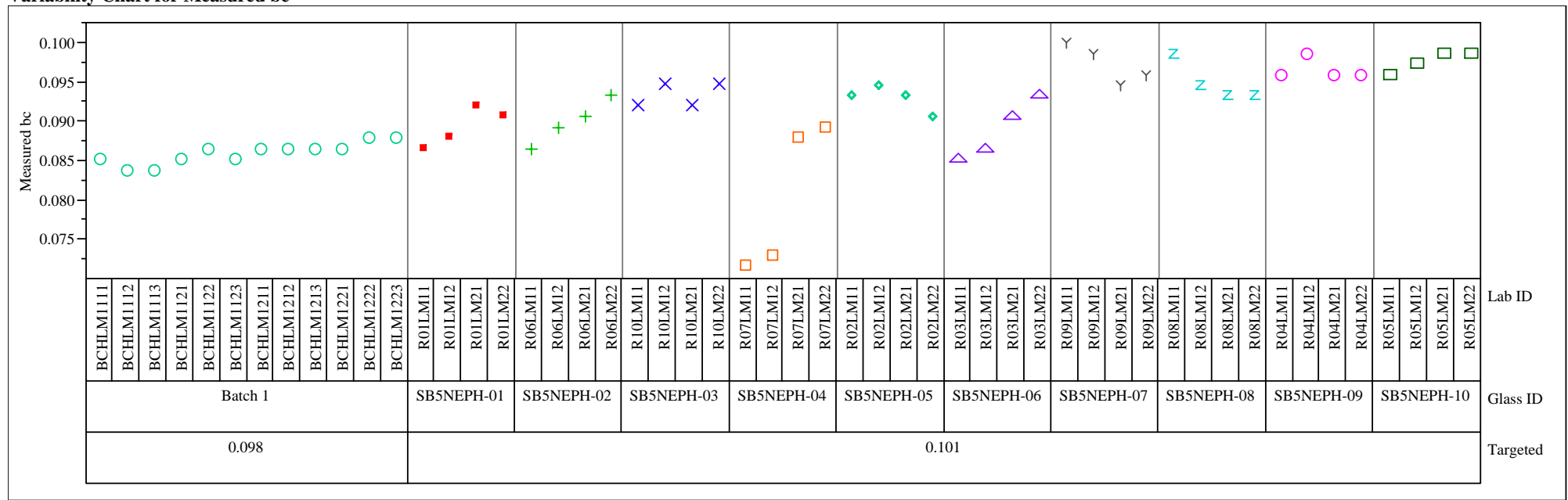


Exhibit A5. Measured and Measured Bias-Corrected Oxide Weight Percents

by Lab ID within Glass ID for the Glasses Prepared Using the LM Method. (continued)

Variability Gage Set $=2$, Oxide=Al2O3 (wt $\%$ )

Variability Chart for Measured

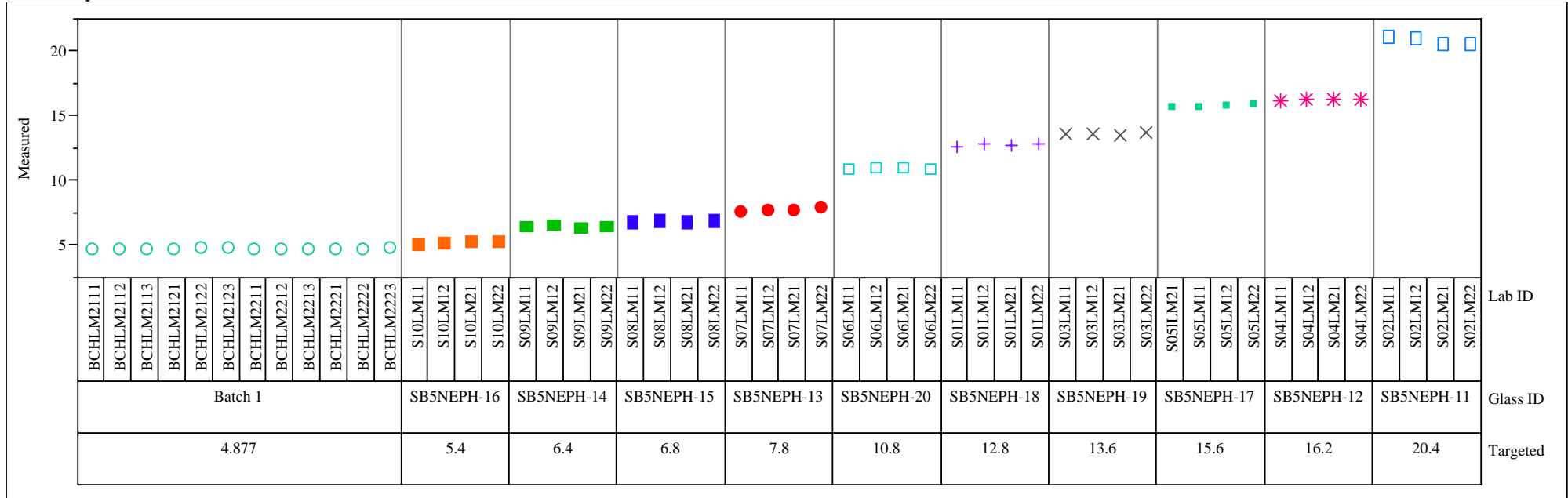

Variability Chart for Measured bc

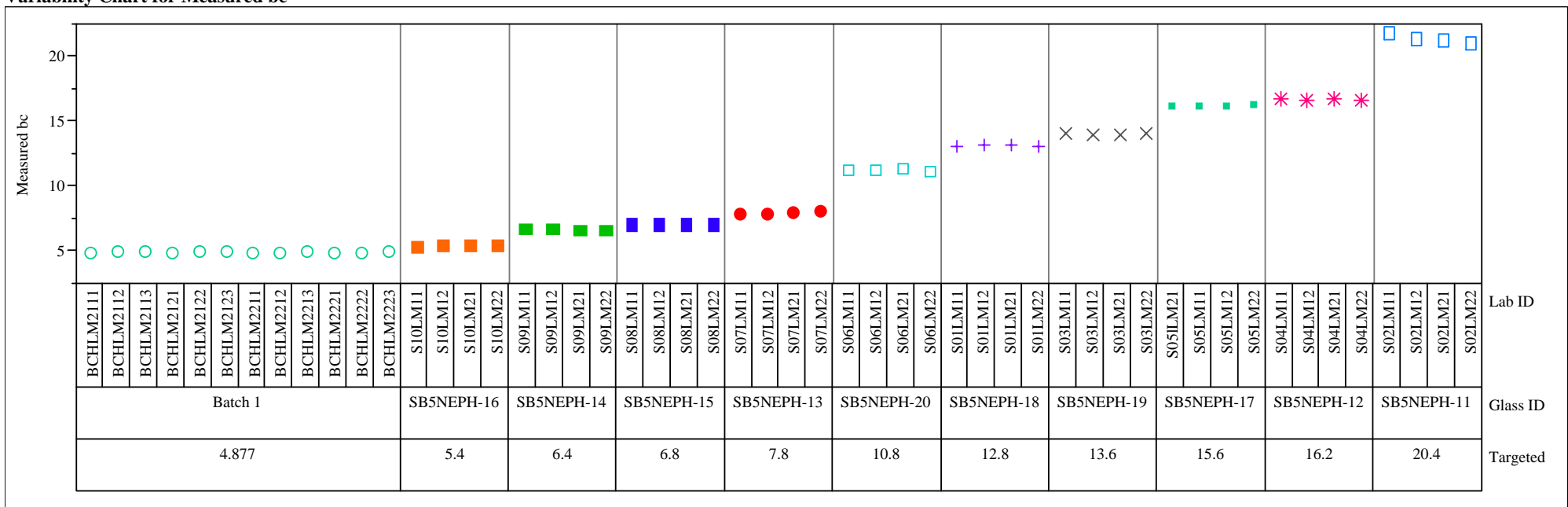


Exhibit A5. Measured and Measured Bias-Corrected Oxide Weight Percents

by Lab ID within Glass ID for the Glasses Prepared Using the LM Method. (continued)

Variability Gage Set $=2$, Oxide $=\mathrm{BaO}$ ( wt $\%$ )

Variability Chart for Measured
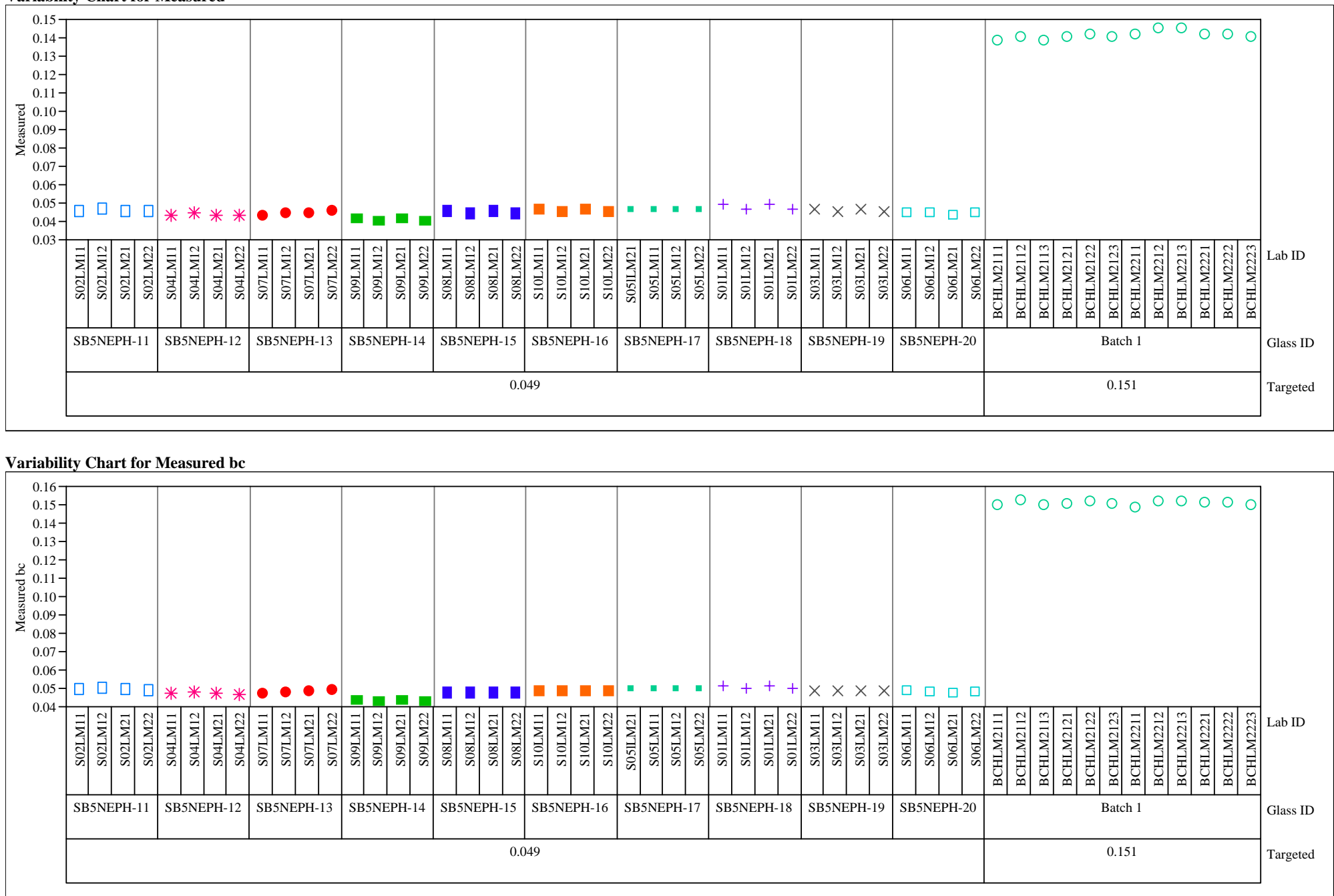
Exhibit A5. Measured and Measured Bias-Corrected Oxide Weight Percents

by Lab ID within Glass ID for the Glasses Prepared Using the LM Method. (continued)

Variability Gage Set $=2$, Oxide $=\mathrm{CaO}$ ( $w \mathrm{t} \%$ )

Variability Chart for Measured

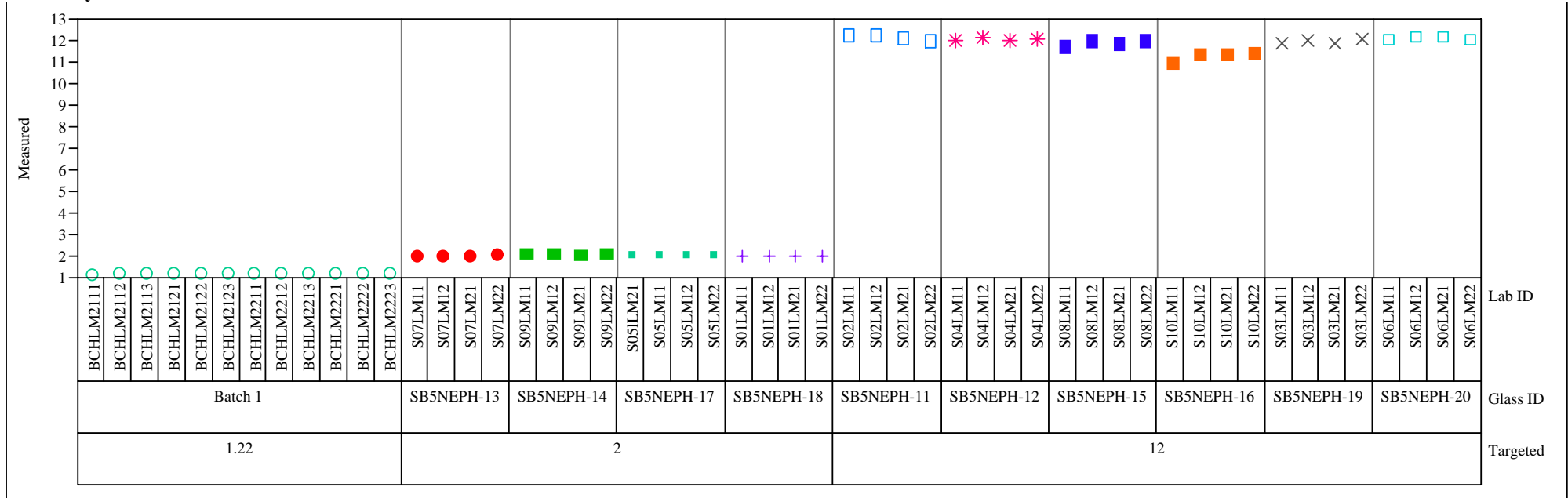

Variability Chart for Measured bc

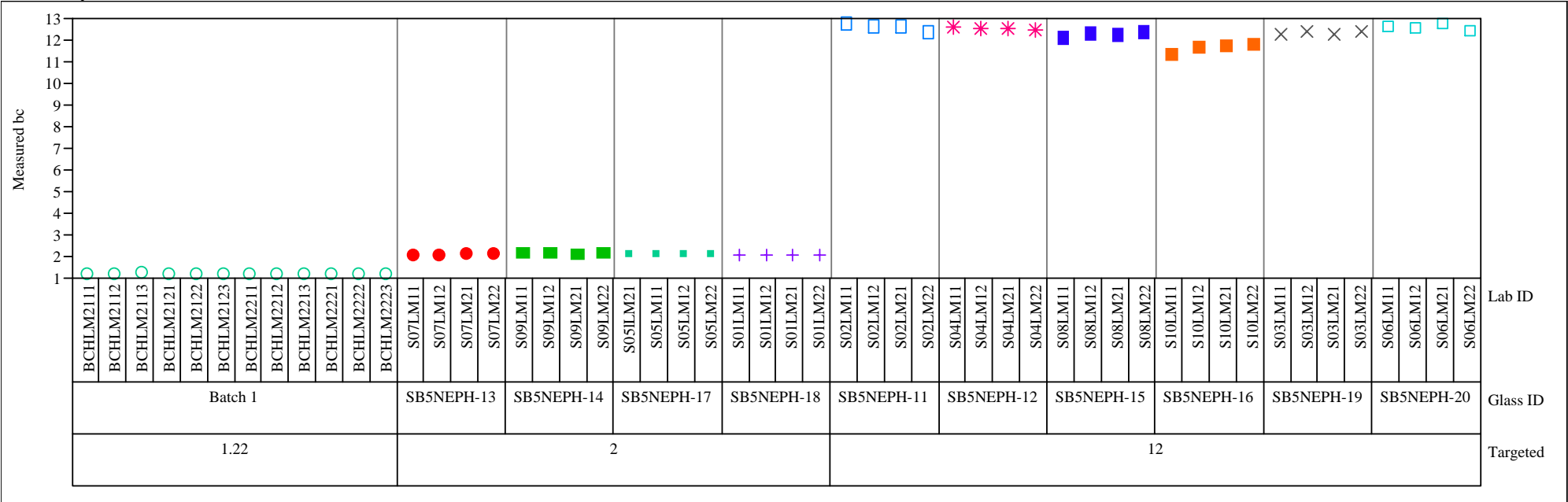


Exhibit A5. Measured and Measured Bias-Corrected Oxide Weight Percents

by Lab ID within Glass ID for the Glasses Prepared Using the LM Method. (continued)

Variability Gage Set $=2$, Oxide $=\mathrm{Ce} 2 \mathrm{O} 3(\mathrm{wt} \%)$

Variability Chart for Measured

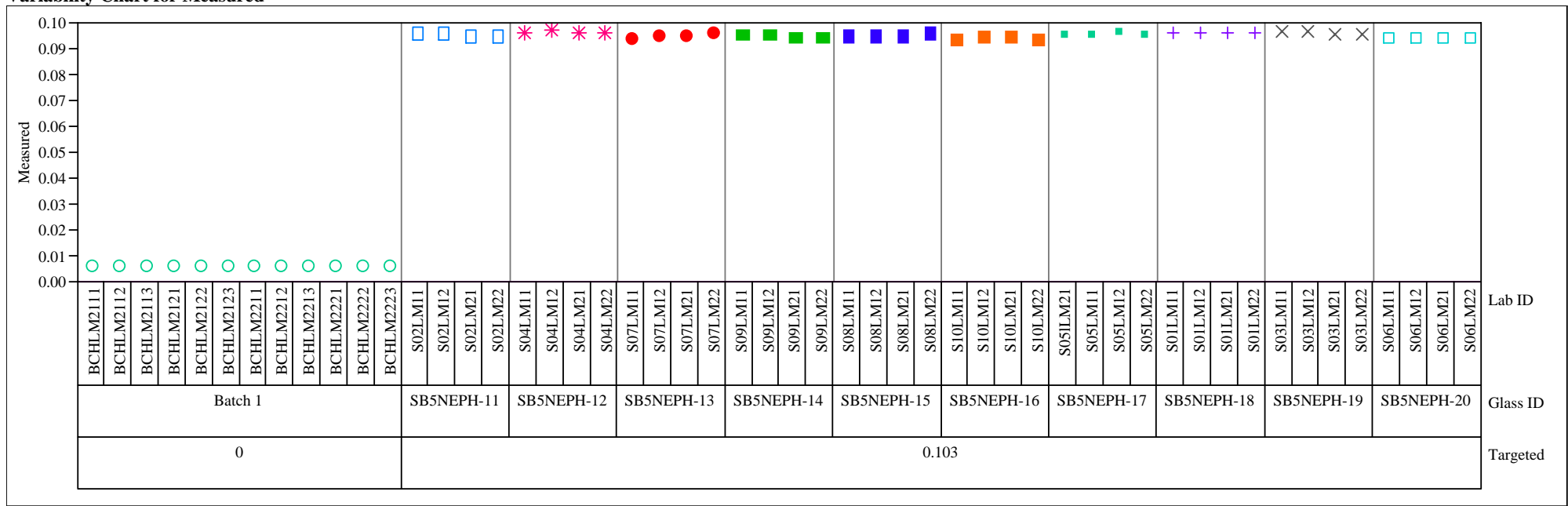

Variability Chart for Measured bc

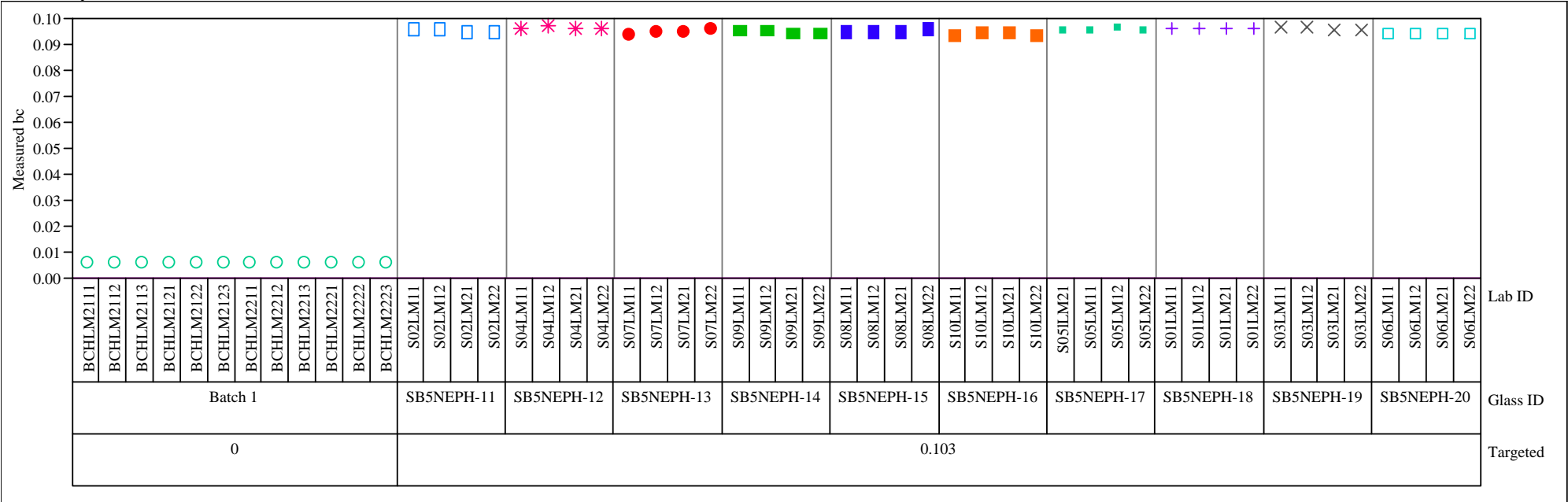


Exhibit A5. Measured and Measured Bias-Corrected Oxide Weight Percents

by Lab ID within Glass ID for the Glasses Prepared Using the LM Method. (continued)

Variability Gage Set $=2$, Oxide $=\mathrm{Cr} 2 \mathrm{O} 3(\mathrm{wt} \%)$

Variability Chart for Measured
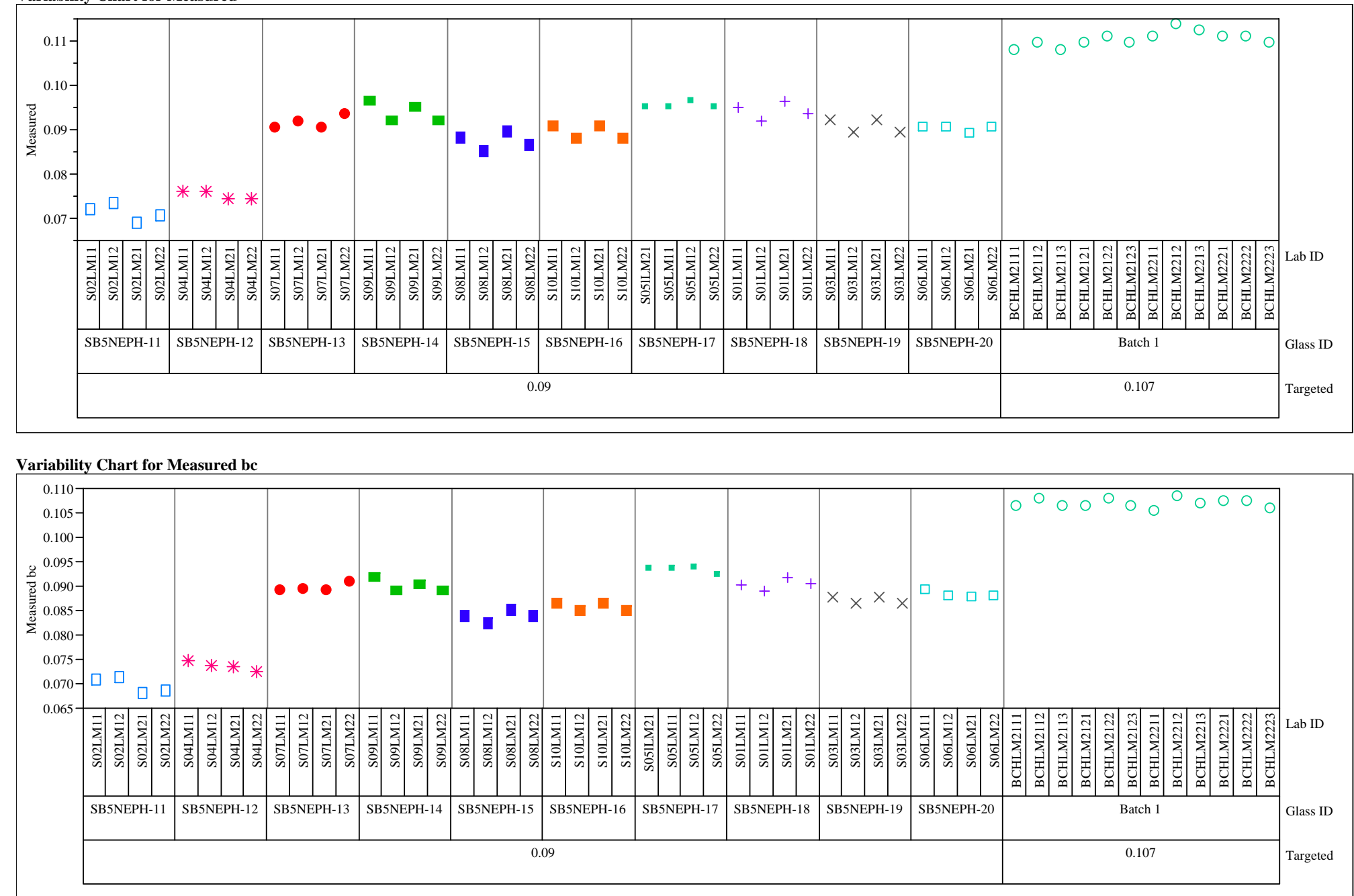
Exhibit A5. Measured and Measured Bias-Corrected Oxide Weight Percents

by Lab ID within Glass ID for the Glasses Prepared Using the LM Method. (continued)

Variability Gage Set $=2$, Oxide $=\mathrm{CuO}(\mathrm{wt} \%)$

Variability Chart for Measured

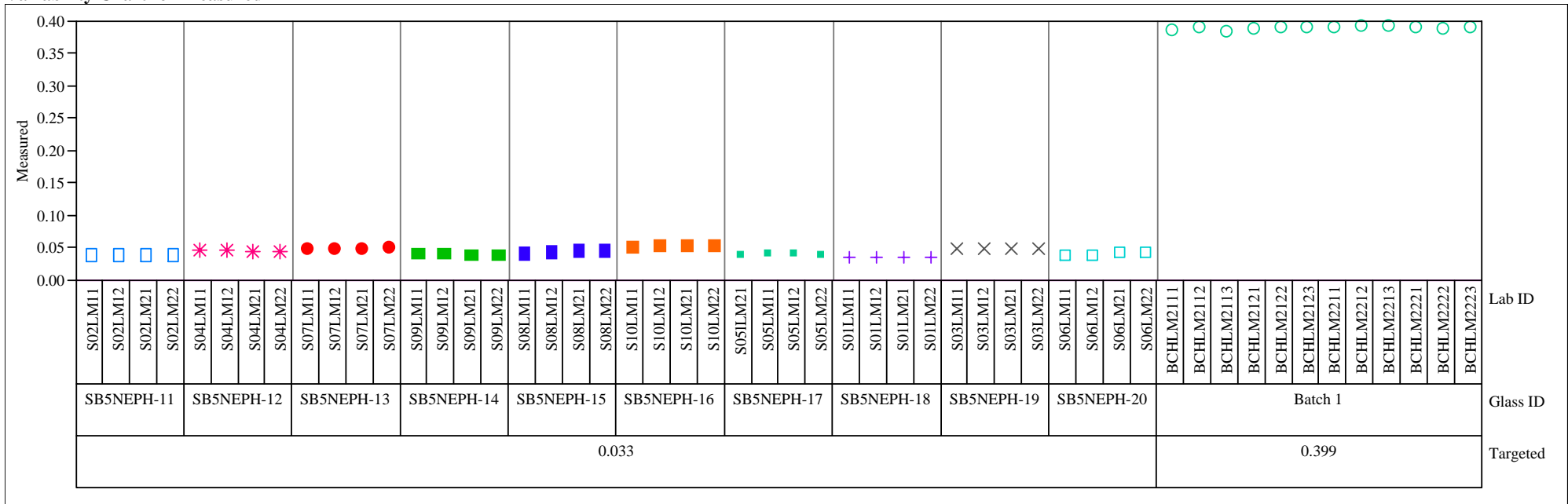

Variability Chart for Measured bc

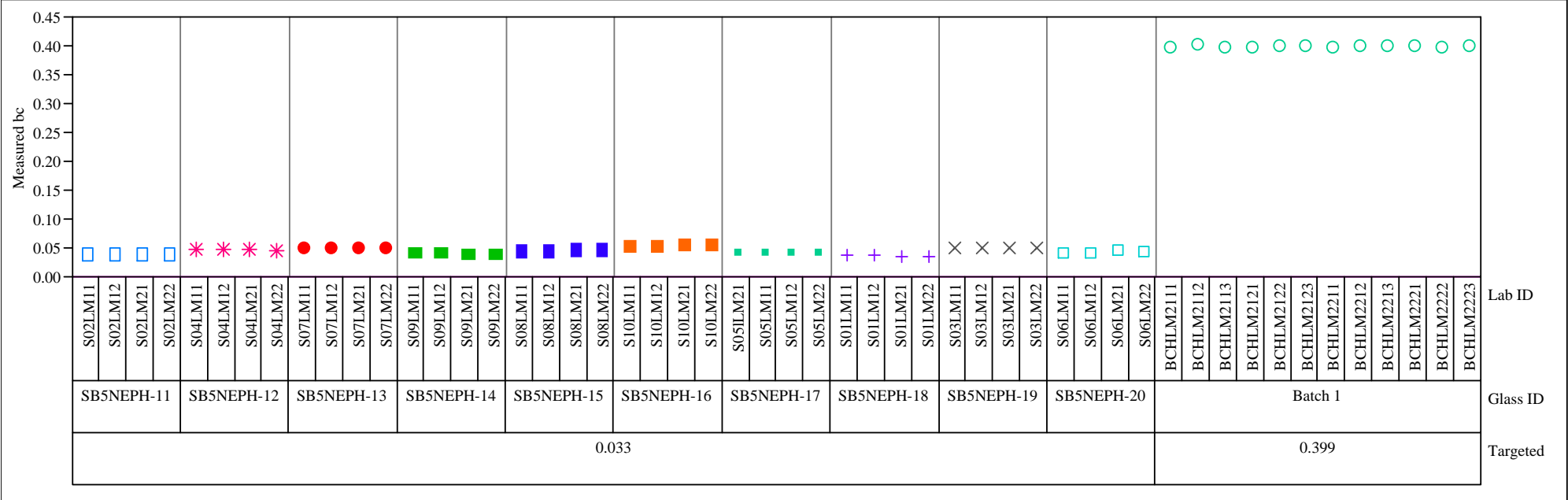


Exhibit A5. Measured and Measured Bias-Corrected Oxide Weight Percents

by Lab ID within Glass ID for the Glasses Prepared Using the LM Method. (continued)

Variability Gage Set $=2$, Oxide $=$ K2O $(w t \%)$

Variability Chart for Measured

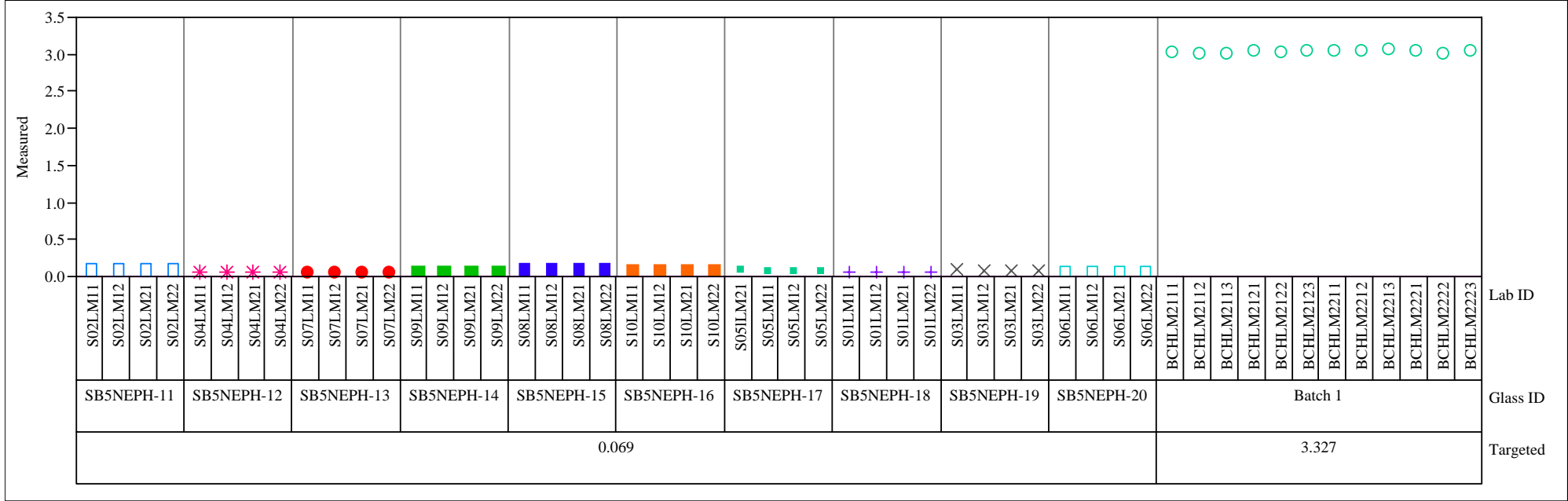

Variability Chart for Measured bc

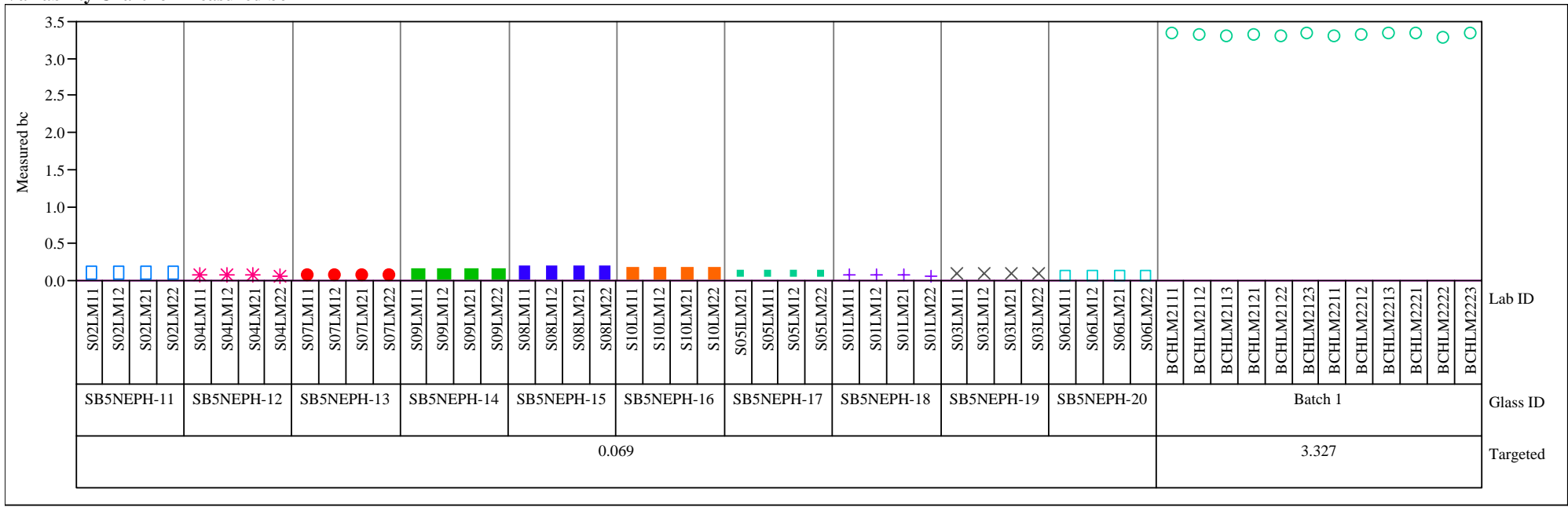


Exhibit A5. Measured and Measured Bias-Corrected Oxide Weight Percents

by Lab ID within Glass ID for the Glasses Prepared Using the LM Method. (continued)

Variability Gage Set $=2$, Oxide $=$ La2O3 (wt $\%$ )

Variability Chart for Measured

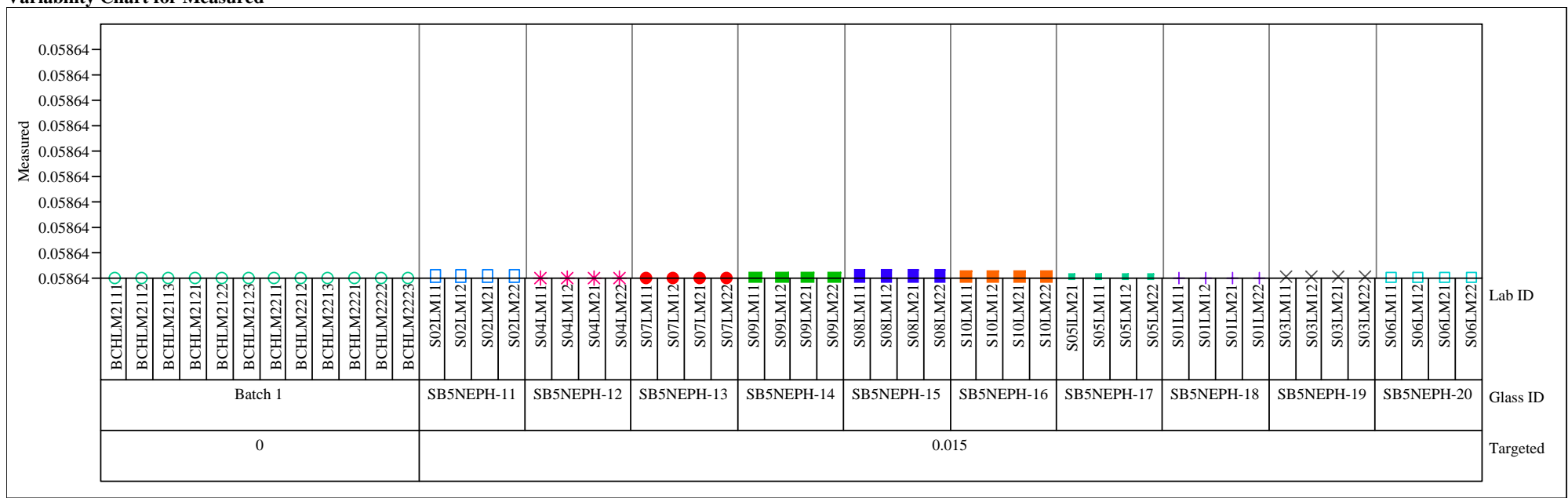

Variability Chart for Measured bc

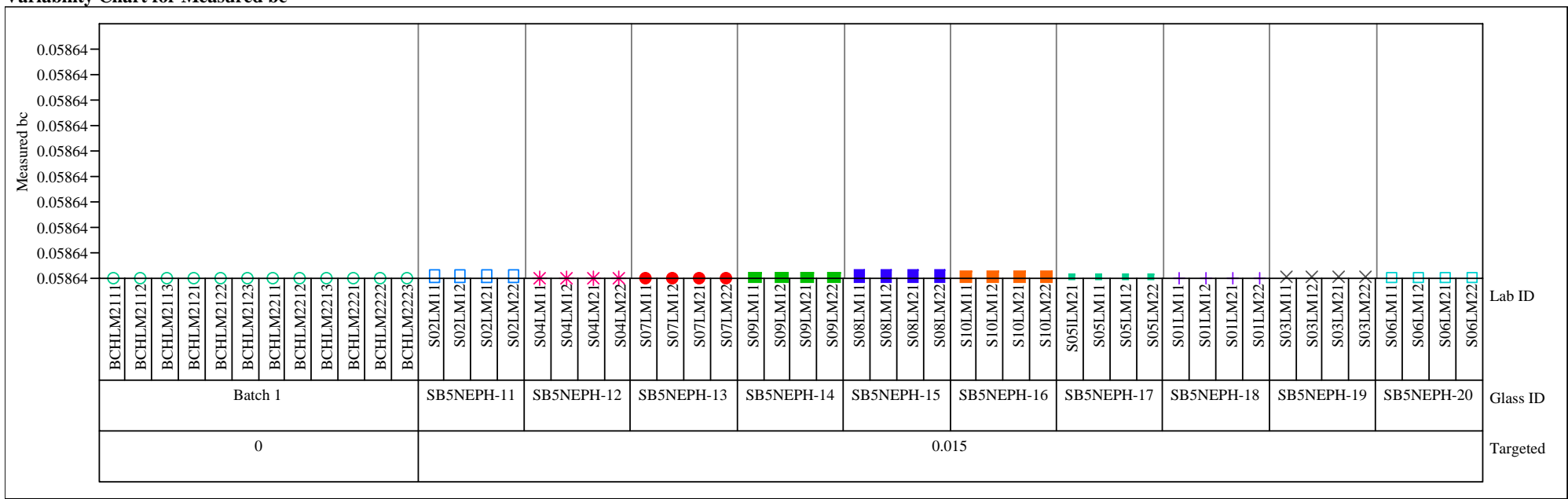


Exhibit A5. Measured and Measured Bias-Corrected Oxide Weight Percents

by Lab ID within Glass ID for the Glasses Prepared Using the LM Method. (continued)

Variability Gage Set=2, Oxide $=\mathrm{MgO}(\mathrm{wt} \%)$

Variability Chart for Measured

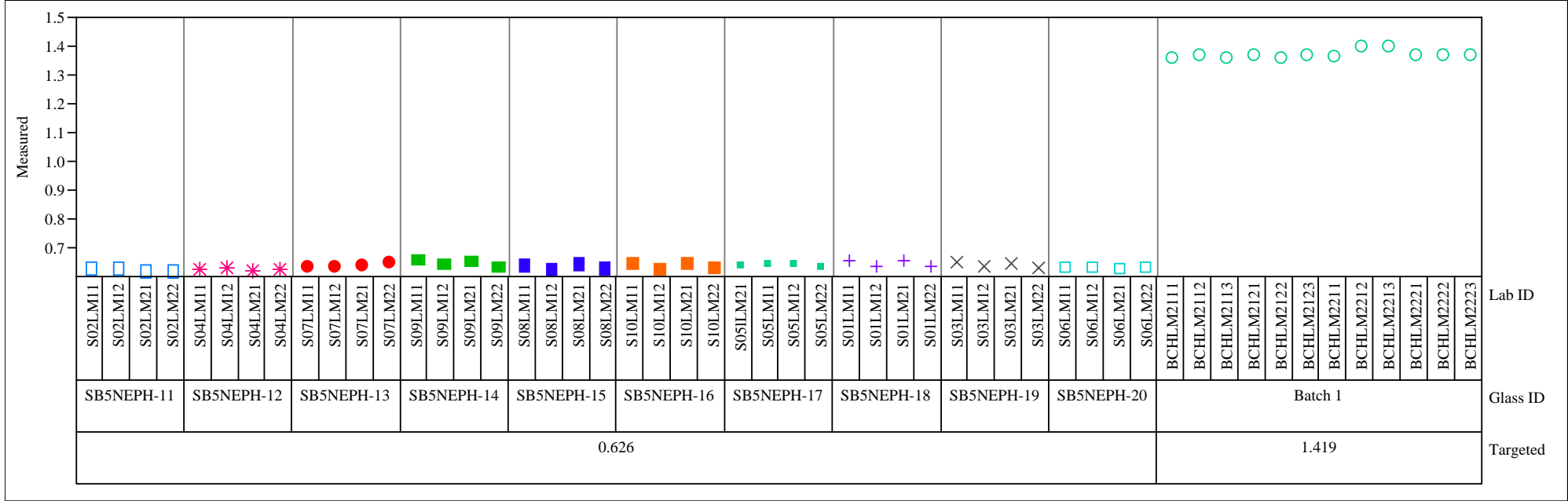

Variability Chart for Measured bc

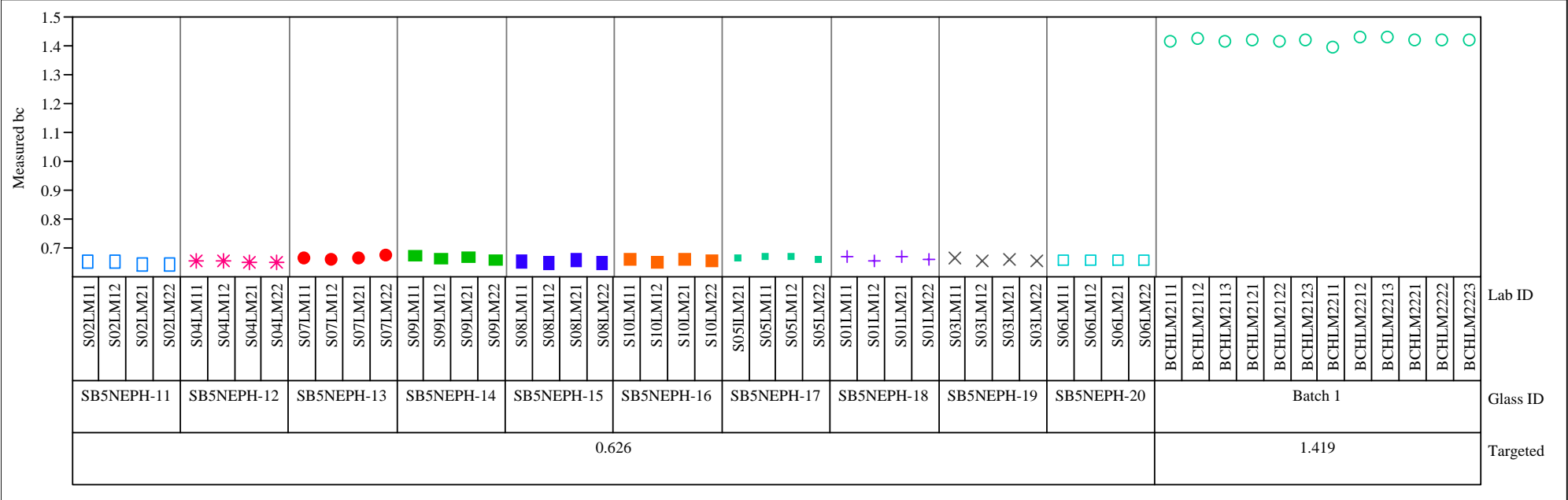


Exhibit A5. Measured and Measured Bias-Corrected Oxide Weight Percents

by Lab ID within Glass ID for the Glasses Prepared Using the LM Method. (continued)

Variability Gage Set=2, Oxide $=\mathrm{MnO}(\mathrm{wt} \%)$

Variability Chart for Measured

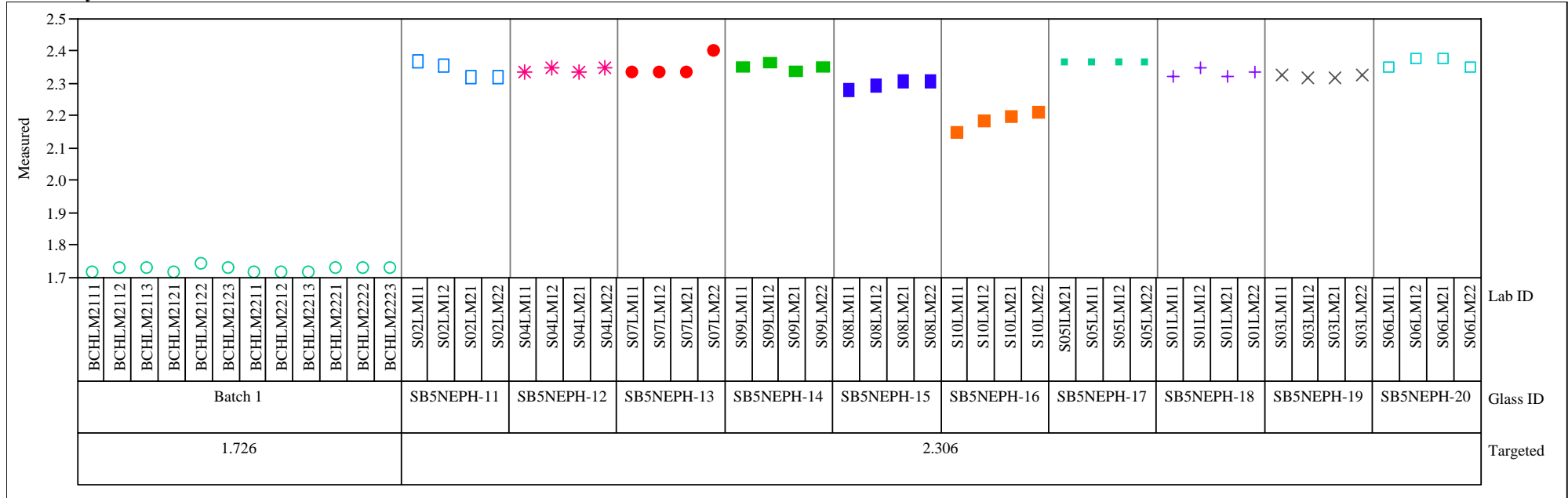

Variability Chart for Measured bc

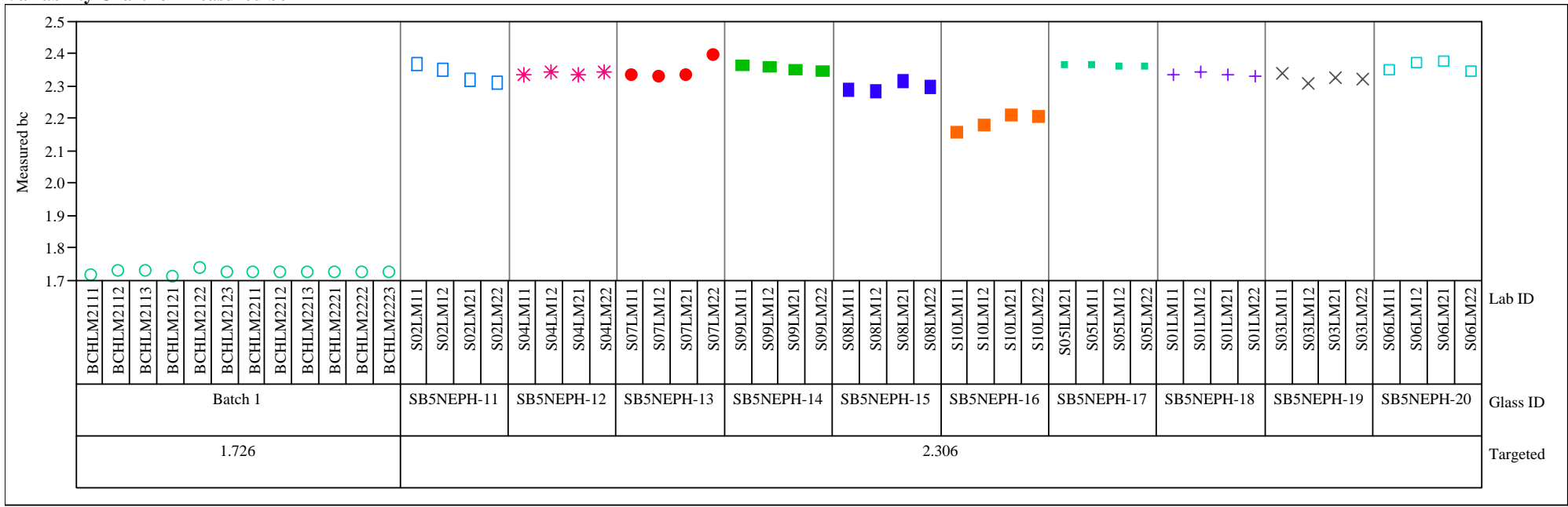


Exhibit A5. Measured and Measured Bias-Corrected Oxide Weight Percents

by Lab ID within Glass ID for the Glasses Prepared Using the LM Method. (continued)

Variability Gage Set $=2$, Oxide $=\mathrm{Na} 2 \mathrm{O}(\mathrm{wt} \%)$

Variability Chart for Measured

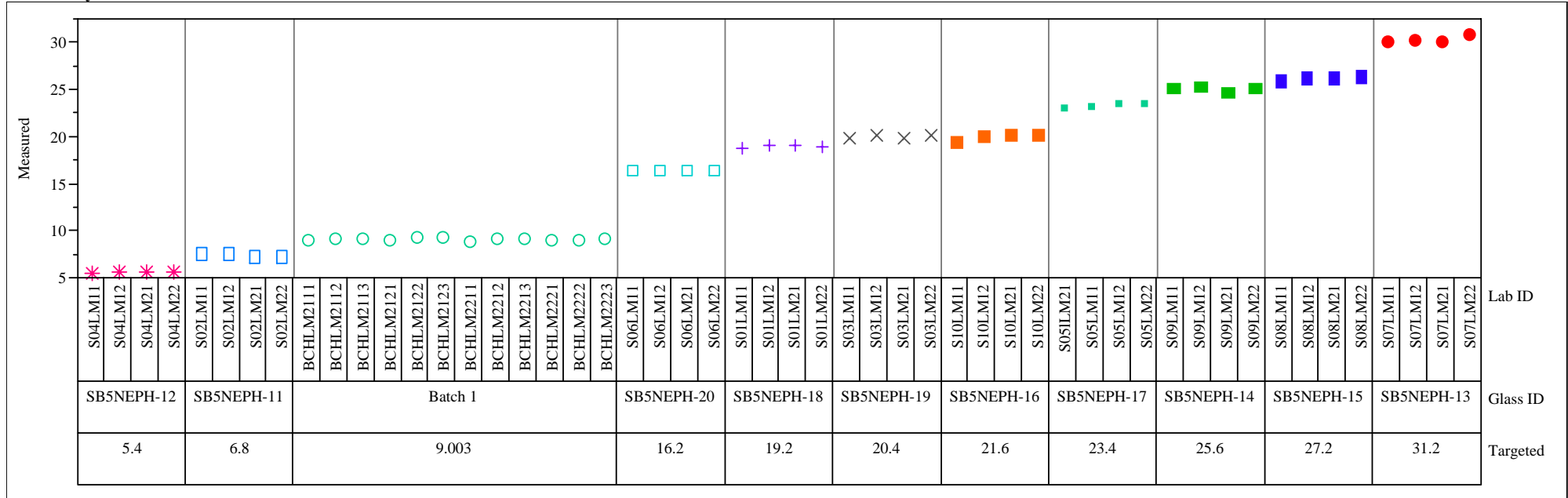

Variability Chart for Measured bc

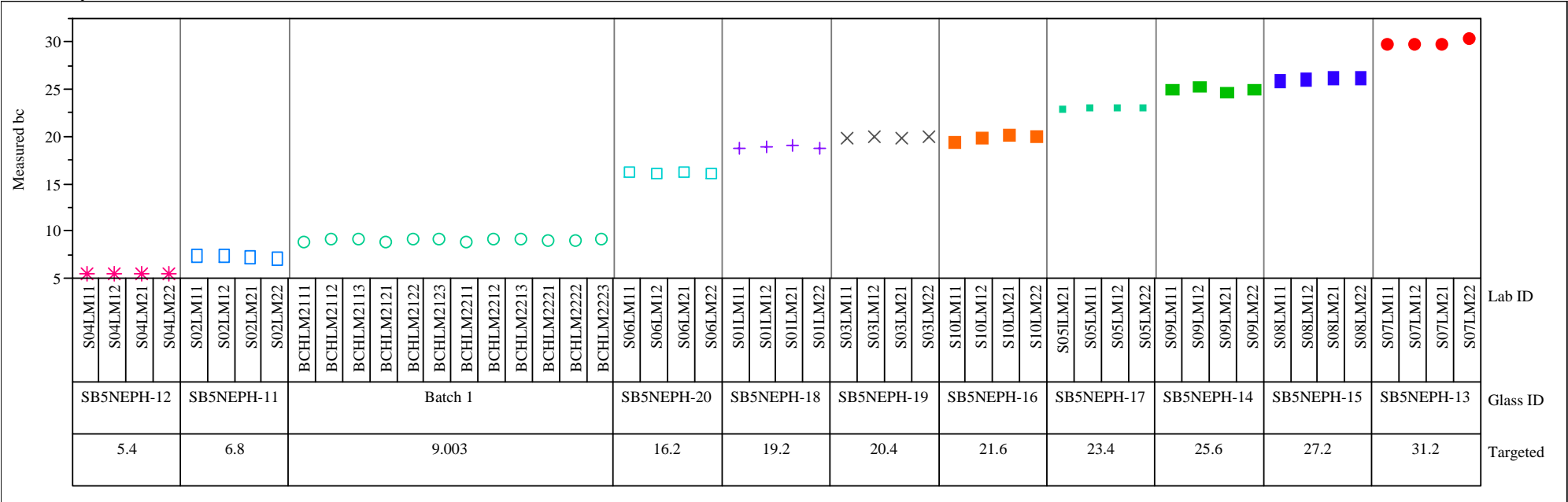


Exhibit A5. Measured and Measured Bias-Corrected Oxide Weight Percents

by Lab ID within Glass ID for the Glasses Prepared Using the LM Method. (continued)

Variability Gage Set $=2$, Oxide $=\mathrm{NiO}$ (wt $\%$ )

Variability Chart for Measured

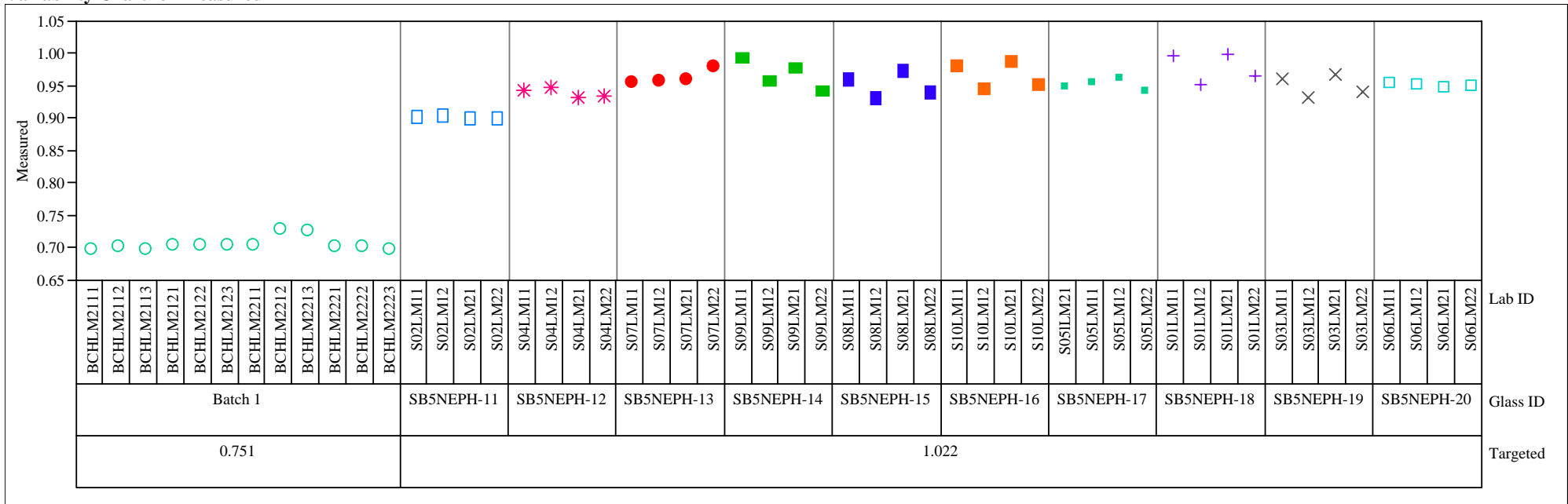

Variability Chart for Measured bc

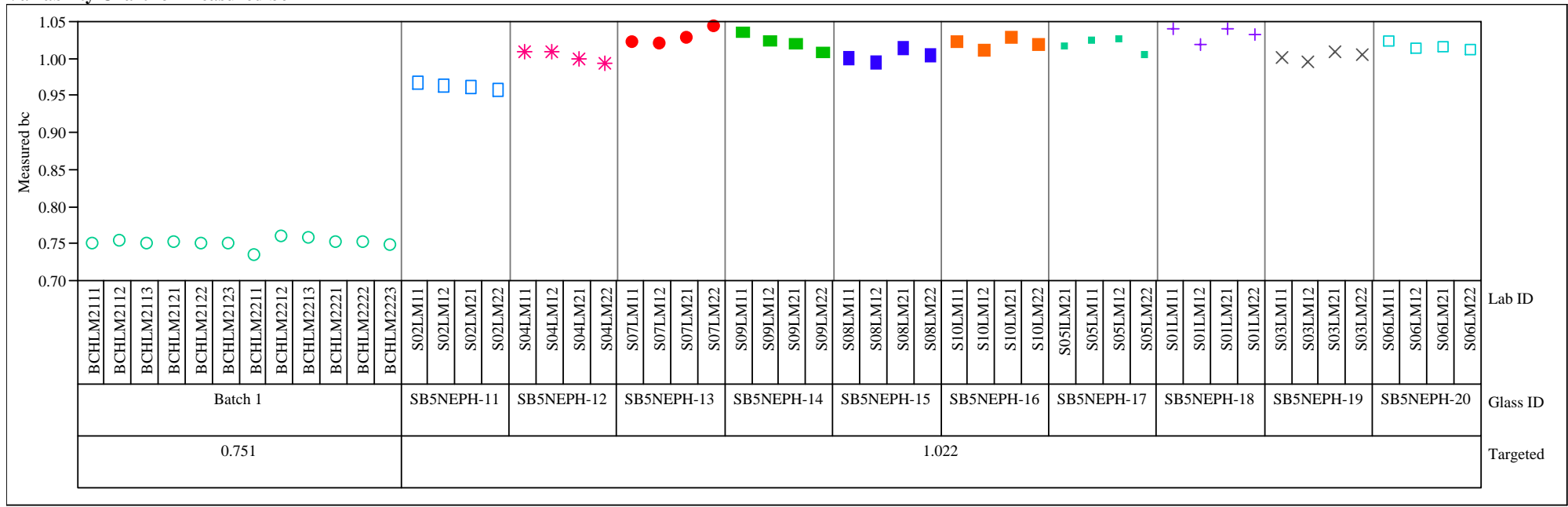


Exhibit A5. Measured and Measured Bias-Corrected Oxide Weight Percents

by Lab ID within Glass ID for the Glasses Prepared Using the LM Method. (continued)

Variability Gage Set $=2$, Oxide $=\mathbf{P b O}$ (wt $\%$ )

Variability Chart for Measured
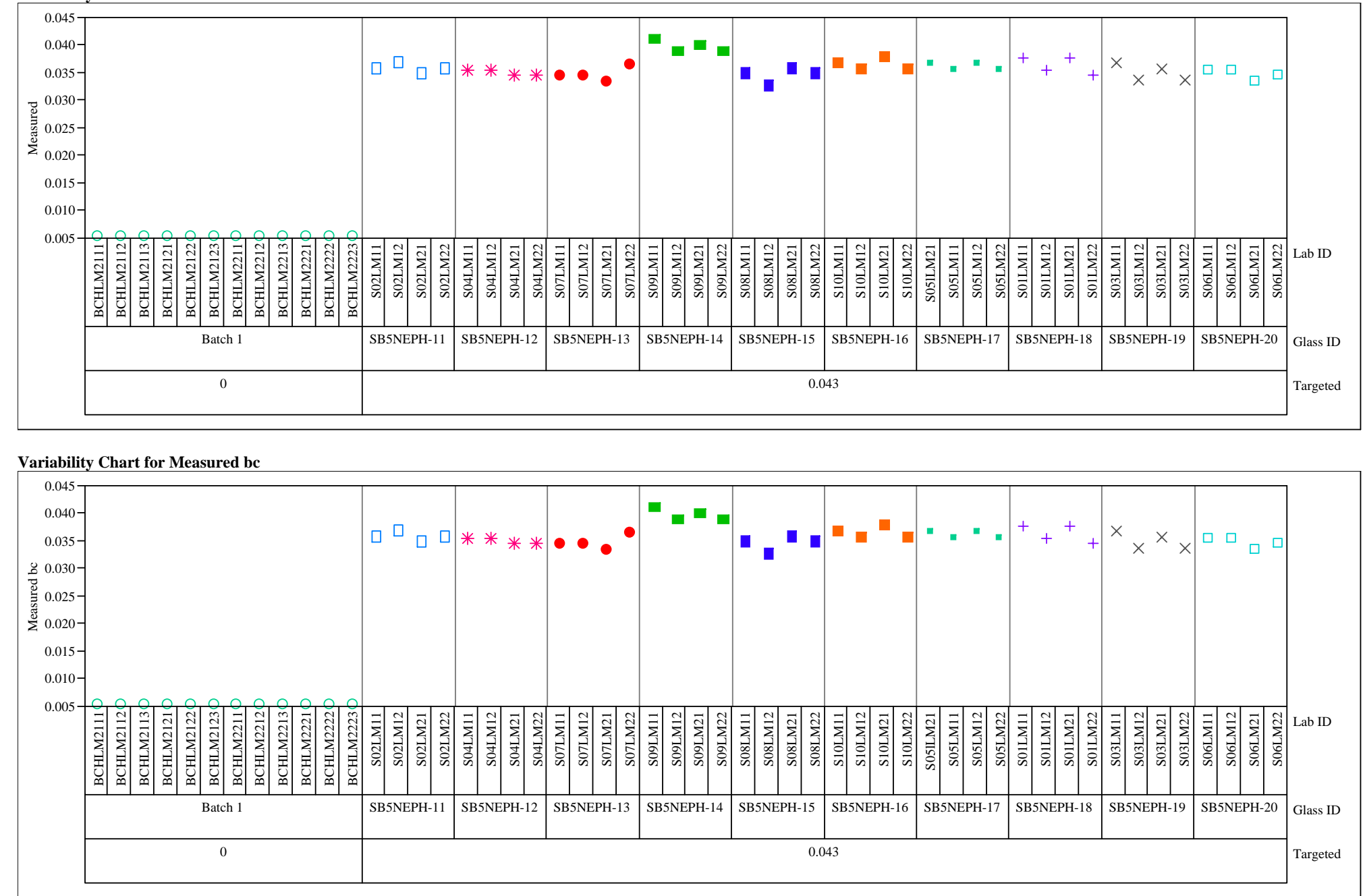
Exhibit A5. Measured and Measured Bias-Corrected Oxide Weight Percents

by Lab ID within Glass ID for the Glasses Prepared Using the LM Method. (continued)

Variability Gage Set=2, Oxide=SO4 (wt \%)

Variability Chart for Measured

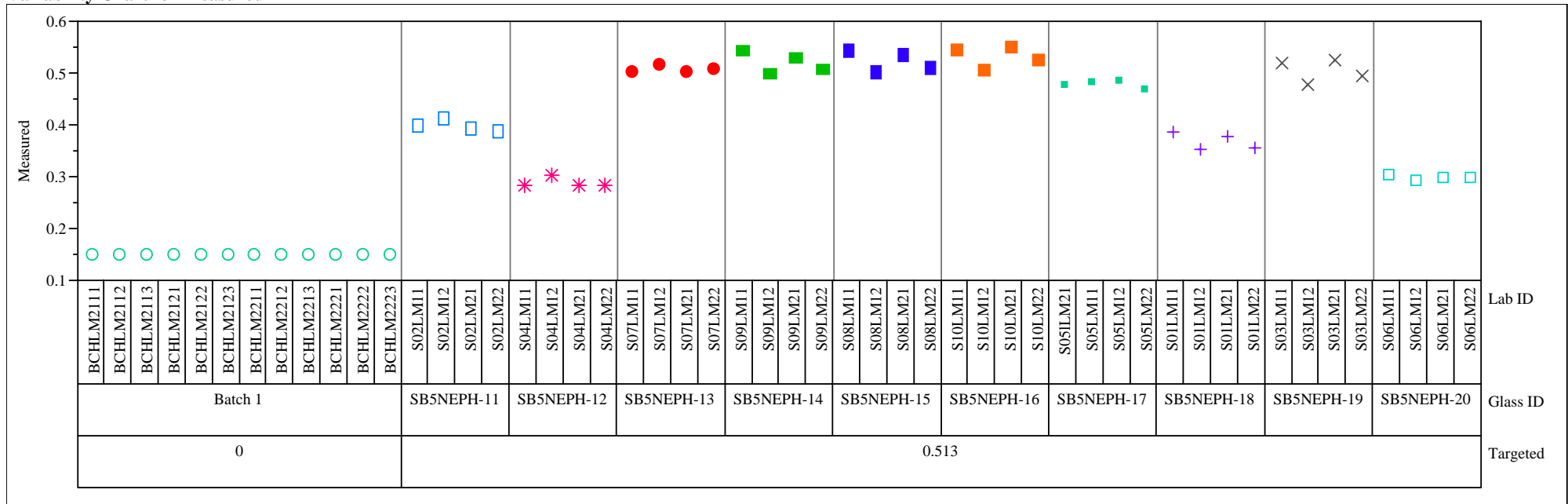

Variability Chart for Measured bc

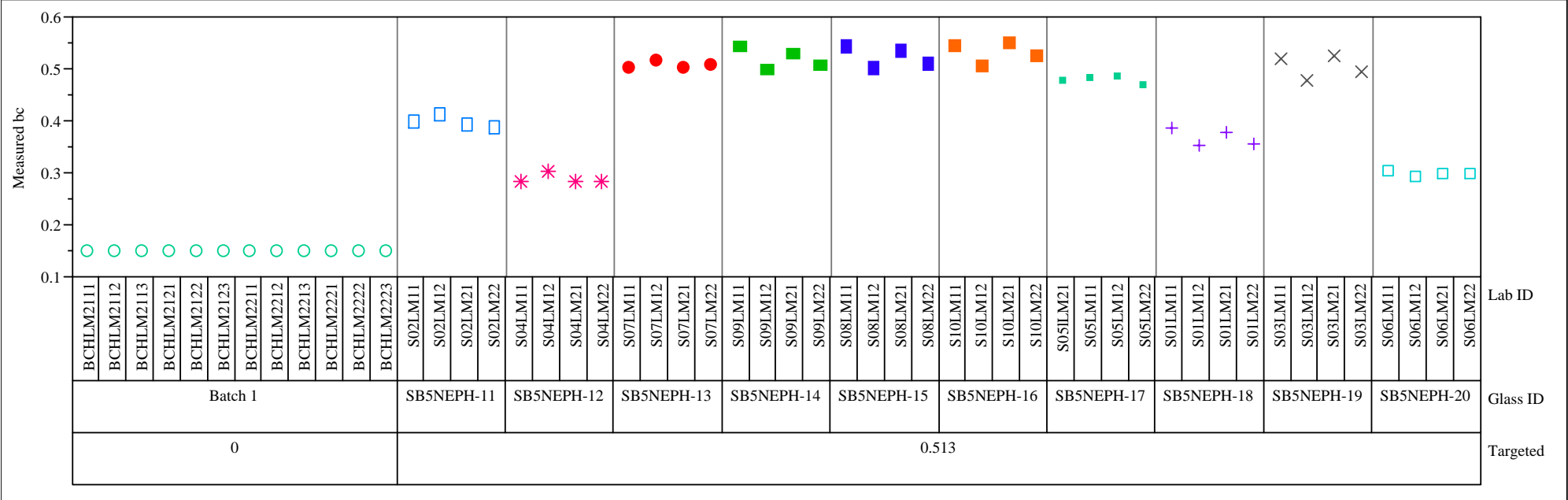


Exhibit A5. Measured and Measured Bias-Corrected Oxide Weight Percents

by Lab ID within Glass ID for the Glasses Prepared Using the LM Method. (continued)

Variability Gage Set $=2$, Oxide=TiO2 (wt $\%)$

Variability Chart for Measured

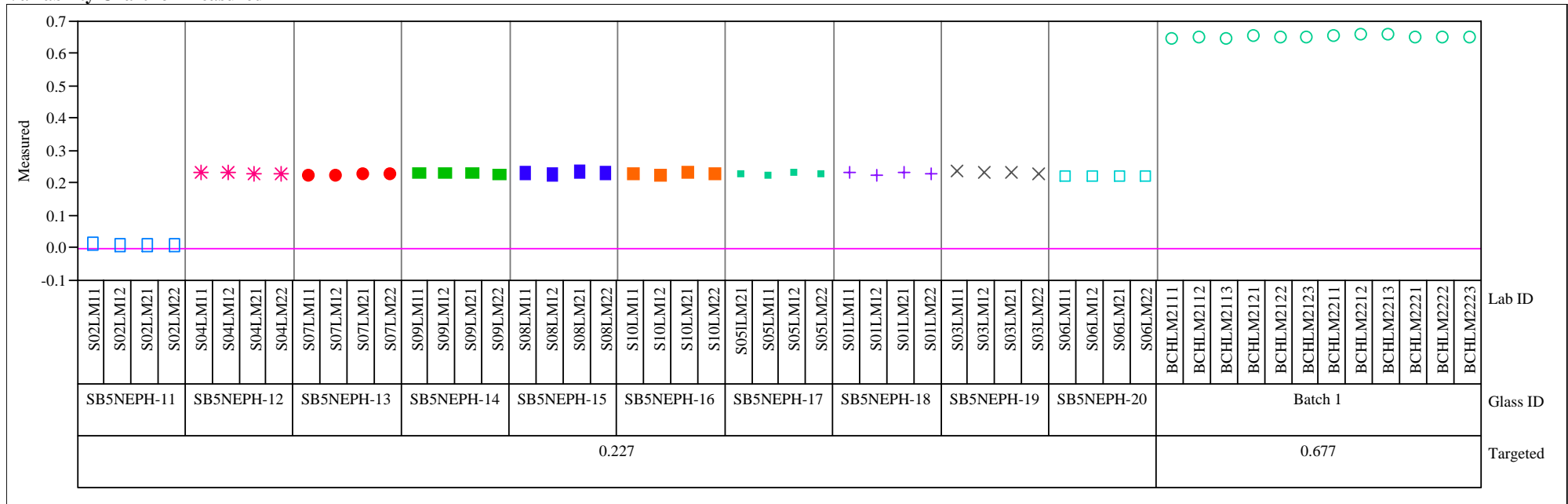

Variability Chart for Measured bc

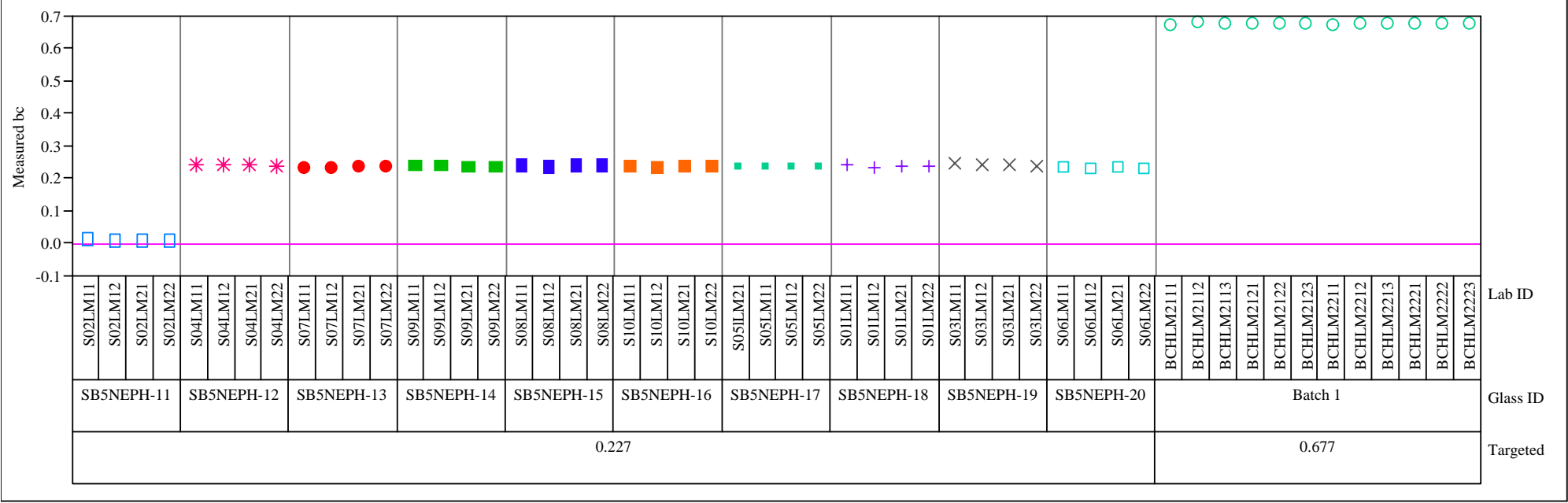


Exhibit A5. Measured and Measured Bias-Corrected Oxide Weight Percents

by Lab ID within Glass ID for the Glasses Prepared Using the LM Method. (continued)

Variability Gage Set $=2$, Oxide $=\mathrm{ZnO}$ (wt $\%$ )

Variability Chart for Measured
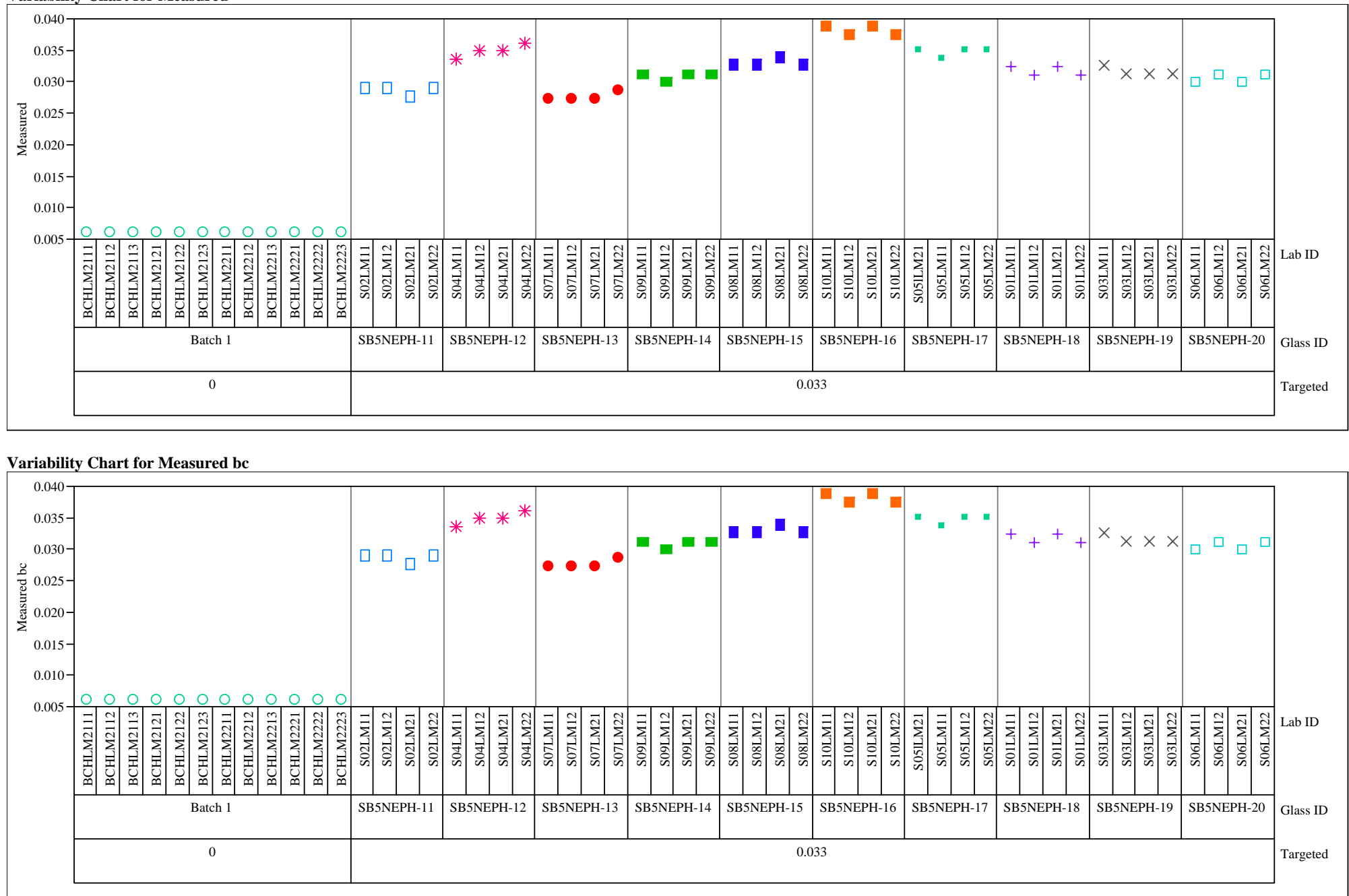
Exhibit A5. Measured and Measured Bias-Corrected Oxide Weight Percents

by Lab ID within Glass ID for the Glasses Prepared Using the LM Method. (continued)

Variability Gage Set $=2$, Oxide $=\mathrm{ZrO} 2(\mathrm{wt} \%)$

Variability Chart for Measured
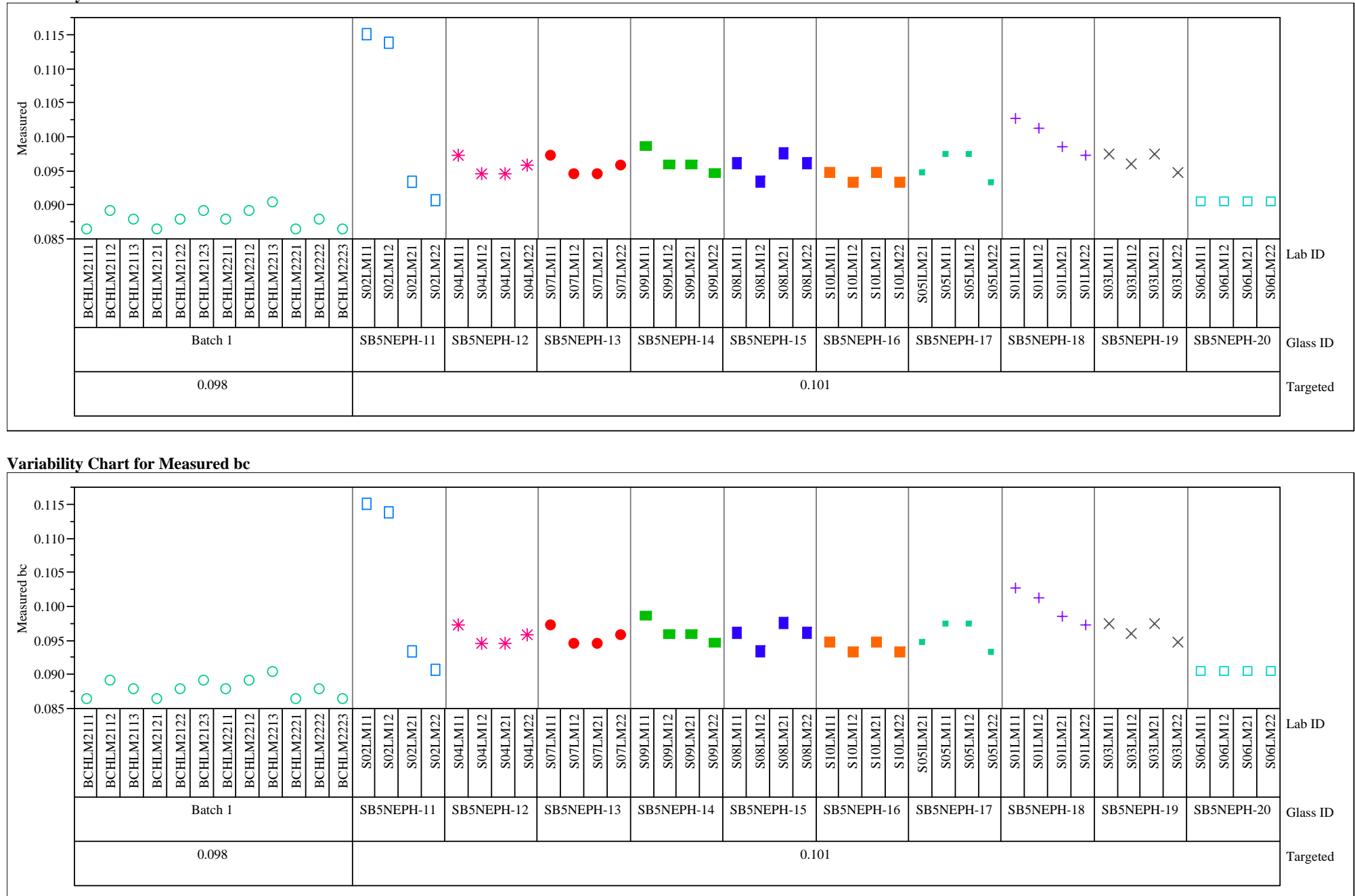
Exhibit A5. Measured and Measured Bias-Corrected Oxide Weight Percents

by Lab ID within Glass ID for the Glasses Prepared Using the LM Method. (continued)

Variability Gage Set=3, Oxide=Al2O3 (wt\%)

Variability Chart for Measured

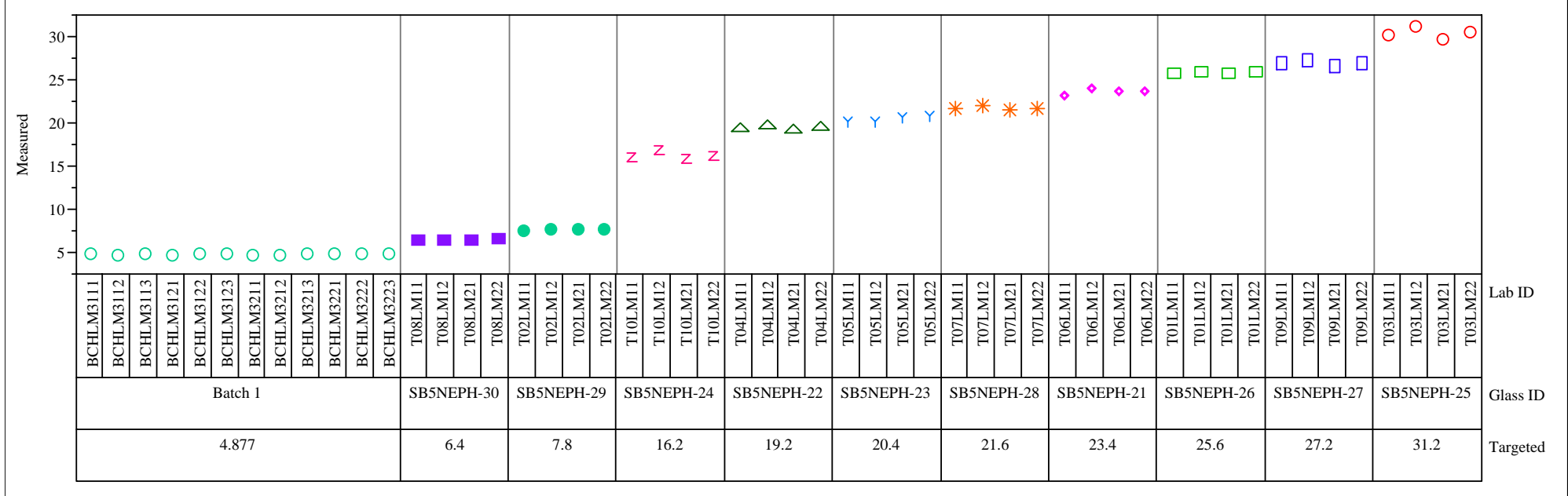

Variability Chart for Measured bc

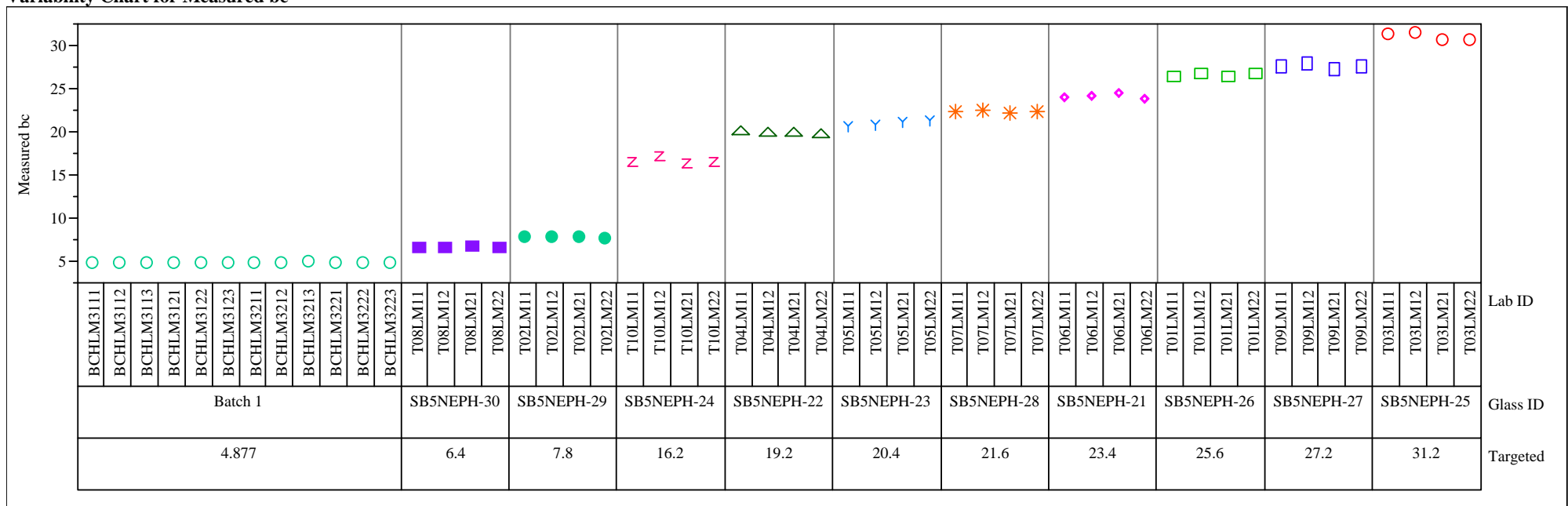


Exhibit A5. Measured and Measured Bias-Corrected Oxide Weight Percents

by Lab ID within Glass ID for the Glasses Prepared Using the LM Method. (continued)

Variability Gage Set $=3$, Oxide $=\mathrm{BaO}$ ( wt $\%$ )

Variability Chart for Measured
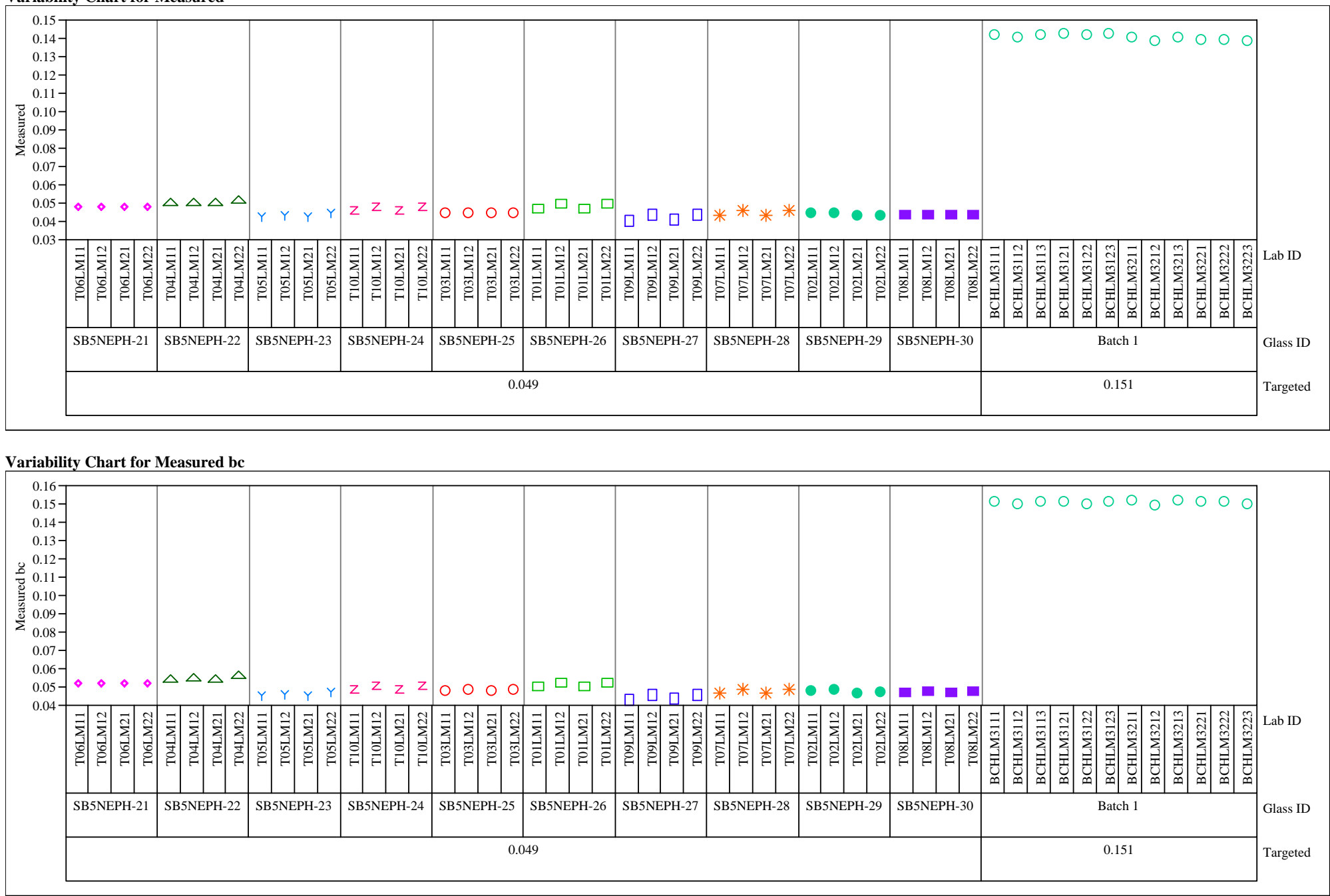
Exhibit A5. Measured and Measured Bias-Corrected Oxide Weight Percents

by Lab ID within Glass ID for the Glasses Prepared Using the LM Method. (continued)

Variability Gage Set $=3$, Oxide $=\mathrm{CaO}$ ( $w \mathrm{t} \%$ )

Variability Chart for Measured

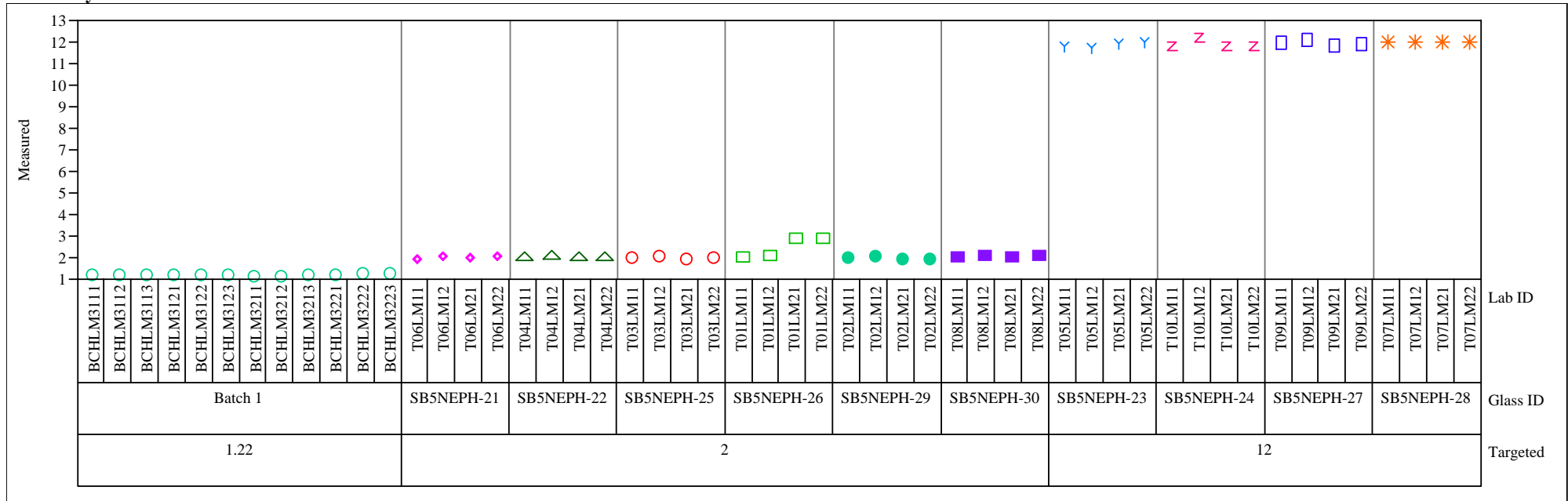

Variability Chart for Measured bc

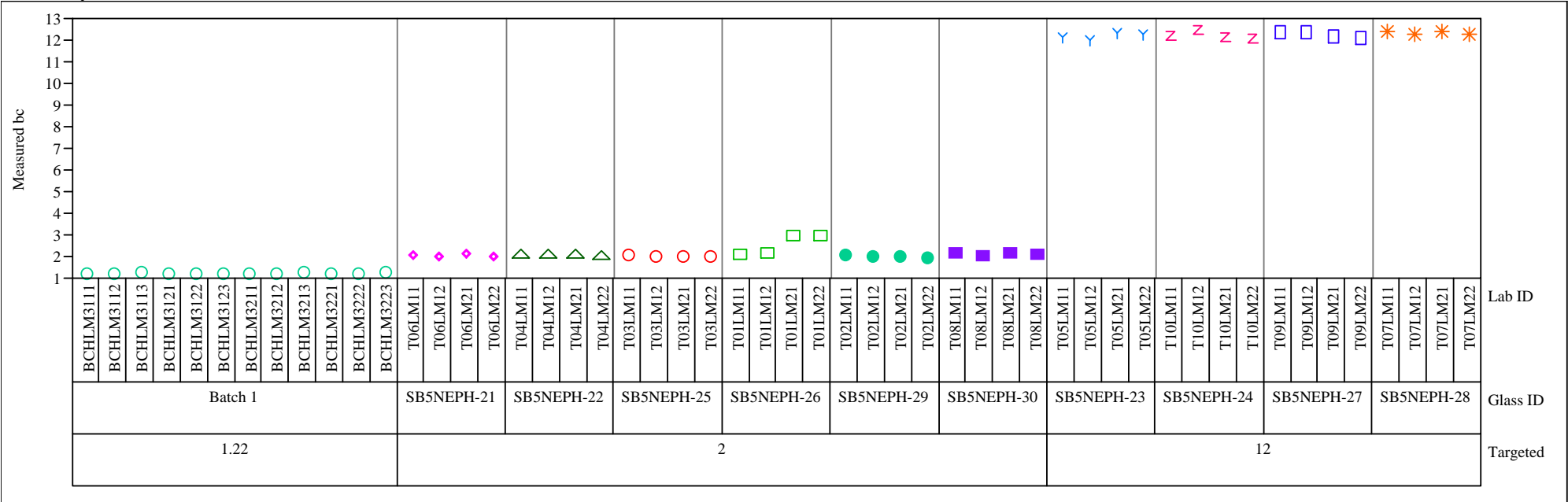


Exhibit A5. Measured and Measured Bias-Corrected Oxide Weight Percents

by Lab ID within Glass ID for the Glasses Prepared Using the LM Method. (continued)

Variability Gage Set $=3$, Oxide $=\mathrm{Ce} 2 \mathrm{O} 3(\mathrm{wt} \%)$

Variability Chart for Measured

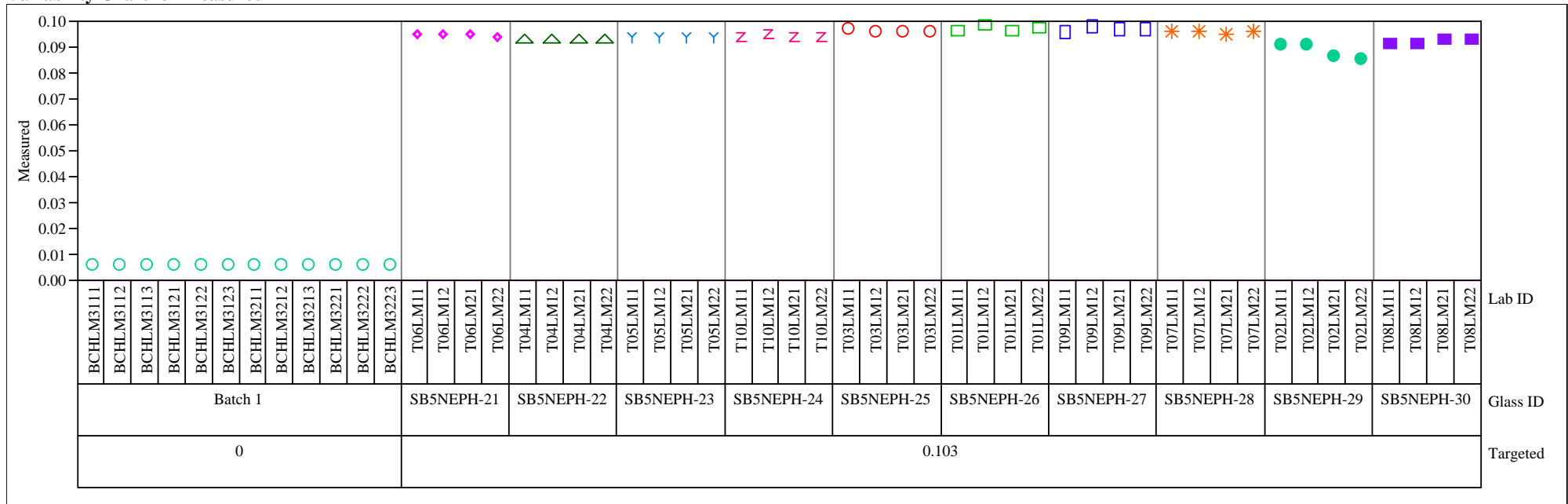

Variability Chart for Measured bc

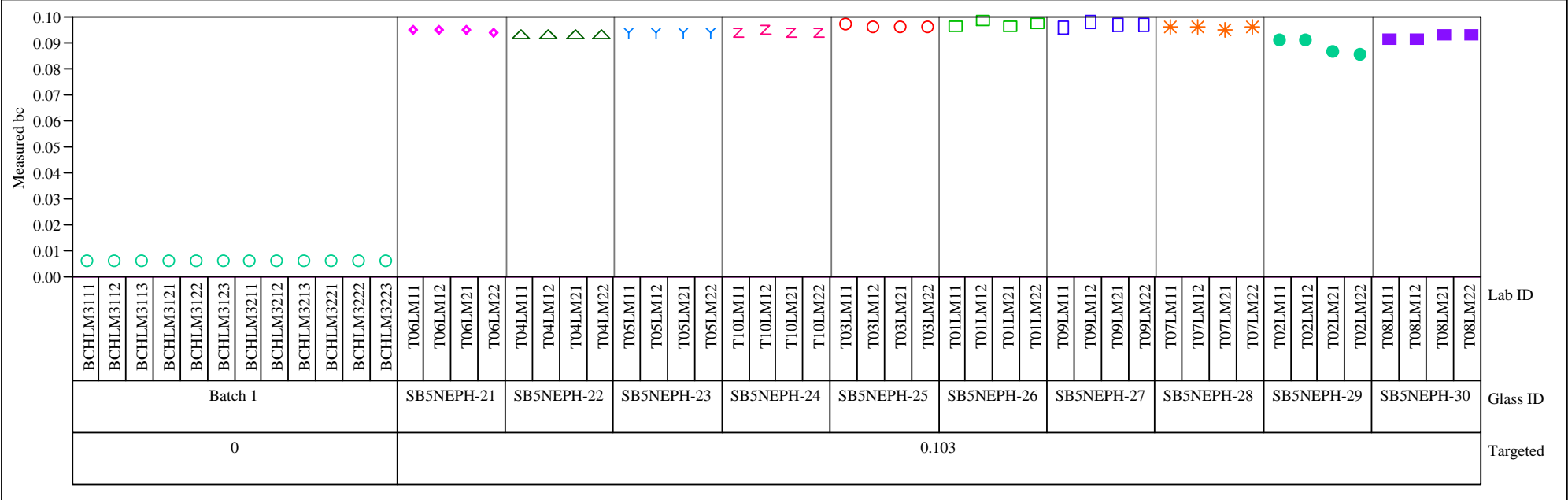


Exhibit A5. Measured and Measured Bias-Corrected Oxide Weight Percents

by Lab ID within Glass ID for the Glasses Prepared Using the LM Method. (continued)

Variability Gage Set $=3$, Oxide $=\mathrm{Cr} 2 \mathrm{O} 3(\mathrm{wt} \%)$

Variability Chart for Measured

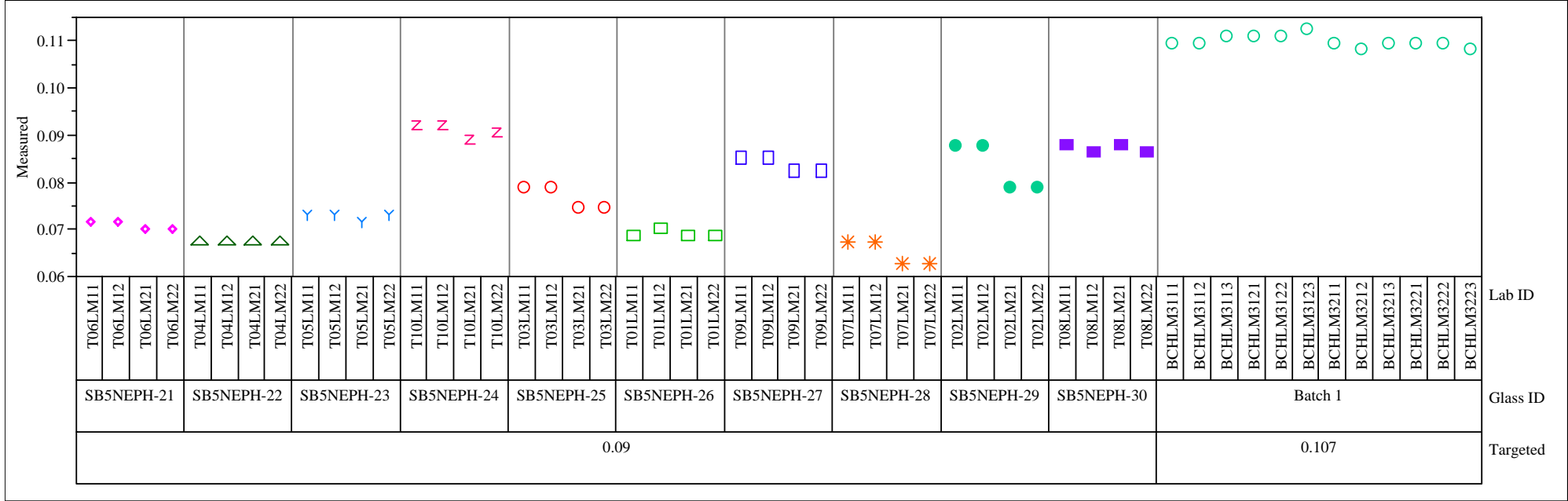

Variability Chart for Measured bc

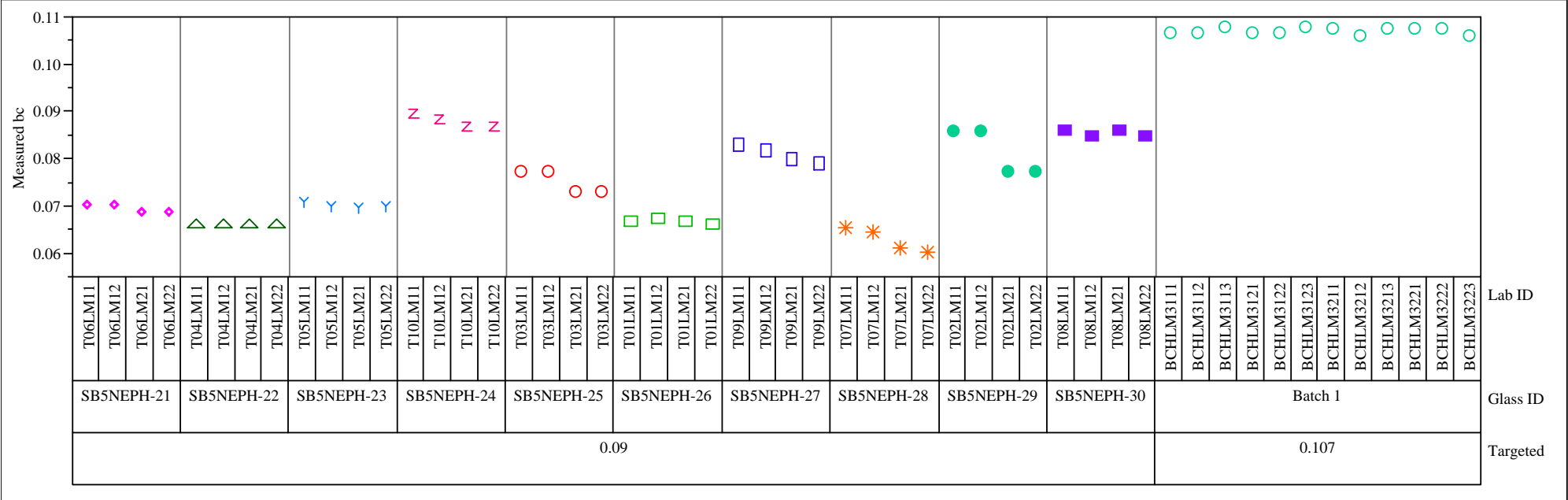


Exhibit A5. Measured and Measured Bias-Corrected Oxide Weight Percents

by Lab ID within Glass ID for the Glasses Prepared Using the LM Method. (continued)

Variability Gage Set $=3$, Oxide $=\mathrm{CuO}(\mathrm{wt} \%)$

Variability Chart for Measured

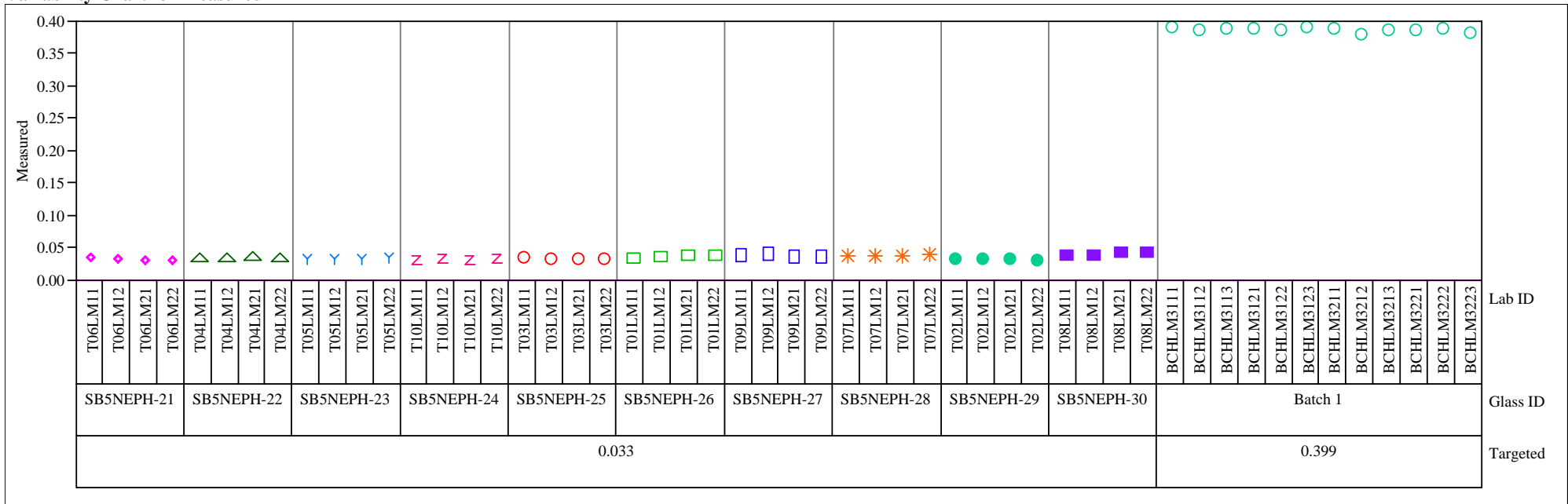

Variability Chart for Measured bc

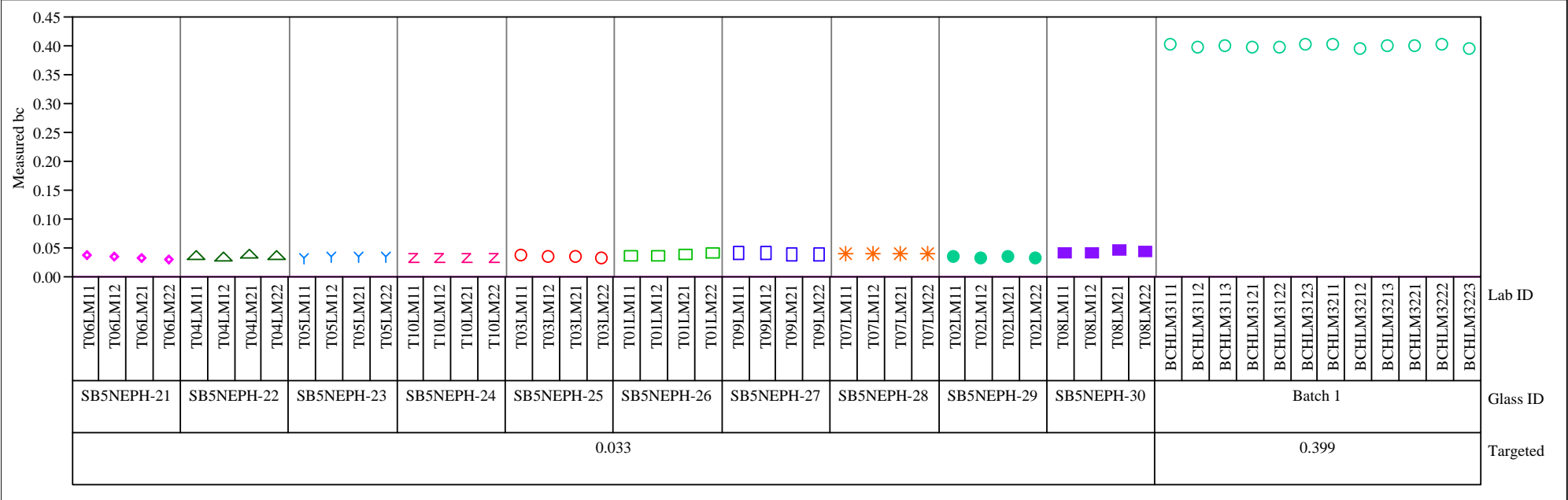


Exhibit A5. Measured and Measured Bias-Corrected Oxide Weight Percents

by Lab ID within Glass ID for the Glasses Prepared Using the LM Method. (continued)

Variability Gage Set $=3$, Oxide=K2O (wt\%)

Variability Chart for Measured

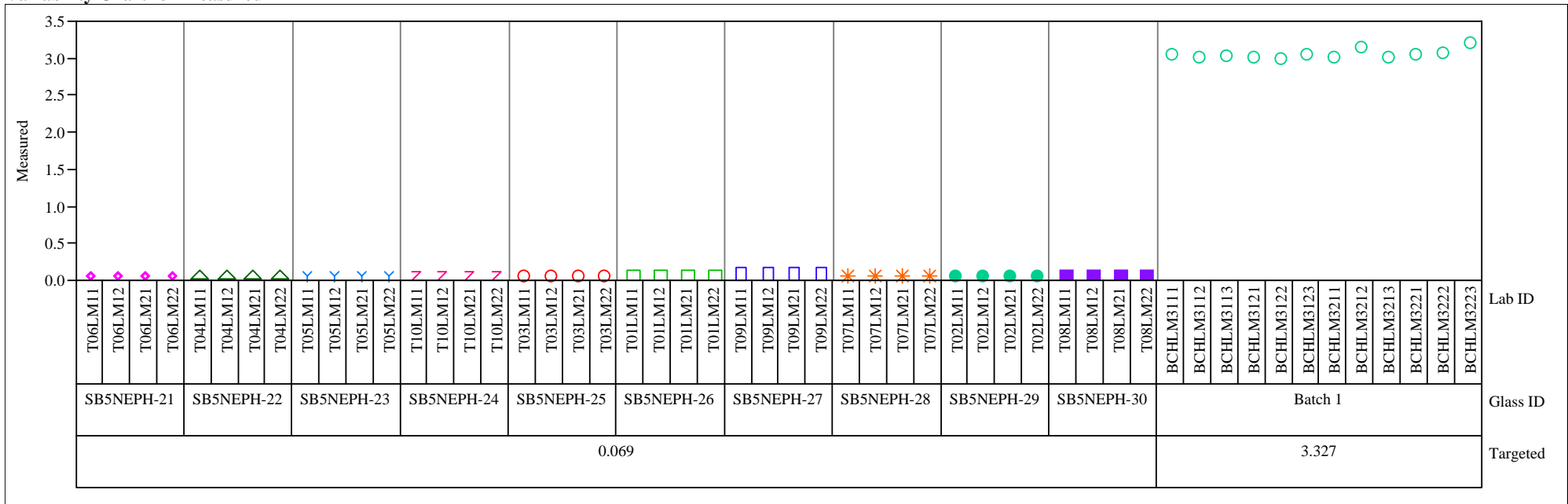

Variability Chart for Measured bc

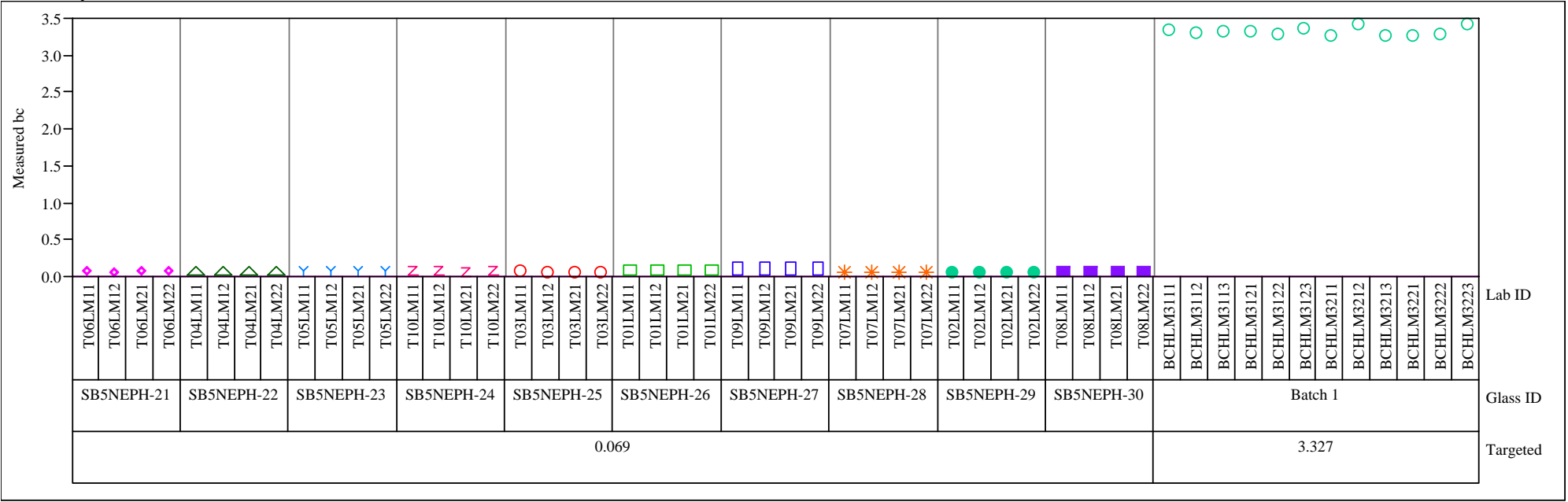


Exhibit A5. Measured and Measured Bias-Corrected Oxide Weight Percents

by Lab ID within Glass ID for the Glasses Prepared Using the LM Method. (continued)

Variability Gage Set $=3$, Oxide $=$ La2O3 (wt \%)

Variability Chart for Measured

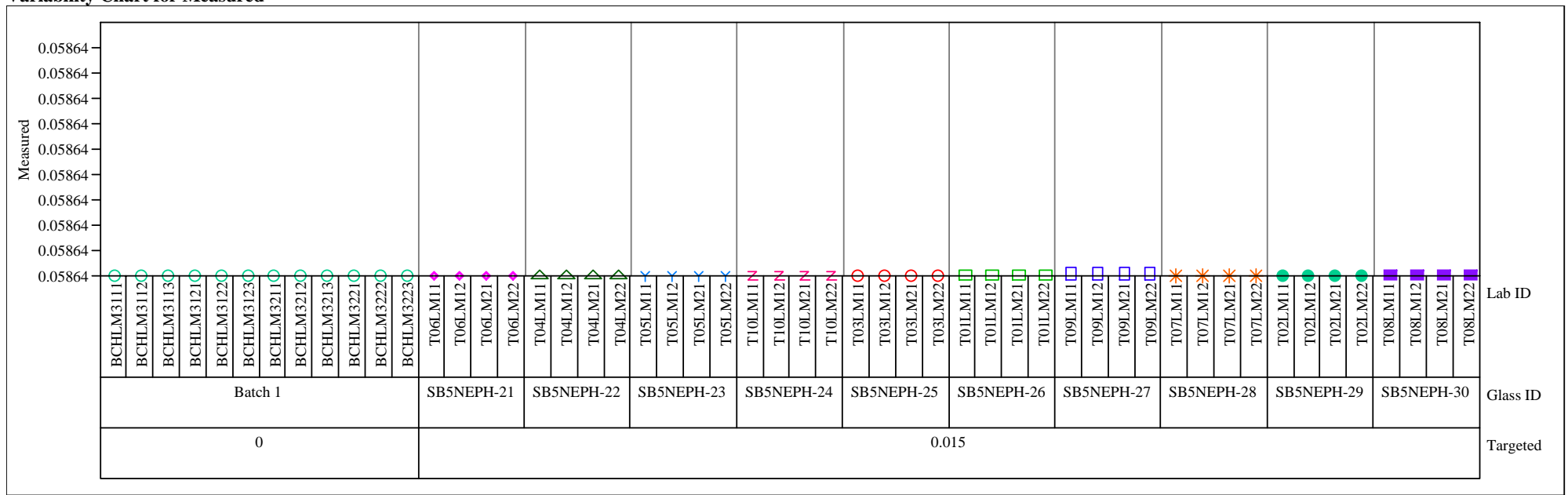

Variability Chart for Measured bc

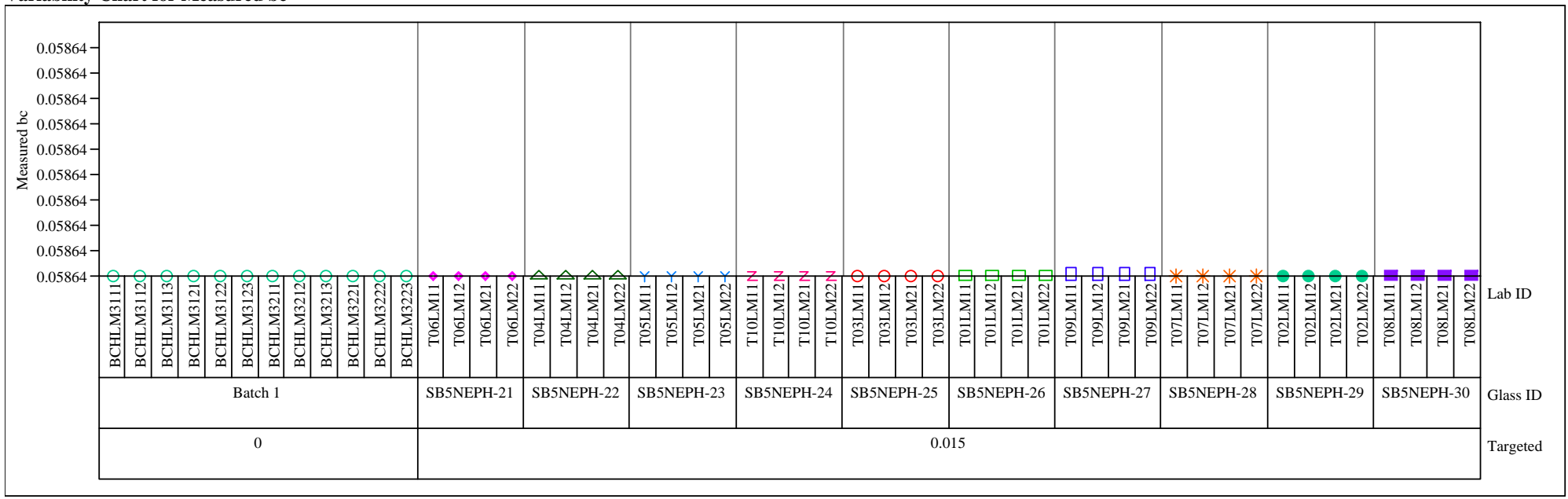


Exhibit A5. Measured and Measured Bias-Corrected Oxide Weight Percents

by Lab ID within Glass ID for the Glasses Prepared Using the LM Method. (continued)

Variability Gage Set $=3$, Oxide $=\mathrm{MgO}(\mathrm{wt} \%)$

Variability Chart for Measured
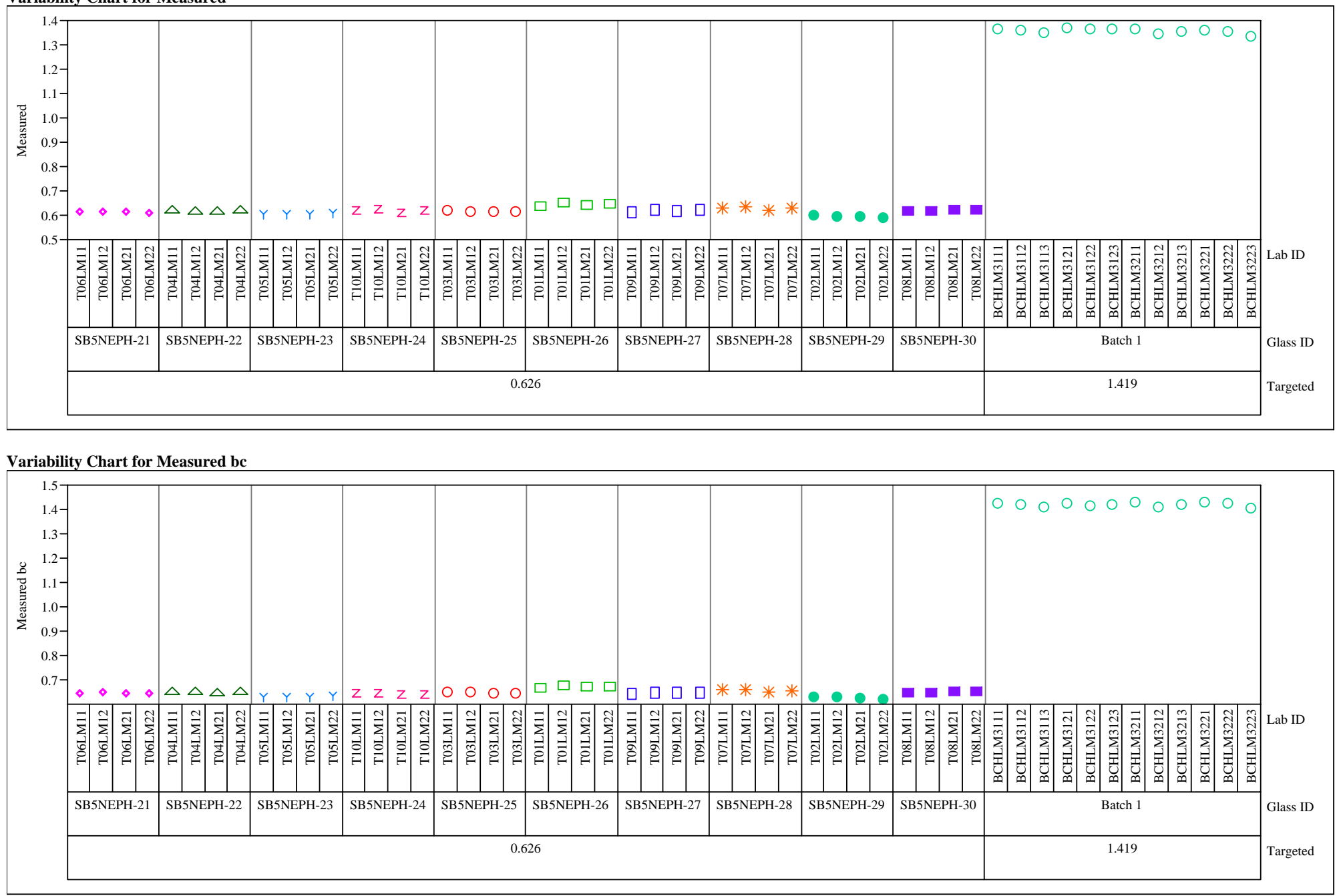
Exhibit A5. Measured and Measured Bias-Corrected Oxide Weight Percents

by Lab ID within Glass ID for the Glasses Prepared Using the LM Method. (continued)

Variability Gage Set $=3$, Oxide $=\mathrm{MnO}(\mathrm{wt} \%)$

Variability Chart for Measured
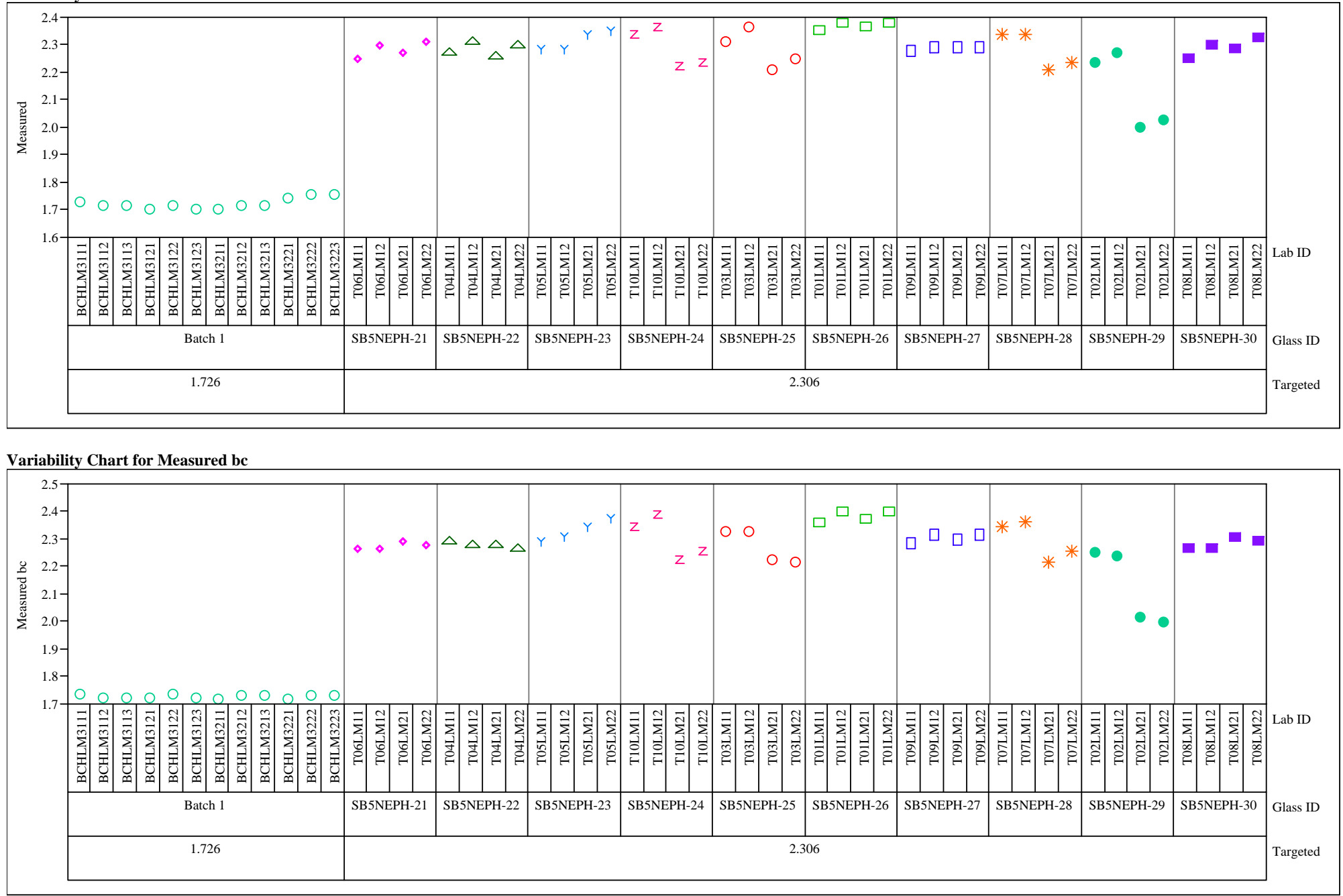
Exhibit A5. Measured and Measured Bias-Corrected Oxide Weight Percents

by Lab ID within Glass ID for the Glasses Prepared Using the LM Method. (continued)

Variability Gage Set $=3$, Oxide $=\mathrm{Na} 2 \mathrm{O}(\mathrm{wt} \%)$

Variability Chart for Measured
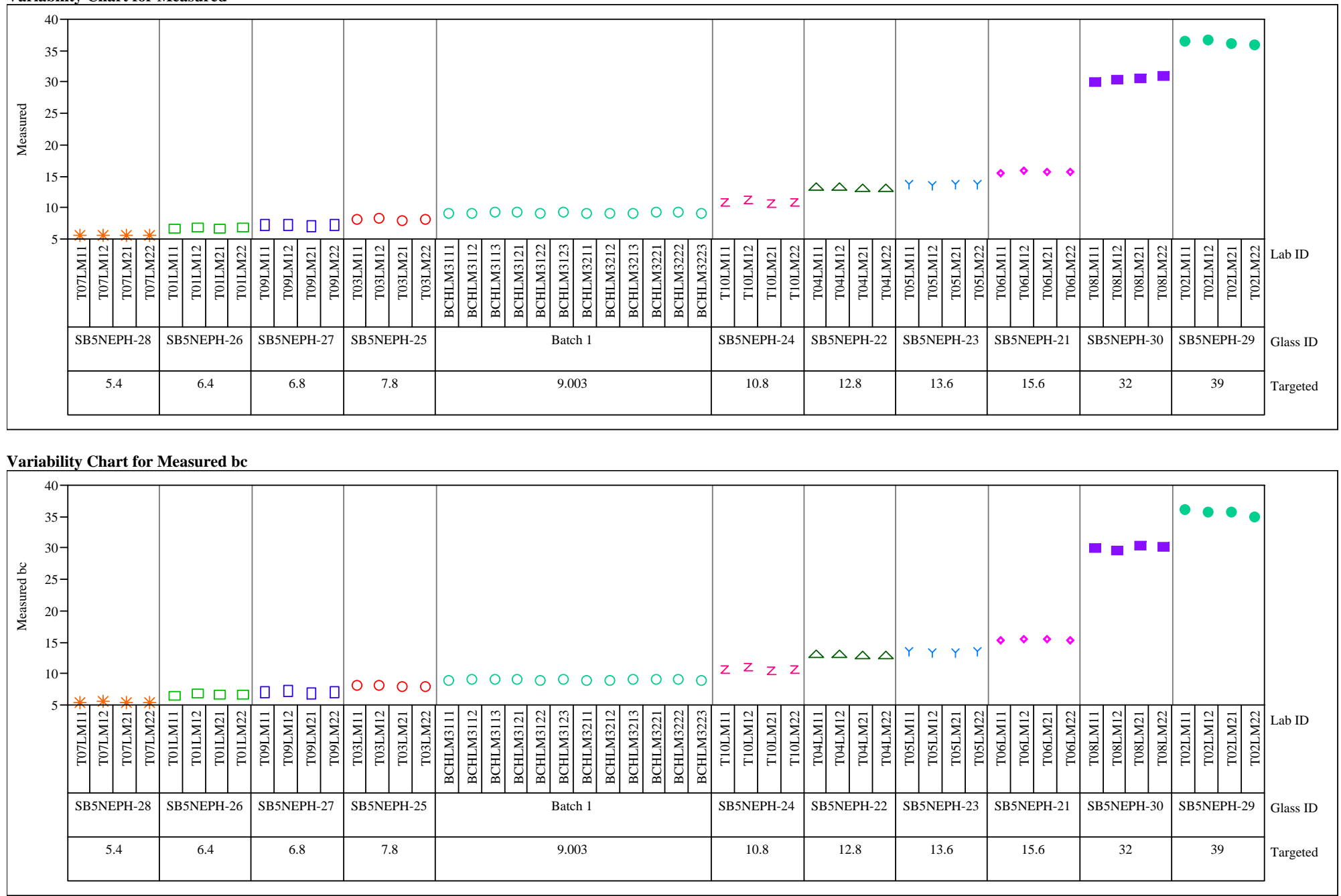
Exhibit A5. Measured and Measured Bias-Corrected Oxide Weight Percents

by Lab ID within Glass ID for the Glasses Prepared Using the LM Method. (continued)

Variability Gage Set $=3$, Oxide $=\mathrm{NiO}(\mathrm{wt} \%)$

Variability Chart for Measured
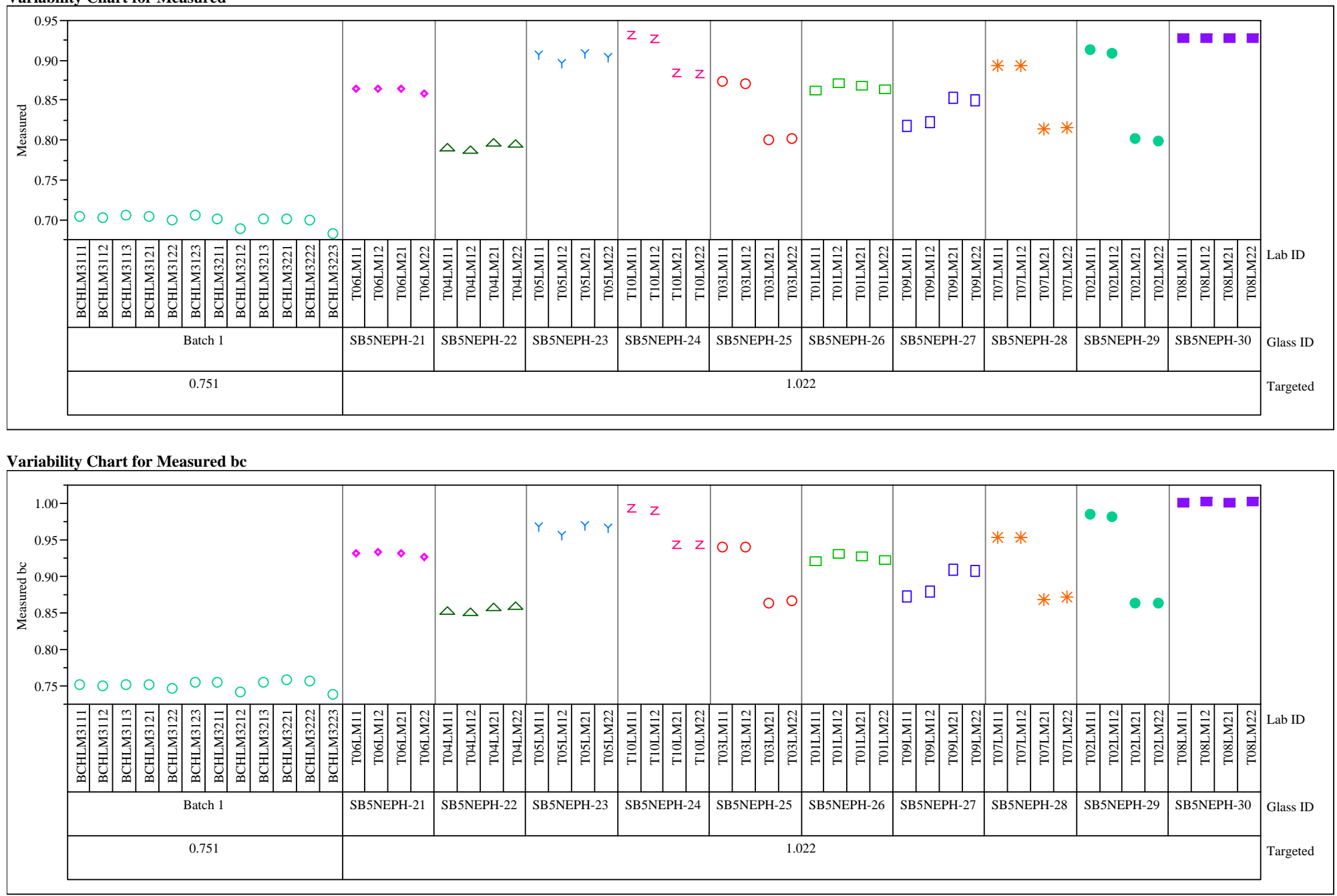
Exhibit A5. Measured and Measured Bias-Corrected Oxide Weight Percents

by Lab ID within Glass ID for the Glasses Prepared Using the LM Method. (continued)

Variability Gage Set $=3$, Oxide $=\mathbf{P b O}$ (wt $\%$ )

Variability Chart for Measured
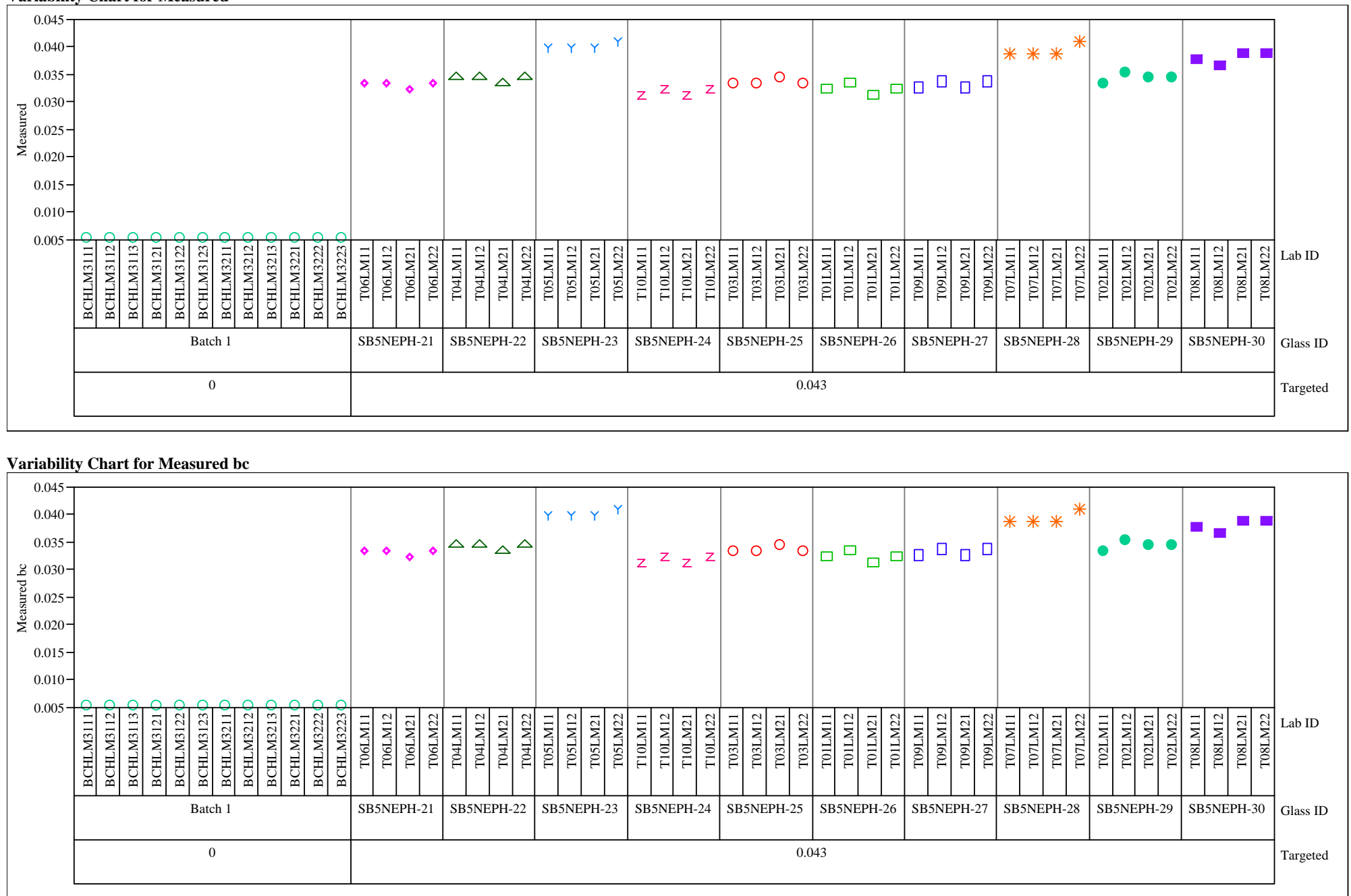
Exhibit A5. Measured and Measured Bias-Corrected Oxide Weight Percents

by Lab ID within Glass ID for the Glasses Prepared Using the LM Method. (continued)

Variability Gage Set $=3$, Oxide=SO4 (wt $\%$ )

Variability Chart for Measured
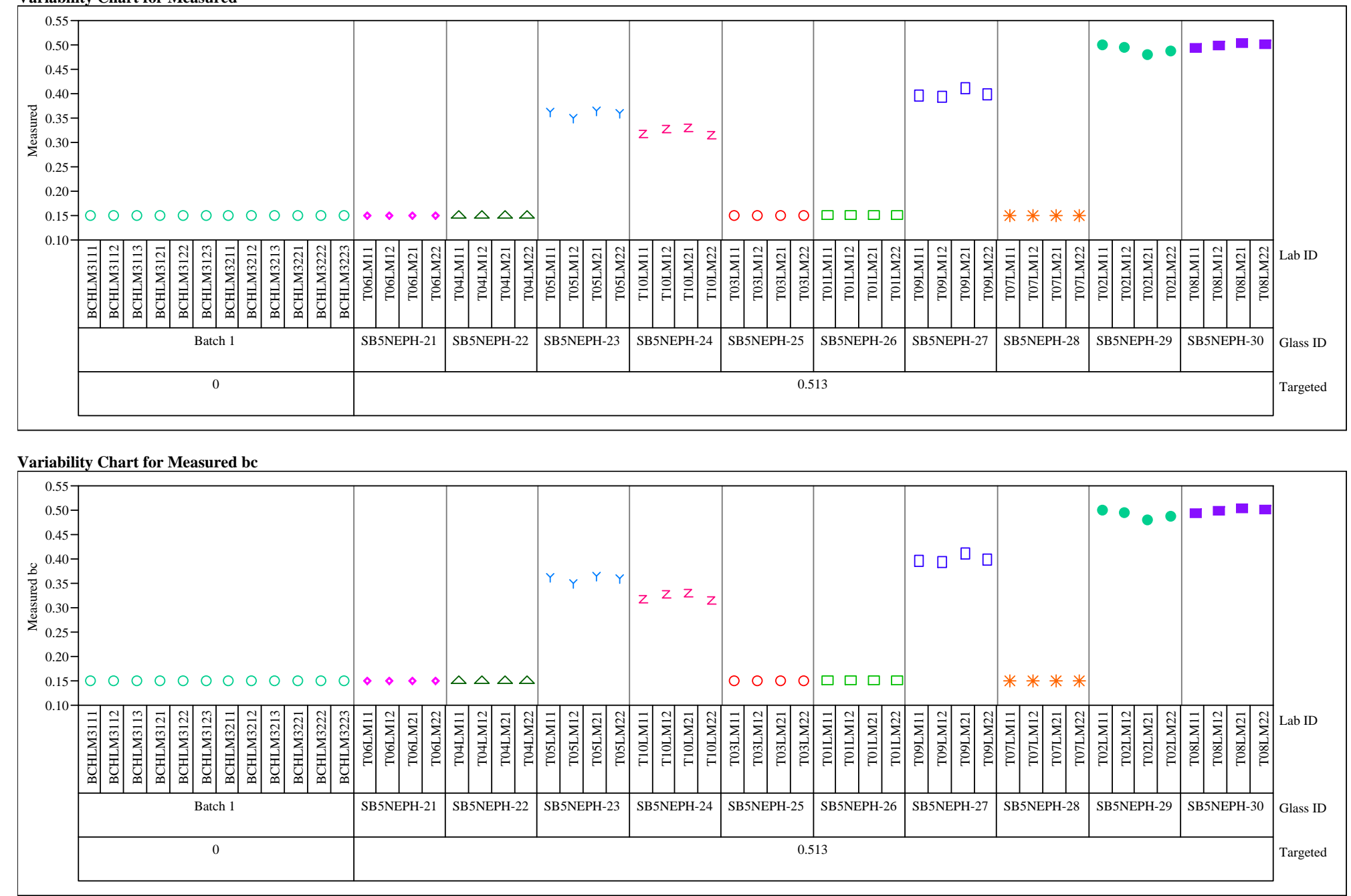
Exhibit A5. Measured and Measured Bias-Corrected Oxide Weight Percents

by Lab ID within Glass ID for the Glasses Prepared Using the LM Method. (continued)

Variability Gage Set $=3$, Oxide $=$ TiO2 $(w t \%)$

Variability Chart for Measured
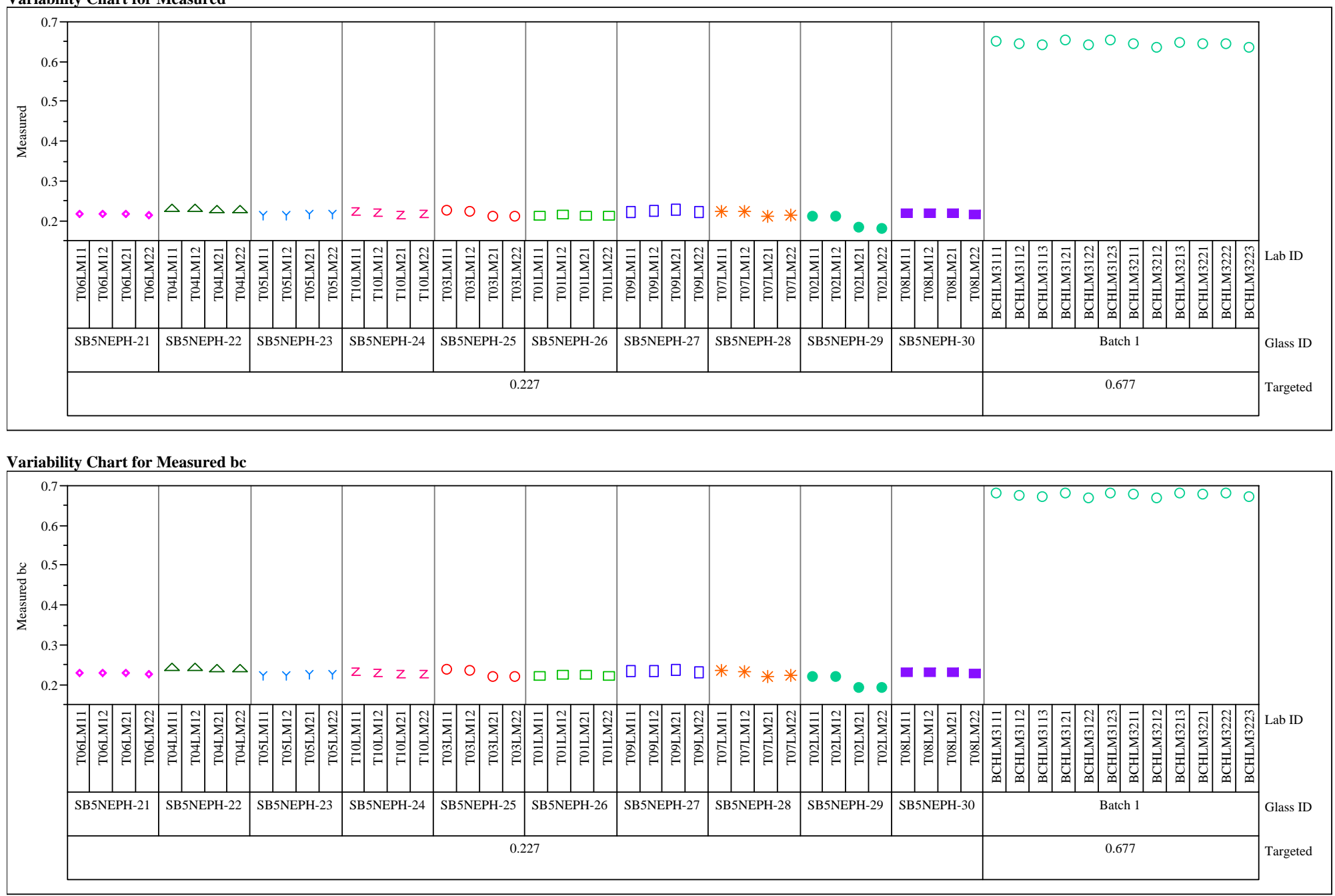
Exhibit A5. Measured and Measured Bias-Corrected Oxide Weight Percents

by Lab ID within Glass ID for the Glasses Prepared Using the LM Method. (continued)

Variability Gage Set $=3$, Oxide $=\mathrm{ZnO}$ (wt\%)

Variability Chart for Measured
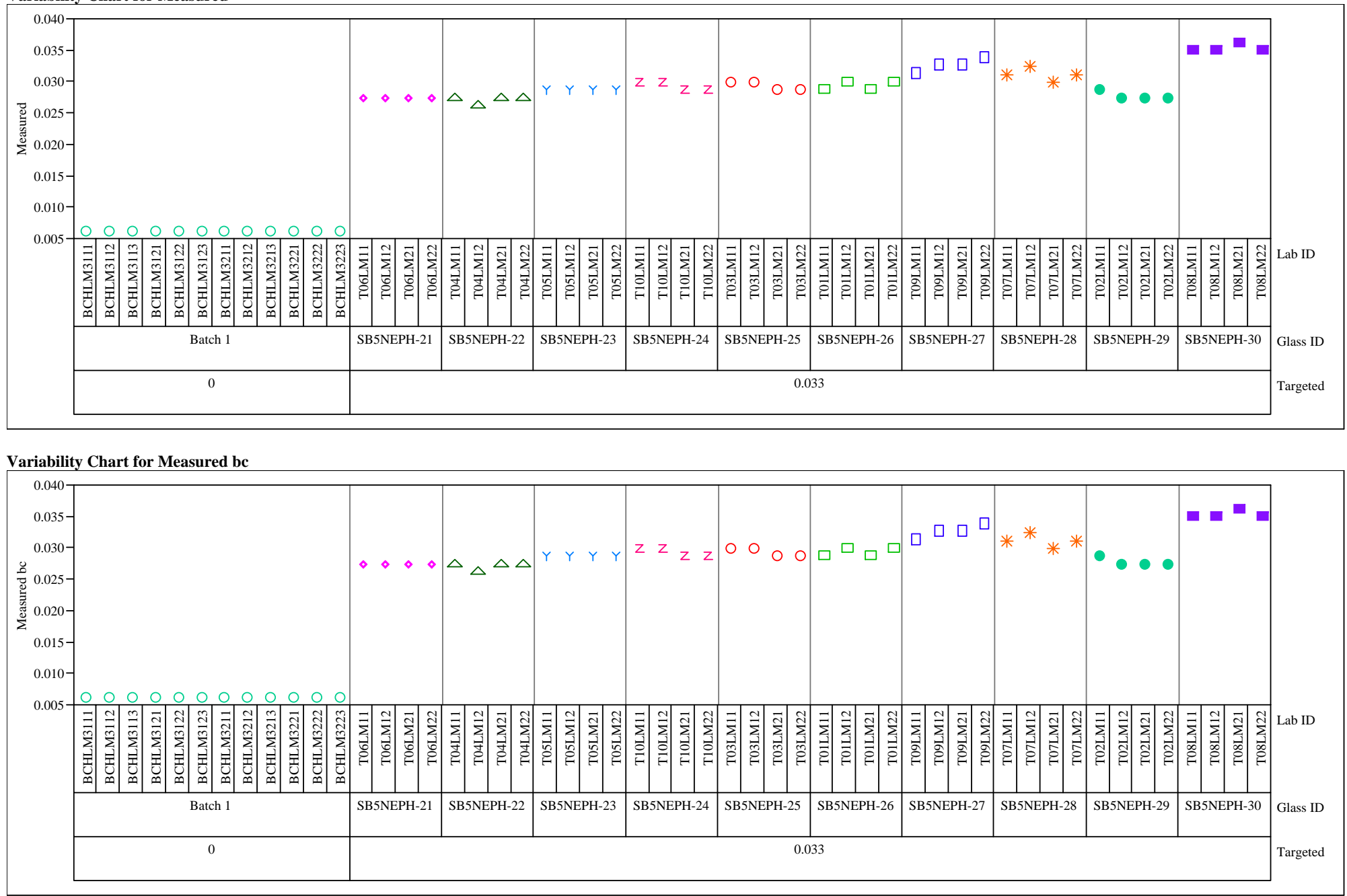
Exhibit A5. Measured and Measured Bias-Corrected Oxide Weight Percents

by Lab ID within Glass ID for the Glasses Prepared Using the LM Method. (continued)

Variability Gage Set=3, Oxide $=\mathrm{ZrO} 2(w t \%)$

Variability Chart for Measured

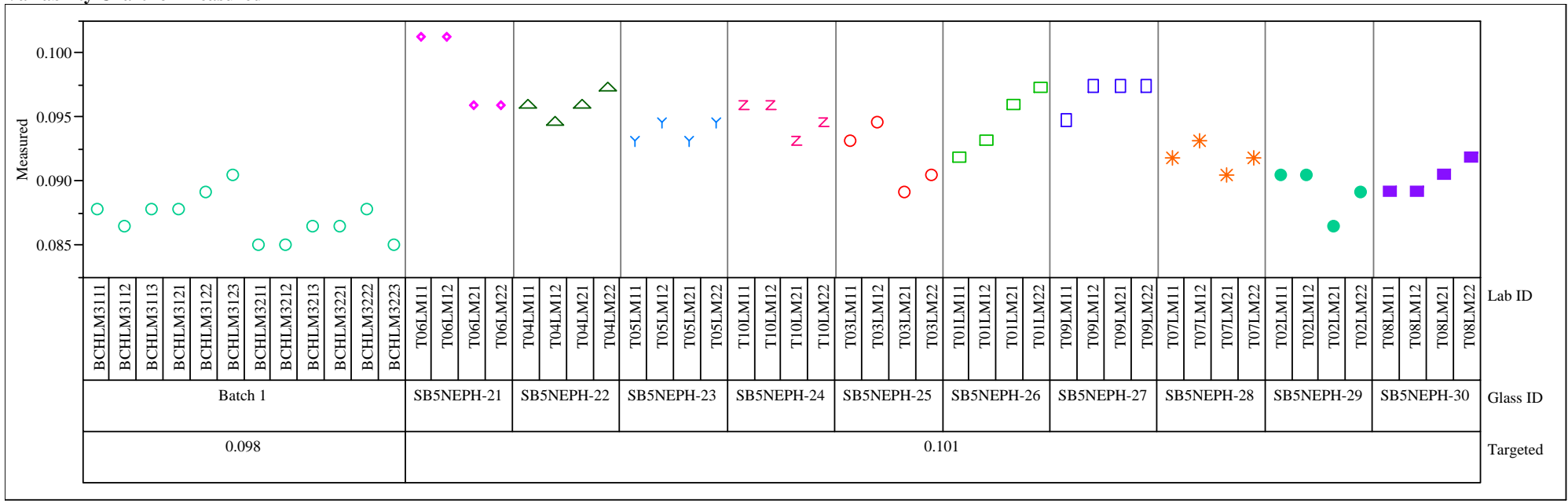

Variability Chart for Measured bc

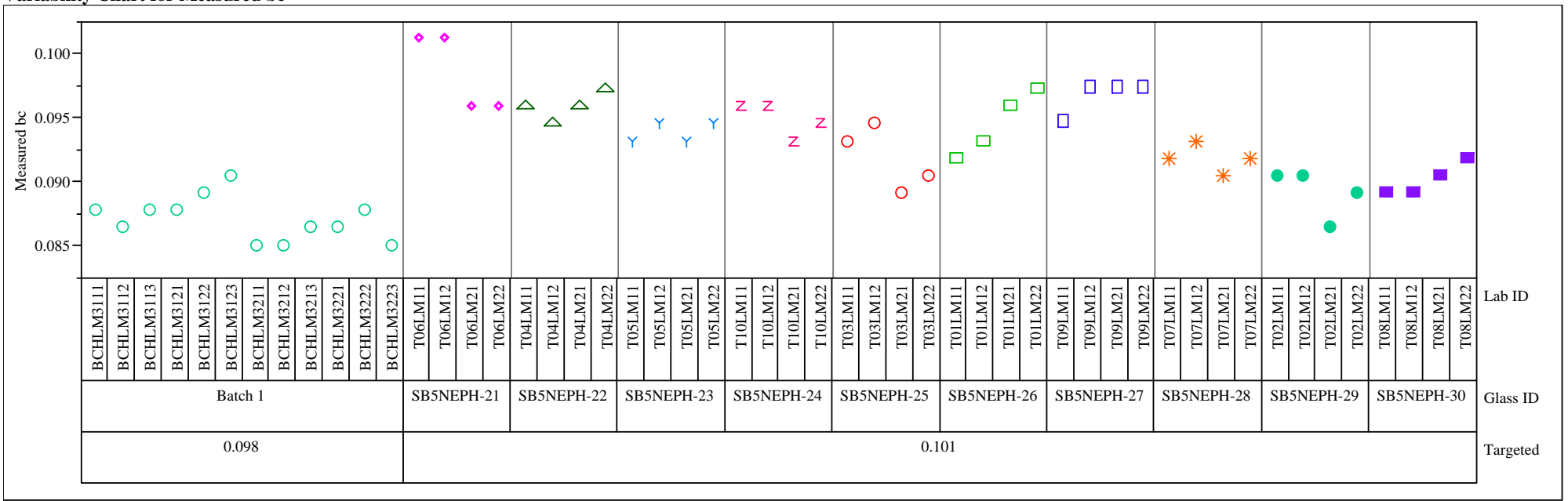


Exhibit A5. Measured and Measured Bias-Corrected Oxide Weight Percents

by Lab ID within Glass ID for the Glasses Prepared Using the LM Method. (continued)

Variability Gage Set $=4$, Oxide $=$ Al2O3 (wt $\%)$

Variability Chart for Measured

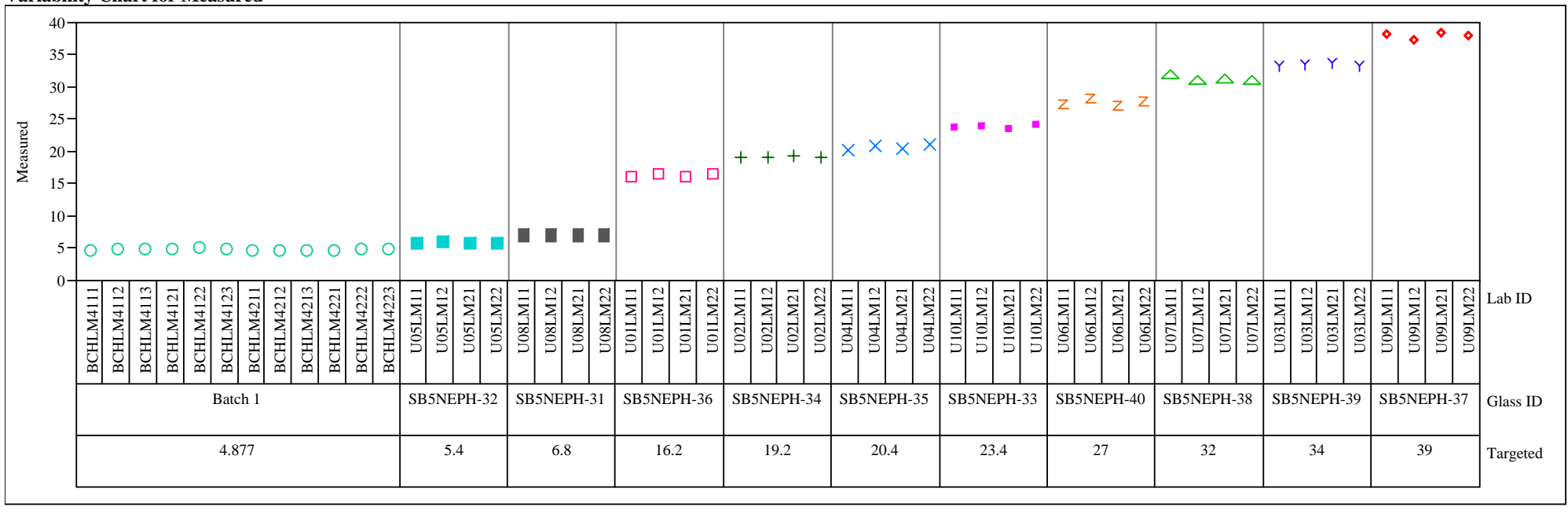

Variability Chart for Measured bc

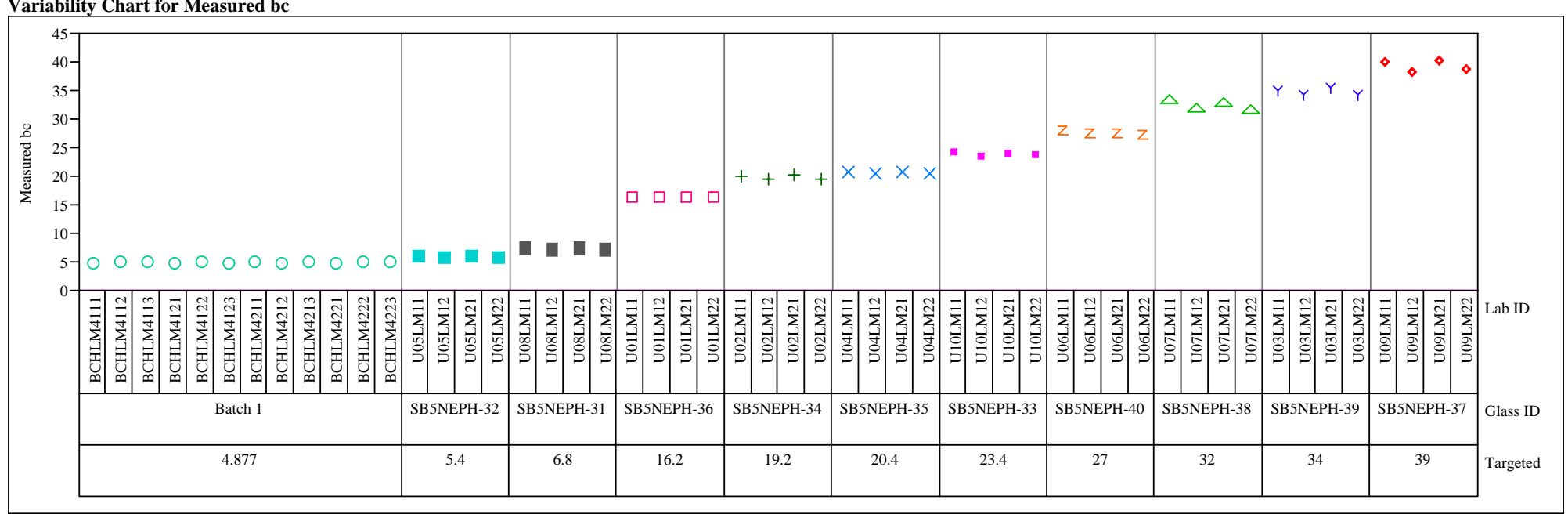


Exhibit A5. Measured and Measured Bias-Corrected Oxide Weight Percents

by Lab ID within Glass ID for the Glasses Prepared Using the LM Method. (continued)

Variability Gage Set $=4$, Oxide $=\mathrm{BaO}$ ( $w$ t $\%$ )

Variability Chart for Measured
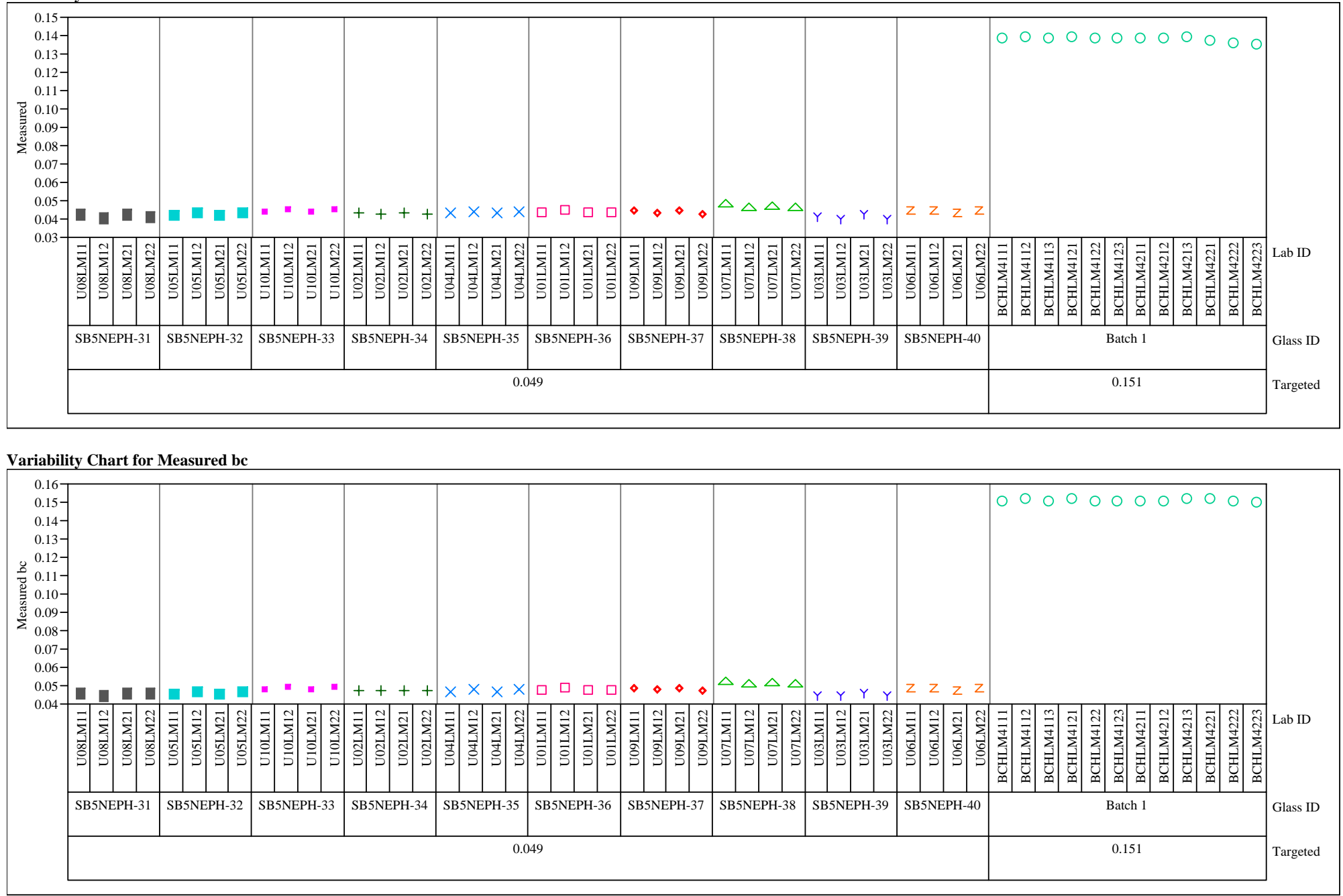
Exhibit A5. Measured and Measured Bias-Corrected Oxide Weight Percents

by Lab ID within Glass ID for the Glasses Prepared Using the LM Method. (continued)

Variability Gage Set $=4$, Oxide $=\mathrm{CaO}(\mathrm{wt} \%)$

Variability Chart for Measured
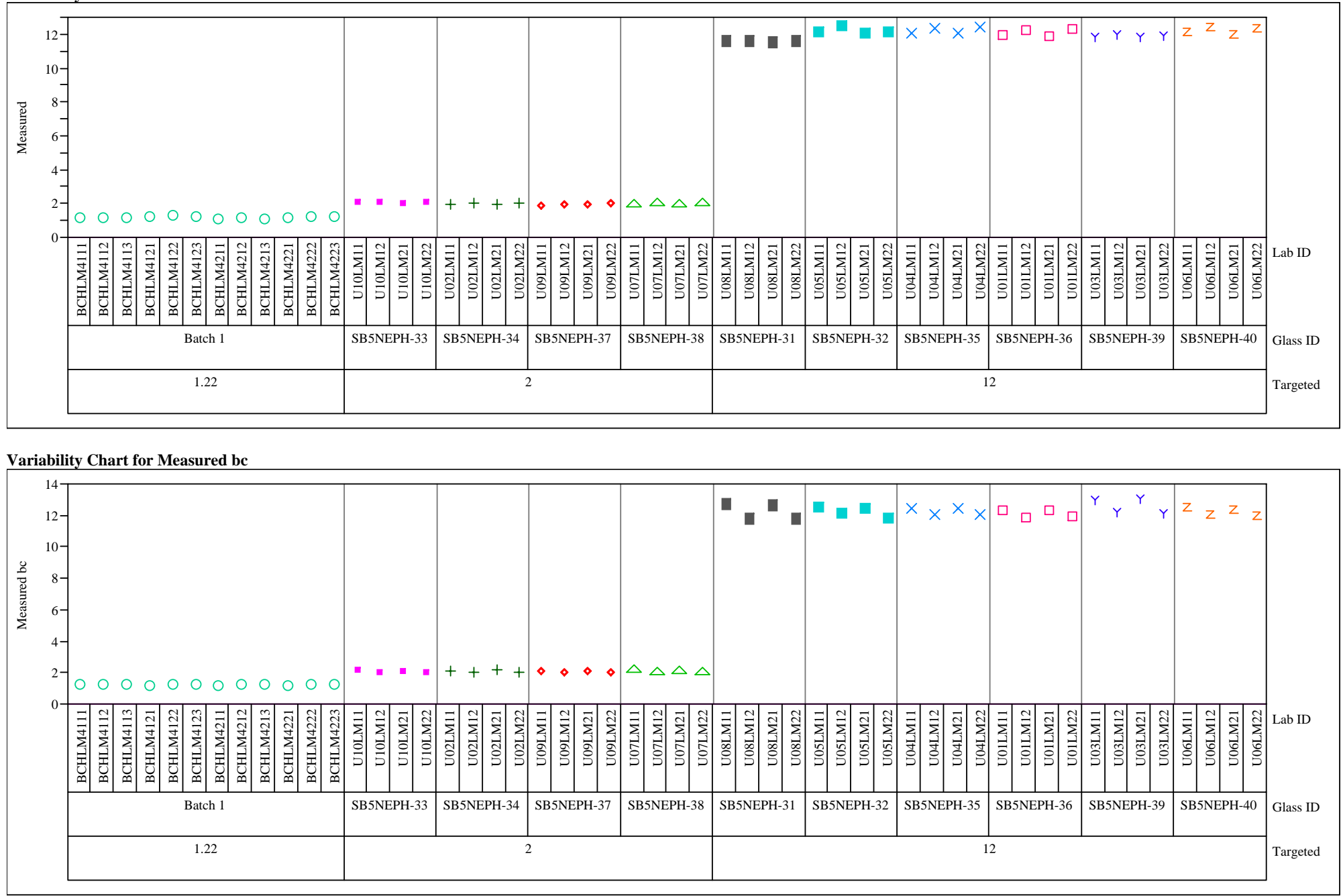
Exhibit A5. Measured and Measured Bias-Corrected Oxide Weight Percents

by Lab ID within Glass ID for the Glasses Prepared Using the LM Method. (continued)

Variability Gage Set $=4$, Oxide $=\mathrm{Ce} 2 \mathrm{O} 3(\mathrm{wt} \%)$

Variability Chart for Measured
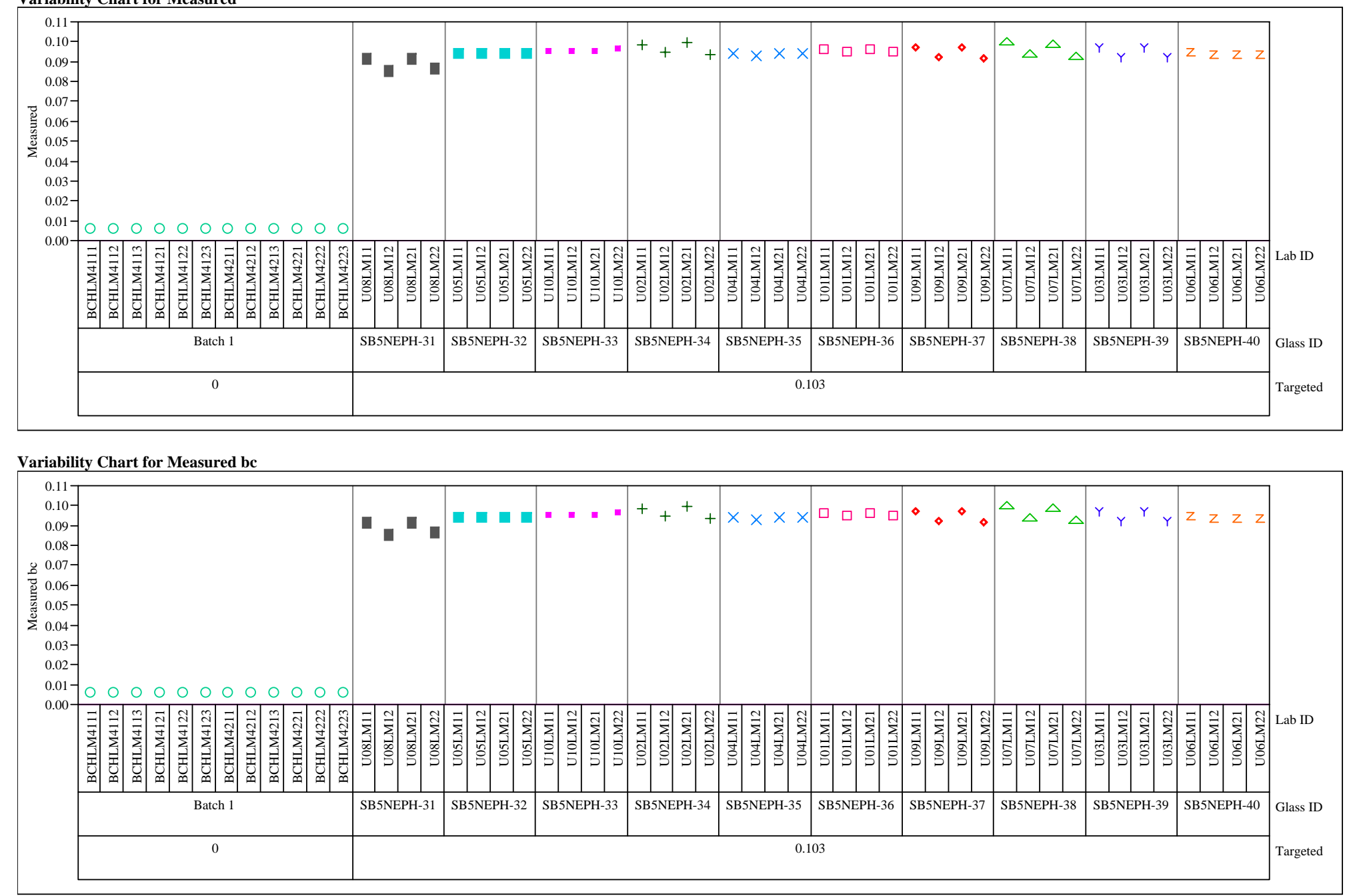
Exhibit A5. Measured and Measured Bias-Corrected Oxide Weight Percents

by Lab ID within Glass ID for the Glasses Prepared Using the LM Method. (continued)

Variability Gage Set $=4$, Oxide $=\mathrm{Cr} 2 \mathrm{O} 3(\mathrm{wt} \%)$

Variability Chart for Measured
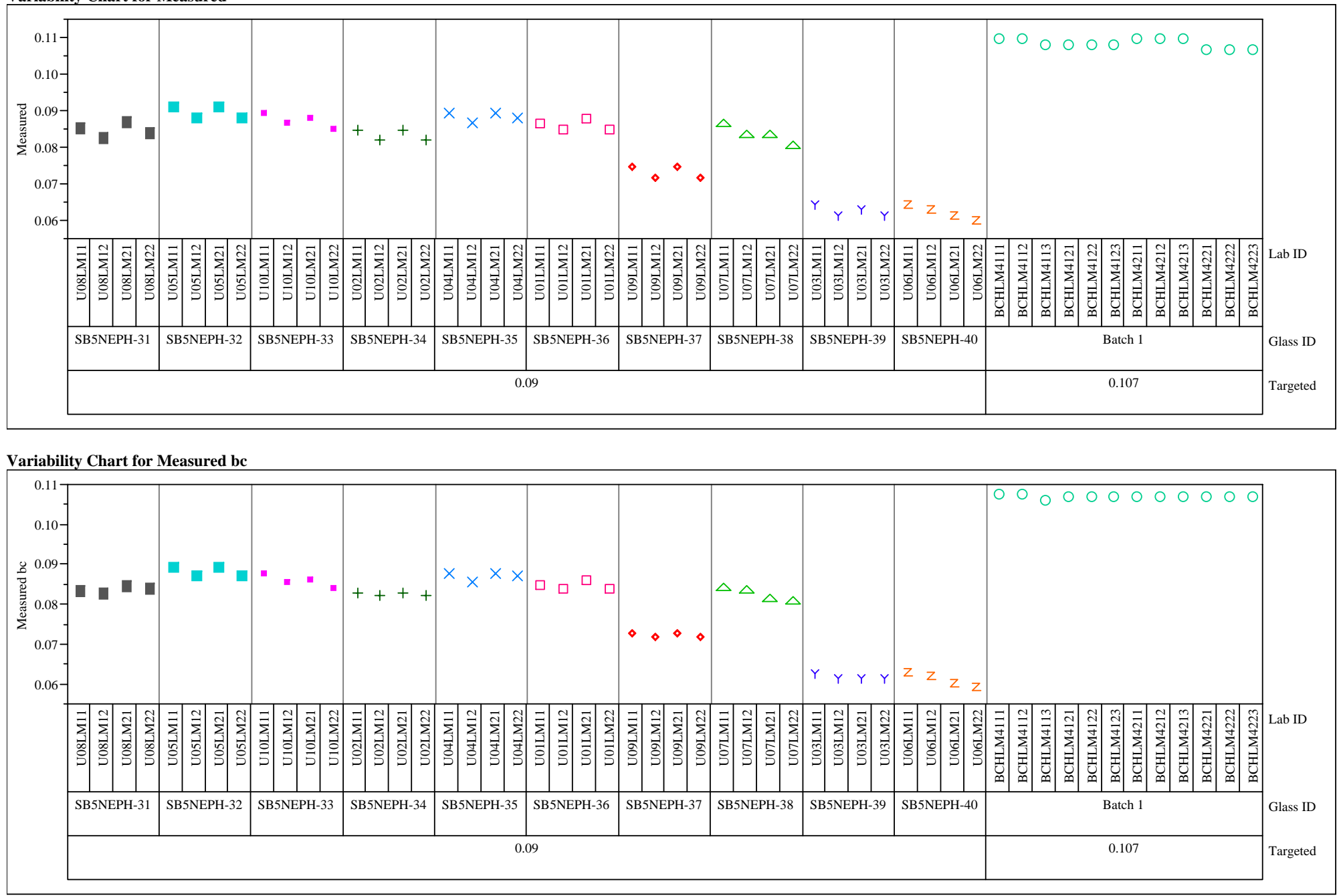
Exhibit A5. Measured and Measured Bias-Corrected Oxide Weight Percents

by Lab ID within Glass ID for the Glasses Prepared Using the LM Method. (continued)

Variability Gage Set $=4$, Oxide $=\mathrm{CuO}(\mathrm{wt} \%)$

Variability Chart for Measured
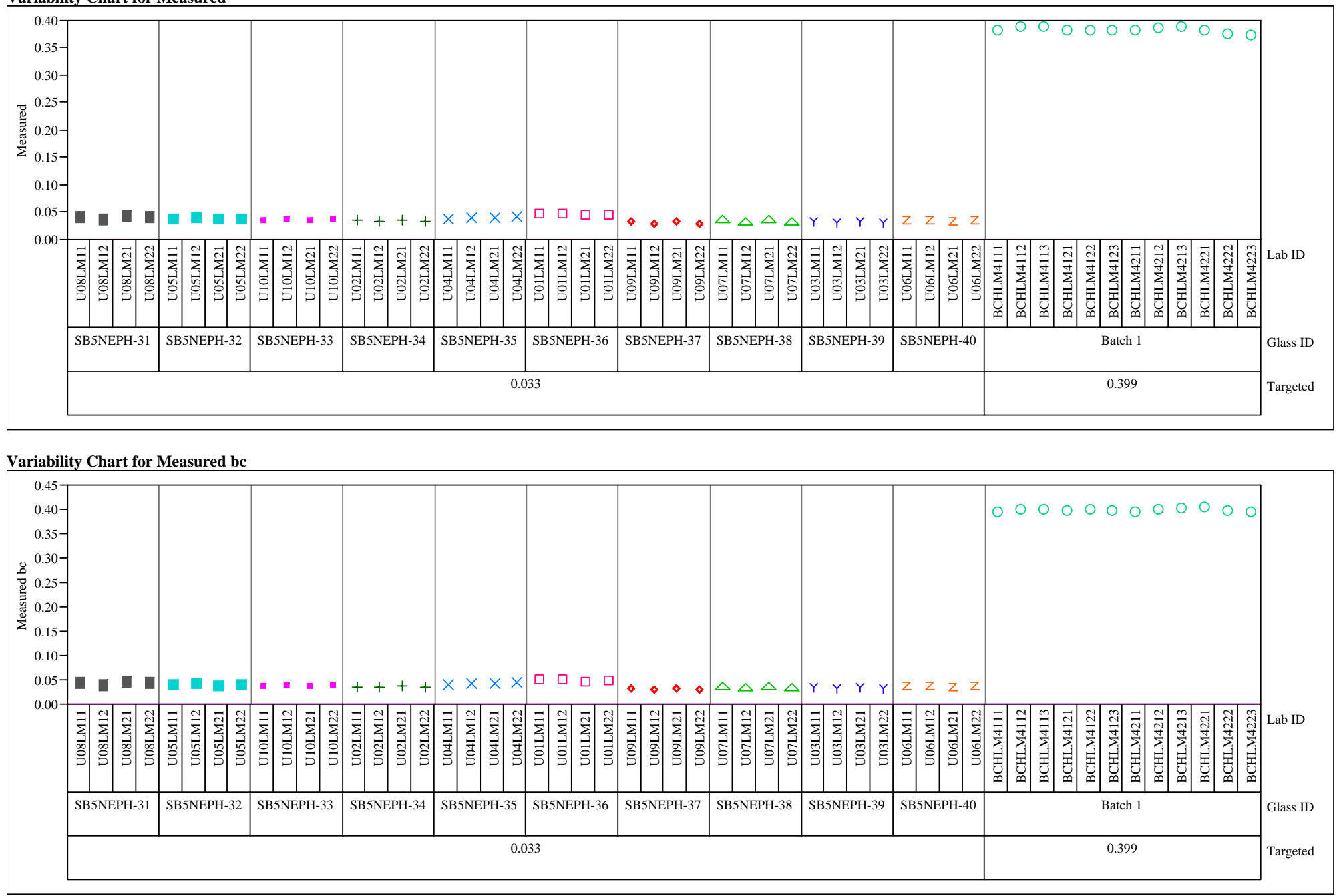
Exhibit A5. Measured and Measured Bias-Corrected Oxide Weight Percents

by Lab ID within Glass ID for the Glasses Prepared Using the LM Method. (continued)

Variability Gage Set $=4$, Oxide $=$ K2O (wt $\%)$

Variability Chart for Measured
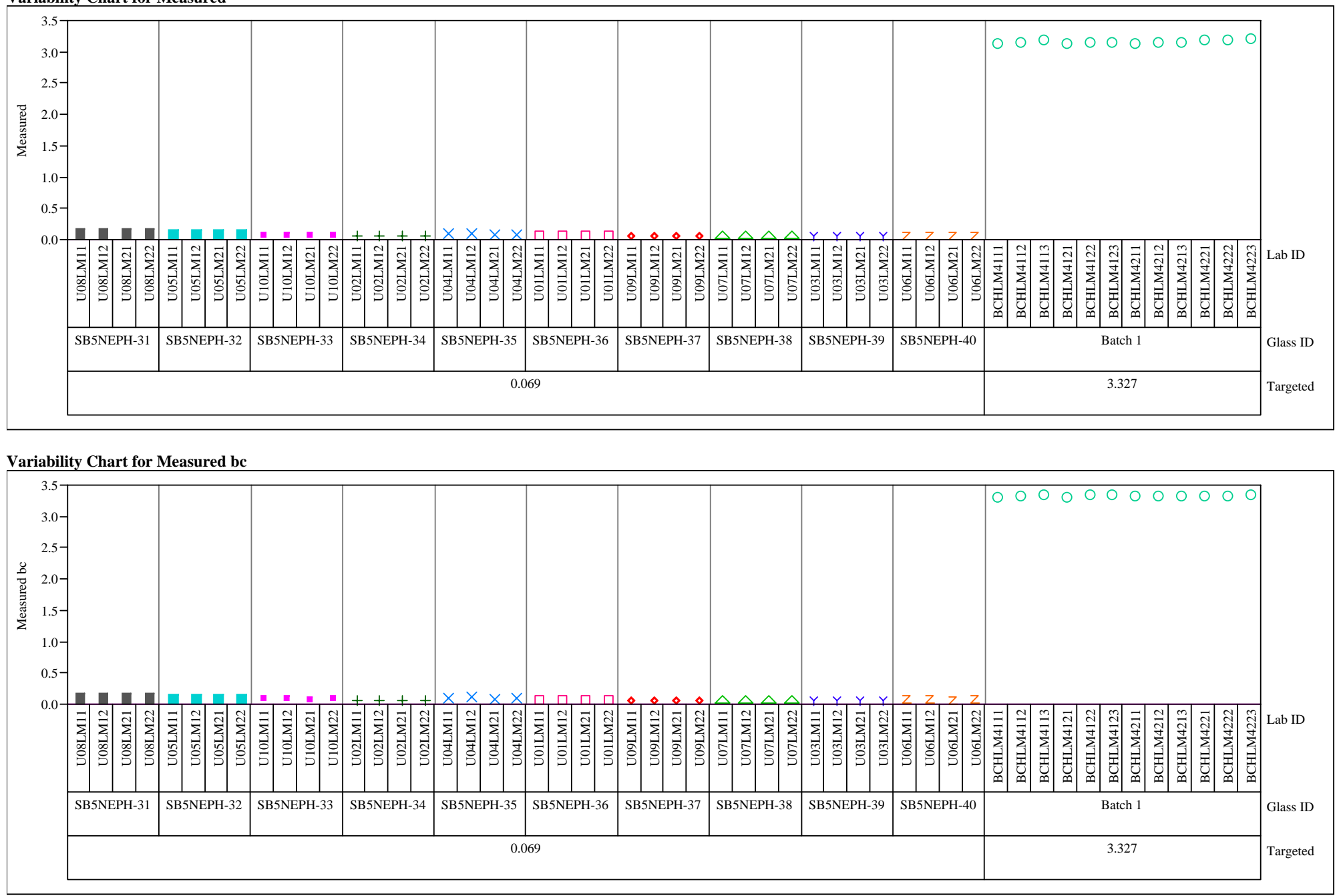
Exhibit A5. Measured and Measured Bias-Corrected Oxide Weight Percents

by Lab ID within Glass ID for the Glasses Prepared Using the LM Method. (continued)

Variability Gage Set $=4$, Oxide $=$ La2O3 (wt $\%)$

Variability Chart for Measured

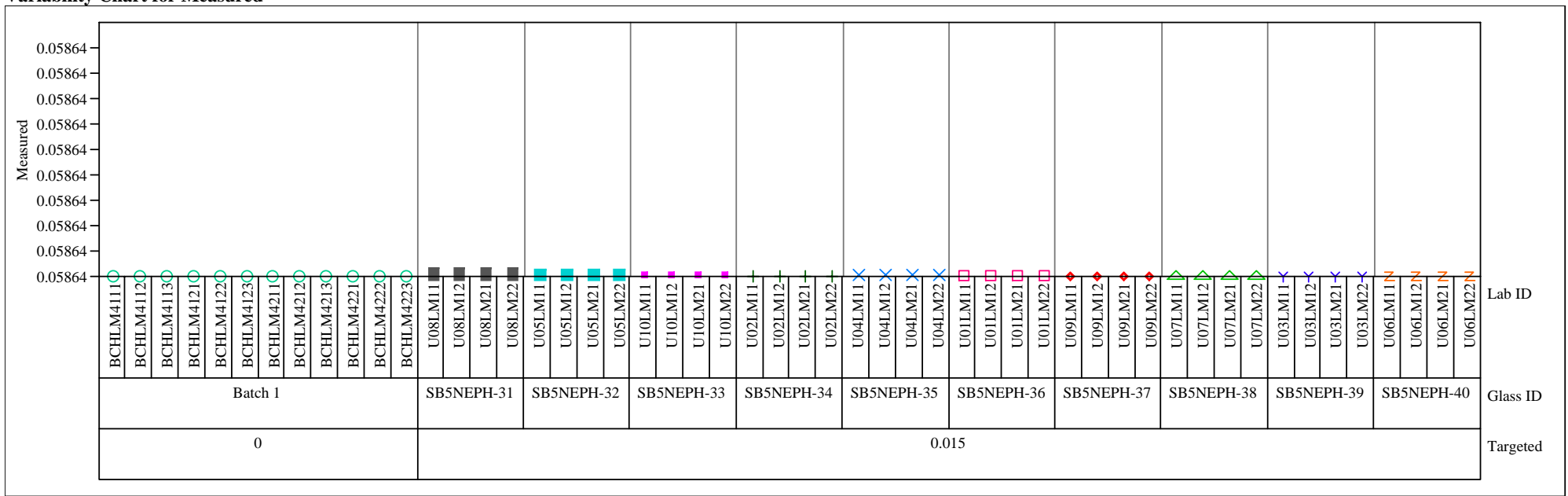

Variability Chart for Measured bc

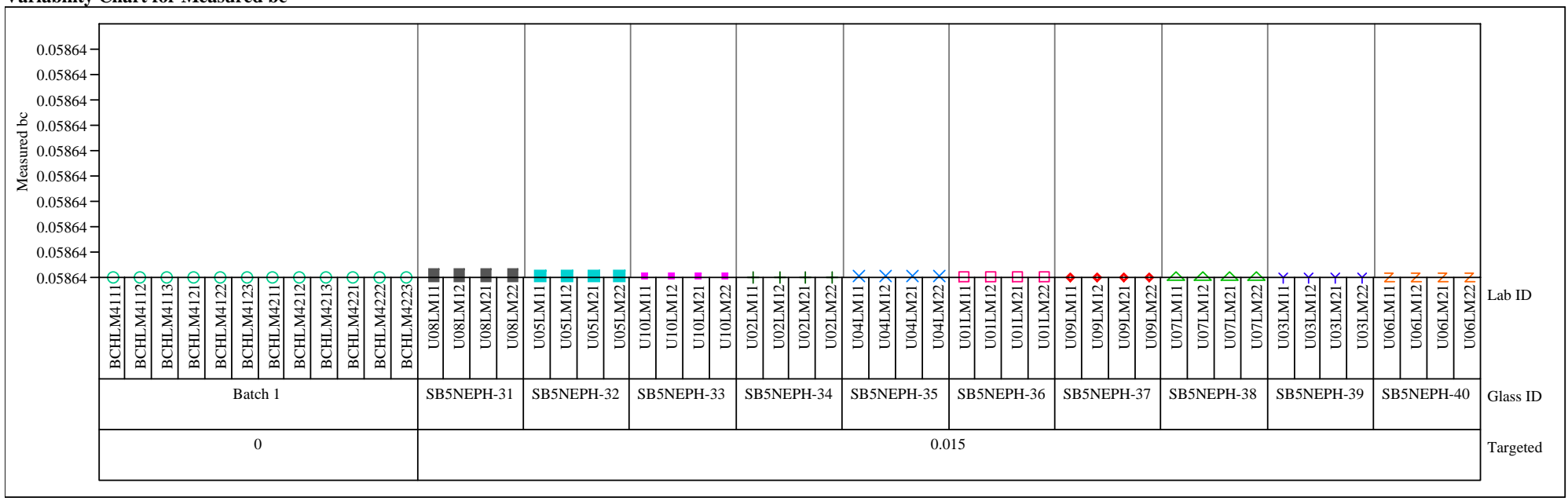


Exhibit A5. Measured and Measured Bias-Corrected Oxide Weight Percents

by Lab ID within Glass ID for the Glasses Prepared Using the LM Method. (continued)

Variability Gage Set $=4$, Oxide $=\mathrm{MgO}(\mathrm{wt} \%)$

Variability Chart for Measured

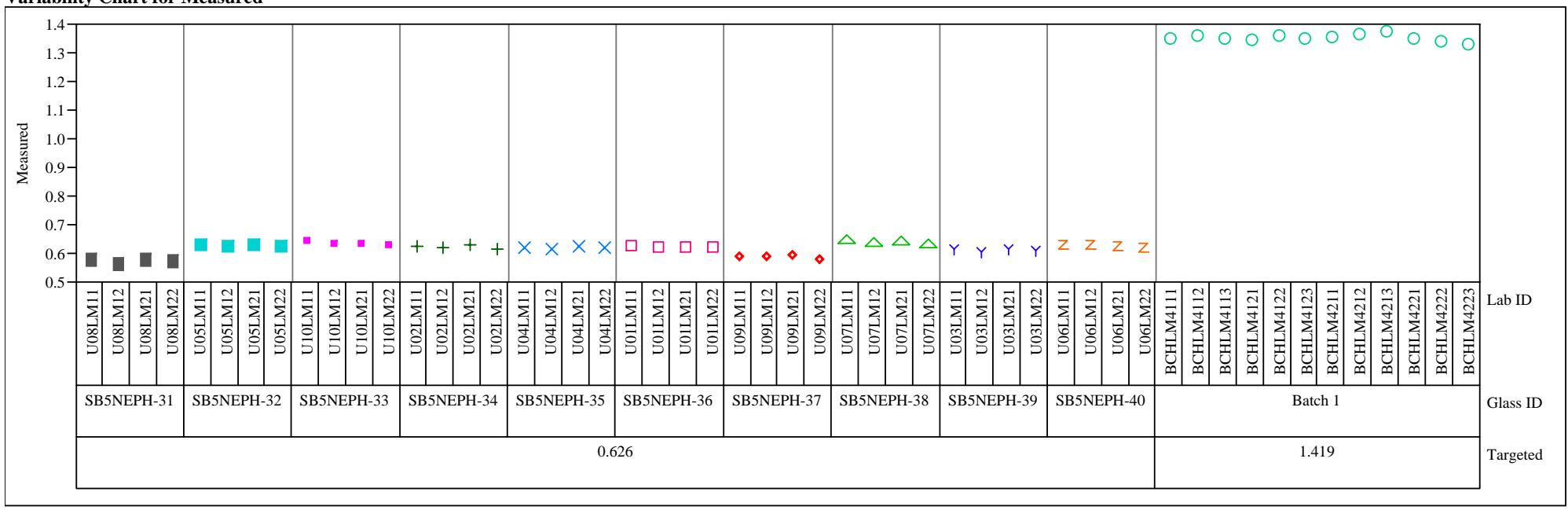

Variability Chart for Measured bc

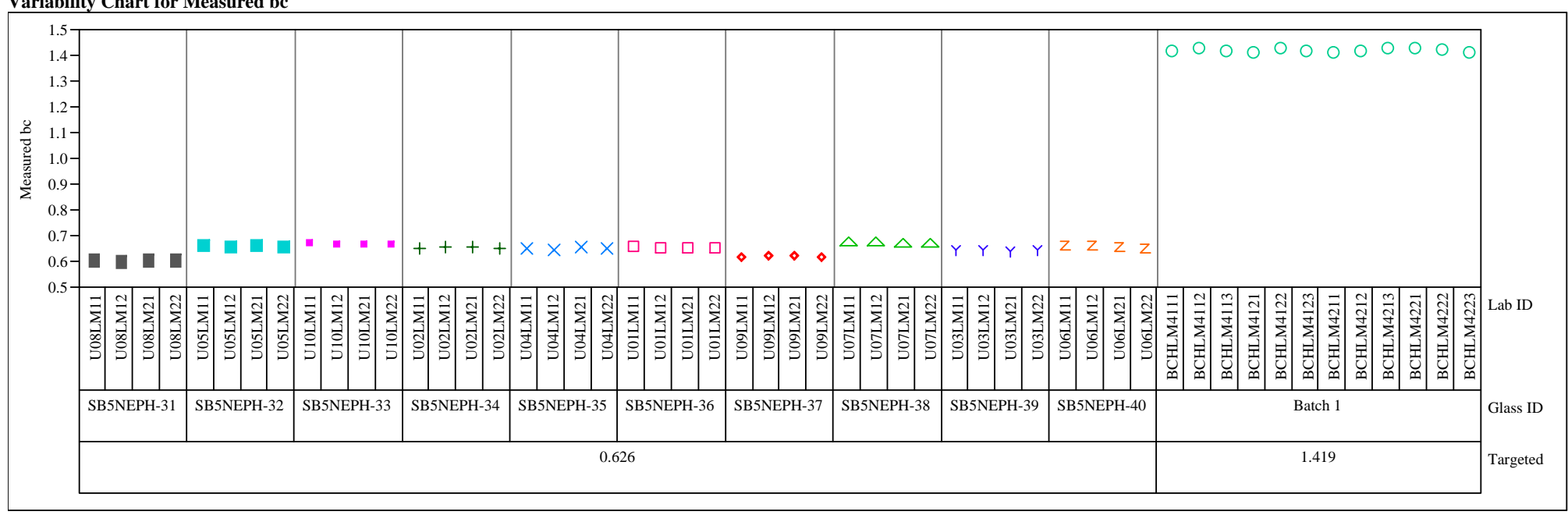


Exhibit A5. Measured and Measured Bias-Corrected Oxide Weight Percents

by Lab ID within Glass ID for the Glasses Prepared Using the LM Method. (continued)

Variability Gage Set $=4$, Oxide $=\mathrm{MnO}(\mathrm{wt} \%)$

Variability Chart for Measured

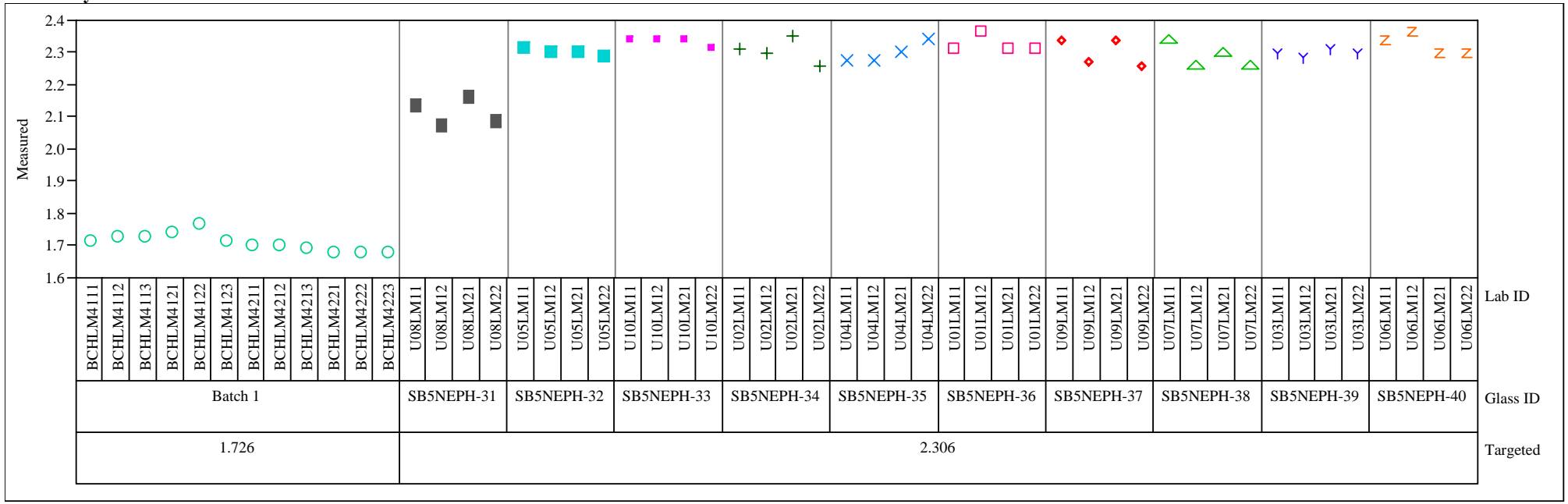

Variability Chart for Measured bc

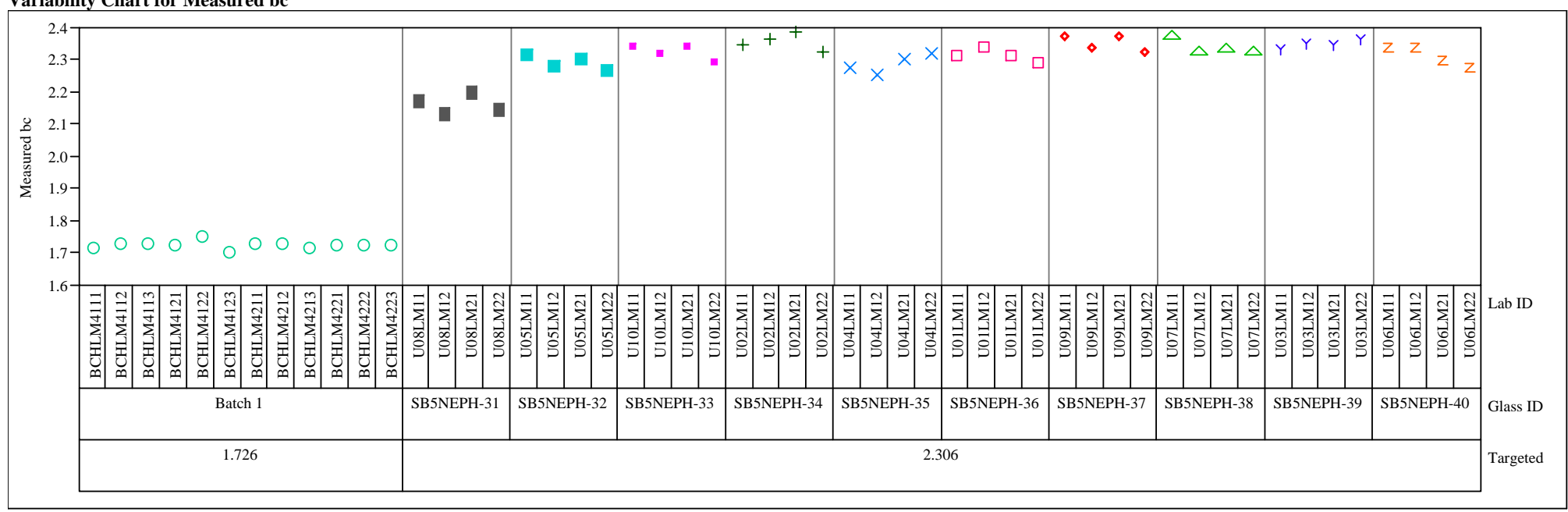


Exhibit A5. Measured and Measured Bias-Corrected Oxide Weight Percents

by Lab ID within Glass ID for the Glasses Prepared Using the LM Method. (continued)

Variability Gage Set $=4$, Oxide $=\mathrm{Na} 2 \mathrm{O}(\mathrm{wt} \%)$

Variability Chart for Measured

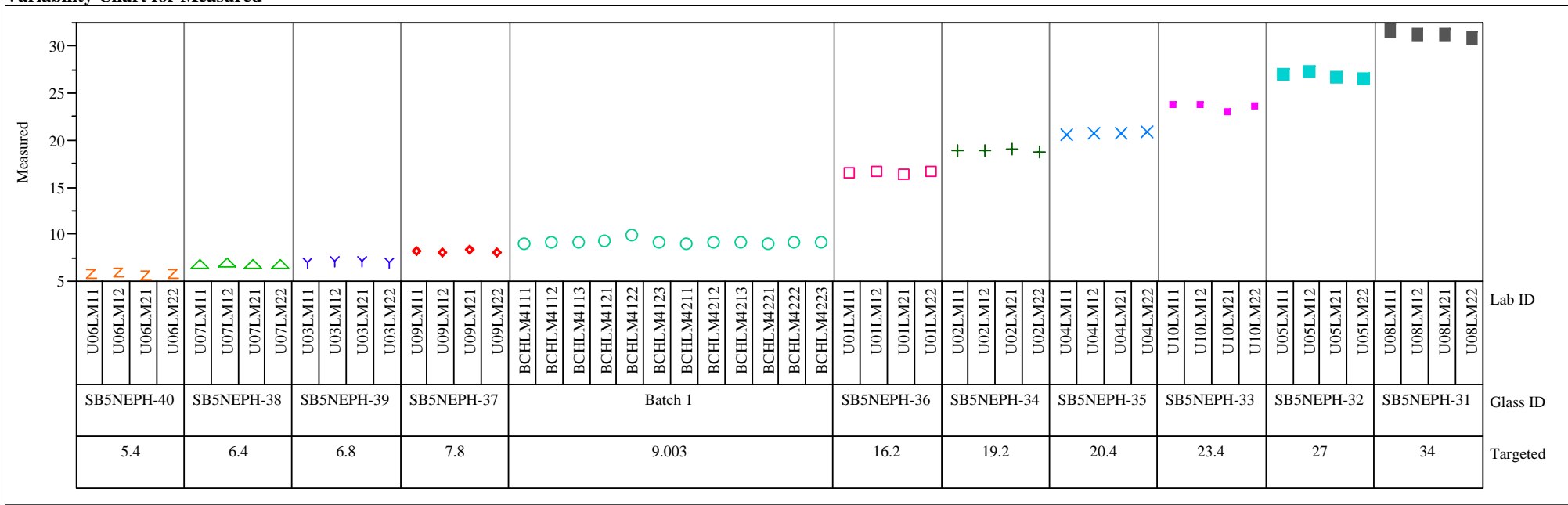

Variability Chart for Measured bc

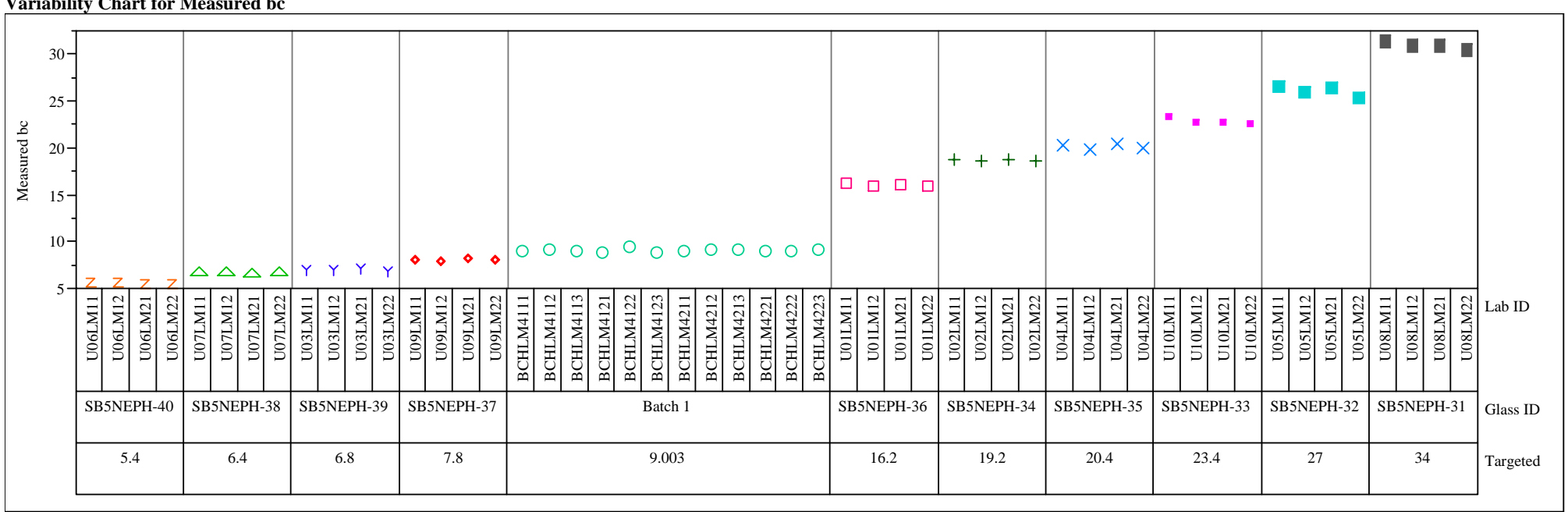


Exhibit A5. Measured and Measured Bias-Corrected Oxide Weight Percents

by Lab ID within Glass ID for the Glasses Prepared Using the LM Method. (continued)

Variability Gage Set $=4$, Oxide $=\mathrm{NiO}(\mathrm{wt} \%)$

Variability Chart for Measured

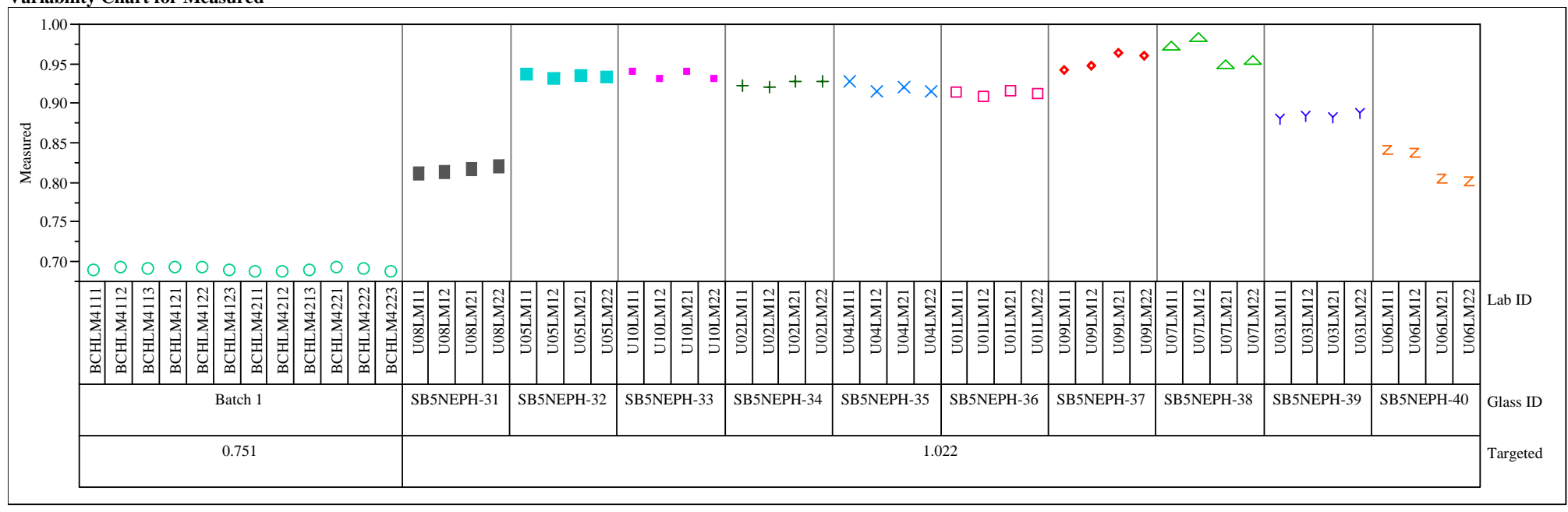

Variability Chart for Measured bc

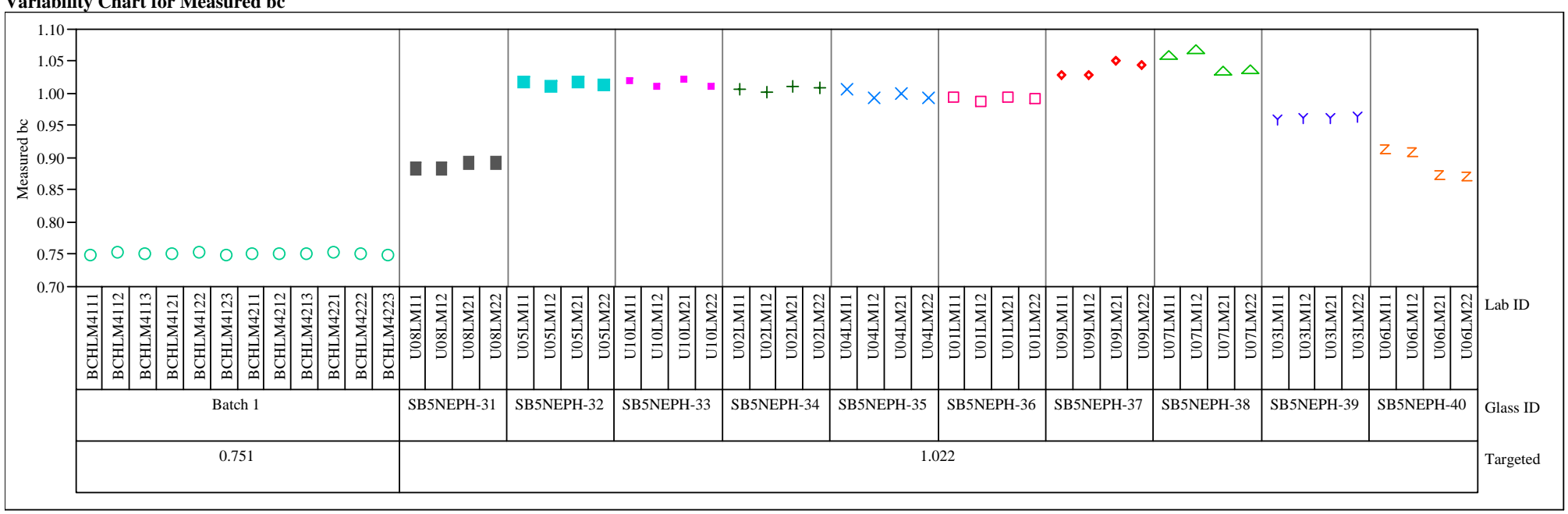


Exhibit A5. Measured and Measured Bias-Corrected Oxide Weight Percents

by Lab ID within Glass ID for the Glasses Prepared Using the LM Method. (continued)

Variability Gage Set $=4$, Oxide $=\mathbf{P b O}$ ( $w$ t $\%$ )

Variability Chart for Measured
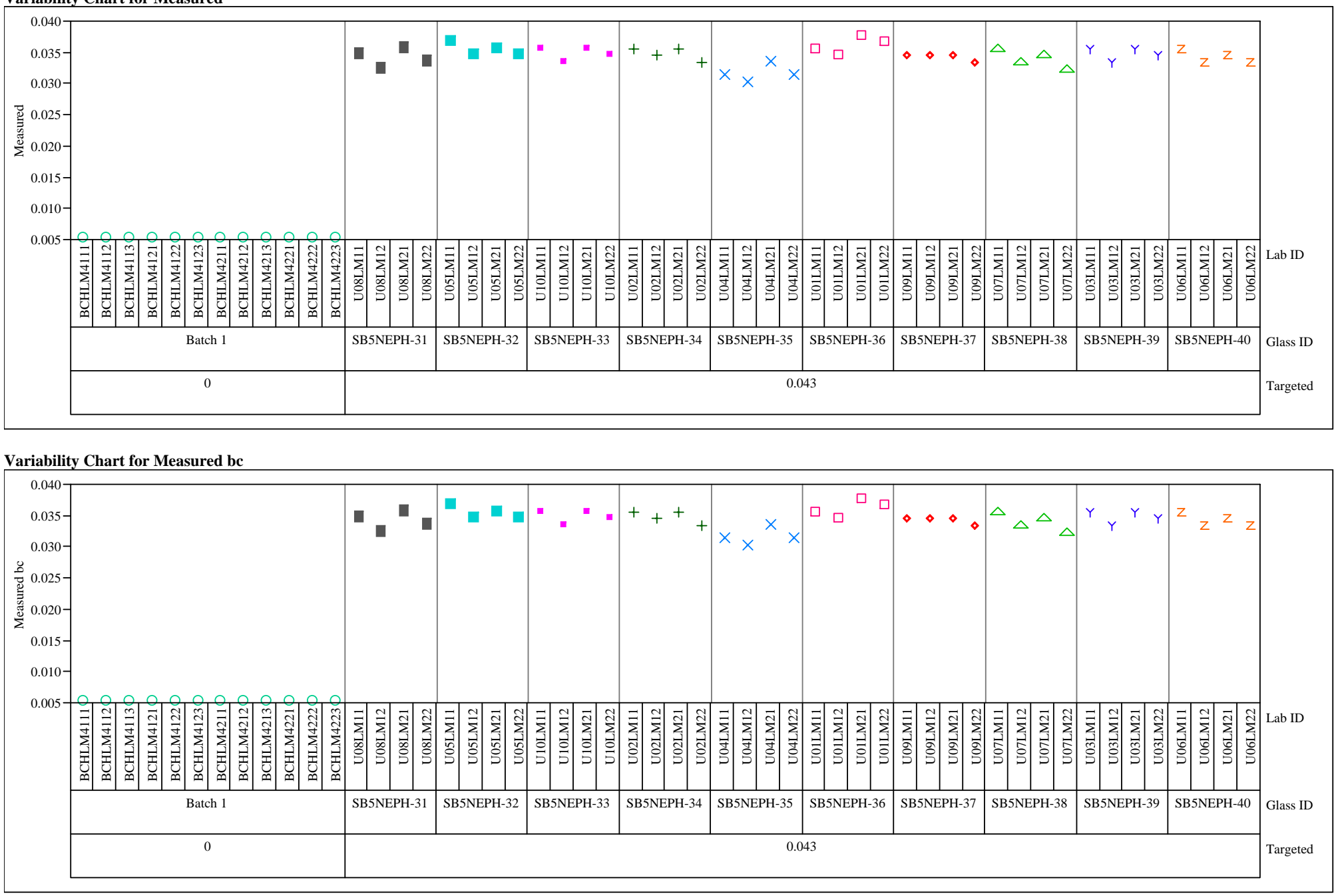
Exhibit A5. Measured and Measured Bias-Corrected Oxide Weight Percents

by Lab ID within Glass ID for the Glasses Prepared Using the LM Method. (continued)

Variability Gage Set $=4$, Oxide $=\mathrm{SO} 4$ (wt $\%$ )

Variability Chart for Measured
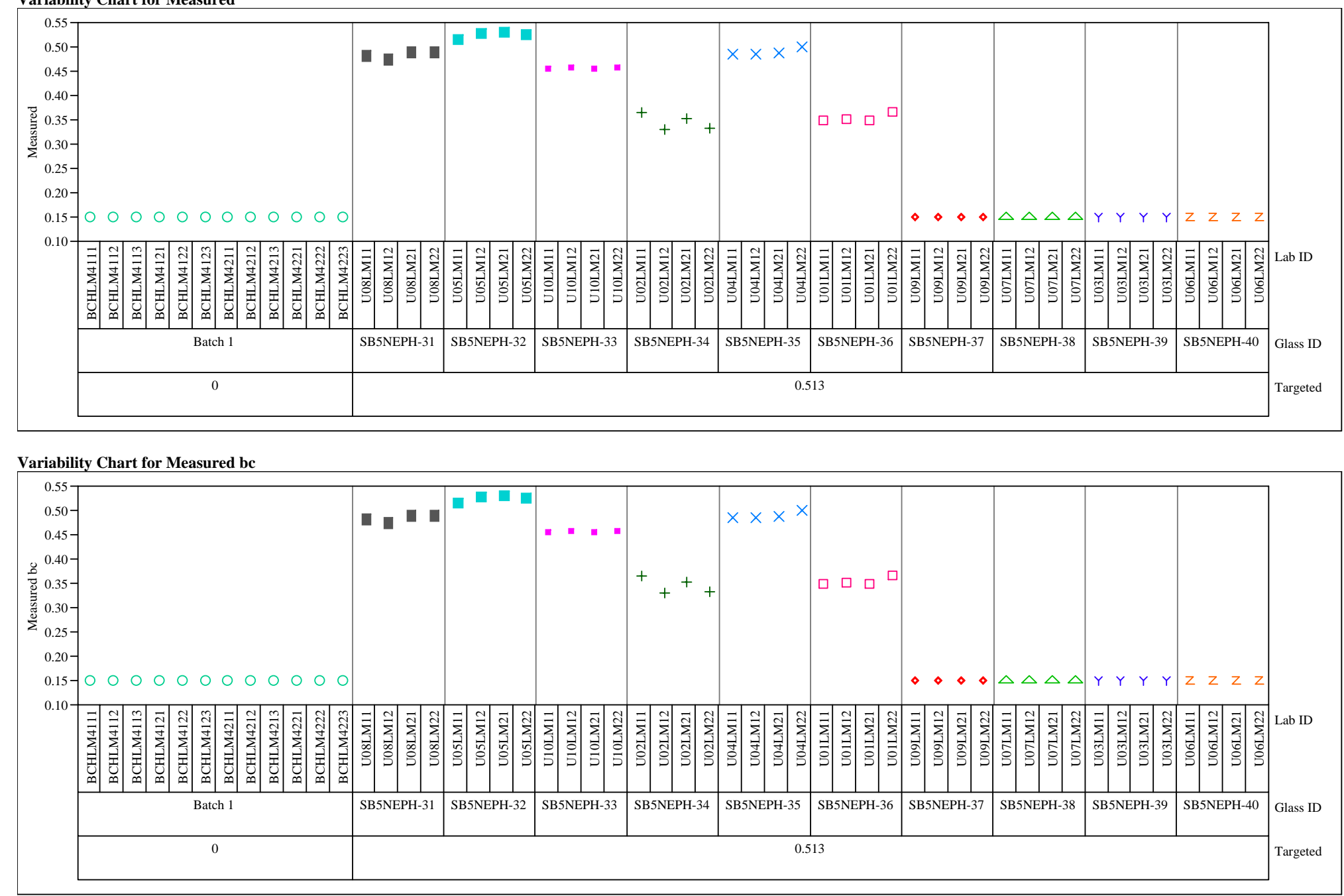
Exhibit A5. Measured and Measured Bias-Corrected Oxide Weight Percents

by Lab ID within Glass ID for the Glasses Prepared Using the LM Method. (continued)

Variability Gage Set=4, Oxide $=\mathrm{TiO} 2(\mathrm{wt} \%)$

Variability Chart for Measured

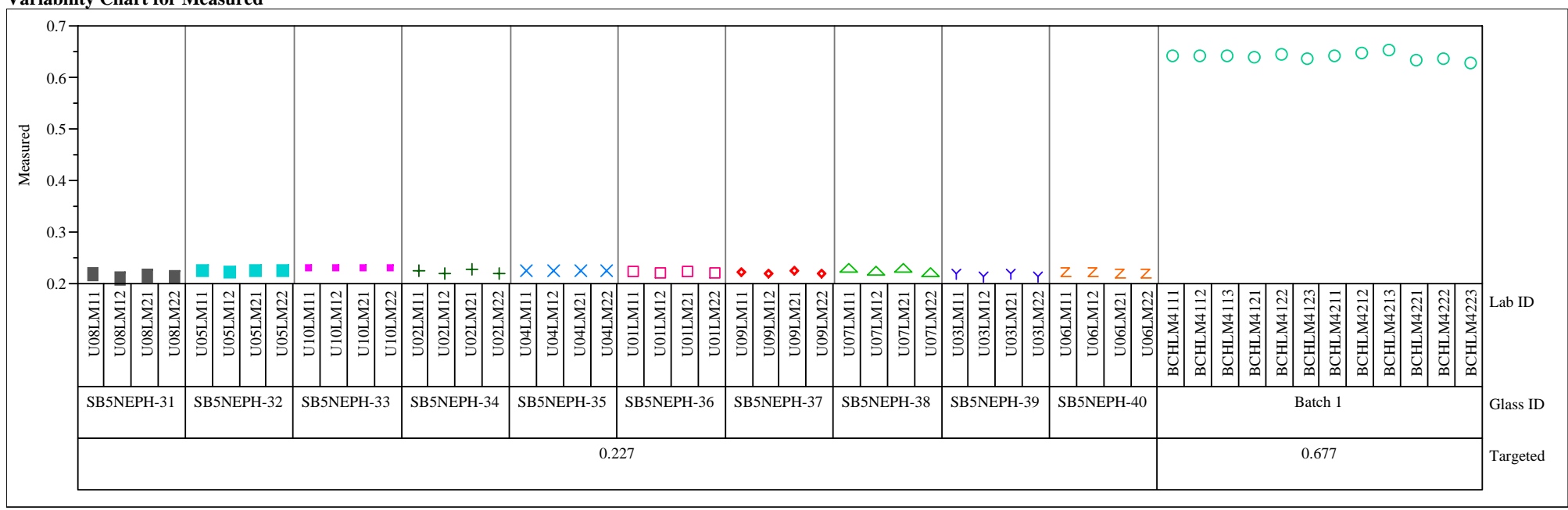

Variability Chart for Measured bc

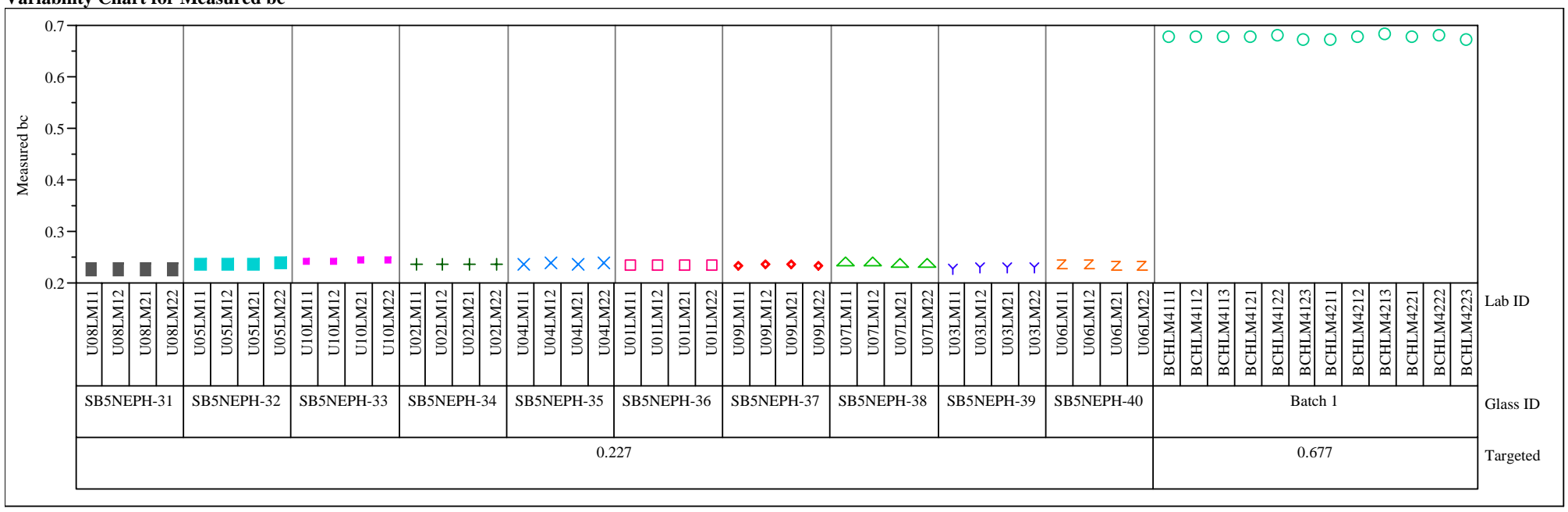


Exhibit A5. Measured and Measured Bias-Corrected Oxide Weight Percents

by Lab ID within Glass ID for the Glasses Prepared Using the LM Method. (continued)

Variability Gage Set $=4$, Oxide $=\mathrm{ZnO}($ wt $\%)$

Variability Chart for Measured
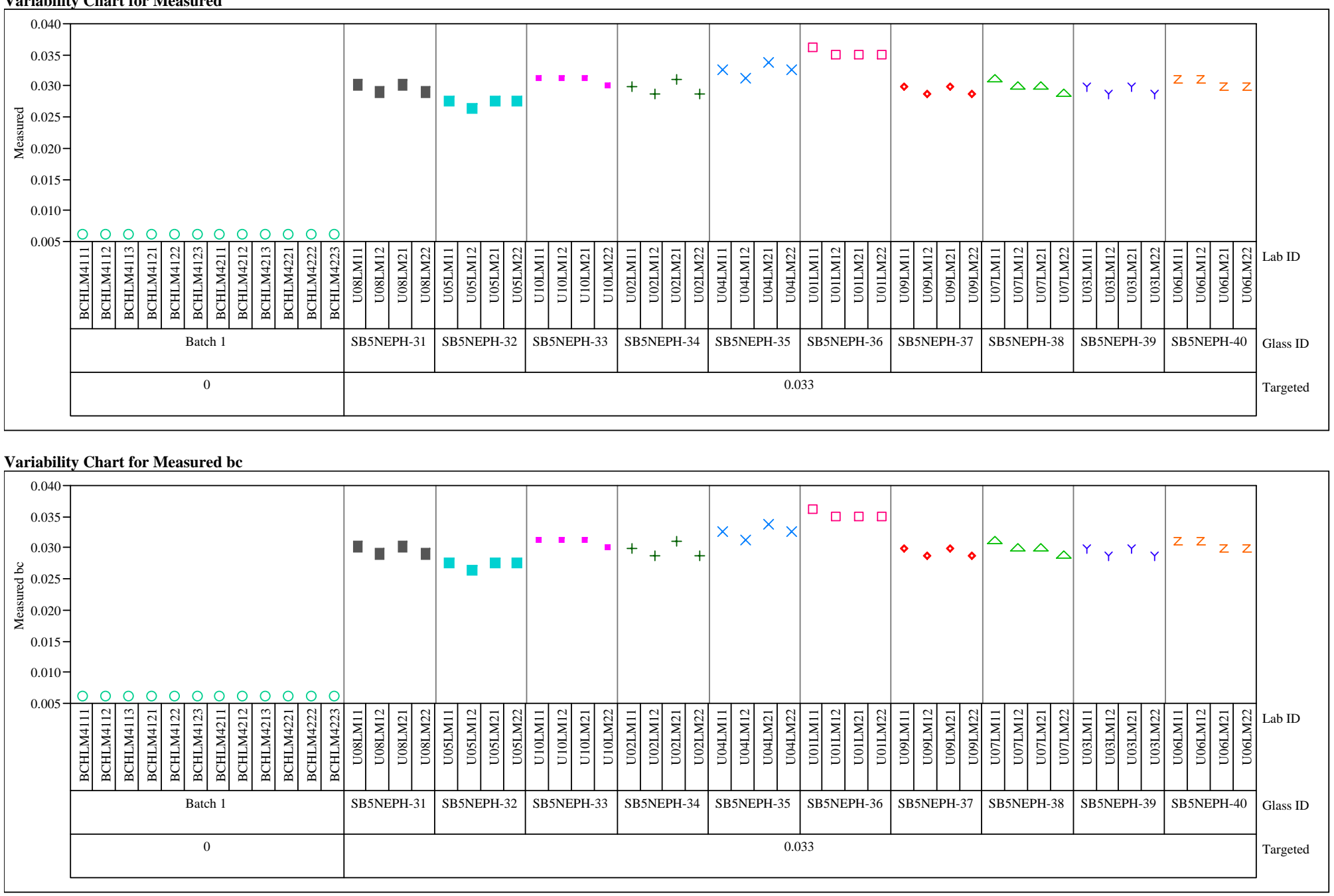
Exhibit A5. Measured and Measured Bias-Corrected Oxide Weight Percents

by Lab ID within Glass ID for the Glasses Prepared Using the LM Method. (continued)

Variability Gage Set $=4$, Oxide $=\mathrm{ZrO} 2(w t \%)$

Variability Chart for Measured
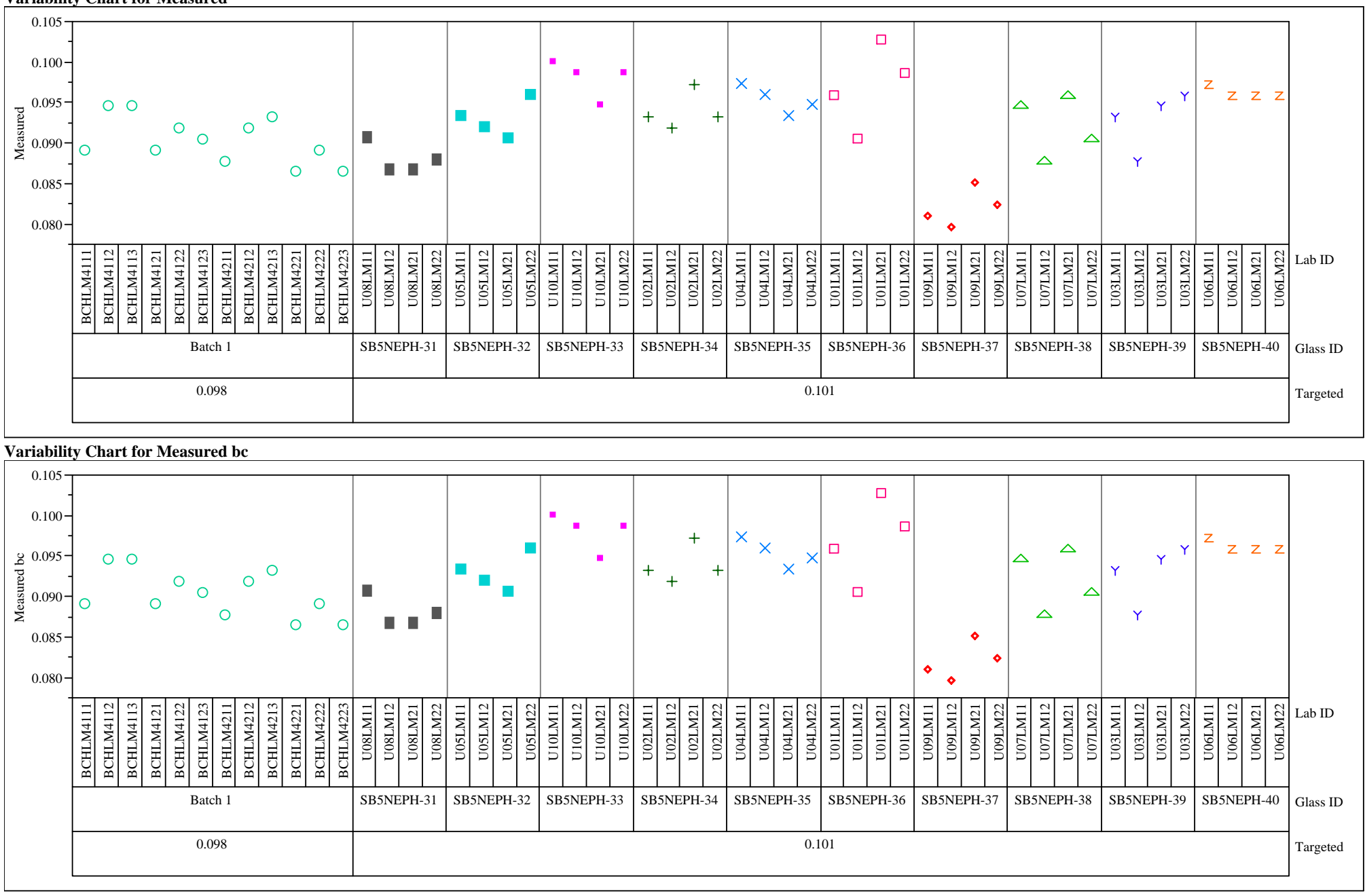
Exhibit A6. Measured and Measured Bias-Corrected Oxide Weight Percents

by Lab ID within Glass ID for the Glasses Prepared Using the PF Method. (continued)

Variability Gage Set=1, Oxide=B2O3 (wt $\%$ )

Variability Chart for Measured
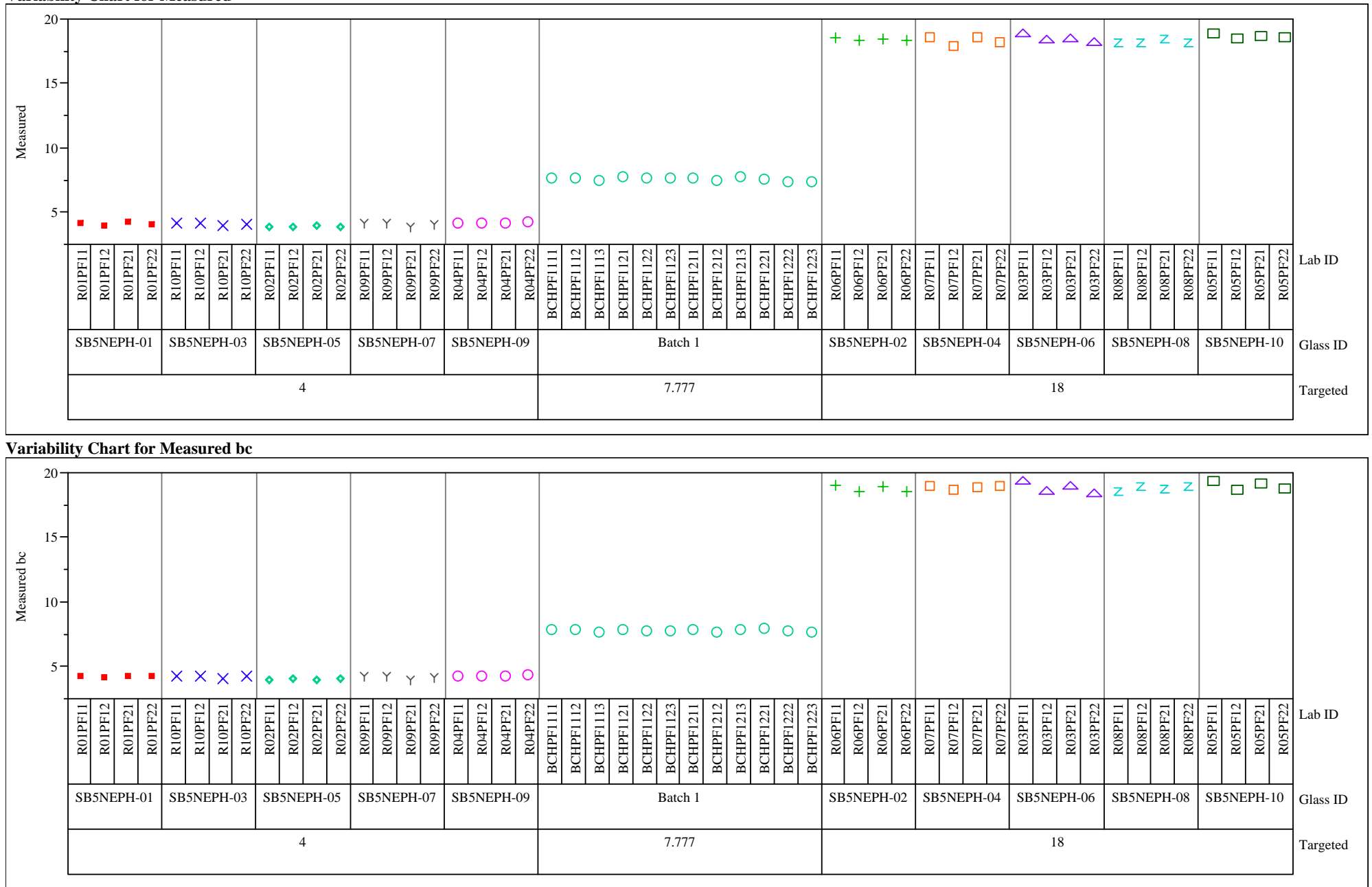
Exhibit A6. Measured and Measured Bias-Corrected Oxide Weight Percents

by Lab ID within Glass ID for the Glasses Prepared Using the PF Method. (continued)

Variability Gage Set $=1$, Oxide=Fe2O3 (wt\%)

Variability Chart for Measured
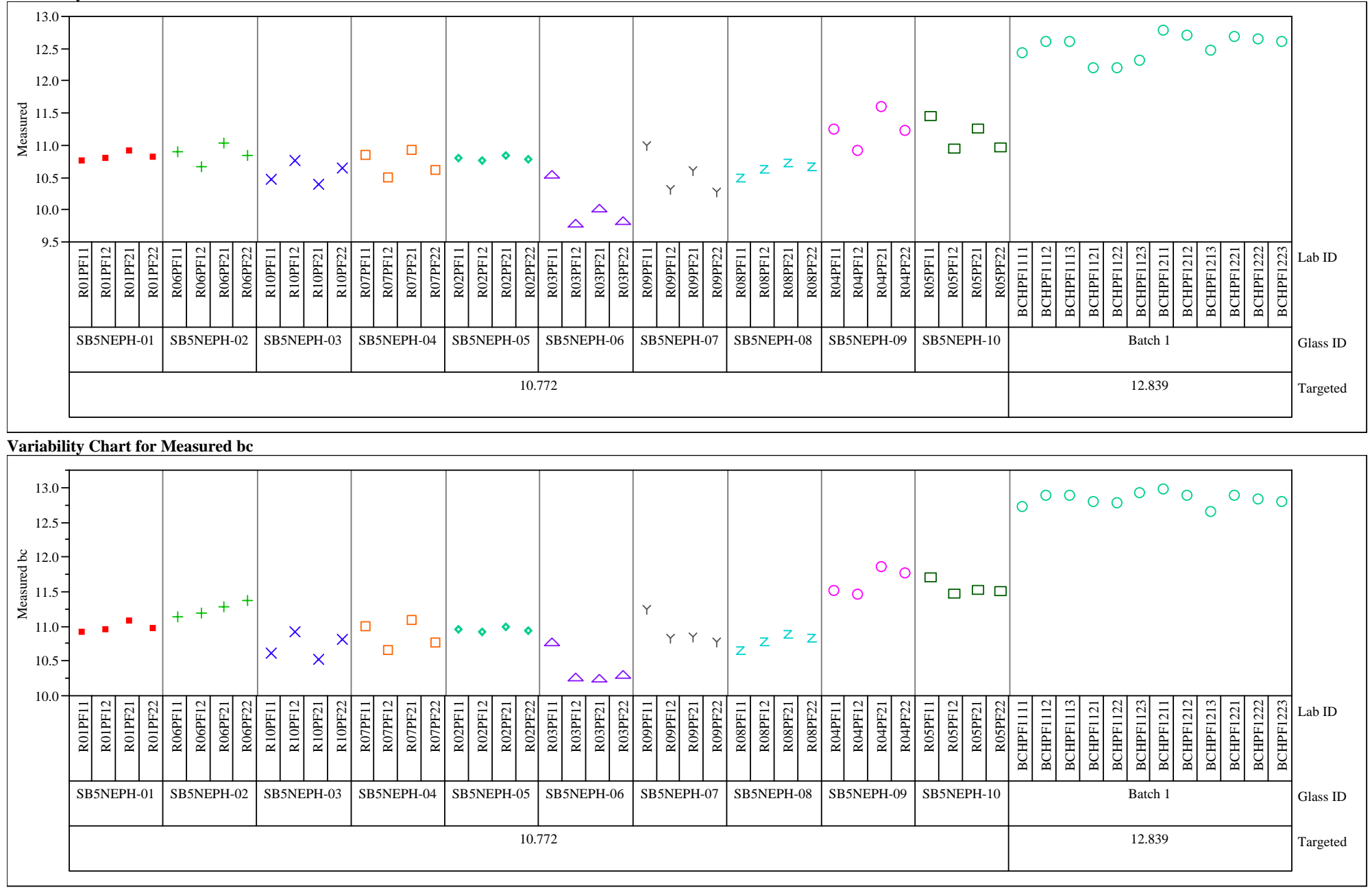
Exhibit A6. Measured and Measured Bias-Corrected Oxide Weight Percents

by Lab ID within Glass ID for the Glasses Prepared Using the PF Method. (continued)

Variability Gage Set $=1$, Oxide $=$ Li2O (wt\%)

Variability Chart for Measured

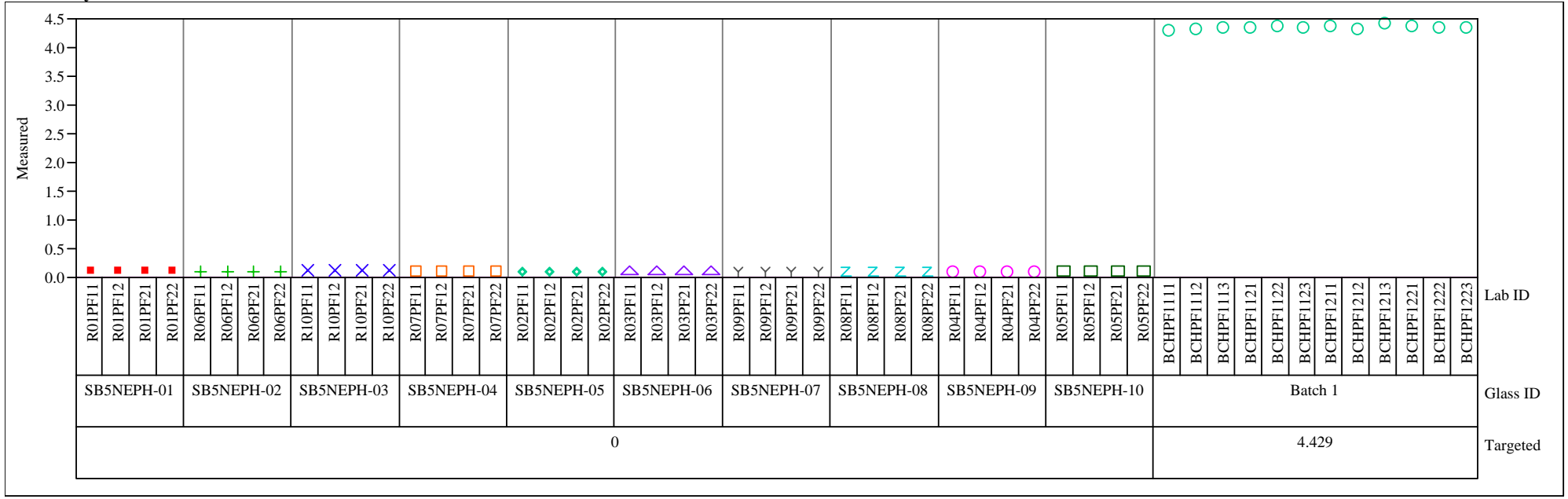

Variability Chart for Measured bc

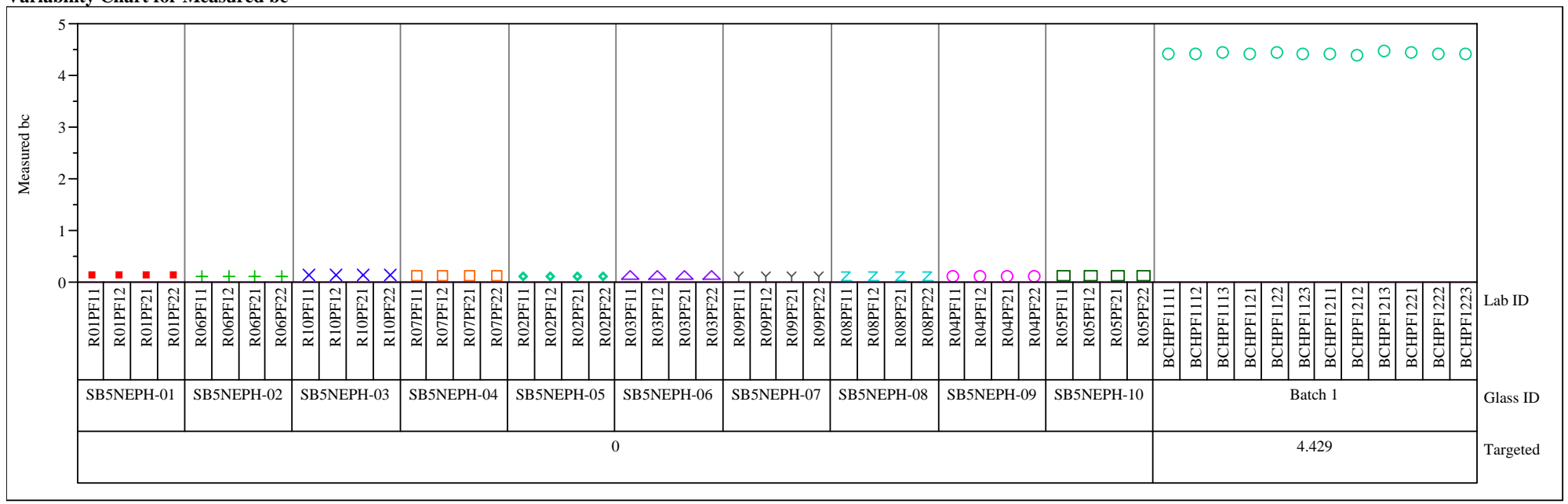


Exhibit A6. Measured and Measured Bias-Corrected Oxide Weight Percents

by Lab ID within Glass ID for the Glasses Prepared Using the PF Method. (continued)

Variability Gage Set $=1$, Oxide $=\mathrm{SiO} 2(\mathrm{wt} \%)$

Variability Chart for Measured

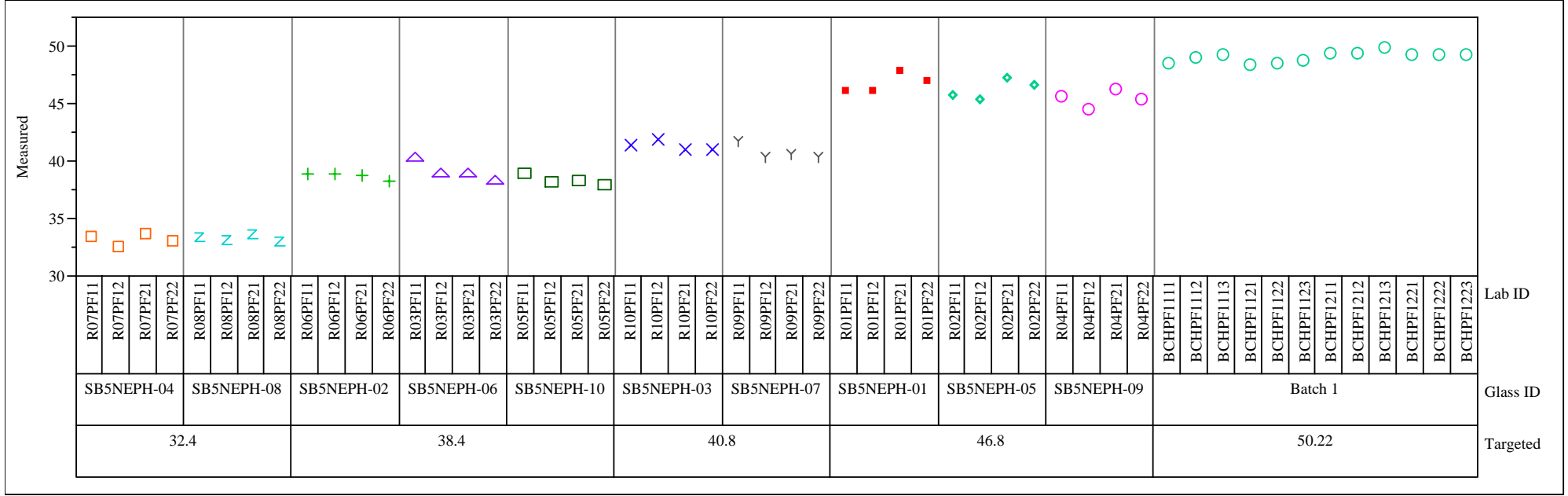

Variability Chart for Measured bc

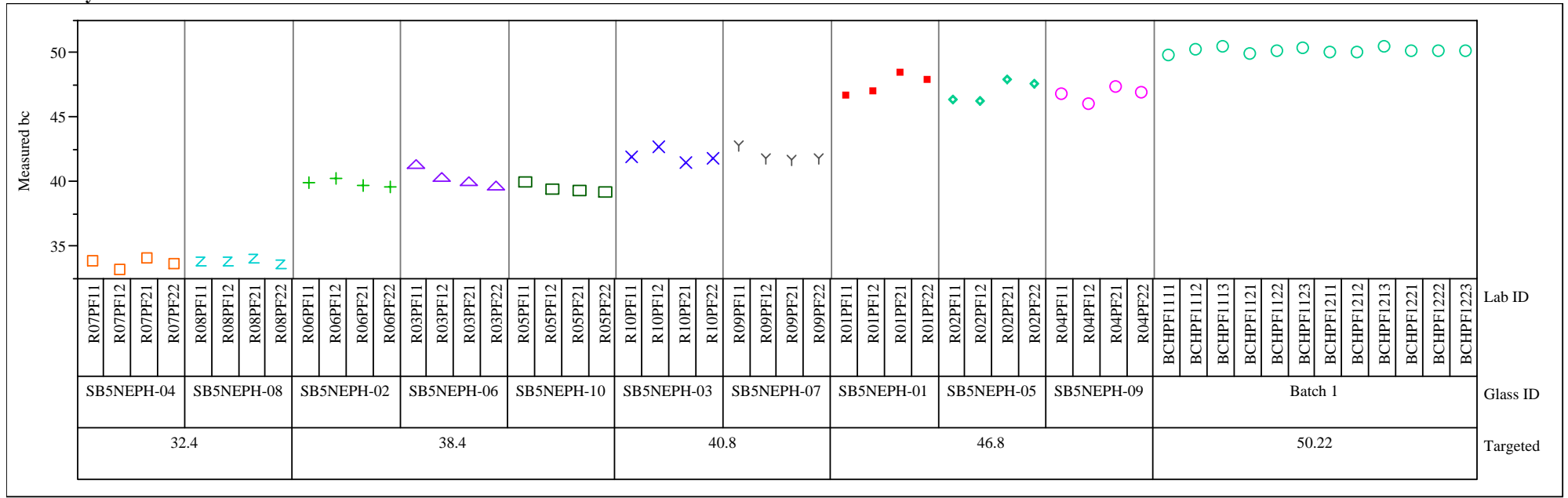


Exhibit A6. Measured and Measured Bias-Corrected Oxide Weight Percents

by Lab ID within Glass ID for the Glasses Prepared Using the PF Method. (continued)

Variability Gage Set $=2$, Oxide $=\mathbf{B} 2 \mathrm{O} 3(\mathrm{wt} \%)$

Variability Chart for Measured

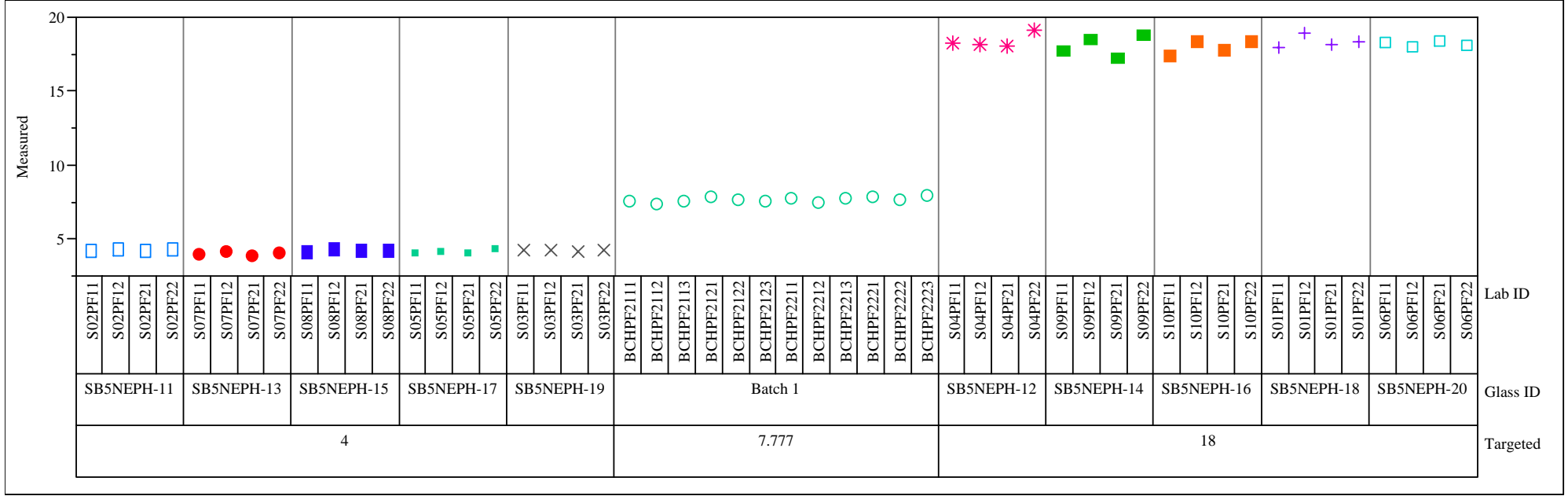

Variability Chart for Measured bc

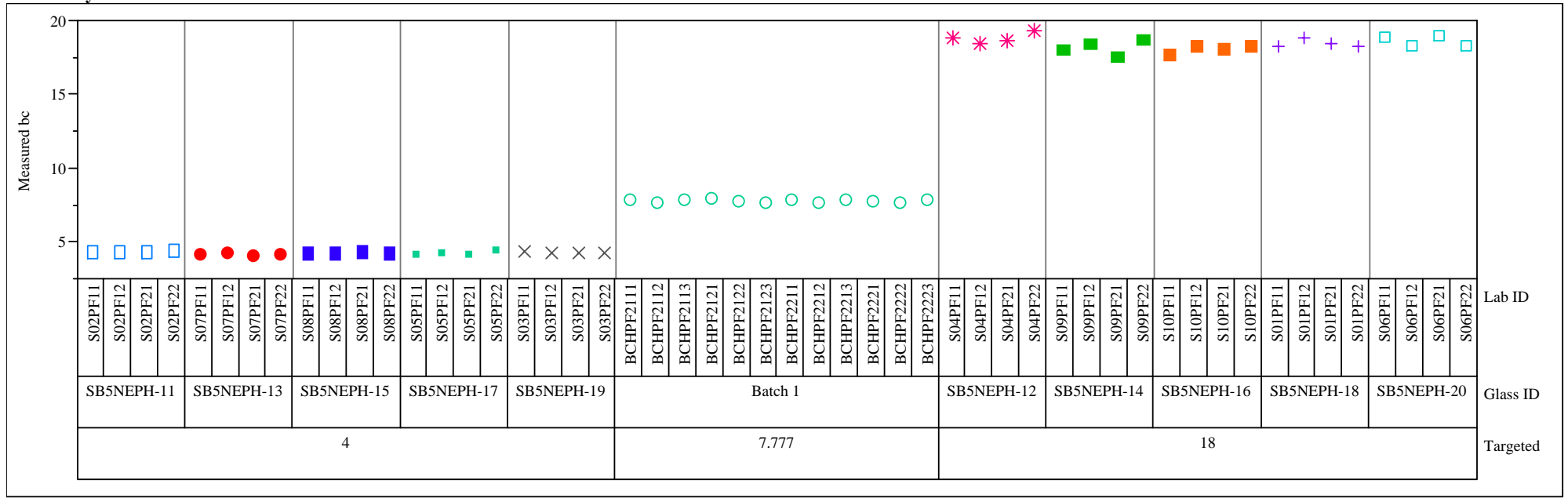


Exhibit A6. Measured and Measured Bias-Corrected Oxide Weight Percents

by Lab ID within Glass ID for the Glasses Prepared Using the PF Method. (continued)

Variability Gage Set=2, Oxide=Fe2O3 (wt $\%$ )

Variability Chart for Measured

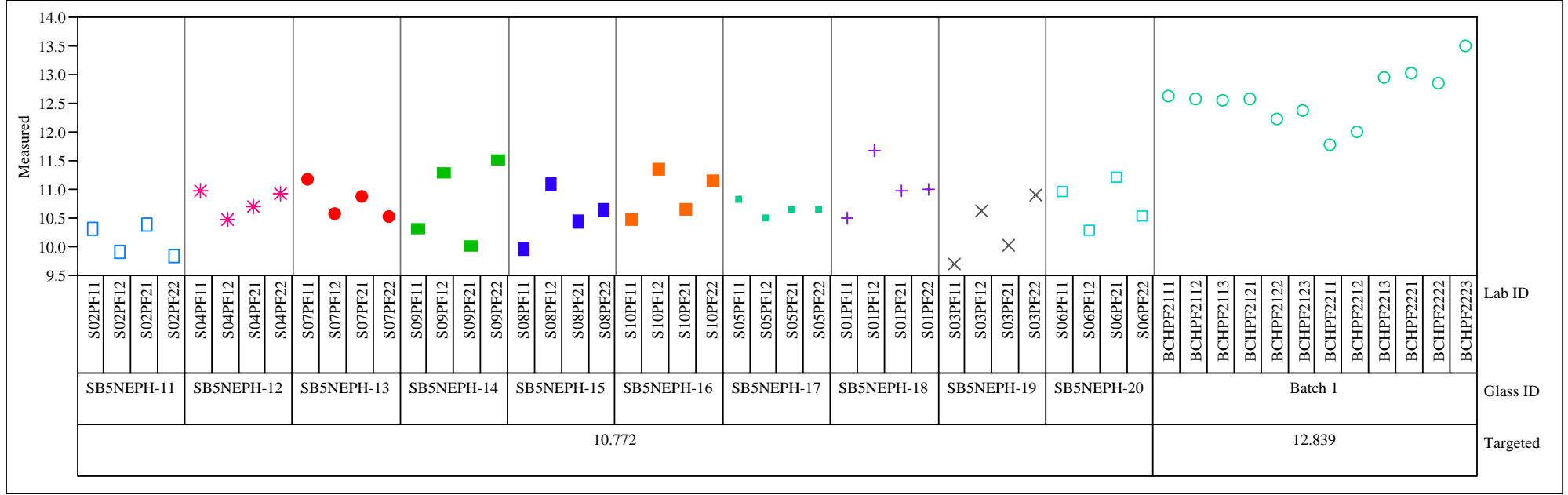

Variability Chart for Measured bc

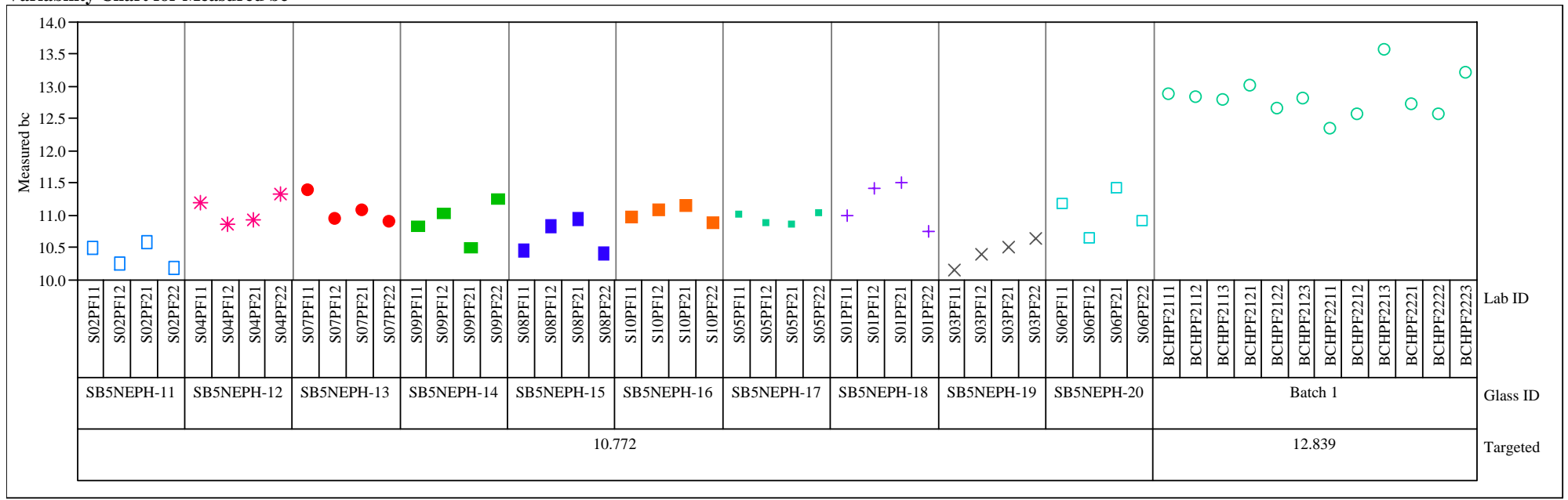


Exhibit A6. Measured and Measured Bias-Corrected Oxide Weight Percents

by Lab ID within Glass ID for the Glasses Prepared Using the PF Method. (continued)

Variability Gage Set=2, Oxide $=$ Li2O $(w t \%)$

Variability Chart for Measured

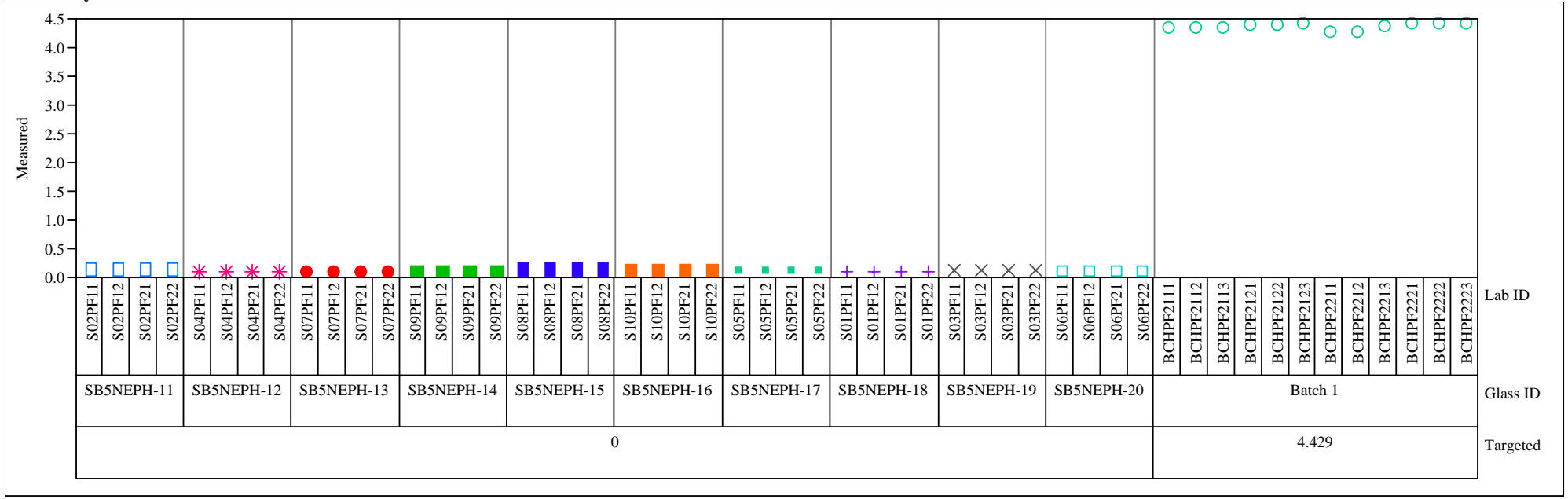

Variability Chart for Measured bc

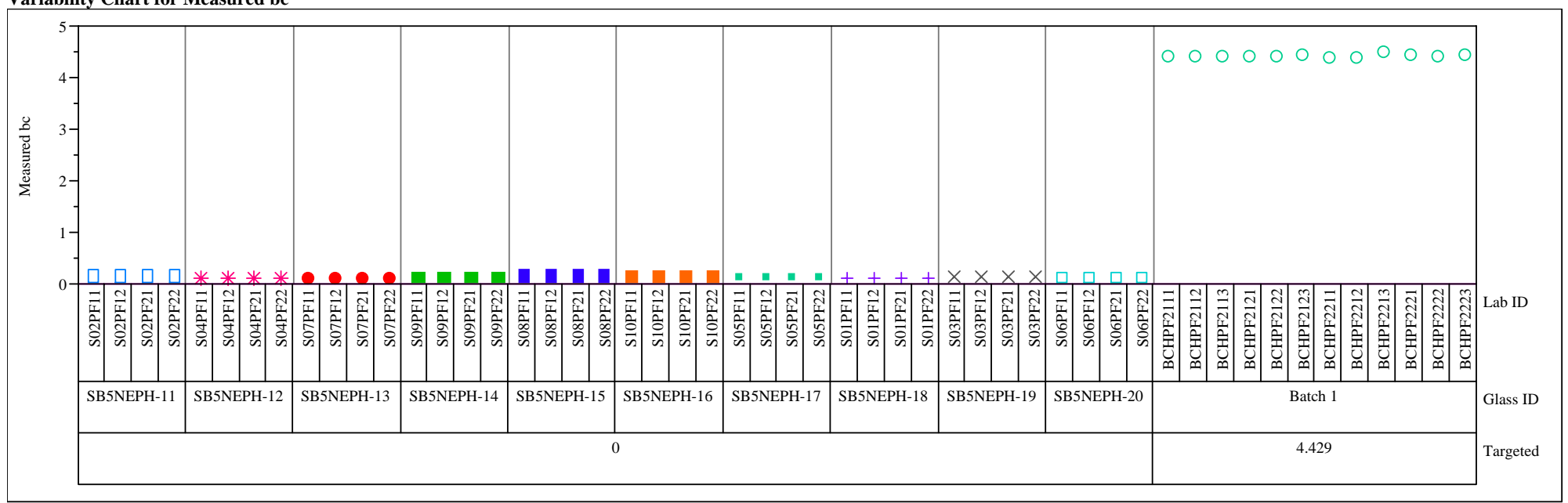


Exhibit A6. Measured and Measured Bias-Corrected Oxide Weight Percents

by Lab ID within Glass ID for the Glasses Prepared Using the PF Method. (continued)

Variability Gage Set $=2$, Oxide $=\mathrm{SiO} 2(\mathrm{wt} \%)$

Variability Chart for Measured

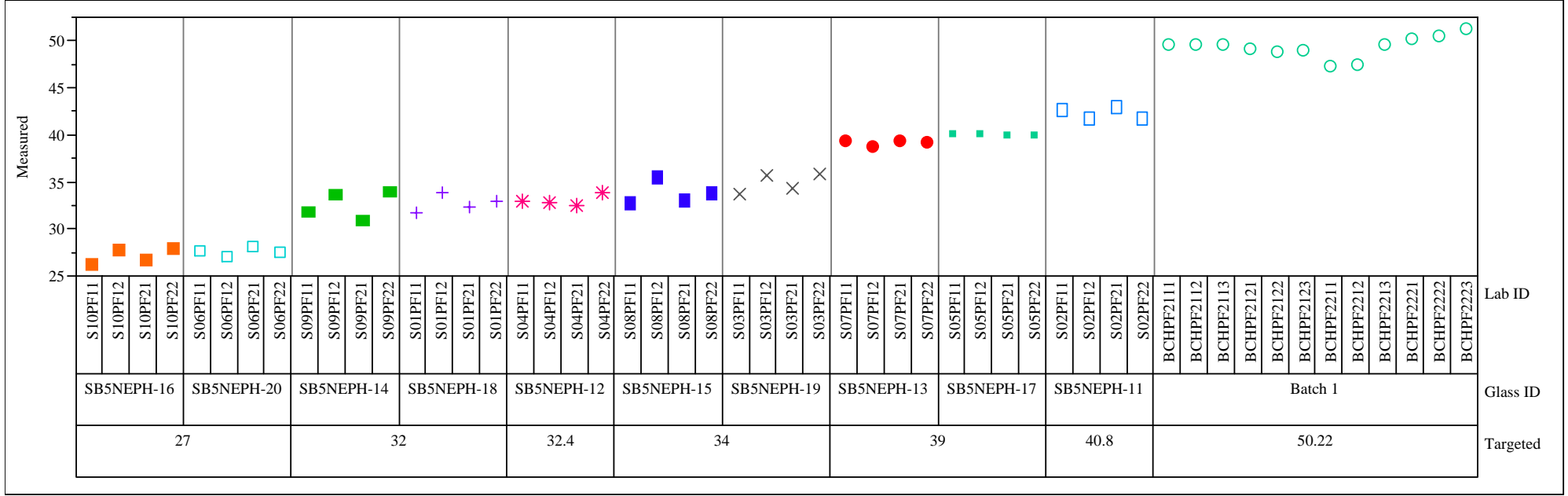

Variability Chart for Measured bc

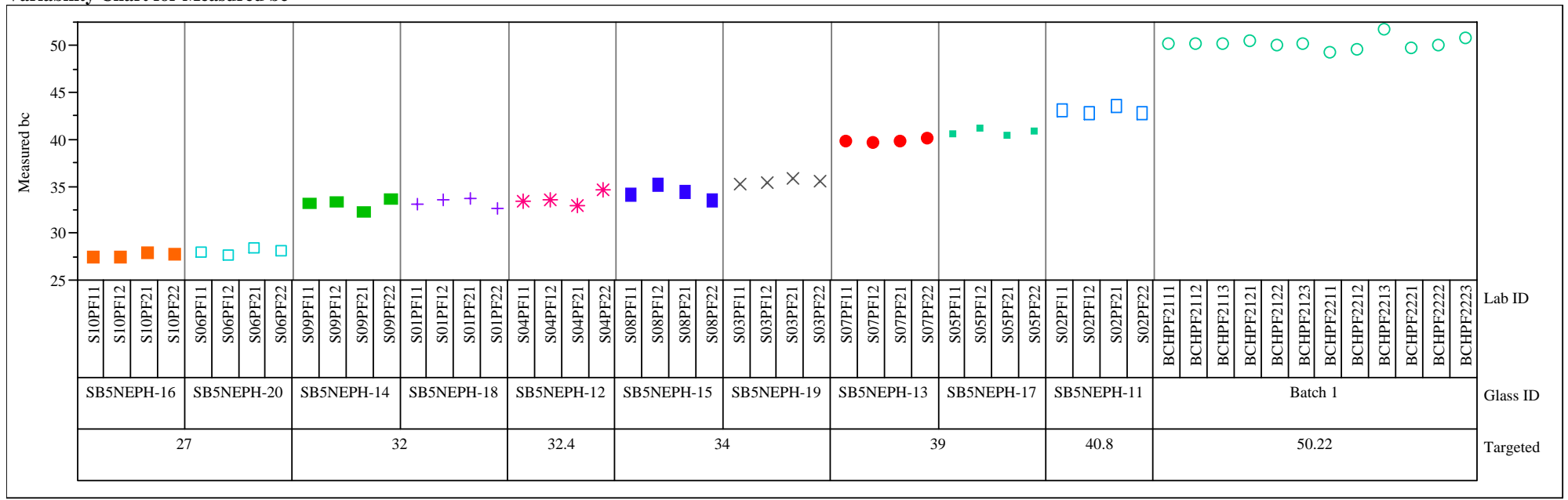


Exhibit A6. Measured and Measured Bias-Corrected Oxide Weight Percents

by Lab ID within Glass ID for the Glasses Prepared Using the PF Method. (continued)

Variability Gage Set=3, Oxide=B2O3 (wt $\%$ )

Variability Chart for Measured

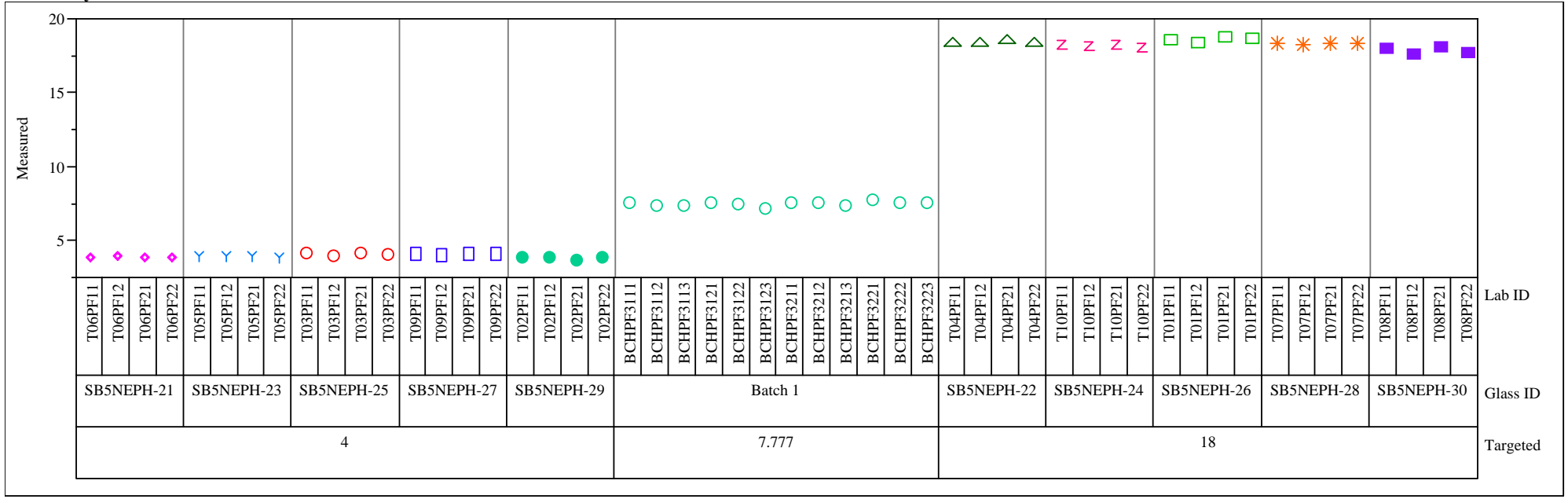

Variability Chart for Measured bc

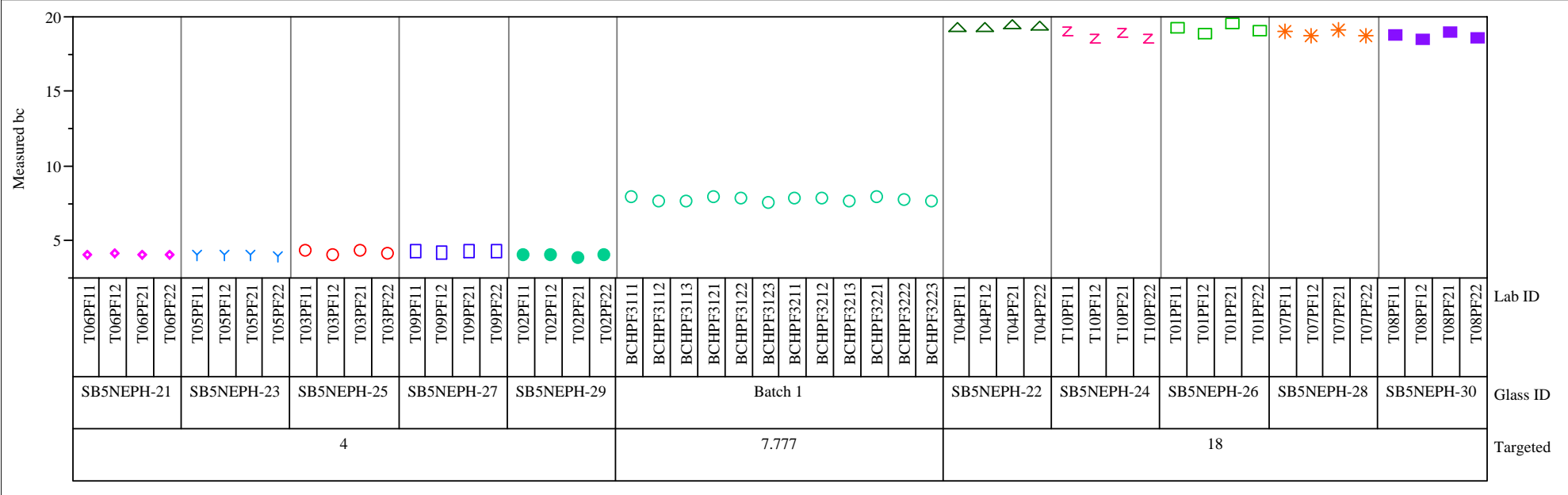


Exhibit A6. Measured and Measured Bias-Corrected Oxide Weight Percents

by Lab ID within Glass ID for the Glasses Prepared Using the PF Method. (continued)

Variability Gage Set=3, Oxide=Fe2O3 (wt\%)

Variability Chart for Measured

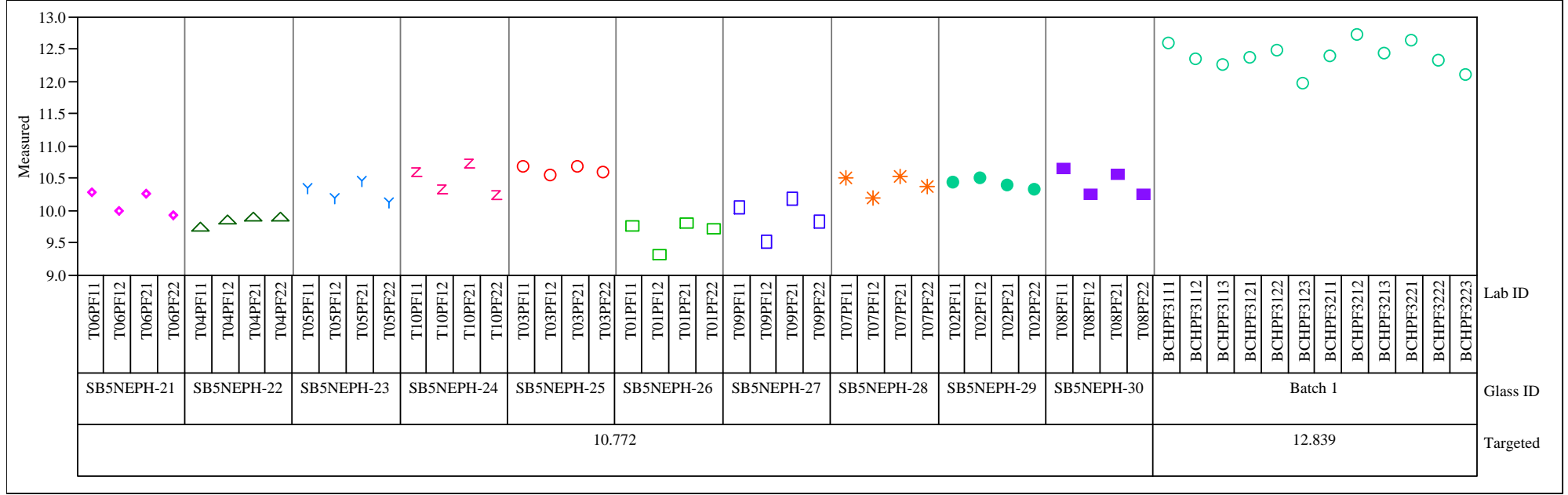

Variability Chart for Measured bc

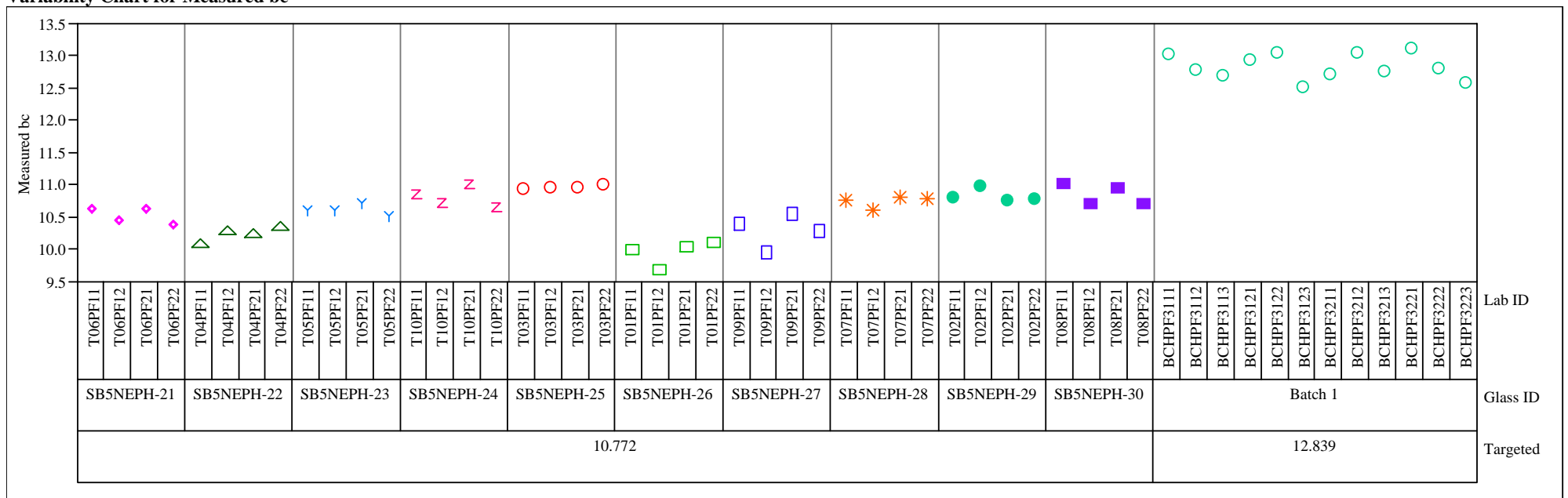


Exhibit A6. Measured and Measured Bias-Corrected Oxide Weight Percents

by Lab ID within Glass ID for the Glasses Prepared Using the PF Method. (continued)

Variability Gage Set=3, Oxide $=$ Li2O (wt $\%$ )

Variability Chart for Measured

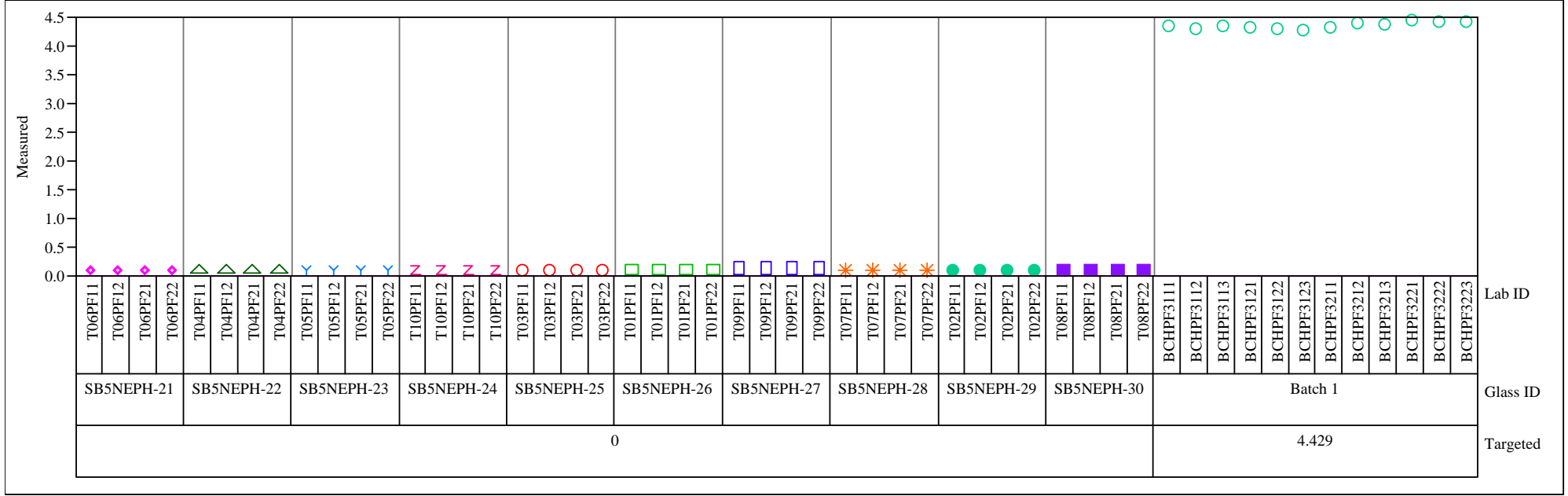

Variability Chart for Measured bc

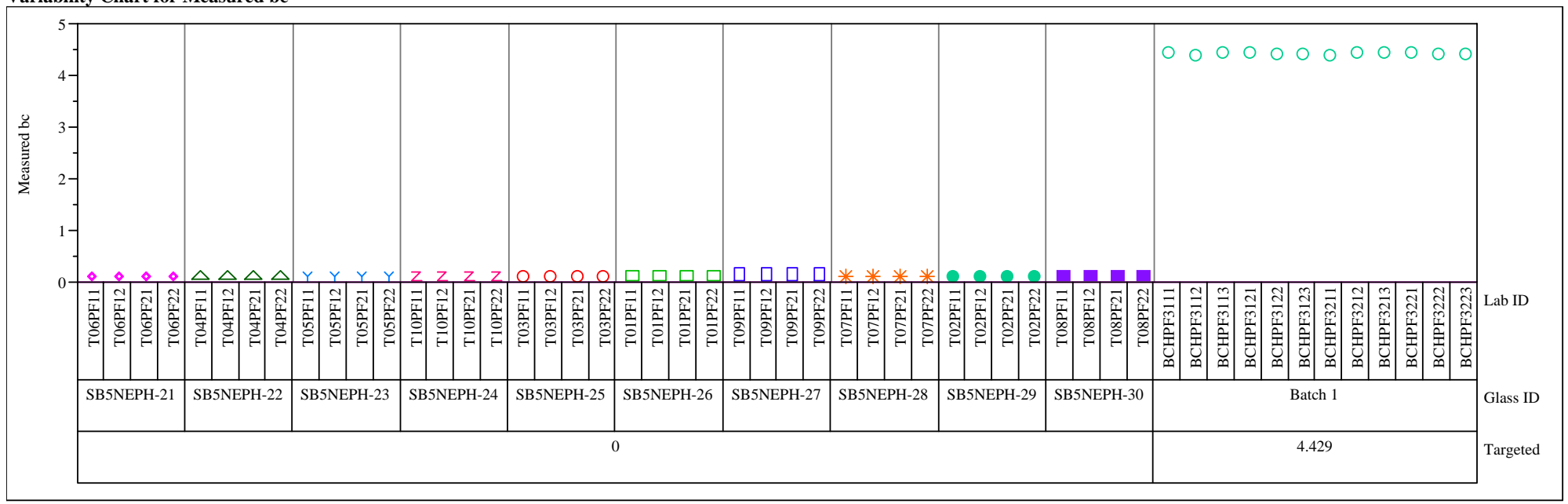


Exhibit A6. Measured and Measured Bias-Corrected Oxide Weight Percents

by Lab ID within Glass ID for the Glasses Prepared Using the PF Method. (continued)

Variability Gage Set $=3$, Oxide $=\mathrm{SiO} 2(\mathrm{wt} \%)$

Variability Chart for Measured

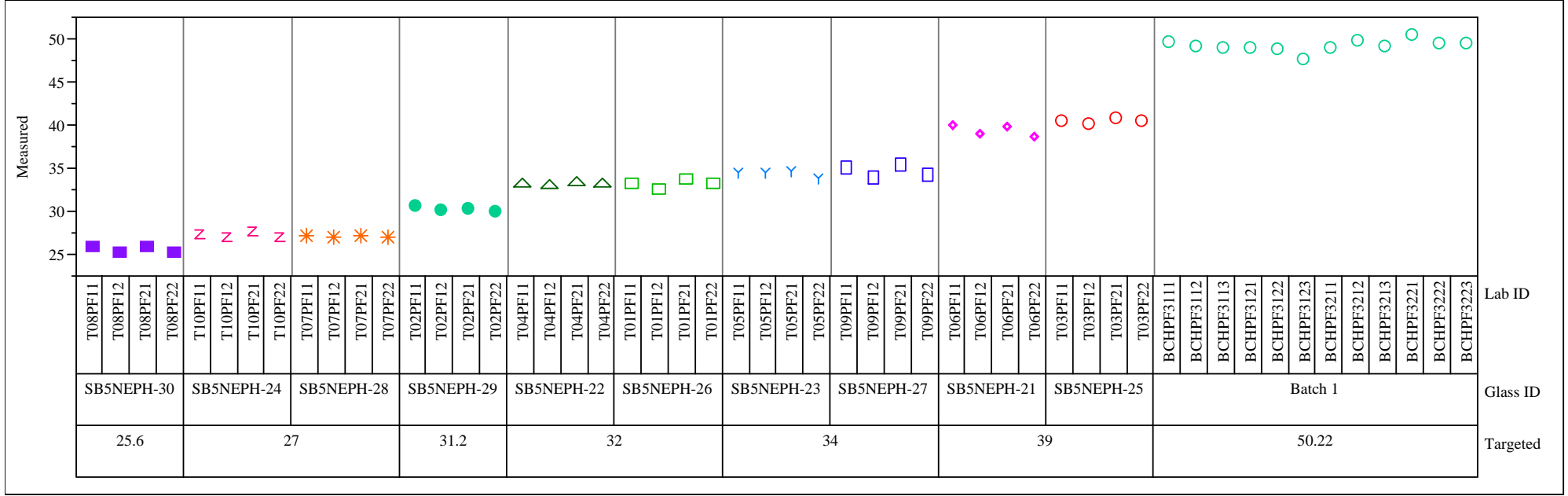

Variability Chart for Measured bc

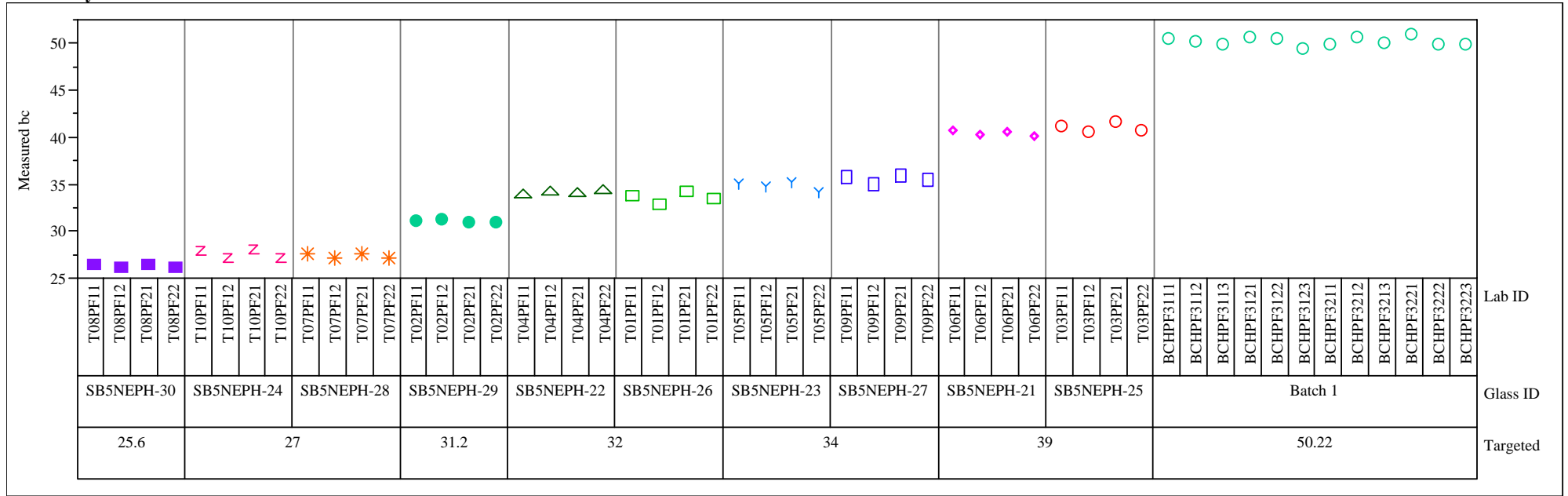


Exhibit A6. Measured and Measured Bias-Corrected Oxide Weight Percents

by Lab ID within Glass ID for the Glasses Prepared Using the PF Method. (continued)

Variability Gage Set=4, Oxide=B2O3 (wt $\%)$

Variability Chart for Measured

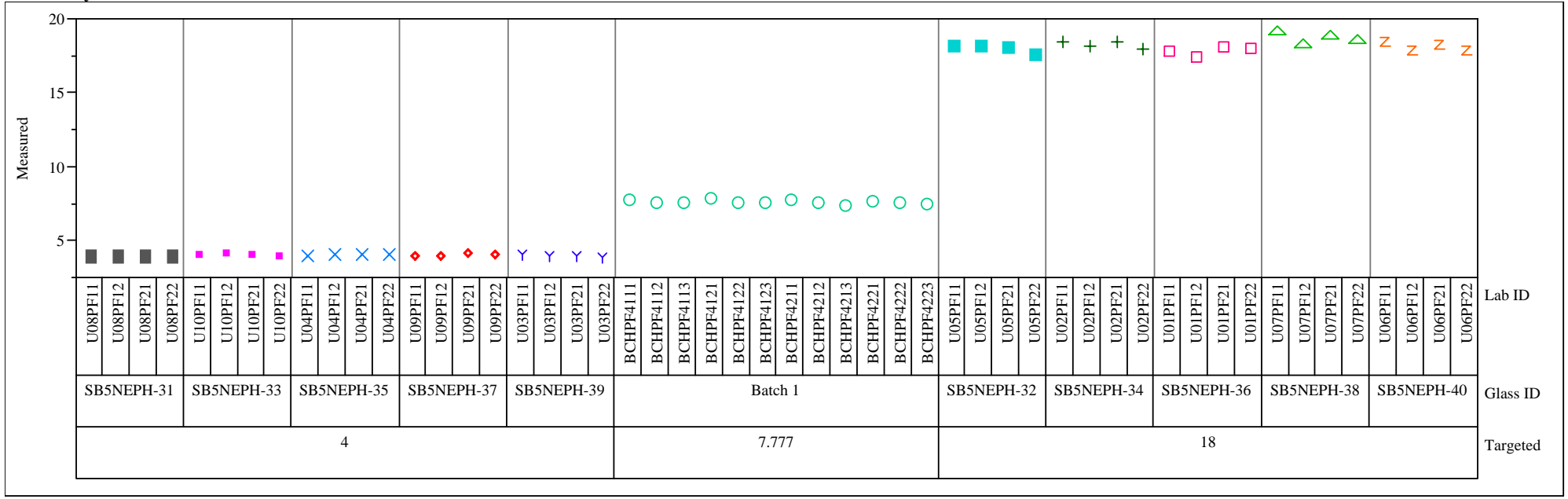

Variability Chart for Measured bc

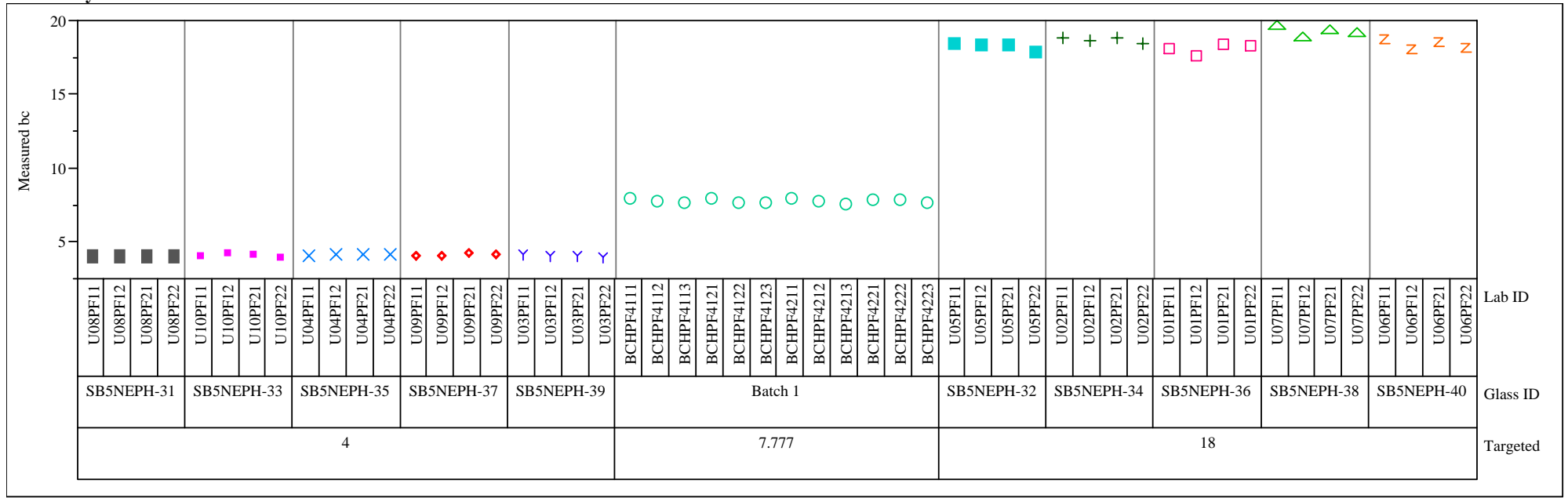


Exhibit A6. Measured and Measured Bias-Corrected Oxide Weight Percents

by Lab ID within Glass ID for the Glasses Prepared Using the PF Method. (continued)

Variability Gage Set=4, Oxide=Fe2O3 (wt\%)

Variability Chart for Measured

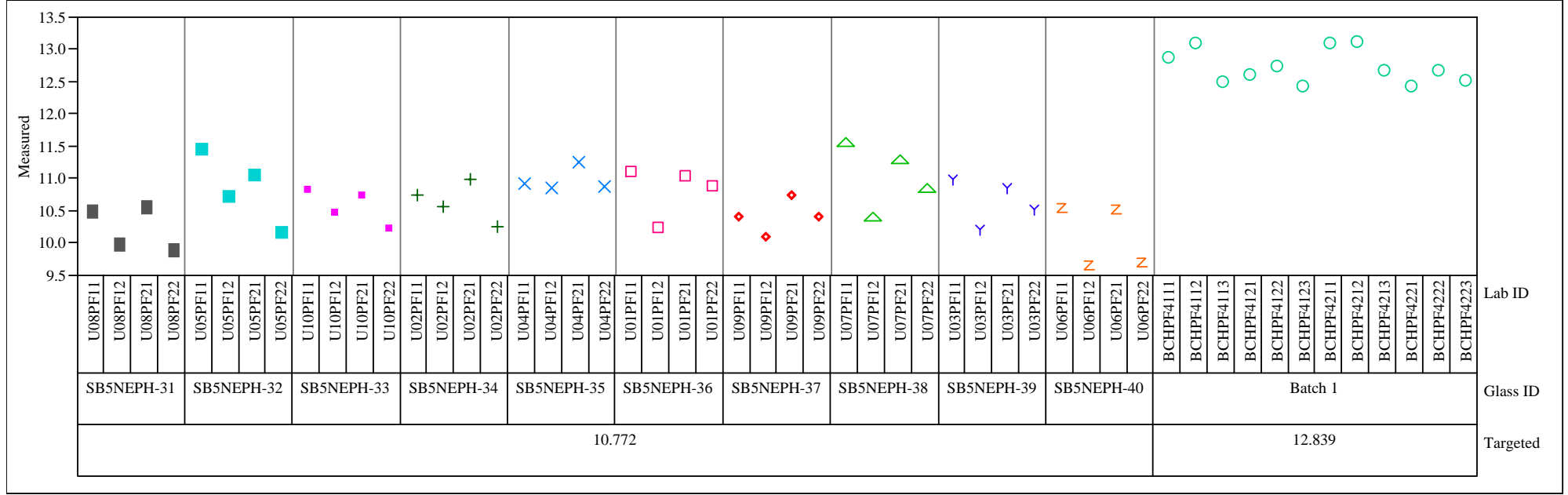

Variability Chart for Measured bc

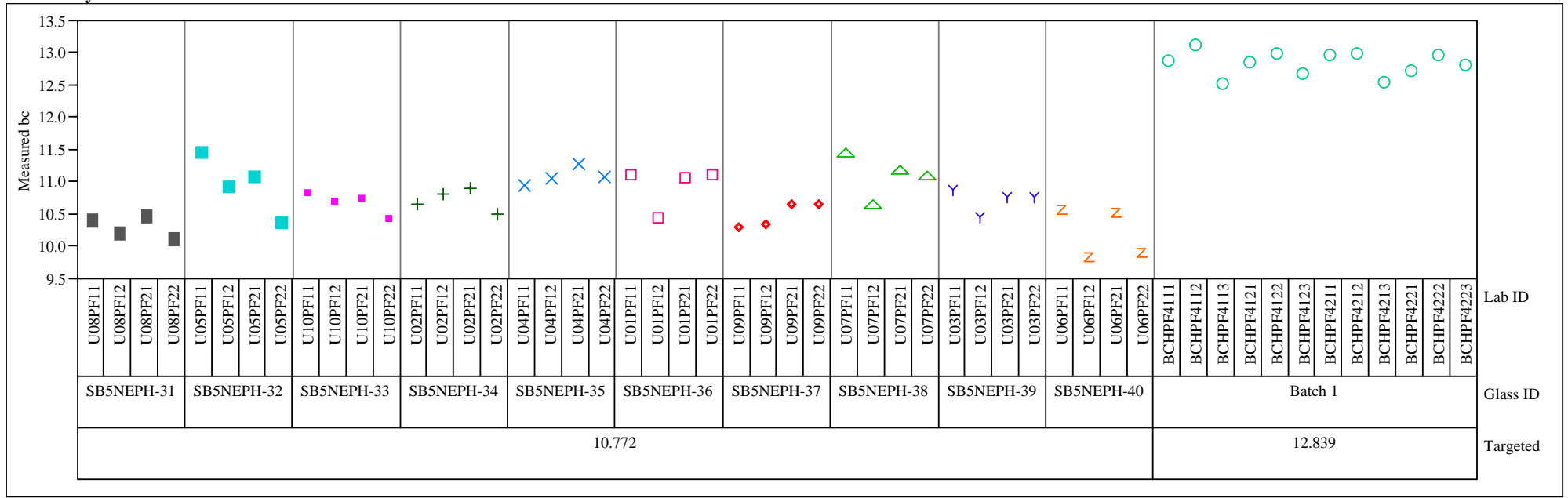


Exhibit A6. Measured and Measured Bias-Corrected Oxide Weight Percents

by Lab ID within Glass ID for the Glasses Prepared Using the PF Method. (continued)

Variability Gage Set=4, Oxide=Li2O (wt\%)

Variability Chart for Measured

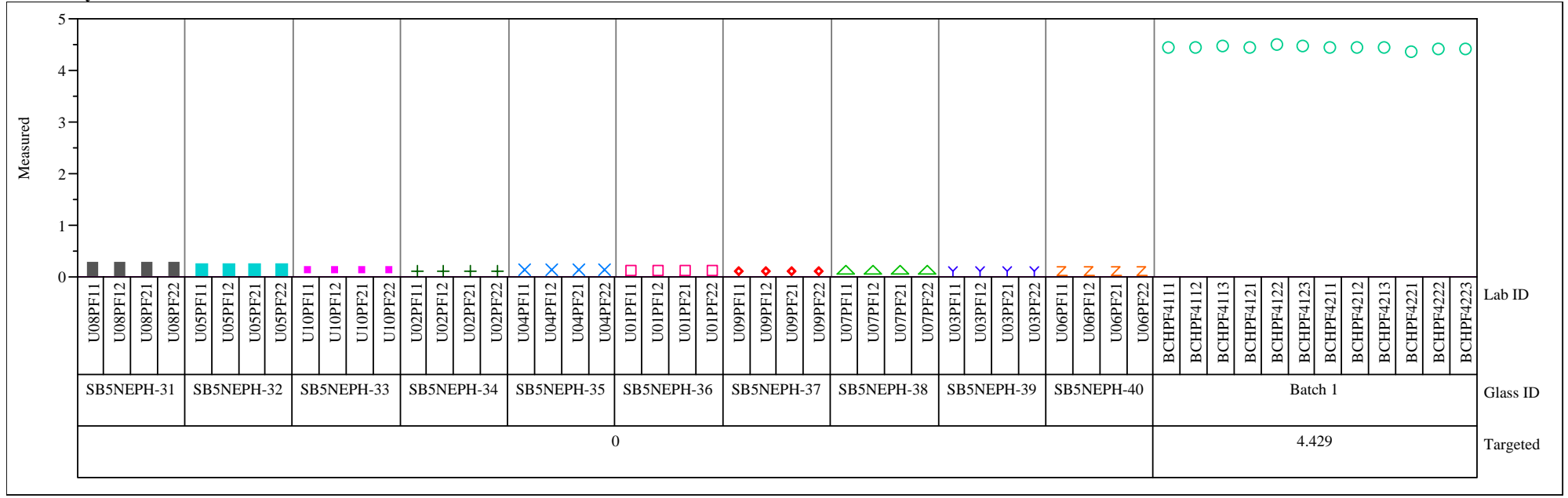

Variability Chart for Measured bc

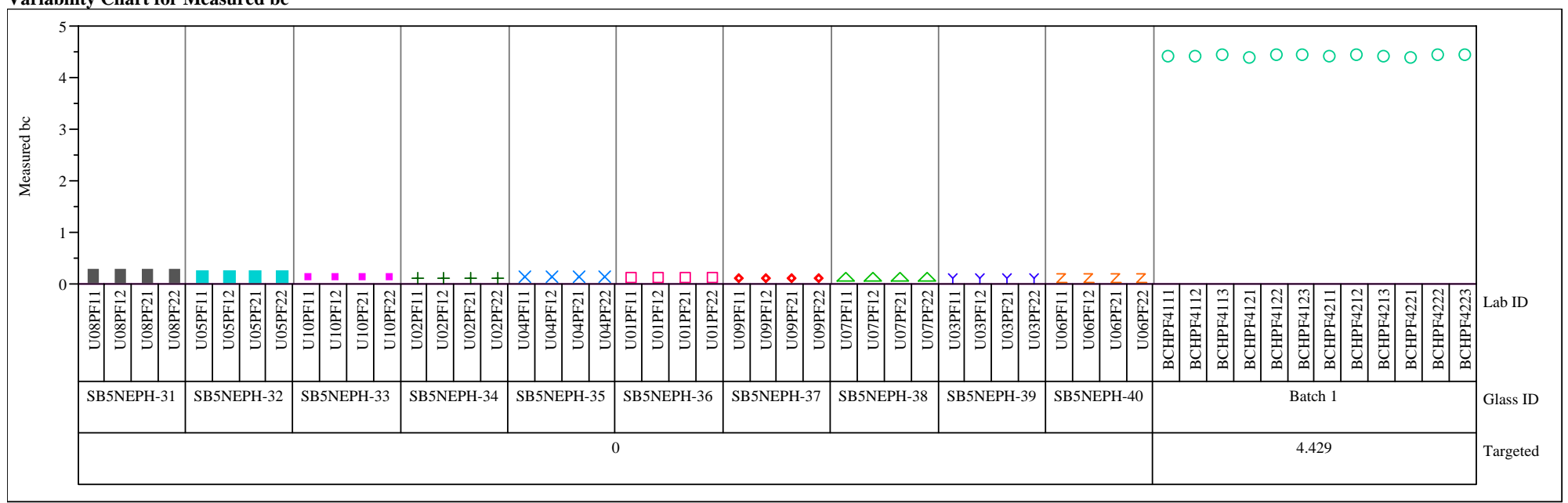


Exhibit A6. Measured and Measured Bias-Corrected Oxide Weight Percents

by Lab ID within Glass ID for the Glasses Prepared Using the PF Method. (continued)

Variability Gage Set $=4$, Oxide $=\mathrm{SiO} 2(\mathrm{wt} \%)$

Variability Chart for Measured

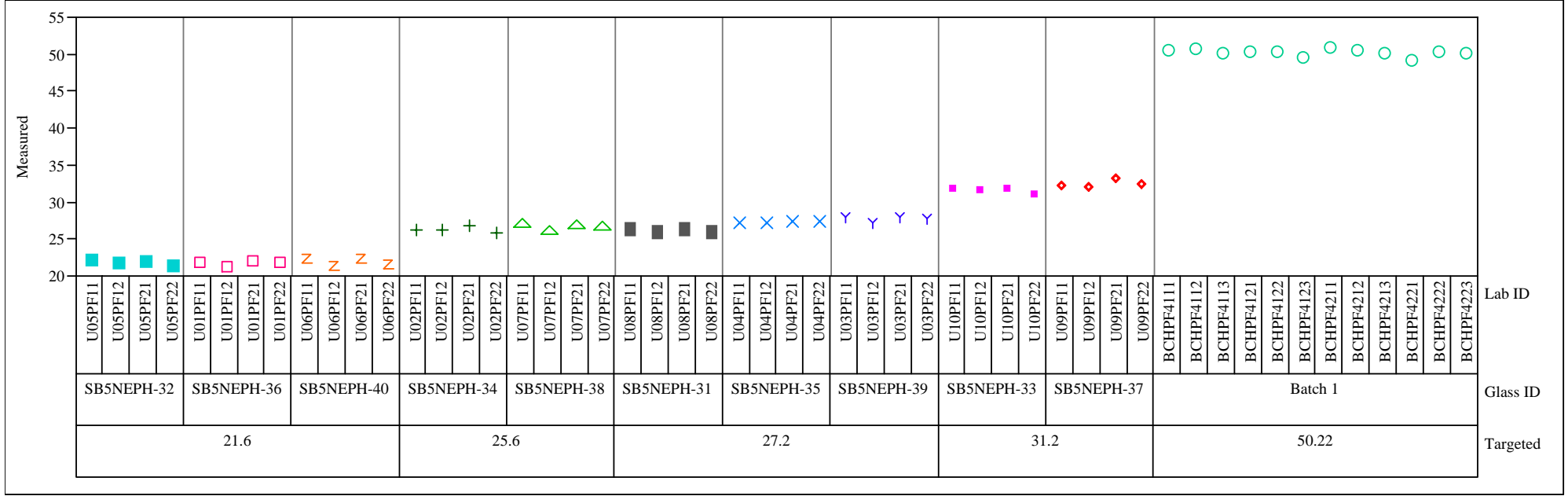

Variability Chart for Measured bc

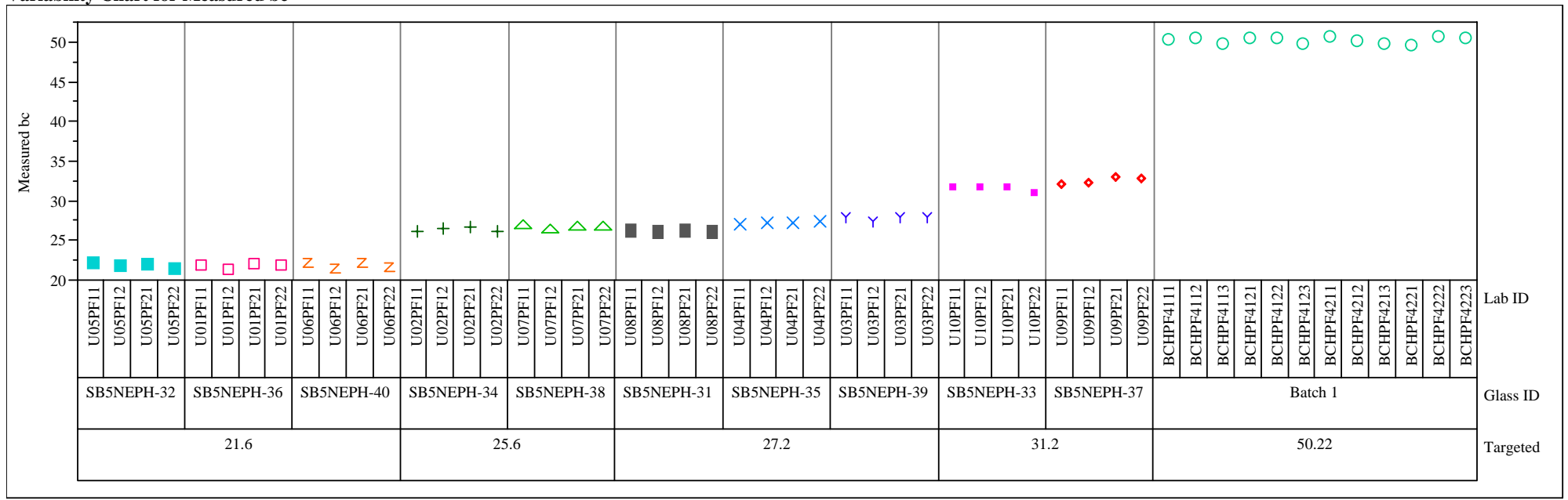


Exhibit A7. Average Measured and Bias-Corrected (bc) Versus

Targeted Compositions by Glass \# by Oxide. (continued)

(100 - Batch 1 for Sets 1, 2, 3, and 4)

Overlay Plot Oxide=Al2O3 (wt \%)

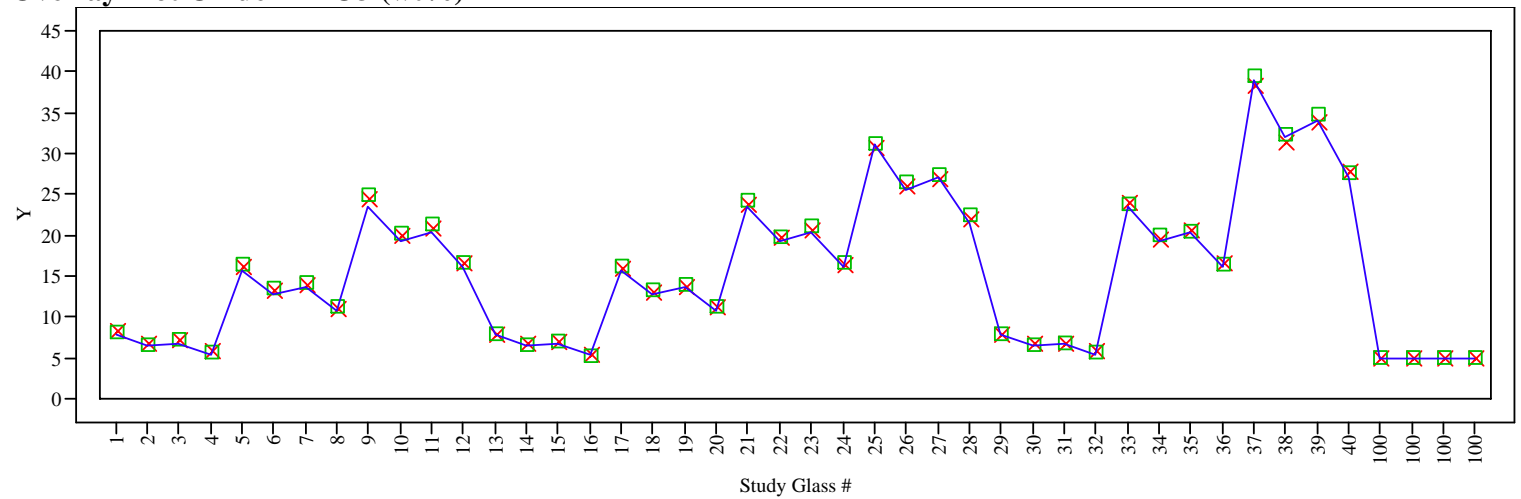

Overlay Plot Oxide=B2O3 (wt \%)

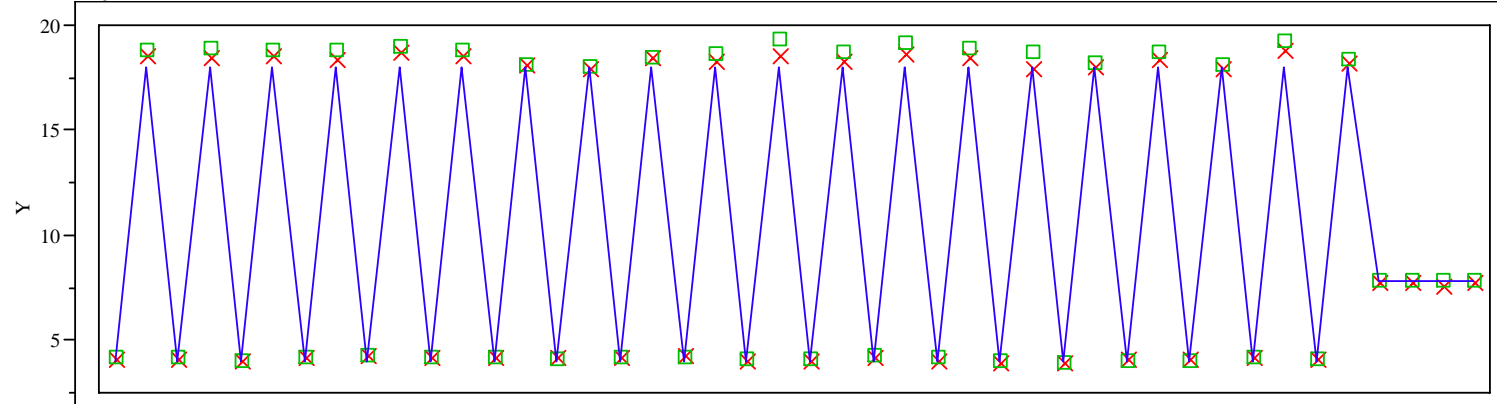

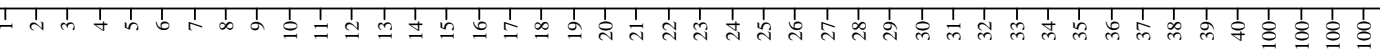

Overlay Plot Oxide $=\mathrm{BaO}(\mathrm{wt} \%)$

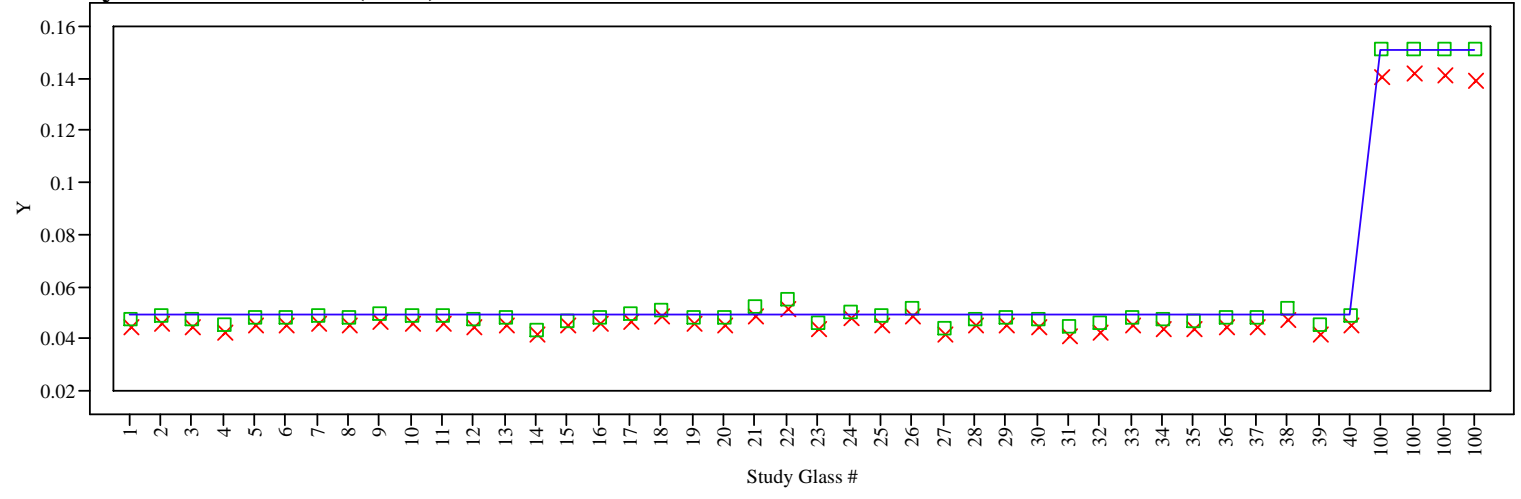

$\mathrm{Y} \times$ Measured $\square$ Measured bc - Targeted 
Exhibit A7. Average Measured and Bias-Corrected (bc) Versus

Targeted Compositions by Glass \# by Oxide. (continued)

(100 - Batch 1 for Sets 1, 2, 3, and 4)

Overlay Plot Oxide $=\mathrm{CaO}($ wt \%)

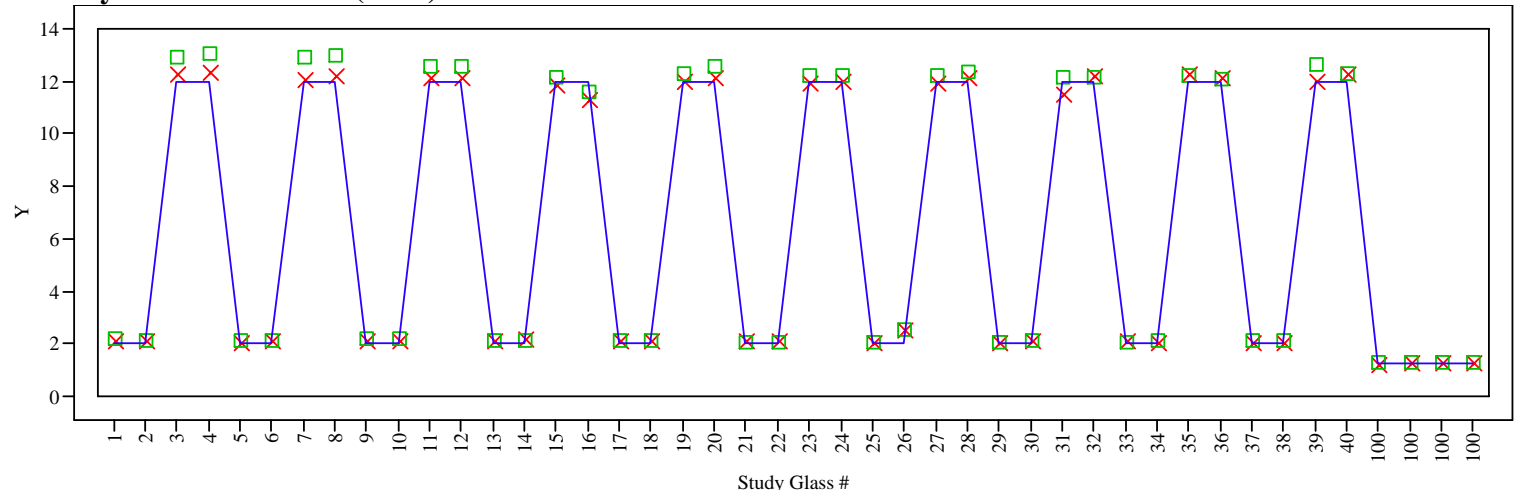

Overlay Plot Oxide $=\mathrm{Ce} 2 \mathrm{O} 3(\mathrm{wt} \%)$

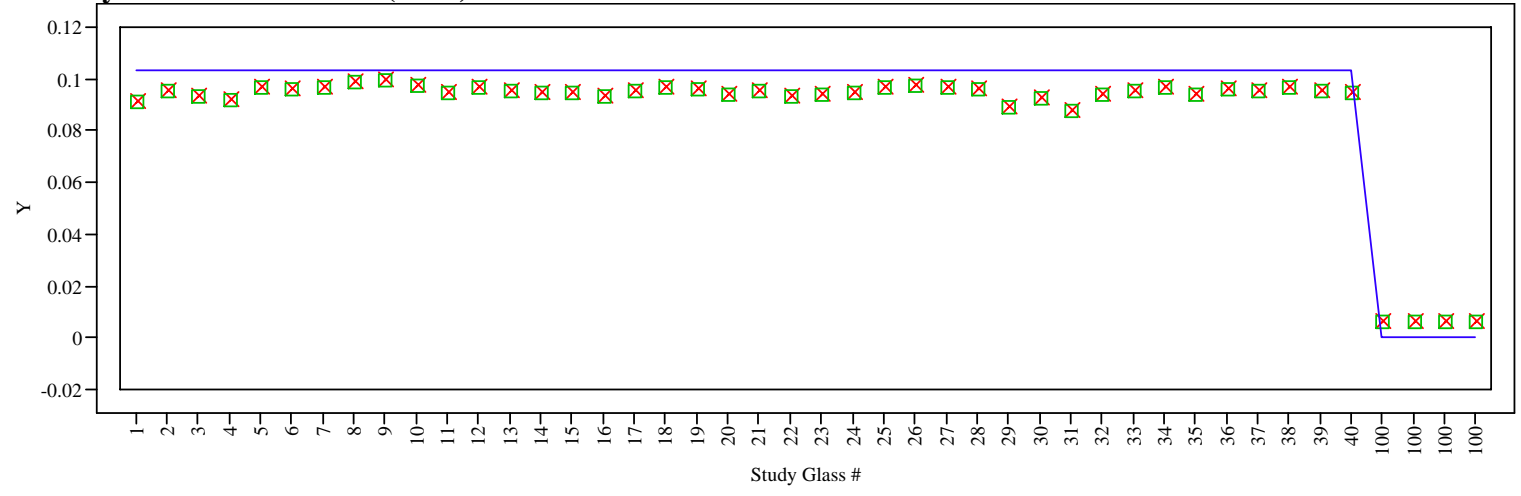

Overlay Plot Oxide $=$ Cr2O3 (wt \%)

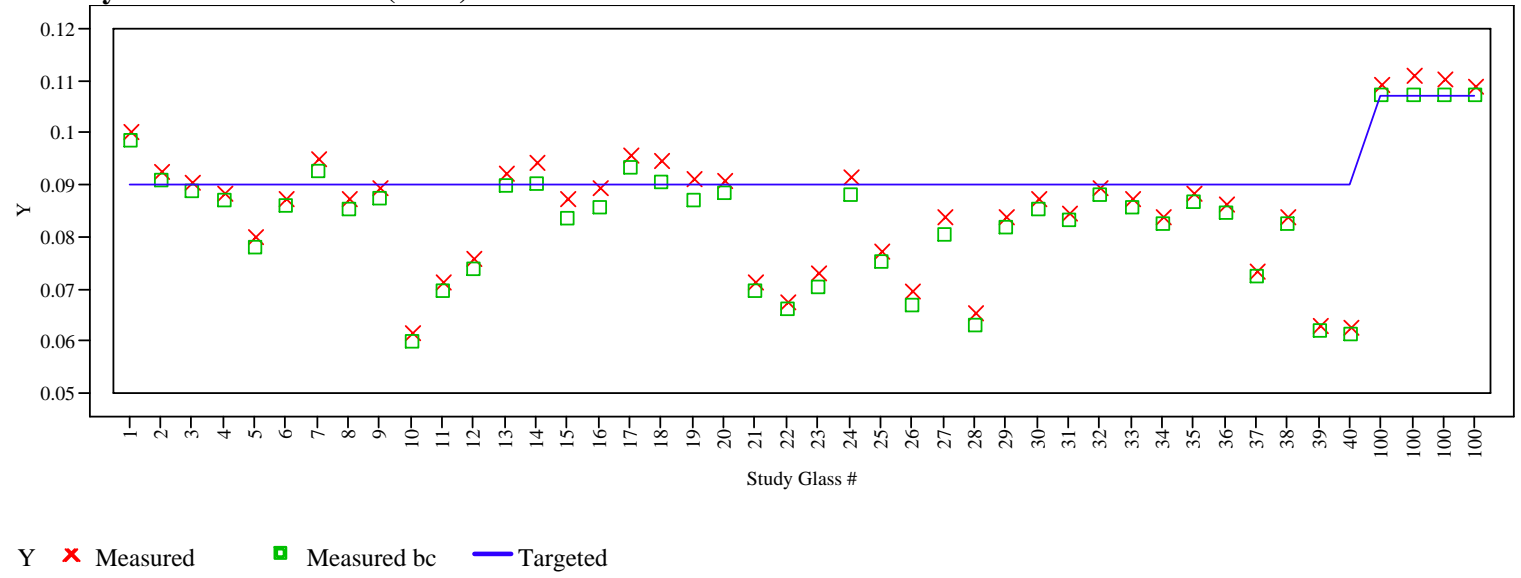


Exhibit A7. Average Measured and Bias-Corrected (bc) Versus

Targeted Compositions by Glass \# by Oxide. (continued)

(100 - Batch 1 for Sets 1, 2, 3, and 4)

Overlay Plot Oxide $=\mathrm{CuO}(\mathrm{wt} \%)$

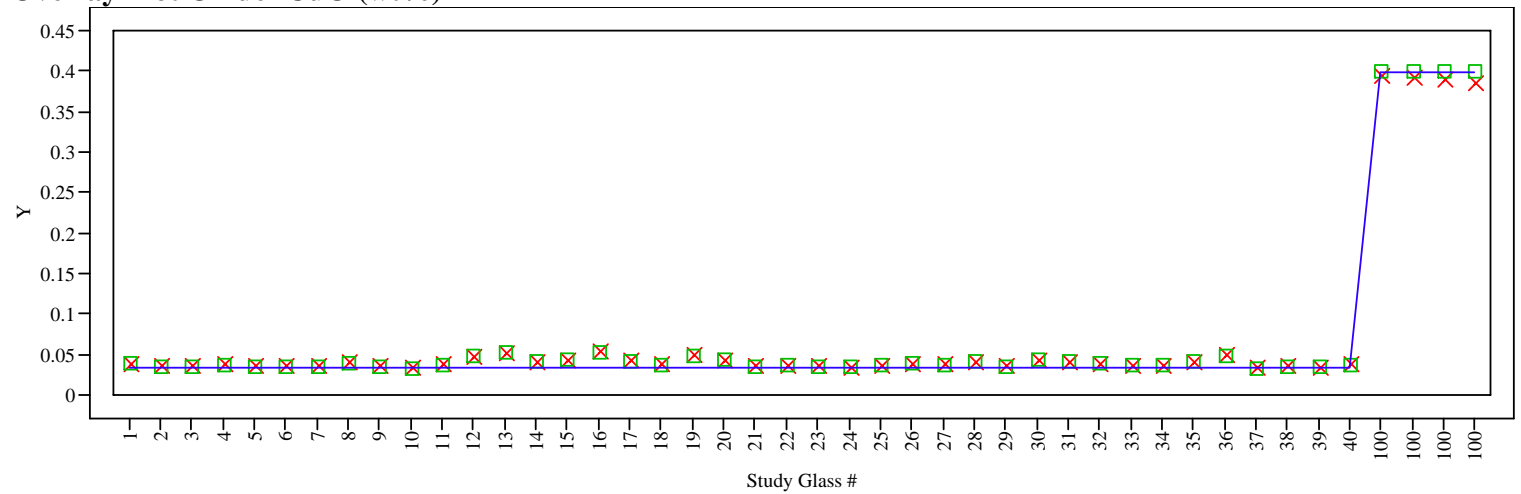

Overlay Plot Oxide=Fe2O3 (wt\%)

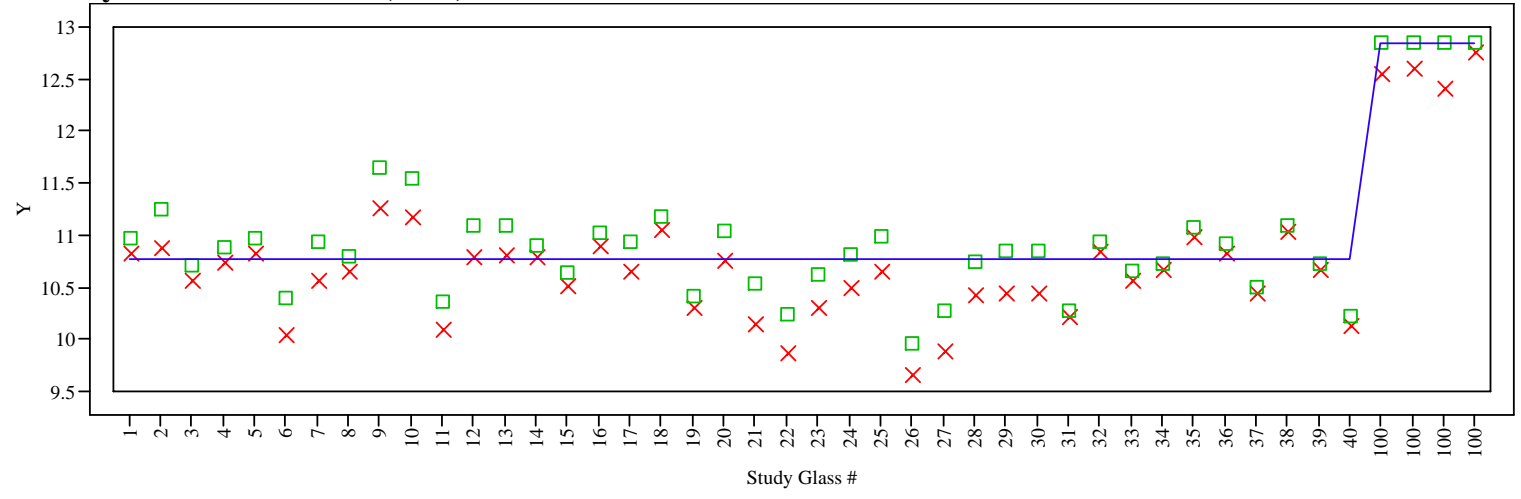

Overlay Plot Oxide=K2O (wt\%)

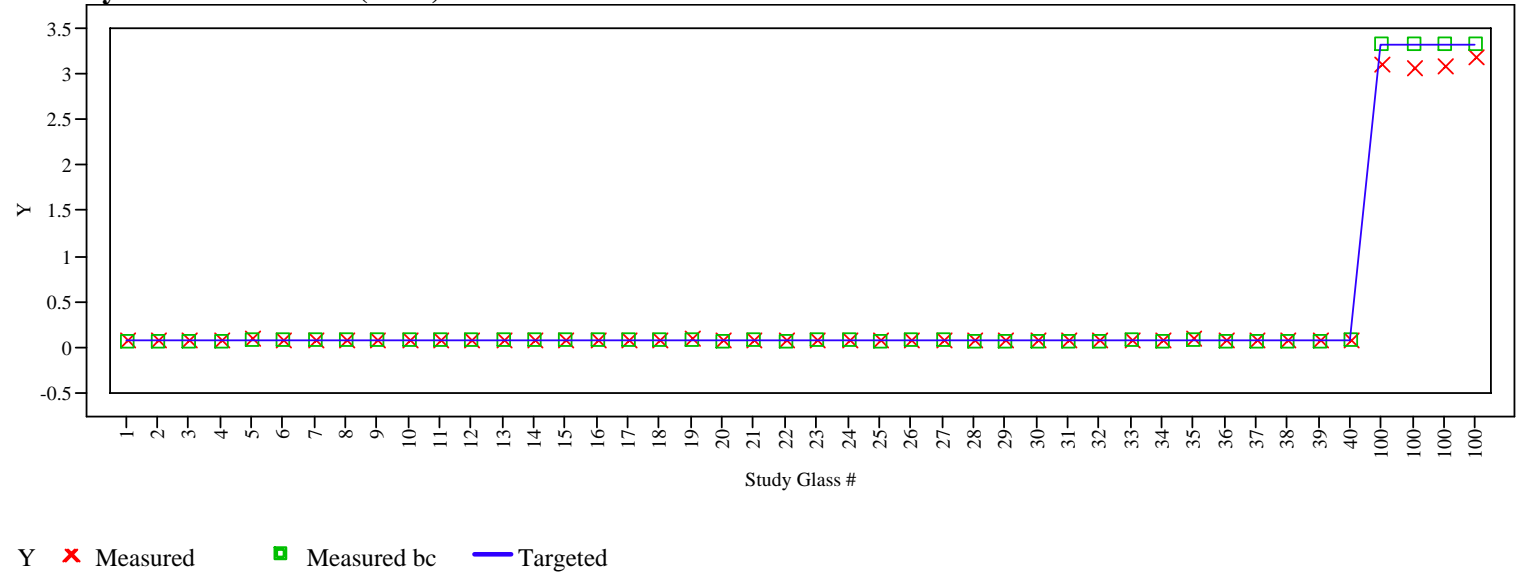


Exhibit A7. Average Measured and Bias-Corrected (bc) Versus

Targeted Compositions by Glass \# by Oxide. (continued)

(100 - Batch 1 for Sets 1, 2, 3, and 4)

Overlay Plot Oxide=La2O3 (wt\%)

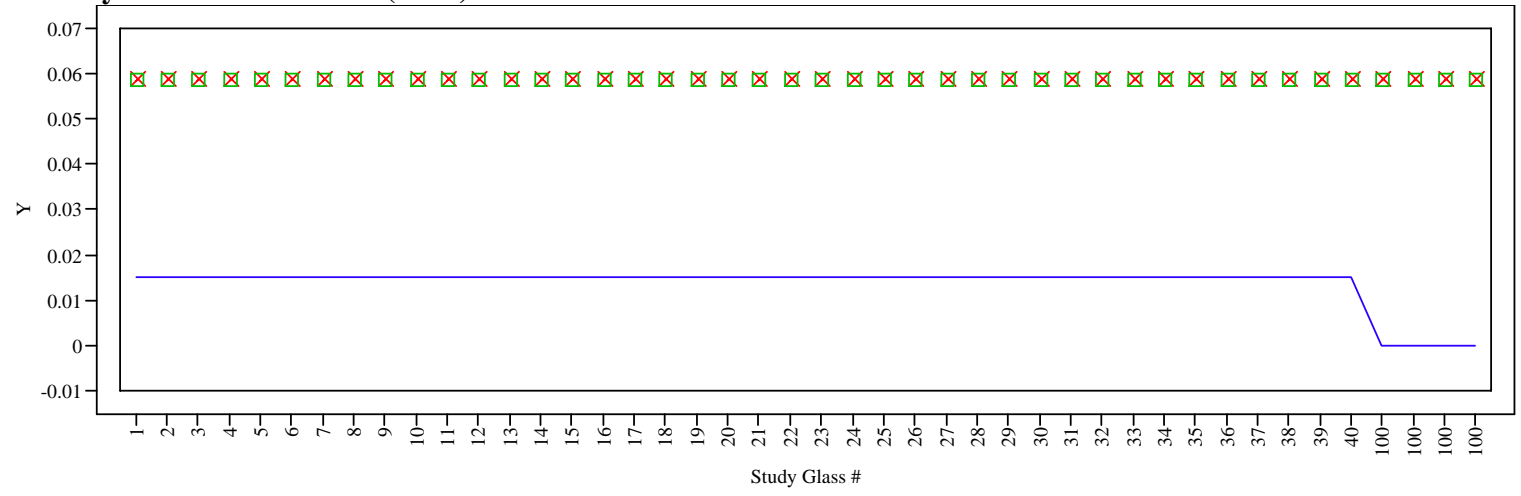

Overlay Plot Oxide=Li2O (wt\%)

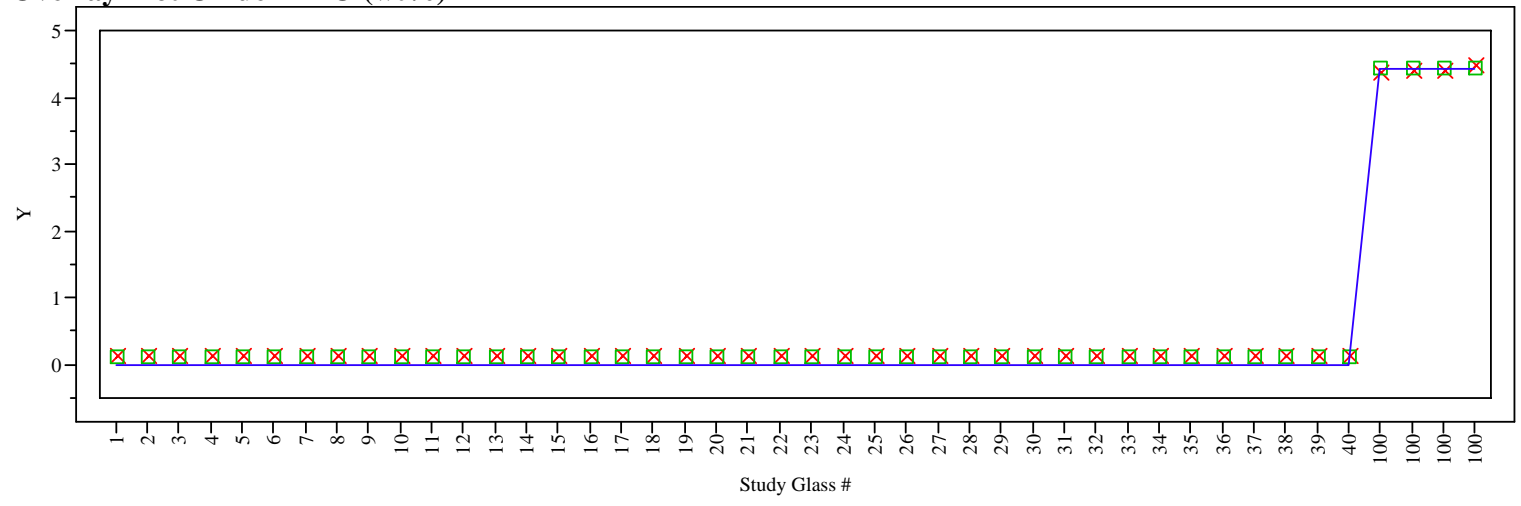

Overlay Plot Oxide=MgO (wt\%)

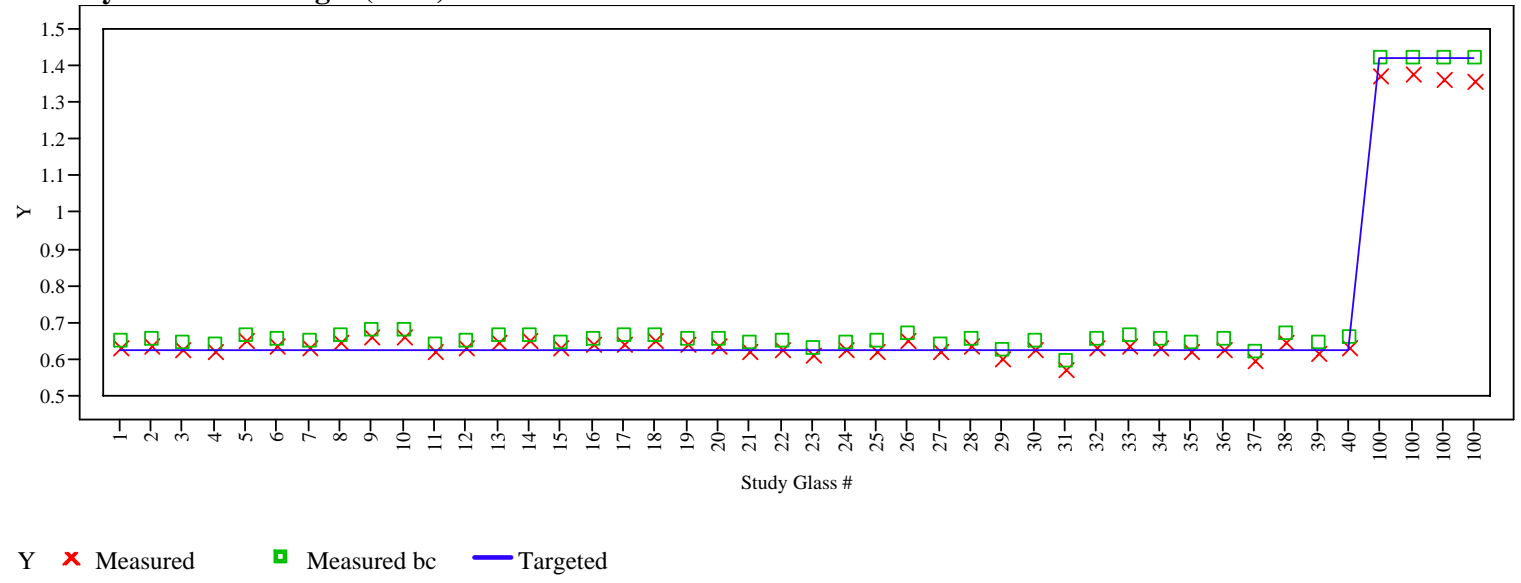


Exhibit A7. Average Measured and Bias-Corrected (bc) Versus

Targeted Compositions by Glass \# by Oxide. (continued)

(100 - Batch 1 for Sets 1, 2, 3, and 4)

Overlay Plot Oxide=MnO (wt\%)

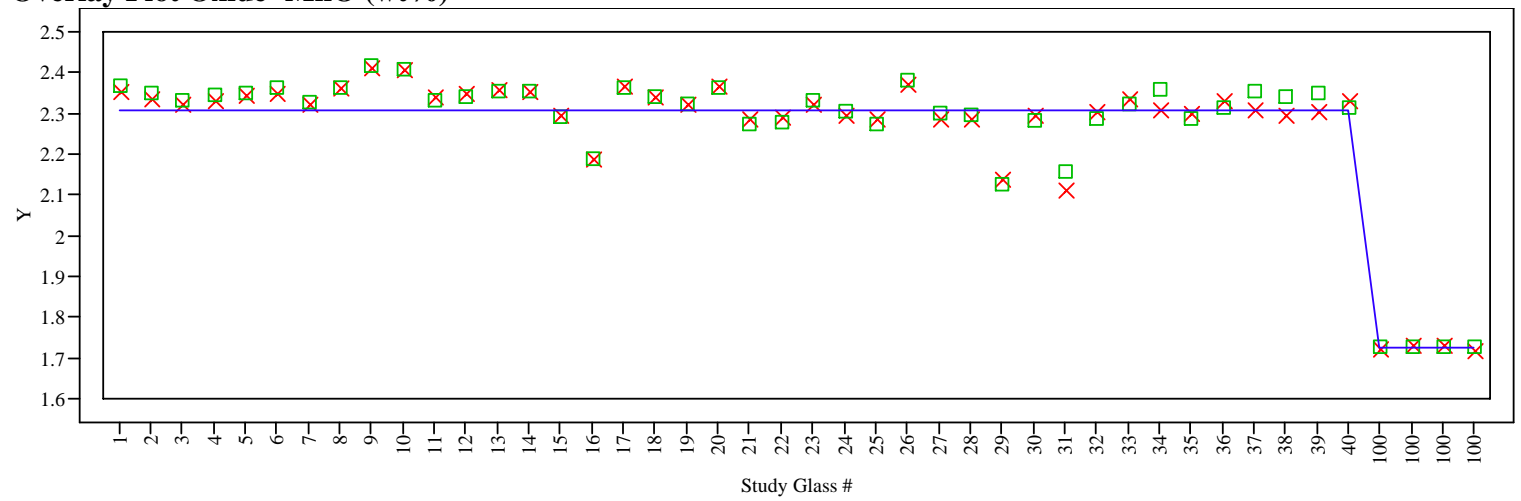

Overlay Plot Oxide=Na2O (wt\%)

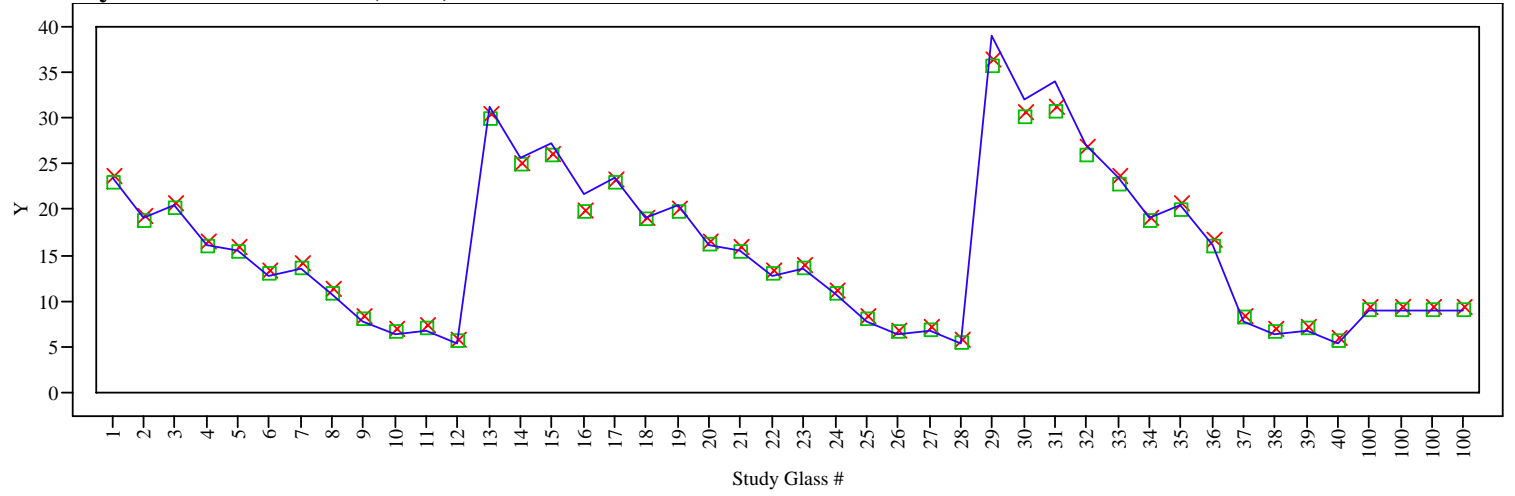

Overlay Plot Oxide=NiO (wt\%)

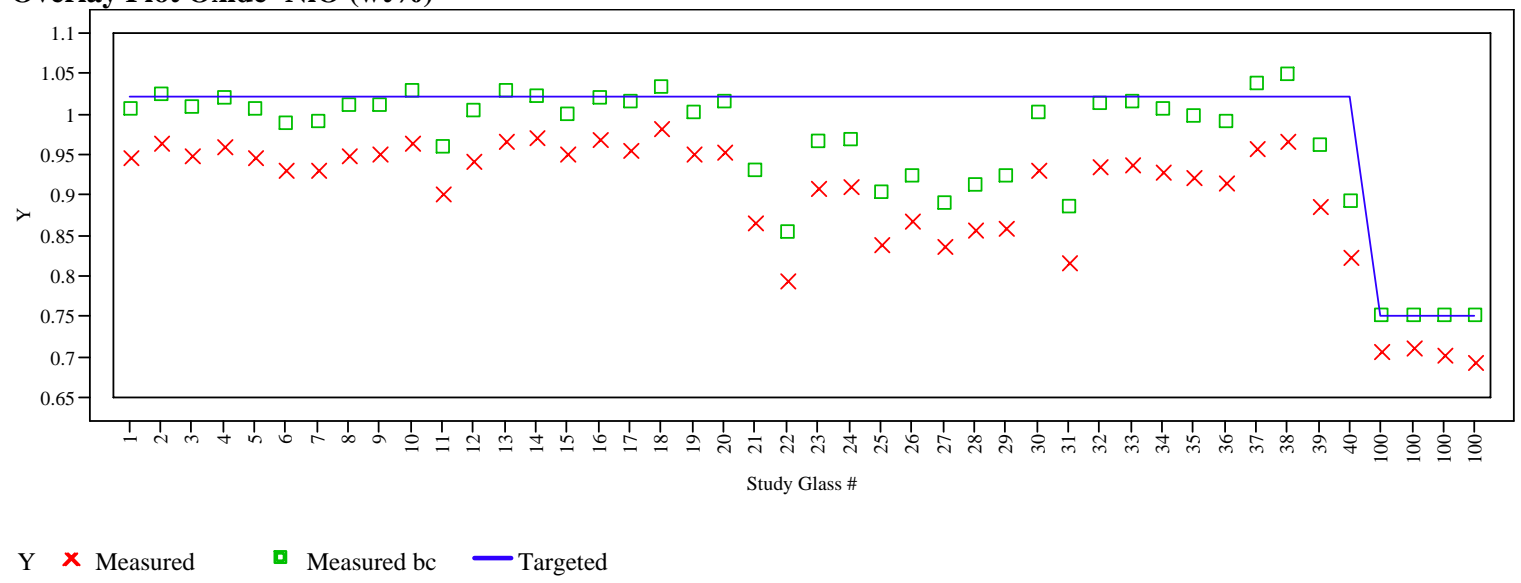


Exhibit A7. Average Measured and Bias-Corrected (bc) Versus

Targeted Compositions by Glass \# by Oxide. (continued)

(100 - Batch 1 for Sets 1, 2, 3, and 4)

Overlay Plot Oxide=PbO (wt \%)

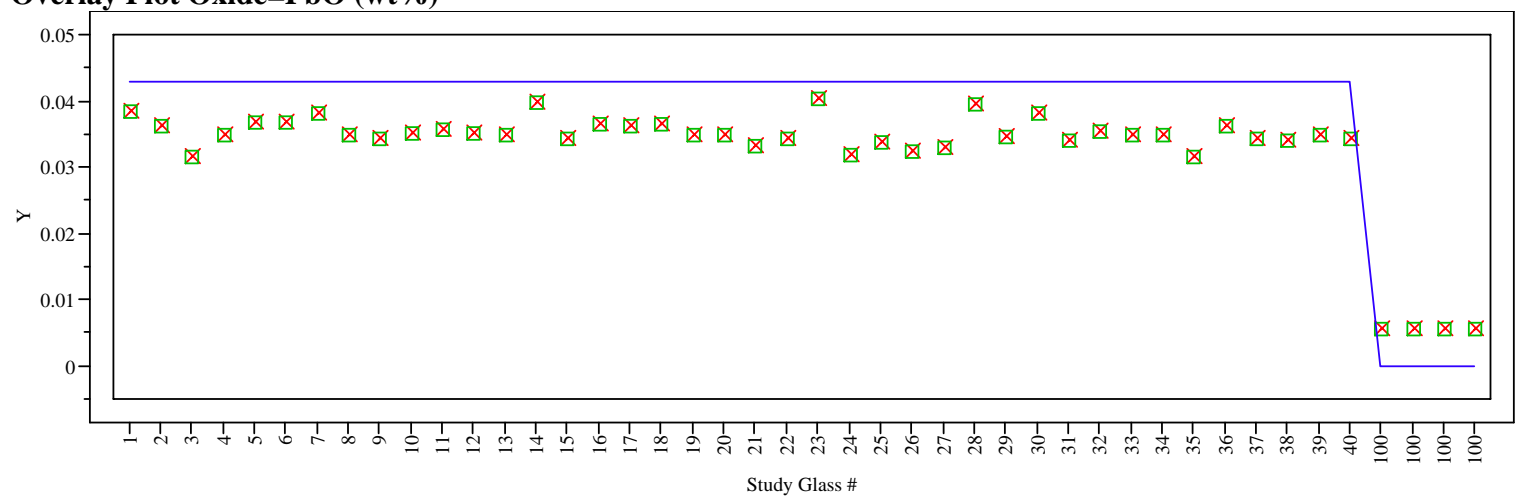

Overlay Plot Oxide=SiO2 (wt\%)

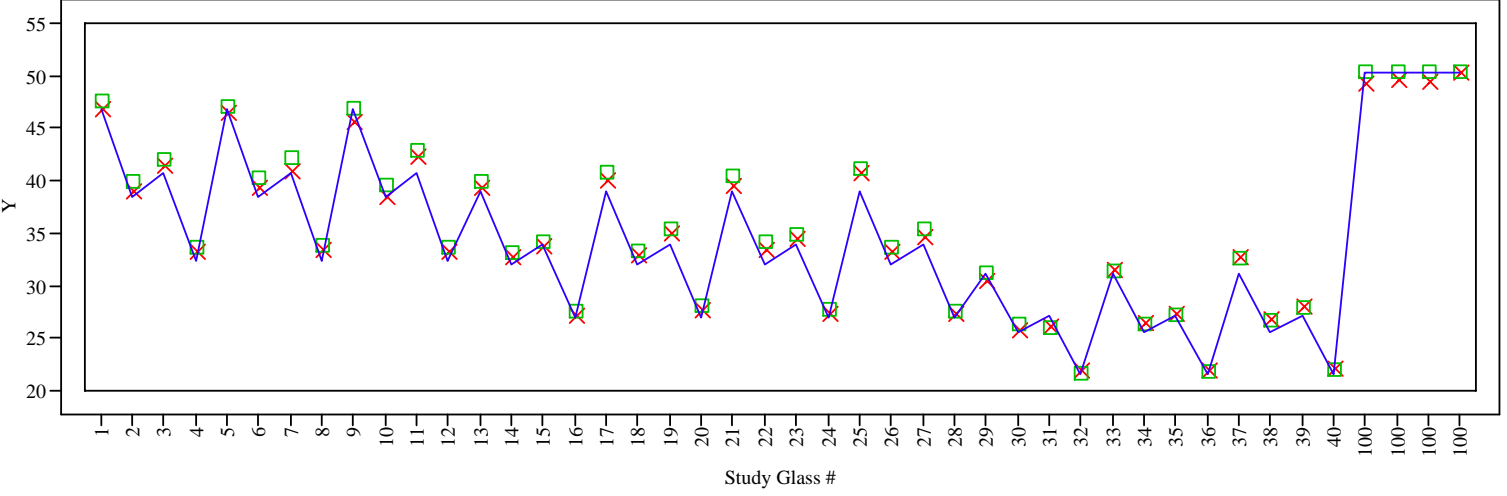

Overlay Plot Oxide=SO4 (wt \%)

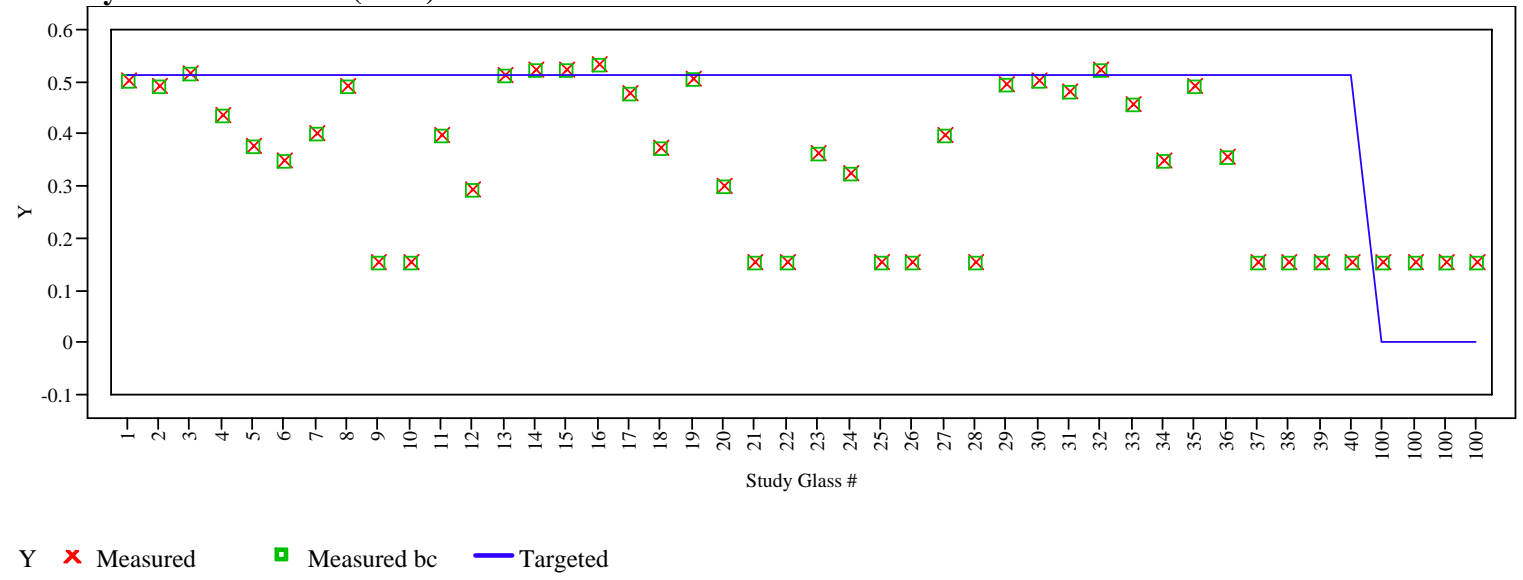


Exhibit A7. Average Measured and Bias-Corrected (bc) Versus

Targeted Compositions by Glass \# by Oxide. (continued)

(100 - Batch 1 for Sets 1, 2, 3, and 4)

Overlay Plot Oxide=TiO2 (wt\%)

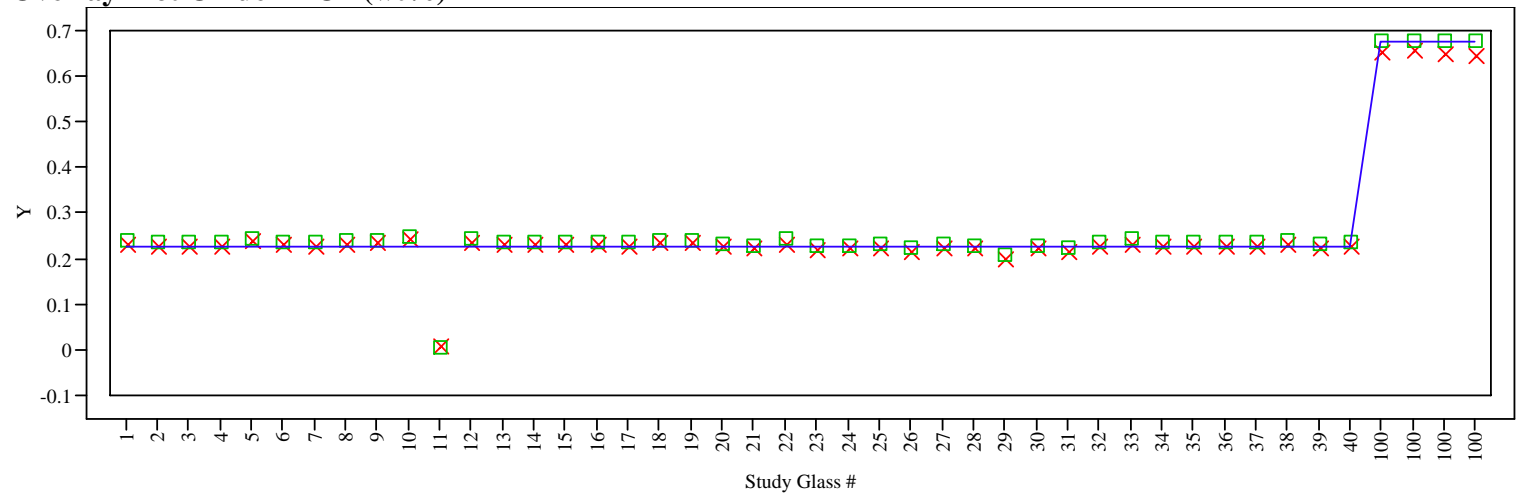

Overlay Plot Oxide $=\mathrm{ZnO}$ (wt \%)

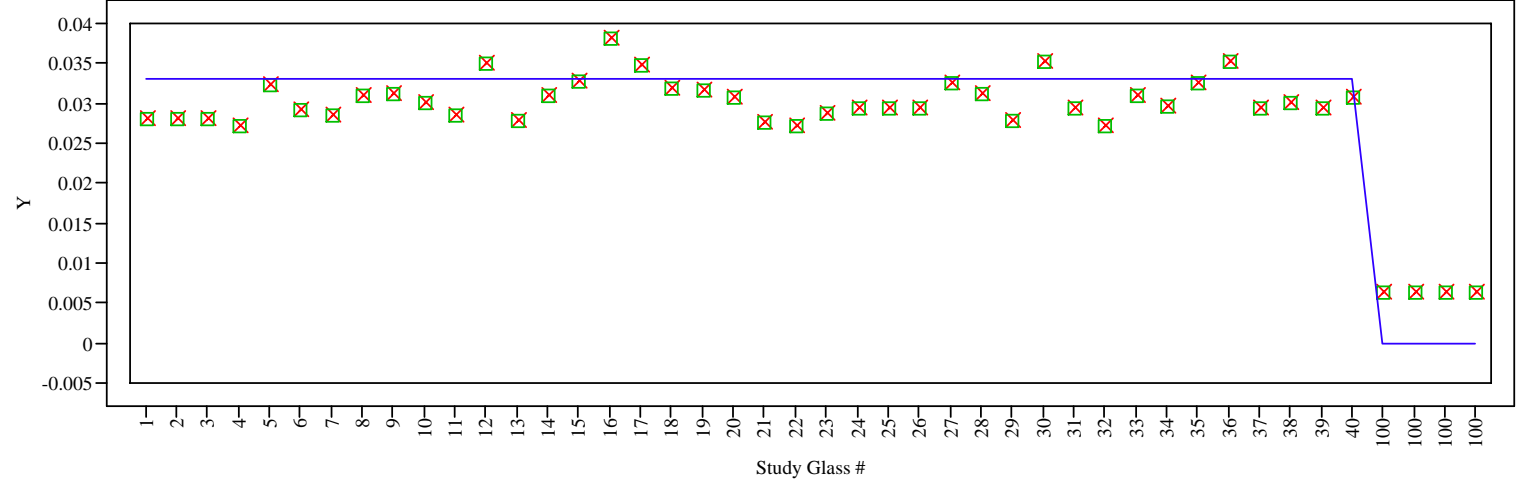

Overlay Plot Oxide=ZrO2 (wt\%)

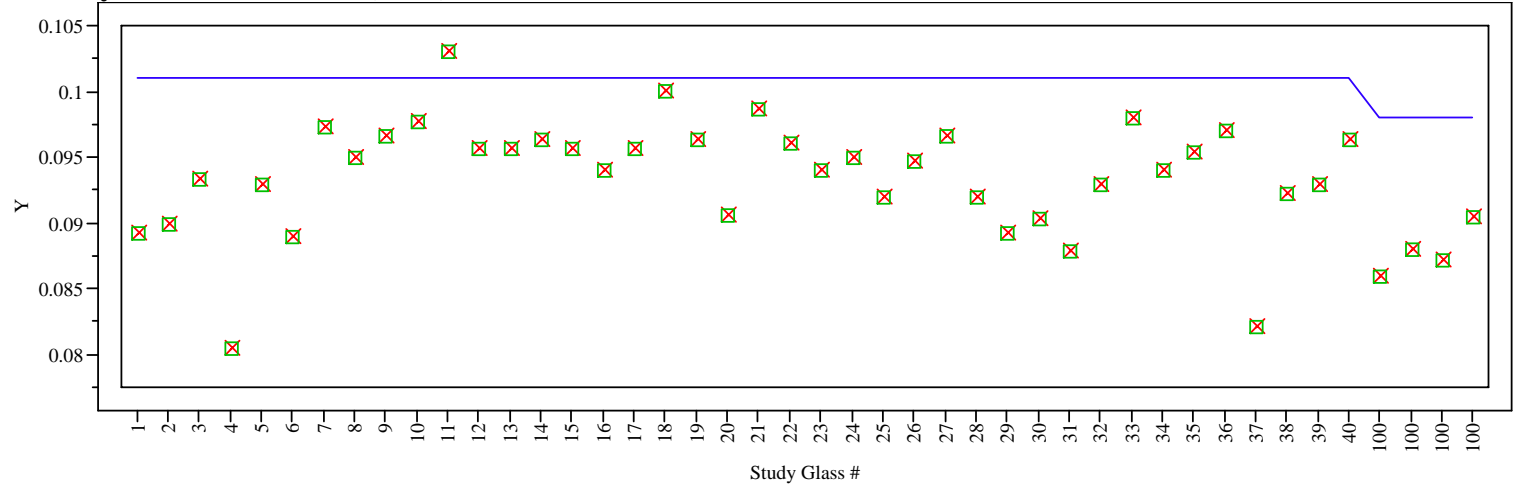

Y $\times$ Measured $\square$ Measured bc - Targeted 
Exhibit A7. Average Measured and Bias-Corrected (bc) Versus

Targeted Compositions by Glass \# by Oxide. (continued) (100 - Batch 1 for Sets 1, 2, 3, and 4)

Overlay Plot Oxide=Sum of Oxides

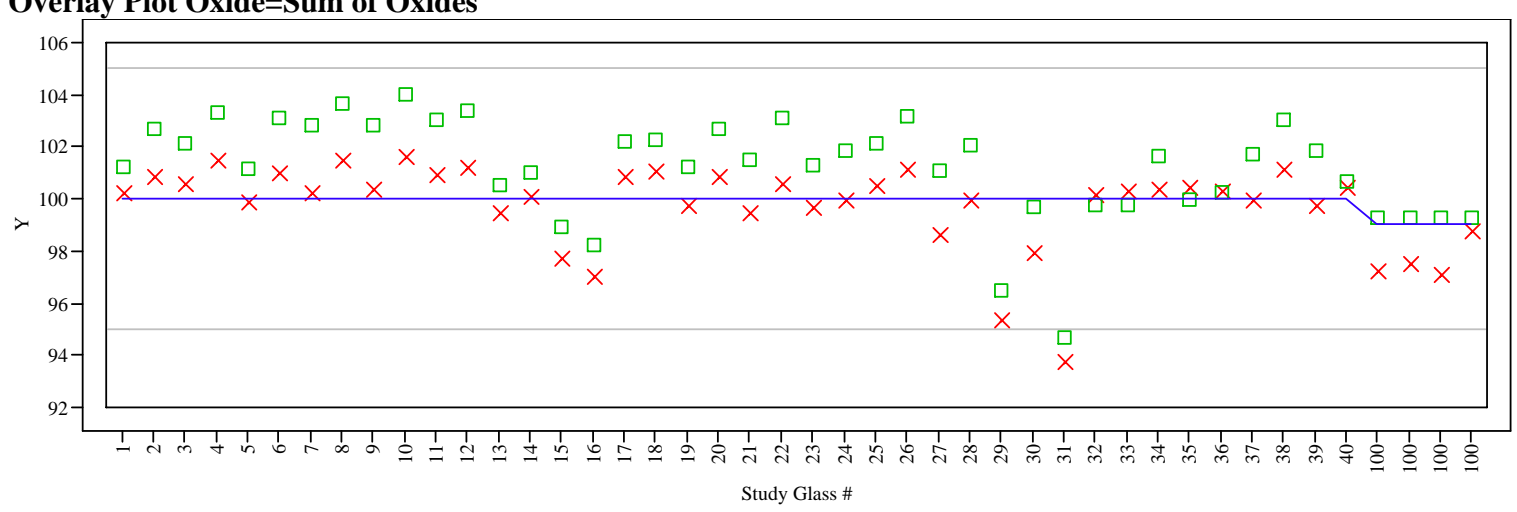


WSRC-STI-2007-00659

Revision 0

Appendix B

Tables and Exhibits Supporting the Analysis of the PCT Results for the Nepheline Study Glasses 
WSRC-STI-2007-00659

Revision 0

Appendix B

Table B1. Laboratory Measurements of the PCT Solutions for the Nepheline Study Glasses. (continued)

\begin{tabular}{|c|c|c|c|c|c|c|c|c|c|}
\hline Set & Glass ID & Heat Treatment & Block & Seq & Lab ID & $\mathrm{B}$ ar & $\mathrm{Li}$ ar & $\mathrm{Na}$ ar & Si ar \\
\hline 1 & Soln Std & ref & 1 & 1 & STD-11-1 & 21.2 & 9.77 & 82 & 50.3 \\
\hline 1 & SB5NEPH-05 & quenched & 1 & 2 & H29 & 3.22 & $<1.00$ & 32.7 & 32.2 \\
\hline 1 & SB5NEPH-08 & quenched & 1 & 3 & H32 & 20.6 & $<1.00$ & 35.2 & 9.09 \\
\hline 1 & SB5NEPH-05сcC & CCC & 1 & 4 & H42 & 2.03 & $<1.00$ & 25.3 & 26.1 \\
\hline 1 & SB5NEPH-03cCC & CCC & 1 & 5 & $\mathrm{H} 44$ & 11.7 & $<1.00$ & 205 & 43.5 \\
\hline 1 & ARM-1 & ref & 1 & 6 & H38 & 10.1 & 7.8 & 21.4 & 34.7 \\
\hline 1 & EA & ref & 1 & 7 & H63 & 36.5 & 10.9 & 106 & 53 \\
\hline 1 & SB5NEPH-01 & quenched & 1 & 8 & H56 & 7.55 & $<1.00$ & 202 & 90.5 \\
\hline 1 & SB5NEPH-04 & quenched & 1 & 9 & $\mathrm{H} 28$ & 107 & $<1.00$ & 269 & 33.6 \\
\hline 1 & SB5NEPH-02 & quenched & 1 & 10 & H33 & 275 & $<1.00$ & 531 & 37.5 \\
\hline 1 & SB5NEPH-03 & quenched & 1 & 11 & $\mathrm{H} 50$ & 7.16 & $<1.00$ & 151 & 29.4 \\
\hline 1 & SB5NEPH-07ccC & CCC & 1 & 12 & H36 & 2.58 & $<1.00$ & 21.9 & 12 \\
\hline 1 & Soln Std & ref & 1 & 13 & STD-11-2 & 20.6 & 9.65 & 79.3 & 49.5 \\
\hline 1 & SB5NEPH-02ccC & CCC & 1 & 14 & H41 & 243 & $<1.00$ & 503 & 38.2 \\
\hline 1 & SB5NEPH-01CCC & CCC & 1 & 15 & H08 & 10.1 & $<1.00$ & 185 & 87.7 \\
\hline 1 & SB5NEPH-04ccC & CCC & 1 & 16 & H18 & 109 & $<1.00$ & 278 & 35.3 \\
\hline 1 & SB5NEPH-10 & quenched & 1 & 17 & H14 & 7.21 & $<1.00$ & 4.38 & 10 \\
\hline 1 & SB5NEPH-06сcC & $\mathrm{CCC}$ & 1 & 18 & $\mathrm{H} 20$ & 38.8 & $<1.00$ & 49.6 & 25 \\
\hline 1 & SB5NEPH-10сcC & $\mathrm{CCC}$ & 1 & 19 & $\mathrm{H} 25$ & 8.8 & $<1.00$ & 5.94 & 13.1 \\
\hline 1 & SB5NEPH-07 & quenched & 1 & 20 & $\mathrm{H} 12$ & 2.29 & $<1.00$ & 23.1 & 11.6 \\
\hline 1 & SB5NEPH-08ccC & $\mathrm{CCC}$ & 1 & 21 & H09 & 16.9 & $<1.00$ & 26.3 & 8.91 \\
\hline 1 & SB5NEPH-09сcC & CCC & 1 & 22 & H11 & 1.19 & $<1.00$ & 4.46 & 11.5 \\
\hline 1 & blank & ref & 1 & 23 & H15 & $<0.100$ & $<1.00$ & $<0.100$ & $<0.100$ \\
\hline 1 & SB5NEPH-06 & quenched & 1 & 24 & $\mathrm{H} 40$ & 30.4 & $<1.00$ & 42.2 & 23.1 \\
\hline 1 & SB5NEPH-09 & quenched & 1 & 25 & $\mathrm{H} 49$ & 1.17 & $<1.00$ & 4.26 & 10.3 \\
\hline 1 & Soln Std & ref & 1 & 26 & STD-11-3 & 20.7 & 9.63 & 84.3 & 49.6 \\
\hline 1 & Soln Std & ref & 2 & 1 & STD-12-1 & 21.5 & 10.2 & 85.9 & 51.8 \\
\hline 1 & SB5NEPH-09ссC & CCC & 2 & 2 & H61 & 1.28 & $<1.00$ & 45.1 & 14.6 \\
\hline 1 & SB5NEPH-01 & quenched & 2 & 3 & $\mathrm{H} 23$ & 7.22 & $<1.00$ & 208 & 90.4 \\
\hline 1 & SB5NEPH-05 & quenched & 2 & 4 & H43 & 2.97 & $<1.00$ & 42 & 34.6 \\
\hline 1 & SB5NEPH-03сcC & $\mathrm{CCC}$ & 2 & 5 & H19 & 12.3 & $<1.00$ & 195 & 48 \\
\hline 1 & SB5NEPH-04ccC & $\mathrm{CCC}$ & 2 & 6 & $\mathrm{H} 21$ & 99 & $<1.00$ & 264 & 38.6 \\
\hline 1 & SB5NEPH-10ccC & CCC & 2 & 7 & H26 & 8.52 & $<1.00$ & 8.79 & 15.4 \\
\hline 1 & SB5NEPH-02 & quenched & 2 & 8 & $\mathrm{H} 48$ & 251 & $<1.00$ & 517 & 41 \\
\hline 1 & SB5NEPH-02ccC & $\mathrm{CCC}$ & 2 & 9 & H62 & 215 & $<1.00$ & 434 & 39.6 \\
\hline 1 & SB5NEPH-01cCC & CCC & 2 & 10 & H34 & 9.21 & $<1.00$ & 211 & 90.3 \\
\hline 1 & SB5NEPH-08ccC & CCC & 2 & 11 & H13 & 17.9 & $<1.00$ & 30.5 & 11.3 \\
\hline 1 & SB5NEPH-07cCC & CCC & 2 & 12 & $\mathrm{H} 03$ & 2.39 & $<1.00$ & 19 & 14.6 \\
\hline 1 & Soln Std & ref & 2 & 13 & STD-12-2 & 20.7 & 10.2 & 83 & 51.5 \\
\hline 1 & SB5NEPH-03 & quenched & 2 & 14 & H01 & 4.94 & $<1.00$ & 140 & 33.2 \\
\hline 1 & SB5NEPH-05сcC & CCC & 2 & 15 & H58 & 2.1 & $<1.00$ & 23.5 & 28.5 \\
\hline 1 & SB5NEPH-10 & quenched & 2 & 16 & H39 & 5.34 & $<1.00$ & 3.4 & 11.7 \\
\hline 1 & EA & ref & 2 & 17 & H16 & 38.4 & 11.7 & 98.7 & 56.1 \\
\hline 1 & SB5NEPH-04 & quenched & 2 & 18 & $\mathrm{H} 02$ & 97.1 & $<1.00$ & 261 & 35.2 \\
\hline 1 & SB5NEPH-09 & quenched & 2 & 19 & H35 & 1.38 & $<1.00$ & 7.7 & 12.6 \\
\hline 1 & SB5NEPH-06 & quenched & 2 & 20 & H65 & 32.6 & $<1.00$ & 45.1 & 26.1 \\
\hline 1 & SB5NEPH-06сcC & $\mathrm{CCC}$ & 2 & 21 & H17 & 40 & $<1.00$ & 48.7 & 27.8 \\
\hline 1 & ARM-1 & ref & 2 & 22 & H64 & 10.6 & 8.27 & 24.4 & 37.2 \\
\hline 1 & SB5NEPH-07 & quenched & 2 & 23 & H57 & 2.18 & $<1.00$ & 22.8 & 13.9 \\
\hline 1 & SB5NEPH-08 & quenched & 2 & 24 & $\mathrm{H} 10$ & 20.7 & $<1.00$ & 36.3 & 11.2 \\
\hline 1 & Soln Std & ref & 2 & 25 & STD-12-3 & 20.9 & 10.5 & 82.6 & 52.3 \\
\hline 1 & Soln Std & ref & 3 & 1 & STD-13-1 & 19.9 & 9.63 & 82.7 & 47.9 \\
\hline 1 & SB5NEPH-02cCC & CCC & 3 & 2 & H53 & 228 & $<1.00$ & 444 & 36.7 \\
\hline 1 & SB5NEPH-09сcC & CCC & 3 & 3 & H54 & 1.96 & $<1.00$ & 4.77 & 11.2 \\
\hline 1 & SB5NEPH-04 & quenched & 3 & 4 & H06 & 100 & $<1.00$ & 249 & 32.4 \\
\hline 1 & SB5NEPH-08ccC & CCC & 3 & 5 & $\mathrm{H} 24$ & 17.1 & $<1.00$ & 27.8 & 7.83 \\
\hline 1 & SB5NEPH-07 & quenched & 3 & 6 & H07 & 2.3 & $<1.00$ & 23.9 & 10.6 \\
\hline 1 & SB5NEPH-08 & quenched & 3 & 7 & H05 & 21.2 & $<1.00$ & 36.8 & 8.31 \\
\hline 1 & EA & ref & 3 & 8 & H27 & 37.4 & 10.9 & 99.6 & 51.7 \\
\hline
\end{tabular}


WSRC-STI-2007-00659

Revision 0

Appendix B

Table B1. Laboratory Measurements of the PCT Solutions for the Nepheline Study Glasses. (continued)

\begin{tabular}{|c|c|c|c|c|c|c|c|c|c|}
\hline Set & "Glass ID & "Heat Treatment & Block & Seq & Lab ID & $\mathrm{B}$ ar & Li ar & $\mathrm{Na}$ ar & Si ar \\
\hline 1 & SB5NEPH-01 & quenched & 3 & 9 & H46 & 7.43 & $<1.00$ & 181 & 86.6 \\
\hline 1 & SB5NEPH-01CCC & CCC & 3 & 10 & H52 & 7.13 & $<1.00$ & 169 & 85.6 \\
\hline 1 & SB5NEPH-03сcC & CCC & 3 & 11 & H30 & 13.1 & $<1.00$ & 196 & 44.8 \\
\hline 1 & SB5NEPH-03 & quenched & 3 & 12 & H37 & 4.01 & $<1.00$ & 141 & 28.9 \\
\hline 1 & Soln Std & ref & 3 & 13 & STD-13-2 & 20.4 & 9.87 & 85.9 & 49 \\
\hline 1 & SB5NEPH-05CCC & CCC & 3 & 14 & H51 & 2.05 & $<1.00$ & 26.1 & 24.1 \\
\hline 1 & SB5NEPH-10ccC & $\mathrm{CCC}$ & 3 & 15 & H60 & 7.78 & $<1.00$ & 6.33 & 11.6 \\
\hline 1 & SB5NEPH-05 & quenched & 3 & 16 & H45 & 2.93 & $<1.00$ & 35.4 & 31.1 \\
\hline 1 & SB5NEPH-06сcC & CCC & 3 & 17 & H68 & 38.6 & $<1.00$ & 53.8 & 23.3 \\
\hline 1 & blank & ref & 3 & 18 & H31 & $<0.100$ & $<1.00$ & $<0.100$ & $<0.100$ \\
\hline 1 & SB5NEPH-10 & quenched & 3 & 19 & H59 & 5.13 & $<1.00$ & 4.54 & 8.35 \\
\hline 1 & SB5NEPH-07cCC & $\mathrm{CCC}$ & 3 & 20 & H55 & 1.35 & $<1.00$ & 23.1 & 10.4 \\
\hline 1 & SB5NEPH-06 & quenched & 3 & 21 & H66 & 0.653 & $<1.00$ & 5.68 & 9.06 \\
\hline 1 & SB5NEPH-09 & quenched & 3 & 22 & H22 & 1.45 & $<1.00$ & 2.61 & 6.42 \\
\hline 1 & SB5NEPH-02 & quenched & 3 & 23 & H67 & 253 & $<1.00$ & 533 & 31.9 \\
\hline 1 & ARM-1 & ref & 3 & 24 & $\mathrm{H} 04$ & 10.9 & 7.96 & 22 & 34.1 \\
\hline 1 & SB5NEPH-04cCC & CCC & 3 & 25 & H47 & 100 & $<1.00$ & 268 & 35.2 \\
\hline 1 & Soln Std & ref & 3 & 26 & STD-13-3 & 20.6 & 9.66 & 86.5 & 47.1 \\
\hline 2 & Soln Std & ref & 1 & 1 & STD-21-1 & 20.6 & 9.89 & 80.9 & 49.3 \\
\hline 2 & SB5NEPH-17 & quenched & 1 & 2 & I 55 & 6.5 & $<1.00$ & 136 & 57.5 \\
\hline 2 & SB5NEPH-13сcC & CCC & 1 & 3 & I 18 & 39.9 & $<1.00$ & 1320 & 221 \\
\hline 2 & blank & ref & 1 & 4 & I 02 & $<1.00$ & $<1.00$ & $<1.00$ & $<1.00$ \\
\hline 2 & SB5NEPH-14cCC & CCC & 1 & 5 & I 06 & 532 & $<1.00$ & 1430 & 125 \\
\hline 2 & SB5NEPH-19 & quenched & 1 & 6 & I 61 & 8.93 & $<1.00$ & 196 & 25.5 \\
\hline 2 & EA & ref & 1 & 7 & I 25 & 41.2 & 11.6 & 163 & 54.4 \\
\hline 2 & SB5NEPH-11 & quenched & 1 & 8 & I 56 & 2.77 & $<1.00$ & 4.71 & 6.03 \\
\hline 2 & SB5NEPH-18cCC & $\mathrm{CCC}$ & 1 & 9 & I 42 & 133 & $<1.00$ & 273 & 19.9 \\
\hline 2 & SB5NEPH-20 & quenched & 1 & 10 & I 65 & 94.4 & $<1.00$ & 250 & 12.5 \\
\hline 2 & SB5NEPH-11CCC & CCC & 1 & 11 & I 64 & 7.35 & $<1.00$ & 10.2 & 6.53 \\
\hline 2 & SB5NEPH-12 & quenched & 1 & 12 & I 05 & 10.3 & $<1.00$ & 8.43 & 4.32 \\
\hline 2 & Soln Std & ref & 1 & 13 & STD-21-2 & 20.9 & 9.95 & 89.6 & 48.8 \\
\hline 2 & SB5NEPH-12ccC & CCC & 1 & 14 & I 39 & 6.78 & $<1.00$ & 5.96 & 5.41 \\
\hline 2 & SB5NEPH-16 & quenched & 1 & 15 & I 07 & 182 & $<1.00$ & 597 & 43.4 \\
\hline 2 & SB5NEPH-18 & quenched & 1 & 16 & I 29 & 145 & $<1.00$ & 281 & 20 \\
\hline 2 & SB5NEPH-15 & quenched & 1 & 17 & I 44 & 21.3 & $<1.00$ & 1040 & 102 \\
\hline 2 & SB5NEPH-19cCC & CCC & 1 & 18 & I 63 & 38.7 & $<1.00$ & 902 & 50.1 \\
\hline 2 & SB5NEPH-20cCC & CCC & 1 & 19 & I 17 & 37.4 & $<1.00$ & 114 & 11.2 \\
\hline 2 & SB5NEPH-13 & quenched & 1 & 20 & I 20 & 28.3 & $<1.00$ & 1250 & 217 \\
\hline 2 & SB5NEPH-14 & quenched & 1 & 21 & I 68 & 724 & $<1.00$ & 1860 & 154 \\
\hline 2 & ARM-1 & ref & 1 & 22 & I 30 & 13.2 & 8.41 & 24 & 36.7 \\
\hline 2 & SB5NEPH-15cCC & CCC & 1 & 23 & I 03 & 78.4 & $<1.00$ & 4100 & 261 \\
\hline 2 & SB5NEPH-17cCC & CCC & 1 & 24 & I 12 & 97.6 & $<1.00$ & 816 & 142 \\
\hline 2 & SB5NEPH-16сcC & CCC & 1 & 25 & I 01 & 191 & $<1.00$ & 618 & 44.6 \\
\hline 2 & Soln Std & ref & 1 & 26 & STD-21-3 & 23.6 & 10.3 & 86 & 50.9 \\
\hline 2 & Soln Std & ref & 2 & 1 & STD-22-1 & 20 & 9.63 & 82.7 & 49.5 \\
\hline 2 & SB5NEPH-16cCC & CCC & 2 & 2 & I 31 & 198 & $<1.00$ & 606 & 47 \\
\hline 2 & SB5NEPH-16 & quenched & 2 & 3 & I 10 & 178 & $<1.00$ & 577 & 42.4 \\
\hline 2 & SB5NEPH-12cCC & CCC & 2 & 4 & I 28 & 7.39 & $<1.00$ & 7.51 & 5.21 \\
\hline 2 & SB5NEPH-12 & quenched & 2 & 5 & I 62 & 9.6 & $<1.00$ & 6.55 & 4.14 \\
\hline 2 & SB5NEPH-15 & quenched & 2 & 6 & I 48 & 20.3 & $<1.00$ & 1090 & 105 \\
\hline 2 & SB5NEPH-17сCC & $\mathrm{CCC}$ & 2 & 7 & I 38 & 90.7 & $<1.00$ & 761 & 135 \\
\hline 2 & SB5NEPH-18cCC & CCC & 2 & 8 & I 15 & 134 & $<1.00$ & 272 & 20.4 \\
\hline 2 & SB5NEPH-13 & quenched & 2 & 9 & I 34 & 27.7 & $<1.00$ & 1260 & 212 \\
\hline 2 & SB5NEPH-20ccC & $\mathrm{CCC}$ & 2 & 10 & I 57 & 38.2 & $<1.00$ & 106 & 11.2 \\
\hline 2 & SB5NEPH-11CCC & CCC & 2 & 11 & I 13 & 6.2 & $<1.00$ & 9.57 & 6.41 \\
\hline 2 & SB5NEPH-18 & quenched & 2 & 12 & I 11 & 146 & $<1.00$ & 295 & 20.2 \\
\hline 2 & Soln Std & ref & 2 & 13 & STD-22-2 & 21.1 & 9.72 & 87.3 & 49.5 \\
\hline 2 & SB5NEPH-19 & quenched & 2 & 14 & I 24 & 5.8 & $<1.00$ & 198 & 25.3 \\
\hline 2 & SB5NEPH-15cCC & CCC & 2 & 15 & I 52 & 99.1 & $<1.00$ & 3980 & 269 \\
\hline
\end{tabular}


WSRC-STI-2007-00659

Revision 0

Appendix B

Table B1. Laboratory Measurements of the PCT Solutions for the Nepheline Study Glasses. (continued)

\begin{tabular}{|c|c|c|c|c|c|c|c|c|c|}
\hline Set & "Glass ID & "Heat Treatment & Block & Seq & Lab ID & $\mathrm{B}$ ar & Li ar & $\mathrm{Na}$ ar & Si ar \\
\hline 2 & SB5NEPH-19ссс & CCC & 2 & 16 & I 33 & 37 & $<1.00$ & 834 & 50.2 \\
\hline 2 & SB5NEPH-17 & quenched & 2 & 17 & I 23 & 6.29 & $<1.00$ & 145 & 56.4 \\
\hline 2 & SB5NEPH-14 & quenched & 2 & 18 & I 36 & 694 & $<1.00$ & 1850 & 151 \\
\hline 2 & SB5NEPH-14ccC & CCC & 2 & 19 & I 45 & 534 & $<1.00$ & 1480 & 124 \\
\hline 2 & EA & ref & 2 & 20 & I 53 & 46.1 & 11.4 & 127 & 55.4 \\
\hline 2 & ARM-1 & ref & 2 & 21 & I 51 & 11.3 & 8.63 & 22.7 & 37.8 \\
\hline 2 & SB5NEPH-11 & quenched & 2 & 22 & I 14 & 4.65 & $<1.00$ & 4.67 & 5.89 \\
\hline 2 & SB5NEPH-20 & quenched & 2 & 23 & I 49 & 99 & $<1.00$ & 283 & 13.6 \\
\hline 2 & SB5NEPH-13сcс & CCC & 2 & 24 & I 66 & 45.8 & $<1.00$ & 1410 & 233 \\
\hline 2 & Soln Std & ref & 2 & 25 & STD-22-3 & 21.4 & 9.51 & 86.6 & 47.5 \\
\hline 2 & Soln Std & ref & 3 & 1 & STD-23-1 & 19.2 & 9.67 & 84.3 & 49 \\
\hline 2 & SB5NEPH-18 & quenched & 3 & 2 & I 41 & 140 & $<1.00$ & 272 & 20.5 \\
\hline 2 & SB5NEPH-18ccC & CCC & 3 & 3 & I 04 & 134 & $<1.00$ & 409 & 21.3 \\
\hline 2 & SB5NEPH-20 & quenched & 3 & 4 & I 16 & 95.7 & $<1.00$ & 250 & 14.1 \\
\hline 2 & SB5NEPH-14 & quenched & 3 & 5 & I 46 & 707 & $<1.00$ & 1750 & 147 \\
\hline 2 & SB5NEPH-16ccC & CCC & 3 & 6 & I 37 & 192 & $<1.00$ & 593 & 44.4 \\
\hline 2 & SB5NEPH-20ccC & CCC & 3 & 7 & I 21 & 39 & $<1.00$ & 127 & 11.9 \\
\hline 2 & SB5NEPH-12ccC & CCC & 3 & 8 & I 26 & 8.13 & $<1.00$ & 5.65 & 6.39 \\
\hline 2 & SB5NEPH-11 & quenched & 3 & 9 & I 35 & 2.36 & $<1.00$ & 4.32 & 7.24 \\
\hline 2 & blank & ref & 3 & 10 & I 50 & $<1.00$ & $<1.00$ & $<1.00$ & $<1.00$ \\
\hline 2 & SB5NEPH-13 & quenched & 3 & 11 & I 40 & 27.2 & $<1.00$ & 1190 & 210 \\
\hline 2 & SB5NEPH-19ccC & $\mathrm{CCC}$ & 3 & 12 & I 58 & 36.6 & $<1.00$ & 855 & 48.9 \\
\hline 2 & Soln Std & ref & 3 & 13 & STD-23-2 & 19.5 & 9.63 & 85.2 & 48.4 \\
\hline 2 & SB5NEPH-19 & quenched & 3 & 14 & I 59 & 4.78 & $<1.00$ & 196 & 26.8 \\
\hline 2 & SB5NEPH-15сcC & CCC & 3 & 15 & I 22 & 77.3 & $<1.00$ & 3820 & 256 \\
\hline 2 & SB5NEPH-17сcC & CCC & 3 & 16 & I 47 & 94 & $<1.00$ & 841 & 140 \\
\hline 2 & SB5NEPH-11CCC & CCC & 3 & 17 & I 32 & 5.23 & $<1.00$ & 10.6 & 7.86 \\
\hline 2 & ARM-1 & ref & 3 & 18 & I 67 & 10.2 & 8.29 & 23.1 & 37.3 \\
\hline 2 & SB5NEPH-16 & quenched & 3 & 19 & I 54 & 180 & $<1.00$ & 589 & 43 \\
\hline 2 & SB5NEPH-15 & quenched & 3 & 20 & I 60 & 21.7 & $<1.00$ & 1020 & 110 \\
\hline 2 & SB5NEPH-13сcC & $\mathrm{CCC}$ & 3 & 21 & I 08 & 40.5 & $<1.00$ & 1330 & 223 \\
\hline 2 & EA & ref & 3 & 22 & I 19 & 36.7 & 11.3 & 113 & 52.8 \\
\hline 2 & SB5NEPH-14ccC & CCC & 3 & 23 & I 09 & 547 & $<1.00$ & 1450 & 123 \\
\hline 2 & SB5NEPH-17 & quenched & 3 & 24 & I 43 & 8.39 & $<1.00$ & 147 & 57.8 \\
\hline 2 & SB5NEPH-12 & quenched & 3 & 25 & I 27 & 9.88 & $<1.00$ & 8.67 & 5.39 \\
\hline 2 & Soln Std & ref & 3 & 26 & STD-23-3 & 20.5 & 9.73 & 86.6 & 49.2 \\
\hline 3 & Soln Std & ref & 1 & 1 & STD-31-1 & 20.4 & 9.63 & 79 & 48.7 \\
\hline 3 & SB5NEPH-22ccC & CCC & 1 & 2 & J38 & 32.8 & $<1.00$ & 47 & 25.9 \\
\hline 3 & SB5NEPH-22 & quenched & 1 & 3 & J35 & 16.4 & $<1.00$ & 28 & 21.7 \\
\hline 3 & SB5NEPH-29ccC & $\mathrm{CCC}$ & 1 & 4 & J22-missing & & & & \\
\hline 3 & SB5NEPH-21 & quenched & 1 & 5 & J26 & 1.36 & $<1.00$ & 26.3 & 21.3 \\
\hline 3 & SB5NEPH-30сcс & $\mathrm{CCC}$ & 1 & 6 & J39 & 125 & $<1.00$ & 2130 & 220 \\
\hline 3 & SB5NEPH-23ccC & CCC & 1 & 7 & J01 & 4.74 & $<1.00$ & 11.3 & 6.24 \\
\hline 3 & SB5NEPH-27 & quenched & 1 & 8 & J29 & 0.642 & $<1.00$ & 3.95 & 4.75 \\
\hline 3 & blank & ref & 1 & 9 & $\mathrm{~J} 50$ & $<0.100$ & $<1.00$ & $<0.100$ & $<0.100$ \\
\hline 3 & SB5NEPH-29 & quenched & 1 & 10 & J19 & 739 & $<1.00$ & 12500 & 1390 \\
\hline 3 & SB5NEPH-27cCC & $\mathrm{CCC}$ & 1 & 11 & J36 & 138 & $<1.00$ & 58.8 & 0.225 \\
\hline 3 & SB5NEPH-25сcC & CCC & 1 & 12 & J51 & 4.7 & $<1.00$ & 5.92 & 10.3 \\
\hline 3 & Soln Std & ref & 1 & 13 & STD-31-2 & 22.4 & 9.92 & 83.8 & 49.5 \\
\hline 3 & SB5NEPH-24 & quenched & 1 & 14 & J04 & 30.4 & $<1.00$ & 57.3 & 3.88 \\
\hline 3 & SB5NEPH-23 & quenched & 1 & 15 & J59 & 2.33 & $<1.00$ & 24.4 & 8.14 \\
\hline 3 & SB5NEPH-24ccC & CCC & 1 & 16 & J55 & 11 & $<1.00$ & 18.6 & 4.76 \\
\hline 3 & SB5NEPH-21CCC & CCC & 1 & 17 & $\mathrm{~J} 24$ & 112 & $<1.00$ & 159 & 14.6 \\
\hline 3 & SB5NEPH-30 & quenched & 1 & 18 & J62 & 3280 & $<1.00$ & 10400 & 534 \\
\hline 3 & SB5NEPH-28ccC & $\mathrm{CCC}$ & 1 & 19 & J46 & 28.5 & $<1.00$ & 4.3 & 3.87 \\
\hline 3 & ARM-1 & ref & 1 & 20 & $\mathrm{~J} 54$ & 23.8 & 7.63 & 22 & 33.3 \\
\hline 3 & SB5NEPH-28 & quenched & 1 & 21 & $\mathrm{~J} 40$ & 19.7 & $<1.00$ & 6.9 & 2.82 \\
\hline 3 & SB5NEPH-25 & quenched & 1 & 22 & J42 & 8.24 & $<1.00$ & 5.54 & 7.94 \\
\hline 3 & SB5NEPH-26сcC & CCC & 1 & 23 & $\mathrm{~J} 23$ & 18.4 & $<1.00$ & 13.7 & 14.8 \\
\hline
\end{tabular}


WSRC-STI-2007-00659

Revision 0

Appendix B

Table B1. Laboratory Measurements of the PCT Solutions for the Nepheline Study Glasses. (continued)

\begin{tabular}{|c|c|c|c|c|c|c|c|c|c|}
\hline Set & "Glass ID & "Heat Treatment & Block & Seq & Lab ID & $\mathrm{B}$ ar & Li ar & $\mathrm{Na}$ ar & Si ar \\
\hline 3 & SB5NEPH-26 & quenched & 1 & 24 & J20 & 8.55 & $<1.00$ & 6.28 & 6.59 \\
\hline 3 & EA & ref & 1 & 25 & J37 & 41.5 & 11.6 & 120 & 53.5 \\
\hline 3 & Soln Std & ref & 1 & 26 & STD-31-3 & 23.2 & 9.89 & 85.5 & 48.6 \\
\hline 3 & Soln Std & ref & 2 & 1 & STD-32-1 & 19.5 & 9.71 & 79.4 & 49.3 \\
\hline 3 & SB5NEPH-21cCC & CCC & 2 & 2 & J64 & 109 & $<1.00$ & 142 & 14.5 \\
\hline 3 & SB5NEPH-28ccC & CCC & 2 & 3 & J03 & 4.66 & $<1.00$ & 3.28 & 4.25 \\
\hline 3 & SB5NEPH-29 & quenched & 2 & 4 & J17 & 733 & $<1.00$ & 12500 & 1410 \\
\hline 3 & SB5NEPH-30сcс & CCC & 2 & 5 & $\mathrm{~J} 10$ & 130 & $<1.00$ & 1960 & 206 \\
\hline 3 & ARM-1 & ref & 2 & 6 & J66 & 11.8 & 7.51 & 19.9 & 33.7 \\
\hline 3 & SB5NEPH-29сcC & CCC & 2 & 7 & J58-missing & & & & \\
\hline 3 & SB5NEPH-21 & quenched & 2 & 8 & J30 & 3.01 & $<1.00$ & 24.6 & 20.9 \\
\hline 3 & SB5NEPH-22 & quenched & 2 & 9 & $\mathrm{~J} 15$ & 16.4 & $<1.00$ & 28.4 & 21 \\
\hline 3 & SB5NEPH-23ccC & CCC & 2 & 10 & $\mathrm{~J} 12$ & 3.92 & $<1.00$ & 10.8 & 6.38 \\
\hline 3 & SB5NEPH-27 & quenched & 2 & 11 & J11 & $<0.100$ & $<1.00$ & 4.45 & 4.82 \\
\hline 3 & SB5NEPH-30 & quenched & 2 & 12 & J34 & 3350 & $<1.00$ & 10300 & 545 \\
\hline 3 & Soln Std & ref & 2 & 13 & STD-32-2 & 21.2 & 9.65 & 79 & 47.2 \\
\hline 3 & SB5NEPH-25 & quenched & 2 & 14 & J65 & 1.86 & $<1.00$ & 4.81 & 8.04 \\
\hline 3 & SB5NEPH-23 & quenched & 2 & 15 & J28 & 1.54 & $<1.00$ & 23.2 & 8.11 \\
\hline 3 & SB5NEPH-24 & quenched & 2 & 16 & J08 & 28.5 & $<1.00$ & 54 & 4.05 \\
\hline 3 & EA & ref & 2 & 17 & J44 & 37.3 & 11.3 & 109 & 52.6 \\
\hline 3 & SB5NEPH-26сcC & CCC & 2 & 18 & J02 & 11.6 & $<1.00$ & 11.6 & 15.1 \\
\hline 3 & SB5NEPH-22ccC & CCC & 2 & 19 & J49 & 35.2 & $<1.00$ & 49.1 & 25 \\
\hline 3 & SB5NEPH-26 & quenched & 2 & 20 & J32 & 2.87 & $<1.00$ & 4.88 & 6.76 \\
\hline 3 & SB5NEPH-25сcC & $\mathrm{CCC}$ & 2 & 21 & J68 & $<0.100$ & $<1.00$ & 5.67 & 10.3 \\
\hline 3 & SB5NEPH-24ccC & CCC & 2 & 22 & J61 & 9.24 & $<1.00$ & 17.7 & 4.97 \\
\hline 3 & SB5NEPH-28 & quenched & 2 & 23 & J09 & 6.78 & $<1.00$ & 6.18 & 2.88 \\
\hline 3 & SB5NEPH-27cCC & $\mathrm{CCC}$ & 2 & 24 & J16 & 147 & $<1.00$ & 57.5 & 0.341 \\
\hline 3 & Soln Std & ref & 2 & 25 & STD-32-3 & 19.7 & 9.79 & 82.3 & 49.6 \\
\hline 3 & Soln Std & ref & 3 & 1 & STD-33-1 & 19.8 & 9.73 & 81.2 & 48.7 \\
\hline 3 & SB5NEPH-28 & quenched & 3 & 2 & J56 & 8.2 & $<1.00$ & 6.07 & 3.11 \\
\hline 3 & SB5NEPH-22 & quenched & 3 & 3 & J67 & 17.3 & $<1.00$ & 28.8 & 22.5 \\
\hline 3 & SB5NEPH-30 & quenched & 3 & 4 & J25 & 3310 & $<1.00$ & 10200 & 782 \\
\hline 3 & SB5NEPH-26 & quenched & 3 & 5 & J05 & 19.5 & $<1.00$ & 4.56 & 6.74 \\
\hline 3 & SB5NEPH-27 & quenched & 3 & 6 & J60 & 11.1 & $<1.00$ & 3.81 & 5.01 \\
\hline 3 & blank & ref & 3 & 7 & J53 & 8.11 & $<1.00$ & $<0.100$ & $<0.100$ \\
\hline 3 & SB5NEPH-28cCC & CCC & 3 & 8 & J06 & 11.9 & $<1.00$ & 3.62 & 4.43 \\
\hline 3 & SB5NEPH-23сcс & $\overline{C C C}$ & 3 & 9 & J14 & 9.75 & $<1.00$ & 11.4 & 6.56 \\
\hline 3 & SB5NEPH-27ccC & CCC & 3 & 10 & J18 & 165 & $<1.00$ & 59.2 & 0.434 \\
\hline 3 & SB5NEPH-25 & quenched & 3 & 11 & J57 & 5.44 & $<1.00$ & 4.88 & 8.19 \\
\hline 3 & SB5NEPH-25ccC & $\mathrm{CCC}$ & 3 & 12 & J52 & 4.5 & $<1.00$ & 5.6 & 10.7 \\
\hline 3 & Soln Std & ref & 3 & 13 & STD-33-2 & 22.3 & 9.69 & 83 & 48.6 \\
\hline 3 & EA & ref & 3 & 14 & J07 & 39.3 & 11.3 & 103 & 52.8 \\
\hline 3 & SB5NEPH-22cсC & CCC & 3 & 15 & J21 & 37.7 & $<1.00$ & 49.6 & 26 \\
\hline 3 & SB5NEPH-24 & quenched & 3 & 16 & J63 & 31.6 & $<1.00$ & 54.8 & 4.39 \\
\hline 3 & ARM-1 & ref & 3 & 17 & $\mathrm{~J} 47$ & 11.4 & 7.88 & 21.8 & 35.1 \\
\hline 3 & SB5NEPH-29ccC & CCC & 3 & 18 & J33-missing & & & & \\
\hline 3 & SB5NEPH-21 & quenched & 3 & 19 & $\mathrm{~J} 13$ & 2.84 & $<1.00$ & 25.4 & 20.9 \\
\hline 3 & SB5NEPH-26сcC & $\mathrm{CCC}$ & 3 & 20 & J27 & 12.4 & $<1.00$ & 11.2 & 14.6 \\
\hline 3 & SB5NEPH-21ccC & CCC & 3 & 21 & $\mathrm{~J} 45$ & 111 & $<1.00$ & 154 & 15.3 \\
\hline 3 & SB5NEPH-23 & quenched & 3 & 22 & J43 & 2.35 & $<1.00$ & 23.6 & 8.28 \\
\hline 3 & SB5NEPH-29 & quenched & 3 & 23 & J41 & 813 & $<1.00$ & 12300 & 1410 \\
\hline 3 & SB5NEPH-24cCC & $\mathrm{CCC}$ & 3 & 24 & $\mathrm{~J} 48$ & 15.2 & $<1.00$ & 17.9 & 5.23 \\
\hline 3 & SB5NEPH-30cCC & CCC & 3 & 25 & J31 & 147 & $<1.00$ & 2060 & 231 \\
\hline 3 & Soln Std & ref & 3 & 26 & STD-33-3 & 20 & 9.66 & 81.1 & 46.9 \\
\hline 4 & Soln Std & ref & 1 & 1 & STD-41-1 & 20.8 & 9.92 & 81.3 & 51 \\
\hline 4 & SB5NEPH-34cCC & CCC & 1 & 2 & K17 & 222 & $<1.00$ & 315 & 8.43 \\
\hline 4 & ARM-1 & ref & 1 & 3 & K09 & 12 & 8.04 & 21.2 & 36.6 \\
\hline 4 & SB5NEPH-39 & quenched & 1 & 4 & K30 & 2.31 & $<1.00$ & 3.59 & 3.91 \\
\hline 4 & SB5NEPH-37 & quenched & 1 & 5 & K21 & 2.49 & $<1.00$ & 8.05 & 10.3 \\
\hline
\end{tabular}


WSRC-STI-2007-00659

Revision 0

Appendix B

Table B1. Laboratory Measurements of the PCT Solutions for the Nepheline Study Glasses. (continued)

\begin{tabular}{|c|c|c|c|c|c|c|c|c|c|}
\hline Set & Glass ID & Heat Treatment & Block & Seq & Lab ID & $\mathrm{B}$ ar & $\mathrm{Li}$ ar & $\mathrm{Na}$ ar & Si ar \\
\hline 4 & SB5NEPH-32ccC & CCC & 1 & 6 & K57 & 94.2 & $<1.00$ & 692 & 61.7 \\
\hline 4 & SB5NEPH-31 & quenched & 1 & 7 & K27 & 509 & $<1.00$ & 10800 & 412 \\
\hline 4 & SB5NEPH-40 & quenched & 1 & 8 & K29 & 13.3 & $<1.00$ & 6.49 & 2.01 \\
\hline 4 & SB5NEPH-32 & quenched & 1 & 9 & K22 & 635 & $<1.00$ & 2280 & 61.4 \\
\hline 4 & SB5NEPH-31ccC & CCC & 1 & 10 & K48 & 322 & $<1.00$ & 8020 & 455 \\
\hline 4 & SB5NEPH-38 & quenched & 1 & 11 & K46 & 14.9 & $<1.00$ & 7.84 & 8.04 \\
\hline 4 & EA & ref & 1 & 12 & K40 & 42.6 & 11.4 & 104 & 55.1 \\
\hline 4 & Soln Std & ref & 1 & 13 & STD-41-2 & 22.2 & 9.85 & 82.1 & 51.9 \\
\hline 4 & SB5NEPH-39ссс & CCC & 1 & 14 & K14 & 180 & $<1.00$ & 59 & $<1.00$ \\
\hline 4 & SB5NEPH-36сcC & CCC & 1 & 15 & K67 & 1100 & $<1.00$ & 1960 & 2.66 \\
\hline 4 & SB5NEPH-38cCC & $\mathrm{CCC}$ & 1 & 16 & K65 & 57.8 & $<1.00$ & 35.4 & 22.2 \\
\hline 4 & SB5NEPH-33 & quenched & 1 & 17 & K32 & 637 & $<1.00$ & 2220 & 38.5 \\
\hline 4 & SB5NEPH-37cCC & CCC & 1 & 18 & K26 & 10.6 & $<1.00$ & 7.61 & 11.1 \\
\hline 4 & SB5NEPH-34 & quenched & 1 & 19 & K42 & 99.3 & $<1.00$ & 215 & 17.6 \\
\hline 4 & SB5NEPH-36 & quenched & 1 & 20 & K05 & 95.5 & $<1.00$ & 239 & 5.53 \\
\hline 4 & SB5NEPH-40ccC & $\mathrm{CCC}$ & 1 & 21 & K06 & 12.1 & $<1.00$ & 4.46 & 3.52 \\
\hline 4 & SB5NEPH-33сCC & CCC & 1 & 22 & K38 & 233 & $<1.00$ & 1960 & 111 \\
\hline 4 & SB5NEPH-35 & quenched & 1 & 23 & K60 & 11.5 & $<1.00$ & 115 & 10.6 \\
\hline 4 & blank & ref & 1 & 24 & K59 & 4.71 & $<1.00$ & $<1.00$ & $<1.00$ \\
\hline 4 & SB5NEPH-35сcC & CCC & 1 & 25 & K44 & 51.4 & $<1.00$ & 922 & 76 \\
\hline 4 & Soln Std & ref & 1 & 26 & STD-41-3 & 22.5 & 9.94 & 84.4 & 51.5 \\
\hline 4 & Soln Std & ref & 2 & 1 & STD-42-1 & 20.3 & 9.68 & 81.5 & 48.5 \\
\hline 4 & SB5NEPH-33cCC & CCC & 2 & 2 & K50 & 223 & $<1.00$ & 1920 & 104 \\
\hline 4 & SB5NEPH-38 & quenched & 2 & 3 & K36 & 10.4 & $<1.00$ & 8.13 & 7.6 \\
\hline 4 & SB5NEPH-33 & quenched & 2 & 4 & K33 & 614 & $<1.00$ & 2210 & 36.8 \\
\hline 4 & ARM-1 & ref & 2 & 5 & K12 & 15.8 & 7.92 & 20.4 & 35.1 \\
\hline 4 & SB5NEPH-37 & quenched & 2 & 6 & K51 & 4.57 & $<1.00$ & 8.57 & 9.66 \\
\hline 4 & SB5NEPH-36 & quenched & 2 & 7 & K54 & 88.1 & $<1.00$ & 225 & 5.1 \\
\hline 4 & SB5NEPH-36ccC & CCC & 2 & 8 & K39 & 1130 & $<1.00$ & 2000 & 2.29 \\
\hline 4 & SB5NEPH-37ccC & CCC & 2 & 9 & K37 & 11.8 & $<1.00$ & 7.84 & 10.3 \\
\hline 4 & SB5NEPH-31 & quenched & 2 & 10 & K43 & 531 & $<1.00$ & 11000 & 429 \\
\hline 4 & SB5NEPH-39ссс & CCC & 2 & 11 & K45 & 195 & $<1.00$ & 59.5 & $<1.00$ \\
\hline 4 & SB5NEPH-32 & quenched & 2 & 12 & K34 & 639 & $<1.00$ & 2310 & 58.3 \\
\hline 4 & Soln Std & ref & 2 & 13 & STD-42-2 & 20.2 & 9.58 & 83.9 & 46.8 \\
\hline 4 & SB5NEPH-40cCC & CCC & 2 & 14 & K47 & 11.8 & $<1.00$ & 4.08 & 3.05 \\
\hline 4 & SB5NEPH-35ccC & $\mathrm{CCC}$ & 2 & 15 & K56 & 49.9 & $<1.00$ & 958 & 69.8 \\
\hline 4 & SB5NEPH-34 & quenched & 2 & 16 & K08 & 98.1 & $<1.00$ & 195 & 16.7 \\
\hline 4 & SB5NEPH-40 & quenched & 2 & 17 & K61 & 13.2 & $<1.00$ & 7 & 1.72 \\
\hline 4 & SB5NEPH-39 & quenched & 2 & 18 & K68 & 4.15 & $<1.00$ & 4.05 & 3.81 \\
\hline 4 & EA & ref & 2 & 19 & K16 & 40.3 & 11.2 & 102 & 52.5 \\
\hline 4 & SB5NEPH-32сcс & CCC & 2 & 20 & K13 & 86.8 & $<1.00$ & 685 & 58.7 \\
\hline 4 & SB5NEPH-34ccC & CCC & 2 & 21 & K24 & 215 & $<1.00$ & 330 & 7.38 \\
\hline 4 & SB5NEPH-35 & quenched & 2 & 22 & K64 & 9.53 & $<1.00$ & 116 & 9.31 \\
\hline 4 & SB5NEPH-31cCC & CCC & 2 & 23 & K58 & 326 & $<1.00$ & 8120 & 457 \\
\hline 4 & SB5NEPH-38ccc & CCC & 2 & 24 & K53 & 51.8 & $<1.00$ & 36 & 20.5 \\
\hline 4 & Soln Std & ref & 2 & 25 & STD-42-3 & 23.1 & 9.55 & 83.4 & 47 \\
\hline 4 & Soln Std & ref & 3 & 1 & STD-43-1 & 19.9 & 9.77 & 80.6 & 48.9 \\
\hline 4 & SB5NEPH-36сcC & CCC & 3 & 2 & K03 & 1150 & $<1.00$ & 2160 & 2.46 \\
\hline 4 & SB5NEPH-32ccc & CCC & 3 & 3 & K19 & 110 & $<1.00$ & 722 & 63.2 \\
\hline 4 & SB5NEPH-40 & quenched & 3 & 4 & K63 & 14.9 & $<1.00$ & 6.77 & 1.92 \\
\hline 4 & EA & ref & 3 & 5 & K25 & 42 & 11.3 & 103 & 54.3 \\
\hline 4 & SB5NEPH-40сcC & CCC & 3 & 6 & K02 & 8.32 & $<1.00$ & 4.35 & 3.41 \\
\hline 4 & SB5NEPH-33сCC & CCC & 3 & 7 & K23 & 228 & $<1.00$ & 2010 & 106 \\
\hline 4 & SB5NEPH-37ccC & $\mathrm{CCC}$ & 3 & 8 & K31 & 3.57 & $<1.00$ & 7.99 & 10.8 \\
\hline 4 & blank & ref & 3 & 9 & K01 & 1.04 & $<1.00$ & $<1.00$ & $<1.00$ \\
\hline 4 & SB5NEPH-34cCC & $\mathrm{CCC}$ & 3 & 10 & K66 & 229 & $<1.00$ & 317 & 7.82 \\
\hline 4 & SB5NEPH-39 & quenched & 3 & 11 & K11 & 2.74 & $<1.00$ & 4.06 & 3.94 \\
\hline 4 & ARM-1 & ref & 3 & 12 & K28 & 11.6 & 8.48 & 23.3 & 37.2 \\
\hline 4 & Soln Std & ref & 3 & 13 & STD-43-2 & 20.2 & 9.75 & 81.9 & 48.5 \\
\hline
\end{tabular}


WSRC-STI-2007-00659

Revision 0

Appendix B

Table B1. Laboratory Measurements of the PCT Solutions for the Nepheline Study Glasses. (continued)

\begin{tabular}{|c|c|c|c|c|c|c|c|c|c|}
\hline Set & Glass ID & Heat Treatment & Block & Seq & Lab ID & $\mathrm{B}$ ar & Li ar & $\mathrm{Na}$ ar & Si ar \\
\hline 4 & SB5NEPH-35сcс & CCC & 3 & 14 & $\mathrm{~K} 10$ & 46.2 & $<1.00$ & 921 & 71.7 \\
\hline 4 & SB5NEPH-34 & quenched & 3 & 15 & K49 & 98.9 & $<1.00$ & 200 & 17 \\
\hline 4 & SB5NEPH-39ccC & CCC & 3 & 16 & K35 & 179 & $<1.00$ & 61 & $<1.00$ \\
\hline 4 & SB5NEPH-31 & quenched & 3 & 17 & K15 & 560 & $<1.00$ & 11500 & 452 \\
\hline 4 & SB5NEPH-35 & quenched & 3 & 18 & K55 & 10.4 & $<1.00$ & 117 & 10.7 \\
\hline 4 & SB5NEPH-37 & quenched & 3 & 19 & K20 & 3.06 & $<1.00$ & 9.81 & 9.73 \\
\hline 4 & SB5NEPH-38ccc & CCC & 3 & 20 & K07 & 48.8 & $<1.00$ & 37.7 & 21.1 \\
\hline 4 & SB5NEPH-38 & quenched & 3 & 21 & K18 & 8.93 & $<1.00$ & 9.29 & 7.82 \\
\hline 4 & SB5NEPH-36 & quenched & 3 & 22 & K41 & 88.7 & $<1.00$ & 246 & 5.44 \\
\hline 4 & SB5NEPH-32 & quenched & 3 & 23 & K62 & 574 & $<1.00$ & 2380 & 59.8 \\
\hline 4 & SB5NEPH-31ccC & CCC & 3 & 24 & K04 & 373 & $<1.00$ & 8590 & 511 \\
\hline 4 & SB5NEPH-33 & quenched & 3 & 25 & K52 & 613 & $<1.00$ & 2330 & 38 \\
\hline 4 & Soln Std & ref & 3 & 26 & STD-43-3 & 20.2 & 9.78 & 80.3 & 49.4 \\
\hline
\end{tabular}


WSRC-STI-2007-00659

Revision 0

Appendix B

Table B2. PSAL Measurements of the PCT Solutions for the Study Glasses After Appropriate Adjustments.

\begin{tabular}{|c|c|c|c|c|c|c|c|c|c|}
\hline Set & Glass ID & Heat Treatment & Block & Seq & Lab ID & B (ppm) & $\mathrm{Li}(\mathrm{ppm})$ & $\mathrm{Na}(\mathrm{ppm})$ & Si (ppm) \\
\hline 1 & Soln Std & ref & 1 & 1 & STD-11-1 & 21.20 & 9.77 & 82.00 & 50.30 \\
\hline 1 & SB5NEPH-05 & quenched & 1 & 2 & H29 & 5.37 & 0.83 & 54.50 & 53.67 \\
\hline 1 & SB5NEPH-08 & quenched & 1 & 3 & H32 & 34.33 & 0.83 & 58.67 & 15.15 \\
\hline 1 & SB5NEPH-05сcс & CCC & 1 & 4 & H42 & 3.38 & 0.83 & 42.17 & 43.50 \\
\hline 1 & SB5NEPH-03сcC & CCC & 1 & 5 & $\mathrm{H} 44$ & 19.50 & 0.83 & 341.67 & 72.50 \\
\hline 1 & ARM-1 & ref & 1 & 6 & H38 & 16.83 & 13.00 & 35.67 & 57.83 \\
\hline 1 & EA & ref & 1 & 7 & H63 & 608.33 & 181.67 & 1766.67 & 883.34 \\
\hline 1 & SB5NEPH-01 & quenched & 1 & 8 & H56 & 12.58 & 0.83 & 336.67 & 150.84 \\
\hline 1 & SB5NEPH-04 & quenched & 1 & 9 & $\mathrm{H} 28$ & 178.34 & 0.83 & 448.34 & 56.00 \\
\hline 1 & SB5NEPH-02 & quenched & 1 & 10 & H33 & 458.34 & 0.83 & 885.02 & 62.50 \\
\hline 1 & SB5NEPH-03 & quenched & 1 & 11 & $\mathrm{H} 50$ & 11.93 & 0.83 & 251.67 & 49.00 \\
\hline 1 & SB5NEPH-07ccC & $\mathrm{CCC}$ & 1 & 12 & H36 & 4.30 & 0.83 & 36.50 & 20.00 \\
\hline 1 & Soln Std & ref & 1 & 13 & STD-11-2 & 20.60 & 9.65 & 79.30 & 49.50 \\
\hline 1 & SB5NEPH-02ccC & $\mathrm{CCC}$ & 1 & 14 & H41 & 405.01 & 0.83 & 838.35 & 63.67 \\
\hline 1 & SB5NEPH-01ccC & $\mathrm{CCC}$ & 1 & 15 & H08 & 16.83 & 0.83 & 308.34 & 146.17 \\
\hline 1 & SB5NEPH-04ccC & $\mathrm{CCC}$ & 1 & 16 & H18 & 181.67 & 0.83 & 463.34 & 58.83 \\
\hline 1 & SB5NEPH-10 & quenched & 1 & 17 & H14 & 12.02 & 0.83 & 7.30 & 16.67 \\
\hline 1 & SB5NEPH-06сcс & CCC & 1 & 18 & $\mathrm{H} 20$ & 64.67 & 0.83 & 82.67 & 41.67 \\
\hline 1 & SB5NEPH-10сcс & $\mathrm{CCC}$ & 1 & 19 & H25 & 14.67 & 0.83 & 9.90 & 21.83 \\
\hline 1 & SB5NEPH-07 & quenched & 1 & 20 & H12 & 3.82 & 0.83 & 38.50 & 19.33 \\
\hline 1 & SB5NEPH-08ccC & $\mathrm{CCC}$ & 1 & 21 & H09 & 28.17 & 0.83 & 43.83 & 14.85 \\
\hline 1 & SB5NEPH-09сcс & CCC & 1 & 22 & H11 & 1.98 & 0.83 & 7.43 & 19.17 \\
\hline 1 & blank & ref & 1 & 23 & H15 & 0.08 & 0.83 & 0.08 & 0.08 \\
\hline 1 & SB5NEPH-06 & quenched & 1 & 24 & $\mathrm{H} 40$ & 50.67 & 0.83 & 70.33 & 38.50 \\
\hline 1 & SB5NEPH-09 & quenched & 1 & 25 & H49 & 1.95 & 0.83 & 7.10 & 17.17 \\
\hline 1 & Soln Std & ref & 1 & 26 & STD-11-3 & 20.70 & 9.63 & 84.30 & 49.60 \\
\hline 1 & Soln Std & ref & 2 & 1 & STD-12-1 & 21.50 & 10.20 & 85.90 & 51.80 \\
\hline 1 & SB5NEPH-09сcC & CCC & 2 & 2 & H61 & 2.13 & 0.83 & 75.17 & 24.33 \\
\hline 1 & SB5NEPH-01 & quenched & 2 & 3 & $\mathrm{H} 23$ & 12.03 & 0.83 & 346.67 & 150.67 \\
\hline 1 & SB5NEPH-05 & quenched & 2 & 4 & $\mathrm{H} 43$ & 4.95 & 0.83 & 70.00 & 57.67 \\
\hline 1 & SB5NEPH-03сcC & $\mathrm{CCC}$ & 2 & 5 & H19 & 20.50 & 0.83 & 325.01 & 80.00 \\
\hline 1 & SB5NEPH-04ccC & $\mathrm{CCC}$ & 2 & 6 & $\mathrm{H} 21$ & 165.00 & 0.83 & 440.01 & 64.33 \\
\hline 1 & SB5NEPH-10сcс & $\mathrm{CCC}$ & 2 & 7 & H26 & 14.20 & 0.83 & 14.65 & 25.67 \\
\hline 1 & SB5NEPH-02 & quenched & 2 & 8 & H48 & 418.34 & 0.83 & 861.68 & 68.33 \\
\hline 1 & SB5NEPH-02ccC & CCC & 2 & 9 & H62 & 358.34 & 0.83 & 723.35 & 66.00 \\
\hline 1 & SB5NEPH-01ccC & $\mathrm{CCC}$ & 2 & 10 & H34 & 15.35 & 0.83 & 351.67 & 150.50 \\
\hline 1 & SB5NEPH-08ccC & CCC & 2 & 11 & H13 & 29.83 & 0.83 & 50.83 & 18.83 \\
\hline 1 & SB5NEPH-07ccC & $\mathrm{CCC}$ & 2 & 12 & H03 & 3.98 & 0.83 & 31.67 & 24.33 \\
\hline 1 & Soln Std & ref & 2 & 13 & STD-12-2 & 20.70 & 10.20 & 83.00 & 51.50 \\
\hline 1 & SB5NEPH-03 & quenched & 2 & 14 & H01 & 8.23 & 0.83 & 233.34 & 55.33 \\
\hline 1 & SB5NEPH-05ccC & CCC & 2 & 15 & H58 & 3.50 & 0.83 & 39.17 & 47.50 \\
\hline 1 & SB5NEPH-10 & quenched & 2 & 16 & H39 & 8.90 & 0.83 & 5.67 & 19.50 \\
\hline 1 & EA & ref & 2 & 17 & H16 & 640.00 & 195.00 & 1645.00 & 935.00 \\
\hline 1 & SB5NEPH-04 & quenched & 2 & 18 & $\mathrm{H} 02$ & 161.84 & 0.83 & 435.01 & 58.67 \\
\hline 1 & SB5NEPH-09 & quenched & 2 & 19 & H35 & 2.30 & 0.83 & 12.83 & 21.00 \\
\hline 1 & SB5NEPH-06 & quenched & 2 & 20 & H65 & 54.33 & 0.83 & 75.17 & 43.50 \\
\hline 1 & SB5NEPH-06сcс & $\mathrm{CCC}$ & 2 & 21 & H17 & 66.67 & 0.83 & 81.17 & 46.33 \\
\hline 1 & ARM-1 & ref & 2 & 22 & H64 & 17.67 & 13.78 & 40.67 & 62.00 \\
\hline 1 & SB5NEPH-07 & quenched & 2 & 23 & H57 & 3.63 & 0.83 & 38.00 & 23.17 \\
\hline 1 & SB5NEPH-08 & quenched & 2 & 24 & $\mathrm{H} 10$ & 34.50 & 0.83 & 60.50 & 18.67 \\
\hline 1 & Soln Std & ref & 2 & 25 & STD-12-3 & 20.90 & 10.50 & 82.60 & 52.30 \\
\hline 1 & Soln Std & ref & 3 & 1 & STD-13-1 & 19.90 & 9.63 & 82.70 & 47.90 \\
\hline 1 & SB5NEPH-02ccc & $\mathrm{CCC}$ & 3 & 2 & H53 & 380.01 & 0.83 & 740.01 & 61.17 \\
\hline 1 & SB5NEPH-09ссс & CCC & 3 & 3 & H54 & 3.27 & 0.83 & 7.95 & 18.67 \\
\hline 1 & SB5NEPH-04 & quenched & 3 & 4 & H06 & 166.67 & 0.83 & 415.01 & 54.00 \\
\hline 1 & SB5NEPH-08ccC & $\mathrm{CCC}$ & 3 & 5 & $\mathrm{H} 24$ & 28.50 & 0.83 & 46.33 & 13.05 \\
\hline 1 & SB5NEPH-07 & quenched & 3 & 6 & H07 & 3.83 & 0.83 & 39.83 & 17.67 \\
\hline 1 & SB5NEPH-08 & quenched & 3 & 7 & H05 & 35.33 & 0.83 & 61.33 & 13.85 \\
\hline 1 & EA & ref & 3 & 8 & $\mathrm{H} 27$ & 623.33 & 181.67 & 1660.00 & 861.67 \\
\hline
\end{tabular}


WSRC-STI-2007-00659

Revision 0

Appendix B

Table B2. PSAL Measurements of the PCT Solutions for the Study Glasses After Appropriate Adjustments. (continued)

\begin{tabular}{|c|c|c|c|c|c|c|c|c|c|}
\hline Set & Glass ID & Heat Treatment & Block & Seq & Lab ID & B (ppm) & Li (ppm) & $\mathrm{Na}(\mathrm{ppm})$ & Si (ppm) \\
\hline 1 & SB5NEPH-01 & quenched & 3 & 9 & H46 & 12.38 & 0.83 & 301.67 & 144.34 \\
\hline 1 & SB5NEPH-01ccc & $\mathrm{CCC}$ & 3 & 10 & H52 & 11.88 & 0.83 & 281.67 & 142.67 \\
\hline 1 & SB5NEPH-03ccC & CCC & 3 & 11 & H30 & 21.83 & 0.83 & 326.67 & 74.67 \\
\hline 1 & SB5NEPH-03 & quenched & 3 & 12 & H37 & 6.68 & 0.83 & 235.00 & 48.17 \\
\hline 1 & Soln Std & ref & 3 & 13 & STD-13-2 & 20.40 & 9.87 & 85.90 & 49.00 \\
\hline 1 & SB5NEPH-05CCC & $\mathrm{CCC}$ & 3 & 14 & H51 & 3.42 & 0.83 & 43.50 & 40.17 \\
\hline 1 & SB5NEPH-10ccC & $\mathrm{CCC}$ & 3 & 15 & H60 & 12.97 & 0.83 & 10.55 & 19.33 \\
\hline 1 & SB5NEPH-05 & quenched & 3 & 16 & $\mathrm{H} 45$ & 4.88 & 0.83 & 59.00 & 51.83 \\
\hline 1 & SB5NEPH-06ccC & $\mathrm{CCC}$ & 3 & 17 & H68 & 64.33 & 0.83 & 89.67 & 38.83 \\
\hline 1 & blank & ref & 3 & 18 & H31 & 0.08 & 0.83 & 0.08 & 0.08 \\
\hline 1 & SB5NEPH-10 & quenched & 3 & 19 & H59 & 8.55 & 0.83 & 7.57 & 13.92 \\
\hline 1 & SB5NEPH-07cCC & $\mathrm{CCC}$ & 3 & 20 & H55 & 2.25 & 0.83 & 38.50 & 17.33 \\
\hline 1 & SB5NEPH-06 & quenched & 3 & 21 & H66 & 1.09 & 0.83 & 9.47 & 15.10 \\
\hline 1 & SB5NEPH-09 & quenched & 3 & 22 & H22 & 2.42 & 0.83 & 4.35 & 10.70 \\
\hline 1 & SB5NEPH-02 & quenched & 3 & 23 & H67 & 421.68 & 0.83 & 888.35 & 53.17 \\
\hline 1 & ARM-1 & ref & 3 & 24 & H04 & 18.17 & 13.27 & 36.67 & 56.83 \\
\hline 1 & SB5NEPH-04cCC & $\mathrm{CCC}$ & 3 & 25 & H47 & 166.67 & 0.83 & 446.68 & 58.67 \\
\hline 1 & Soln Std & ref & 3 & 26 & STD-13-3 & 20.60 & 9.66 & 86.50 & 47.10 \\
\hline 2 & Soln Std & ref & 1 & 1 & STD-21-1 & 20.60 & 9.89 & 80.90 & 49.30 \\
\hline 2 & SB5NEPH-17 & quenched & 1 & 2 & I 55 & 10.83 & 0.83 & 226.67 & 95.84 \\
\hline 2 & SB5NEPH-13cCC & CCC & 1 & 3 & I 18 & 66.50 & 0.83 & 2200.04 & 368.34 \\
\hline 2 & blank & ref & 1 & 4 & I 02 & 0.83 & 0.83 & 0.83 & 0.83 \\
\hline 2 & SB5NEPH-14cCC & $\mathrm{CCC}$ & 1 & 5 & I 06 & 886.68 & 0.83 & 2383.38 & 208.34 \\
\hline 2 & SB5NEPH-19 & quenched & 1 & 6 & I 61 & 14.88 & 0.83 & 326.67 & 42.50 \\
\hline 2 & EA & ref & 1 & 7 & I 25 & 686.67 & 193.33 & 2716.67 & 906.67 \\
\hline 2 & SB5NEPH-11 & quenched & 1 & 8 & I 56 & 4.62 & 0.83 & 7.85 & 10.05 \\
\hline 2 & SB5NEPH-18ccC & $\mathrm{CCC}$ & 1 & 9 & I 42 & 221.67 & 0.83 & 455.01 & 33.17 \\
\hline 2 & SB5NEPH-20 & quenched & 1 & 10 & I 65 & 157.34 & 0.83 & 416.68 & 20.83 \\
\hline 2 & SB5NEPH-11cCC & $\mathrm{CCC}$ & 1 & 11 & I 64 & 12.25 & 0.83 & 17.00 & 10.88 \\
\hline 2 & SB5NEPH-12 & quenched & 1 & 12 & I 05 & 17.17 & 0.83 & 14.05 & 7.20 \\
\hline 2 & Soln Std & ref & 1 & 13 & STD-21-2 & 20.90 & 9.95 & 89.60 & 48.80 \\
\hline 2 & SB5NEPH-12ccc & $\mathrm{CCC}$ & 1 & 14 & I 39 & 11.30 & 0.83 & 9.93 & 9.02 \\
\hline 2 & SB5NEPH-16 & quenched & 1 & 15 & I 07 & 303.34 & 0.83 & 995.02 & 72.33 \\
\hline 2 & SB5NEPH-18 & quenched & 1 & 16 & I 29 & 241.67 & 0.83 & 468.34 & 33.33 \\
\hline 2 & SB5NEPH-15 & quenched & 1 & 17 & I 44 & 35.50 & 0.83 & 1733.37 & 170.00 \\
\hline 2 & SB5NEPH-19ccc & $\mathrm{CCC}$ & 1 & 18 & I 63 & 64.50 & 0.83 & 1503.36 & 83.50 \\
\hline 2 & SB5NEPH-20ccC & $\mathrm{CCC}$ & 1 & 19 & I 17 & 62.33 & 0.83 & 190.00 & 18.67 \\
\hline 2 & SB5NEPH-13 & quenched & 1 & 20 & I 20 & 47.17 & 0.83 & 2083.38 & 361.67 \\
\hline 2 & SB5NEPH-14 & quenched & 1 & 21 & I 68 & 1206.69 & 0.83 & 3100.06 & 256.67 \\
\hline 2 & ARM-1 & ref & 1 & 22 & I 30 & 22.00 & 14.02 & 40.00 & 61.17 \\
\hline 2 & SB5NEPH-15ccC & $\mathrm{CCC}$ & 1 & 23 & I 03 & 130.67 & 0.83 & 6833.47 & 435.01 \\
\hline 2 & SB5NEPH-17ccC & CCC & 1 & 24 & I 12 & 162.67 & 0.83 & 1360.03 & 236.67 \\
\hline 2 & SB5NEPH-16сcC & CCC & 1 & 25 & I 01 & 318.34 & 0.83 & 1030.02 & 74.33 \\
\hline 2 & Soln Std & ref & 1 & 26 & STD-21-3 & 23.60 & 10.30 & 86.00 & 50.90 \\
\hline 2 & Soln Std & ref & 2 & 1 & STD-22-1 & 20.00 & 9.63 & 82.70 & 49.50 \\
\hline 2 & SB5NEPH-16сcC & $\mathrm{CCC}$ & 2 & 2 & I 31 & 330.01 & 0.83 & 1010.02 & 78.33 \\
\hline 2 & SB5NEPH-16 & quenched & 2 & 3 & I 10 & 296.67 & 0.83 & 961.69 & 70.67 \\
\hline 2 & SB5NEPH-12ccC & CCC & 2 & 4 & I 28 & 12.32 & 0.83 & 12.52 & 8.68 \\
\hline 2 & SB5NEPH-12 & quenched & 2 & 5 & I 62 & 16.00 & 0.83 & 10.92 & 6.90 \\
\hline 2 & SB5NEPH-15 & quenched & 2 & 6 & I 48 & 33.83 & 0.83 & 1816.70 & 175.00 \\
\hline 2 & SB5NEPH-17cCC & $\mathrm{CCC}$ & 2 & 7 & I 38 & 151.17 & 0.83 & 1268.36 & 225.00 \\
\hline 2 & SB5NEPH-18ccc & $\mathrm{CCC}$ & 2 & 8 & I 15 & 223.34 & 0.83 & 453.34 & 34.00 \\
\hline 2 & SB5NEPH-13 & quenched & 2 & 9 & I 34 & 46.17 & 0.83 & 2100.04 & 353.34 \\
\hline 2 & SB5NEPH-20ccC & CCC & 2 & 10 & I 57 & 63.67 & 0.83 & 176.67 & 18.67 \\
\hline 2 & SB5NEPH-11ccC & $\mathrm{CCC}$ & 2 & 11 & I 13 & 10.33 & 0.83 & 15.95 & 10.68 \\
\hline 2 & SB5NEPH-18 & quenched & 2 & 12 & I 11 & 243.34 & 0.83 & 491.68 & 33.67 \\
\hline 2 & Soln Std & ref & 2 & 13 & STD-22-2 & 21.10 & 9.72 & 87.30 & 49.50 \\
\hline 2 & SB5NEPH-19 & quenched & 2 & 14 & I 24 & 9.67 & 0.83 & 330.01 & 42.17 \\
\hline 2 & SB5NEPH-15сCC & $\mathrm{CCC}$ & 2 & 15 & I 52 & 165.17 & 0.83 & 6633.47 & 448.34 \\
\hline
\end{tabular}


WSRC-STI-2007-00659

Revision 0

Appendix B

Table B2. PSAL Measurements of the PCT Solutions for the Study Glasses After Appropriate Adjustments. (continued)

\begin{tabular}{|c|c|c|c|c|c|c|c|c|c|}
\hline Set & Glass ID & Heat Treatment & Block & Seq & Lab ID & B (ppm) & $\mathrm{Li}(\mathrm{ppm})$ & $\mathrm{Na}(\mathrm{ppm})$ & Si (ppm) \\
\hline 2 & SB5NEPH-19ссс & $\mathrm{CCC}$ & 2 & 16 & I 33 & 61.67 & 0.83 & 1390.03 & 83.67 \\
\hline 2 & SB5NEPH-17 & quenched & 2 & 17 & I 23 & 10.48 & 0.83 & 241.67 & 94.00 \\
\hline 2 & SB5NEPH-14 & quenched & 2 & 18 & I 36 & 1156.69 & 0.83 & 3083.40 & 251.67 \\
\hline 2 & SB5NEPH-14ccC & CCC & 2 & 19 & I 45 & 890.02 & 0.83 & 2466.72 & 206.67 \\
\hline 2 & EA & ref & 2 & 20 & I 53 & 768.33 & 190.00 & 2116.67 & 923.34 \\
\hline 2 & ARM-1 & ref & 2 & 21 & I 51 & 18.83 & 14.38 & 37.83 & 63.00 \\
\hline 2 & SB5NEPH-11 & quenched & 2 & 22 & I 14 & 7.75 & 0.83 & 7.78 & 9.82 \\
\hline 2 & SB5NEPH-20 & quenched & 2 & 23 & I 49 & 165.00 & 0.83 & 471.68 & 22.67 \\
\hline 2 & SB5NEPH-13ссс & CCC & 2 & 24 & I 66 & 76.33 & 0.83 & 2350.05 & 388.34 \\
\hline 2 & Soln Std & ref & 2 & 25 & STD-22-3 & 21.40 & 9.51 & 86.60 & 47.50 \\
\hline 2 & Soln Std & ref & 3 & 1 & STD-23-1 & 19.20 & 9.67 & 84.30 & 49.00 \\
\hline 2 & SB5NEPH-18 & quenched & 3 & 2 & I 41 & 233.34 & 0.83 & 453.34 & 34.17 \\
\hline 2 & SB5NEPH-18ccc & CCC & 3 & 3 & I 04 & 223.34 & 0.83 & 681.68 & 35.50 \\
\hline 2 & SB5NEPH-20 & quenched & 3 & 4 & I 16 & 159.50 & 0.83 & 416.68 & 23.50 \\
\hline 2 & SB5NEPH-14 & quenched & 3 & 5 & I 46 & 1178.36 & 0.83 & 2916.73 & 245.00 \\
\hline 2 & SB5NEPH-16сcс & CCC & 3 & 6 & I 37 & 320.01 & 0.83 & 988.35 & 74.00 \\
\hline 2 & SB5NEPH-20сcC & $\mathrm{CCC}$ & 3 & 7 & I 21 & 65.00 & 0.83 & 211.67 & 19.83 \\
\hline 2 & SB5NEPH-12ccc & $\mathrm{CCC}$ & 3 & 8 & I 26 & 13.55 & 0.83 & 9.42 & 10.65 \\
\hline 2 & SB5NEPH-11 & quenched & 3 & 9 & I 35 & 3.93 & 0.83 & 7.20 & 12.07 \\
\hline 2 & blank & ref & 3 & 10 & I 50 & 0.83 & 0.83 & 0.83 & 0.83 \\
\hline 2 & SB5NEPH-13 & quenched & 3 & 11 & I 40 & 45.33 & 0.83 & 1983.37 & 350.01 \\
\hline 2 & SB5NEPH-19ссс & CCC & 3 & 12 & I 58 & 61.00 & 0.83 & 1425.03 & 81.50 \\
\hline 2 & Soln Std & ref & 3 & 13 & STD-23-2 & 19.50 & 9.63 & 85.20 & 48.40 \\
\hline 2 & SB5NEPH-19 & quenched & 3 & 14 & I 59 & 7.97 & 0.83 & 326.67 & 44.67 \\
\hline 2 & SB5NEPH-15сcс & CCC & 3 & 15 & I 22 & 128.84 & 0.83 & 6366.79 & 426.68 \\
\hline 2 & SB5NEPH-17ссс & CCC & 3 & 16 & I 47 & 156.67 & 0.83 & 1401.69 & 233.34 \\
\hline 2 & SB5NEPH-11ccC & CCC & 3 & 17 & I 32 & 8.72 & 0.83 & 17.67 & 13.10 \\
\hline 2 & ARM-1 & ref & 3 & 18 & I 67 & 17.00 & 13.82 & 38.50 & 62.17 \\
\hline 2 & SB5NEPH-16 & quenched & 3 & 19 & I 54 & 300.01 & 0.83 & 981.69 & 71.67 \\
\hline 2 & SB5NEPH-15 & quenched & 3 & 20 & I 60 & 36.17 & 0.83 & 1700.03 & 183.34 \\
\hline 2 & SB5NEPH-13ссс & $\mathrm{CCC}$ & 3 & 21 & I 08 & 67.50 & 0.83 & 2216.71 & 371.67 \\
\hline 2 & EA & ref & 3 & 22 & I 19 & 611.67 & 188.33 & 1883.34 & 880.00 \\
\hline 2 & SB5NEPH-14ccc & $\mathrm{CCC}$ & 3 & 23 & I 09 & 911.68 & 0.83 & 2416.72 & 205.00 \\
\hline 2 & SB5NEPH-17 & quenched & 3 & 24 & I 43 & 13.98 & 0.83 & 245.00 & 96.34 \\
\hline 2 & SB5NEPH-12 & quenched & 3 & 25 & I 27 & 16.47 & 0.83 & 14.45 & 8.98 \\
\hline 2 & Soln Std & ref & 3 & 26 & STD-23-3 & 20.50 & 9.73 & 86.60 & 49.20 \\
\hline 3 & Soln Std & ref & 1 & 1 & STD-31-1 & 20.40 & 9.63 & 79.00 & 48.70 \\
\hline 3 & SB5NEPH-22ccC & CCC & 1 & 2 & J38 & 54.67 & 0.83 & 78.33 & 43.17 \\
\hline 3 & SB5NEPH-22 & quenched & 1 & 3 & J35 & 27.33 & 0.83 & 46.67 & 36.17 \\
\hline 3 & SB5NEPH-29сcс & CCC & 1 & 4 & J22-missing &. &. & . &. \\
\hline 3 & SB5NEPH-21 & quenched & 1 & 5 & J26 & 2.27 & 0.83 & 43.83 & 35.50 \\
\hline 3 & SB5NEPH-30сcC & CCC & 1 & 6 & J39 & 208.34 & 0.83 & 3550.07 & 366.67 \\
\hline 3 & SB5NEPH-23сcC & $\mathrm{CCC}$ & 1 & 7 & $\mathrm{~J} 01$ & 7.90 & 0.83 & 18.83 & 10.40 \\
\hline 3 & SB5NEPH-27 & quenched & 1 & 8 & J29 & 1.07 & 0.83 & 6.58 & 7.92 \\
\hline 3 & blank & ref & 1 & 9 & $\mathrm{~J} 50$ & 0.08 & 0.83 & 0.08 & 0.08 \\
\hline 3 & SB5NEPH-29 & quenched & 1 & 10 & J19 & 1231.69 & 0.83 & 20833.75 & 2316.71 \\
\hline 3 & SB5NEPH-27ccс & $\mathrm{CCC}$ & 1 & 11 & J36 & 230.00 & 0.83 & 98.00 & 0.38 \\
\hline 3 & SB5NEPH-25сcC & CCC & 1 & 12 & J51 & 7.83 & 0.83 & 9.87 & 17.17 \\
\hline 3 & Soln Std & ref & 1 & 13 & STD-31-2 & 22.40 & 9.92 & 83.80 & 49.50 \\
\hline 3 & SB5NEPH-24 & quenched & 1 & 14 & J04 & 50.67 & 0.83 & 95.50 & 6.47 \\
\hline 3 & SB5NEPH-23 & quenched & 1 & 15 & J59 & 3.88 & 0.83 & 40.67 & 13.57 \\
\hline 3 & SB5NEPH-24ccC & $\mathrm{CCC}$ & 1 & 16 & $\mathrm{~J} 55$ & 18.33 & 0.83 & 31.00 & 7.93 \\
\hline 3 & SB5NEPH-21ccc & $\mathrm{CCC}$ & 1 & 17 & $\mathrm{~J} 24$ & 186.67 & 0.83 & 265.01 & 24.33 \\
\hline 3 & SB5NEPH-30 & quenched & 1 & 18 & J62 & 5466.78 & 0.83 & 17333.68 & 890.02 \\
\hline 3 & SB5NEPH-28ccC & $\mathrm{CCC}$ & 1 & 19 & $\mathrm{~J} 46$ & 47.50 & 0.83 & 7.17 & 6.45 \\
\hline 3 & ARM-1 & ref & 1 & 20 & $\mathrm{~J} 54$ & 39.67 & 12.72 & 36.67 & 55.50 \\
\hline 3 & SB5NEPH-28 & quenched & 1 & 21 & $\mathrm{~J} 40$ & 32.83 & 0.83 & 11.50 & 4.70 \\
\hline 3 & SB5NEPH-25 & quenched & 1 & 22 & $\mathrm{~J} 42$ & 13.73 & 0.83 & 9.23 & 13.23 \\
\hline 3 & SB5NEPH-26сCC & CCC & 1 & 23 & $\mathrm{~J} 23$ & 30.67 & 0.83 & 22.83 & 24.67 \\
\hline
\end{tabular}


WSRC-STI-2007-00659

Revision 0

Appendix B

Table B2. PSAL Measurements of the PCT Solutions for the Study Glasses After Appropriate Adjustments. (continued)

\begin{tabular}{|c|c|c|c|c|c|c|c|c|c|}
\hline Set & Glass ID & Heat Treatment & Block & Seq & Lab ID & B (ppm) & $\mathrm{Li}(\mathrm{ppm})$ & $\mathrm{Na}(\mathrm{ppm})$ & Si (ppm) \\
\hline 3 & SB5NEPH-26 & quenched & 1 & 24 & $\mathrm{~J} 20$ & 14.25 & 0.83 & 10.47 & 10.98 \\
\hline 3 & EA & ref & 1 & 25 & J37 & 691.67 & 193.33 & 2000.00 & 891.67 \\
\hline 3 & Soln Std & ref & 1 & 26 & STD-31-3 & 23.20 & 9.89 & 85.50 & 48.60 \\
\hline 3 & Soln Std & ref & 2 & 1 & STD-32-1 & 19.50 & 9.71 & 79.40 & 49.30 \\
\hline 3 & SB5NEPH-21ccC & CCC & 2 & 2 & J64 & 181.67 & 0.83 & 236.67 & 24.17 \\
\hline 3 & SB5NEPH-28ccC & $\mathrm{CCC}$ & 2 & 3 & $\mathrm{~J} 03$ & 7.77 & 0.83 & 5.47 & 7.08 \\
\hline 3 & SB5NEPH-29 & quenched & 2 & 4 & $\mathrm{~J} 17$ & 1221.69 & 0.83 & 20833.75 & 2350.05 \\
\hline 3 & SB5NEPH-30сcC & CCC & 2 & 5 & $\mathrm{~J} 10$ & 216.67 & 0.83 & 3266.73 & 343.34 \\
\hline 3 & ARM-1 & ref & 2 & 6 & $\mathrm{~J} 66$ & 19.67 & 12.52 & 33.17 & 56.17 \\
\hline 3 & SB5NEPH-29ссс & $\mathrm{CCC}$ & 2 & 7 & J58-missing &. & . &. & . \\
\hline 3 & SB5NEPH-21 & quenched & 2 & 8 & J30 & 5.02 & 0.83 & 41.00 & 34.83 \\
\hline 3 & SB5NEPH-22 & quenched & 2 & 9 & $\mathrm{~J} 15$ & 27.33 & 0.83 & 47.33 & 35.00 \\
\hline 3 & SB5NEPH-23сcс & CCC & 2 & 10 & $\mathrm{~J} 12$ & 6.53 & 0.83 & 18.00 & 10.63 \\
\hline 3 & SB5NEPH-27 & quenched & 2 & 11 & $\mathrm{~J} 11$ & 0.08 & 0.83 & 7.42 & 8.03 \\
\hline 3 & SB5NEPH-30 & quenched & 2 & 12 & J34 & 5583.45 & 0.83 & 17167.01 & 908.35 \\
\hline 3 & Soln Std & ref & 2 & 13 & STD-32-2 & 21.20 & 9.65 & 79.00 & 47.20 \\
\hline 3 & SB5NEPH-25 & quenched & 2 & 14 & J65 & 3.10 & 0.83 & 8.02 & 13.40 \\
\hline 3 & SB5NEPH-23 & quenched & 2 & 15 & $\mathrm{~J} 28$ & 2.57 & 0.83 & 38.67 & 13.52 \\
\hline 3 & SB5NEPH-24 & quenched & 2 & 16 & $\mathrm{~J} 08$ & 47.50 & 0.83 & 90.00 & 6.75 \\
\hline 3 & EA & ref & 2 & 17 & $\mathrm{~J} 44$ & 621.67 & 188.33 & 1816.67 & 876.67 \\
\hline 3 & SB5NEPH-26сcс & $\mathrm{CCC}$ & 2 & 18 & $\mathrm{~J} 02$ & 19.33 & 0.83 & 19.33 & 25.17 \\
\hline 3 & SB5NEPH-22ccc & $\mathrm{CCC}$ & 2 & 19 & $\mathrm{~J} 49$ & 58.67 & 0.83 & 81.83 & 41.67 \\
\hline 3 & SB5NEPH-26 & quenched & 2 & 20 & J32 & 4.78 & 0.83 & 8.13 & 11.27 \\
\hline 3 & SB5NEPH-25сcC & $\mathrm{CCC}$ & 2 & 21 & J68 & 0.08 & 0.83 & 9.45 & 17.17 \\
\hline 3 & SB5NEPH-24ccC & $\mathrm{CCC}$ & 2 & 22 & $\mathrm{~J} 61$ & 15.40 & 0.83 & 29.50 & 8.28 \\
\hline 3 & SB5NEPH-28 & quenched & 2 & 23 & J09 & 11.30 & 0.83 & 10.30 & 4.80 \\
\hline 3 & SB5NEPH-27ccC & $\mathrm{CCC}$ & 2 & 24 & J16 & 245.00 & 0.83 & 95.84 & 0.57 \\
\hline 3 & Soln Std & ref & 2 & 25 & STD-32-3 & 19.70 & 9.79 & 82.30 & 49.60 \\
\hline 3 & Soln Std & ref & 3 & 1 & STD-33-1 & 19.80 & 9.73 & 81.20 & 48.70 \\
\hline 3 & SB5NEPH-28 & quenched & 3 & 2 & J56 & 13.67 & 0.83 & 10.12 & 5.18 \\
\hline 3 & SB5NEPH-22 & quenched & 3 & 3 & $\mathrm{~J} 67$ & 28.83 & 0.83 & 48.00 & 37.50 \\
\hline 3 & SB5NEPH-30 & quenched & 3 & 4 & $\mathrm{~J} 25$ & 5516.78 & 0.83 & 17000.34 & 1303.36 \\
\hline 3 & SB5NEPH-26 & quenched & 3 & 5 & $\mathrm{~J} 05$ & 32.50 & 0.83 & 7.60 & 11.23 \\
\hline 3 & SB5NEPH-27 & quenched & 3 & 6 & $\mathrm{~J} 60$ & 18.50 & 0.83 & 6.35 & 8.35 \\
\hline 3 & blank & ref & 3 & 7 & $\mathrm{~J} 53$ & 13.52 & 0.83 & 0.08 & 0.08 \\
\hline 3 & SB5NEPH-28ccC & $\mathrm{CCC}$ & 3 & 8 & J06 & 19.83 & 0.83 & 6.03 & 7.38 \\
\hline 3 & SB5NEPH-23сcC & CCC & 3 & 9 & $\mathrm{~J} 14$ & 16.25 & 0.83 & 19.00 & 10.93 \\
\hline 3 & SB5NEPH-27ccC & CCC & 3 & 10 & $\mathrm{~J} 18$ & 275.01 & 0.83 & 98.67 & 0.72 \\
\hline 3 & SB5NEPH-25 & quenched & 3 & 11 & $\mathrm{~J} 57$ & 9.07 & 0.83 & 8.13 & 13.65 \\
\hline 3 & SB5NEPH-25сcC & CCC & 3 & 12 & $\mathrm{~J} 52$ & 7.50 & 0.83 & 9.33 & 17.83 \\
\hline 3 & Soln Std & ref & 3 & 13 & STD-33-2 & 22.30 & 9.69 & 83.00 & 48.60 \\
\hline 3 & EA & ref & 3 & 14 & J07 & 655.00 & 188.33 & 1716.67 & 880.00 \\
\hline 3 & SB5NEPH-22ccC & $\mathrm{CCC}$ & 3 & 15 & $\mathrm{~J} 21$ & 62.83 & 0.83 & 82.67 & 43.33 \\
\hline 3 & SB5NEPH-24 & quenched & 3 & 16 & $\mathrm{~J} 63$ & 52.67 & 0.83 & 91.34 & 7.32 \\
\hline 3 & ARM-1 & ref & 3 & 17 & $\mathrm{~J} 47$ & 19.00 & 13.13 & 36.33 & 58.50 \\
\hline 3 & SB5NEPH-29сcс & CCC & 3 & 18 & J33-missing &. &. &. &. \\
\hline 3 & SB5NEPH-21 & quenched & 3 & 19 & $\mathrm{~J} 13$ & 4.73 & 0.83 & 42.33 & 34.83 \\
\hline 3 & SB5NEPH-26сcC & CCC & 3 & 20 & $\mathrm{~J} 27$ & 20.67 & 0.83 & 18.67 & 24.33 \\
\hline 3 & SB5NEPH-21ccC & CCC & 3 & 21 & $\mathrm{~J} 45$ & 185.00 & 0.83 & 256.67 & 25.50 \\
\hline 3 & SB5NEPH-23 & quenched & 3 & 22 & $\mathrm{~J} 43$ & 3.92 & 0.83 & 39.33 & 13.80 \\
\hline 3 & SB5NEPH-29 & quenched & 3 & 23 & $\mathrm{~J} 41$ & 1355.03 & 0.83 & 20500.41 & 2350.05 \\
\hline 3 & SB5NEPH-24ccC & $\mathrm{CCC}$ & 3 & 24 & $\mathrm{~J} 48$ & 25.33 & 0.83 & 29.83 & 8.72 \\
\hline 3 & SB5NEPH-30сcс & $\mathrm{CCC}$ & 3 & 25 & J31 & 245.00 & 0.83 & 3433.40 & 385.01 \\
\hline 3 & Soln Std & ref & 3 & 26 & STD-33-3 & 20.00 & 9.66 & 81.10 & 46.90 \\
\hline 4 & Soln Std & ref & 1 & 1 & STD-41-1 & 20.80 & 9.92 & 81.30 & 51.00 \\
\hline 4 & SB5NEPH-34ccC & CCC & 1 & 2 & K17 & 370.01 & 0.83 & 525.01 & 14.05 \\
\hline 4 & ARM-1 & ref & 1 & 3 & K09 & 20.00 & 13.40 & 35.33 & 61.00 \\
\hline 4 & SB5NEPH-39 & quenched & 1 & 4 & K30 & 3.85 & 0.83 & 5.98 & 6.52 \\
\hline 4 & SB5NEPH-37 & quenched & 1 & 5 & $\mathrm{~K} 21$ & 4.15 & 0.83 & 13.42 & 17.17 \\
\hline
\end{tabular}


WSRC-STI-2007-00659

Revision 0

Appendix B

Table B2. PSAL Measurements of the PCT Solutions for the Study Glasses After Appropriate Adjustments. (continued)

\begin{tabular}{|c|c|c|c|c|c|c|c|c|c|}
\hline Set & Glass ID & Heat Treatment & Block & Seq & Lab ID & B (ppm) & $\mathrm{Li}(\mathrm{ppm})$ & $\mathrm{Na}(\mathrm{ppm})$ & Si (ppm) \\
\hline 4 & SB5NEPH-32ccC & $\mathrm{CCC}$ & 1 & 6 & K57 & 157.00 & 0.83 & 1153.36 & 102.84 \\
\hline 4 & SB5NEPH-31 & quenched & 1 & 7 & K27 & 848.35 & 0.83 & 18000.36 & 686.68 \\
\hline 4 & SB5NEPH-40 & quenched & 1 & 8 & K29 & 22.17 & 0.83 & 10.82 & 3.35 \\
\hline 4 & SB5NEPH-32 & quenched & 1 & 9 & K22 & 1058.35 & 0.83 & 3800.08 & 102.34 \\
\hline 4 & SB5NEPH-31ccc & CCC & 1 & 10 & K48 & 536.68 & 0.83 & 13366.93 & 758.35 \\
\hline 4 & SB5NEPH-38 & quenched & 1 & 11 & K46 & 24.83 & 0.83 & 13.07 & 13.40 \\
\hline 4 & EA & ref & 1 & 12 & K40 & 710.00 & 190.00 & 1733.34 & 918.34 \\
\hline 4 & Soln Std & ref & 1 & 13 & STD-41-2 & 22.20 & 9.85 & 82.10 & 51.90 \\
\hline 4 & SB5NEPH-39сcс & $\mathrm{CCC}$ & 1 & 14 & K14 & 300.01 & 0.83 & 98.34 & 0.83 \\
\hline 4 & SB5NEPH-36ссс & CCC & 1 & 15 & K67 & 1833.37 & 0.83 & 3266.73 & 4.43 \\
\hline 4 & SB5NEPH-38ccC & $\mathrm{CCC}$ & 1 & 16 & K65 & 96.34 & 0.83 & 59.00 & 37.00 \\
\hline 4 & SB5NEPH-33 & quenched & 1 & 17 & K32 & 1061.69 & 0.83 & 3700.07 & 64.17 \\
\hline 4 & SB5NEPH-37сcс & CCC & 1 & 18 & K26 & 17.67 & 0.83 & 12.68 & 18.50 \\
\hline 4 & SB5NEPH-34 & quenched & 1 & 19 & K42 & 165.50 & 0.83 & 358.34 & 29.33 \\
\hline 4 & SB5NEPH-36 & quenched & 1 & 20 & K05 & 159.17 & 0.83 & 398.34 & 9.22 \\
\hline 4 & SB5NEPH-40сcс & CCC & 1 & 21 & K06 & 20.17 & 0.83 & 7.43 & 5.87 \\
\hline 4 & SB5NEPH-33ссC & $\mathrm{CCC}$ & 1 & 22 & K38 & 388.34 & 0.83 & 3266.73 & 185.00 \\
\hline 4 & SB5NEPH-35 & quenched & 1 & 23 & K60 & 19.17 & 0.83 & 191.67 & 17.67 \\
\hline 4 & blank & ref & 1 & 24 & K59 & 7.85 & 0.83 & 0.83 & 0.83 \\
\hline 4 & SB5NEPH-35сcс & CCC & 1 & 25 & K44 & 85.67 & 0.83 & 1536.70 & 126.67 \\
\hline 4 & Soln Std & ref & 1 & 26 & STD-41-3 & 22.50 & 9.94 & 84.40 & 51.50 \\
\hline 4 & Soln Std & ref & 2 & 1 & STD-42-1 & 20.30 & 9.68 & 81.50 & 48.50 \\
\hline 4 & SB5NEPH-33ссс & $\mathrm{CCC}$ & 2 & 2 & K50 & 371.67 & 0.83 & 3200.06 & 173.34 \\
\hline 4 & SB5NEPH-38 & quenched & 2 & 3 & K36 & 17.33 & 0.83 & 13.55 & 12.67 \\
\hline 4 & SB5NEPH-33 & quenched & 2 & 4 & K33 & 1023.35 & 0.83 & 3683.41 & 61.33 \\
\hline 4 & ARM-1 & ref & 2 & 5 & K12 & 26.33 & 13.20 & 34.00 & 58.50 \\
\hline 4 & SB5NEPH-37 & quenched & 2 & 6 & K51 & 7.62 & 0.83 & 14.28 & 16.10 \\
\hline 4 & SB5NEPH-36 & quenched & 2 & 7 & K54 & 146.84 & 0.83 & 375.01 & 8.50 \\
\hline 4 & SB5NEPH-36ccc & CCC & 2 & 8 & K39 & 1883.37 & 0.83 & 3333.40 & 3.82 \\
\hline 4 & SB5NEPH-37ccC & $\mathrm{CCC}$ & 2 & 9 & K37 & 19.67 & 0.83 & 13.07 & 17.17 \\
\hline 4 & SB5NEPH-31 & quenched & 2 & 10 & K43 & 885.02 & 0.83 & 18333.70 & 715.01 \\
\hline 4 & SB5NEPH-39сcс & $\mathrm{CCC}$ & 2 & 11 & K45 & 325.01 & 0.83 & 99.17 & 0.83 \\
\hline 4 & SB5NEPH-32 & quenched & 2 & 12 & K34 & 1065.02 & 0.83 & 3850.08 & 97.17 \\
\hline 4 & Soln Std & ref & 2 & 13 & STD-42-2 & 20.20 & 9.58 & 83.90 & 46.80 \\
\hline 4 & SB5NEPH-40ccC & $\mathrm{CCC}$ & 2 & 14 & K47 & 19.67 & 0.83 & 6.80 & 5.08 \\
\hline 4 & SB5NEPH-35сcC & $\mathrm{CCC}$ & 2 & 15 & K56 & 83.17 & 0.83 & 1596.70 & 116.34 \\
\hline 4 & SB5NEPH-34 & quenched & 2 & 16 & K08 & 163.50 & 0.83 & 325.01 & 27.83 \\
\hline 4 & SB5NEPH-40 & quenched & 2 & 17 & K61 & 22.00 & 0.83 & 11.67 & 2.87 \\
\hline 4 & SB5NEPH-39 & quenched & 2 & 18 & K68 & 6.92 & 0.83 & 6.75 & 6.35 \\
\hline 4 & EA & ref & 2 & 19 & K16 & 671.67 & 186.67 & 1700.00 & 875.00 \\
\hline 4 & SB5NEPH-32ccC & CCC & 2 & 20 & K13 & 144.67 & 0.83 & 1141.69 & 97.84 \\
\hline 4 & SB5NEPH-34ccC & CCC & 2 & 21 & K24 & 358.34 & 0.83 & 550.01 & 12.30 \\
\hline 4 & SB5NEPH-35 & quenched & 2 & 22 & K64 & 15.88 & 0.83 & 193.34 & 15.52 \\
\hline 4 & SB5NEPH-31ccC & $\mathrm{CCC}$ & 2 & 23 & K58 & 543.34 & 0.83 & 13533.60 & 761.68 \\
\hline 4 & SB5NEPH-38сcс & $\mathrm{CCC}$ & 2 & 24 & K53 & 86.34 & 0.83 & 60.00 & 34.17 \\
\hline 4 & Soln Std & ref & 2 & 25 & STD-42-3 & 23.10 & 9.55 & 83.40 & 47.00 \\
\hline 4 & Soln Std & ref & 3 & 1 & STD-43-1 & 19.90 & 9.77 & 80.60 & 48.90 \\
\hline 4 & SB5NEPH-36ссC & CCC & 3 & 2 & K03 & 1916.71 & 0.83 & 3600.07 & 4.10 \\
\hline 4 & SB5NEPH-32ccc & $\mathrm{CCC}$ & 3 & 3 & K19 & 183.34 & 0.83 & 1203.36 & 105.34 \\
\hline 4 & SB5NEPH-40 & quenched & 3 & 4 & K63 & 24.83 & 0.83 & 11.28 & 3.20 \\
\hline 4 & EA & ref & 3 & 5 & K25 & 700.00 & 188.33 & 1716.67 & 905.00 \\
\hline 4 & SB5NEPH-40сcс & $\mathrm{CCC}$ & 3 & 6 & K02 & 13.87 & 0.83 & 7.25 & 5.68 \\
\hline 4 & SB5NEPH-33ссс & $\mathrm{CCC}$ & 3 & 7 & K23 & 380.01 & 0.83 & 3350.07 & 176.67 \\
\hline 4 & SB5NEPH-37сcс & CCC & 3 & 8 & K31 & 5.95 & 0.83 & 13.32 & 18.00 \\
\hline 4 & blank & ref & 3 & 9 & K01 & 1.73 & 0.83 & 0.83 & 0.83 \\
\hline 4 & SB5NEPH-34ccC & $\mathrm{CCC}$ & 3 & 10 & K66 & 381.67 & 0.83 & 528.34 & 13.03 \\
\hline 4 & SB5NEPH-39 & quenched & 3 & 11 & K11 & 4.57 & 0.83 & 6.77 & 6.57 \\
\hline 4 & ARM-1 & ref & 3 & 12 & K28 & 19.33 & 14.13 & 38.83 & 62.00 \\
\hline 4 & Soln Std & ref & 3 & 13 & STD-43-2 & 20.20 & 9.75 & 81.90 & 48.50 \\
\hline
\end{tabular}


WSRC-STI-2007-00659

Revision 0

Appendix B

Table B2. PSAL Measurements of the PCT Solutions for the Study Glasses After Appropriate Adjustments. (continued)

\begin{tabular}{|c|c|c|c|c|c|c|c|c|c||}
\hline Set & Glass ID & Heat Treatment & Block & Seq & Lab ID & B (ppm) & Li (ppm) & Na (ppm) & Si (ppm) \\
\hline 4 & SB5NEPH-35ccc & ccc & 3 & 14 & K10 & 77.00 & 0.83 & 1535.03 & 119.50 \\
\hline 4 & SB5NEPH-34 & quenched & 3 & 15 & K49 & 164.84 & 0.83 & 333.34 & 28.33 \\
\hline 4 & SB5NEPH-39ccc & ccc & 3 & 16 & K35 & 298.34 & 0.83 & 101.67 & 0.83 \\
\hline 4 & SB5NEPH-31 & quenched & 3 & 17 & K15 & 933.35 & 0.83 & 19167.05 & 753.35 \\
\hline 4 & SB5NEPH-35 & quenched & 3 & 18 & K55 & 17.33 & 0.83 & 195.00 & 17.83 \\
\hline 4 & SB5NEPH-37 & quenched & 3 & 19 & K20 & 5.10 & 0.83 & 16.35 & 16.22 \\
\hline 4 & SB5NEPH-38ccC & ccc & 3 & 20 & K07 & 81.33 & 0.83 & 62.83 & 35.17 \\
\hline 4 & SB5NEPH-38 & quenched & 3 & 21 & K18 & 14.88 & 0.83 & 15.48 & 13.03 \\
\hline 4 & SB5NEPH-36 & quenched & 3 & 22 & K41 & 147.84 & 0.83 & 410.01 & 9.07 \\
\hline 4 & SB5NEPH-32 & quenched & 3 & 23 & K62 & 956.69 & 0.83 & 3966.75 & 99.67 \\
\hline 4 & SB5NEPH-31ccC & ccc & 3 & 24 & K04 & 621.68 & 0.83 & 14316.95 & 851.68 \\
\hline 4 & SB5NEPH-33 & quenched & 3 & 25 & K52 & 1021.69 & 0.83 & 3883.41 & 63.33 \\
\hline 4 & Soln Std & ref & 3 & 26 & STD-43-3 & 20.20 & 9.78 & 80.30 & 49.40 \\
\hline
\end{tabular}


Exhibit B1. Laboratory PCT Measurements in Analytical Sequence for Study Glasses, EA, ARM, Blanks, and Solution Standards.

\section{B (ppm) By Analytical Sequence}

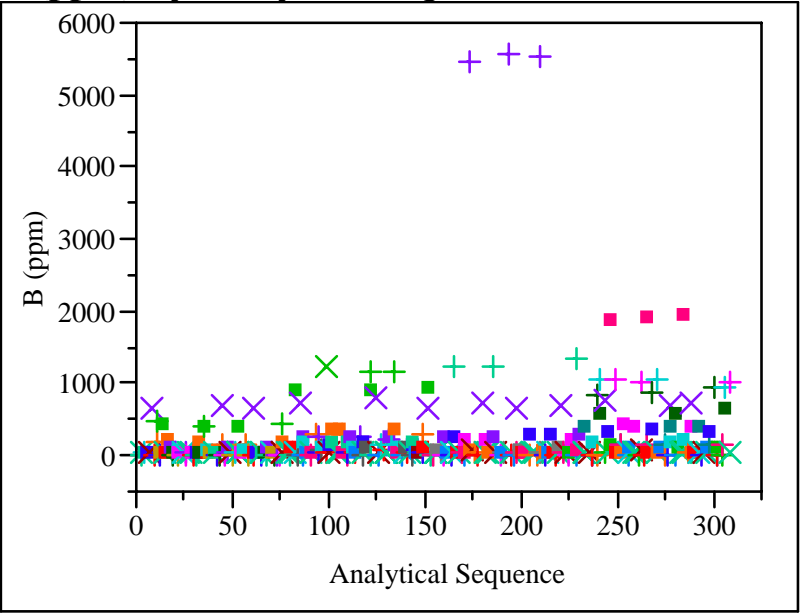

\section{Li (ppm) By Analytical Sequence}

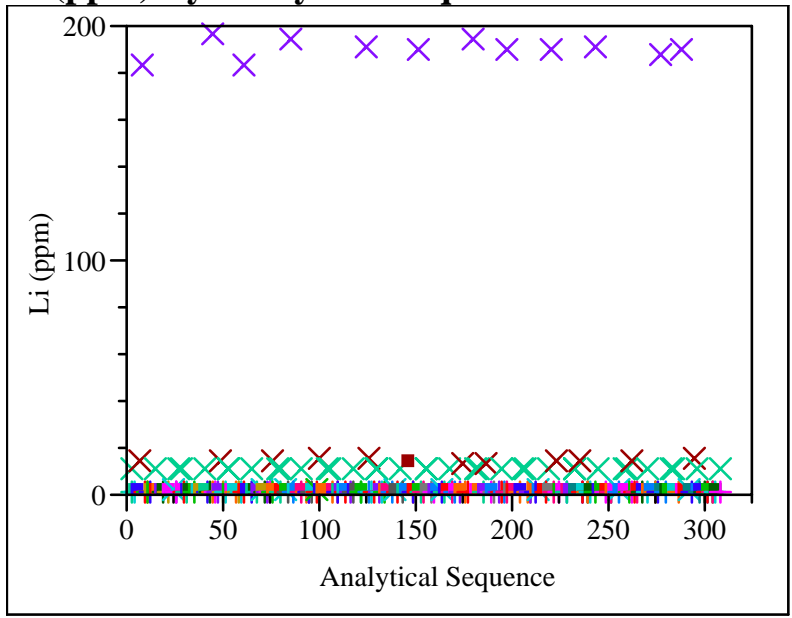

Na (ppm) By Analytical Sequence

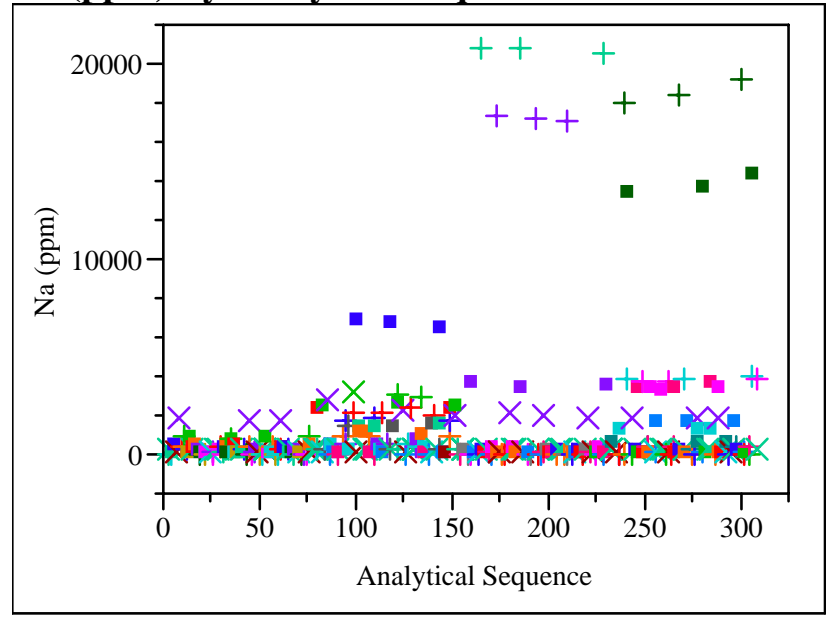

Si (ppm) By Analytical Sequence

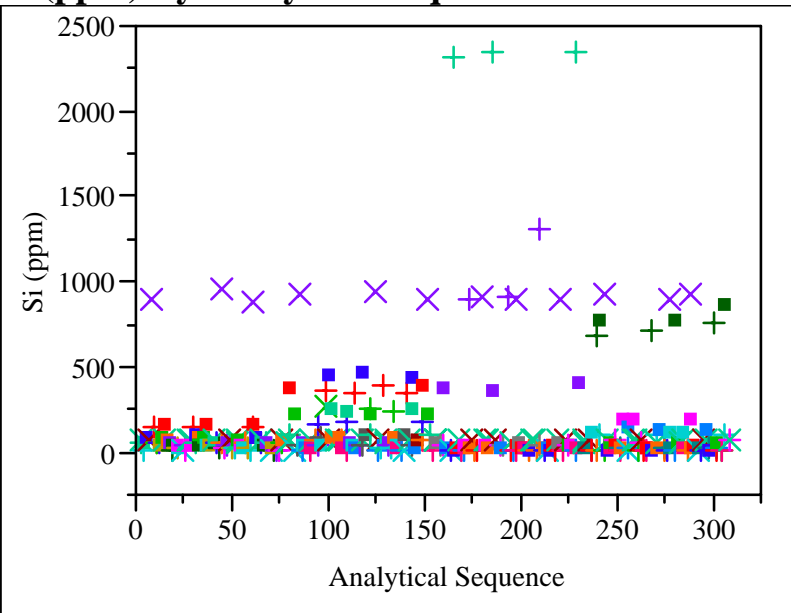


Exhibit B2. Laboratory PCT Measurements in Analytical Sequence for Study Glasses.

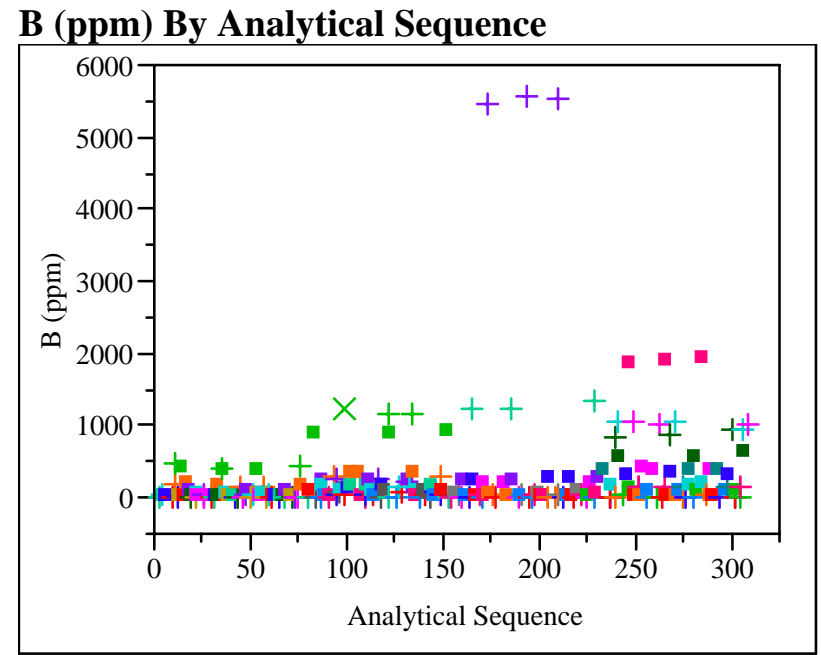

\section{Li (ppm) By Analytical Sequence}

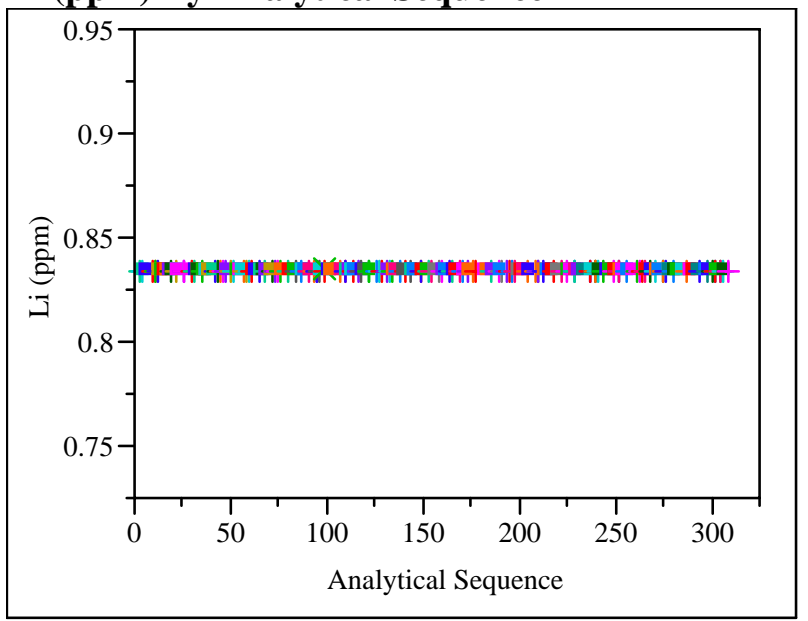

Na (ppm) By Analytical Sequence

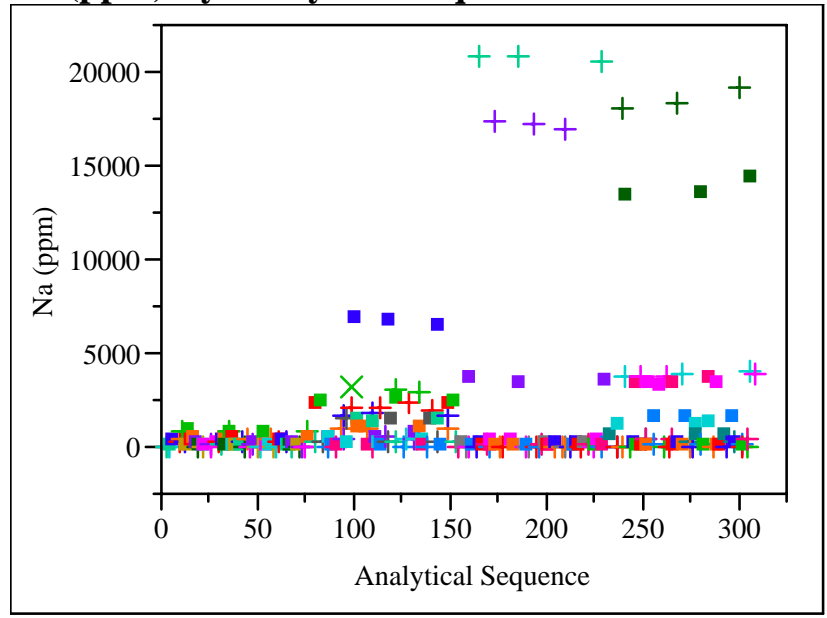

Si (ppm) By Analytical Sequence

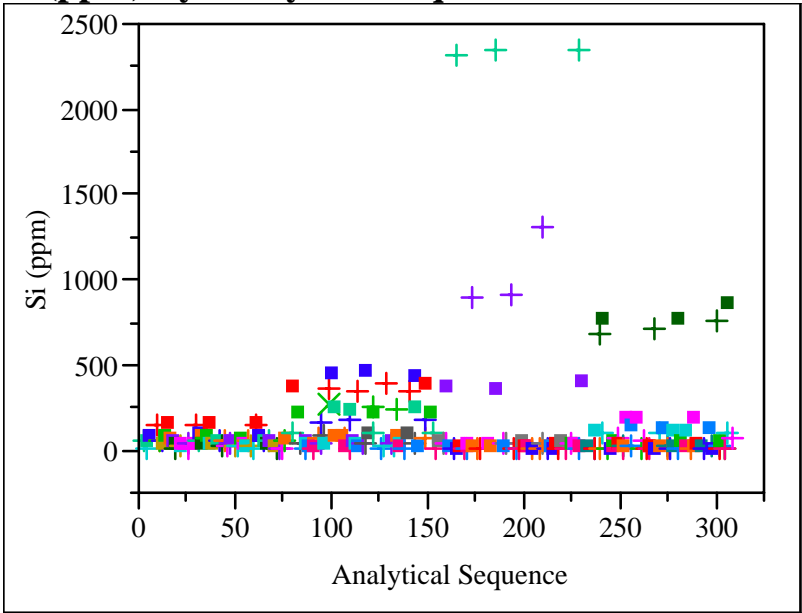


WSRC-STI-2007-00659

Revision 0

Appendix B

\section{Exhibit B3. Measurements of the Multi-Element Solution Standard by ICP Block.}

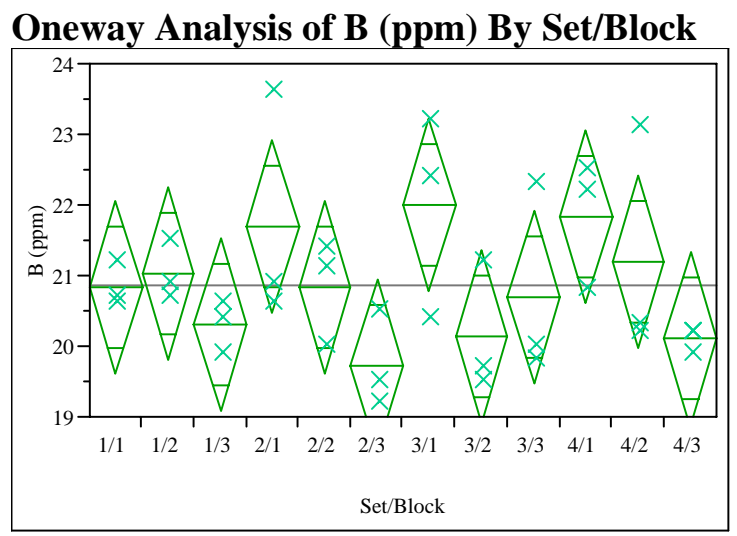

\section{Oneway Anova \\ Summary of Fit}

$\begin{array}{lr}\text { Rsquare } & 0.40939 \\ \text { Adj Rsquare } & 0.138693 \\ \text { Root Mean Square Error } & 1.023882 \\ \text { Mean of Response } & 20.86667 \\ \text { Observations (or Sum Wgts) } & 36\end{array}$

\section{Analysis of Variance}

Source DF Sum of Squares Mean Square F Ratio Prob $>$ F

$\begin{array}{llllll}\text { Set/Block } 11 & 17.440000 & 1.58545 & 1.5124 & 0.1912\end{array}$

$\begin{array}{llll}\text { Error } & 24 & 25.160000 & 1.04833\end{array}$

C. Total $35 \quad 42.600000$

\section{Means for Oneway Anova}

Level Number Mean Std Error Lower 95\% Upper 95\%

$\begin{array}{lllllr}1 / 1 & 3 & 20.8333 & 0.59114 & 19.613 & 22.053\end{array}$

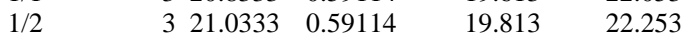

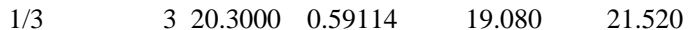

$\begin{array}{llllll}2 / 1 & 3 & 21.7000 & 0.59114 & 20.480 & 22.920\end{array}$

$\begin{array}{llllll}2 / 2 & 3 & 20.8333 & 0.59114 & 19.613 & 22.053\end{array}$

$\begin{array}{llllll}2 / 3 & 3 & 19.7333 & 0.59114 & 18.513 & 20.953\end{array}$

$\begin{array}{llllll}3 / 1 & 3 & 22.0000 & 0.59114 & 20.780 & 23.220\end{array}$

$\begin{array}{llllll}3 / 2 & 3 & 20.1333 & 0.59114 & 18.913 & 21.353\end{array}$

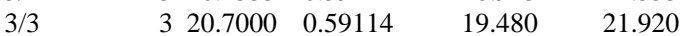

$\begin{array}{llllll}4 / 1 & 3 & 21.8333 & 0.59114 & 20.613 & 23.053\end{array}$

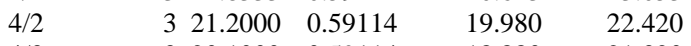

$\begin{array}{llllll}4 / 3 & 3 & 20.1000 & 0.59114 & 18.880 & 21.320\end{array}$

Std Error uses a pooled estimate of error variance

\section{Oneway Analysis of Li (ppm) By} Set/Block

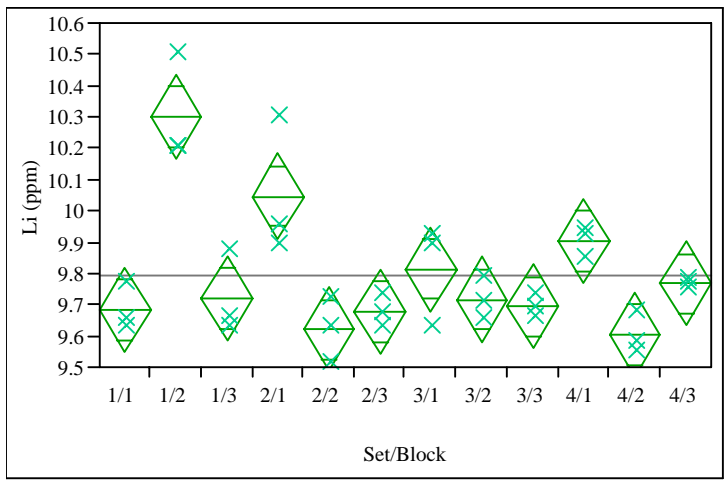

Oneway Anova

Summary of Fit

$\begin{array}{lr}\text { Rsquare } & 0.813079 \\ \text { Adj Rsquare } & 0.727407 \\ \text { Root Mean Square Error } & 0.113358 \\ \text { Mean of Response } & 9.795278 \\ \text { Observations (or Sum Wgts) } & 36\end{array}$

Analysis of Variance

Source DF Sum of Squares Mean Square F Ratio Prob $>$ F

$\begin{array}{llllll}\text { Set/Block } & 11 & 1.3414972 & 0.121954 & 9.4906 & <.0001\end{array}$

$\begin{array}{llll}\text { Error } & 24 & 0.3084000 & 0.012850\end{array}$

C. Total $35 \quad 1.6498972$

\section{Means for Oneway Anova}

Level Number Mean Std Error Lower 95\% Upper 95\%

$\begin{array}{llllll}1 / 1 & 3 & 9.6833 & 0.06545 & 9.548 & 9.818\end{array}$

$\begin{array}{llllll}1 / 2 & 3 & 10.3000 & 0.06545 & 10.165 & 10.435\end{array}$

$\begin{array}{llllll}1 / 3 & 3 & 9.7200 & 0.06545 & 9.585 & 9.855\end{array}$

$\begin{array}{llllll}2 / 1 & 3 & 10.0467 & 0.06545 & 9.912 & 10.182\end{array}$

$\begin{array}{llllll}2 / 2 & 3 & 9.6200 & 0.06545 & 9.485 & 9.755\end{array}$

$\begin{array}{llllll}2 / 3 & 3 & 9.6767 & 0.06545 & 9.542 & 9.812\end{array}$

$\begin{array}{llllll}3 / 1 & 3 & 9.8133 & 0.06545 & 9.678 & 9.948\end{array}$

$\begin{array}{llllll}3 / 2 & 3 & 9.7167 & 0.06545 & 9.582 & 9.852\end{array}$

$\begin{array}{llllll}3 / 3 & 3 & 9.6933 & 0.06545 & 9.558 & 9.828\end{array}$

$\begin{array}{llllll}4 / 1 & 3 & 9.9033 & 0.06545 & 9.768 & 10.038\end{array}$

$\begin{array}{llllll}4 / 2 & 3 & 9.6033 & 0.06545 & 9.468 & 9.738\end{array}$

$\begin{array}{llllll}4 / 3 & 3 & 9.7667 & 0.06545 & 9.632 & 9.902\end{array}$

Std Error uses a pooled estimate of error variance 
WSRC-STI-2007-00659

Revision 0

Appendix B

\section{Exhibit B3. Measurements of the Multi-Element Solution Standard by ICP Block. (continued)}

Oneway Analysis of Na (ppm) By

Set/Block

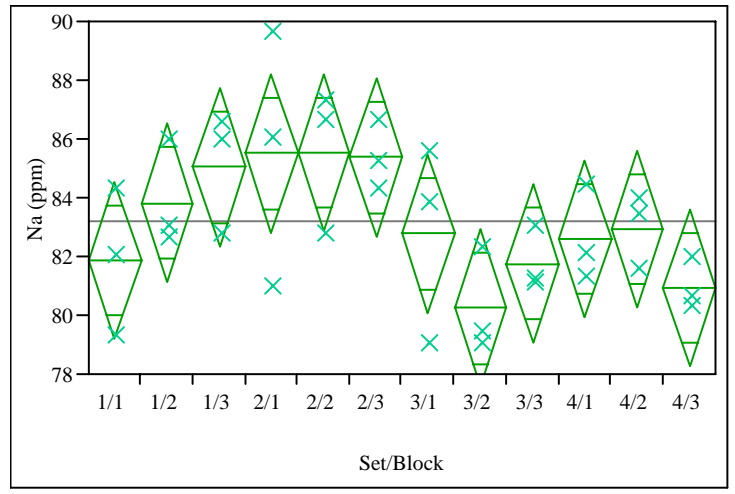

Oneway Anova

Summary of Fit

$\begin{array}{lr}\text { Rsquare } & 0.480515 \\ \text { Adj Rsquare } & 0.242417 \\ \text { Root Mean Square Error } & 2.253577 \\ \text { Mean of Response } & 83.19722 \\ \text { Observations (or Sum Wgts) } & 36\end{array}$

\section{Analysis of Variance}

Source

Set/Block

Error

C. Total

$\begin{array}{rr}\text { DF } & \text { Sum of Squares } \\ 11 & 112.74306 \\ 24 & 121.88667 \\ 35 & 234.62972\end{array}$

\section{Means for Oneway Anova}

Level Number Mean Std Error Lower 95\% Upper 95\%

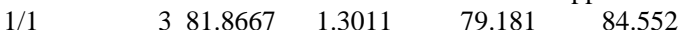

$\begin{array}{llllll}1 / 2 & 3 & 83.8333 & 1.3011 & 81.148 & 86.519\end{array}$

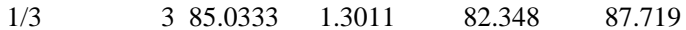

$\begin{array}{llllll}2 / 1 & 3 & 85.5000 & 1.3011 & 82.815 & 88.185\end{array}$

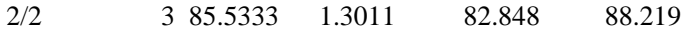

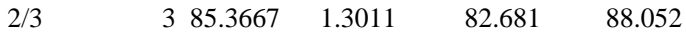

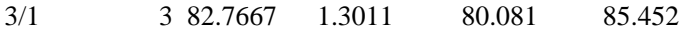

$\begin{array}{llllll}3 / 2 & 3 & 80.2333 & 1.3011 & 77.548 & 82.919\end{array}$

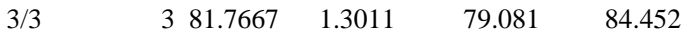

$\begin{array}{llllll}4 / 1 & 3 & 82.6000 & 1.3011 & 79.915 & 85.285\end{array}$

$\begin{array}{llllll}4 / 2 & 3 & 82.9333 & 1.3011 & 80.248 & 85.619\end{array}$

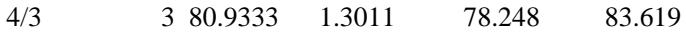

Std Error uses a pooled estimate of error variance
Oneway Analysis of Si (ppm) By Set/Block

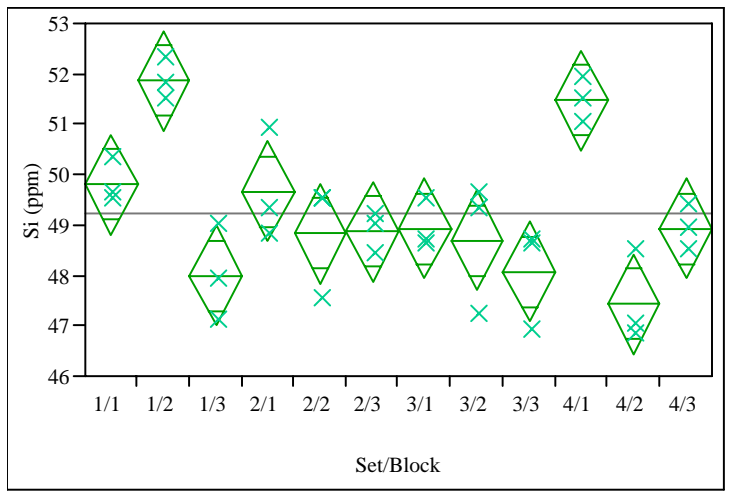

Oneway Anova
Summary of Fit

Rsquare $\quad 0.778922$

Adj Rsquare $\quad 0.677595$

Root Mean Square Error $\quad 0.827647$

Mean of Response 49.21389

Observations (or Sum Wgts) 36

Analysis of Variance

MEmurquare DF Sum of Squ RatioMean SquarePFolatib Prob > F $\begin{array}{llllll}\text { Set1Bl3484 } 11 & 57.9280381 & 5.26573 & \emptyset .6829 & <.0001\end{array}$

Errō̄.0786 $24 \quad 16.440000 \quad 0.68500$

$\begin{array}{lll}\text { C. Total } & 35 & 74.363056\end{array}$

\section{Means for Oneway Anova}

Level Number Mean Std Error Lower 95\% Upper 95\%

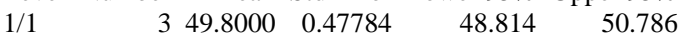

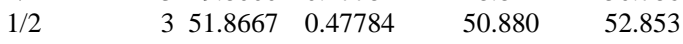

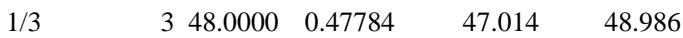

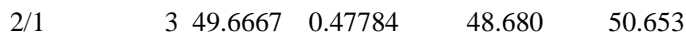

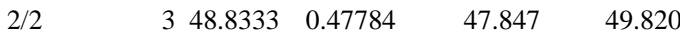

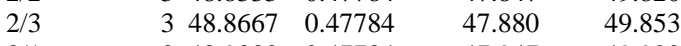

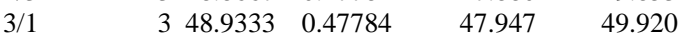

$\begin{array}{llllll}3 / 2 & 3 & 48.7000 & 0.47784 & 47.714 & 49.686\end{array}$

$\begin{array}{llllll}3 / 3 & 3 & 48.0667 & 0.47784 & 47.080 & 49.053\end{array}$

$\begin{array}{llllll}4 / 1 & 3 & 51.4667 & 0.47784 & 50.480 & 52.453\end{array}$

$\begin{array}{llllll}4 / 2 & 3 & 47.4333 & 0.47784 & 46.447 & 48.420\end{array}$

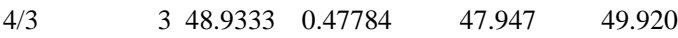

Std Error uses a pooled estimate of error variance 
Exhibit B4. Laboratory PCT Measurements by Glass Number for Study Glasses and Standards.

(100 - Solution Standard; 101 - EA; 102 - ARM; 103 - Blanks)

Variability Gage Set $=1$

Variability Chart for B (ppm)

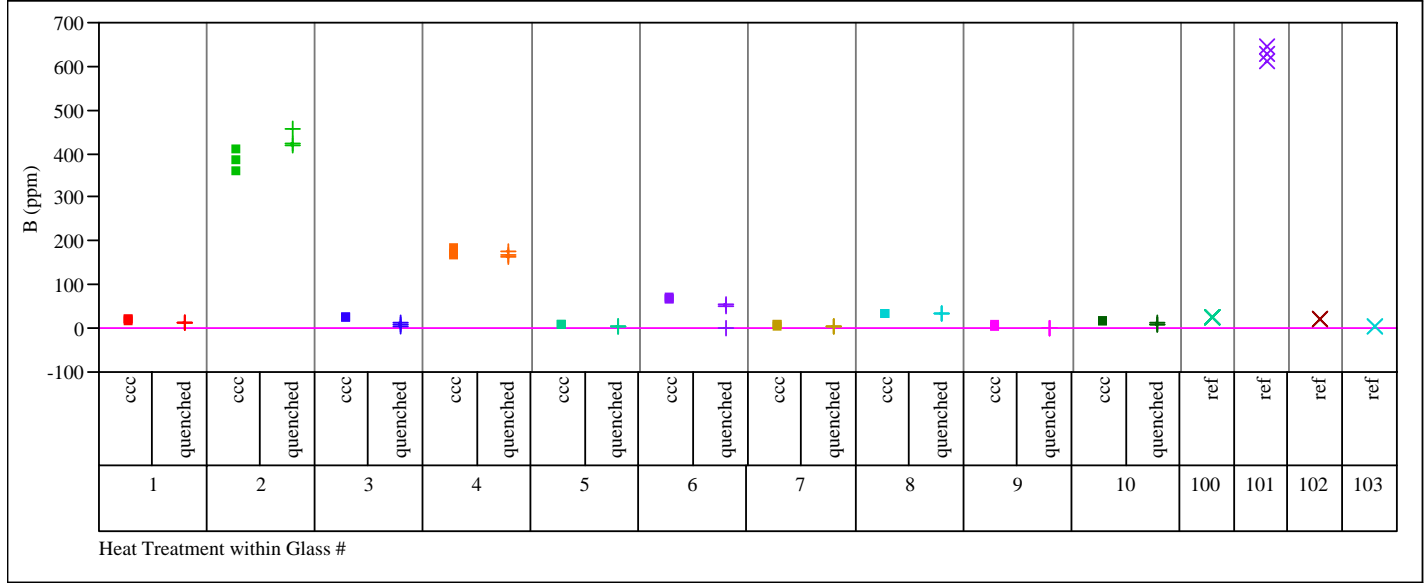

Variability Gage Set $=1$

Variability Chart for Li (ppm)

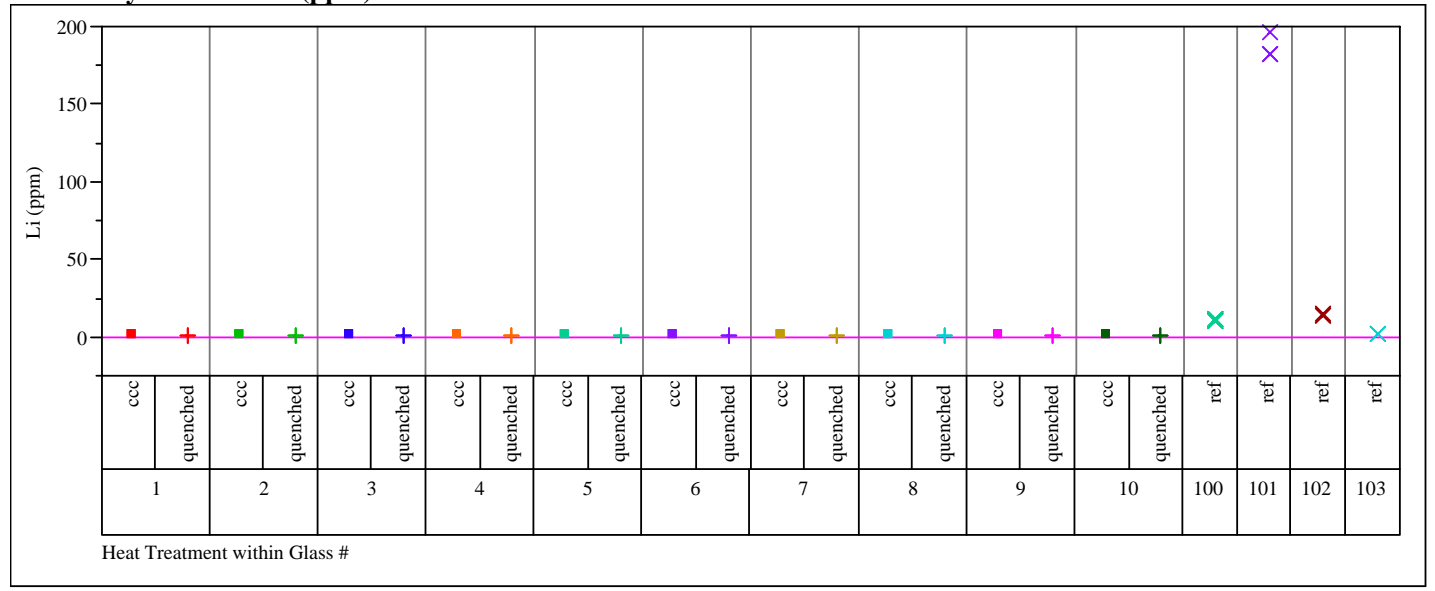

Variability Gage Set $=1$

Variability Chart for Na (ppm)

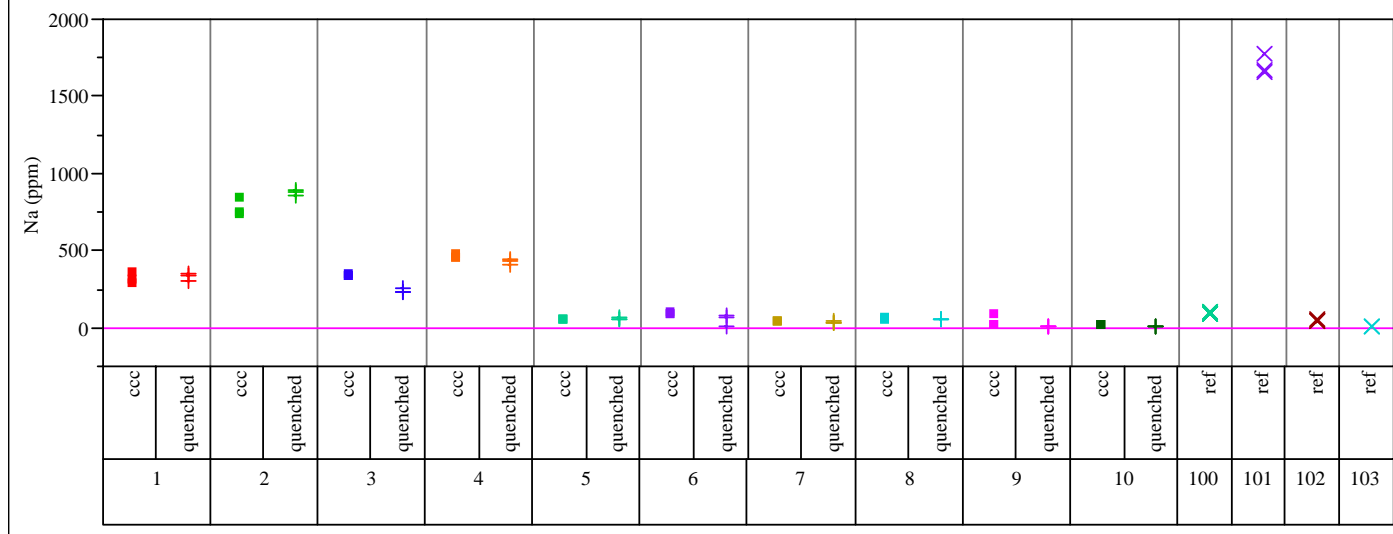

Heat Treatment within Glass \# 
Exhibit B4. Laboratory PCT Measurements by Glass Number for Study Glasses and Standards. (continued)

(100 - Solution Standard; 101 - EA; 102 - ARM; 103 - Blanks)

Variability Gage Set $=1$

Variability Chart for Si (ppm)

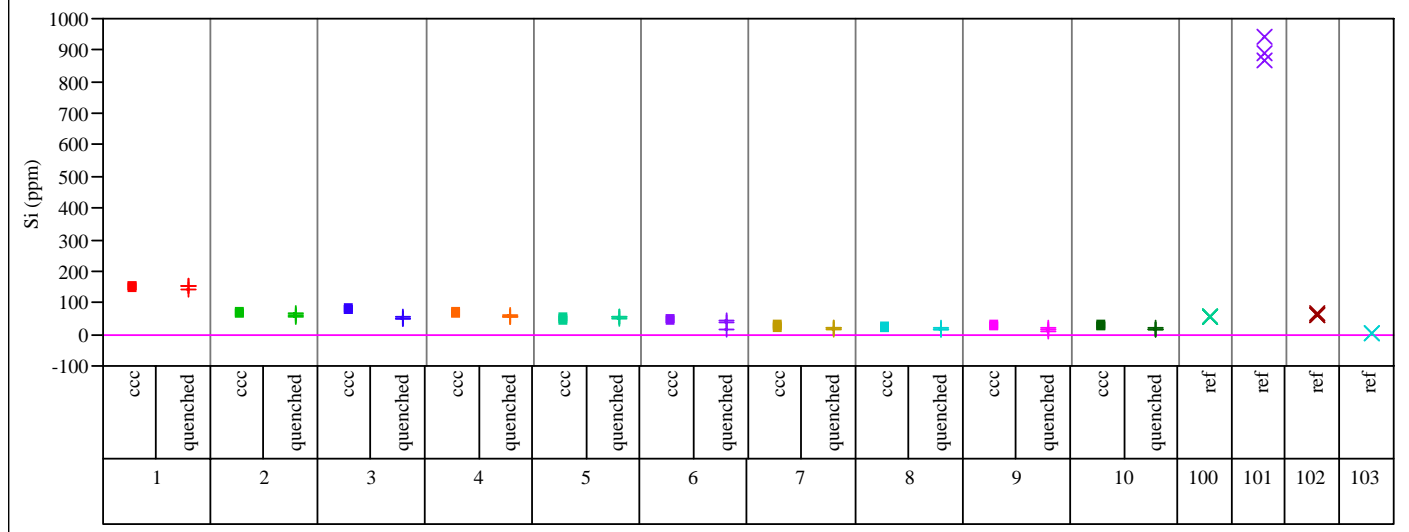

Heat Treatment within Glass \#

Variability Gage Set $=2$

Variability Chart for B (ppm)

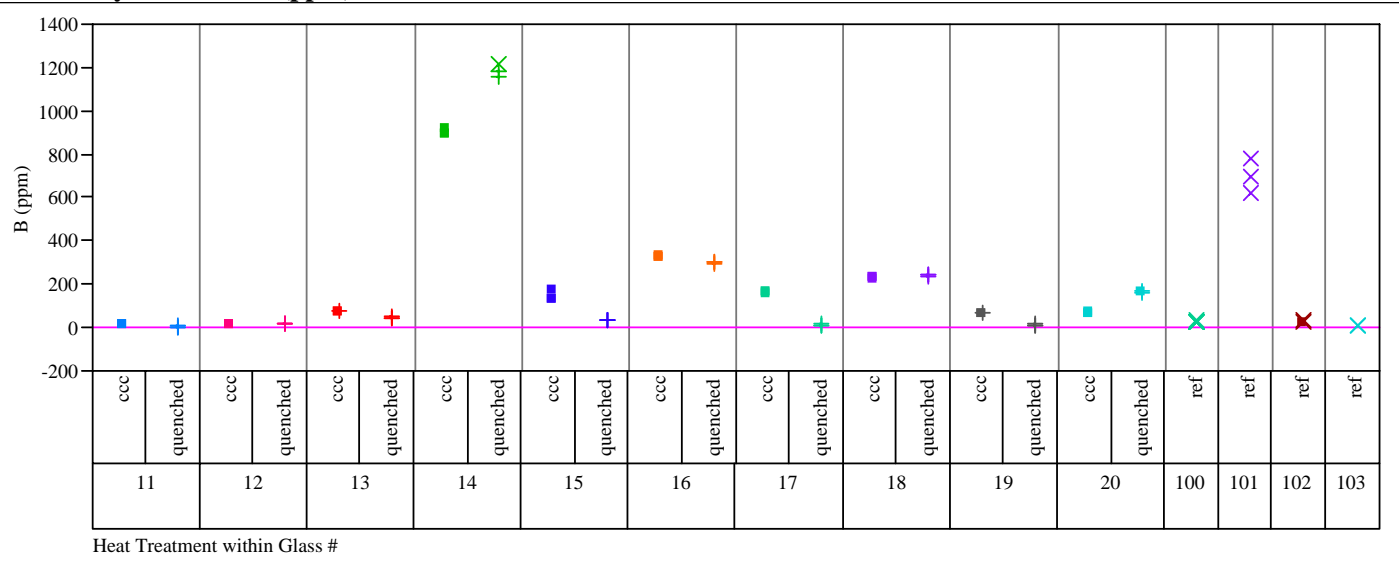

Variability Gage Set $=2$

Variability Chart for Li (ppm)

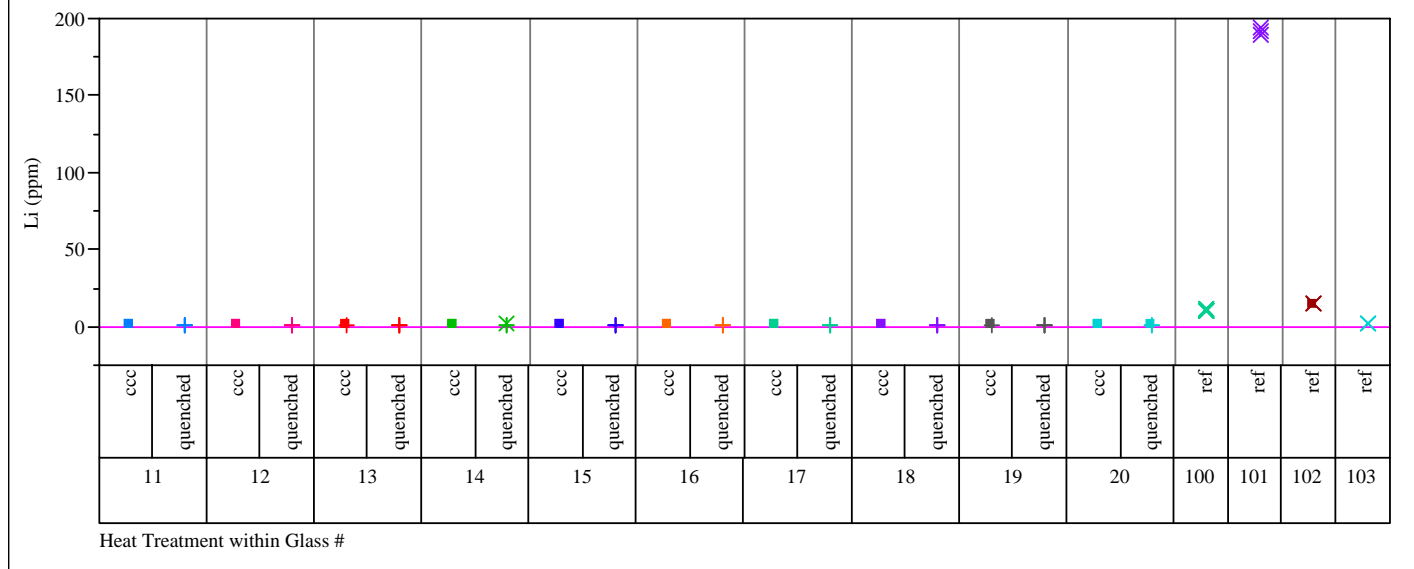


Exhibit B4. Laboratory PCT Measurements by Glass Number for Study Glasses and Standards. (continued)

(100 - Solution Standard; 101 - EA; 102 - ARM; 103 - Blanks)

Variability Gage Set $=2$

Variability Chart for Na (ppm)

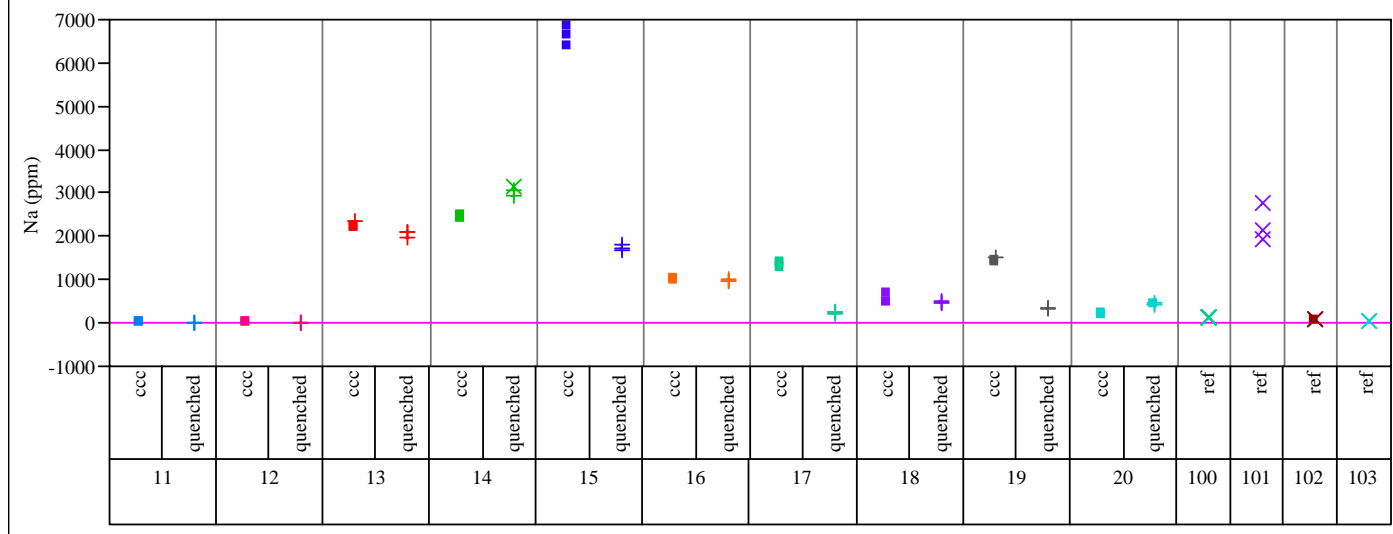

Heat Treatment within Glass \#

Variability Gage Set $=2$

Variability Chart for Si (ppm)

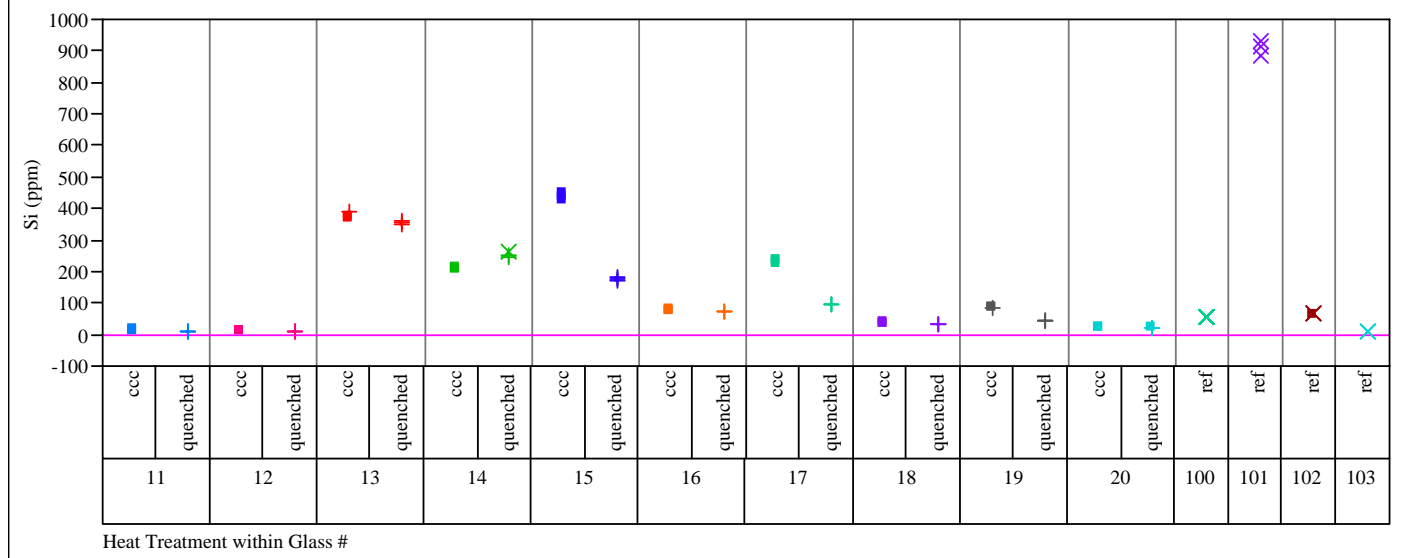

Variability Gage Set $=3$

Variability Chart for B (ppm)

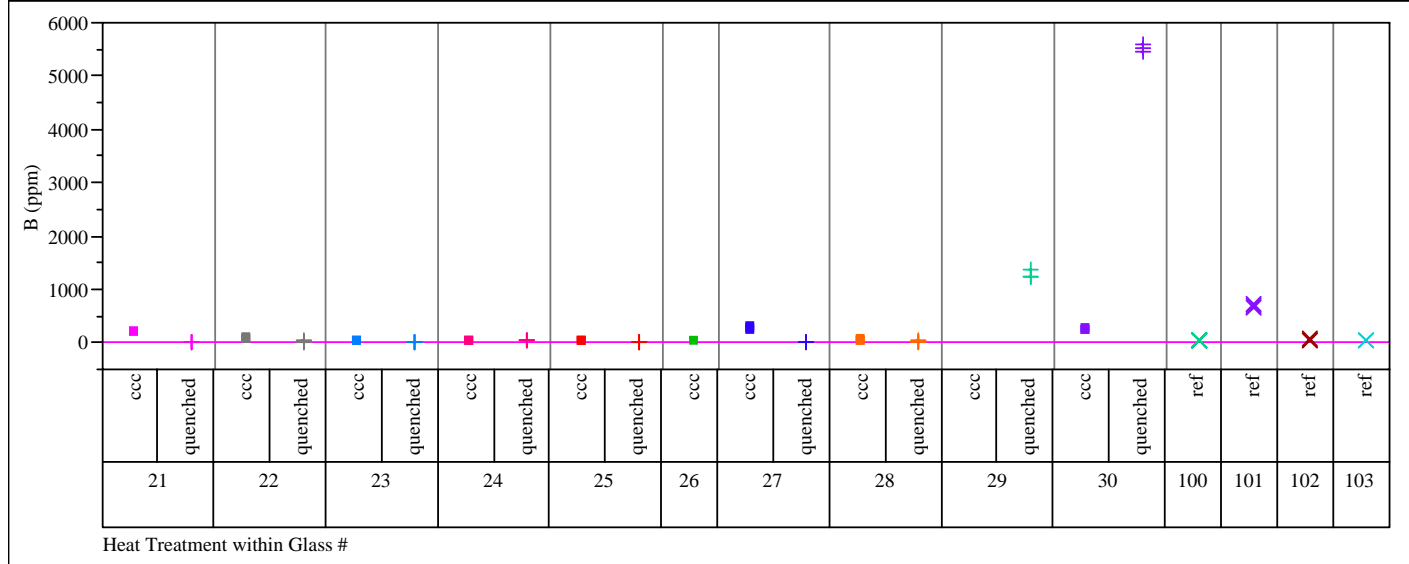


Exhibit B4. Laboratory PCT Measurements by Glass Number for Study Glasses and Standards. (continued)

(100 - Solution Standard; 101 - EA; 102 - ARM; 103 - Blanks)

Variability Gage Set $=3$

Variability Chart for Li (ppm)

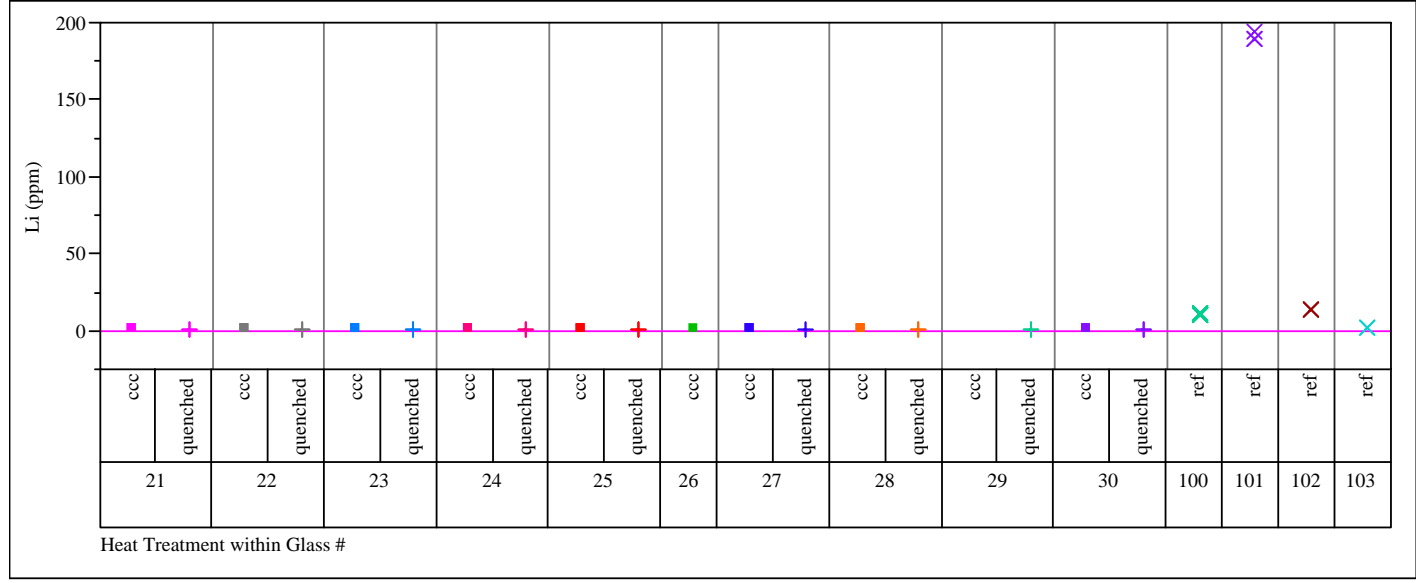

Variability Gage Set $=3$

Variability Chart for Na (ppm)

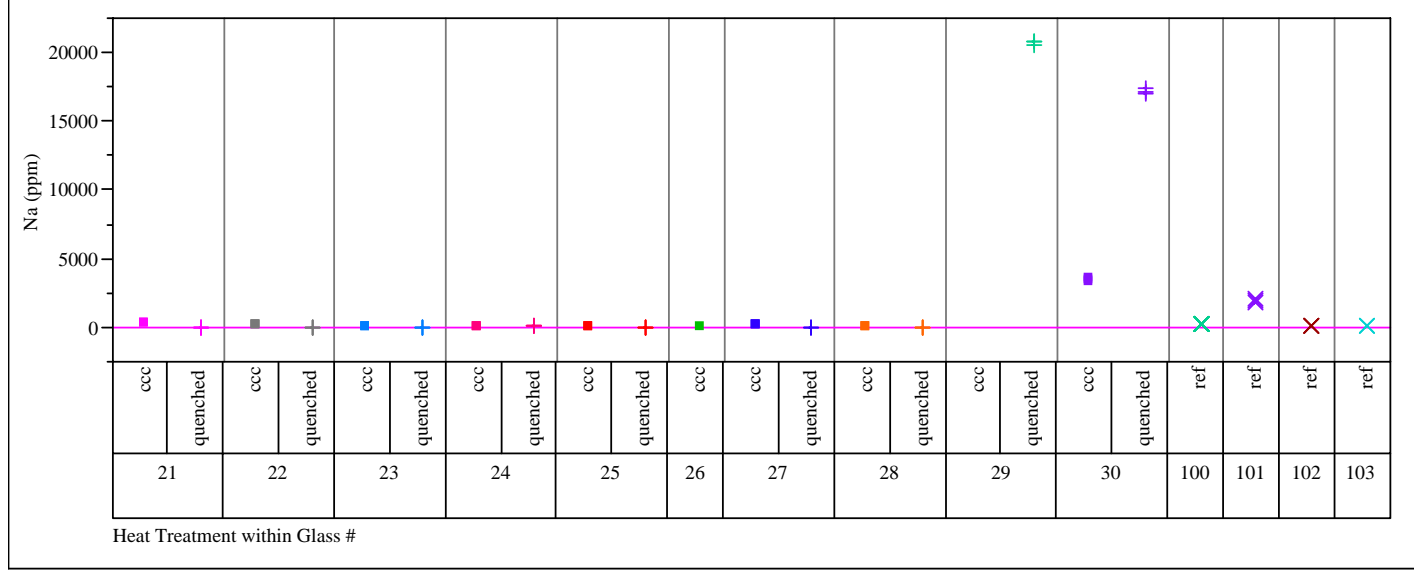

Variability Gage Set $=3$

Variability Chart for Si (ppm)

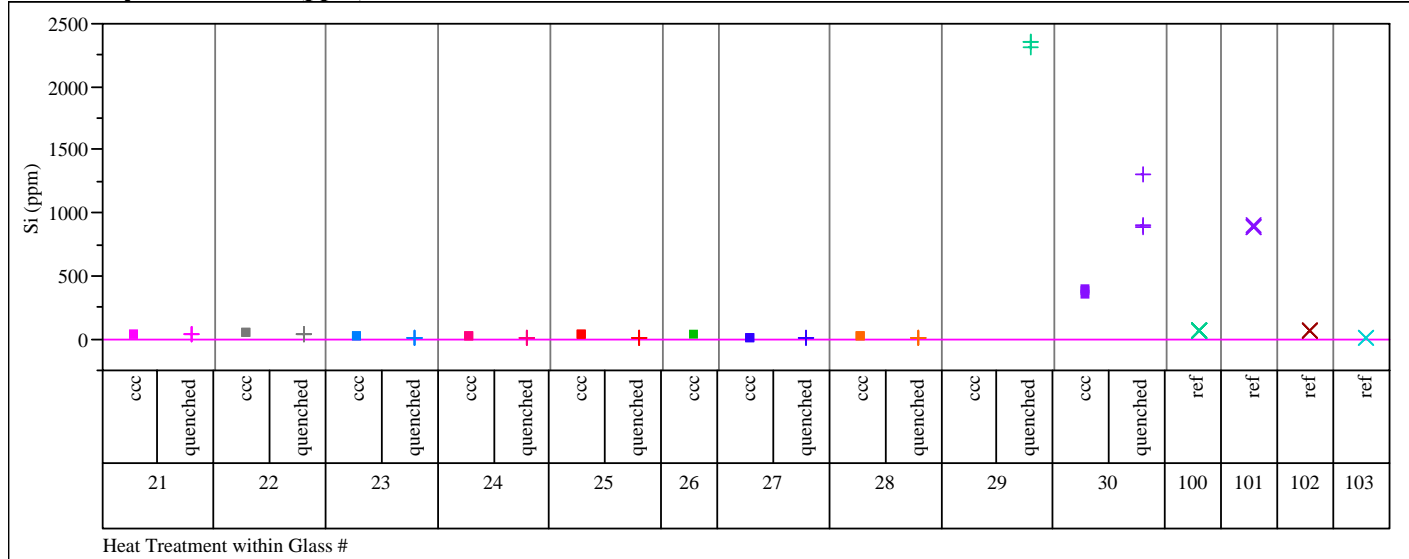


Exhibit B4. Laboratory PCT Measurements by Glass Number for Study Glasses and Standards. (continued)

(100 - Solution Standard; 101 - EA; 102 - ARM; 103 - Blanks)

Variability Gage Set $=4$

Variability Chart for B (ppm)

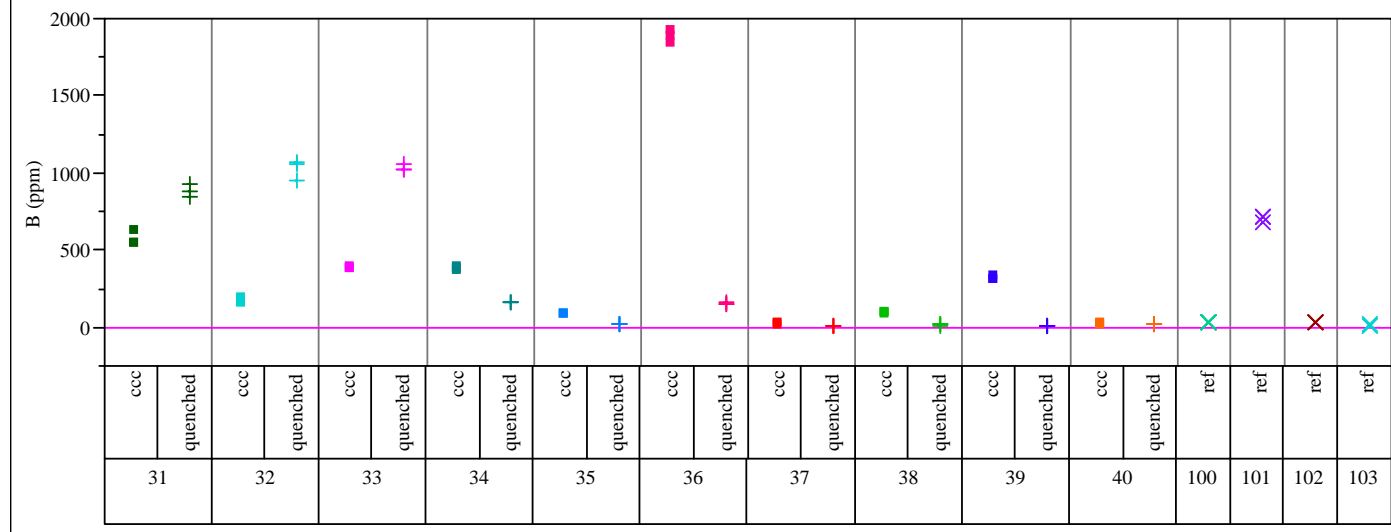

Heat Treatment within Glass \#

Variability Gage Set $=4$

Variability Chart for Li (ppm)

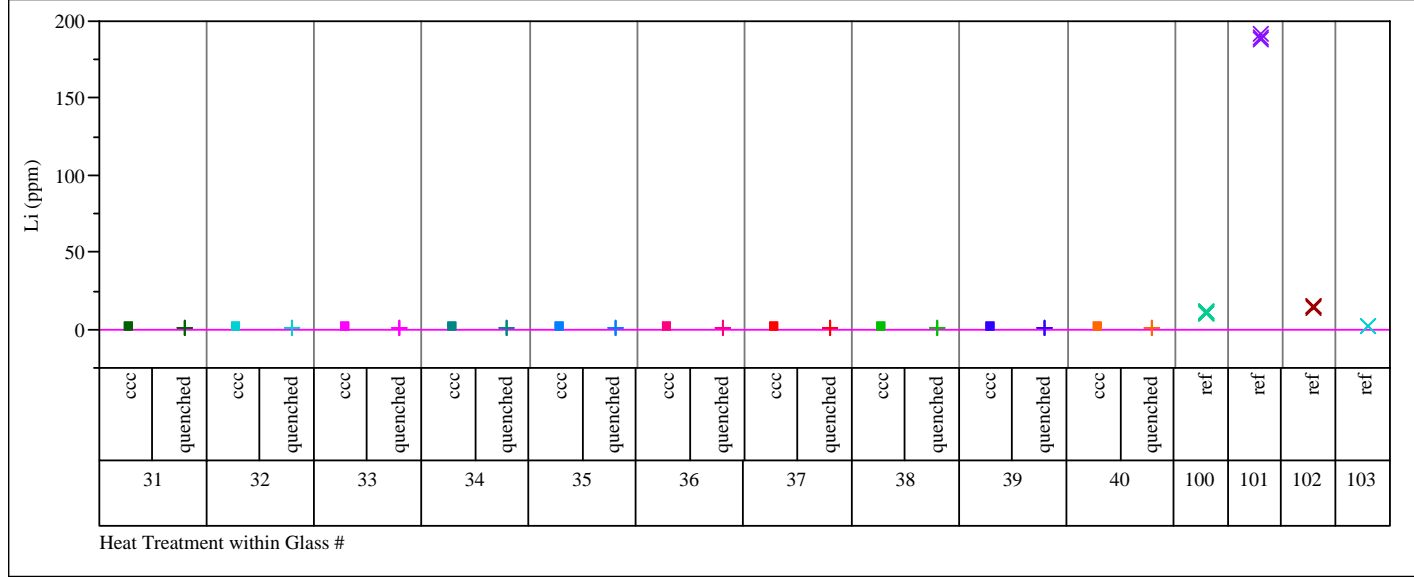

Variability Gage Set $=4$

Variability Chart for Na (ppm)

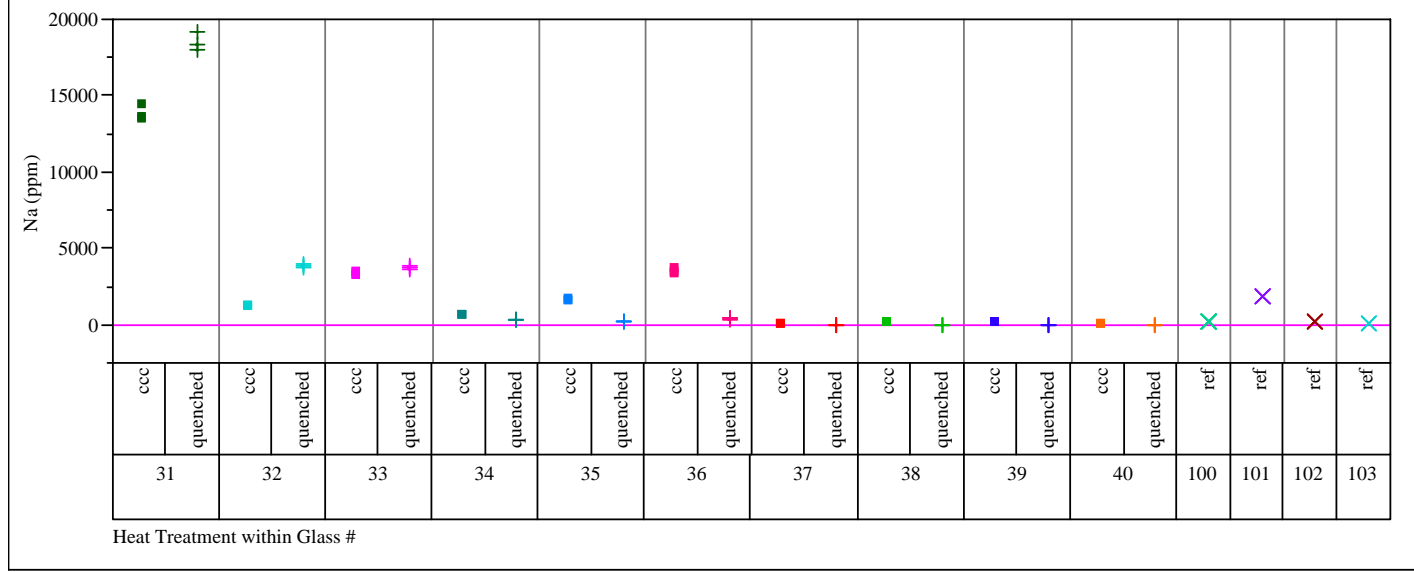


Exhibit B4. Laboratory PCT Measurements by Glass Number for Study Glasses and Standards. (continued)

(100 - Solution Standard; 101 - EA; 102 - ARM; 103 - Blanks)

Variability Gage Set $=4$

Variability Chart for Si (ppm)

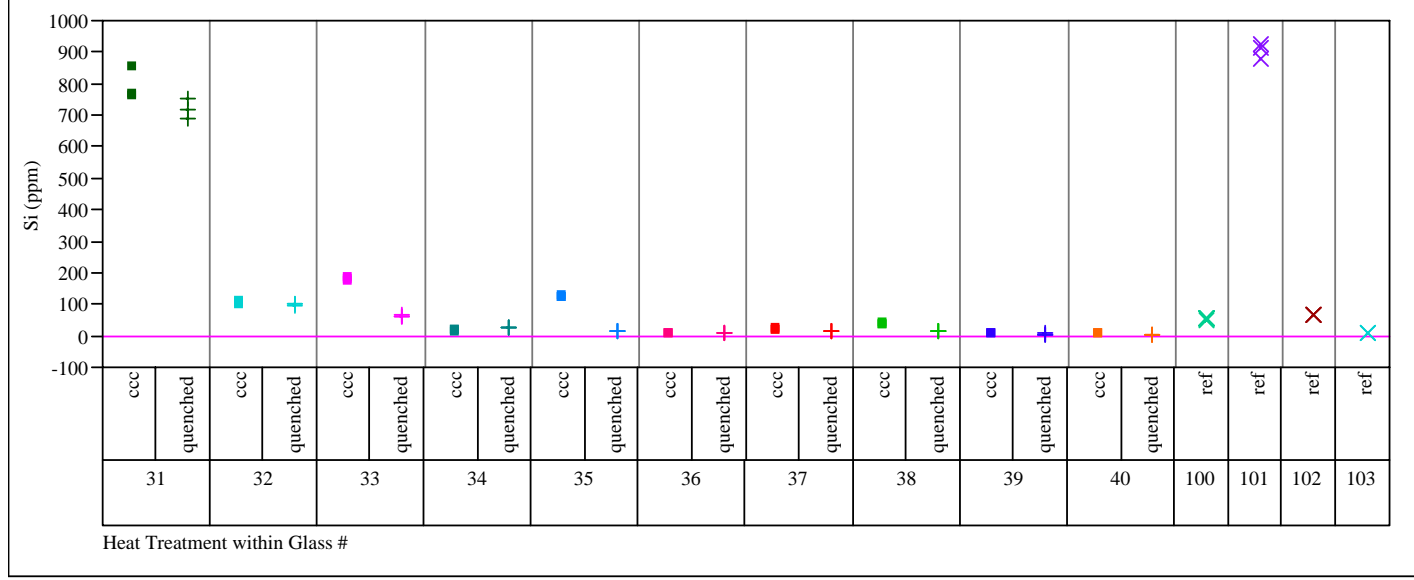


WSRC-STI-2007-00659

Revision 0

Appendix B

\section{Exhibit B5. Correlations and Scatter Plots of Normalized PCTs Over All Compositional Views and Heat Treatments.}

\section{Correlations}

$\log \mathrm{NL}[\mathrm{B}(\mathrm{g} / \mathrm{L})]$

$\log \mathrm{NL}[\mathrm{Na}(\mathrm{g} / \mathrm{L})]$

$\log \mathrm{NL}[\mathrm{Si}(\mathrm{g} / \mathrm{L})]$

$$
\begin{array}{r}
\log \mathrm{NL}[\mathrm{B}(\mathrm{g} / \mathrm{L})] \\
1.0000 \\
0.9186
\end{array}
$$

$$
\begin{array}{r}
\log \mathrm{NL}[\mathrm{Na}(\mathrm{g} / \mathrm{L})] \\
0.9186 \\
1.0000 \\
0.7640
\end{array}
$$$$
\begin{array}{r}
\log \mathrm{NL}[\mathrm{Si}(\mathrm{g} / \mathrm{L})] \\
0.5812 \\
0.7640 \\
1.0000
\end{array}
$$

6 rows not used due to missing or excluded values or frequency or weight variables missing, negative or less than one.

\section{Scatterplot Matrix}

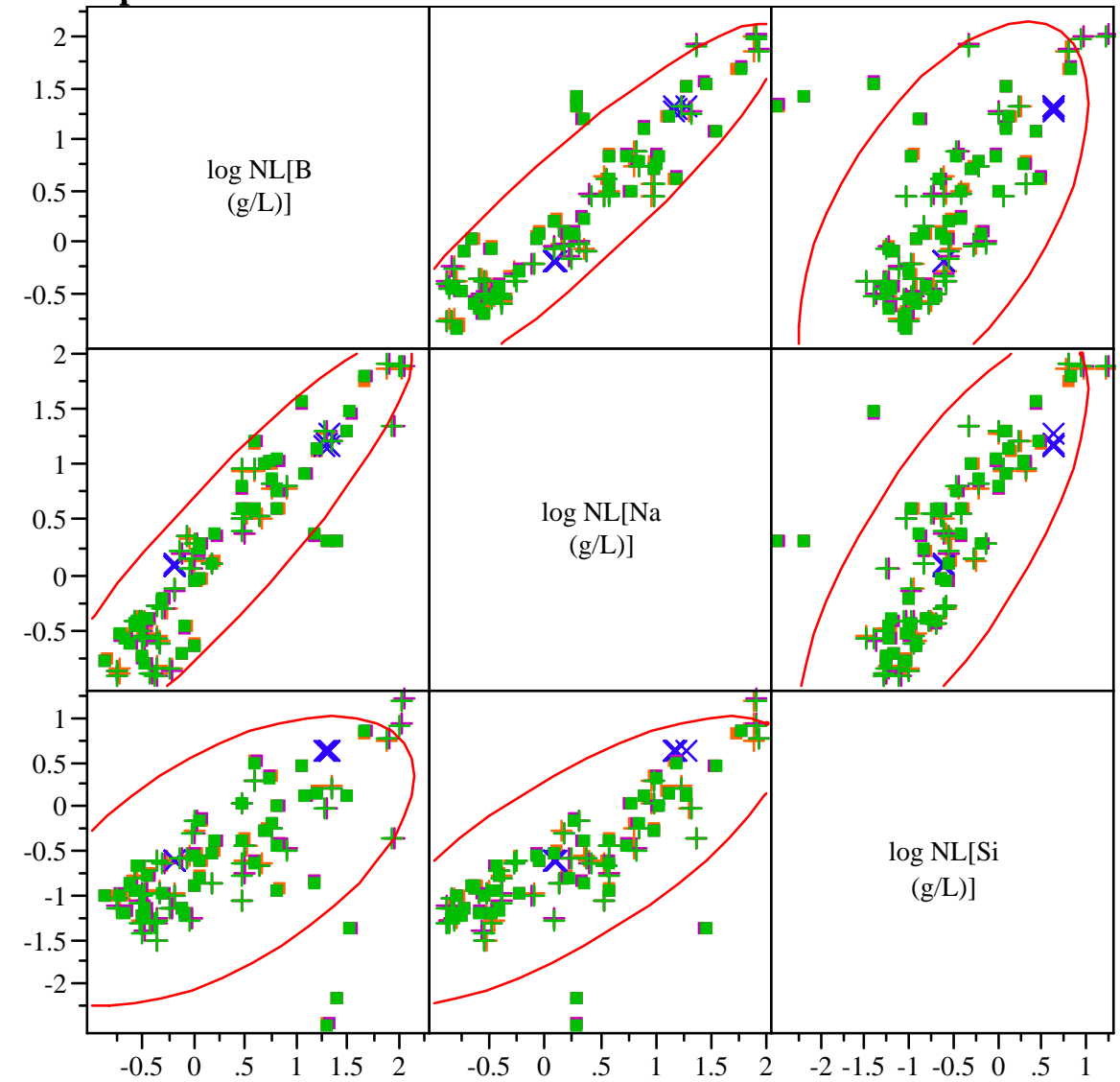




\section{Distribution:}

A.B. Barnes, 999-W

D.R. Best, 786-1A

N.E. Bibler, 773-A

D.B. Burns, 786-5A

D.A. Crowley, 999-W

B.A. Davis, 704-27S

R.E. Edwards, 773-A

T.B. Edwards, 999-W

K.M. Fox, 999-W

J.M. Gillam, 766-H

J.C. Griffin, 773-A

B.A. Hamm, 766-H

C.C. Herman, 999-W

J.F. Iaukea, 704-30S

C.M. Jantzen, 773-A

T.M. Jones, 999-W
J.E. Marra, 773-A

R.T. McNew, 704-27S

D.H. Miller, 999-W

T.A. Nance, 773-42A

J.D. Newell, 999-W

J.E. Occhipinti, 704-S

D.K. Peeler, 999-W

F.C. Raszewski, 999-W

J.W. Ray, 704-S

I.A. Reamer, 999-1W

H.B. Shah, 766-H

M.E. Smith, 999-W

M.E. Stone, 999-W

R.J. Workman, 999-1W

A.L. Youchak, 999-W 